\title{
Report of the \\ American Physical Society Study Group on Boost-Phase Intercept Systems for National Missile Defense: Scientific and Technical Issues *
}

David K. Barton

Hanover, NH

Roger Falcone

University of California, Berkeley

Daniel Kleppner, Co-Chair

Massachusetts Institute of Technology

Frederick K. Lamb, Co-Chair

University of Illinois at Urbana-Champaign

Ming K. Lau

Sandia National Laboratories

Harvey L. Lynch

Stanford Linear Accelerator Center
David Moncton ${ }^{\dagger}$

Argonne National Laboratory

David Montague

LDM Associates, Menlo Park, CA

David E. Mosher, Staff Director

RAND, Washington, DC

William Priedhorsky

Los Alamos National Laboratory

Maury Tigner

Cornell University

David R. Vaughan

RAND, Santa Monica, CA

Institutions are listed for purposes of identification only. Any opinions, findings, conclusions, or recommendations expressed in this report are those of the Study Group members, and do not necessarily represent the official views, opinions, or policies of their institutions.

Published 5 October 2004;

publisher errors corrected 13 October 2004 and 16 February 2005.

${ }^{*}$ D. K. Barton et al., Rev. Mod. Phys. 76, S1 (2004)

${ }^{\dagger}$ Now at Massachusetts Institute of Technology. 



\section{Editor's Preface}

The Report of the American Physical Society Study Group on Boost-Phase Intercept (BPI) Systems for National Missile Defense, appearing in this special issue of Reviews of Modern Physics, represents an effort of the APS that is both normal and extraordinary. The APS has periodically produced reports on matters of public interest that require technical understanding, and for which an impartial and authoritative analysis would be of particular use to the public and to policy makers. The BPI Study, as it is informally called, represents the latest in this series of technical studies.

The extraordinary part of the effort is the extended commitment of time and energy that the Study Group applied to its work. Considerable original research was required to explore some aspects of the issue. Great care was taken to make the study broadly comprehensible to an audience of non-experts.

In view of the procedures for review and approval of an APS Study, the report has not been subjected to the usual review process employed for RMP. Instead the Study was examined by a Review Committee chaired by James Langer, University of California, Santa Barbara (Chair), and including Thomas Appelquist, Yale University; Will Happer, Princeton University; Claire Max, Lawrence Livermore National Laboratory; Burton Richter, Stanford Linear Accelerator Center; and James Tsang, IBM T.J. Watson Research Center. We thank the members of the Review Committee for their helpful and timely critiques.

The APS released an earlier version of this report to the press in 2003. That version appeared on the APS web site and in a limited number of printed copies. This Reviews of Modern Physics version, supported financially by the American Physical Society, contains corrections and revisions for clarity with respect to the July 2003 version. There are no major changes.

The acknowledgments section contains thanks to many individuals and institutions that have contributed substantially to the report and I add my thanks to them here.

Martin Blume

Editor in Chief 



\section{Acknowledgments}

The final preparation of the lengthy and complex report was arduous for all. We express our deep appreciation to APS Presidents James Langer (2000), George Trilling (2001), William Brinkman (2002), Myriam Sarachik (2003), and Helen Quinn (2004) and especially to APS Executive Officer Judy Franz, who have all played essential roles in advising and encouraging the study and Study Group.

The Study Group wishes to thank the many experts who generously presented briefings and provided technical advice.

We offer additional and heartfelt gratitude to Ken Cole of the APS, who served as staff liaison to the study group; to George F. Bertsch, Editor of Reviews of Modern Physics, for his early offer to publish the report in RMP; and to Stanley Brown, APS Editorial Director, for facilitating the final publication.

For their generous assistance in the production of the Study Report, we wish to thank Carol A. Costa, Celia M. Elliott, Sue L. Herdle, Sharon Jensen, Andrew N. Kleppner, Katherine M. Lamb, Daniel S. Lewart, Mary-Pat McNally, Anita Spiess, Martin Stock, and Linda S. Thorman.

Last but hardly least we are especially grateful for the generous financial support received from the John D. and Catherine T. MacArthur Foundation, the W. Alton Jones Foundation, the Carnegie Corporation of New York, and from the American Physical Society. 



\section{Contents}

Preface $\quad$ iii

Acknowledgments $\quad v$

Executive Summary $\quad$ xxi

$\begin{array}{ll}\text { Findings } & \text { xxix }\end{array}$

1 Introduction to the Report $\quad$ S1

1.1 Boost-Phase Intercept . . . . . . . . . . . . . . . . . . . . S S1

1.2 The American Physical Society Call for the Study . . . . . . . . . . S3

1.3 Scope of the Study . . . . . . . . . . . . . . . . . . . S3

1.4 Issues Not Addressed . . . . . . . . . . . . . . . . . . . . . . S3

1.5 The Varieties of Boost-Phase Intercept Systems . . . . . . . . . . . . S4

1.6 Requirements for Success . . . . . . . . . . . . . . . . S4

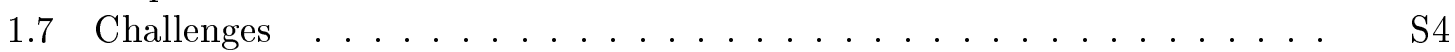

1.8 A Guide to the Report . . . . . . . . . . . . . . S5

Part A. Boost-Phase Missile Defenses $\quad$ S9

2 Overview of the Analysis of Boost-Phase Intercept Systems S11

2.1 Boost-Phase Intercept Compared to Alternative Approaches . . . . . . . S S11

2.2 Overview of the Analysis of Hit-to-Kill Systems . . . . . . . . . . . S13

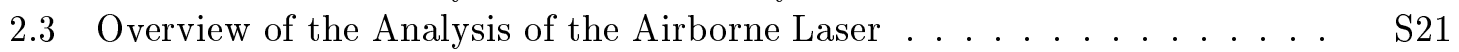

2.4 Key Issues . . . . . . . . . . . . . . . . . . . . . . . . . . . . . . . . . . . . . . .

2.5 Summary of Assumptions . . . . . . . . . . . . . . . S25

3 Ballistic Missile Threats to the United States S29

3.1 Ballistic Missile Capabilities of Selected States _. . . . . . . . . . . . . S32

3.2 Historical Patterns of ICBM Development . . . . . . . . . . . S S34

3.3 The Changing Context of Missile Development Programs . . . . . . . S35

3.4 Implications for the Study . . . . . . . . . . . . . . S39

4 Kinetic-Kill Engagement Fundamentals $\quad$ S47

4.1 ICBM Characteristics Key to This Analysis . . . . . . . . . . . . . . . . . . . . 48

4.2 Effect on Impact Range of Terminating Missile Thrust . . . . . . . . . . S S52

4.3 Surface-Based Interceptors _ . . . . . . . . . . . . . . S53

4.4 Engagement Timelines . . . . . . . . . . . . . . . S54 
4.5 Terrestrial Planar Engagements _. . . . . . . . . . . . . S55

4.6 Non-planar Engagements and Interceptor Basing Areas . . . . . . . . . S58

4.7 Space-Based Engagement of ICBMs . . . . . . . . . . . . . . S60

4.8 Summary . . . . . . . . . . . . . . . . . . . . S61

5 Defending the United States Using Surface-Based Interceptors S63

5.1 Key Assumptions for the Basing Analysis . . . . . . . . . . . . . . . S65

5.2 Analysis of Options for Basing Interceptors . . . . . . . . . . . . S70

5.3 Basing Analysis Conclusions . . . . . . . . . . . . . . . S77

5.4 Defending Against ICBMs Launched from North Korea . . . . . . . . . S79

5.5 Defending Against ICBMs Launched from Iraq . . . . . . . . . . . . . . . . . . . . . . . . . . . .

5.6 Defending Against ICBMs Launched from Iran . . . . . . . . . . . . S90

5.7 Other Engagements Considered . . . . . . . . . . . . . . . . S94

5.8 Avoiding Harming Other Countries . . . . . . . . . . . . S96

5.9 Sensitivity to Other Assumptions f . . . . . . . . . . . S S99

6 Defending the United States Using Space-Based Interceptors S103

6.1 Coverage by a Single Interceptor . . . . . . . . . . . . . . . . . . S105

6.2 Operational Altitudes for SBIs . . . . . . . . . . . . . S106

6.3 Calculating the Number of Interceptors for Earth Coverage . . . . . . . . S108

6.4 Sizing the SBI Satellite: Kill Vehicle, Interceptor, and Life Jacket . . . . . S110

6.5 Optimizing System Mass-in-Orbit . . . . . . . . . . . . . . . . . S114

6.6 The Effect of Decision and Intercept Time on System Mass _ . . . . . . S S116

6.7 The Effect of High-Acceleration Interceptors on System Mass . . . . . . . S S117

6.8 Space-Launch Requirements for SBIs . . . . . . . . . . . . . . . . . S124

6.9 The Effect of Reducing Kill-Vehicle Mass . . . . . . . . . . . . . . . $\quad$ S125

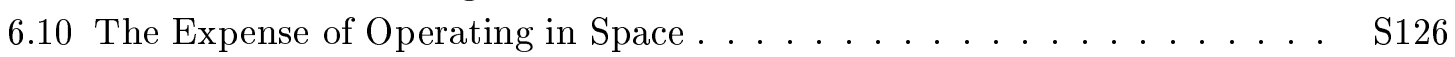

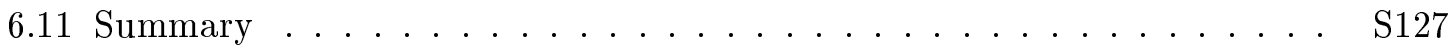

7 Airborne Laser Engagement Fundamentals $\quad$ S131

7.1 Differences Between Laser and Hit-to-Kill Intercepts . . . . . . . . . . . . S131

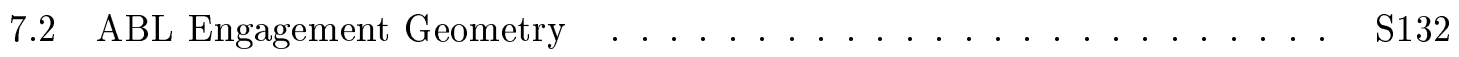

7.3 ABL Performance Parameters . . . . . . . . . . . . . . S132

8 Defending the United States Using the Airborne Laser S135

8.1 Geometrical Considerations . . . . . . . . . . . . . S136

8.2 Determining the Flying Area . . . . . . . . . . . . . . . . S137

8.3 Defending Against Missiles Launched from North Korea . . . . . . . . . . S137

8.4 Defending Against Missiles Launched from Iraq . . . . . . . . . . . . . . . . . . . . . . . . . . . . .

8.5 Defending Against Missiles Launched from Iran . . . . . . . . . . . . . . . S140

8.6 Discussion . . . . . . . . . . . . . . . . . . . . . . S142

8.7 Controlling Shortfall . . . . . . . . . . . . . . . . S143

9 Countermeasures to Boost-Phase Intercept $\mathbf{S 1 4 5}$

9.1 Countermeasures to Kinetic Kill . . . . . . . . . . . . . . . S146

9.2 Countermeasures to the ABL . . . . . . . . . . . . . S150 
Part B. Requirements to Hit an Accelerating Missile

10 Detecting and Tracking Missiles in Powered Flight $\quad$ S157

10.1 Space-Based Detection and Tracking . . . . . . . . . . . . . S159

10.2 Radar Tracking . . . . . . . . . . . . . . . . . . . S171

10.3 Sensors on the Kill Vehicle . . . . . . . . . . . . . . . . . . S185

10.4 Applications of Missile Detection and Tracking . . . . . . . . . . . S196

11 Kill Vehicles for Boost-Phase Defense $\quad$ S203

11.1 Background: The Evolution of Kill Vehicles . . . . . . . . . . . . . . . S203

11.2 Three Kill-Vehicle Configurations . . . . . . . . . . . . . . . . S204

11.3 Endoatmospheric Kill Vehicles . . . . . . . . . . . . . . . . . S208

11.4 Kill Mechanisms Other than Body-to-Body Hit . . . . . . . . . . . . . . S208

11.5 Key Requirements for Boost-Phase Kinetic Kill Above the Atmosphere - S209

11.6 Summary . . . . . . . . . . . . . . . . . . . S210

12 Hitting the Target $\quad$ S211

12.1 Overview of the Analysis . . . . . . . . . . . . . . . . S212

12.2 Guidance Laws . . . . . . . . . . . . . . . . . . . . . S216

12.3 Required Velocity Change Capability . . . . . . . . . . . . . . . . S222

12.4 The Endgame . . . . . . . . . . . . . . . . . . . S229

12.5 Summary of Requirements for Hitting the Target $\ldots \ldots \ldots$. . . . . S235

13 Disabling the Target $\quad$ S239

13.1 Damage Caused by Collision with a Booster . . . . . . . . . . . S S240

13.2 Warhead Destruction . . . . . . . . . . . . . . . . S241

13.3 Effects of Incomplete Warhead Kill on Other Layers of a Defense . . . . . S243

13.4 Enhancing the Lethality of the Kill Vehicle . . . . . . . . . . . . S243

13.5 Summary of Conclusions . . . . . . . . . . . . . . . S244

14 Size of the Kill Vehicle $\quad$ S247

14.1 Performance Requirements for Boost-Phase Kill Vehicles . . . . . . . . S248

14.2 Natural and Induced Environmental Considerations . . . . . . . . . . S248

14.3 Design Concept for a Notional Kill Vehicle . . . . . . . . . . . . . . . S249

14.4 Final Kill-Vehicle Sizing . . . . . . . . . . . . . . . . . . . . S249

Part C. Supporting Analysis for Hit-to-Kill Engagements $\quad$ S257

15 Ballistic Missile and Trajectory Models $\quad$ S259

15.1 ICBM Models . . . . . . . . . . . . . . . . . . . . S260

15.2 ICBM Trajectories . . . . . . . . . . . . . . . . . . S264

15.3 Models of Medium-Range Ballistic Missiles . . . . . . . . . . . . . S274

16 Terrestrial-Based Interceptor Models $\quad$ S277

16.1 Analytical Approach . . . . . . . . . . . . . . . . . . . . . S277

16.2 Models of Unconstrained Interceptors . . . . . . . . . . . . . . . . . S279

16.3 Models of Constrained Interceptors f . . . . . . . . . . . . . . S282

16.4 Summary of Interceptor Models . . . . . . . . . . . . . . . . . . . S284 
16.5 Interceptor Basing Options . . . . . . . . . . . . . . S285

Part D. Supporting Analysis for Airborne Laser Engagements $\quad$ S291

17 Overview of the Airborne Laser Analysis $\quad$ S293

18 Technology of the Airborne Laser $\quad$ S297

18.1 Overview of the Airborne Laser f . . . . . . . . . . . . . . . S297

18.2 Description of COIL . . . . . . . . . . . . . . . . . . . S299

18.3 Chronology of the Airborne Laser . . . . . . . . . . . . . . . . . . S300

18.4 Airborne Laser Performance . . . . . . . . . . . . . . . . . . S301

19 Airborne Laser Beam Propagation S303

19.1 Vacuum Propagation . . . . . . . . . . . . . . . . . S303

19.2 Atmospheric Effects . . . . . . . . . . . . . . . . . . S304

19.3 Characterization of Turbulence . . . . . . . . . . . . . S305

19.4 Adaptive Optics . . . . . . . . . . . . . . . . . . S306

19.5 Benchmarking Adaptive Optics Hardware and Algorithms . . . . . . . S307

19.6 Other Issues . . . . . . . . . . . . . . . . . . . . . . S309

19.7 Engagement Envelope . . . . . . . . . . . . . . . . S309

20 Disabling Missiles Using the Airborne Laser $\quad$ S313

20.1 Effects of Laser Beams on Boosters and Warheads . . . . . . . . . . . . S314

20.2 Kill Assessment . . . . . . . . . . . . . . . . . . . S316

21 Airborne Laser Engagements $\quad$ S319

21.1 Engagement Steps . . . . . . . . . . . . . . . . . S320

21.2 Parameter Choices and Constraints . . . . . . . . . . . . S322

21.3 Optical Engagement . . . . . . . . . . . . . . . . . . . . S324

21.4 Limiting Cases . . . . . . . . . . . . . . . . . . . . . . S326

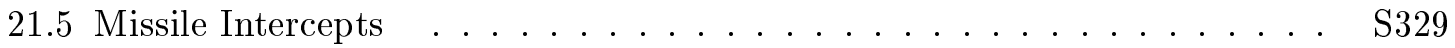

22 Deployment Considerations for the Airborne Laser S339

22.1 Parameters of Operations . . . . . . . . . . . . . S339

22.2 Operating Altitude and Cloud Cover . . . . . . . . . . . . S340

22.3 ABL Defense Against Short-Range Missiles . . . . . . . . . . . . . . S340 
A Ballistic Missile Types and Phases of Flight S343

A.1 Classes of Ballistic Missiles . . . . . . . . . . . . . . . . . S343

A.2 Phases of Ballistic Missile Flight . . . . . . . . . . . . . S345

$\begin{array}{lr}\text { B Calculating Missile Trajectories } & \mathbf{S 3 4 9}\end{array}$

B.1 Forces . . . . . . . . . . . . . . . . . . . . S S350

B.2 Computing Trajectories . . . . . . . . . . . . . . . S352

B.3 Simple Scaling Relations . . . . . . . . . . . . . . . . . . . S354

B.4 Discussion . . . . . . . . . . . . . . . . . S355

C Boost-Phase Tracking Simulations $\quad$ S359

C.1 Engagement of ICBM S1 and the 10-km/s Interceptor I-5 . . . . . . . S S360

C.2 Engagement of ICBM L and the $5-\mathrm{km} / \mathrm{s}$ Interceptor I-5 . . . . . . . . . S381

D Beam Propagation and Corrections $\quad$ S391

D.1 Atmospheric Effects . . . . . . . . . . . . . . . . . . . . . . S392

D.2 Characterization of $C_{n}^{2} \ldots \ldots \ldots \ldots \ldots \ldots \ldots \ldots \ldots \ldots$ S394

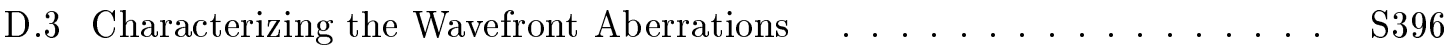

D.4 Anisoplanatic Effects . . . . . . . . . . . . . . . . . S397

D.5 Strehl Ratio Details . . . . . . . . . . . . . . . . . . S405

D.6 Minor Issues . . . . . . . . . . . . . . . . . . . . . . . . . . . . . . . . . . . S S408

$\begin{array}{ll}\text { Glossaries } & \text { S415 }\end{array}$

Acronyms . . . . . . . . . . . . . . . . . . . . . S415

Technical Terms . . . . . . . . . . . . . . . . S420 



\section{List of Tables}

$3.1 \quad$ Ballistic Missiles of Selected States of Concern . . . . . . . . . . . S S32

3.2 Characteristics of Early ICBMs . . . . . . . . . . . . . . S35

4.1 Model Interceptors . . . . . . . . . . . . . . . . . . S53

5.1 Model ICBMs Considered . . . . . . . . . . . . . . . . . S65

5.2 Great-Circle Ranges and Azimuths to Various U.S. Cities . . . . . . . S68

5.3 Interceptors Considered . . . . . . . . . . . . . . . . S69

5.4 Range and Velocity for Early Thrust Termination . . . . . . . . . . . S71

5.5 Available Decision Times for Defending All 50 States . . . . . . . . S S78

5.6 Intercept Times and ICBM Ground Ranges from North Korea . . . . . . $\quad$ S81

5.7 Ground Ranges of Interceptors Fired Against ICBMs Launched from North Korea to the United States . . . . . . . . . . . . . . . . . S81

5.8 Ground Ranges of Interceptors Fired Against ICBMs Launched from Iraq or Iran to the United States . . . . . . . . . . . . . . . . . . . S88

5.9 Model Medium-Range Ballistic Missiles . . . . . . . . . . . . . . . S95

5.10 Ground Speed and Increment in Range . . . . . . . . . . . . . . . S97

6.1 Components of an SBI Life Jacket . . . . . . . . . . . . . . . . . S113

6.2 Number of Interceptors as a Function of $v_{\text {flyout }}$, Decision Time, and Geography against Solid-Propellant ICBM S1 . . . . . . . . . . . S116

6.3 Mass-in-Orbit as a Function of $v_{\text {flyout }}$, Decision Time, and Geography against Solid-Propellant ICBM S1 . . . . . . . . . . . . . . . . S117

6.4 Number of Interceptors as a Function of $v_{\text {flyout }}$, Decision Time, and Geography against Liquid-Propellant ICBM L . . . . . . . . . . . . . . . S118

6.5 Mass-in-Orbit as a Function of $v_{\text {flyout }}$, Decision Time, and Geography against Liquid-Propellant ICBM L . . . . . . . . . . . . . . . . . . . . . . S118

6.6 Effect of Possible Mass Reduction Strategies for Kill Vehicles _ . . . . . S126

10.1 ICBM Trajectory Parameters for Land- and Ship-Based Radars . . . . . S174

10.2 Theater High-Altitude Area Defense (THAAD) Radar Characteristics . S175

10.3 THAAD Performance Requirements for ICBM Searches . . . . . . . . S175

10.4 AN/SPY-1B Radar Characteristics . . . . . . . . . . . . . . . S177

10.5 AN/SPY-1B Performance Requirements for ICBM Searches . . . . . . . S177

10.6 AN/APY-2 Radar Characteristics . . . . . . . . . . . . . . . . S178

10.7 AN/APY-2 Performance Requirements for ICBM Searches . . . . . . . . . S178

10.8 AN/APY-2 Performance Requirements for Searches at $300 \mathrm{~km}$. . . . . . S S179

10.9 A Phased-Array AWACS Radar . . . . . . . . . . . . . . . . S179

10.10 Phased-Array AWACS Performance Requirements for ICBM Searches . . S180 
10.11 Characteristics of Space Lasers _. . . . . . . . . . . . . . . . S191

10.12 Return Signal from a Notional LIDAR . . . . . . . . . . . . . . S192

12.1 ICBM Engagements Simulated in the Study . . . . . . . . . . . S S215

12.2 Uncertainties in Estimates of the State of the Target ICBM . . . . . . S225

14.1 Properties of a surface-based kill vehicle . . . . . . . . . . . . . S251

14.2 Properties of terrestrial- and space-based kill vehicles . . . . . . . . . S253

14.3 Surface-based kill vehicles . . . . . . . . . . . . . . . . S S253

15.1 Model ICBMs Considered in the Study . . . . . . . . . . . . . . . . . S261

15.2 Single-Stage Model MRBMs Considered in the Study . . . . . . . . . . . S261

15.3 Characteristics of Liquid-Propellant ICBM L . . . . . . . . . . . . . S262

15.4 Characteristics of Solid-Propellant ICBM S1 . . . . . . . . . . . . . . . . S262

15.5 Characteristics of Solid-Propellant ICBM S2 . . . . . . . . . . . . . . S263

15.6 Characteristics of 1300-km M1 . . . . . . . . . . . S275

15.7 Characteristics of $600-\mathrm{km} \mathrm{M} 2 \ldots \ldots \ldots \ldots$ S276

16.1 Characteristics of the $6.7-\mathrm{km} / \mathrm{s}$ Interceptor I-3 . . . . . . . . . . S S 279

16.2 Characteristics of the $6.5-\mathrm{km} / \mathrm{s}$ Interceptor I-4 . . . . . . . . . . . S281

16.3 Characteristics of the $10-\mathrm{km} / \mathrm{s}$ Interceptor I-5 . . . . . . . . . . . . S S282

16.4 Characteristics of the VLS $5-\mathrm{km} / \mathrm{s}$ Interceptor I-2 . . . . . . . . . . S S284

16.5 Interceptor Models Used in the Study . . . . . . . . . . . . . . . . S284

20.1 Summary of Missile Properties . . . . . . . . . . . . . . . . . . S313

20.2 Some Material Properties of Threat Missiles . . . . . . . . . . . . S315

C.1 Summary of state-estimate uncertainties for the different sensors used during the target-tracking problem ................. . . S372

D.1 Zernike Functions . . . . . . . . . . . . . . . . . . S398 


\section{List of Figures}

2.1 Great circles from North Korea and Iraq or Iran to the United States . . S17

2.2 Timelines for engaging liquid- and solid-propellant ICBMs launched from Iran against cities on the East Coast of the United States . . . . . . . S S23

4.1 Liquid-propellant ICBM model L maximum-range boost-phase trajectory in the altitude-range plane . . . . . . . . . . . . . . .

4.2 Solid-propellant ICBM model S1 maximum-range and lofted boost-phase trajectories in the altitude-range plane . . . . . . . . . . . . S50

4.3 Solid-propellant ICBM model S2 maximum-range and lofted boost-phase trajectories in the altitude-range plane . . . . . . . . . . S50

4.4 Examples of possible planar trajectories for solid-propellant ICBM model S1

4.5 Payload range of model ICBMs as a function of the number of seconds before burnout that their thrust is terminated . . . . . . . . . . S52

4.6 Interceptor models used in the Study . . . . . . . . . . . . . . S54

4.7 Flyout fan of trajectories and the acceleration profile for the $6.5-\mathrm{km} / \mathrm{s}$ interceptor I-4 . . . . . . . . . . . . . . . . . . . S55

4.8 Geometry of a planar engagement . . . . . . . . . . . S56

4.9 Engagement diagrams for planar engagements, $1000-\mathrm{km}$ standoff distance $\quad$ S57

4.10 Geometry of a non-planar engagement . . . . . . . . . . . S59

4.11 Projection of the trajectories of a non-planar engagement onto the Earth's

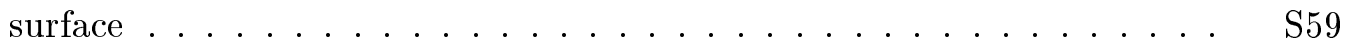

4.12 Coverage provided by a single space-based interceptor . . . . . . . S60

$5.1 \quad$ Great circles from North Korea and Iraq or Iran to U.S. cities . . . . . S67

5.2 Payload range of model ICBMs as a function of the number of seconds before burnout that their thrust is terminated . . . . . . . . . S71

5.3 Ground tracks of illustrative trajectories of liquid- and solid-propellant ICBM models from North Korea to U.S. cities . . . . . . . . . . . . . . S73

5.4 Planar missile and interceptor trajectories and feasible interceptor basing area for an idealized boost-phase engagement . . . . . . . . . . S74

5.5 Ground range vs. time for the liquid and solid-propellant ICBM models and candidate interceptors at a standoff distance of $1000 \mathrm{~km} \ldots \ldots . .$.

5.6 The effects of uncertainties in azimuth, launch location, and missile trajectory on basing areas . . . . . . . . . . . . . S76

5.7 Map of North Korea and adjacent countries and azimuths for missiles launched from North Korea to the United States . . . . . . . . . . . . . . . . . S79 
5.8 Timelines for engaging liquid-propellant ICBM model L and solid-propellant ICBM model S1 launched from North Korea toward the central United

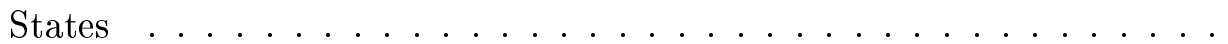

5.9 Interceptor basing areas for defending the contiguous United States against the liquid-propellant missile ICBM model L launched from North Korea

5.10 Interceptor basing areas for defending the contiguous United States against the solid-propellant missile ICBM model S1 launched from North Korea

5.11 Basing areas for defending Hawaii from the solid-propellant missile model S1 launched by North Korea . . . . . . . . . . . . . . . . . S85

5.12 Map showing Iraq and Iran and nearby countries and bodies of water . . S86

5.13 Ground tracks of the trajectories of the liquid- and solid-propellant ICBM models from Iraq to U.S. cities . . . . . . . . . . . . . . . S87

5.14 Interceptor basing areas for defending the contiguous United States against ICBMs launched from Iraq . . . . . . . . . . . . . . . . . . .

5.15 Defending all of the United States against missiles launched from Iraq, using interceptors based at two sites . . . . . . . . . . . . .

5.16 Interceptor basing areas for defending the contiguous United States against ICBMs launched from Iran . . . . . . . . . . . . . . . . .

5.17 Defending all of the United States against missiles launched from Iran, using interceptors based at two sites . . . . . . . . . . . . .

5.18 Illustrative planar engagements of medium-range ballistic missiles by interceptor I-1 shown in the altitude-range plane . . . . . . . . . . . .

6.1 The coverage of a single space-based interceptor . . . . . . . . . . S106

6.2 Flyout range $r_{\text {flyout }}$ as a function of time for a range of accelerations and burnout velocities . . . . . . . . . . . . . . . S107

6.3 Range, mass, and required number of hypothesized space-based interceptors as a function of flyout velocity for a kill vehicle with a total divert velocity

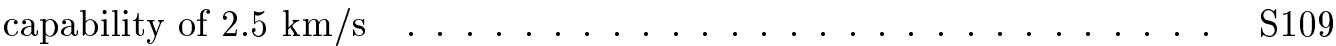

6.4 The effect of flyout and divert velocities on total mass of a single SBI with a two-stage booster . . . . . . . . . . . . . . . S113

6.5 Total mass of an orbiting constellation for the baseline case, including lifejackets, for one- and two-stage SBIs against solid- and liquid-propellant missiles . . . . . . . . . . . . . . . . . . . . S114

6.6 Total mass-in-orbit as a function of divert velocity and flyout velocity for a two-stage interceptor having an acceleration of $10 \mathrm{~g} \ldots \ldots$. . . . . S115

6.7 Variation of the number of interceptors, mass of an individual SBI, and onorbit mass as a function of SBI burn time, including the estimated "divert penalty" . . . . . . . . . . . . . . . . . S120

6.8 Mass-on-orbit for a family of one- and two-stage interceptors carrying the baseline kill vehicle as a function of SBI burn time, including the estimated "divert penalty." . . . . . . . . . . . . . . .

6.9 The variation of SBI mass as a function of flyout velocity for different accelerations of an SBI with a 136-kg kill vehicle . . . . . . . . . . . . S122

6.10 Variation of the required number of interceptors, the individual mass of an interceptor, and the mass-in-orbit for a one-stage, $3.5-\mathrm{km} / \mathrm{s}$ interceptor as a function of SBI burn time, including the estimated "acceleration penalty" 
6.11 Mass-on-orbit for a family of one- and two-stage interceptors carrying the baseline kill vehicle as a function of SBI burn time, including the estimated

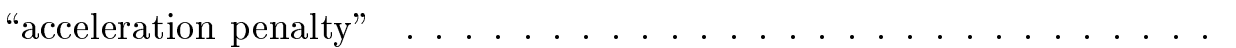

8.1 Geometry of an ABL intercept . . . . . . . . . . . . . . S136

8.2 Illustrative flying areas of the ABL against missiles launched from North Korea to the United States . . . . . . . . . . . . . . . . . S139

8.3 Illustrative flying areas of the ABL against liquid-propellant ICBMs launched from Iraq and Iran to the United States . . . . . . . . . . . . . . . . . . S141

8.4 Illustrative flying areas of the ABL against a solid-propellant missile (ICBM S2) launched from Iraq and Iran to the United States . . . . . . . . . . . S142

9.1 Illustration of concept of ejected decoys and jammers . . . . . . . . . . S148

9.2 Illustration of concept of multiple third stages . . . . . . . . . . . . . S149

10.1 Illustrative spectra of the IR emission from the exhaust plume of a rocket burning hydrazine fuels in a ground test . . . . . . . . . . . . . S160

10.2 Characteristic variation with altitude of the luminosity per unit solid angle of rocket exhaust plumes . . . . . . . . . . . . . . . . S161

10.3 Probability that the optical path length of cloud at mid-latitudes exceeds a given value as a function of cloud optical thickness . . . . . . . . . . S163

10.4 Transmission of Earth's atmosphere from ground to space and from 10-km altitude to space as a function of wavelength in the 1-10 $\mu \mathrm{m}$ region for a typical mid-latitude atmosphere . . . . . . . . . . . . . . S164

10.5 Comparison of the IR spectral luminosity of a representative missile plume at an altitude of $20 \mathrm{~km}$ with the equivalent intensity of Earth's atmosphere, clouds, and terrain, as viewed from space . . . . . . . . . . S165

10.6 Single-pixel false alarm probability as a function of threshold luminosity for the IR scanner considered, when viewing the stressing background scene

10.7 Outlines of the two ICBMs used to illustrate missile radar cross sections and analyze radar performance requirements . . . . . . . . . . . S173

10.8 Estimated average S-band radar cross sections of the liquid- and solidpropellant ICBM models as functions of aspect angle from the nose . . . S174

10.9 THAAD tracking errors vs. time for the liquid- and solid-propellant ICBM models . . . . . . . . . . . . . . . . . . . S183

10.10 Aegis tracking errors vs. time for the liquid- and solid-propellant ICBM models . . . . . . . . . . . . . . . . . S183

10.11 AWACS phased array radar tracking errors vs. time for the liquid- and solid-propellant ICBM models . . . . . . . . . . . . . . . . . . . S184

10.12 Contours of constant SWIR spectral radiance in the exhaust plume of a Titan IIIC at an altitude of $110 \mathrm{~km}$. . . . . . . . . . . . . . S187

10.13 The geometry involved in detecting and tracking a rocket body using a LIDAR system on the kill vehicle . . . . . . . . . . . . . . . . S191

10.14 The plume from a liquid-propellant Delta launcher . . . . . . . . . . . S194

10.15 The plume from a solid-propellant Pegasus rocket, just after first-stage separation . . . . . . . . . . . . . . . . . S195

11.1 Line-of-sight behavior in intercepts: illustration of the principle of proportional navigation . . . . . . . . . . . . . . S205 
11.2 Homing Overlay Experiment . . . . . . . . . . . . . . . . S206

11.3 Kill-vehicle designed by Raytheon for use in a midcourse intercept system and interceptor rocket designed by Boeing . . . . . . . . . . . . S206

11.4 Artist's conception of a lethality enhancement device on the ERIS experiment . . . . . . . . . . . . . . . . . S207

12.1 Block diagram of an idealized proportional navigation scheme for a twodimensional engagement, formulated in terms of the zero effort miss (ZEM)

12.2 The time evolution of an engagement in which a kill vehicle using proportional navigation attempts to hit a target with a time-varying acceleration

12.3 Block diagram of an idealized augmented proportional navigation scheme

12.4 Block diagram of the guidance scheme used in simulating the engagements

12.5 Illustration of estimates of target acceleration using polynomial filters, with and without sensor noise . . . . . . . . . . . . . . S225

12.6 Overview of the engagement of ICBM model S2 by interceptor model I-4 in the range-altitude plane . . . . . . . . . . . . . . S226

12.7 Vertical component of the kill vehicle's acceleration and cumulative velocity change with and without sensor noise . . . . . . . . . . . S227

12.8 Total kill-vehicle velocity change required to reach the endgame as a function of the uncertainty in the measurements of the target's position . . . S228

12.9 Guidance signal and kill-vehicle response to an 8-g lunge maneuver by the target missile $0.9 \mathrm{~s}$ before the predicted intercept time, in the absence of sensor noise . . . . . . . . . . . . . . . . .

12.10 The miss distance produced by the 8 -g lunge maneuver by the target missile as a function of the time that the missile executes the maneuver, neglecting sensor noise . . . . . . . . . . . . . . . .

12.11 Simulation of the endgame for an 8-g lunge by the target missile one second before the predicted intercept time with sensor noise included . . . . . . S234

12.12 Target acceleration and response of the kill vehicle to a $1-\mathrm{Hz}, 2-\mathrm{g}$ target jinking maneuver with and without sensor noise . . . . . . . . S235

13.1 Artist's conception of a lethality enhancement device on the ERIS experiment . . . . . . . . . . . . . . . . . .

14.1 Assumed KV structure . . . . . . . . . . . . . . . . S250

15.1 Maximum-range boost-phase trajectory and acceleration profiles of liquidpropellant ICBM model L in the altitude-range plane . . . . . . . . . . S267

15.2 Maximum-range and lofted boost-phase trajectories and acceleration profiles of solid-propellant ICBM model S1 in the altitude-range plane . . . S268

15.3 Maximum-range and lofted boost-phase trajectories and acceleration profiles of solid-propellant ICBM model S2 plotted in the altitude-range plane S268

15.4 Comparison of the missile's altitude as a function of its range for the maximum-range boost-phase trajectories of the three primary ICBM mod-

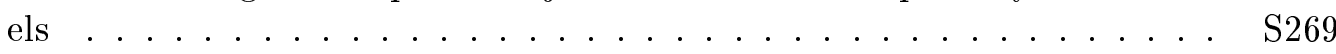

15.5 Comparison of time after launch as a function of range for the maximumrange boost-phase trajectories of the three primary ICBM models . . . . S270

15.6 Examples of the variety of planar trajectories that are possible for solidpropellant ICBM model S1 . . . . . . . . . . . . . . . . . . . S271 
15.7 Illustrative GEMS maneuver for solid-propellant ICBM S2 third stage . S272

15.8 Components of the acceleration of the third stage of ICBM model S2 during an illustrative GEMS maneuver . . . . . . . . . . . . . . . . S272

15.9 Maximum-range trajectories of the two medium-range missile models . . S275

16.1 A fan of planar flyout trajectories computed for the notional $6.7-\mathrm{km} / \mathrm{s}$ interceptor I-3 . . . . . . . . . . . . . . . . . . S278

16.2 Notional profiles of the interceptor models used in the Study . . . . . . S280

16.3 A flyout fan of planar trajectories and the acceleration profile computed for the $6.5-\mathrm{km} / \mathrm{s}$ interceptor $\mathrm{I}-4 \ldots \ldots \ldots$. . . . . . . . . . . . . 281

16.4 A flyout fan of planar trajectories and the acceleration profile computed for the $10-\mathrm{km} / \mathrm{s}$ interceptor I-5 . . . . . . . . . . . . . . S283

16.5 A flyout fan of planar trajectories and the acceleration profile computed for the $5-\mathrm{km} / \mathrm{s}$ interceptor $\mathrm{I}-2 \ldots \ldots \ldots$. . . . . . . . . . . . . S285

16.6 Comparison of model interceptors with various existing missiles . . . . S286

18.1 Schematic drawing of ABL engagement, showing the four laser beams onto the target . . . . . . . . . . . . . . . . . S298

18.2 Elements of the High Energy Laser . . . . . . . . . . . . . . . S301

21.1 Estimated Strehl ratio vs. $\sigma_{R}^{2}$ achievable for adaptive optics . . . . . . . S323

21.2 Schematic of steps of an optical engagement . . . . . . . . . . . . S324

21.3 Effect on maximum slant range by applying various limits to the optical engagement of a liquid-propellant missile vs. altitude of the target . . . . S328

21.4 Effect on maximum slant range by applying various limits to the optical engagement of a solid-propellant missile vs. altitude of the target . . . . S329

21.5 Acceleration vs. altitude for liquid-propellant ICBM L and solid-propellant ICBM S2 . . . . . . . . . . . . . . . . . S330

21.6 Speed versus altitude for liquid-propellant ICBM L and solid-propellant ICBM S2 . . . . . . . . . . . . . . . . . S331

21.7 Engagement of liquid- and solid-propellant ICBMs for $t_{e}=20 \mathrm{~s}$, for laser powers of $6 \mathrm{MW}, 3 \mathrm{MW}$, and $1.5 \mathrm{MW} \ldots \ldots \ldots$. . . . . . . S331

21.8 Engagement of liquid- and solid-propellant ICBMs for $t_{e}=5 \mathrm{~s}$, for laser powers of $6 \mathrm{MW}, 3 \mathrm{MW}$, and $1.5 \mathrm{MW} \ldots \ldots \ldots$. . . . . . . . S332

21.9 Altitude vs. time since launch for the liquid-propellant ICBM L and the solid-propellant ICBM S2 . . . . . . . . . . . . . . . . . S332

21.10 Engagement of liquid- and solid-propellant missile boosters for $t_{e}=20 \mathrm{~s} \quad \mathrm{~S} 334$

21.11 Engagement of liquid- and solid-propellant missiles for $t_{e}=5 \mathrm{~s} \quad \ldots \quad$ S335

21.12 Typical engagement geometries for a liquid-propellant missile, ICBM L, and a solid-propellant missile, ICBM S2, for a planar engagement . . . . . . S335

C.1 Engagement of ICBM S1 by multiple 10-km/s interceptors . . . . . . . S S361

C.2 Downrange and vertical velocities and accelerations of ICBM S1 . . . . S S362

C.3 Downrange and vertical velocities and accelerations of the $10-\mathrm{km} / \mathrm{s}$ inter-

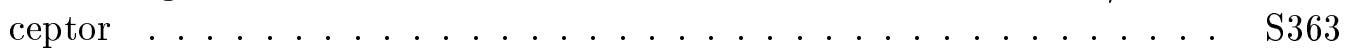

C.4 Five target missile trajectories . . . . . . . . . . . . . . S363

C.5 Estimated average S-band radar cross sections of the liquid-propellant ICBM model L and the solid-propellant ICBM model S1, as functions of aspect angle from the nose . . . . . . . . . . . . . . . . 
C.6 Signal-to-noise ratio for acquisition and tracking Aegis radar for ICBM L and ICBM S1 . . . . . . . . . . . . . . . . . . . . S365

C.7 Aegis tracking errors vs. time for the liquid- and solid-propellant ICBM

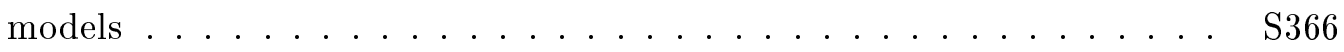

C.8 Sample sequence of the noise (random) and bias (correlated) components of the radar azimuth and elevation measurement errors . . . . . . . . . S370

C.9 Baseline case: Downrange, crossrange, and altitude errors in position and velocity and filter estimates of $1-\sigma$ values . . . . . . . . . . . . S373

C.10 Baseline case: Downrange, crossrange, and altitude acceleration errors and filter estimates of $1-\sigma$ values . . . . . . . . . . . . . . . S374

C.11 Poor-initial-estimates case: Downrange, crossrange, and altitude errors in position and velocity and filter estimates of $1-\sigma$ values $\ldots . . . . . \quad$ S377

C.12 Poor-initial-estimates case: Downrange, crossrange, and altitude acceleration errors and filter estimates of $1-\sigma$ values . . . . . . . . . . . S S378

C.13 Large-measurement-errors case: Downrange, crossrange, and altitude errors in position and velocity and filter estimates of $1-\sigma$ values . . . . . . . S S379

C.14 Large-measurement-errors case: Downrange, crossrange, and altitude accel-

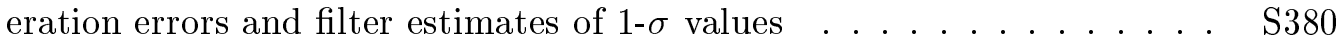

C.15 Engagement between the target missile ICBM L and three $5-\mathrm{km} / \mathrm{s}$ interceptors . . . . . . . . . . . . . . . . . . S382

C.16 Downrange and vertical velocities and accelerations of the ICBM L target missile . . . . . . . . . . . . . . . . . . S383

C.17 Downrange and vertical velocities and acceleration of the $5-\mathrm{km} / \mathrm{s}$ intercep-

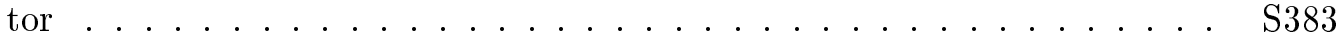

C.18 Downrange, crossrange, and altitude errors in position and velocity and filter estimates of $1-\sigma$ values . . . . . . . . . . . . . S387

C.19 Downrange, crossrange, and altitude acceleration errors and filter estimates of $1-\sigma$ values . . . . . . . . . . . . . . . . . . . S388

D.1 $C_{n}^{2}$ vs. target altitude in Clear-1 Night model . . . . . . . . . . . S394

D.2 Rytov variance vs. target altitude for ranges of 100, 200,300,400, and $500 \mathrm{~km} \ldots \ldots \ldots \ldots \ldots \ldots \ldots \ldots \ldots \ldots \ldots$

D.3 Fried parameter vs. target altitude for ranges of 100, 200, 300, 400, and $500 \mathrm{~km} \ldots \ldots \ldots \ldots \ldots \ldots \ldots \ldots \ldots \ldots \ldots$

D.4 Root mean square tilt angle vs. target altitude for ranges of 100, 200, 300, 400 , and $500 \mathrm{~km} \ldots \ldots \ldots \ldots \ldots$. . . . . . . . . . . . . . . . . . . . .

D.5 Isoplanatic angle vs. target altitude for ranges of 100, 200,300,400, and $500 \mathrm{~km} \ldots \ldots \ldots \ldots \ldots \ldots \ldots \ldots \ldots$. . . . . . . . . . . . . . . . . . . . . .

D.6 Filter functions for Anisoplanatism, Piston, Tilt, Piston Removed, and Higher Order (beyond Tilt) . . . . . . . . . . . . . . . . S399

D.7 Contributions to the anisoplanatic integrand of Eq. D.21 . . . . . . . S400

D.8 Variance reduction factor vs. normalized variance for piston removed mode S401

D.9 $\sigma_{\varphi c B W}^{2}$ for $n_{f}=1$ vs. target altitude for ranges of 100, 200, 300, 400, and

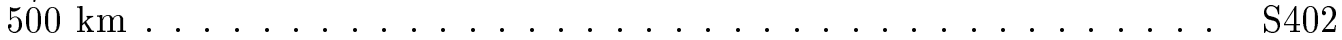

D.10 Variance correction factor vs. normalized variance for tilt mode . . . . S405 


\section{Executive Summary}

Boost-phase intercept systems for defending the United States against ballistic missile attack are being actively considered as a major part of a national missile defense strategy. Spending on such systems by the U.S. Department of Defense is growing, and there is a prospect of much larger expenditures in the future. Boost-phase intercept weapons would seek to disable attacking missiles during the first few minutes of flight, while the missiles' boosters are still burning and before they have released nuclear, chemical, or biological munitions. The technical aspects and feasibility of such weapons are the subject of this report.

In spite of the growing interest in boost-phase intercept systems and the increasing resources being committed to developing them, little quantitative information about their technical feasibility, required performance, and potential advantages and disadvantages is available to the public. Consequently, the American Physical Society (APS) convened a study group of physicists and engineers, including individuals with expertise in sensors, missiles, rocket interceptors, guidance and control, high-powered lasers, and missile-defense-related systems, to assess the technical feasibility of boost-phase intercept systems.

The Study Group has based its assessments solely on information found in the open literature about ballistic missiles and missile defense. We have supplemented this information by our expertise in science and engineering and have confined the assessments reported here to those that can be made with confidence by applying the fundamental principles of rocket propulsion, signal detection and processing, guidance and control, and laser beam propagation. In many instances, as documented throughout this report, we have performed our own analyses to address important issues and to assure ourselves of the validity of our conclusions.

Our main conclusions are the following:

1. Boost-phase defense against intercontinental ballistic missiles (ICBMs) hinges on the burn time of the attacking missile and the speed of the defending interceptor rocket. Defense of the entire United States against liquid-propellant ICBMs, such as those deployed early by the Soviet Union and the People's Republic of China (China), launched from countries such as the Democratic People's Republic of Korea (North Korea) and Iran, may be technically feasible using terrestrial (land-, sea-, or airbased) interceptors. However, the interceptor rockets would have to be substantially faster (and therefore necessarily larger) than those usually proposed in order to reach the ICBMs in time from international waters or neighboring countries willing to host the interceptors. The system would also require the capability to cope with at least the simplest of countermeasures.

2. Boost-phase defense of the entire United States against solid-propellant ICBMs, which have shorter burn times than liquid-propellant ICBMs, is unlikely to be practical 
when all factors are considered, no matter where or how interceptors are based. Even with optimistic assumptions, a terrestrial-based system would require very large interceptors with extremely high speeds and accelerations to defeat a solid-propellant ICBM launched from even a small country such as North Korea. Even such highperformance interceptors could not defend against solid-propellant ICBMs launched from Iran, because they could not be based close enough to disable the missiles before they deployed their munitions.

3. If interceptor rockets were based in space, their coverage would not be constrained by geography, but they would confront the same time constraints and engagement uncertainties as terrestrial-based interceptors. Consequently, their kill vehicles (the final homing stage of the interceptors) would have to be similar in size to those of terrestrial-based interceptors. With the technology we judge could become available within the next 15 years, defending against a single ICBM would require a thousand or more interceptors for a system having the lowest possible mass and providing realistic decision time. Deploying such a system would require at least a five- to tenfold increase over current U.S. space-launch rates.

4. The Airborne Laser now under development could have some capability against liquidpropellant missiles, but it would be ineffective against solid-propellant ICBMs, which are more heat-resistant.

5. The existing U.S. Navy Aegis system, using an interceptor rocket similar to the Standard Missile 2, should be capable of defending against short- or medium-range missiles launched from ships, barges, or other platforms off U.S. coasts. However, interceptor rockets would have to be positioned within a few tens of kilometers of the launch location of the attacking missile.

6. A key problem inherent in boost-phase defense is munitions shortfall: although a successful intercept would prevent munitions from reaching their target, it could cause live nuclear, chemical, or biological munitions to fall on populated areas short of the target, in the United States or other countries. Timing intercepts accurately enough to avoid this problem would be difficult.

\section{The Charge}

Boost-phase missile defense systems would disable attacking missiles while their rocket motors are burning by hitting them with an interceptor rocket or a laser beam. For ICBMs, this phase of flight typically lasts 3 or 4 minutes. Boost-phase defense has been proposed as a way to avoid the problems faced by midcourse defense systems, which are intended to disable the attacking missile's warheads after they have been deployed. The midcourse approach is complicated by the need to counter multiple warheads, submunitions ("bomblets"), lightweight decoys, and other countermeasures.

The Study Group was asked to evaluate boost-phase intercept systems that would defend the United States using land-, sea-, air-, or space-based interceptor rockets or an airborne laser now being developed. Space-based laser systems were not included because the technology needed for such systems would not be ready within the 10- to 15-year period considered. The Study did not consider the feasibility of the communications, command, control, and battle management that would be required. Nor did it consider policy issues, 
such as the arms control, strategic stability, or foreign policy implications of testing or deploying a boost-phase defense.

\section{The Focus}

Developing and deploying a reliable boost-phase missile defense would be a major undertaking likely to require a decade or more to complete. We therefore considered missiles that might be developed or acquired by North Korea and Iran during the next 10 to 15 years. These countries were the focus of the Study because the U.S. government has expressed concern specifically about them. According to U.S. intelligence estimates, neither of them currently has a credible ICBM capability but they are projected to develop or acquire ICBMs within the next 10 to 15 years. The Study Group also considered defense against ICBMs launched from Iraq. With the changed political situation arising from the events of the Spring of 2003, an ICBM threat from Iraq appears unlikely for the foreseeable future. We have nevertheless retained the analysis of the Iraq threat in the body of our report, to illustrate the requirements for defending against ICBMs from a country that is intermediate in size between North Korea and Iran.

We began by identifying boost-phase intercept systems that could work in principle and then determined the system performance that would be required to defend the entire United States, the contiguous 48 states, or only the largest U.S. cities. The attacking missiles were assumed to be similar to the first ICBMs developed 30 to 40 years ago by the United States, the Soviet Union, and China. Both liquid- and solid-propellant missiles were considered, because either type might be developed or acquired within 10 to 15 years and the Study Group therefore concluded that it would be imprudent not to consider both in evaluating the feasibility of boost-phase defense.

\section{Key Issues}

Hitting the Missile. An important question in boost-phase defense is whether the kill vehicle carried by the interceptor could actually hit a long-range missile, given the inherently unpredictable acceleration that is normal for an ICBM in powered flight and the possibility of programmed trajectory-shaping or evasive maneuvers. Assuming interceptors can reach the missile during its boost phase, we find no fundamental obstacle to homing on the missile accurately enough to hit it. To do so, however, the kill vehicle would have to be very agile and would need to carry enough fuel to continue adjusting to the missile's acceleration until the moment of impact. We determined that kill vehicles capable of meeting these requirements would be substantially heavier than those that some have suggested for boostphase intercept. Our analysis of this agility requirement and its implications for the weight of the interceptor are key new aspects of this study.

Time. Time is short for boost-phase defense because ICBMs burn out quickly: in roughly 3 minutes for solid-propellant missiles and 4 minutes for liquid-propellant missiles. But the time actually available is substantially shorter than the duration of the burn. Even systems with state-of-the-art tracking sensors would require 45 to 65 seconds or longer to detect the launch of a potentially threatening rocket and determine its direction of flight well enough to fire an interceptor (that is, obtain a firing solution).

Additional time must also be allowed for the decision to fire. We have analyzed the decision times that would be provided by various boost-phase defenses. "Decision time" as used here also includes any additional time required for communication between system 
elements, estimating the performance characteristics of the attacking missile and its trajectory, resolving uncertainties in the performance of the defense system, and other operational factors.

To be successful, the intercept would have to occur before the missile gives its munitions the velocity needed to reach the United States. This velocity could be attained as early as 40 seconds before the missile would normally burn out.

Due to the potentially similar flight profiles of ICBMs and space launchers, in many cases the defense system would not be able to distinguish a peaceful space launch from an ICBM attack. In these cases, the defense would have to shoot at every rocket, unless it had been established as nonthreatening before it was launched.

Extending the time for intercept beyond the boost phase into the ascent phase (defined here as the period after the missile's final stage has burned out or its thrust has been terminated but before it has deployed all its munitions and decoys) would not increase the available time significantly. The reason is that once the missile's thrust has been terminated, it could deploy its munitions and any decoys or countermeasures quickly, possibly in less than a second.

With so little time available, interceptors would need to reach high speeds very quickly. Taken together, the short time available for intercept and the size of the kill vehicle needed to hit an unpredictably accelerating ICBM would require large interceptors. In some cases, they would have to be larger and faster than the ICBMs themselves and would have to accelerate four times more quickly. Such interceptors have never been built and would push the state of the art.

Range. The useful range of interceptor rockets is restricted by practical limits on rocket speeds and by the short time available for intercepting the attacking missile. The range of the Airborne Laser is also limited, both by constraints on its power and by the distance its beam can propagate through the atmosphere and remain focused. Consequently, boostphase defense would be possible using interceptor rockets only if they could be positioned close enough to the required intercept locations, generally within 400 to 1000 kilometers. Defense would be possible using the Airborne Laser only if it could be stationed within 300 to 600 kilometers of the intercept points. The required intercept locations are typically hundreds of kilometers downrange from the missile launch site, which would further restrict interceptor basing options.

In general, boost-phase defense using terrestrial (land-, sea-, or air-based) rocket interceptors or the Airborne Laser requires that the missile's flight path during its boost phase be accessible from international waters or from neighboring countries willing to host U.S. interceptors. The feasibility of boost-phase defense therefore depends not only on the performance of the attacking missile and the speed of the interceptor, but also on the size of the country that launches the missile, the direction of the missile's flight, the distance to its target, and the local physical and political geography.

Shortfall. If a missile were hit during its boost phase by an interceptor, it would probably lose thrust quickly, but the missile (perhaps in fragments) and its munitions would not fall straight down. Instead they would continue on ballistic trajectories, falling to Earth short of their target but possibly on populated areas. Thus, unless the missile's munitions were disabled by the collision - which cannot be assumed because they are loosely coupled to the missile and hardened to withstand re-entry at hypersonic speeds - a successful intercept could cause live munitions to fall on populated areas. These areas would not be in the attacking country but might well be in countries friendly to the United States or in the United States itself. 
This problem is inherent in boost-phase intercept. Our analysis indicates that it would be extremely difficult to time intercepts to avoid causing live munitions or debris to hit populated areas. This problem would be eliminated if the interceptor could reliably destroy the missile's munitions, but doing so would be much more difficult than simply disabling the missile's booster rocket.

Space-Based Interceptor Requirements. Boost-phase interceptors fired from orbiting satellites could in principle defend the United States against ICBMs launched from anywhere on Earth. While their coverage would not be constrained by geography, spacebased interceptors would have the same time constraints and engagement uncertainties as terrestrial-based interceptors. As a result, their kill vehicles would have to be at least as massive as the kill vehicles of terrestrial-based interceptors. Because a satellite orbiting at low altitude spends so little time over a single spot on Earth, many interceptor-carrying satellites would be needed to defend against even a single missile. The precise number of satellites and the total mass that would have to be placed into orbit would depend on the type of ICBM as well as the speeds, accelerations, and masses of the interceptors and their kill vehicles, which would in turn depend on the technology available. Based on the technology that could, in our judgment, be developed within the next 10 to 15 years, we find that a thousand or more interceptors would be needed for a system having the lowest possible mass and providing a realistic decision time. Even so, the total mass that would have to be orbited would require at least a five- to tenfold increase over current U.S. space-launch rates, making such a system impractical.

The Airborne Laser's Performance. A laser weapon now in development has also been proposed for boost-phase defense. The Airborne Laser is being developed to disable short- or medium-range ballistic missiles by illuminating them with a powerful laser beam from distances of several hundred kilometers, heating them sufficiently to cause the structure of the missiles to fail. In principle, this weapon could also disable long-range missiles during their boost phase. Because the laser beam could reach an ICBM within a fraction of a second, its speed is not an issue. However, the range of the Airborne Laser is limited by the distance its beam can propagate through the atmosphere and remain focused. Assuming that it works as planned, its useful range would be about 600 kilometers against a typical liquid-propellant ICBM. This range would be sufficient to defend the United States against such ICBMs launched from North Korea but insufficient to defend against such missiles launched from Iran, unless the laser could be stationed over the Caspian Sea or Turkmenistan. Because solid-propellant ICBMs are more heat-resistant, the Airborne Laser's ground range against them would be only about 300 kilometers, too short to defend against solid-propellant ICBMs from either Iran or North Korea.

Countermeasures. While boost-phase intercept would not be susceptible to some of the countermeasures to midcourse intercept that have been proposed, there is no reason to think it would not face any countermeasures. Effective countermeasures to boost-phase intercept by interceptor rockets could include launching several ICBMs at nearly the same time or deploying rocket-propelled decoys and jammers. Furthermore, ICBMs could be programmed to fly evasive maneuvers that might overwhelm the agility and guidance and control capabilities of the interceptor or exhaust its propellant. Shortening the boost phase would also be an effective countermeasure: it would be practically impossible for any interceptor rocket to reach an ICBM with a boost phase of 2 minutes or less, even if it were launched from a very small country. Countermeasures against the Airborne Laser could include applying ablative coatings or rotating the ICBM to reduce the amount of heat the missile absorbs, launching multiple missiles to overwhelm the Airborne Laser's capabilities, 
or attacking the aircraft carrying the laser.

\section{Defending the United States}

We considered the effectiveness of boost-phase intercept for defending the United States against ICBMs from the two specific countries of concern, North Korea and Iran, using appropriate physical laws and engineering principles to compute the feasibility of particular intercepts. The results summarized here are based on a series of generally optimistic, specific assumptions. For example, we have made optimistic assumptions about the missile detection and tracking capabilities available to the defense. Moreover, we did not account for many of the real world factors that would have to be considered to make a realistic assessment of the capability of such a system. This includes uncertainties about the performance of the attacking missile and its trajectory, ignorance of the missile's target, the unpredictable nature of variations in any missile's flight, and uncertainties in how quickly an intercept would terminate an ICBM's thrust. Nor did we account for possible operational delays in processing and transmitting information. All of these factors would make boost-phase intercept more difficult than our simulations suggest. Consequently, our results reflect the theoretical possibility of an intercept, but this cannot be equated with certainty.

We found that terrestrial-based interceptors that burn out in 40 to 50 seconds and reach speeds of 6.5 to $10 \mathrm{~km} / \mathrm{s}$ would generally be required to defend against ICBMs launched from North Korea or Iran depending on the type of ICBM. In many cases, interceptors with significantly longer burn times are likely to be ineffective. As noted above, such interceptors would have to be substantially larger and capable of higher performance than any that have yet been built or deployed. In a few situations, a $5-\mathrm{km} / \mathrm{s}$ interceptor would work against slow-burning liquid-propellant ICBMs. The time available would be significantly greater for very slowly burning liquid-propellant ICBMs having burn times of 5 minutes or longer, but a defense that would work only against such missiles, which would be as slow as the slowest-burning missiles ever built, would risk being ineffective.

North Korea. Defense of all 50 states against typical liquid-propellant ICBMs launched from North Korea would require interceptors with speeds of $6.5 \mathrm{~km} / \mathrm{s}$ (almost as fast as ICBMs) based in Russia or the Sea of Japan and fired within about 40 seconds of obtaining a firing solution. The intercept locations for most ICBM trajectories from North Korea would be over China, hundreds of kilometers inside its border. Such interceptors would have ranges as long as ICBMs. Consequently, firing them toward China to intercept a North Korean missile could be mistaken for an attack on China, Russia, or other countries. The Airborne Laser might provide an alternative defense against liquid-propellant ICBMs.

To defend against typical solid-propellant ICBMs and provide more than a few seconds of decision time would require interceptors that could reach speeds of about $10 \mathrm{~km} / \mathrm{s}$, 50 percent faster than a typical ICBM, in one-quarter of the time it would take an ICBM to reach its maximum speed. Such interceptors would push the limits of what would be practical and should be considered a bounding case. The interceptors would have to be based in Russia or the Sea of Japan and fired within 30 to 40 seconds after a firing solution was obtained. Such interceptors could be mistaken for offensive weapons.

Iran. To defend the entire United States against liquid-propellant ICBMs launched from Iran using interceptors based in conventional locations would require basing $10-\mathrm{km} / \mathrm{s}$ interceptors in the Persian Gulf, and even this deployment would provide only about 15 sec-

onds of decision time. More decision time would be possible only if interceptors could be based in unconventional locations, such as Turkmenistan or the land-locked Caspian Sea. A 
system with $6.5-\mathrm{km} / \mathrm{s}$ interceptors based in either of these locations could provide a decision time of about 30 seconds.

Defense of the entire United States against solid-propellant ICBMs launched from Iran appears impractical; even a system with $10-\mathrm{km} / \mathrm{s}$ interceptors based both in the Caspian Sea and in Turkmenistan or Afghanistan would provide less than 10 seconds of decision time, which is unlikely to be adequate for an operational system.

Defending Only a Portion of the United States. We also considered the feasibility of defending only the contiguous 48 states or only the largest U.S. cities against ICBMs launched from North Korea or Iran. In most cases, this would be no easier than defending all 50 states. If, however, a boost-phase defense were not solely responsible for intercepting all missiles from these countries, the required system performance would be less demanding. Interceptors could hit liquid- or solid-propellant missiles launched from these countries toward some U.S. targets. Such a system could provide a partial defense; for instance, for one U.S. coast but not the other. Coupled with an effective midcourse system, a partially effective boost-phase defense could improve protection of some targets by hitting missiles before they deploy decoys that could overwhelm the midcourse layer. This possibility, however, depends on the midcourse system's being able to handle the unpredictable debris generated by a boost-phase intercept while engaging the warheads, which most likely would survive the intercept. Such a capability would be difficult to achieve.

Defending Against Short- or Medium-Range Missiles Launched from Offshore. Missiles that could be used for a sea-based attack probably are already available to nations of concern to the United States. The Aegis radar system is adequate for tracking such missiles provided it is within a few tens of kilometers of the missile launch location, and a missile similar to the Navy's Standard Missile 2 is adequate for such an engagement without significant modification. 



\section{Findings}

The Study Group analyzed boost-phase defense against liquid-propellant ICBMs, which the United States may face initially, and against solid-propellant ICBMs, which the nation may face later. The basic parameters of systems that could counter these threats in a variety of geographical situations were identified. In the course of analyzing these systems, the Study Group identified many significant limitations to boost-phase intercept, especially when confronting solid-propellant ICBMs. However, it made no judgment as to whether any or all of these limitations would rule out deployment of such systems on operational, political, or economic grounds. The analysis in the main body of this report supports the following findings. A number (or letter) in parentheses indicates the relevant chapter (or appendix), section, or subsection of the supporting material.

\section{Intercepting missiles during their boost phase presents major challenges not faced by midcourse-intercept systems.}

- Midcourse systems have 20 to 25 minutes to observe and intercept threatening warheads (A.2); boost-phase intercept systems could have 4 minutes or less to detect, track, and intercept potentially threatening missiles $(4.4,5.4-5.6,10.4,15)$.

- In midcourse flight, the trajectory of a warhead is ballistic and highly predictable (B); in powered flight, the trajectory of a missile is inherently unpredictable. This unpredictability results from uncertainty about the intended target, the effects of the missile's maneuvers to manage its energy, shape its trajectory, or evade intercept, and its unpredictable thrust variations $(4,12.4,15.2)$.

2. The effective ranges of boost-phase hit-to-kill interceptors, whether land-, sea-, air-, or space-based, are limited by the short duration of ICBM boost phases and practical limits on interceptor fly-out velocities. The range of the Airborne Laser is limited primarily by the distance its beam can propagate through the atmosphere while remaining focused, and to a lesser extent on its power.

These limitations have the following consequences:

- In a hit-to-kill boost-phase defense, the time remaining after an interceptor is fired is so short-less than 170 seconds for a liquid-propellant threat missile and less than 120 seconds for a solid-propellant threat missile - that the defense could fire only once, either a single interceptor or a salvo of interceptors fired virtually simultaneously. There would be no opportunity to recover from a misfire or failure of an intercept attempt (5.4-5.6).

- Boost-phase defense with interceptor rockets would be possible only if the rockets could be positioned close to the intended intercept point. The intercept point is 
typically 400 to 500 kilometers from the missile launch point. The interceptors typically must travel at least 500 kilometers from the interceptor base to reach the intercept point (5.4-5.6).

- Terrestrial-based boost-phase defense - both by interceptors and airborne lasersalso depends on the size of the country that launches the missile, the direction of the missile's flight, the distance to its target, and access to areas adjacent to that country, determined by local physical and political geography (5).

- Boost-phase defense using terrestrial-based interceptors could not defend the United States against accidental or unauthorized launches of ICBMs from the interiors of large countries such as Russia or China (5).

3. The large and unpredictable variations of ICBM boost-phase trajectories and the short time available for engaging them drive the requirements for any boost-phase kinetic kill interceptor.

Factors contributing to uncertainties in the intercept point include:

- Random and systematic errors in the defense detection and tracking system's measurement of position and velocity and estimate of acceleration of the attacking missile $(10.1 .4,12.3 .1)$.

- Lack of knowledge of the missile's target (15.2).

- Normal or induced thrust-time variations of the threat booster (15.2).

- Intentional trajectory shaping, including lofting or depressing the trajectory and maneuvering to manage energy (15.2).

- Intentional evasive maneuvers, such as dog-legs or other maneuvers (12.4).

- Lack of knowledge of the potential type or characteristics of the threat (3.3).

- Uncertainties in the method and times at which the missiles' warheads or submunitions would be deployed (15.2, A.2.2).

These uncertainties reduce the time available for the engagement and require kill-vehicle maneuver velocity and acceleration substantially greater than is generally recognized. These effects are discussed in Chapters 5 and 12.

4. The only way a boost-phase defense can assure that lethal warheads will not strike a defended area is to disable the attacking missile before the earliest time it can achieve the velocity needed to carry its munitions to that area, because the defense does not know the particular target. This time is uncertain because the missile may fly various trajectories and execute a variety of maneuvers to manage its energy or evade the defense $(4.1,5.1 .3,5.2 .1, A .2)$.

5. A robust boost-phase defense against ICBMs would require modern space-based sensors to detect launches and provide initial tracking information needed to launch interceptors. Even so, it would take at least 45 to 65 seconds to detect the launch of an ICBM and establish a track of its trajectory accurate enough to launch an interceptor. Such sensors would also be needed to provide continually updated tracking information to the interceptors as they fly to the target. A system such as the high-altitude Space-Based Infrared System (SBIRS-High) now under development 
could perform these functions if the boost-phase defense requirement is included in its design (10.4).

- While radars with sufficient sensitivity exist, for early detection and initial tracking, horizon limitations, clutter problems, and geographic constraints that require stand-off distances greater than $300 \mathrm{~km}$ would preclude their use. Consequently, a modern space-based missile warning and tracking system, such as the planned SBIRS-High system, would be needed in order to achieve the earliest detection and initial tracking (10.4). The existing Defense Support Program (DSP) system could provide launch detection and initial tracking, but it would take 30 seconds longer to obtain a firing solution than a system such as SBIRS-High (10.4). Consequently DSP would be useful only against slow missiles, and only if the fastest interceptors were used (5.9.2).

- Additional time margin would be required to allow for the decision to fire and any other intentional or system delays. We use the term "decision time" to encompass any time required beyond the zero decision time case (5.1.3).

6. While boost-phase defense against slow-burning liquid-propellant ICBMs not employing countermeasures appears technically feasible in principle for some geographic scenarios, the much shorter burn times typical of solid-propellant ICBMs using even 40-year-old technology call into question the fundamental feasibility of boost-phase intercept of such threats at useful ranges-no matter where or how the interceptors are based-even with very optimistic assumptions about detection and track times $(5.3,6.11)$.

- While liquid-propellant ICBMs typically have powered flight times of 4 minutes or more, solid-propellant missiles typically have three boost stages that burn a total of 3 minutes or less (3.4). This difference is crucial.

- No matter where or how they are based, interceptors would typically have to travel 500 kilometers or more in about two minutes to reach solid-propellant ICBMs before they have achieved the velocity required to deliver their payloads to the United States (5.3-5.6). This would require interceptors with extremely high flyout velocities (in excess of orbital velocities and as high as $10 \mathrm{~km} / \mathrm{s}$ ) and very high accelerations. Such interceptors would push the state of the art and may not be feasible.

- By comparison, against liquid-propellant ICBMs, small two-stage terrestrial-based interceptors having modest burnout velocities of only about $5 \mathrm{~km} / \mathrm{s}$, such as the largest-sized interceptor that could meet the constraints of the Aegis cruiser vertical launchers or deployment by bombers, could marginally engage threats at about 500 kilometers (5.3). Interceptors having velocities similar to those of ICBMs would provide greater decision time and range for this case but still could not engage solid-propellant ICBMs.

7. Based on unclassified summaries of U.S. intelligence estimates, the Study Group concluded that countries of concern might acquire or develop solid-propellant ICBMs within the next 10-15 years and that it would be imprudent not to consider them in evaluating the feasibility of boost-phase defense systems (3.4).

- Proliferation of solid-propellant technology has been rapid (3.3). 
- A boost-phase defense would create incentives to develop or acquire solid-propellant ICBMs (3.4).

- Boost-phase defenses not able to defend against solid-propellant ICBMs risk being obsolete when deployed.

8. The time constraints imposed on any boost-phase defense system by the short duration of ICBM boost phases would pose significant real-time decision issues.

- In most situations, interceptors would have to be fired within a few seconds after confirmation of the launch of a large rocket to intercept it in time to defend the United States (5.3). The decision to fire interceptors would have to be almost automatic (5.3-5.6).

- Because of the potentially similar flight profiles of ICBMs and space launchers, in many cases the defense system would have difficulty distinguishing a space launch from an ICBM attack. In these cases, the defense would have to shoot at every rocket, unless it had been identified as non-threatening before it was launched (10.4).

9. Despite the variations and uncertainties inherent in the boost-phase trajectories of ICBMs, our analysis indicates that a kill vehicle incorporating current sensor and guidance technology could home on ICBMs in powered flight with a precision compatible with direct hit-to-kill requirements, assuming the kill vehicle's booster could place it on a trajectory that would take it within homing range of the ICBM. The kill vehicle would also have to meet certain critical performance requirements.

Critical kill-vehicle performance requirements include:

- Capacity to shift from guiding on the rocket's exhaust plume to guiding on the rocket body. The Study Group believes this requirement in particular requires more investigation (10.4).

- Ability to acquire and track the rocket body within the plume at ranges of at least 200 kilometers and with sufficient precision, using sensors on board the kill vehicle (12.3).

- Sufficient kill-vehicle acceleration (7-8 g initially and $15 \mathrm{~g}$ in the end game), velocity for maneuvering $(2 \mathrm{~km} / \mathrm{s}$ for terrestrial-based and $2.5 \mathrm{~km} / \mathrm{s}$ for space-based kill vehicles), and guidance system response (0.1 second or less) (12.5).

These requirements would result in kill vehicles with masses substantially greater than is generally appreciated. In our judgment, kill vehicles using technology that would be available in the next few years would have masses on the order of 90 kilograms to 140 kilograms: 90 kilograms for the total divert velocities of $2 \mathrm{~km} / \mathrm{s}$ that would be required for most ground- and air-based interceptors and roughly 140 kilograms for 2.5$\mathrm{km} / \mathrm{s}$ divert velocities that would be appropriate for space-based interceptors and the fastest ground-based interceptors (14.4).

10. Although a successful intercept would prevent munitions from reaching their target, live nuclear, chemical, or biological munitions could fall on populated areas short of the target, in the United States or other countries. This problem of shortfall is inherent in boost-phase missile defense. 
- Warheads and submunitions are loosely coupled to the final stage of the ICBM and cannot be assumed to be destroyed by an intercept that destroys or disables the ICBM booster, as borne out by numerous destruct events during flight tests (13.1).

- After an intercept, the munitions and debris will continue on a ballistic trajectory, albeit one that is shorter than intended by the attacker (5.8).

- The warheads or munitions and debris of an intercepted missile will not fall on the country that launched it (5.8).

- Preventing warheads or submunitions and debris of intercepted missiles from hitting the territory of U.S. friends and allies would sometimes require the defense to intercept missiles within a time window as small as 5 to 10 seconds, greatly complicating the already daunting intercept management problem (5.8.1).

- Given the unpredictable variations in trajectories and thrust that characterize ICBMs in powered flight, it is not clear that the intercept can be timed to occur within the narrow window required (5.8.2).

The problem of controlling shortfall could be avoided if the boost-phase defense system could destroy the missile's warheads or submunitions during boost, rather than simply disabling the booster. This is a much more difficult task, and it has not been established that it can be accomplished (13).

11. Airborne interceptors offer some unique advantages for boost-phase defense, but they also have significant limitations in defending against ICBMs. They could be deployed more quickly than land- or sea-based interceptors in response to new threats, but several backup aircraft equipped with interceptors, as well as refueling aircraft and defensive air cover, would be required for every airborne-interceptor aircraft on station (16.5.3).

- An interceptor of any given size has a slightly greater range if launched from a highaltitude platform, because it uses less energy to overcome gravity and aerodynamic drag as it flies out toward its target. However, the constraints on the size and weight of missiles that an aircraft can carry limit the flyout velocity of high-acceleration airborne interceptors to about $5 \mathrm{~km} / \mathrm{s}(16.5 .3)$.

- Because of their limited flyout velocity, airborne interceptors could engage ICBMs only in situations comparable to the situations in which a $5 \mathrm{~km} / \mathrm{s}$ surface-based interceptor could engage them. Consequently, using airborne interceptors to defend the United States against long-burning liquid-propellant ICBMs would be possible only if the required intercept locations are within about 500 kilometers of the interceptor-carrying aircraft (5.5.1).

12. A constellation of space-based interceptors (SBIs) could, in principle, overcome the geographic limitations of terrestrial-based interceptors and intercept ICBMs launched from much of the Earth's surface. However, they would be subject to range and time constraints similar to those that constrain terrestrial-based systems. Consequently achieving reasonable coverage between latitudes 30 degrees and 45 degrees North would come at a very high cost.

- Because a satellite in low-Earth orbit spends so little time over a single spot on Earth, a system having the minimum mass-in-orbit and providing a realistic time 
to construct a firing solution would require a thousand or more interceptors to intercept even a single liquid-propellant ICBM 5 seconds before it burns out (6.6). ${ }^{1}$

- The SBI kill vehicles would be similar to those of terrestrial-based interceptors. Because of the high closing velocities of SBI engagements, space-based kill vehicles would require divert velocities of about $2.5 \mathrm{~km} / \mathrm{s}$ (14.1). Such a kill vehicle would have a mass of roughly 140 kilograms $(6.11,14.4)$. We estimate that an interceptor that combined the kill vehicle with a two-stage booster to impart the required flyout velocity of $4 \mathrm{~km} / \mathrm{s}$ in 30 to 40 seconds would have a mass, including its on-orbit support systems, of about 1200 kilograms (6.11).

- To intercept a solid-propellant ICBM launched from North Korea or Iran 5 seconds before burnout, at least 1600 interceptors would be required for a system having the lowest possible on-orbit mass and providing an optimistically short time to construct a firing solution (6.11). Such a system would have a mass in orbit of at least 2000 tonnes. To deploy it would require at least a five- to ten-fold increase in the current annual U.S. launch capacity.

- In practice, more interceptors and mass would be required in orbit because solidpropellant ICBMs launched from North Korea or Iran would usually have to be intercepted before 5 seconds prior to their burnout. The number of interceptors would also increase if the system were designed to assure that two interceptors could be fired against each ICBM, provide more decision time, or have the capability to defend against ICBMs launched nearly simultaneously from closely spaced launch sites $(6.6,6.11)$.

- Defending against a liquid-propellant ICBM would cut the number of interceptors required to about 700, with a corresponding reduction in the mass of the system, because such missiles burn much longer (15.2.1). However, a system designed to counter only liquid-propellant ICBMs could become obsolete quickly, given the time that would be required to develop and deploy an SBI system (Finding 15), the incentives it would create for emerging missile states to build or procure solidpropellant missiles, and the rate at which solid-propellant technology is proliferating (3.4.2).

13. Although boost-phase missile defense systems using hit-to-kill interceptors could avoid some of the countermeasures to midcourse intercept that have been proposed, there are effective countermeasures to such boost-phase systems. Many of them have been demonstrated in past U.S. programs for other purposes $(5,9,12,15)$.

- Shortening the boost phase of ICBMs. Switching from liquid-propellant to typical solid-propellant ICBMs would cut the boost phase by a minute or more (Finding 6). Boost phases as short as 130 seconds are certainly possible; such missiles would be practically impossible to intercept (5.1.1).

- Maneuvering the ICBM (15.2).

- Fractionating the payload during final-stage boost $(9.1 .2,9.1 .5)$.

\footnotetext{
${ }^{1}$ Interceptors in low-Earth orbits revolve around Earth at high speeds while the Earth rotates beneath them. As a result, at any instant almost all the interceptors in a space-based system would be too far away to engage a rocket from any particular launch site. A constellation of a thousand or more interceptors would therefore be required to ensure that at least one would always be within range of a hostile launch.
} 
- Deploying small, rocket-propelled decoys from the missile designed to mask or mimic the radar and electro-optical characteristics of the booster (9.1.3).

- Launching multiple missiles within a short time. Launching tactical ballistic missiles before launching ICBMs could exhaust the defense's supply of interceptors (9.1.6).

14. The Airborne Laser ( $A B L)$ has been designed to intercept theater ballistic missiles and is scheduled to achieve initial operational capability in about $\mathbf{1 0}$ years. It could offer some capability for intercepting ICBMs, but would have less range than large ground-based hit-to-kill interceptors. ABL aircraft could be rapidly deployed, but several $A B L$ aircraft, as well as tanker support aircraft and defensive air cover, would be required to maintain one $A B L$ aircraft continuously on station. While the $A B L$ has some self-defense capability, without supporting tactical air cover $A B L$ aircraft would be vulnerable to attack by enemy aircraft or surface-to-air missiles.

- Performance requirements for the ABL are driven largely by the construction materials of the missile and the distance to the target missile - engagement time and uncertainty about the target's trajectory are not issues (21).

- The laser fluence needed to disable ICBMs is currently rather uncertain, making it difficult to estimate accurately the ABL's range if used against ICBMs. The ABL's range is expected to be roughly similar to that of the modest-sized interceptors that could be carried by aircraft (21.5). If so, it could engage only long-burning liquid-propellant ICBMs launched from geographically small, accessible countries (8.3-8.5).

- Defense would be possible using the ABL only if it can be stationed within 600 kilometers of the intercept point of a liquid-propellant missile or within 300 kilometers of the intercept point of a solid-propellant missile. The ABL's laser beam would have to heat an ICBM for several seconds to disable it; hence ABL engagements would have to be timed to avoid the brief periods during which one stage burns out and is discarded as the next ignites (8.7).

- The ABL would have substantial ability to defend the U.S. against liquid-propellant ICBMs launched from North Korea; however, it would have no utility in defending the U.S. against these missiles launched from geographically large, less-vulnerable countries such as Iran. Because of the greater heat resistance of solid-propellant missiles, the ABL could not defend against these missiles launched from either North Korea or Iran. (8.3-8.5).

- The ABL could not disable nuclear warheads or biological or chemical submunitions that have been hardened to survive re-entry at ICBM speeds (20.1).

15. Few of the components that would be required for early deployment (i.e., within 5 years) of a boost-phase defense currently exist. Moreover, we see no means for deploying an effective boost-phase defense against ICBMs within 10 years. Several key components are lacking and are unlikely to be developed in much less than a decade.

- Large, high-acceleration interceptors $(5,16)$ having the physical characteristics and performance that would be needed for a surface-based boost-phase intercept system have never been built. To counter short- or medium-range missiles launched from 
platforms off U.S. coasts a missile similar to the U.S. Navy's Standard Missile 2 could be used (5.7.1). We know of no other booster in existence or under development that offers any utility for boost-phase intercept of ICBMs.

- No kill vehicle currently under development has the acceleration and maneuverability required for ICBM boost-phase intercept $(11.6,12.5)$.

- While radars with sufficient sensitivity exist, such as the THAAD ground-based radar and the Aegis AN/SPY-1 radar, their horizon limitations and geographical constraints would require space-based infrared sensors for detection and initial tracking of threatening missiles (10.2). If SBIRS-High were available and had sufficient capability, it could perform this function (10.4); however, recent reports indicate that SBIRS-High is unlikely to be deployed before 2010 (10.1.2).

- The ABL is currently not expected to be ready for deployment against theater ballistic missiles before 2012 (18.3). Testing and evaluation of the ABL against ICBMs probably would not occur until after it has been tested for its intended mission, intercepting theater ballistic missiles.

- Given the U.S. space launch capability and the high cost of putting mass in orbit, space-based intercept is not practical because small, lightweight sensors, interceptors, and kill vehicles are not currently available (6.11).

16. Much of the public discussion of missile defense has focused on ICBM attacks, but the threat posed by existing short- or medium-range tactical ballistic missiles launched from ships or other platforms positioned off U.S. coasts is more immediate. It appears that a missile similar to the existing U.S. Navy Aegis Standard Missile 2 could engage short- or medium-range ballistic missiles launched from sea platforms without significant modification, provided that the Aegis ship is within a few tens of kilometers of the launch platform (5.7.1).

- According to the U.S. intelligence community, launching short- or medium-range ballistic missiles from platforms a few hundred kilometers off U.S. coasts would be much less demanding technologically than launching ICBMs. The missiles that would be needed for such an attack are already available (A.1).

- Many of the challenges that make ICBM defense difficult - such as geographic constraints that prevent the defense from positioning interceptors close to the missile's boost trajectory, delays in detecting and tracking the target missile, uncertainties about the exact target, and the problem of controlling shortfall-are absent when the threatening missile is launched from a ship near the United States.

- The Airborne Laser might also be able to counter this threat, but the Study Group did not analyze this possibility.

17. In our view, there are many issues for a boost-phase intercept system that require further study before the true capabilities and deployment timelines of boost-phase missile defense can be determined.

These issues include:

- The communications, command, and control networks and systems that would be required for a boost-phase intercept system to function with the reliability required 
under the extreme time pressures that a defense system would face, particularly one using space-based interceptors.

- The capability for transferring the interceptor's guidance from tracking the missile's luminous plume to tracking the missile itself ("plume-to-hardbody handover") (10.4). This task is technically challenging and not well understood. More realistic modeling, testing, and evaluation would be required to demonstrate that it can be done reliably under all engagement conditions.

- The effects on liquid- and solid-propellant boosters of a body-to-body collision with a kill vehicle need more extensive modeling and realistic testing (13).

- The realistic capabilities that would be needed to deploy, maintain, and control a space-based system must be understood before an informed decision can be made about the feasibility of such a concept. Given the extreme sensitivity of system costs to changes in the mass of space-based interceptors, a careful assessment of the effects of countermeasures should be included (6).

- The lethality of the ABL when used against ICBMs, especially solid-propellant ICBMs. Further modeling and realistic testing are needed under the wide range of conditions that would be encountered in intercepting ICBMs during their boost phase (20).

\section{Concluding remarks}

In assessing the feasibility of boost-phase missile defense using hit-to-kill interceptors or the ABL, we attempted to make optimistic assumptions to bound the performance of such systems. In some cases we made assumptions that appear technically possible but may not be realistic on other grounds. An important example is the assumption in some of our analyses that interceptors could be fired as soon as a target track has been constructed, without allowing additional time for decision or assessment. In other cases we simply examined the performance that would be required to make the system workable, without making any judgment about whether such components could realistically be deployed. An example of this kind is our consideration of an interceptor capable of reaching a flyout velocity 40 percent higher than an ICBM's velocity in only 45 seconds. Consequently, with those optimistic assumptions our results reflect the theoretical possibility, rather than the certainty, of an intercept. Real-world factors would make boost-phase intercept more difficult than our results suggest. Moreover, the choices made in this study were used to obtain upper bounds on performance; their use does not necessarily imply that the Study Group judged these choices to be realistic.

Given the results that follow from our assumptions, we conclude that while the boost phase technologies we studied are potentially capable of defending the United States against liquid-propellant ICBMs in certain geographic scenarios, at least in the absence of countermeasures, when all factors are considered none of the boost-phase defense concepts studied is likely to be viable for the foreseeable future to defend the 50 states against even firstgeneration solid-propellant ICBMs $(5,6.11,8.6)$. 



\section{Chapter 1}

\section{Introduction to the Report}

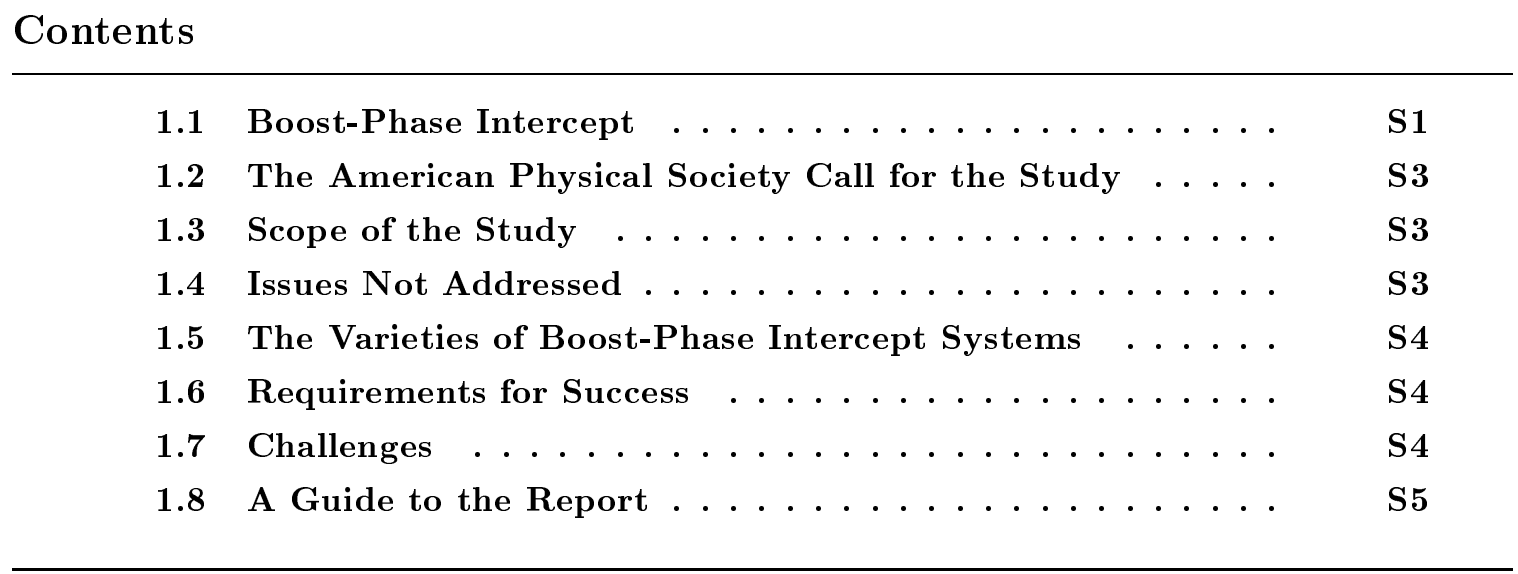

\subsection{Boost-Phase Intercept}

Boost-phase intercept systems for defending the United States against ballistic missile attack are the focus of increasing public discussion [1,2, 3, 4, 5], and Department of Defense spending on such systems is growing, with the prospect of much larger expenditures in the future. ${ }^{1}$ Boost-phase weapons would seek to destroy attacking missiles while their boosters are still burning and before they have released nuclear, chemical, or biological munitions. The technical aspects and feasibility of such weapons are the subject of this report.

For more than four decades, the United States has invested substantial resources in developing anti-ballistic missile systems [7]. During most of this period, the focus of the effort was on developing weapons and systems that could defend against the thousands of intercontinental ballistic missiles (ICBMs) fielded by the Soviet Union. In response to the end of the Cold War and other changes in the international situation, the emphasis of the U.S. program to defend against long-range missiles shifted first to systems that could counter accidental or unauthorized launches of a few ballistic missiles against the United States and, more recently, to systems that could counter missiles that might be deployed by countries that have missile development programs and with which the nation does not currently have friendly relations.

The U.S. intelligence community has identified several countries of concern that are developing long-range missiles [8, 9]. Although the intelligence community judges that U.S. territory is currently more likely to be attacked by nuclear, chemical, and biological weapons

\footnotetext{
${ }^{1}$ The budget request for boost-phase intercept systems for FY $04-F Y 05$ is $\$ 12.6$ billion [6].
} 
delivered by means other than missiles, it is the assessment of most of the intelligence community that the United States is likely to face ICBM threats from some of the countries of concern during the next 15 years [8,9]. The growing sale and transfer of ballistic-missilerelated technologies, materials, and expertise has generated concern that some unfriendly countries might be able to deploy full-range ICBMs, including solid-propellant ICBMs, with little warning $[10,11]$. The U.S. intelligence community has judged that several countries of concern are technically capable of developing within the next decade the ability to launch short- or medium-range ballistic missiles against coastal regions of the United States from ships or other platforms positioned hundreds of kilometers offshore [12, 13, 14]. To counter these potential threats, the United States is actively pursuing a variety of missile defense options.

The principal focus of the nation's current national missile defense program is on weapons that would destroy warheads after they have been launched by ICBMs but before they re-enter the atmosphere. ${ }^{2}$ During this so-called midcourse phase of flight, which lasts approximately 25 minutes, warheads follow predictable ballistic trajectories outside Earth's atmosphere. Using ground-based interceptors located at only a few sites, a midcourse intercept system potentially could defend the United States from missiles launched from almost anywhere on Earth.

Midcourse-intercept systems, however, must contend with two challenges. A single missile could launch multiple warheads or even dozens of chemical or biological submunitions, thereby overwhelming the defense. In addition, many argue that the system could be defeated by countermeasures and penetration aids, including large numbers of lightweight decoys that would be difficult to discern from real warheads outside the atmosphere $[15,16]$. For a contrary view, see [17].

The difficulty of meeting these challenges has led to growing advocacy of boost-phase intercept as a possible alternative $[18,19,20]$. Boost-phase systems potentially offer three important advantages: the possibility of destroying missiles before they can deploy multiple warheads or submunitions, the presumed difficulty of developing countermeasures, and the ease of tracking the bright exhaust plumes of ICBMs in powered flight. A boost-phase defense that used surface- or air-based interceptors or the Airborne Laser (ABL) would also be attractive, some argue, because their limited ranges would not threaten Russian or Chinese land-based nuclear missile forces. Space-based boost-phase weapons have also been proposed as the first layer in a layered defense system [21, 22, 23].

The boost phase of an ICBM typically lasts no more than 3 or 4 minutes. Consequently, even fast interceptor missiles would have to be fired from bases close to anticipated ICBM intercept points [24]. Therefore, surface- and air-based interceptors would be capable of countering only those missiles launched from countries that are sufficiently small geographically, and on boost-phase flight paths that are within range of interceptors stationed in international waters or in neighboring countries willing to host them. The Airborne Laser is similarly constrained because of its own engagement range limitations.

\footnotetext{
${ }^{2}$ We use the phrase "national missile defense" to indicate a defense system intended to defend the national territory of the United States rather than, for example, U.S. allies or troops based outside the United States, because it describes the goal of such a defense concisely. We note, however, that the present administration no longer uses this terminology.
} 


\subsection{The American Physical Society Call for the Study}

In spite of the government's growing interest in boost-phase intercept systems and the resources being committed to developing them, little quantitative information on the technical feasibility, required performance, and potential advantages and disadvantages of such systems is available to the public. To increase public understanding of these matters, the American Physical Society convened a study group of physicists and engineers, including individuals with expertise in sensors, missiles, rocket interceptors, guidance and control, high-powered lasers, and missile-defense-related systems, to assess the technical feasibility of boost-phase intercept systems.

Many of the key questions concerning the technical feasibility and required performance of boost-phase intercept systems can be answered by considering basic physics and engineering principles. The American Physical Society therefore asked the Study Group to produce an unclassified report based on publicly available information. The intention was to provide the membership of the Society, other scientists and engineers, policy makers, and the public with basic information about the science and technology of boost-phase intercept systems. The American Physical Society hopes that this report, which describes the technologies and technical requirements of these systems, their advantages and limitations, and the technological challenges in developing and deploying them, will help in evaluating proposals to build such systems.

\subsection{Scope of the Study}

The Study Group was asked to consider primarily boost-phase intercept systems that could defend the United States from attack by ballistic missiles of intercontinental range. In particular, the Study Group was asked to evaluate the potential of systems using terrestrialbased or space-based hit-to-kill rocket interceptors, or the ABL, for this purpose. Spacebased laser systems were not considered because the technology that would be required for such systems is at a much earlier stage of development. We also considered briefly the feasibility of defending against attacks by ship-based ballistic missiles launched off U.S. coasts using short-range interceptor rockets. The Study focused on technology that could, in principle, begin to be deployed in about 10 years.

\subsection{Issues Not Addressed}

A number of important technical issues were identified but not analyzed in detail, either because the necessary information was not available to us or because they lay outside the scope of the Study. These include the feasibility of building and deploying long-range, high-acceleration interceptors; the beam quality and certain other performance characteristics of the ABL; the effectiveness of kinetic-energy weapons or laser beams in disabling boosters, warheads, and submunitions; communications, command, control and battle management; survivability; and system complexity. Nor did the Study consider nuclear-tipped interceptors for boost-phase defense, or midcourse or terminal intercept systems.

Finally, the Study did not consider policy issues, such as the economic, arms control, strategic stability, or foreign policy implications of developing, testing, or deploying boostphase intercept weapons $[25,26,27,28]$. 


\subsection{The Varieties of Boost-Phase Intercept Systems}

The Study examined systems that would use interceptor missiles based either on land, on ships at sea, on aircraft, or on satellites in space. Small interceptors could be carried by satellites in low-Earth orbits. Somewhat larger interceptors could be carried by large aircraft or bombers. Still larger interceptors could be based on ships or on land. The range of an interceptor is limited by the highest speed that is technically feasible and the time available to complete the intercept. Systems that utilize surface- or air-based interceptors could potentially defend against missiles launched from limited geographical areas. In contrast, a space-based system could in principle defend against missiles launched from anywhere on Earth. However, due to Earth's rotation and the motions of orbiting satellites, a system of thousands of satellites armed with interceptors would be required to defend against missiles launched from a single launch site.

The Study also considered the Airborne Laser, which was originally planned as a weapon for theater missile defense but is now also being considered for national missile defense. It uses a high-powered chemical laser beam. Mirrors direct the beam toward the target missile. The beam travels at the speed of light and would therefore reach the target in a fraction of a second. Such a beam could potentially disable a missile in powered flight by heating its body long enough (at least several seconds) to cause structural failure. The effectiveness of the ABL against ICBMs would depend on the power and quality of its beam and the degree to which the beam could be focused on the target in the presence of the atmospheric turbulence at the altitudes at which it would operate.

\subsection{Requirements for Success}

In a boost-phase intercept, the largest and most fragile targets are the ICBM's boost stages. Moreover, a boost stage that is burning produces a bright exhaust plume that is easily spotted by sensors in orbit or on the interceptor, although the interceptor must eventually home on the body of the booster and not its plume. If a burning boost stage were hit by an interceptor, it would quickly lose thrust, but the collision would not necessarily disable all the missile's munitions, and the missile (perhaps in fragments) and its munitions would not fall straight down. Instead, they would continue on ballistic trajectories, falling to Earth short of the intended target. Consequently, to ensure that a missile's munitions do not strike the United States, a boost-phase defense must disable the missile before it has reached a speed sufficient to carry its munitions to the United States. Later interception may be too late, because the munitions may have already separated from the missile. The system would then have to contend with the problems of a midcourse intercept.

Unless the boost-phase intercept system is able to destroy the missile's munitions, they will strike somewhere outside the boundaries of the country that launched the missile. Consequently, an intercept could cause live nuclear warheads or biological or chemical munitions to fall on populated areas of the United States or other countries. This risk is inherent in boost-phase defense.

\subsection{Challenges}

The greatest challenge for a boost-phase defense system is the very short time within which the intercept must be completed. The boost-phase of a liquid-propellant ICBM typically lasts about 4 minutes. (Five-minute burn time missiles are also considered, though these 
are regarded as a less likely threat.) The shorter duration of the boost phases of solidpropellant ICBMs - typically about 3 minutes - is even more daunting. The narrow time window dominates every aspect of boost-phase intercept, driving the required performance of the detection and tracking systems, the interceptors, and the kill vehicle. About 1 minute is required to confirm the launch of a potentially threatening rocket, leaving slightly less than 3 minutes to decide whether it is an attacking ICBM and if so, to fire an interceptor and disable or destroy the ICBM. The interceptor must give its kill vehicle a velocity that will carry it sufficiently close to the expected intercept point, and the kill vehicle must then be able to home on the missile and maneuver to collide with it. The missile would be destroyed or disabled by the kinetic energy of the collision. (The kinetic energy released in the collision of an interceptor with an ICBM is greater than the chemical energy that could be released by explosives carried by the interceptor.)

While it delivers energy at the speed of light and is therefore not constrained by flyout time, the ABL has engagement range limitations caused by atmospheric effects and the durability of the target. The challenges are to preserve the focus of the high-energy beam on the target after propagation through the atmosphere and to maintain the beam on the target long enough to disable the missile. The time required varies greatly depending on the structural material used in the target missile.

\subsection{A Guide to the Report}

Part A of the Report provides an overview of boost-phase missile defense and compares it with other approaches to missile defense in the context of the anticipated threat. It first describes the analytical approach and key assumptions made in our analysis of boostphase intercept systems that would use terrestrial-based or space-based interceptor rockets employing the kinetic energy of a collision to disable or destroy the target missile. It then describes the analytical approach and key assumptions made in our analysis of potential utility of the Airborne Laser for boost-phase intercept of ICBMs. Part A analyzes the performance each of these three types of systems would require to defend the United States against missiles launched from three geographical locales and ends with a discussion of possible countermeasures to these approaches to boost-phase missile defense.

Chapters 2 and 3 frame the boost-phase defense problem in relation to alternative approaches to missile defense and describe the rationale for the missile models used in the Study. Chapters 4 and 5 analyze the basic requirements for engaging a missile in time to defend the United States and then examine the relationship between target missile characteristics, interceptor performance, and allowable basing areas for surface-based interceptors. Chapter 4 describes the engagement assumptions and analytical methods we adopted and develops the approach we used to simulate engagements. Chapter 5 uses these results to determine where the different interceptors would have to be based to defend the United States against ICBMs launched from North Korea, Iran, or Iraq. ${ }^{3}$ This chapter also discusses whether it is possible to avoid causing possibly live munitions to strike other countries.

In Chapter 6, the methods developed and some of the previous results are applied to analyze the feasibility of a global missile defense system employing space-based boost-phase interceptors. Here, the methodology previously used in analyzing terrestrial-based intercept

\footnotetext{
${ }^{3}$ As explained in the Executive Summary, although Iraq is not considered likely to pose an ICBM threat to the United States in the foreseeable future, the Study Group has retained the analysis of defense against ICBMs from Iraq to illustrate the requirements for defending against a country that is intermediate in size between North Korea and Iran.
} 
systems is applied to analyze the required performance and size of a system of space-based interceptors intended to defend the United States against ICBMs. This analysis shows how the total mass that must be launched into orbit depends on the number and size of interceptors required for coverage, which in turn depends on the burn time of the attacking missiles, the flyout capability of the interceptors, and the mass of the kill vehicle.

In these analyses, we assumed that if the kill vehicle could reach the target missile in time, the intercept attempt would succeed, deferring to Part B of the Report the question of the capabilities the kill vehicle would require to home on the interceptor and hit it, and resulting size and mass of the kill vehicle. This "best case" approach allowed us to determine the minimum requirements for intercepting different types of missiles and to establish the tracking geometric and measurement uncertainties and geographic constraints on engagements, including possible interceptor basing locations for specific scenarios.

Chapter 7 introduces the methodology for analyzing boost-phase engagements of liquidand solid-propellant ICBMs by the ABL. The performance parameters and their relationship to the engagement are discussed. Chapter 8 analyzes the missile-defense capabilities of the ABL in actual geographical scenarios comparable with the approach used for surfacebased intercepts in Chapter 5.

Part A concludes with Chapter 9, a discussion of countermeasures to boost-phase intercept likely to be encountered by kinetic-kill intercept systems and the ABL.

Part B addresses what it would take for a kinetic-kill boost-phase defense-regardless of its basing mode - to acquire, track, hit, and destroy a target missile. Chapters 10 through 14 summarize the sensor and kill-vehicle flyout and homing performance that would be required for terrestrial- and space-based interceptors to have a high probability of hitting an ICBM during its boost phase. Chapter 10 analyzes the potential performance of missile acquisition and tracking sensors. We estimated the uncertainties in determining the trajectory of an attacking ICBM caused by tracking system limitations and variations in the trajectory of the ICBM - both intended and unintended - when the kill vehicle relies on data from offboard and on-board sensors to maneuver to hit the target. We then analyzed the kill-vehicle performance - including divert velocity, acceleration, and guidance-and-control system response time - that would be required to hit a maneuvering ICBM. The issues associated with confidently disabling an ICBM are examined in Chapter 13. Based on these results, we were able to estimate the mass of a kill vehicle with the required performance. The mass of the kill vehicle ultimately determines the total mass of any hit-to-kill interceptor system.

In Part C, we describe our modeling of illustrative threat missiles in Chapter 15 and interceptors to defend against them in Chapter 16. These models were used in the analyses presented throughout the Report.

Part D of the Report explores the physics and technology requirements for successfully utilizing the ABL to disable ICBMs during their boost phase. The analysis of the ABL described there relies on some of the assumptions and portions of the analysis in Part A. Part D analyzes propagation of high-power laser beams through the atmosphere, lasertargeting issues, and the effective range of the ABL when used against various types of ICBMs, assuming that the planned performance of the ABL is achieved. An overview to the analysis of the ABL for boost-phase defense is presented in Chapter 17. Chapter 18 describes the operation of the laser itself, and Chapter 19 discusses the propagation of the laser beam through the atmosphere. The factors involved in disabling a missile using the ABL are described in Chapter 20, and ABL engagements are discussed in Chapter 21. Deployment issues are described in Chapters 22.

Appendices provide supporting information on the categories and characteristics of bal- 
listic missiles, how the missile and interceptor trajectories used in the Study were computed, the tracking of missiles during boost-phase engagements, and the propagation of the ABL's beam through the atmosphere. Glossaries of acronyms and technical terms are provided at the end of the Report.

\section{References for Chapter 1}

[1] Richard L. Garwin, Hearing of the Senate Foreign Relations Committee, May 4, 1999.http://www.access.gpo.gov/congress/senate/senate11sh106.html

[2] John Deutch, Harold Brown, and John P. White, "National Missile Defense: Is There Another Way?", Foreign Policy, p. 91, Summer 2000.

[3] President George W. Bush, speech at the National Defense University, Washington, D.C., May 1, 2001.

http://www.fas.org/nuke/control/abmt/text/treaty-abm-010501.htm

[4] Robert Wall, "Growing Interest Bolsters BPI", Aviation Week 6 Space Technology, August 13, 2001.

[5] Geoffrey Forden, "Laser Defenses: What if They Work?", Bulletin of the Atomic Scientists, v. 58, 48-53, 2002.

[6] Missile Defense Agency, Fiscal Year (FY) 2005 Budget Estimate, February 2, 2004.

[7] Stephen Daggett and Robert D. Shuey, "National Missile Defense: Status of the Debate," Congressional Research Service Report for Congress, 97-862 F, updated May 29, 1998. http://countingcalifornia.cdlib.org/crs/ascii/97-862

[8] National Intelligence Council, "Foreign Missile Developments and the Ballistic Missile Threat to the United States Through 2015," unclassified summary of a National Intelligence Estimate, December 2001. http://www.cia.gov/nic/pubs/

[9] Robert Walpole, Testimony by the National Intelligence Officer for Strategic and Nuclear Programs before the International Security, Proliferation, and Federal Services Subcommittee of the Governmental Affairs Committee, U.S. Senate, February 11, 2002, available at http://govt-aff.senate.gov/031102witness.htm .

[10] Donald Rumsfeld et al., "Executive Summary of the Report of the Commission to Assess the Ballistic Missile Threat to the United States," July, 1998.

http://www.fas.org/irp/threat-bm-threat.htm

[11] Robert H. Schmucker, "Engineering and Proliferation Analysis of Third World Theatre to Intermediate Range Ballistic Missiles," contribution to Year 2000 Multinational BMD Conference, Philadelphia, PA, 5-8 June, 2000 (unpublished).

[12] National Intelligence Council, "Foreign Missile Developments and the Ballistic Missile Threat to the United States Through 2015," unclassified summary of a National Intelligence Estimate, September 1999, available at: http://www.cia.gov/nic/pubs/

[13] National Intelligence Council, "Foreign Missile Developments and the Ballistic Missile Threat to the United States Through 2015," unclassified summary of a National Intelligence Estimate, December 2001, available at: http://www.cia.gov/nic/pubs/ 
[14] Robert D. Walpole, "The ballistic missile threat to the United States," speech by the National Intelligence Officer for Strategic and Nuclear Programs at the Carnegie Endowment for International Peace, 17 September 1998, available at http://www.cia.gov/cia/public_affairs/speeches/1998/walpole_speech_091798.html

[15] George N. Lewis and Theodore A. Postol, "Future Challenges to Ballistic Missile Defense," IEEE Spectrum, September 1997, p. 60.

[16] Andrew Sessler et al., "Countermeasures: A Technical Evaluation of the Operational Effectiveness of the Planned U.S. National Missile Defense System," Union of Concerned Scientists/MIT Security Studies Program, April 2000. http://www.ucsusa.org/global_security/missile_defense/page.cfm?pageID=586

[17] William A. Davis, "Why Missile Defense Will Work," Inside Missile Defense, October 3, 2001, p. 10.

[18] Richard L.Garwin, "Boost-Phase Intercept: A Better Alternative," ArmsControl Today, September 2000, p. 8.

[19] Theodore A. Postol, "Boost-Phase Missile Defense Concepts for Protecting the U.S. from Postulated Rogue-State ICBMs," 2001, unpublished.

[20] Hans Mark, "A White Paper on Defense Against Ballistic Missiles," The Bridge, Vol. 31, No. 2, Summer 2001, p. 17.

[21] Jeffrey A. Isaacson, "There's a Better Way to Missile Defense," Los Angeles Times, May 3, 2001, p. A13.

[22] Donald Rumsfeld, Memorandum on Missile Defense Program Direction, January 2, 2002, available at http://fas/org/ssp/bmd/d20020102mda.pdf

[23] James Dao, "Plan to Stop Missile Threat Could Cost $\$ 238$ Billion," New York Times, February 1, 2002.

[24] George R. Pitman, "Boost-Phase Intercept Missile Defense: A Critical Analysis," 2001, unpublished.

[25] Dean Wilkening, "Ballistic Missile Defense and Strategic Stability," Adelphi Paper No. 334, International Institute for Strategic Studies, London, 2000, p. 59.

[26] William J. Perry, San Jose Mercury News, September 10, 2000.

[27] Pugwash Workshop on Nuclear Stability and Missile Defense, Pugwash Occasional Papers, Vol. 2, No. 2, March 2001.

[28] Camille Grand, "Ballistic Missile Threats, Missile Defenses, Deterrence, and Strategic Stability," Mark Smith, "Missile Proliferation, Missile Defenses, and Arms Control," and James Clay Moltz, "Forecasting the Strategic-Military Implications of NMD Deployment," in International Perspectives on Missile Proliferation and Defenses, Occasional Paper No. 5, Center for Nonproliferation Studies, Monterey Institute of International Studies, March 2001. 
Part A. Boost-Phase Missile Defenses 



\section{Chapter 2}

\section{Overview of the Analysis of Boost-Phase Intercept Systems}

\section{Contents}

2.1 Boost-Phase Intercept Compared to Alternative Approaches S11

2.1.1 Boost-phase defense using hit-to-kill interceptors . . . . . . . . S12

2.1.2 Boost-phase defense using the Airborne Laser . . . . . . . . . . S13

2.1 .3 Criteria for success . . . . . . . . . . . . . . . . S13

2.2 Overview of the Analysis of Hit-to-Kill Systems _ . . . . S13

2.2.1 Walk-through of a hit-to-kill boost-phase engagement . . . . . S13

2.2.2 Analytical process: Hit-to-kill systems . . . . . . . . . . S16

2.3 Overview of the Analysis of the Airborne Laser . . . . S S21

2.3.1 Walk-through of an Airborne Laser boost-phase engagement . S21

2.3.2 Analytical process: Airborne Laser defense . . . . . . . . . . . S21

2.4 Key Issues . . . . . . . . . . . . . . . . . S S22

2.4 .1 Hit-to-kill engagement timeline . . . . . . . . . . . . S22

2.4.2 Airborne Laser energy delivery . . . . . . . . . . . . . S24

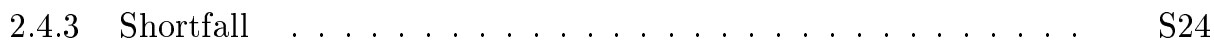

2.4.4 Disabling the booster or the warhead . . . . . . . . S24

2.4.5 Countermeasures . . . . . . . . . . . . . S25

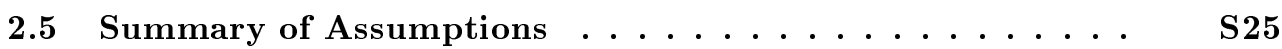

This chapter provides an overview of the boost-phase intercept problem and outlines the methods that the Study Group employed to analyze boost-phase intercept systems. This chapter also summarizes the assumptions made by the Study Group in its analysis, to facilitate comparisons with other studies that might arrive at different conclusions based upon different assumptions.

\subsection{Boost-Phase Intercept Compared to Alternative Approaches}

An ICBM is a multi-stage rocket having a payload that includes the missile's weapons and may also include devices intended to help the weapons penetrate any defenses. The weapons may consist of one or more nuclear warheads or dozens of submunitions ("bomblets") filled 
with chemical or biological warfare agents. The ICBM accelerates its munitions until they are moving in the proper direction with the speed required to reach the target. The total duration of an ICBM's boost phase is typically 3 or 4 minutes.

When the final stage of a full-range ICBM shuts down or burns out, its altitude is typically 150 to $200 \mathrm{~km}$. At this altitude the missile is well outside the atmosphere, and aerodynamic forces are negligible. If it has only a single warhead, the warhead is usually deployed when the final stage shuts down or burns out, but it could be deployed before the missile's boost phase ends. If the ICBM carries multiple warheads, these could be deployed when the final stage shuts down or burns out, or by a post-boost vehicle that separates from the final stage and then maneuvers using small, on-board thrusters. Single or multiple warheads or munitions could also be deployed while the final stage is still in powered flight. ${ }^{1}$

From the time an ICBM's munitions are deployed until they re-enter the atmosphere, the only force acting on them is gravity, and thus they travel through space on ballistic trajectories. During this so-called midcourse phase, which typically lasts about 25 minutes, they can be tracked and their future positions predicted accurately. The terminal phase of their flight begins when the munitions re-enter the atmosphere at an altitude of roughly $100 \mathrm{~km}$. The terminal phase lasts only a minute or two.

Each of the three phases of missile flight-boost, midcourse, and terminal — is best addressed by different types of missile defense systems, each of which has advantages and disadvantages. In recent years the focus of the U.S. program has been on midcourse intercept. The major advantages of midcourse intercept are that the midcourse lasts a relatively long time, and the midcourse trajectory of the warhead or other munitions can be determined and predicted accurately. In addition, the long time available to midcourse interceptors to engage their targets allows them to fly long distances. Consequently midcourse interceptors sited at just a few locations could defend the entire nation. However, midcourse-intercept systems face several serious challenges. An attacking missile could deploy dozens of separate submunitions carrying chemical or biological weapons capable of surviving re-entry, which would overwhelm the defense. An attacking missile could also deploy decoys and other countermeasures to confound the defense.

Intercepting warheads or other munitions during the terminal phase of their flight has the advantage that aerodynamic forces quickly separate light decoys from heavy munitions. A terminal defense system could defend a very small area against munitions launched from anywhere in the world, but every defended area would require its own system of radars and interceptors. And, as with midcourse intercept, deployment of multiple warheads by the attacking missile would complicate the task of the defense, while deployment of submunitions would require an unacceptably large number of interceptors.

The major attraction of boost-phase intercept is that it could in principle avoid the problems posed by multiple warheads, submunitions, and decoys by intercepting the missile before it deploys them. In contrast to a terminal defense, a boost-phase defense could defend the entire United States against attack from each missile launch area covered by the defense.

\subsubsection{Boost-phase defense using hit-to-kill interceptors}

Because of the short time between detection of the launch of a potentially threatening rocket and the end of its powered flight, the interceptors of a hit-to-kill boost-phase defense sys-

\footnotetext{
${ }^{1}$ The warheads of one of the earliest U.S. long-range missiles, the Polaris A3, were deployed during powered flight.
} 
tem would have to be based close to the powered flight portion of the missile's trajectory. Consequently, unless they were space-based, interceptor rockets could intercept only those missiles launched from relatively small countries that border international waters or have neighbors willing to host interceptor bases. Some view this requirement as a significant advantage of systems that use terrestrial-based interceptors, because these would not threaten the land-based nuclear missile forces of Russia and China. On the other hand, such systems could not defend against accidental or unauthorized launches of Russian or Chinese ICBMs.

\subsubsection{Boost-phase defense using the Airborne Laser}

The Airborne Laser was originally designed for defense against theater ballistic missiles, but it is now being considered for use in defense against ICBMs. Because it delivers its energy at the speed of light, the short time available to intercept a missile during its boost phasewhich is a serious challenge for rocket interceptors - is not an issue. Instead, the challenge is to deliver sufficient energy to disable the missile. Whether this is possible depends on the laser's performance and the ability of the beam to propagate through the atmosphere. The ABL's ability to fire at a missile soon after it has been launched provides options for defense not available to hit-to-kill systems. However, the relatively modest range of the ABL limits its effectiveness against ICBMs.

\subsubsection{Criteria for success}

The primary criterion for success of a boost-phase intercept system is whether it can prevent live munitions from falling on the area to be defended. A second criterion, whether the defense can avoid causing live munitions to strike other areas of the United States or on U.S. friends and neighbors, may also be important. Thus, the criteria for success depend on what areas the system is supposed to defend. We considered four possible objectives for a U.S. boost-phase defense system, and the performance required to achieve each objective:

- Defense of all 50 states.

- Defense of the contiguous 48 states.

- Defense of major cities within the contiguous 48 states.

- Defense of Hawaii.

We turn now to a description of the analytical process we used to evaluate both hit-to-kill systems and the ABL for boost-phase intercept of ICBMs.

\subsection{Overview of the Analysis of Hit-to-Kill Systems}

We describe here the elements of a hit-to-kill boost-phase defense system and the methods by which we analyzed its essential elements. To provide a context for the description, we start with a walk-through of a hit-to-kill boost-phase engagement.

\subsubsection{Walk-through of a hit-to-kill boost-phase engagement}

The elements of a boost-phase intercept system can be described by considering a hypothetical engagement by a defense system that utilizes hit-to-kill interceptor rockets. In the 
following account, a single interceptor hits a single target missile. In reality, an attacker would probably launch several missiles at once. To increase the chance of success, the defense would probably fire two or more interceptors at each missile.

Standby mode The boost-phase defense system would normally be in a standby mode in which the system is "cocked", but the interceptor is not "aimed". Earth would be under continual surveillance by infrared sensors on early-warning satellites. These sensors would continuously search for a bright spot that might indicate the exhaust plume of a large rocket rising through the upper atmosphere.

Detection of a rocket launch Current space-based missile-warning sensors monitor Earth in specific infrared wavelength bands, where infrared radiation is strongly absorbed by water vapor in the atmosphere. This absorption prevents the sensors from "seeing" the heat of fires on land, glints of sunlight off ocean waves, and sunlight that is reflected from most cloud tops. Consequently, current early warning satellites usually do not detect the radiation from the exhaust of a rocket rising through the atmosphere until it has reached an altitude of about $10 \mathrm{~km}$. Future space-based early-warning sensors may monitor Earth in wavebands where they could see to the ground on a clear day. However, the cloud cover over midlatitude launch sites is sufficiently frequent and heavy that such sensors would not see the exhaust of a large rocket with high probability until it had reached an altitude of about $7 \mathrm{~km}$. Liquid-propellant ICBMs attain this altitude about 40 to $50 \mathrm{~s}$ after launch; solid-propellant ICBMs, which rise more quickly, attain this altitude about $30 \mathrm{~s}$ after launch.

A bright spot is not necessarily the signature of a missile; it might, for instance, come from the afterburner of a jet airplane. The unique signature of a rocket is its high speed. By analyzing for about 15 to $20 \mathrm{~s}$ the data on the motion of the bright spot that could be provided by a modern space-based infrared missile-tracking system, the existence of a large rocket in powered flight could be confirmed and the rocket's direction of motion could be roughly estimated. However, in some situations it would not be possible to determine from the tracking data before an interceptor would have to be fired whether the rocket indicated by the bright spot was an innocuous space launch or was instead an attacking missile.

Deadline for the intercept The last moment for intercepting an ICBM aimed at the United States during its boost phase depends on the characteristics of the ICBM, its target, and its trajectory. Interceptors must be based close enough to the missile's expected flight path to reach the missile before its munitions have sufficient velocity to reach the area to be defended, regardless of the trajectory the missile follows. The possible locations for interceptor bases are determined by this constraint, the known performance of the interceptors, and the earliest time after the missile has been launched that an interceptor could confidently be fired.

From the moment the signature of a potentially threatening rocket was detected, data from space-based sensors and possibly from ground- or air-based radars would be accumulated. The rocket's trajectory up to its current position and velocity would be repeatedly updated with increasing refinement as new data were received. Before interceptors could be fired, sufficient tracking information would have to be collected to construct a firing solution.

A firing solution tells the interceptor how to fly from its base to reach the potentially threatening rocket at the desired time by estimating the future behavior of the rocket during 
the remainder of its powered flight and the uncertainty in this estimate. The interceptor must be fired at a time and in a direction that will permit it to reach the target rocket no matter what path the rocket follows. For a large rocket, the earliest a firing solution may be available is 15 to $20 \mathrm{~s}$ after the rocket has been detected.

Firing the interceptor Firing an interceptor the instant a firing solution is obtained is the fastest response possible. In most situations, interceptors are unlikely to be fired until the situation has been assessed for some additional time, which we refer to as the decision time. As the required decision time increases, the possible interceptor basing locations rapidly decrease, but the defensive system's knowledge of the nature of the threat improves. In determining possible interceptor basing locations for a given boost-phase defense system, we considered the limiting case of firing interceptors with zero decision time and, alternatively, firing them with a decision time of $30 \mathrm{~s}$. From the results for zero and 30-s decision times, we were able to estimate the decision time that would actually be available in various geographical scenarios.

Interceptor boost The interceptor is a multi-stage rocket that first accelerates and then deploys its kill vehicle, which flies onward to intercept the target rocket. As the interceptor flies out toward the predicted intercept point, it is guided by its inertial guidance system and possibly also by an on-board Global Positioning System (GPS) receiver. In addition, it receives regular updates from remote sensors that track its position and that of the target rocket. Using these updates, the interceptor continually revises the predicted intercept point, taking into account the changes in the trajectory of the target missile, and adjusts its course accordingly, until its final stage burns out.

Flight of the kill vehicle The kill vehicle is a small, highly maneuverable rocket-propelled stage that would have a sophisticated guidance system and sensors that would enable it to home on a target rocket. The sensors might include infrared and ultraviolet detectors, which would detect and track radiation from the target's plume, and LIDAR, a radar-like system that would employ a laser beam to enable the kill vehicle to home on the body of the target rocket. At the altitude at which surface-based boost-phase interceptors typically burn out, the atmosphere is still too dense for a kill vehicle to begin operating. As they coast upward, the interceptor and its kill vehicle would continue to receive updates from remote sensors on the position of the target, including changes in its trajectory. However, the kill vehicle cannot adjust its trajectory until it has reached a sufficiently high altitude (typically 80 to $100 \mathrm{~km}$ ) to begin to operate autonomously. At this point, it would separate from the final stage of the interceptor and begin maneuvering to try to collide with the target rocket.

The kill vehicle would use the information provided by its homing sensors to refine its estimate of the position of the target rocket and determine what maneuvers would be needed to intercept it. By the time it has closed to within several hundred kilometers of the target rocket, the kill vehicle must detect the rocket's body (as distinguished from its much larger plume) and begin to home on it. A missile in powered flight is not easy to hit because its acceleration changes abruptly as each stage burns out and the next ignites. Furthermore, the acceleration and velocity of a missile can vary greatly as it performs trajectory-shaping or energy-management maneuvers. A missile can also maneuver as a countermeasure, changing its acceleration in the final seconds before collision would have occurred. To assure a high 
probability of success, the kill vehicle must be able to accelerate at $15 \mathrm{~g}$ or more in the final moments. The required performance of the kill vehicle determines its size and mass, which in turn determines the size and total mass of the entire interceptor.

\subsubsection{Analytical process: Hit-to-kill systems}

To gain insights into the dynamic relationships involved in hit-to-kill boost-phase engagements and their implications for system requirements, we conducted an end-to-end analysis of representative engagements, from the launch of a threatening ICBM until the kill vehicle either hit the ICBM or missed it. The analysis began with the selection of illustrative scenarios based on recent National Intelligence Estimates (see Chapter 3), namely, the launch of an ICBM from one of the three countries considered: either North Korea, Iraq, or Iran. From these scenarios, we derived top-level assumptions about the objectives and tactics of offense and defense to the extent they would influence the engagements. These are discussed later in this chapter.

Using the illustrative scenarios and related assumptions, we developed a global geographical picture that reflected possible ICBM launch areas and the locations of their potential targets. Next, we established the ranges and firing azimuths associated with ballistic missile threats for each scenario. Figure 2.1 illustrates possible trajectories from North Korea and the Middle East to cities in the United States. In both cases, the launch azimuths of trajectories to major U.S. cities span about 40 degrees of arc.

Modeling of representative threat missiles Representative missiles with ranges great enough to reach the United States and similar to those that might be deployed in the future by countries of concern were modeled and their flight simulated in sufficient detail to establish realistic spatial and temporal trajectory characteristics under varying conditions during their boost phases and ballistic flight. The rationale for the selection and modeling of these missiles is discussed in detail in Chapters 3 and 15. The target missiles that were modeled have the characteristics that missiles based on 30- or 40-year-old technology would have. We included missiles utilizing liquid propellants, which are likely to be used in the first long-range missiles deployed by the countries of concern. Because it would take approximately 10 years to deploy boost-phase intercept systems, and the U.S. intelligence community has concluded that North Korea and Iran could develop or acquire solid-propellant ICBMs within the next 10 to 15 years, we also included solid-propellant missiles. Most of our analysis considered defense against long-range missiles, but we also modeled short-range ballistic missiles, which have also been judged to be a potential threat to the United States if launched from an off-shore ship.

Synthesis of preliminary defense architectures With these models of notional threat missiles in hand, we synthesized several preliminary conceptual architectures of boost-phase defense systems utilizing terrestrial-based interceptors. A similar approach was used to formulate the space-based architectures studied in Chapter 6. The system architectures we studied generally reflect concepts that have been proposed by advocates of boost-phase intercept. In analyzing the performance of these systems, we assumed they would have acquisition and tracking sensors similar to those known to exist or that are in the process of being developed.

We created computer models of interceptors having a variety of speeds and sizes to investigate different scenarios. These ranged in size from an interceptor judged to be have 

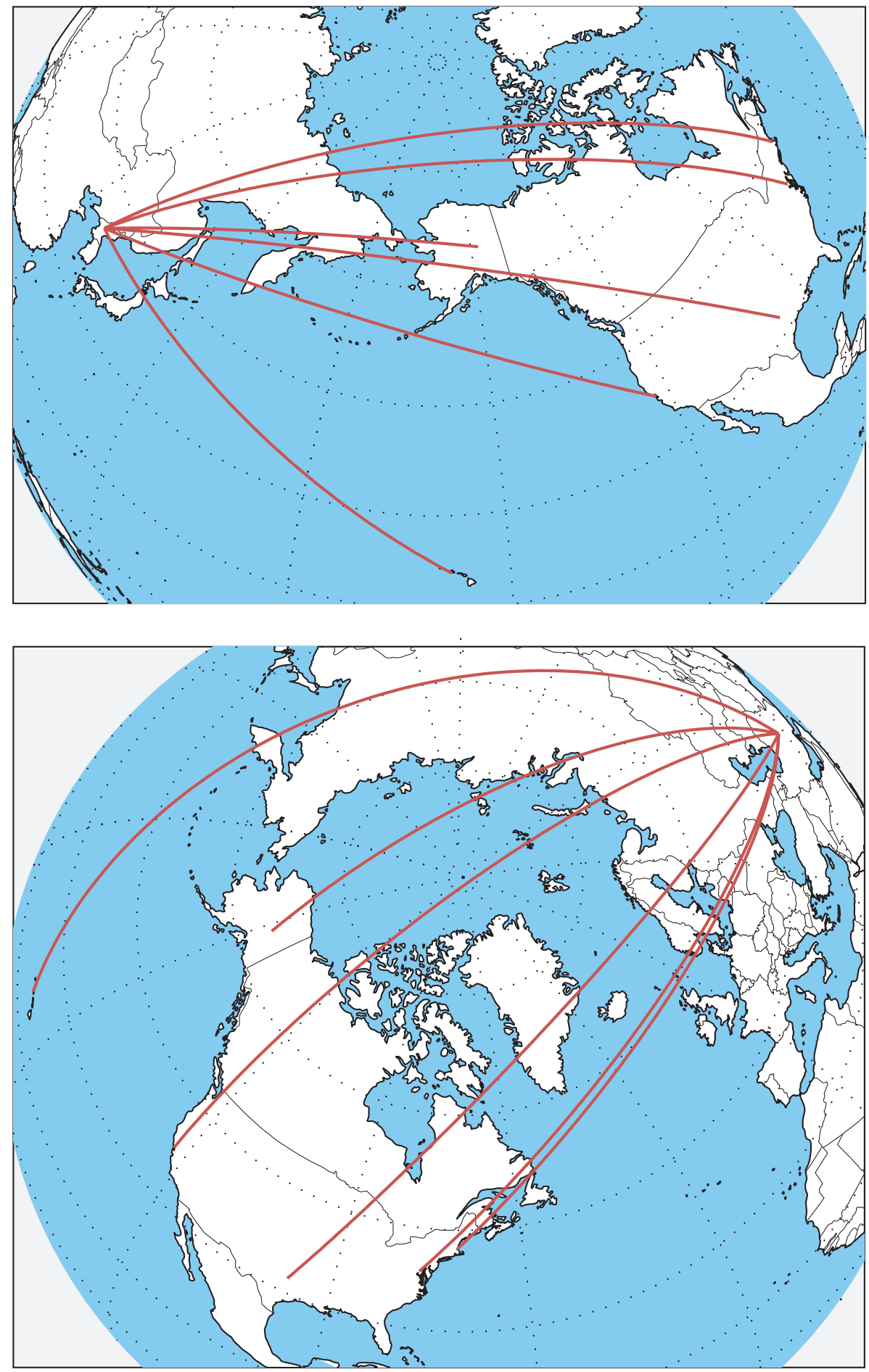

Figure 2.1. Great circles from North Korea (top) and Iraq or Iran (bottom) to Boston, Washington, D.C., Dallas, San Francisco, Fairbanks, and Honolulu. 
the highest performance that could be deployed in an existing ship-based launching system or in an air-based launcher, to one larger than an ICBM with a flyout velocity of $10 \mathrm{~km} / \mathrm{s}$, greater than the velocity required to achieve orbit (see Chapter 16). The 10-km/s interceptor was included to explore the upper bound on the feasible performance of boost-phase systems using interceptors.

In addition to these interceptors, we considered an interceptor similar to the U.S. Navy's existing Standard Missile 2 as a possible counter to medium- or short-range ballistic missiles launched from ships or other platforms off U.S. coasts.

Using these threat objectives, trajectories, and first-order physical constraints on sensors, such as radar horizons and cloud cover, we studied the kinematic requirements for an interceptor to engage the notional threat missiles at various times during their boost phase. To establish when an intercept should occur during the boost trajectory of the threat missile, the impact range as a function of cutoff time was determined for each of the threat trajectories modeled. The earliest and latest desired intercept times were determined for each scenario and threat missile, based on assumptions about areas to be defended and areas where munitions and debris from the intercepted missile should not fall (Chapter 5).

From this preliminary analysis, we were able to choose a range of interceptor flyout capabilities based on our initial estimates of the performance required to meet the geographical constraints of each scenario, as well as the performance requirements that have been suggested by advocates of specific boost-phase intercept concepts. We then considered a variety of engagement scenarios for the target missiles and interceptors launched from geographically reasonable launch sites that reflected the ranges and viewing capabilities of the detection and tracking sensors. The outputs of this initial analysis were (1) nominal timelines for engaging the various threats, (2) engagement geometry variations, and (3) expected closing velocities. This analysis is discussed in Chapter 4.

Up to this point the engagement analysis assumed a perfect endgame - the kill vehicle had sufficient sensor and maneuver capability to achieve an intercept when placed on a ballistic path to the predicted intercept point. It also initially assumed that perfect threat acquisition and tracking sensors would acquire the threat after physical viewing constraints, such as those imposed by radar horizons and cloud cover, were met. The interceptors were given a nominal kill vehicle size and mass at this point to allow a characteristic area for drag calculations to be established for simulating realistic constraints on interceptor flyout trajectories. The interceptor performance capabilities were later adjusted if necessary as the engagement analysis proceeded. The interceptor sizes were also adjusted later to reflect the results of the kill vehicle analysis summarized in the next section and detailed in Chapter 12 .

Using the results of this initial investigation as input, we divided the further analysis into three parallel tracks to estimate the basic requirements for each element of the system:

1. The off-board sensor performance required for acquisition and tracking.

2. The interceptor reach and basing required to meet the geographic constraints imposed by each scenario.

3. The on-board sensor, guidance, control, and maneuver capability required for the kill vehicle to deal with tracking and threat uncertainties and successfully intercept the target.

Acquisition and tracking analysis The speed with which missiles can be identified and the precision with which they can be tracked are critical to the effectiveness of any boost- 
phase intercept system. Thus, detecting and tracking sensors play essential roles at every stage of the intercept. The acquisition and tracking analysis, therefore, provided key inputs for the other two parts of the analysis. From the initial analysis, we could model the evolution of the engagement geometry in time, and knowing this, we could evaluate the systematic and random measurement uncertainties expected from representative sensors. We determined the minimum interceptor firing time that is required for the geographic analysis by examining detection thresholds for the notional space-based infrared sensors and the minimum time needed to establish an acceptable track for each ICBM type.

Based on initial estimates of the engagement geometry and postulated sensor characteristics for each scenario, we made estimates of a number of key parameters, including tracking precision and uncertainties for threat state-vectors. ${ }^{2}$ The resulting measurement uncertainties for the trajectory were then used in the third portion of the analysis - examining the kill vehicle midcourse and endgame requirements, which depend on the combined effects of measurement uncertainties and actual threat trajectory variations.

Constant surveillance of all potential launch areas is essential for detecting the launch as early as possible. We considered two types of surveillance sensors for detecting missile launches: short-wavelength infrared sensors based high in space and on aircraft, and radars based on the ground and on aircraft. We analyzed the capabilities of current and possible future space-based sensors for detecting and tracking ICBM exhaust plumes (Chapter 10). We also considered the missile-tracking capabilities of a variety of land-based, ship-based, and airborne radars and analyzed the likely near-term performance of passive infrared and active LIDAR seekers on the kill vehicle. For the ranges likely to be required for boostphase interceptors to defend the United States against long-range missiles, we concluded that space-based sensors are the only viable option for detecting the launch quickly; the horizon would block the view of the other three sensor options until too late in the threat missile's flight.

The existing DSP (Defense Support Program) surveillance system was examined. However, the low scan rate, once every 10 seconds, discourages its use in a boost-phase intercept system. A replacement for DSP is planned, the SBIRS-High system. Because the properties of SBIRS-High are not in the public domain, we constructed a notional space-based sensor system based on technology that is currently available or that seems plausible for deployment within a few years. The analysis is based on this system, which was found to provide about the same accuracy as a radar once the threat was above the horizon.

A critical part of the tracking problem is the final stage of the engagement when the kill vehicle has separated from its booster. In addition to the tracking data provided by off-board sensors, the capabilities of the kill vehicle's own sensors, which may include infrared detectors, optical-imaging systems, and LIDAR-ranging systems, are important in analyzing the kill-vehicle guidance and control problem. Several possible kill-vehicle homing sensors were considered (Chapter 10) and their capabilities used in analyzing kill-vehicle performance requirements (Chapter 12).

Geographic analysis for these scenarios We used the results of our analysis of engagements (Chapter 4) to examine the geographical constraints in our illustrative scenarios. Engagements of ICBMs launched from North Korea, Iraq, and Iran were analyzed to determine the geographical areas within which interceptors would have to be based to disable a missile before it could give its munitions sufficient speed to reach the United States. We

\footnotetext{
${ }^{2}$ The state is specified by the vector components of the missile's position, velocity, and acceleration.
} 
considered potential ICBM launch sites and examined the effects of specific geographic constraints on the feasibility of using each of the model interceptors in each scenario. As part of this analysis, we examined the effects of key assumptions, such as the time delay before firing the interceptor, areas to be defended, and intercept debris impact constraints. We also varied these key assumptions to explore the sensitivity of our results to changes in our baseline assumptions about all phases of the engagement and to assist in understanding the effects of potential countermeasures. Chapter 5 derives and summarizes the conclusions of the Study concerning the feasibility of boost-phase intercept utilizing terrestrial-based interceptors.

Kill-vehicle requirements for boost-phase engagements The kill-vehicle analysis examined what would be required of the kill vehicle to handle the two primary uncertainties: measurement errors from the off-board and on-board sensors and unexpected variations in the trajectory of the target missile. Together, these uncertainties establish the maneuverability the kill vehicle would require during the divert and endgame phases of an engagement to intercept the target missile. The key parameters are the kill vehicle's velocity change capability, acceleration, and response time.

Estimates of closing geometries and velocities derived from the engagement analysis provided the initial conditions for the kill-vehicle performance analysis. This part of the Study explored parametrically the relationship between the velocity change and acceleration capabilities of the kill vehicle and the resulting miss distance, for different guidance schemes. We analyzed the performance requirements for the kill vehicle and the off-board and onboard sensors by first modeling the errors expected in tracking large rockets using spacebased infrared sensors, surface- and air-based radars, and passive infrared and active LIDAR sensors on the kill vehicle, based on the analyses of these sensors (Chapter 10). Using these error models, we numerically simulated a variety of engagements to explore the dependence of the required total kill-vehicle velocity change on the precision of the off-board missiletracking sensors, and the dependence of the miss distance (relative to the aim point) on the precision of the on-board homing sensors, the closing velocity, and the kill vehicle's agility. The model of the kill vehicle incorporated the guidance algorithm, the latency in the kill vehicle's information about the target missile, the delay in its dynamical response to the accelerations commanded by the guidance system, and its maximum acceleration. We studied the effects of typical trajectory-shaping and evasive maneuvers by the target missile. Performance requirements for the off-board sensors and the kill vehicle were estimated by requiring the miss distance to be small enough to ensure a collision of the kill vehicle with the rocket body. This analysis is described in Chapter 12 and Appendix C.

Size of the kill vehicle The size of the kill vehicle is driven by two sets of parameters: first by the total velocity change capability required during the homing phase and the acceleration required during the endgame, and second by the technology that can be confidently brought to bear in the on-board sensors and avionics. We determined the size of the kill vehicle that would be required by utilizing our analysis of kill-vehicle performance requirements (Chapter 12), estimating the mass of each required component, and combining them. This analysis is described in Chapter 14. 


\subsection{Overview of the Analysis of the Airborne Laser}

The Airborne Laser is a directed-energy weapon that delivers its energy at the speed of light. In analyzing the possibilities for boost-phase defense against ICBMs using the ABL, we used many of the same elements employed in the analysis of hit-to-kill interceptors, including the suite of model ICBMs and the times by which their acceleration would have to be terminated to protect the United States. However, the crucial issue of whether an interceptor could reach the missile within the required time is not relevant to an ABL defense. Instead, the key issue is whether the ABL can deliver enough energy to the target missile to disable it. A target ICBM could be engaged any time after it has risen sufficiently high in the atmosphere until the latest time to intercept. The success of an ABL engagement depends on the power of the laser, the properties of the laser beam, the distance to the target, the energy required to disable the target, and the ability of the ABL to focus its energy on the target for the required time in the presence of atmospheric fluctuations. An overview of the analysis of the ABL is presented in Chapter 17.

\subsubsection{Walk-through of an Airborne Laser boost-phase engagement}

The scenario for detecting a rocket's launch and establishing its trajectory with sufficient accuracy to support an ABL engagement is similar to that of a hit-to-kill engagement. One difference is that the ABL could either acquire this information itself or receive it from external sources. The ABL's sensors may be able to acquire tracking information earlier than the space-based surveillance system, but doing so would not affect the engagement time, because the ICBMs considered in the Study could be engaged only when the missile has risen to an altitude of at least $30 \mathrm{~km}$.

The ABL uses three lasers - the tracking illuminator laser (TILL), the beacon illuminator laser (BILL), and the high energy laser (HEL). Once a missile is detected, the TILL would be directed to the nose of the missile to establish a geometric reference point. The images produced by the TILL and the BILL would be used for adaptive optics corrections to the HEL beam. The HEL beam is focused onto the target and dwells on the designated aim point for several seconds, until the energy density (the energy per unit area or fluence) that has been deposited on the missile body is great enough to cause structural failure.

\subsubsection{Analytical process: Airborne Laser defense}

Airborne Laser properties In analyzing the ABL, we adopted ABL performance characteristics based on the best publicly available information. However, because some of the technical specifications of the ABL are classified, a number of important parameters were unknown. These include the laser's power and the amount of energy required to disable a missile. Consequently, we made what we consider to be reasonable estimates and show the sensitivity of our results to changes in these estimates. In cases of doubt, we adopted the best-case scenario. The properties of the ABL are described in Chapter 18.

Airborne Laser engagement analysis As noted above, the performance of the ABL for boost-phase intercept of an ICBM would be insensitive to the time required to detect the missile and determine its direction of flight. To analyze the possibilities for boost-phase intercept by an ABL aircraft stationed at a given location, we computed the range from the ABL to the missile at which the ABL could be effective against both liquid- and solidpropellant missiles. Basic issues related to ABL engagements are described in Chapter 7. 
Airborne Laser energy delivery Our assessment of energy delivery by the Airborne Laser rests on theoretical analyses of beam propagation through the atmosphere and of the performance of adaptive optics. These issues are closely coupled to the problem of tracking the missile so that the laser beam could focus continuously on one spot. As the missile gains altitude early in its flight, the density of the air through which the beam must pass decreases, and atmospheric effects become less important. As the distance to the missile increases, the energy delivery is limited by the falloff of beam power density on the target. At large distances, the finite propagation time must be taken into account and can limit the capability of the adaptive optics system. Beam propagation is analyzed in Chapter 19, the requirements for disabling the missile are described in Chapter 20, and the interplay of missile position and energy delivery is analyzed in Chapter 21.

Airborne Laser engagements Analyzing a real ABL engagement involves first calculating the energy density delivered to the target by the laser as the missile accelerates along its trajectory. With this information, the maximum slant range from the ABL aircraft to the target missile can be determined throughout the engagement. From the slant range, the distance on the ground between the ABL and the missile, which is important for geographical considerations, can be computed. For the ABL performance we assume, the time the ABL beam must dwell on an ICBM to disable it is estimated to vary between 5 and $20 \mathrm{~s}$, depending on the missile's type and the distance from the ABL to the missile. The dwell time must be taken into account, because the target would move a significant distance during the engagement.

Geographic analysis of an ABL defense The area within which the ABL must be flying throughout the engagement is called the ABL flying area and is analogous to the interceptor basing area for a hit-to-kill engagement. Because an ICBM could be engaged any time after it has risen to an altitude greater than $30 \mathrm{~km}$ and the laser's range would vary with the altitude of the ICBM, the allowed flying areas would be oblong in shape even for a single ICBM trajectory, in contrast to the circular shape of the allowed interceptor basing area of a rocket interceptor for a single ICBM trajectory. ABL flying areas for ICBMs launched from North Korea, Iraq, and Iran are displayed in Chapter 8. Based on these flying areas, the possibilities for defending the United States against ICBMs launched from these countries are described.

\subsection{Key Issues}

\subsubsection{Hit-to-kill engagement timeline}

The pressure of time is the overriding consideration for any boost-phase intercept system that utilizes rocket interceptors. The time available for the interceptor to reach the target is determined on the front end by how quickly a firing solution can be generated. It is determined on the back end by the latest time at which the interceptor must hit the missile to ensure that its munitions cannot strike any point in the defended area, no matter which of its many potential trajectories the missile follows.

Based on our tracking analysis, we concluded that an adequate firing solution for the interceptors could be constructed once the direction of the velocity vector of the target rocket is known to within 7 degrees, provided in-flight updates were continued during the interceptor's boost phase. Using estimates of the possible capabilities of the next generation 


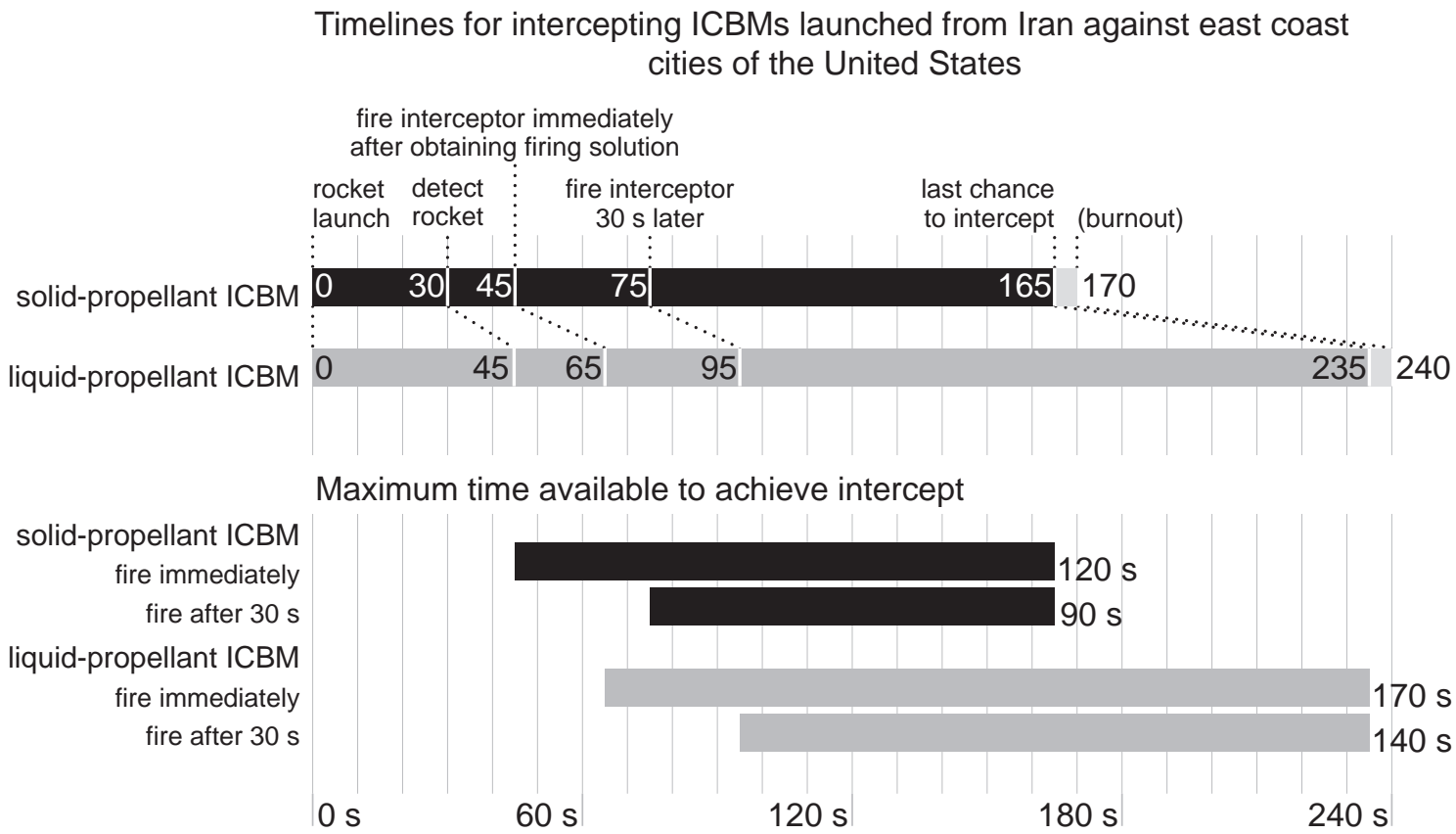

Figure 2.2. Top: Timelines for engaging liquid-propellant ICBM model L and solid-propellant ICBM model S1 when launched from Iran against cities on the East Coast of the United States. Bottom: Maximum times available to complete the intercept. All times are rounded to five seconds.

of missile warning and tracking satellites, we calculated the time that would be needed after the infrared signal of a rocket had been detected to determine that the signal indeed came from a large rocket that could be a long-range missile, and to estimate the rocket's direction of flight with this precision, i.e., within 7 degrees. These times are $20 \mathrm{~s}$ for the liquid- and $15 \mathrm{~s}$ for the solid-propellant missiles under consideration. We took the sum of the detection time and the additional time needed to construct a firing solution as the earliest possible time that an interceptor could be fired. This time would be $65 \mathrm{~s}$ after the liquid-propellant ICBM was launched or $45 \mathrm{~s}$ after launch of either solid-propellant ICBM.

In most circumstances the defense is likely to want additional time to assess the situation before firing. (At the minimum firing time, the defense might not be able to determine whether the rocket that had been launched was an ICBM, a space launch, or a theater ballistic missile, or even whether it was a liquid- or a solid-propellant rocket, differences that would strongly affect the predicted intercept point.) To investigate the effects of delaying the decision to fire an interceptor, we also analyzed system performance for a 30second decision time that would, for example, allow the defense to see the burnout of the first boost stage of most solid ICBMs and would therefore help in typing the target missile.

Within the available time, the interceptor would have to fly toward the projected intercept point and deploy the kill vehicle, which would then have to fly onward toward the projected intercept point, home on the body of the missile using its on-board sensors, and hit the missile. During its fly out, the interceptor and then the kill vehicle would have to respond to maneuvers of the target missile using data from off- and on-board sensors. Figure 2.2 shows the timelines for defending the United States against a notional liquid- and a notional solid-propellant ICBM launched from Iran against cities on the East Coast of the 
United States. The entire East Coast is within range of the three model missiles, which all have ranges of $12,000 \mathrm{~km}$, and, as discussed in Chapter 5, is a likely target. Timelines are shown for both zero and a 30-second decision time.

\subsubsection{Airborne Laser energy delivery}

A key issue for the ABL is the energy per unit area that must be delivered to the target ICBM to cause structural failure. The energy density required to disable a solid-propellant ICBM is considerably greater than that required for a liquid-propellant ICBM, because of their different mechanical construction. A second key issue is whether the ABL can deliver sufficient power, focus it on the target, and maintain focus on the aim point long enough to induce failure. The actual laser power the ABL can produce is classified, but we have made a reasonable estimate of it. The ability of the ABL to focus the beam depends on how close the beam is to its diffraction limit and the ability of the ABL to correct the beam for distortions induced by atmospheric inhomogeneities. We have estimated the dwell time that is possible, which is set by practical considerations.

We analyzed the sensitivity of our results to variations of these parameters and find our results to be robust.

\subsubsection{Shortfall}

With respect to defending the United States, it makes no difference whether intercept merely causes the target missile to lose propulsion or also disables its munitions: in neither case will munitions strike the United States. However, if the missile's munitions are not destroyed, the intercept would have to be carefully timed to avoid causing potentially live munitions to strike other nations. This problem is discussed in Chapter 5. To avoid causing munitions to strike other countries, intercepts may have to be timed to occur with in a time window as narrow as 10 to $20 \mathrm{~s}$, and in some cases even less. It is unclear whether this is possible, given the difficulty of predicting the exact future position of an ICBM as a function of time during its boost phase, even if the ICBM does not execute any trajectory-shaping or evasive maneuvers.

Giving the kill vehicle the capability to change its flyout speed enough to delay or hasten an intercept would improve the ability of the defense to time an intercept but could significantly increase the kill vehicle's size, and thus the size of the entire interceptor. Without a detailed analysis of the uncertainties in generating a firing solution and predicting the target missile's trajectory, there is no way of determining whether there is a solution to this problem. We are not aware of any systematic analysis of this problem in the publicly available literature.

Boost-phase intercept using the ABL has the same problem, but for a different reason. The dwell time of the laser on an ICBM that would be required to disable it is longseconds - and it is difficult to know exactly when during the dwell the missile would lose thrust.

\subsubsection{Disabling the booster or the warhead}

As explained above, having the ability to disable an attacking missile's warheads or submunitions would be crucial if a goal of the boost-phase intercept system is to avoiding causing possibly live munitions to strike countries other than the United States. Having the ability 
to disable an attacking missile's munitions would also be desirable if the boost-phase intercept system is the first segment of a layered missile defense system, provided this can be done in a way that decreases the burden on the succeeding layers of the system.

It is much more difficult for hit-to-kill interceptors to destroy a missile's warheads or submunitions than to disable its booster. Lacking access to any data on tests that might have been carried out on collisions between kill vehicles and missiles in space at the high closing speed - typically $10 \mathrm{~km} / \mathrm{s}$ - of an intercept, the Study Group was unable to analyze the likelihood that such a collision would destroy a missile's warheads or submunitions. However, a missile's warhead is much more durable than its booster, to which it is usually coupled only loosely, and there is ample evidence from intentional and accidental destruction of missiles during testing to support the view that the warheads will survive catastrophic booster destruction. The Airborne Laser would have no ability to destroy warheads or submunitions.

If causing live warheads to strike other countries is a concern, the defense has three choices: design the boost-phase intercept system to destroy the warhead with high confidence, build a midcourse defense to intercept warheads and submunitions that would be capable of handling the possibly unpredictable nature of the debris cloud produced by a boost-phase intercept, or time the boost-phase intercept carefully so that the debris lands in the ocean, a problem that is discussed in Chapter 5.

\subsubsection{Countermeasures}

As with every defense system, countermeasures to a boost-phase intercept system could be developed. In the course of its analysis, the Study Group identified several sensitivities of boost-phase intercept systems that could potentially be exploited to degrade the effectiveness of such a defense. In addition, the Study Group identified several countermeasures that could be adapted from existing technologies and must be seriously considered. These are discussed in Chapter 9.

\subsection{Summary of Assumptions}

The conclusions of the Study follow from assumptions made by the Study Group. Because these are scattered throughout different sections of the Report, we summarize here those assumptions we judge to be most crucial to our findings. In the course of the Study, our key assumptions were revisited to investigate the sensitivity of our results to changes in them. Our principal assumptions are discussed below.

Defense posture The defense is on full alert and its missile warning sensors are operating in their surveillance mode, with the DSP early warning system or a modern space-based infrared sensor system tasked to detect launches from the geographical areas of concern.

Objective of the adversary The objective of the adversary is to use his weapons to deter the United States from taking some actions against his interests or to retaliate if the United States is not deterred. Consequently the adversary's targeting objective is to inflict large numbers of casualties without particular regard to where in the United States the casualties occur. Implied is the use of nuclear, chemical, or biological weapons without requiring that the delivery system strike a particular target with high accuracy. The defense therefore 
cannot presume to know a missile's target beyond what can be inferred from tracking data obtained during the early part of its boost phase.

Defense knowledge The defense's knowledge is not perfect. Other than general intelligence information it may have, it knows only what its sensors tell it about the nature of the attack and the attacking missiles. Because the attacker's objective may be to strike anywhere within the United States, the defense cannot presume to know a missile's target, beyond what can be inferred from tracking it during the early part of its boost phase. If the attacking missiles have been tested many times, as U.S. and Russian missiles have, the defense may know some of their performance characteristics, such as their average thrust profiles, but not the specific details of the attack.

Criteria for success Success requires that the defense prevent any munitions from striking the defended area. If the additional goal of avoiding causing munitions to strike other nations is adopted, the challenges for the defense would increase significantly.

Scenarios analyzed Guided by recent assessments by the U.S. intelligence community and other experts, the Study Group selected four geographic scenarios to frame the Study:

- A small country situated near international waters, using North Korea as the model.

- Two mid-size countries, using Iran and Iraq as the models.

- An attack by a short- or medium-range ballistic missile launched from a ship off a U.S. coast.

Area to be defended We examined the requirements for defending four areas: (1) all 50 states, (2) only the contiguous 48 states, (3) only major cities within the contiguous 48 states, and (4) only Hawaii.

Launch detection times Geographical areas of concern are continually monitored by modern, space-based see-to-the ground sensors. Given the cloud cover over mid-latitude launch sites, these sensors would have a high probability of detecting the exhaust of a large rocket only after it reaches an altitude of about $7 \mathrm{~km}$. We assume that any potentially threatening rocket is detected as soon as it reaches this altitude. The liquid-propellant ICBM reaches this altitude $45 \mathrm{~s}$ after launch; 20 more seconds are required to determine that the signal detected is being produced by a large rocket and estimate its direction of flight. The solid-propellant ICBMs, which accelerate more quickly, reach $7 \mathrm{~km} 30 \mathrm{~s}$ after launch; only 15 more seconds are required to determine that the signal detected is being produced by a large rocket and estimate its direction of flight.

Interceptor firing time The earliest time an interceptor can be fired (zero decision time) is $65 \mathrm{~s}$ after the liquid-propellant ICBM has been launched and $45 \mathrm{~s}$ after the solid-propellant ICBMs have been launched. These are the bounding cases. If the defense requires 30 more seconds to decide whether to fire, the earliest time an interceptor can be fired is $95 \mathrm{~s}$ after the liquid-propellant ICBM has been launched and $75 \mathrm{~s}$ after the solid-propellant ICBMs have been launched. No approval from the National Command Authority (with its associated time delay) would be possible in either case. 
Airborne Laser performance Critical performance parameters of the ABL are classified, but we have made assumptions - based on unclassified descriptions - that we believe are credible, although possibly optimistic. In particular, we have assumed a beam power of $3 \mathrm{MW}$, and that the adaptive optics system will perform as well as in the laboratory tests of scale models. We considered laser dwell times ranging from 5 to $20 \mathrm{~s}$. To determine the energy per unit area the ABL would have to deliver to the target missile to disable it, we have made simple estimates.

Criterion for disabling a target missile A body-to-body hit on a booster is assumed to disable the missile but not its warheads or submunitions. The ABL is assumed to be capable of disabling the booster of a missile, but not its warheads or submunitions, by supplying a sufficiently large amount of radiant energy to the missile's body. 



\section{Chapter 3}

\section{Ballistic Missile Threats to the United States}

\section{Contents}

3.1 Ballistic Missile Capabilities of Selected States _...... S32

3.1 .1 North Korea . . . . . . . . . . . . . . . . . . S33

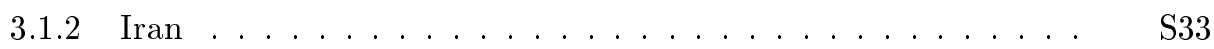

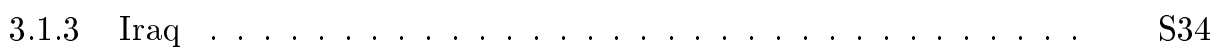

3.2 Historical Patterns of ICBM Development . . . . . . . S34

3.3 The Changing Context of Missile Development Programs $\quad$ S35

3.3.1 Differences in possible goals . . . . . . . . . . S36

3.3.2 Transfer of ballistic missile technology and systems . . . . . S S36

3.3.3 Spread of solid-propellant technology _. . . . . . . . . S S37

3.3 .4 Munitions . . . . . . . . . . . . . . . S37

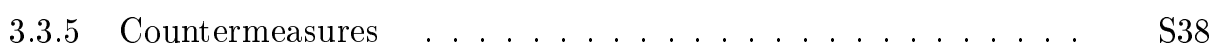

3.3 .6 Uncertainties . . . . . . . . . . . . . . . . $\quad$ S38

3.3.7 Forward-based sea-launch of Short-Range Ballistic Missiles (SRBMs) or Medium-Range Ballistic Missiles (MRBMs) . . . . . . S S39

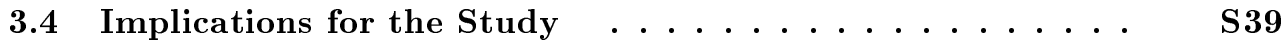

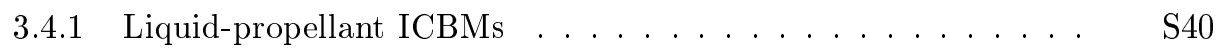

3.4 .2 Solid-propellant ICBMs $\ldots \ldots \ldots \ldots$ S . . . . . . . . . . . . . . . . . . . . . . .

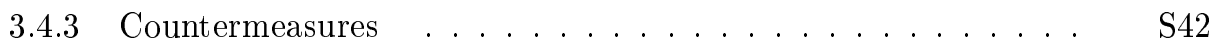

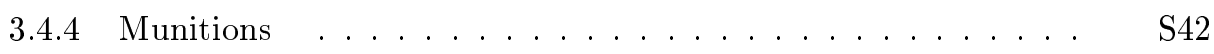

3.4 .5 Uncertainties . . . . . . . . . . . . . . S42

3.4.6 Forward-based sea-launch of SRBMs or MRBMs . . . . . . S S43

This chapter summarizes assessments by the U.S. intelligence community of the ballistic missile threat that the United States could face within the next 10 to 15 years and explains how these assessments were used by the Study Group in its analyses. Section 3.1 summarizes briefly the ballistic missile programs of North Korea, Iran, and Iraq. More detailed discussions of the status of these programs and those of other countries of concern may be found in the unclassified summaries of recent National Intelligence Estimates [29, 30] and in official statements to the U.S. Congress [31, 32] and private organizations [33, 34]. The historical evolution of the performance characteristics of the first long-range ballistic missiles deployed by the countries that have deployed such missiles is described in Section 3.2. The changed context within which countries of concern are currently developing long-range 
ballistic missiles is discussed in Section 3.3. Section 3.4 explains how this information was used by the Study Group in its analysis of boost-phase missile defense systems.

Russia and China are the only potential adversaries of the United States that possess long-range ballistic missiles capable of striking the United States. The U.S. intelligence community judges [34] that an unauthorized or accidental launch of a Russian or Chinese strategic missile is highly unlikely, as long as current security procedures and systems are in place. (Russia employs an extensive array of technical and procedural safeguards, and China keeps its missiles unfueled and without warheads mated [34], although this may change as it deploys solid-propellant ICBMs.)

Among the countries that have programs to develop long-range missiles, the U.S. intelligence community has expressed special concern about North Korea and Iran [30]. 1 The Study's emphasis on these countries reflects this concern; the Study Group itself has made no judgment about which countries or missile programs pose a risk to the United States. None of these three countries presently have an overt, credible, ICBM capability. However, as their missile programs advance, they may develop or acquire long-range ballistic missiles that could threaten the United States with nuclear, biological, or chemical weapons $[30,35]$. According to the most recent U.S. National Intelligence Estimate of the ballistic missile threat to the United States [30], most U.S. intelligence agencies projected that before 2015, the United States most likely will face ICBM threats from North Korea and Iran, and possibly from Iraq - barring significant changes in their political orientations - in addition to the longstanding missile forces of Russia and China.

A fundamental difference between the approach that emerging missile states are currently using to field ballistic missiles and the approach taken by the Soviet Union and the United States during the Cold War is that the newcomers are not as likely to place a high priority on accuracy, safety, reliability, survivability, and numbers [29]. Consequently, countries of concern may not conduct a series of tests before deploying a missile, but may instead deploy after as little as one flight test, as North Korea did with its No Dong missile [31].

Deployment times are being shortened substantially by foreign assistance and technology transfer. Evidence suggests that Russia, China, and North Korea are providing assistance to states of concern. Foreign assistance can play a pivotal role in the development of ballistic missiles [30, 35, 36, 37]. Ballistic missile systems could also be acquired by purchase and launched with little or no warning, according to the intelligence community and the Rumsfeld Commission [30, 36, 37]. The potential for significantly shortened development times and the possibility of little or no warning means that to be effective, a defense must be robust when confronted with missiles that have characteristics that differ significantly from the characteristics of previous missiles tested or deployed by a particular country.

This study focuses on the feasibility of boost-phase defenses against ICBMs that might be deployed by countries that have relatively new missile development programs and do not presently have friendly relations with the United States. As explained in Chapters 2 and 15, any analysis of the feasibility of such a defense must consider the performance characteristics of the missiles it is expected to confront. The Study Group used the information summarized in Sections 3.2 and 3.3 to guide its analysis of possible ballistic missile threats, the effects of uncertainties, and the effects of possible countermeasures to boost-phase defenses. The

\footnotetext{
${ }^{1}$ As stated in the Executive Summary, the Study Group also considered defense against ICBMs launched from Iraq. With the changed political situation arising from the events of the spring of 2003, an ICBM threat from Iraq seems unlikely for the foreseeable future. Nevertheless, we have retained the analysis of the history of Iraq's ballistic missile programs to illustrate the path to ballistic missiles it had taken and the types of threats that could emerge from other countries.
} 
basis for the choices made by the Study Group is explained in detail in Section 3.4. Here we summarize these choices and the reasons for them.

Development and deployment of a boost-phase missile defense system would be a major investment and would probably take a decade or more to complete. The Study Group therefore considered long-range missiles and countermeasures that might be fielded by countries of concern during the next 10 to 15 years, including missiles that might be acquired in response to prospective or actual deployment of a boost-phase missile defense by the United States.

Guided by the assessments summarized in Sections 3.2 and 3.3, the Study Group chose a liquid-propellant model ICBM having a 240-second boost phase as its baseline liquidpropellant threat missile. This computer model is similar to the first liquid-propellant ICBMs deployed by the Soviet Union and China. The Group also considered a liquidpropellant ICBM having a 300-second boost phase to facilitate comparison with the results of previous studies.

The Study Group concluded from the assessments summarized in Sections 3.2 and 3.3 that solid-propellant ICBMs may be deployed by countries of concern within the next 10 to 15 years, partly as a response to U.S. missile defense programs, and that solidpropellant ICBMs should therefore be considered in the analysis of boost-phase defense systems presented in this report. The Study Group constructed detailed computer models of two solid-propellant ICBMs, each with a boost phase of $170 \mathrm{~s}$. These computer models are based on 1960s missile technology and are similar to the first solid-propellant ICBMs deployed by the United States and the Soviet Union. The Study Group also considered briefly a solid-propellant ICBM having a burn time of $130 \mathrm{~s}$, to explore the implications for boost-phase defense systems of faster-burning ICBMs.

As discussed in Section 3.3, the U.S. intelligence community estimates that many countries of potential concern have considered ballistic missile defense countermeasures and that some of these countries will develop countermeasures to U.S. national missile defense over the next 15 years. Therefore, the Study Group explored possible countermeasures to boost-phase defense in addition to deployment of solid-propellant ICBMs, which is a natural countermeasure. The Group identified several countermeasures that could be developed and deployed using technologies or techniques that have already been used.

The U.S. intelligence community has judged that countries of concern may arm the long-range missiles they are developing with biological or chemical munitions, including submunitions ("bomblets"), as well as nuclear weapons (Section 3.3). The Study Group therefore considered missiles armed with chemical, biological, and nuclear warheads, including submunitions.

The U.S. intelligence community has expressed concern that it is becoming increasingly difficult for the United States to gather detailed information about the physical characteristics and performance of missiles that may threaten the nation, as summarized in Section 3.3. The Study Group therefore considered the effects on the performance of boost-phase defenses of uncertainties about the physical characteristics and performance of the attacking missile as well as its intended target and trajectory.

The U.S. intelligence community has judged that several countries of concern are technically capable of developing within the next decade the ability to launch short- or mediumrange ballistic missiles against coastal regions of the United States from ships or other platforms positioned hundreds of kilometers offshore. The Study Group therefore considered briefly the feasibility of countering this potential threat using shorter-range, ship-based interceptor rockets. 


\subsection{Ballistic Missile Capabilities of Selected States}

This section reviews publicly available information about the ballistic missile programs of North Korea, Iran, and Iraq. ${ }^{2}$ These countries are not the only ones that could develop and deploy ballistic missiles that threaten the United States. As noted earlier, the Study Group has made no judgment about which countries or missile programs pose a risk to the United States. The Study's emphasis on these countries simply reflects the concerns that have been expressed by the U.S. government.

North Korea and Iran have tested or deployed short- and medium-range ballistic missiles [29, 30]; Iraq had deployed short-range ballistic missiles [29, 30] and had retained some despite disarmament efforts by the United Nations [30, 38]. These missiles could be adapted to attack the United States from ships or other platforms positioned off-shore [29, 30]. Flight testing from ships of such missiles could be difficult to detect, making it harder for the United States to know whether a state had developed a sea-launch capability [39]. North Korea and Iran also have active programs to develop long-range ballistic missiles [29, 30]. For these reasons, their missile programs have attracted attention and concern (see, for example, Refs. $[29,30,36])$. The missiles discussed in this section, their basic physical characteristics, and some sources of information about them are listed in Table 3.1.

Table 3.1. Ballistic Missiles of Selected States of Concern

\begin{tabular}{|c|c|c|c|c|c|c|c|}
\hline State & Missile $^{a}$ & $\begin{array}{c}\text { Range }^{b} \\
(\mathrm{~km}) \\
\end{array}$ & $\begin{array}{c}\text { Fuel } \\
\text { Type }^{c}\end{array}$ & $\begin{array}{c}\text { Booster } \\
\text { Stages }\end{array}$ & $\begin{array}{c}\text { Payload }^{d} \\
(\mathrm{~kg})\end{array}$ & $\begin{array}{c}\text { Burn } \\
\text { Time }(\mathrm{s}) \\
\end{array}$ & Status $^{e}$ \\
\hline North & No Dong [40] & 1,300 & $\mathrm{~L}$ & 1 & 1200 & $110[41]$ & $\mathrm{D}$ \\
\hline \multirow[t]{2}{*}{ Korea } & $\begin{array}{l}\text { Taepo }[42] \\
\text { Dong-1 }[44]\end{array}$ & $\begin{array}{c}2,000-2,200 \\
(2,500-3,000)\end{array}$ & $\begin{array}{l}\mathrm{L} \\
(\mathrm{S})\end{array}$ & $\begin{array}{c}2 \\
(3)\end{array}$ & $\begin{array}{l}750-1,000 \\
(290-500)\end{array}$ & $\begin{array}{c}266[43] \\
(293)\end{array}$ & $\mathrm{T}$ \\
\hline & $\begin{array}{l}\text { Taepo }[45,34] \\
\text { Dong-2 }\end{array}$ & $4,000-6,000$ & $\mathrm{~L}$ & 2 & $700-1,000$ & $<230[46]$ & $\mathrm{R} \& \mathrm{D}$ \\
\hline \multirow[t]{2}{*}{ Iran } & Shahab-3 [47] & 1,300 & $\mathrm{~L}$ & 1 & 1,200 & $110[48]$ & $\mathrm{T}$ \\
\hline & Shahab-4 ${ }^{f}[47]$ & $2,000-3,000$ & $\mathrm{~L}$ & 1 & $<1,500$ & $266 ?[46]$ & $\mathrm{R} \& \mathrm{D}$ \\
\hline Iraq & Al Hussein [38] & 630 & $\mathrm{~L}$ & 1 & 500 & - & $-g$ \\
\hline
\end{tabular}

${ }^{a}$ Primary reference for each missile; supplemental references are listed in other columns.

${ }^{b}$ Number in parentheses is the estimated range for the three-stage variant of the missile.

${ }^{c}$ L: liquid; S: solid. Parenthetical fuel type refers to the third stage of the missile.

${ }^{d}$ Number in parentheses is the estimated payload for the three-stage variant of the missile.

${ }^{e} \mathrm{R} \& \mathrm{D}$ : research and development; D: deployed; T: flight tested.

${ }^{f}$ Some reports discuss development of Shahab-5 [49] and Shahab-6 [50] missiles, but little information is available to confirm the existence of these programs.

${ }^{g}$ Some reports suggest that 15 to 50 Al Hussein missiles may not have been reported to U.N. inspection teams; Iraq may have covertly retained these missiles [30, 38].

\footnotetext{
${ }^{2}$ As mentioned above, Iraq is included for what it reveals about how countries in the developing world have mounted ballistic-missile programs.
} 


\subsubsection{North Korea}

North Korea probably has the most advanced ballistic missile program of the countries the United States considers to be of concern [51, 34]. North Korea is believed to have benefited - perhaps substantially - from foreign assistance by Russia and possibly China [52, 53]. However, since 1999, it has observed a voluntary moratorium on missile flight tests, although it has continued to work on its Taepo Dong-2 ICBM (see below) [30, 32]. North Korea extended the moratorium in September 2002 [54].

North Korea's No Dong, which is a medium-range ballistic missile having a range of $1,300 \mathrm{~km}$, is an extensive redesign of Scud missile technology [36]. It is the longest-range ballistic missile that North Korea has so far deployed. The Rumsfeld Commission [36] judged that "...the No Dong was operationally deployed long before the U.S. government recognized that fact. There is ample evidence that North Korea has created a sizable missile production infrastructure, and therefore it is highly likely that considerable numbers of No Dongs have been produced." A key reason North Korea's No Dong capability was not recognized earlier by the United States was its limited flight testing. North Korea conducted only one flight test before the missile was considered operational; the missile has also been tested by Pakistan and Iran. [41, 31].

In August of 1998, North Korea launched a Taepo Dong-1 missile with a solid-propellant third stage configured as a space-launch vehicle (SLV) [29, 33, 34]. Although the third stage failed, a three-stage version of the missile could hypothetically reach parts of the United States with a very small payload. The possibility that North Korea might add a third stage was not appreciated by the United States until the August 1998 space launch occurred [29, 31, 32].

North Korea's Taepo Dong-2, currently under development, is a multiple-stage ICBM that in some configurations would be capable of reaching parts of the United States [30]. It is estimated that in its basic two-stage configuration, the missile could deliver a payload of 700-1000 kilograms to a range of 4,000-6,000 km, sufficient to strike Alaska and Hawaii [45]. With a smaller payload, the missile could have a range as long as $10,000 \mathrm{~km}$, according to the intelligence community, allowing it to reach some of the contiguous 48 states [30, 32]. With a third stage similar to the one used on the Taepo Dong-1 for the 1998 space-launch attempt in a ballistic missile configuration, it could deliver a smaller payload of at most a few hundred kilograms up to $15,000 \mathrm{~km}$, sufficient to strike all of North America [30, 32]. The Taepo Dong-2 has not yet been flight tested; a flight test would probably be conducted with it configured as an SLV [30, 32].

One of the most detrimental aspects of North Korea's missile program has been its effect on attempts to limit the spread of ballistic missiles and missile technologies [36]. North Korea has become the missile and manufacturing technology source for the ballistic missile programs of many other countries, especially Iran and Pakistan [30, 32]. The willingness of North Korea to sell complete systems and components has enabled other countries to acquire longer-range capabilities sooner than they otherwise would have [30].

\subsubsection{Iran}

Iran's ballistic missile program has relied extensively on foreign assistance, particularly from Russia, China, and North Korea [30, 32]. Iran has depended particularly on technical expertise from Russia to advance its missile programs [30, 37, 50]. The most advanced ballistic missile tested by Iran is the Shahab-3, a medium-range ballistic missile based on 
the North Korean No Dong [30, 32]. It was first flight tested in July 1998 [47] and was declared ready for service in July 2003 [55].

Some reports indicate that Iran is also pursuing development of the Shahab-4 and possibly the Shahab-5 [47, 56, 49]. Little is known about these missiles. According to one source, the Shahab may be based on the North Korean Taepo Dong [56, 49], although that connection is not a widely held view [47]. In addition to North Korean assistance to the Shahab program, Iran may have benefited from technical information provided by Russia about the Soviet SS-4 [47], particularly its mobile launcher [56]. Iran is also known to have received solid-propellant technology from other countries [36].

\subsubsection{Iraq}

Iraq worked for decades to establish an indigenous capability to produce ballistic missiles. In 1974, Iraq imported the Scud B from the Soviet Union. In 1987, Iraq began to reverseengineer the Scud B to create a longer-range missile, although it is still not clear how successful they were. The results of these efforts were the Al Hussein and Al Abbas [38]. The Al Hussein had a range of $600 \mathrm{~km}$ and was used extensively during the 1991 Persian Gulf War. The Al Abbas was an attempt to extend the range of the Al Hussein to $950 \mathrm{~km}$ [57], but this program is believed to have been terminated to concentrate efforts on improving the Al Hussein [38]. Iraq also began development of the Al Aabed SLV, but it was never tested [58].

Since the Gulf War, Iraq's ballistic missile development program had been hindered by U.N. resolutions and monitoring. However, Iraq continued attempts to gain components, such as gyroscopes from dismantled Russian missiles [59]. It also continued working on missiles such as the Scud and the Al Samoud, which were claimed to have ranges less than $150 \mathrm{~km}$ and were thus allowed under U.N. resolutions, to maintain the technical expertise and infrastructure for missile production [57]. Presumably, Iraq's ballistic missile program ended with the fall of the Hussein regime.

\subsection{Historical Patterns of ICBM Development}

Historical patterns of ICBM development and deployment may be useful in estimating the likely characteristics of the first long-range missiles that would be deployed by North Korea or Iran if they continue their programs to develop such missiles.

Countries beginning development of ICBMs have all used liquid propellants initially, because liquid-propellant rocket technology is easier to master than solid-propellant technology. There has been a steady decrease in the total burn time of the first liquid-propellant ICBM deployed by a given country during the past 45 years; each new country that fielded a liquid-propellant ICBM deployed a missile with a shorter burn time for the same payload mass and range than the first missile deployed by the country that preceded it (see Table 3.2).

The United States was first to field an ICBM, deploying the Atlas D in 1958. It had a payload of roughly 1.5 to 2 tonnes and a total burn time of $309 \mathrm{~s}$ [60], longer than any of the missiles in its class that came after it. The much larger Titan II, which was deployed by the U.S. in 1962, had a payload of 3.5 tonnes and a total burn time of $331 \mathrm{~s}$ [61, pp. 456458], longer than any other missile in its payload class and the longest of any first- or second-generation ICBM. 
Table 3.2. Characteristics of Early ICBMs

\begin{tabular}{lccccc}
\hline \hline ICBM & $\begin{array}{c}\text { Deployed } \\
(\text { year })\end{array}$ & Country & $\begin{array}{c}\text { Range } \\
(\mathrm{km})\end{array}$ & $\begin{array}{c}\text { Payload } \\
(\text { tonnes })\end{array}$ & $\begin{array}{c}\text { Boost } \\
\text { Phase (s) }\end{array}$ \\
\hline Atlas D [60, 62] & 1958 & US & 12,000 & $\sim 1.5-2$ & $309[60]$ \\
Titan II [61, 63] & 1962 & US & 10,000 & $\sim 3.5-4$ & $331[61]$ \\
SS-6 (R-7) [60, 64, 65] & 1960 & USSR & $8,000\left(10,000+^{a}\right)$ & $5.4\left(3^{a}\right)$ & $286[60]$ \\
SS-7 (R-16) [66, 65] & 1961 & USSR & 11,000 & $1.5-2.2$ & - \\
SS-8 (R-9A) [65] & 1965 & USSR & 12,000 & $1.7-2.1$ & - \\
SS-9 (R-36) [67, 65] & 1967 & USSR & $10,000-15,000$ & $4-5.8$ & $280[60]$ \\
SS-11 (UR-100) [65] & 1966 & USSR & $11,000-12,000$ & $0.8-1.5$ & $267[68]$ \\
DF-5 [69] & 1981 & China & 12,000 & 3 & $231[61]$ \\
\hline
\end{tabular}

${ }^{a}$ Range and payload for a longer-range missile.

The Soviet Union was the second country to field long-range missiles. Its first ICBMs had total burn times 20 to $50 \mathrm{~s}$ shorter than the first U.S. ICBMs, for missiles with comparable ranges and payloads. The long-range version of the Soviet SS-6 was deployed in 1960, had a payload of roughly 3 tonnes, and burned out in $286 \mathrm{~s}$, more than $20 \mathrm{~s}$ faster than the Atlas D, even though the SS-6 carried a larger payload. The SS-11, which was fielded in 1966 and carried a 0.8 - to 1.5-tonne payload, had a 267 -second boost phase, a full 40 s shorter than the boost phase of the Atlas D. Larger Soviet missiles followed the same trend. The Soviet SS-9 had a payload and range comparable to those of the Titan II, but completed its boost phase in $280 \mathrm{~s}, 50 \mathrm{~s}$ faster than the U.S. Titan II. After the Titan II, the United States deployed only solid-propellant ICBMs, whereas the Soviet Union continued to develop and deploy liquid-propellant ICBMs.

China was the third country to deploy an ICBM. Its DF-5 (CSS-4) was tested in 1971, but was not fielded until 1981, roughly 20 years after the Soviet Union deployed its first ICBM [70]. The DF-5 had the same range $(12,000 \mathrm{~km})$ and twice the payload (3 tonnes) of the SS-11 but completed its boost phase in only $231 \mathrm{~s}, 36 \mathrm{~s}$ faster than the Soviet SS-11 and nearly $100 \mathrm{~s}$ faster than the U.S. Titan II.

To date, no other country has deployed an ICBM. Several have tested or deployed SLVs that carry only small payloads to ICBM ranges, too small to be considered militarily useful. The closest that any of the three countries considered in this study has come to testing an ICBM was when North Korea attempted to launch a satellite using its Taepo Dong 1 in 1998. The core two-stage liquid-propellant missile used in this space-launch attempt had a range of only about $2,000 \mathrm{~km}$, but it carried a small solid-propellant third stage to place a small satellite into orbit. The attempt failed. The total burn time of this cobbled-together rocket was $293 \mathrm{~s}$, including substantial time between stages 1 and 2, when the missile was coasting [44].

\subsection{The Changing Context of Missile Development Programs}

The context in which countries of concern are currently developing ballistic missiles differs significantly from the context in which they were developed by the United States, the Soviet Union, and China. The countries of concern enjoy a much wider access to technology, 
information, and expertise that can be and is being used to speed the development and deployment of nuclear, chemical, and biological munitions, as well as long-range ballistic missiles capable of delivering them to targets at intercontinental distances [30]. This access is also being used to develop denial and deception techniques that could impede U.S. intelligence gathering about the development and deployment programs of these countries [36]. The change in context increases the importance of several factors related to boost-phase defense, which we summarize here.

\subsubsection{Differences in possible goals}

The motives that North Korea and Iran may have for developing and possibly using ICBMs and the goals they may hope to achieve could be significantly different from the motives and goals the United States, the Soviet Union, and China had when they developed and deployed such missiles [39]. For example, what is required technically for a crude terror weapon is very different from what is required for a weapon that is militarily useful. Accuracy requirements would be much less, as would the numbers needed. Safety and reliability might be less important. Consequently there is a possibility that North Korea or Iran might deploy or even, in some circumstances, launch long-range ballistic missiles against the United States that had been tested very little, if at all $[30,39,36]$.

\subsubsection{Transfer of ballistic missile technology and systems}

The increased transfer of missile technology to and among states that are currently beginning to develop long-range missiles has several important consequences:

- Countries that are just beginning to develop long-range missiles now have easier access to the technologies that were used in early generations of U.S. and Soviet missiles [36].

- Proliferation of ballistic missile-related technologies, materials, and expertise has enabled emerging missile states to accelerate the development timelines for their existing programs, deploy missiles with little, if any, flight testing, acquire turnkey systems to gain previously non-existent capabilities, and lay the groundwork for the expansion of domestic infrastructures to potentially accommodate even more capable and longer-range future systems [30, 37].

- Commerce in ballistic missile and warhead technology and hardware has been growing, which may make proliferation self-sustaining among countries of concern to the United States [36]. North Korea has become the manufacturing and technology source for many missile programs [30]. North Korea has helped countries acquire technologies to serve as the basis for domestic development efforts [30]. Iran is expanding its efforts to sell missile technology [30].

- North Korea has been willing to sell complete systems as well as components and has received help from Russia and probably China [30, 53]. Iran has received crucial assistance from Russia and China, as well as North Korea [30]. Some experts believe there is a possibility that complete, long-range ballistic missile systems could be transferred from one nation to another and that such missiles could be equipped with nuclear, chemical, or biological warheads [36]. 


\subsubsection{Spread of solid-propellant technology}

Since the 1960s, the trend in ballistic missile programs has been toward development and deployment of solid-propellant systems because of their reduced logistical requirements and simplicity of operation. The spread of ballistic missile-related technologies, materials, and expertise has led to increasingly widespread development of solid-propellant missiles:

- Beginning in the mid-1960s, China focused its missile programs on large solidpropellant missiles [37]. It is currently developing three solid-propellant ICBMs: the CSS-X-10 (also called the DF-31), which is now being flight tested; a longer-range version of the DF-31; and the JL-2 submarine-launched ballistic missile [30, 32]. In 2003, China also flight tested a 4-stage version of its solid-propellant ICBM [71]. According to the U.S. intelligence community, China has carried out extensive proliferation of liquid and solid SRBM and MRBS ballistic missiles technology and has provided complete missile systems to countries of concern, including Iran and Pakistan [72]. Some believe China has sought to compete with Russia, which has dominated commerce in liquid-propellant missiles and technology, by marketing solid-propellant technology and missiles [37].

- Iran, with China's assistance, has developed a solid-propellant rocket infrastructure for building short-range missiles. It is able to produce short-range rockets on its own and is seeking long-range solid-propellant missile technology from outside sources [36].

- Pakistan has developed a two-stage, solid-propellant medium-range ballistic missile, the Shaheen II, which reportedly could carry a 1,000-kilogram payload to a range of about 2,500 km [30,32]. (The various types of ballistic missiles are described in Appendix A.) Some experts argue that missiles with significantly increased performance could be based on the more modern, all-solid design of the Shaheen II, or on older liquid-propellant technologies [37, p. 8].

- There has been considerable commerce in medium- and short-range solid-rocket technology from supplier nations particularly Russia, China, and North Korea to Iran, Iraq, Pakistan, and possibly others [30, 72]. According to the intelligence community, the Chinese, who have developed or acquired this technology and are using it in their space and military rocket programs, are providing it to several countries of concern $[72,36,37]$. It is possible that the several decades that it took Russia and China to develop reliable ICBM-class solid rockets could be shortened for those countries if they receive technical assistance.

- The North Korean Taepo Dong 1 was launched with a solid-propellant third stage in 1998, although it failed [30].

Based on these developments and intelligence estimates, the Study Group concluded that countries of concern might deploy solid-propellant ICBMs within 10 to 15 years, if they were able to purchase or otherwise acquire solid-propellant missiles or technology from countries with more advanced missile programs.

\subsubsection{Munitions}

Countries that are developing long-range ballistic missiles could arm them with biological or chemical weapons [52]. These weapons could take the form of a single, large warhead or 
dozens or hundreds of bomblets [36]. The knowledge needed to design and build a nuclear weapon is now widespread [73], and emerging ballistic missile powers may gain access to the needed fissile material through domestic efforts and foreign channels [36].

The U.S. intelligence community judged in the mid-1990s that North Korea had produced enough plutonium for one or possibly two nuclear weapons [73]. The status of North Korea's nuclear program remains an open question. North Korea also has chemical and biological weapons programs [30].

The U.S. intelligence community judges that Iran does not yet have a nuclear weapon [30]. Most agencies assess that it could have one by the end of the decade; the time required could be reduced by several years with foreign assistance [30]. Iran has biological and chemical weapons programs [30].

Prior to 1990, Iraq had a crash program to develop a nuclear weapon for delivery by ballistic missiles, but bombing by coalition forces during the 1991 Gulf War and subsequent disarmament activities by the International Atomic Energy Agency (IAEA) and the United Nations Special Commission (UNSCOM) set back the effort significantly [30]. In 2001, the U.S. intelligence community estimated that, if unrestrained, Iraq would have been able to produce within a few years enough fissile material to make a nuclear weapon [30]. Iraq admitted to having biological and chemical weapons programs before the 1991 Gulf War and was thought to have maintained those programs [30]. However, all of Iraq's nuclear, chemical, and biological weapon programs presumably ended in 2003 with the second Gulf War and the fall of the Hussein regime.

\subsubsection{Countermeasures}

The U.S. intelligence community judges that many countries with ballistic-missile programs, including North Korea, Pakistan, Iran, India, and China, probably have considered ballisticmissile-defense countermeasures [29]. Historically, the development and deployment of missile-defense systems has been accompanied by the development of countermeasures and penetration aids by potential adversaries, either in reaction to the threat or in anticipation of it [29]. The Russians and Chinese have had countermeasures programs for decades and may be willing to transfer some related technology to others [29]. The U.S. intelligence community expects that countries of concern that are developing long-range ballistic missiles will respond to the U.S. national missile defense program by deploying penetration aids and countermeasures [29].

\subsubsection{Uncertainties}

According to recent reports $[29,30,36]$, the U.S. intelligence community's ability to provide timely and accurate estimates of ballistic missile threats to the U.S. is eroding. As a result, the prospects that the U.S. will have advance knowledge of the detailed characteristics and performance of newly deployed threatening ballistic missiles are being reduced. This erosion of warning time has several implications.

- Deception and denial efforts are intense, and U.S. collection and analysis assets are limited. These factors create a high risk of continued surprise [36]. There are plausible scenarios that could result in an increased missile threat to the United States for which there would be little or no warning [34]. 
- In particular, the U.S. intelligence community judges that it may not be able to provide much warning if a country purchases an ICBM or already has a space-launch capability [29, 34]. In some scenarios-including re-basing or transfer of operational missiles, sea- and air-launch options, shortened development programs that might include testing in a third country, or some combination of these - the U.S. might have little or no warning of an operational deployment [36]. The U.S. intelligence community may not be able to provide much, if any, warning of a forward-based ballistic-missile threat to the United States, such as would be posed by forward seabasing of short-range ballistic missiles or medium-range ballistic missiles [29].

- The U.S. intelligence community has more confidence in its ability to warn of efforts by countries to develop ICBMs than to describe accurately the missile configurations that will comprise the threat [29]. For example, North Korea test fired the Taepo Dong-1 missile roughly on the timetable projected by the U.S. intelligence community, but with a completely unanticipated vehicle configuration that included a third stage [29].

\subsubsection{Forward-based sea-launch of Short-Range Ballistic Missiles (SRBMs) or Medium-Range Ballistic Missiles (MRBMs)}

According to the U.S. intelligence community, several countries of concern have the technical expertise required to develop ballistic missiles launchable from a forward-based platform, such as a surface ship positioned off a U.S. coast [29, 30, 34]. Forward-basing on dedicated vessels or freighters could pose a new threat to the United States in the near term-well before 2010 [34].

An SRBM or MRBM could be launched against the United States from a forward-based sea platform positioned in international waters within a few hundred kilometers of U.S. coastal regions. According to the U.S. intelligence community [29, 30, 32], using such a sea platform would not pose major technical problems and would be much less technologically demanding than launching an ICBM attack. Although the accuracy of the missile probably would be reduced significantly because of the movement of the ocean's surface, it would probably still be better than that of some early ICBMs [29, 30]. Adapting missiles for launch from a commercial ship could be accomplished covertly, and probably with little or no warning [34]. Much of the population, commerce, and infrastructure of the United States is located within $100 \mathrm{~km}$ of its East and West coasts and would therefore be vulnerable to such an attack.

Sea launch of shorter-range ballistic missiles could enable a country to pose a direct territorial threat to the United States sooner than it could by developing an ICBM for launch from its own territory [36]. Sea-launching could also allow a country to target a larger area of the United States than it could with a missile fired from its home territory [36].

\subsection{Implications for the Study}

The Study Group used the information summarized in Sections 3.2 and 3.3 to define (1) the characteristics of the ballistic missiles considered in the Study, (2) possible countermeasures that might be employed by states of concern, and (3) the uncertainties a missile defense would likely encounter. This section summarizes these decisions and the basis for them.

The Study Group decided to model a spectrum of ICBMs that might be developed or acquired by countries of concern during the next 10-15 years, including solid-propellant 
ICBMs that might be deployed in response to prospective or actual fielding of a boost-phase intercept missile defense system by the United States.

\subsubsection{Liquid-propellant ICBMs}

The first ICBMs that might be deployed by countries of concern are likely to use liquid propellants, because this technology is easier to master. The Group chose a liquid-propellant model ICBM with a 240-second boost phase as its baseline liquid-propellant long-range threat missile. This model is similar to the first liquid-propellant ICBMs deployed by the Soviet Union and China 20 to 40 years ago.

To facilitate comparisons with previous studies, the Study Group also considered a liquid-propellant ICBM with a 300-second boost phase.

These choices were made for the following reasons:

- Each new country that has fielded a liquid-propellant ICBM has deployed a missile with a shorter burn time for the same payload mass and range than the country that preceded it (Section 3.2).

- The boost phases of the first liquid-propellant ICBMs deployed (in the mid-1960s) by the Soviet Union lasted 265 to $285 \mathrm{~s}$ (Section 3.2). The boost phase of the first liquid-propellant ICBM tested (in 1971) and deployed (in 1981) by China lasted only about $230 \mathrm{~s}$ (Section 3.2).

- The transfer of ballistic-missile technology from Russia and China is playing an important role in the development of missiles by states of concern (Section 3.3).

- The historical trend toward faster-burning liquid-propellant ICBMs combined with the ongoing transfer of ballistic-missile technology from Russia and China to countries of concern suggests that the next new ICBM is likely to have a boost phase no longer than the first ICBMs deployed by Russia and China.

- The studies of boost-phase intercept that have been published in the open literature $[74,75,76]$ considered liquid-propellant ICBMs and examined missiles with boost phases as long as the 330-second boost phase of the U.S. Titan II.

- The boost phase of the Titan II is substantially longer than that of any other first- or second-generation liquid-propellant ICBM (Section 3.2). Such a long boost phase reduces significantly the performance requirements for any boost-phase missile defense system (Chapter 5). It therefore seemed imprudent to base the Study's analysis of liquid-propellant ICBMs on such an extreme example.

- Comparing results with previous studies is useful. Consequently, the Study Group considered - but did not analyze in detail — a liquid-propellant model ICBM with a 300-second boost phase, even though the Group judged that deployment of a new liquid-propellant ICBM with such a long boost phase is unlikely.

\subsubsection{Solid-propellant ICBMs}

Based on unclassified intelligence community statements, the Study Group concluded that countries of concern might develop or acquire solid-propellant ICBMs within 10 to 15 years 
and that it would therefore be imprudent not to consider such ICBMs in evaluating the feasibility of the boost-phase defense systems. The Study Group constructed detailed computer models of two solid-propellant ICBMs having 170-second boost phases. These computer models are based on 1960s solid-propellant missile technology and are similar to the first solid-propellant ICBMs deployed by the United States and the Soviet Union.

The Study Group also considered - but did not analyze in detail-a solid-propellant ICBM with a burn time of $130 \mathrm{~s}$, to explore the implications of faster-burning ICBMs for boost-phase defense systems.

These choices were made for the following reasons:

- Although they are somewhat more technically challenging than liquid-propellant missiles, solid-propellant missiles are inherently attractive because of their reduced logistical requirements and simplicity of operation.

- The short durations of solid-propellant missiles' boost phases increase the performance required of a boost-phase defense system (Chapter 5).

- Solid-propellant ICBMs have shorter boost phases than liquid-propellant ICBMs for the same payload mass and range. Hence acquisition and deployment of solidpropellant ICBMs is an effective countermeasure to boost-phase defenses.

- Countries of concern may seek to deploy solid-propellant ICBMs in response to prospective or actual deployment of a boost-phase intercept missile defense system by the United States, just as all previous countries with ICBMs have developed countermeasures when facing the possible deployment of a ballistic-missile defense.

- China has for more than 30 years concentrated its long-range missile development efforts on solid-propellant ballistic missiles, has tested such ICBMs, and has been a provider of solid-rocket technology to countries of concern (Section 3.3). It is now flight testing the CSS-X-10 ICBM (also called the DF-31) and has recently flight tested a four-stage solid-propellant rocket capable of orbiting a 100-kg payload (Section 3.3).

- The transfer of ballistic-missile-related technologies, materials, and expertise has led to the spread of solid-propellant missile technology (Section 3.3).

- North Korea, Iran, and Iraq have received solid-propellant missile technology for short-range missiles from supplier nations (Section 3.3). North Korea and Iran have developed and tested such rockets (Section 3.3).

- Pakistan has developed a two-stage, solid-propellant medium-range ballistic missile, the Shaheen II, which reportedly could carry a 1,000-kilogram payload to a range of about 2,500 km (Section 3.3).

- Pakistan has received solid-propellant ballistic missile technology from supplier countries and has an extensive short- and medium-range solid-propellant ballistic missile program(Section 3.3).

- Countries of concern could deploy solid-propellant ICBMs within the next 10-15 years if they were able to purchase or otherwise acquire solid-propellant missiles or technology from countries with more advanced missile programs (Section 3.3). It 
is possible that a complete, long-range ballistic missile system could be transferred from one nation to another (Section 3.3).

- The accelerations of the upper stages of solid-propellant ICBMs may vary significantly (Chapter 15). It therefore seemed prudent to analyze the implications for boost-phase defenses of two different upper-stage designs.

\subsubsection{Countermeasures}

In addition to deployment of solid-propellant ICBMs, which is a natural countermeasure to any boost-phase defense, the Study Group identified several other countermeasures that could be developed and deployed using technologies or techniques that have been already implemented for one reason or another (Chapter 9).

Illustrative countermeasures were identified for the following reasons:

- It has been claimed that there are no countermeasures to a boost-phase defense. The countermeasures identified by the Study Group show that this statement is incorrect.

- The U.S. intelligence community estimates that many countries of potential concern have considered ballistic-missile-defense countermeasures and that some of these countries will develop countermeasures to national missile defense over the next 15 years (Section 3.3).

\subsubsection{Munitions}

The Study Group considered missiles armed with chemical and biological warheads, including submunitions ("bomblets"), as well as missiles with nuclear warheads.

These different types of munitions were considered for the following reason:

- The U.S. intelligence community judges that countries of concern that are developing long-range missiles may arm them with biological or chemical munitions, including bomblets, as well as nuclear weapons (Section 3.3).

\subsubsection{Uncertainties}

The Study Group considered the effects on the performance of boost-phase defenses of uncertainties about the physical characteristics and performance of the attacking missile, as well as its intended target and trajectory.

The effects of such uncertainties were considered for the following reasons:

- Such uncertainties can significantly degrade the performance of a boost-phase intercept system (Chs. 4 and 5).

- Some countries of concern appear willing to deploy a missile after just a single test flight, which makes it more difficult for the United States to gather detailed information about the physical characteristics and performance of the missile and its possible countermeasures (Section 3.3).

- The U.S. intelligence community has expressed concern that testing may occur in a third country, that operational missiles may be transferred, and that the United States might therefore not know of a deployment much before a missile is launched (Section 3.3). 


\subsubsection{Forward-based sea-launch of SRBMs or MRBMs}

Motivated by the assessment of the U.S. intelligence community that an attack on U.S. territory by sea-based SRBMs or MRBMs is more likely than an attack by ICBMs (3.3), the Study Group examined the possible role of shorter-range boost-phase intercept systems in countering this threat (Chapters 15 and 5).

\section{References for Chapter 3}

[29] National Intelligence Council, "Foreign Missile Developments and the Ballistic Missile Threat to the United States Through 2015," Unclassified Summary of a National Intelligence Estimate. Available at: http://www.cia.gov/nic/NIC_otherproducts.html, September 1999.

[30] National Intelligence Council, "Foreign Missile Developments and the Ballistic Missile Threat to the United States Through 2015," Unclassified Summary of a National Intelligence Estimate. Available at: http://www.cia.gov/nic/NIC_otherproducts.html, December 2001.

[31] Robert D. Walpole, "The ballistic missile threat to the United States." Statement for the Record by the National Intelligence Officer for Strategic and Nuclear Programs to the International Security, Proliferation, and Federal Services Subcommittee of the Governmental Affairs Committee, U.S. Senate. Available at: http://www.cia.gov/cia/public_affairs/speeches/archives/2000/nio_speech_020900.html, 9 February 2000.

[32] Robert D. Walpole, "The ballistic missile threat to the United States." Testimony by the National Intelligence Officer for Strategic and Nuclear Programs before the International Security, Proliferation, and Federal Services Subcommittee of the Governmental Affairs Committee, U.S. Senate. Available at: http://govt-aff.senate.gov/031102witness.htm, 11 February 2002.

[33] Robert D. Walpole, "North Korea's Taepo Dong launch and some implications on the ballistic missile threat to the United States." Speech by the National Intelligence Officer for Strategic and Nuclear Programs at the Center for Strategic and International Studies, 8 December 1998.

[34] Robert D. Walpole, "The ballistic missile threat to the United States." Speech by the National Intelligence Officer for Strategic and Nuclear Programs at the Carnegie Endowment for International Peace. Available at: http://www.cia.gov/cia/public_affairs/speeches/1998/walpole_speech_091798.html, 17 September 1998.

[35] Richard T. Cupitt, "Export controls and missile technology transfers." Commission to Assess the Ballistic Missile Threat to the United States, Appendix III: Unclassified Working Papers, 1998. Available at: http://www.fas.org/irp/threat/missile/rumsfeld/pt2_cupitt.htm.

[36] Donald Rumsfeld et al., "Executive Summary of the Report of the Commission to Assess The Ballistic Missile Threat to the United States." Available at: http://www.fas.org/irp/threat/bm-threat.htm, July 1998. 
[37] Robert H. Schmucker, "Engineering and proliferation analysis of third world theater to intermediate range ballistic missiles." Contribution to Year 2000 Multinational BMD Conference, Philadelphia, PA, 5-8 June 2000.

[38] Jane's Strategic Weapons Systems (JSWS) 38, 11 July 2002. Article: Al Hussein.

[39] Gates Panel, Independent panel review of NIE 95-19: "Emerging missile threats to North America during the next 15 years." Available at: http://www.fas.org/irp/threat/missile/oca961908.htm, December 1996.

[40] Jane's Strategic Weapons Systems (JSWS) 38, 11 July 2002. Article: No Dong 1/2.

[41] Charles P. Vick, "North Korean No Dong Missile." Available at: http://www.fas.org/nuke/guide/dprk/missile/nd-1.htm, 2002.

[42] Jane's Strategic Weapons Systems (JSWS) 38, 12 August 2002. Article: Taepo Dong 1.

[43] Charles P. Vick, "North Korean Taepo Dong-1 Missile." Available at: http://www.fas.org/nuke/guide/dprk/missile/td-1.htm, 2002.

[44] David Wright, "Ballistic missile systems." Briefing Presented to the APS Study Group, July 2001.

[45] Jane's Strategic Weapons Systems (JSWS) 38, 12 August 2002. Article: Taepo Dong 2.

[46] Charles P. Vick, "North Korean Taepo Dong-2 Missile." Available at: http://www.fas.org/nuke/guide/dprk/missile/td-2.htm, 2002.

[47] Jane's Strategic Weapons Systems (JSWS) 38, 11 July 2002. Article: Shahab 3/4.

[48] Charles P. Vick, "Iranian Shahab-3 Missile." Available at: http://www.fas.org/nuke/guide/iran/missile/shahab-3.htm, 2002.

[49] Charles P. Vick, "Iranian Shahab-5 Missile." Available at: http://www.fas.org/nuke/guide/iran/missile/shahab-5.htm, 2002.

[50] Charles P. Vick, "Iranian Shahab-6 Missile." Available at: http://www.fas.org/nuke/guide/iran/missile/shahab-6.htm, 2002.

[51] Greg Gerardi and Joseph Bermudez, Jr., "An analysis of North Korean ballistic missile testing." Jane's Intelligence Review 7(4), 190 (1995).

[52] U.S. Central Intelligence Agency, "Unclassified Report to Congress on the Acquisition of Technology Relating to Weapons of Mass Destruction and Advanced Conventional Munitions," 1 January-30 June 2000: http://www.cia.gov/cia/reports/721_reports/jan_jun2000.htm.

[53] Joseph S. Bermudez, Jr., A History of Ballistic Missile Development in the DPRK. Number 2 in Occasional Paper. Center for Non-Proliferation Studies, Monterey Institute of International Studies, Monterey, California, 1999. 
[54] Howard W. French, "Japan and North Korea reach agreement on relations." New York Times, 17 September 2002.

[55] Nazila Fathi, "Iran Confirms Test of Missile That Is Able to Hit Israel," The New York Times, July 8, 2003.

[56] Charles P. Vick, "Iranian Shahab-4 Missile." Available at: http://www.fas.org/nuke/guide/iran/missile/shahab-4.htm, 2002.

[57] Michael Eisenstadt, "Missiles and weapons of mass destruction (WMDs) in Iraq and Iran." Commission to Assess the Ballistic Missile Threat to the United States, Appendix III: Unclassified Working Papers, 1998. Available at: http://www.fas.org/irp/threat/missile/rumsfeld/pt2_eisen.htm.

[58] Jane's Strategic Weapons Systems (JSWS) 38, 12 August 2002. Article: Al Aabed.

[59] Jane's Sentinel Security Assessment, "The Gulf States (GULFS)," January 2002.

[60] S. J. Isakowitz, International Reference Guide to Space Launch Systems. American Institute of Aeronautics and Astronautics, 2nd edition, December 1995.

[61] Steven J. Isakowitz, Joseph P. Hopkins, and Joshua B. Hopkins, International Reference Guide to Space Launch Systems. American Institute of Aeronautics and Astronautics, 3rd edition, December 1999.

[62] Charles P. Vick, "U.S. SM-65 Atlas Missile." Available at: http://www.fas.org/nuke/guide/usa/icbm/sm-65.htm, 1999.

[63] Charles P. Vick, "U.S. SM-68B Titan II Missile." Available at: http://www.fas.org/nuke/guide/usa/icbm/sm-68b.htm, 1997.

[64] John Pike et al., "Soviet SS-6 (R-7) Missile." Available at: http://www.fas.org/nuke/guide/russia/icbm/r-7.htm, 2000.

[65] Pavel Podvig, ed., Russian Strategic Nuclear Forces (MIT Press, Cambridge, MA), 2001.

[66] John Pike et al., "Soviet SS-7 (R-16) Missile." Available at: http://www.fas.org/nuke/guide/russia/icbm/r-16.htm, 2000.

[67] John Pike et al., "Soviet SS-9 (R-36) Missile." Available at: http://www.fas.org/nuke/guide/russia/icbm/r-36.htm, 2000.

[68] Charles P. Vick, "Russian UR-100 (SS-11) Missile." Available at: http://www.fas.org/nuke/guide/russia/icbm/ur-100k.htm, 2000.

[69] Jane's Strategic Weapons Systems (JSWS) 36, 12 July 2001. Article: CSS-4 (DF-5).

[70] John Wilson Lewis and Hua Di, "China's ballistic missile programs: Technologies, strategies, goals," International Security, 17(2), Fall 1992.

[71] "China Develops Its First Solid-Fuel Satellite Rocket," Agence France-Presse, September 24, 2003. Available at http://quickstart.clarinet.net/qs_se/webnews/wed/dt/Qchina-space.Rrh8_DSO.html 
[72] U.S. Central Intelligence Agency, "Unclassified Report to Congress on the Acquisition of Technology Relating to Weapons of mass Destruction and Advanced Conventional Munitions," 1 January-30 June 2003.

[73] U.S. Central Intelligence Agency, "Report of Proliferation-Related Acquisition in 1997." Available at http://www.cia.gov/cia/reports/721_reports/acq1997.html, 1997.

[74] Richard L. Garwin, "Boost-phase intercept: A better alternative." Arms Control Today 30(7), 8-11 September 2000.

[75] Theodore A. Postol, "A preliminary analysis of a Russian-U.S. boost-phase defense to defend Russia and the U.S. from postulated rogue-state ICBMs: A potential option for Russian-U.S. cooperation in missile defense." Technical report, Carnegie Endowment for International Peace, October 1999.

[76] Theodore A. Postol, "Boost-phase missile defense concepts for protecting the U.S. from postulated rogue-state ICBMs." Technical report, MIT Security Studies Program, February 2001. 


\title{
Chapter 4
}

\section{Kinetic-Kill Engagement Fundamentals}

\author{
Contents \\ 4.1 ICBM Characteristics Key to This Analysis . . . . . . S S48

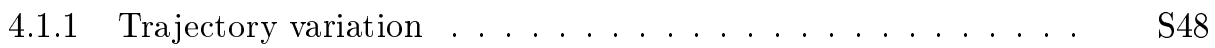 \\ 4.1.2 Payload deployment . . . . . . . . . . . . . . . . . S51 \\ 4.2 Effect on Impact Range of Terminating Missile Thrust _ . $\quad$ S52 \\ 4.3 Surface-Based Interceptors ................ S53 \\ 4.4 Engagement Timelines ................. S54 \\ 4.5 Terrestrial Planar Engagements ............ S55 \\ 4.6 Non-planar Engagements and Interceptor Basing Areas . . S58 \\ 4.7 Space-Based Engagement of ICBMs . . . . . . . . . . S S60

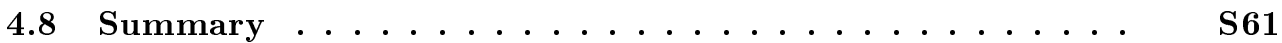

Intercepting a ballistic missile during its boost phase requires, first, an interceptor that can deliver a kill vehicle close to the target quickly enough, and with a velocity that is adequate in both speed and direction, to permit the kill vehicle to maneuver to hit the target. Second, it requires a kill vehicle that can home on and hit the target, disabling or destroying it, even as the target accelerates unpredictably.

This chapter explains how the interceptor performance and basing required to accomplish the first task - getting to the target in time - can be determined. We do this by analyzing engagements of the model ICBMs (Chapter 15) with the model interceptors (Chapter 16), and using the results from the analysis of missile warning and tracking systems (Chapter 10). The trajectories of the missiles and interceptors were computed as described in Appendix B. The sensor and kill-vehicle performance, kill-vehicle sizes and masses, and interceptor masses needed to accomplish the second task-homing on and disabling the target - are investigated in Chapters 11, 12, and 14. The methods described here are used in the next chapter (Chapter 5) to explore the possibilities for terrestrial-based interceptors to protect the United States in various geographical scenarios, and to analyze the problem of debris from an intercept harming other countries. Chapter 6 examines the unique requirements and trade-offs between system performance and requirements for space basing of boost-phase interceptors.

The purpose of the engagement analysis presented here is to determine the spatial and kinematic relationships between the threat trajectories with their variations, interceptor launch-time constraints imposed by threat detection and tracking, interceptor fly-out 
performance, and threat impact constraints that determine when during threat burn the intercept must occur to protect the defended area. This analysis provides a method for determining the interceptor "reach" - the distance that the interceptor can travel within the time available - needed for any combination of interceptor, ICBM, impact constraint, firing time, and geography. Interceptor ground range - the projection of interceptor reach on Earth - can then be used to calculate basing areas, i.e., the area in which an interceptor must be located to intercept an ICBM in time to prevent it from hitting a defended area.

The engagement analysis starts with the models of trajectories for the postulated ICBMs (Chapter 15) and shows how the impact ranges of those missiles are affected when thrust is terminated early. Next it introduces a simplified geometric model of an engagement where the ICBM and terrestrial-based interceptor are in the same plane to demonstrate how interceptor ground range (the distance from the interceptor launch point to a point on Earth directly beneath the intercept point) and target ground range (the distance from the threat missile launch point to that point on Earth directly beneath the intercept point) are developed for any engagement. Applying this model, the chapter then illustrates, for any set of geographical constraints, how to calculate the ground ranges of the interceptor and the target for different intercept times with several engagements, using the model ICBM trajectories and the interceptor flyout envelopes or "fans" developed in Chapter 16. Then, a more generalized model is developed for calculating interceptor ground ranges and basing areas for non-planar engagements, which is applied to postulated geographic scenarios in the next chapter.

Many of the engagement issues are common to all interceptors, but space basing requires a separate discussion of the engagement issues unique to that basing mode. This chapter concludes with a discussion of the methods used to analyze those space-basing issues.

The engagements in this chapter assume that the time delay from threat launch until interceptor launch provides exact information about the threat missile trajectory and that constraints on the location of interceptors relative to the threat are parametric rather than geography-specific. The parametrics must eventually take into account the geographic scenarios used later. In Chapter 5, the procedures developed here are applied to real situations and those assumptions are dropped. Other simplifications used in this chapter, including the assumption that the intercept is successful if the interceptor reaches the target missile, are valid for these analyses, as long as the interceptor kill vehicle has the capabilities described in Chapter 12.

\subsection{ICBM Characteristics Key to This Analysis}

In carrying out this analysis, we used three different models of offensive missiles (Chapter 15). These include a two-stage liquid-propellant ICBM that burns for $240 \mathrm{~s}$ maximum, and two solid-propellant three-stage ICBMs that burn for a maximum of $170 \mathrm{~s}$. The boost trajectory profiles for these three missiles are shown in Figs. 4.1, 4.2, and 4.3.

\subsubsection{Trajectory variation}

Boost-phase defense systems are sometimes analyzed assuming that attacking ICBMs fly their maximum-range trajectories. Figures 4.1, 4.2, and 4.3 show the boost-phase trajectories to burnout. However, a given ICBM model launched from a particular launch site against a target at less than its maximum range generally could terminate its thrust early or fly any of a broad range of boost-phase trajectories. The latter could include trajectories 


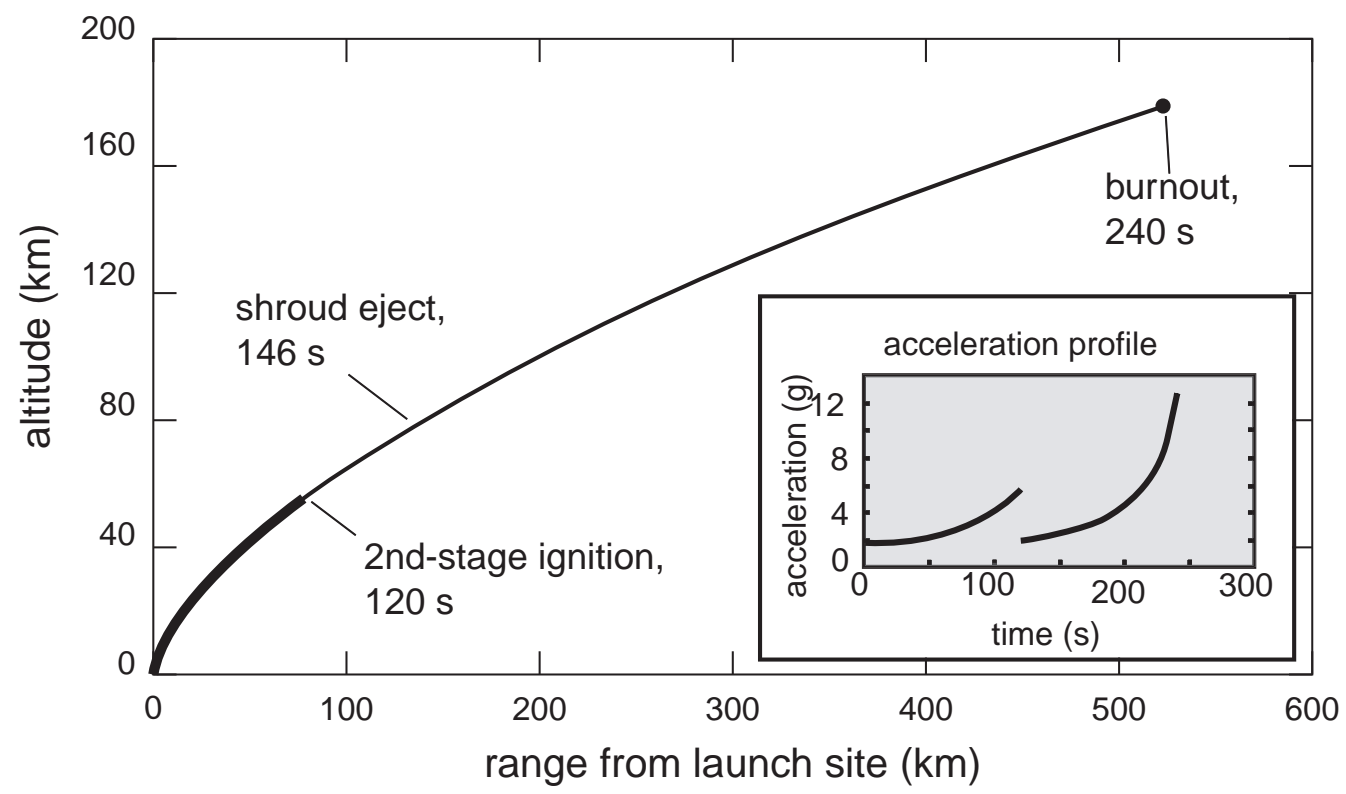

Figure 4.1. Liquid-propellant ICBM model L maximum-range boost-phase trajectory in the altituderange plane. The first- and second-stage boost-phase trajectories are shown respectively by the heavy and light lines. Inset: Acceleration profiles of the two stages. The acceleration of the second stage reaches $12 \mathrm{~g}$ before it burns out.

that are lofted or depressed relative to the maximum-range trajectory and trajectories that have in-plane or out-of-plane dog-legs. Such trajectories may be flown to expend excess energy to hit a particular target. ${ }^{1}$

Evasive maneuvers may be employed to achieve either or both of two basic goals: (1) to force the defensive system's interceptors to travel farther in essentially the same time, restricting the areas where interceptors could be based and/or (2) to cause the interceptors' kill vehicles to exhaust their propellant without achieving intercept. The effect of evasive maneuvers on the reach of interceptors is considered in this chapter; the effect of such maneuvers on the ability of the kill vehicle to hit the target rocket is analyzed in Chapter 12 .

As shown in Fig. 4.4, an attacker could program the ICBM to maneuver after it is out of the atmosphere to fly any trajectory within a fairly large conical volume in space centered on the maximum-range trajectory, with only a modest reduction in the missile's range. For example, any of the model ICBMs considered here could easily change its azimuth by \pm 10 degrees without changing the range to defended area targets dramatically. Consequently, there can be a total uncertainty of 20 degrees in the azimuth of the intended target.

From North Korea, Iran, or Iraq, the azimuth spread for trajectories to the U.S, East and West Coasts is approximately 40 degrees. This means that missiles apparently aimed at the eastern half of the United States could divert to U.S. and Canadian population centers anywhere within that 20 degrees. Similarly a missile aimed at the western half of the nation could divert to within that 20 degree uncertainty to anywhere in the western United States and in addition could divert even more to threaten most of Alaska.

\footnotetext{
${ }^{1}$ Solid-propellant rocket motors normally burn until the propellant is exhausted but to hit a target closer than its maximum range, the thrust of a solid-propellant missile would have to be terminated while its propellant continues to burn. Alternatively, it could fly dog-legs or execute other maneuvers designed to expend excess energy during its boost phase (see Chapter 15), to obfuscate a missile's intended target or flight path, or to evade interceptors.
} 


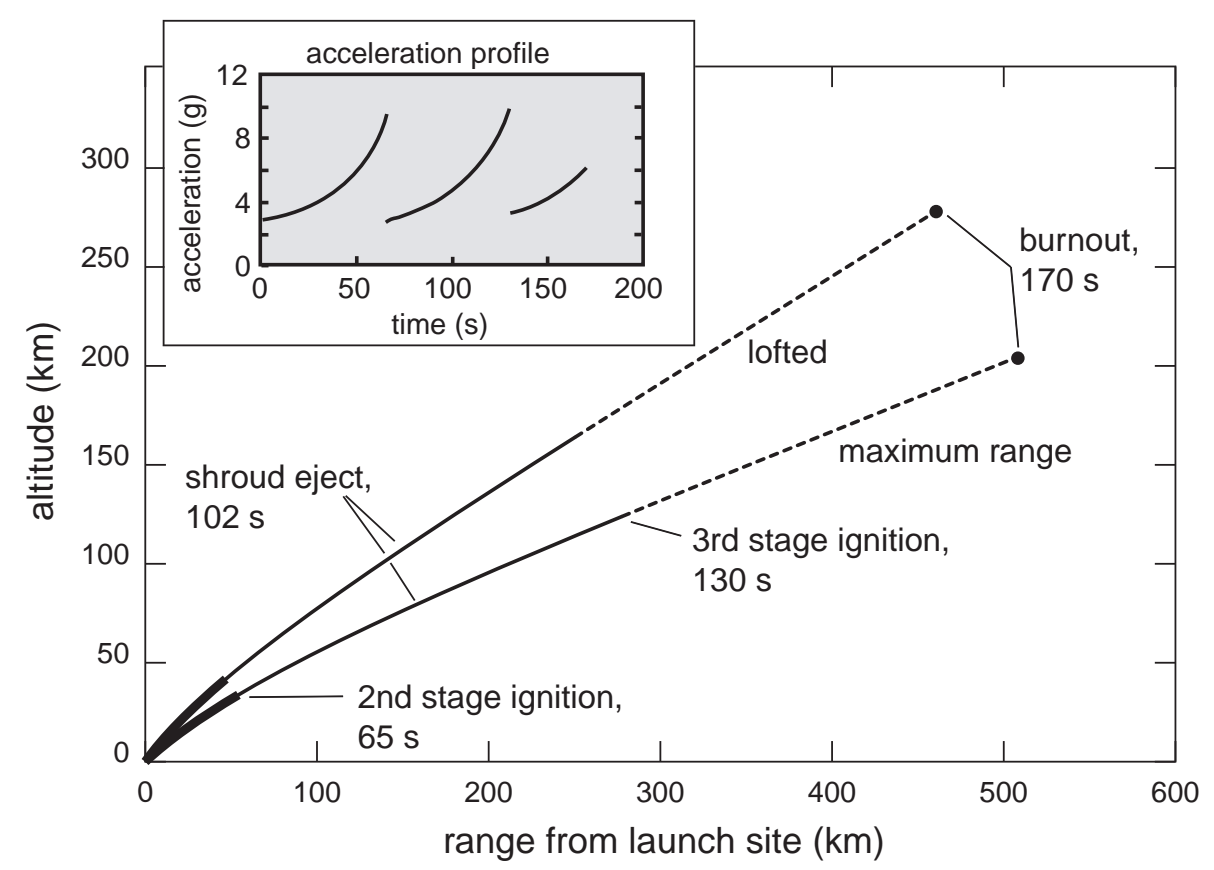

Figure 4.2. Solid-propellant ICBM model S1 maximum-range and lofted boost-phase trajectories in the altitude-range plane. The trajectories during first-, second-, and third-stage boost are indicated by the heavy, light, and dotted lines, respectively. The flight-path angle at the time of burn-out measured from the horizon at launch is $19^{\circ}$ for the maximum-range trajectory and $27^{\circ}$ for the lofted trajectory. Inset: Acceleration profiles of the three stages.

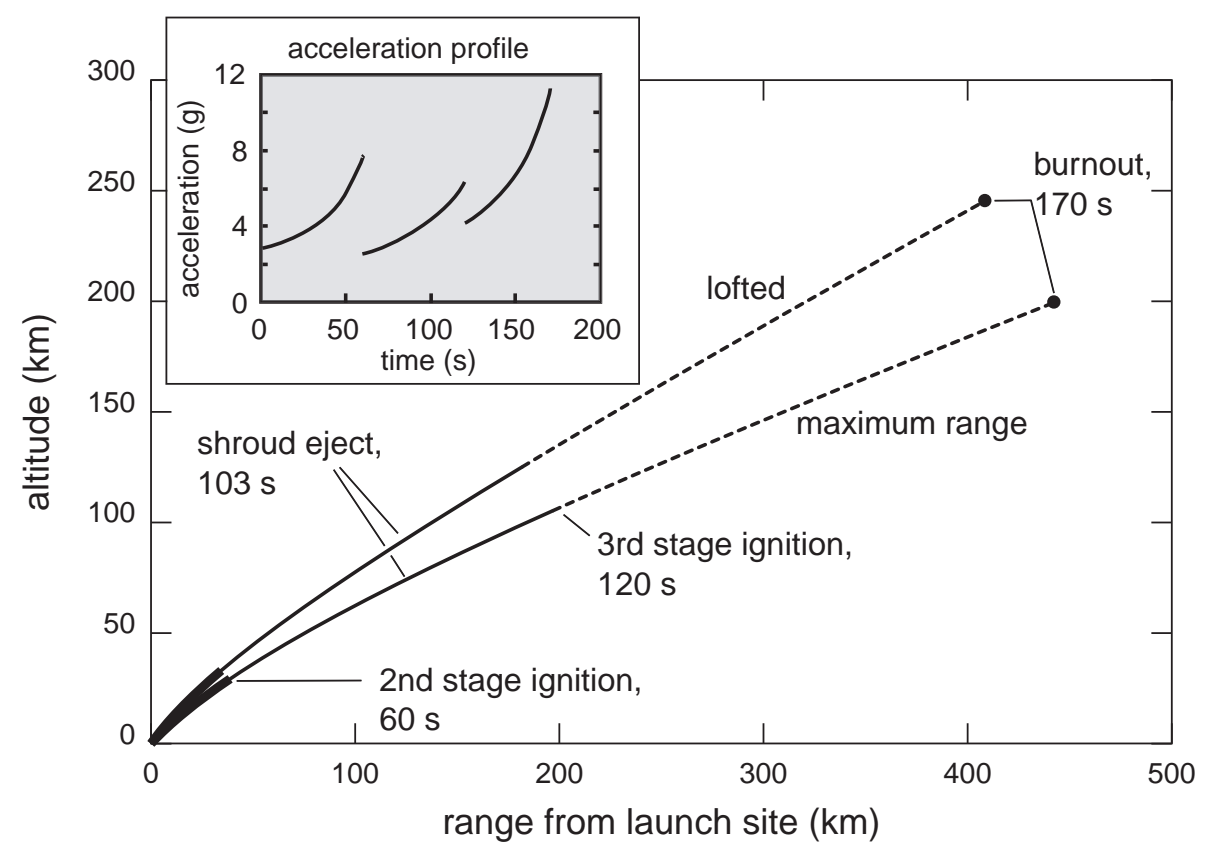

Figure 4.3. Solid-propellant ICBM model S2 maximum-range and lofted boost-phase trajectories in the altitude-range plane. The trajectories during first-, second-, and third-stage boost are indicated by the heavy, light, and dotted lines, respectively. The flight-path angle at the time of burn-out measured from the horizon at launch is $19^{\circ}$ for the maximum-range trajectory and $27^{\circ}$ for the lofted trajectory. Inset: Acceleration profiles of the three stages. 


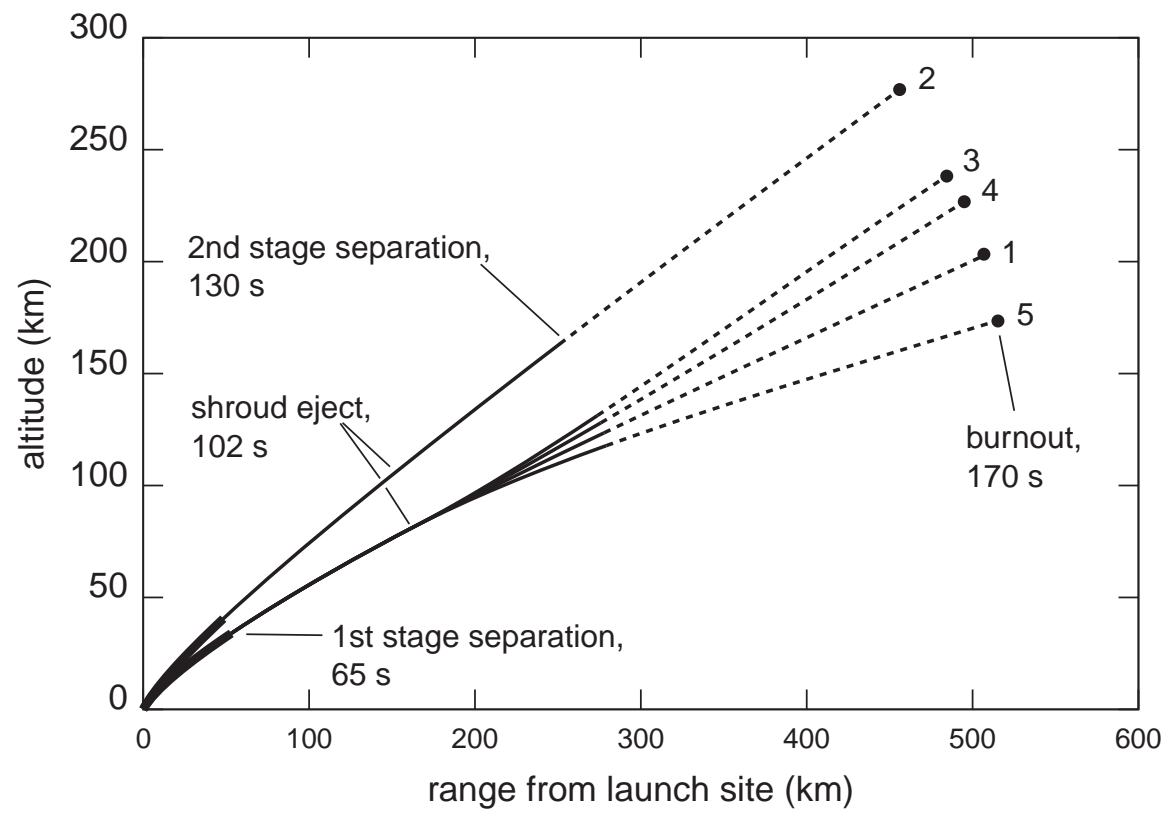

Figure 4.4. Examples of the variety of planar trajectories that are possible for solid-propellant ICBM model S1 by lofting it slightly at launch or by changing its flight path angle after the aerodynamic shield that protects the warhead is ejected. (The numbers for the trajectory are keys for the text in Chapter15.) The large-scale trajectory variations shown reduce the range of the missile by at most 12 percent of its maximum range. Out-of-plane deviations by similar distances reduce the range by a similar amount. Trajectory variations like these are also possible for the liquid-propellant ICBM model L and solid-propellant ICBM model S2, with similar range penalties. The volume in space that these trajectories span represents the volume that interceptors must be able to cover to ensure that a boost-phase intercept is possible.

The point to be made here is that this uncertainty must be considered in the required time of intercept. (A 50 percent reduction would still allow any of the model ICBMs used in the Study to attack targets anywhere in Alaska from launch sites in North Korea.) Dog-legs, like any unpredicted maneuvers, must be dealt with by the kill vehicle's own capability to change its velocity (see Chapter 12).

\subsubsection{Payload deployment}

An ICBM's munitions and devices to aid in penetrating any defenses ("penetration aids") can be deployed in a variety of ways (see Chapters 15 and 9 and Appendix A). The simplest is to release the payload after the propellant of the missile's final boost stage has been exhausted or its thrust has been terminated, but while its attitude is still under control. However, it is also possible to deploy multiple warheads and penetration aids while the rocket motor of the final stage is still burning. Historically this has been done by ejecting warheads from the accelerating final boost stage. Consequently, when determining the latest time a given missile can be intercepted, we assume its warheads could be deployed at any time during final-stage boost, as well as at burnout.

No matter how a missile's munitions are deployed, the only way to be certain of protecting the defended area is to intercept the missile before it has achieved a velocity that would carry its munitions to that area. 


\subsection{Effect on Impact Range of Terminating Missile Thrust}

The fundamental principle of boost-phase defense is that missiles intercepted before they achieve their intended final velocity will fall short of their intended targets. The degree to which a missile's range will be shortened depends on the specific design of the missile and how soon before burnout it is intercepted. For hit-to-kill interceptors, longer threat missile burn times translate into greater interceptor reach and more flexibility in where they can be based.

Figure 4.5 shows the effect on range for our three model missile types as a function of the time when the thrust is cut off, expressed as the time before normal burnout. The range of liquid-propellant ICBM L is much more sensitive to terminating thrust than either of the solids, which is a direct result of the large acceleration that it receives near the end of the second-stage burn, as shown in Chapter 15. For example, terminating the thrust of ICBM L only $20 \mathrm{~s}$ before burnout cuts the range of the missile by 70 percent. In contrast, the range of solid-propellant ICBM S1 is reduced by less than 50 percent.

The curves in Fig. 4.5 are used for the analysis in this chapter and the next to determine when a specific type of missile must be intercepted before it can reach the defended area. The uppermost shaded areas on the right shows when the missiles must be disabled to defend the eastern or western portions of the United States from missiles launched by North Korea or the Middle East. Other shaded areas show when the missile must be disabled to avoid falling on other countries. The darkest areas on the right-hand side of the figure indicate "safe" impact zones where disabled missiles would fall in the open ocean. These safe impact zones can be easily related to intercept times for each threat missile type on the left portion of the figure.

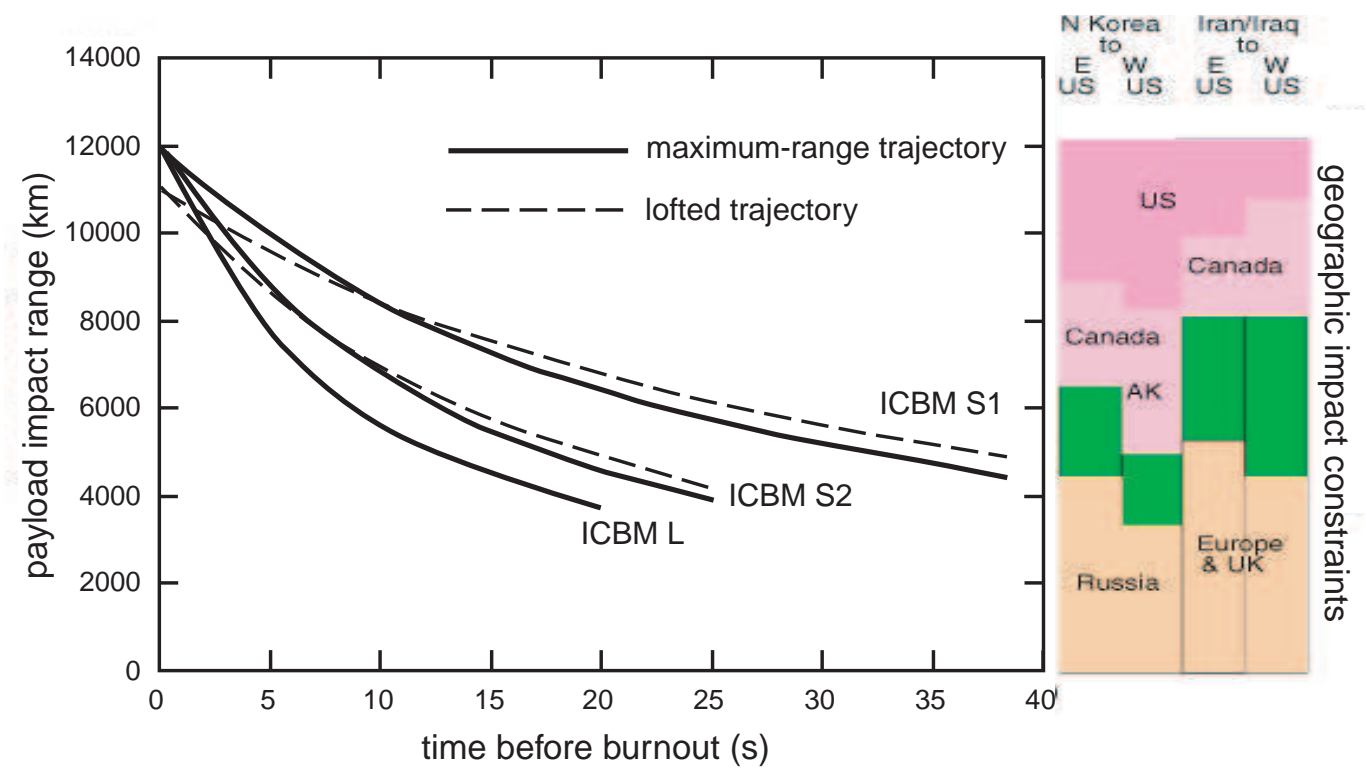

Figure 4.5. Payload range of the missiles modeled in this study as a function of the number of seconds before burnout that their thrust is terminated. The full burn range is $12,000 \mathrm{~km}$. The full burn range for lofted trajectories is $11,000 \mathrm{~km}$ (shown for the solid-propellant missiles). Full burn for ICBM L is $240 \mathrm{~s}$; full burn for ICBMs S1 and S2 is $170 \mathrm{~s}$. Geographic impact constraints for launches from North Korea, Iraq, and Iran against the western and eastern continental United States are also shown. The darkest shaded regions are safe zones where intercepts will cause the threat missile debris to fall into open ocean. 
Table 4.1. Model Interceptors ${ }^{a}$

\begin{tabular}{cccccc}
\hline \hline Model & $\begin{array}{c}V_{\mathrm{bo}} \\
(\mathrm{km} / \mathrm{s})\end{array}$ & $\begin{array}{c}t_{b} \\
(\mathrm{~s})\end{array}$ & $\begin{array}{c}\text { Length } \\
(\mathrm{m})\end{array}$ & $\begin{array}{c}\text { Diameter } \\
(\mathrm{m})\end{array}$ & $\begin{array}{c}\text { Mass } \\
\left(\mathrm{tn}^{b}\right)\end{array}$ \\
\hline I-1 & 1.7 & 47 & 7.9 & 0.34 & 1.6 \\
$\mathrm{I}-2$ & 5.0 & 47 & 6.4 & 0.53 & 2.3 \\
$\mathrm{I}-3$ & 6.7 & 75 & 16.5 & 1.25 & 14.6 \\
$\mathrm{I}-4$ & 6.5 & 40 & 15.5 & 1.6 & 16.9 \\
$\mathrm{I}-5$ & 10 & 45 & 20 & 3 & 65.6 \\
\hline
\end{tabular}

${ }^{a} t_{b}$ is the total duration of the boost phase. $V_{\mathrm{bo}}$ is the burnout velocity if the missile were fired vertically, taking into account the effects of gravitational and aerodynamic drag forces (see Appendix B). For further details, see Chapter 16 .

${ }^{b} \operatorname{tn}=$ metric tonne $=1.1$ ton.

It should be noted that the figure assumes defense knowledge of the acceleration profiles and staging of the attacking missile. In reality, there is uncertainty in that knowledge. For example, the two solid-propellant ICBMs modeled in the figure, S1 and S2, have the same maximum range $(12,000 \mathrm{~km})$ and total burn times $(170 \mathrm{~s})$, yet their impact ranges differ significantly for the same cutoff time before burnout. The reason for this difference is that the missiles have different staging ratios, particularly for the third stage, from which S2 gets more of its acceleration. Secondly, normal variations in propellant burn rates can easily be \pm 10 percent. The defense must provide margin in reach to accommodate such variations. These constraints are considered in detail in Chapter 5 for terrestrial-based engagements and must also be considered for space-based engagements.

\subsection{Surface-Based Interceptors}

Five different computer models of interceptor missiles were developed by the Study Group (see Chapter 16). The basic physical and performance characteristics of the interceptors are listed in Table 4.1. The models were constructed to explore the range of capabilities required in several proposed system architectures. The physical sizes of these interceptors are illustrated in Fig. 4.6. Figure 4.7 shows a flyout fan of planar trajectories and the acceleration profile calculated using the computer model of the $6.5-\mathrm{km} / \mathrm{s}$ interceptor I- 4 .

The engagements we analyzed were selected to illustrate the sensitivity of the outcome to the performance of the ICBMs and interceptors, as well as the firing doctrine adopted. We also investigated engagements of shorter-range ballistic missile models M-1 and M-2 by interceptor I-1 to understand the potential of an existing air-defense interceptor against medium-range missiles launched off U.S. coasts (Chapter 5).

In this chapter, we illustrate our analytical approach with two engagements: interceptor model I-4 against liquid-propellant ICBM model L, and interceptor I-5 against solidpropellant ICBM model S1. 


\subsection{Engagement Timelines}

Our analysis assumes that the missile launch is detected by space-based infrared sensors after the missile achieves an altitude of $7 \mathrm{~km}$ which is above almost all of the water vapor in the atmosphere (see Chapter 10). The liquid-propellant ICBM modeled in this study reaches that altitude $45 \mathrm{~s}$ after launch. The solid-propellant missiles S1 and S2 rise faster, reaching $7 \mathrm{~km}$ in about $30 \mathrm{~s}$. After about $15 \mathrm{~s}$ of tracking the solid-propellant missile, the defense is assumed to have the minimum amount of information to determine the first predicted engagement point and launch an interceptor. (This assumes that the United States has deployed a system similar to the SBIRS-High, as discussed in Chapter 10.) The liquid-propellant ICBM requires $20 \mathrm{~s}$ of tracking, longer than the solid-propellant ICBM, because at an altitude of $7 \mathrm{~km}$, it is moving more slowly and on a more vertical trajectory.

For the most optimistic case, we assume that the interceptors are fired as soon as the missile detection and tracking system has enough information to confirm that a potentially threatening rocket is in flight and to construct a firing solution. We refer to this as the "zero decision time" case. Under this optimistic scenario, the firing time for an interceptor is $65 \mathrm{~s}$ after launch for the liquid-propellant missile (45 s to detect and $20 \mathrm{~s}$ to establish a preliminary track) and $45 \mathrm{~s}$ after launch for the solid-propellant missiles (30 s to detect and $15 \mathrm{~s}$ to track). This firing doctrine would give the interceptors the greatest possible opportunity of achieving an intercept, but provides no margin, or "battlespace," that would allow replacing interceptor failures. In the next chapter we consider both the zero-decisiontime case, as well as the effect of delaying the decision to fire by $30 \mathrm{~s}$.

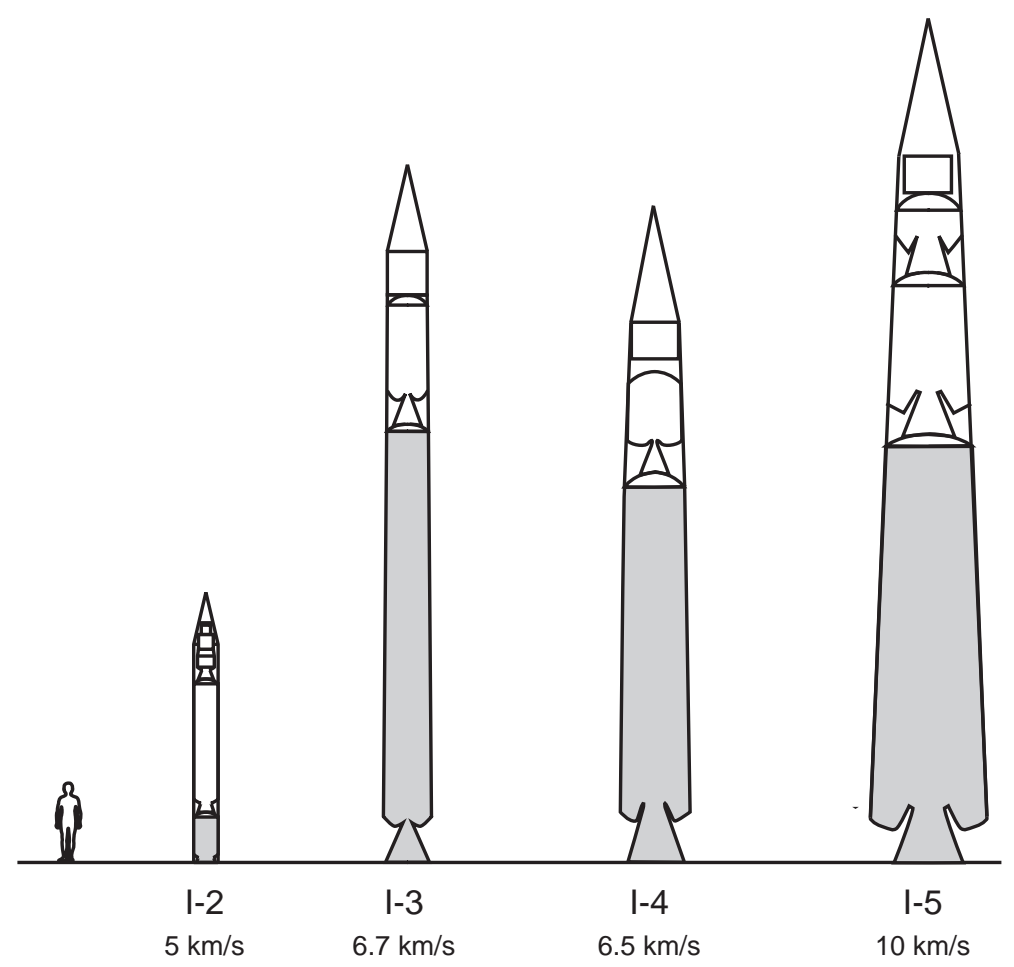

Figure 4.6. Interceptor models used in the Study. The basic physical and performance characteristics of these interceptors are listed in Table 4.1. Further details are given in Chapter 16. 

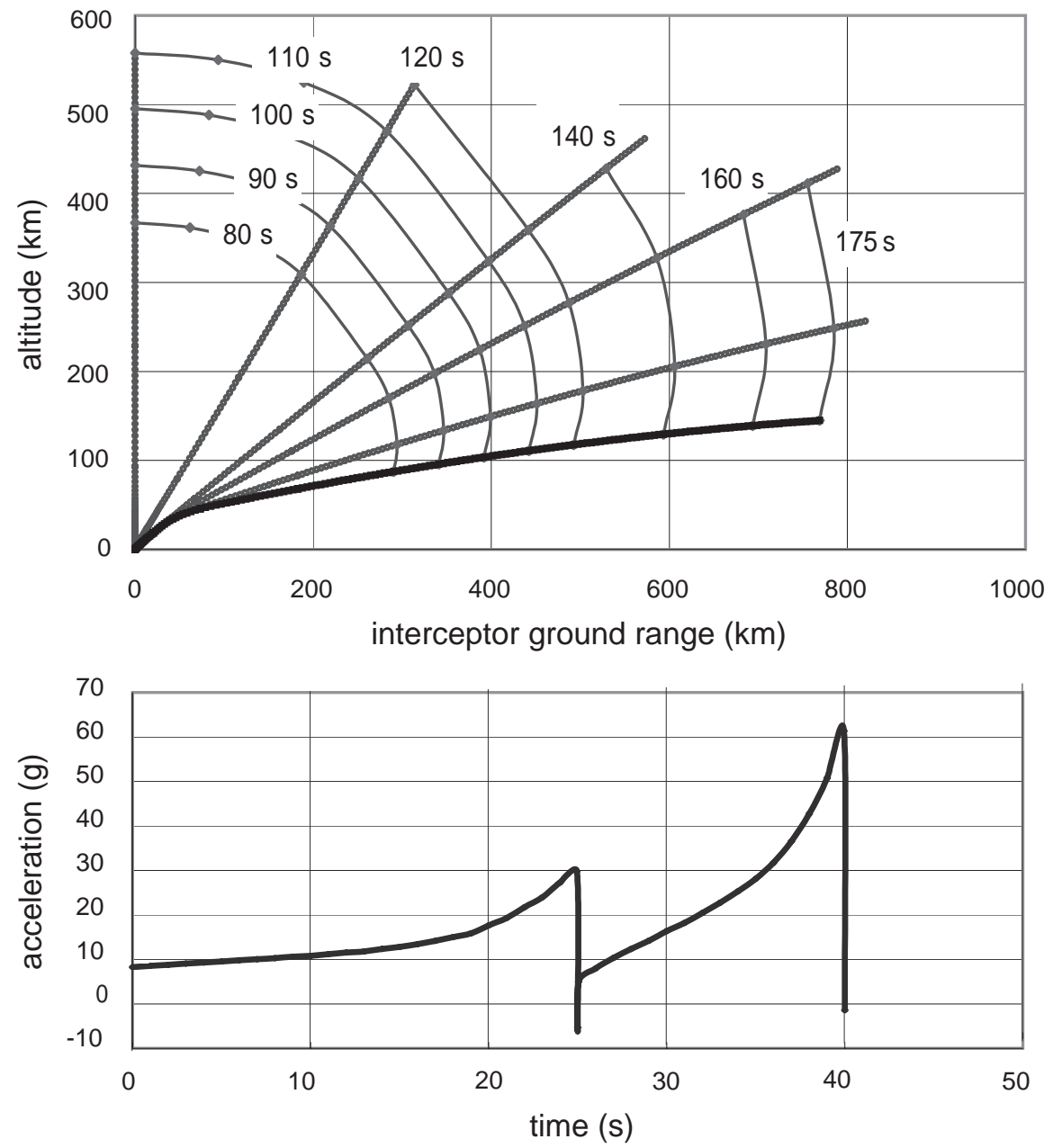

Figure 4.7. Flyout fan of trajectories and the acceleration profile for the $6.5-\mathrm{km} / \mathrm{s}$ interceptor I-4.

\subsection{Terrestrial Planar Engagements}

To intercept an ICBM during boost, the interceptor and the ICBM must arrive at the same point in space at the same time, and that point must be on the threat boost trajectory before the ICBM achieves the velocity necessary to hit a defended area. Modeling this engagement requires solving the complex simultaneous trajectory equations for missile and interceptor in the same reference frame.

The easiest way to both model and visualize the ability of our model interceptors to reach the accelerating target is by simulating a series of planar engagements in the configuration illustrated in Fig. 4.8. These planar engagements allow understanding the engagement space as a function of the distance of the interceptor launch platform and the location of sensors relative to the target missile launch sites, as well as the detection and tracking times required for an initial interceptor firing solution, assuming perfect kill-vehicles performance.

In Fig. 4.8, the threat missile is launched from point M. The interceptor is subsequently fired from the interceptor-basing point, F. If the intercept is successful, the interceptor and the threat missile arrive simultaneously at the intercept point IP. The ground intercept point $\mathrm{P}$ is the point on Earth's surface directly below IP. The threat missile's ground range (TGR) is the distance it moves over the surface of the Earth to the intercept point, and the 


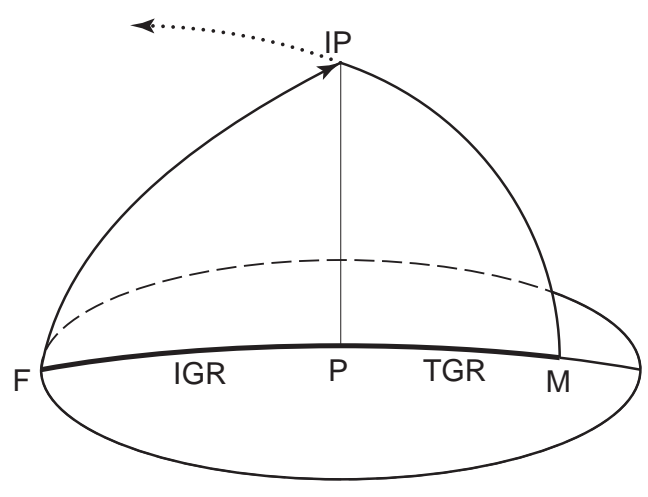

M target missile launch point

$\mathrm{F} \quad$ interceptor firing point

IP intercept point

$P \quad$ ground intercept point

TGR target ground range

IGR interceptor ground range

Figure 4.8. Geometry of a planar engagement.

interceptor ground range (IGR) is the corresponding distance for the interceptor.

The engagement analysis provides input values for the tracking sensor analysis presented in Chapter 10. It also provides the maximum closing velocity and range to the predicted intercept point as a function of time essential for establishing the requirements for the kill vehicle performance, as discussed later in Chapter 12. In this chapter, however, we are interested only in whether the interceptor is capable of reaching the predicted intercept point in the required intercept time.

Two examples of the engagement simulation output are shown in Fig. 4.9. We used the interceptor and ICBM flyout trajectories described above to model planar engagements. Those planar engagements display the flyout fan (range and altitude as a function of time) of candidate interceptors generated with the simulation models. Each is plotted on the same display with an opposing threat trajectory positioned at any standoff range desired. (The standoff range is the distance between the threat missile launch point and the basing location of the interceptor. In the planar case it is simply IGR + TGR.) By adjusting this stand-off range, we can evaluate the ability of any candidate interceptor to intercept a threat at any specific time on the threat trajectory by comparing the 5 -second time ticks on the threat trajectory with the time contours of the interceptor flyout, allowing for the delay in interceptor launch from threat launch.

In Fig. 4.9, the trajectories of the target missile start from their launch point at the right-hand side of the figure and curve upward to the left. The ICBM launch point is at an arbitrarily chosen interceptor standoff distance of $1,000 \mathrm{~km}$. The interceptor is fired from the origin. "Fans" of possible flyout trajectories for the interceptor are shown rising from the lower left-hand corners of the figure. The arcs that traverse the interceptor trajectories are contours of constant elapsed time from the moment the interceptor is fired.

In the particular example shown in Fig. 4.9, top, a $6.5-\mathrm{km} / \mathrm{s}$ interceptor (I-4) is launched against a 240-second, liquid-propellant ICBM model L from a standoff distance of $1000 \mathrm{~km}$. The interceptor is fired $65 \mathrm{~s}$ after the missile is launched, consistent with the zero-decisiontime case for liquid-propellant ICBM L, as described in the previous section. The flyout fan is shown having 10-second contours. Figure 4.9 can be used to determine graphically the earliest intercept that is possible by matching target missile time ticks to interceptor time contours for the desired reach to the intercept point, accounting for the time delay from target missile launch to interceptor launch.

This process can be demonstrated by calculating the earliest intercept point for the set 

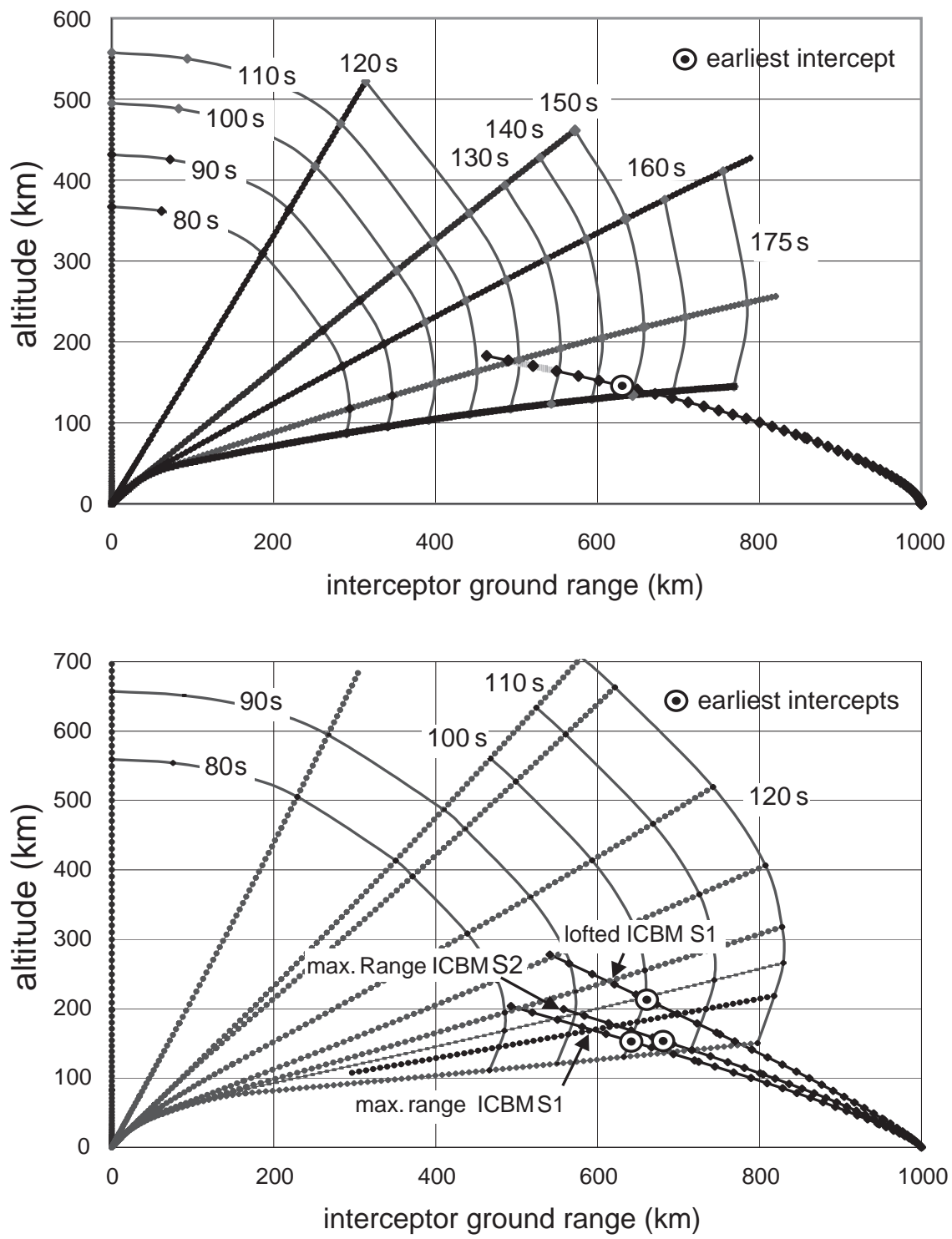

Figure 4.9. Engagement diagrams for planar engagements, 1000-km standoff distance. Top: liquid-propellant ICBM model L engaged by the $6.5 \mathrm{~km} / \mathrm{s}$ interceptor I-4 fired $65 \mathrm{~s}$ after missile launch. The ICBMs are launched from $1000 \mathrm{~km}$, and the interceptors are fired from $0 \mathrm{~km}$. A series of possible interceptor trajectories is shown. The tick marks on the ICBM trajectory are at 5 -second intervals. The earliest possible intercept point is encircled. The curves show the interceptor positions at the times indicated, measured from the time the interceptor is fired. The shaded regions along each trajectory correspond to "safe" zones depicted for the North Korean launches shown in Fig. 4.5 Bottom: solid-propellant ICBM models S1 and S2 engaged by the $10-\mathrm{km} / \mathrm{s}$ interceptor I- 5 fired $45 \mathrm{~s}$ after missile launch.

of parameters in the figure. This point is where the time contour of the interceptor plus the firing delay time crosses the equivalent time tick on the ICBM trajectory. In Fig. 4.9 (top), they are roughly equivalent $210 \mathrm{~s}$ into the ICBM's powered flight and $145 \mathrm{~s}$ after interceptor launch (210 s minus the 65 -s firing delay). The earliest intercept point for each ICBM trajectory is encircled and occurs about $30 \mathrm{~s}$ before the propellant of the ICBM's 
second stage would be depleted. According to the figure, the earliest intercept would be at an interceptor ground range (IGR) of about $620 \mathrm{~km}$. The corresponding target ground range at intercept would be about $380 \mathrm{~km}$.

If intercepting the ICBM $30 \mathrm{~s}$ before burnout is not soon enough to prevent debris and warheads from striking the defended area, the interceptor must be located closer to the ICBM launch point. Graphically, the ICBM launch point would be moved to the left until the equivalent time contours and ticks correspond to the intercept time required to protect the defended area. Conversely, the interceptor could be moved farther away (the ICBM launch point slid to the right in the figure) if the intercept could occur later and still drop the missile debris short of the defended area.

In short, Fig. 4.9 graphically solves the two complex sets of simultaneous equations for the intercept time for a planar engagement. Using this basic approach, a straightforward method for determining the effect of changing the time between target missile launch and interceptor launch will be shown in Chapter 5. Such a change could reflect either an earlier or later detection of the target missile, or a longer decision time. Moreover, by sliding the interceptor flyout fan to greater or lesser distance, approximate interceptor ground range required to intercept this target missile at an earlier or later time can be determined. Note that Fig. 4.9 shows the ICBM trajectory to burnout. In most cases, however, a missile would have to be intercepted before burnout to assure that debris from the collision does not strike the United States. With a suitable map or globe, the country and location from which an ICBM is launched, the missile's azimuth, and trajectory profile and the area to be defended, one can determine when intercept must occur to protect a defended area, and from this one can determine both the TGR and the IGR. By examining the trajectory in detail, one can also determine what intercept times should be avoided to prevent a disabled missile from striking other territory. (These times can be found graphically from the shaded zones in Fig. 4.5).

In general, and interceptor trajectory will not lie in the same plane as the missile trajectory, so that the co-planar geometry is a special case. However, from an analysis of co-planar engagements, it is a straightforward matter to analyze non-planar engagements.

Knowing the required ground ranges, we can use the planar engagement simulation to determine what size interceptor can meet the requirement for candidate defense-basing locations at sea, ashore, or in the air. While interceptor reach is two-dimensional in range and altitude, it is convenient to use IGR and TGR as key engagement measures of interceptor reach and threat position, as illustrated in Fig. 4.8, because ground range can be directly related to basing areas constrained by actual geography in any scenario. A look at any of the interceptor flyouts shows that at the altitudes where boost-phase intercepts occur, the interceptor flyout time contours change very little with altitude, making IGR a valid measure of reach.

\subsection{Non-planar Engagements and Interceptor Basing Areas}

Up to this point we have considered only planar engagements; that is, engagements in which the planes of the target missile and interceptor trajectories coincide. In most cases, however, the trajectories of the target missile and interceptor will lie in different planes.

Fortunately, the planar case can be easily generalized to the non-planar case because the IGRs are the same, defined only by the characteristics of the interceptor and the time available to intercept the missile and independent of the relationship between the trajectory planes of the interceptor and the target. In other words, the interceptor could be rotated 


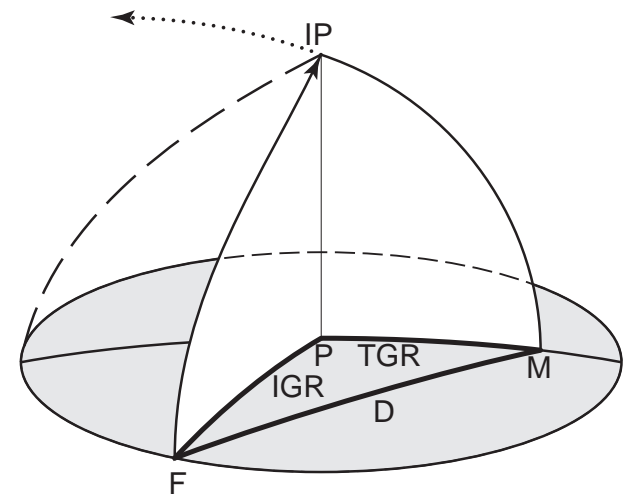

M target missile launch point

$F \quad$ interceptor firing point

IP intercept point

$P$ ground intercept point

TGR target missile ground range

IGR interceptor ground range

D standoff distance

Figure 4.10. Geometry of a non-planar engagement. The standoff distance has its maximum value $D_{\max }$ when the trajectories are in the same plane. The circle centered on $\mathrm{P}$ and passing through $\mathrm{F}$ defines the interceptor basing area.

around the intercept point and still reach the missile at the same time, as long as it remains within the IGR of the ground intercept point. Figure 4.10 shows this basic geometry of such a non-planar engagement. This construction shows that an interceptor based anywhere on or within the circle centered on the ground intercept point, whose radius is the interceptor ground range, can intercept the missile within the required time. This area is called the interceptor basing area. Figure 4.11 shows this same geometry projected onto Earth. Both the threat missile TGR and the IGR depend on the time within which the missile must be intercepted to prevent it from striking the United States. This time depends on the missile type and its intended target. The non-planar model developed here is used extensively in Chapter 5.

Figures 4.10 and 4.11 illustrate the case for which the IGR is greater than the TGR, since interceptors are generally faster than ICBMs in early flight. The reverse could occur if there were a significant delay in the interceptor launch or if the interceptor were slow. In such cases, the missile launch point would lie outside of the interceptor basing area.

The situation described here is highly idealized. In reality, uncertainties in the missile type and trajectory, normal variations in missile performance, the possible need to defend

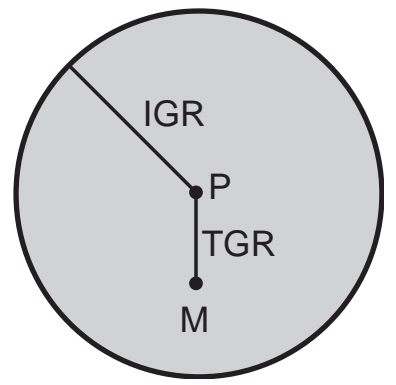

Figure 4.11. Projection of the trajectories of the non-planar engagement shown in Fig. 4.10 onto the Earth's surface. The target missile is launched from M, TGR is the target missile ground range, and IGR is the interceptor ground range. The permitted interceptor basing area for this engagement is the shaded area centered on the ground intercept point P. 


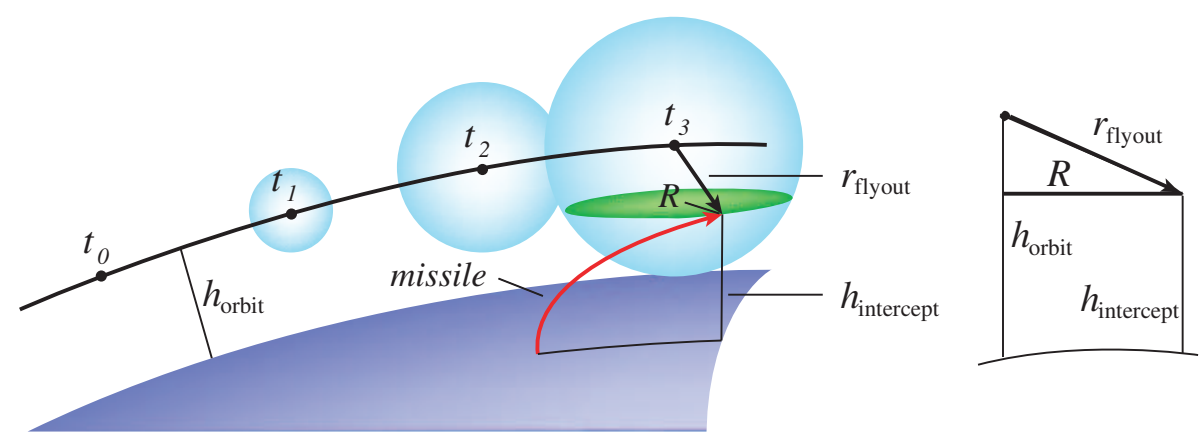

Figure 4.12. Coverage provided by a single space-based interceptor. The height of the intercept is determined by the altitude at which the interceptor is based and the distance it can fly out in the time that is available to intercept the target.

targets on several azimuths from a single interceptor base, and other factors all serve to reduce the area available for effective interceptor basing. These issues are addressed in Chapter 5.

\subsection{Space-Based Engagement of ICBMs}

Space-based hit-to-kill interceptors share many of the same technical issues as terrestrial engagements. However, while the basing of land-, sea-, and air-based interceptors is determined by the geography near the threat ICBM launch points, space-based interceptors (SBIs) are free of this constraint. Instead, space basing is governed by orbital mechanics and the mass of the constellation of interceptors that must be placed in orbit. Space-based interceptors are still subject to two constraints that depend upon the geography- the last time to intercept a missile before it can reach the United States and the time window for a safe impact of the missile's munitions with respect to other countries. The number of SBIs and the reach of each SBI in the time available determine the coverage over the latitudes of interest. A large number of SBI satellites would be needed to ensure that at least one satellite would always be close enough to every potential boost-phase ICBM trajectory to intercept ICBMs during the 100 or so seconds available.

We approached the SBI engagement analysis by creating a "baseline" system that could intercept a single, solid-propellant ICBM $5 \mathrm{~s}$ before burnout with zero decision time. Like terrestrial-based interceptors, the coverage of an SBI is determined by the distance it can fly from the time $t_{0}$ when it is fired to the time when it must intercept the target missile.

The change in the distance of a space-based interceptor from the center of Earth during an engagement is generally very small compared to the radius of its orbit. In this limit, the position at time $t_{n}$ of an SBI fired at an earlier time $t_{0}$ is displaced from the position it would have occupied had it not been fired by the distance $r_{\text {flyout }}$ it has flown in the time interval $t_{n}-t_{0}$. Consequently, the volume it could cover at $t_{n}$ is approximately a sphere centered on the position it would have occupied at $t_{n}$, with its radius equal to $r_{\text {flyout }}$. This is illustrated in Fig. 4.12. Assuming that the altitude of the satellite's orbit is $h_{\text {orbit }}$ and that the intercept occurs at altitude $h_{\text {intercept, }}$ a given SBI could intercept a rocket that rises through this altitude anywhere within the circular area of radius

$$
a=\sqrt{r_{\text {flyout }}^{2}-\left(h_{\text {orbit }}-h_{\text {intercept }}\right)^{2}}
$$


shown in Fig. 4.12.

The flyout distance depends on the acceleration profile and the terminal velocity of the interceptor. Both are important for flyout times in the range $100 \mathrm{~s}$ to $150 \mathrm{~s}$, which are typical for this application, and both are key factors in the size of the SBI. In addition, the interceptor would need to use some of its range in diving down from its parking altitude to the intercept altitude, and this requirement must be considered in coverage calculations. In any case, the range would be measured in hundreds rather than thousands of kilometers, so a large number of interceptors would be needed.

For a given performance, SBIs can be smaller than their terrestrial-based relatives because they are already at high altitude and are not subject to aerodynamic drag or gravity drop as they fly out to their targets. The penalty for overcoming drag and gravity has already been paid by the rocket that put them in orbit. Furthermore, the rocket nozzle on the interceptor can be tuned for a single external pressure (i.e., zero), and no aerodynamic structure, such as a shroud, is needed. On the other hand, a SBI would need life-support (i.e., system support) and station-keeping systems during its multiyear lifetime on orbit that terrestrial-based interceptors do not. These issues are discussed in Chapter6.

Once the interceptor has been boosted toward the intended target missile, the kill vehicle requirements are virtually the same for SBIs and terrestrial-based interceptors, and the same kill-vehicle sizing methodology is used.

\subsection{Summary}

The time at which a given threat missile must be intercepted to prevent it from hitting the United States can be found by calculating how its range varies with intercept time. Given this intercept time plus an estimate of the time required to confirm the missile's launch, the remaining time available for an intercept can be determined. The area where an interceptor would have to be based to intercept an ICBM is defined by the interceptor ground range, which is the distance over the ground that an interceptor can travel in the available time. The analytical methods derived here provide the essential tools needed to analyze engagements in geographic scenarios of interest, which is the subject of Chapter 5 . The unique aspects of space-based engagement issues and the methods used to examine them summarized here are discussed in depth in Chapter 6. 



\section{Chapter 5}

\section{Defending the United States Using Surface-Based Interceptors}

\section{Contents}

5.1 Key Assumptions for the Basing Analysis . . . . . . . S65

5.1 .1 ICBM models, basing, and flight . . . . . . . . . . S65

5.1 .2 Interceptor models, firing, and flight . . . . . . . . . S68

5.1 .3 Margins for uncertainties . . . . . . . . . . . . . S69

5.2 Analysis of Options for Basing Interceptors _....... S70

5.2.1 Determining the latest time to intercept safely . . . . . . . S S70

5.2 .2 Determining required interceptor performance . . . . . . S S72

5.2 .3 Determining interceptor basing areas . . . . . . . . . S S74

5.2 .4 Determining the available decision time . . . . . . . . . S76

5.3 Basing Analysis Conclusions . . . . . . . . . . S77

5.4 Defending Against ICBMs Launched from North Korea . $\quad 579$

5.4 .1 Defending Hawaii . . . . . . . . . . . . . . . S82

5.4.2 Defending against missiles from North Korea with multiple ground

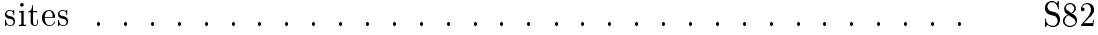

5.4.3 Possibility of an interceptor being mistaken as a threat. . . . S S85

5.5 Defending Against ICBMs Launched from Iraq . . . . . . S85

5.5.1 Defending the contiguous states . . . . . . . . . S S85

5.5.2 Defending Alaska and Hawaii . . . . . . . . . . . S88

5.5.3 Defending against missiles from Iraq with multiple interceptor sites S90

5.6 Defending Against ICBMs Launched from Iran . . . . . . S90

5.6.1 Defending the contiguous states . . . . . . . . . . S90

5.6.2 Defending Alaska and Hawaii . . . . . . . . . . . . . . S93

5.6.3 Defending against missiles from Iran with multiple interceptor sites S94

5.6.4 Possibility of an interceptor being mistaken as a threat . . . S S94

5.7 Other Engagements Considered _............ S S94

5.7.1 Defending against sea-based medium-range ballistic missiles . $\quad$ S94

5.7.2 Defending against a 300-second burn-time missile . . . . . S95

5.8 Avoiding Harming Other Countries . . . . . . . . . S96

5.8.1 Controlling shortfall for North Korea and Iraq or Iran . . . . . S97 
5.8.2 The problem of timing an intercept $\ldots \ldots \ldots \ldots$

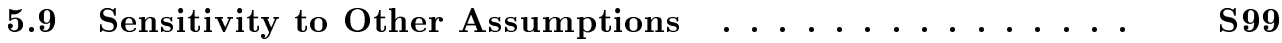

5.9 .1 Reducing the defended area $\ldots \ldots \ldots \ldots$ S99 . . . . . . .

5.9.2 Using the existing early-warning satellite system . . . . . . . S100

5.9.3 Effect of dog-leg trajectories on basing . . . . . . . . . S100

5.9 .4 Achieving warhead kill _. . . . . . . . . . . . . S100

5.9.5 Intercepting the missile during its ascent phase . . . . . . . S101

5.9 .6 Defending by slower interceptors . . . . . . . . . . . S101

This chapter analyzes the feasibility of preventing munitions launched by attacking missiles from landing on U.S. territory by intercepting the missiles during their boost phase using terrestrial (land-, sea-, or air-based) interceptors. In accordance with the stated policy of the current U.S. administration, we take as our baseline goal the defense of all 50 states. $^{1}$ We also consider the effects on interceptor performance and basing requirements if only the contiguous 48 states or, alternatively, only a few major U.S. cities were defended. The defense of Hawaii is treated as a special case. Finally, we discuss the problem of managing intercepts so that munitions do not fall on populated areas short of the target.

We investigate the interceptor performance and basing that would be needed to defend the United States against ICBMs launched from North Korea, Iraq, and Iran. The U.S. intelligence community has judged these countries to be of concern and to be capable of developing or acquiring ICBMs within the next 10 to 15 years (see Chapter 3 ). These three countries also have dimensions and geographies that illustrate the range of problems a boost-phase defense system might have to overcome.

Toward the end of this chapter, we discuss briefly boost-phase defense of the United States against short- or medium-range ballistic missiles launched from ships or other seabased platforms positioned off U.S. coasts. It is the judgment of the U.S. intelligence community that some countries of concern are likely to develop and deploy such systems before 2015 (see Chapter 3). Specifically, we examine the boost-phase intercept capability of the existing Aegis air-defense system in this role.

The following material underlies the discussion in this chapter: the threat missiles and the rationales for their choice that are described in Chapter 15, the speeds and flyout properties of the interceptors that are described in Chapter 16, and the times for detecting a rocket launch and generating a firing solution that are described in Section 10.1.

Also underlying this discussion is the assumption that if the interceptor can reach the threat missile, it can destroy the missile. The analysis that supports this assumption forms Part B of the Report. Its impact on this chapter is indirect: given the assumed interceptor flyout profile and tracking capability, the mass of the kill vehicle is established (Chapter 14), and the kill-vehicle mass then determines the total mass of the interceptor. The interceptor masses listed in Chapter 16 were determined by this procedure. However, interceptor masses are not of primary importance in this chapter, only their flyout properties.

\footnotetext{
1 "Our missile defense must be designed to protect all 50 states - and our friends and allies and deployed forces overseas - from missile attacks by rogue nations, or accidental launches", G. W. Bush, speech at the National Press Club, Washington, D.C., May 23, 2000; see also President G. W. Bush's speech at the National Defense University, Washington, D.C., May 1, 2001.
} 
Table 5.1. Model ICBMs Considered ${ }^{a}$

\begin{tabular}{lrcr}
\hline \hline Model & $t_{b}(\mathrm{~s})$ & $V_{\text {bo }}(\mathrm{km} / \mathrm{s})$ & Payload $(\mathrm{kg})$ \\
\hline $\mathrm{L}$ & 240 & 7.2 & 2545 \\
$\mathrm{~S} 1$ & 170 & 7.2 & 918 \\
$\mathrm{~S} 2$ & 170 & 7.2 & 1040 \\
\hline
\end{tabular}

${ }^{a}$ All these models have nominal ranges of $12,000 \mathrm{~km}$. For further details, see Chapter 15. We also discuss, but do not consider in detail, a liquid-propellant missile with a $300 \mathrm{~s}$ boost phase (ICBM L2) and a fast-burn solid-propellant missile with a 130-second boost phase (ICBM S3). The duration of the boost phase is $t_{b}$, and $V_{\mathrm{bo}}$ is the burnout velocity

\subsection{Key Assumptions for the Basing Analysis}

This section discusses various key assumptions which are part of the analysis. The effect of relaxing these assumptions is discussed in Section 5.9.

\subsubsection{ICBM models, basing, and flight}

ICBM models Since the three states considered in this study do not currently have intercontinental ballistic missiles, our analysis focused on the three illustrative ICBM models developed in Chapter 15: liquid-propellant model L, which has a total boost time of $240 \mathrm{~s}$, and solid-propellant models S1 and S2, both of which have total boost times of $170 \mathrm{~s}$ (see Table 5.1). ${ }^{2}$ All three have nominal non-rotating-Earth maximum ranges of $12,000 \mathrm{~km}$. Their differences illustrate the different performance characteristics that are possible even for ICBMs having identical maximum ranges.

We consider only briefly liquid-propellant ICBMs with the very long burn times $(300 \mathrm{~s}$ or more) used in some previous studies. Obviously an additional $60 \mathrm{~s}$ of burn time would allow a much more optimistic view of boost-phase intercept. We note, however, that all the ICBMs that have been developed during the past three decades, including the first liquid-propellant ICBM developed by China more than 30 years ago, have had boost phases significantly shorter than $300 \mathrm{~s}$ (see Chapter 3). At the other extreme, we discuss only briefly defense against "fast-burn" solid-propellant ICBMs, such as model S3, which has a 130-second boost phase. No terrestrial-based interceptor rockets could reach such an ICBM, even if it were launched from a very small country, which would allow closer basing of the interceptors to the launch point.

ICBM launch sites To reduce the number of cases analyzed, we focus on the launch sites in each country that would be most advantageous for an attacker, but without considering ease of access or the local topography. We generally place launch sites at least $100 \mathrm{~km}$ inside a country's border, to make them less vulnerable to preemptive attack. The exception is North Korea, where we consider a launch site closer to its border with China, because of

\footnotetext{
${ }^{2}$ The reasons for our focus on these three models are explained in more detail in Chs. 2 and 15.
} 
China's historical support of North Korea's missile program and the advantage such a site would have for evading intercept by a boost-phase defense system.

Location of interceptor bases We assume that interceptor bases and aircraft must be at least $100 \mathrm{~km}$ from the borders of potentially hostile countries, or, in the case of sea-based interceptors, at least $100 \mathrm{~km}$ from the coasts of potentially hostile countries, so that ships are beyond the horizons of land-based radars and have adequate room for maneuvering. Basing interceptor batteries at a single site or at multiple sites are both considered.

ICBM trajectories ICBMs launched from a given site in North Korea or the Middle East could attack a variety of targets in the United States. Missiles flying to different targets would fly outward from their launch site along different azimuths, as illustrated by Figure 5.1 for missiles launched from North Korea and Iraq or Iran. The ground tracks shown are great circles from the launch sites to the targets and neglect the effect of the Earth's rotation on the missile's flight path. ${ }^{3}$ Table 5.2 lists the great-circle distances from launch sites in North Korea, Iraq, and Iran to various cities in the United States and the azimuths of these great circles at the launch sites. The spread of possible initial azimuths is about 40 degrees for ICBMs flying from any of these countries to targets in the 48 contiguous states.

It is important to recall that all of these ICBMs can easily change their azimuths during late boost (after interceptor booster burn-out) by 10 degrees with little degradation in range. Thus, a North Korean missile initially on an azimuth to Los Angeles could easily divert to Seattle, or from Dallas to Seattle.

The trajectory ${ }^{4}$ that an ICBM would follow from a given launch site to a specific target depends on the missile's type and performance characteristics. In particular, solidpropellant ICBMs typically accelerate much faster and burn out much sooner than liquidpropellant ICBMs, even if their maximum ranges are the same (see Chapter 15). For example, the boost phase of the solid-propellant ICBM model S2 is $70 \mathrm{~s}$ shorter than the boost phase of the liquid-propellant ICBM model L. Moreover, when both are flying their maximum-range trajectories, ICBM S2 burns out $100 \mathrm{~km}$ closer to the launch site but $50 \mathrm{~km}$ higher than ICBM L. Their trajectories are so different that a boost-phase defense might have to fire two or more interceptors at each potentially threatening rocket, unless the defense knows in advance or can determine within a few seconds after the rocket has been detected whether it is powered by a liquid- or a solid-propellant motor. Even two ICBMs with the same type of propulsion and the same maximum range can have significantly different flight profiles. As an example, solid-propellant ICBM model S1 burns out $50 \mathrm{~km}$ further downrange than model S2, when both are flying their maximum-range trajectories. Consequently, in analyzing interceptor basing areas, launch engagement areas, and the feasibility

\footnotetext{
${ }^{3}$ The most important effects of the Earth's rotation on a missile's flight are the eastward velocity at launch contributed by the motion of the launch site and the motion of the target relative to the point from which the missile was launched during the missile's flight. We have included the Earth's rotation when computing the initial azimuths of trajectories from North Korea to targets in North America and from Iraq and Iran to Hawaii, because Earth's rotation has a significant impact on the feasibility of boost-phase intercept of ICBMs flying these trajectories. We have neglected Earth's rotation when computing other trajectories, because the rotation does not have a significant impact on the feasibility of boost-phase intercept in those cases.

${ }^{4}$ The term "trajectory" is sometimes used to denote the path followed by a missile. Here we use it to denote the path of the missile as a function of time. Time is important, because missiles may follow similar paths but arrive at the same position at different times after they have been launched. The time dimension is critical for intercepting them.
} 

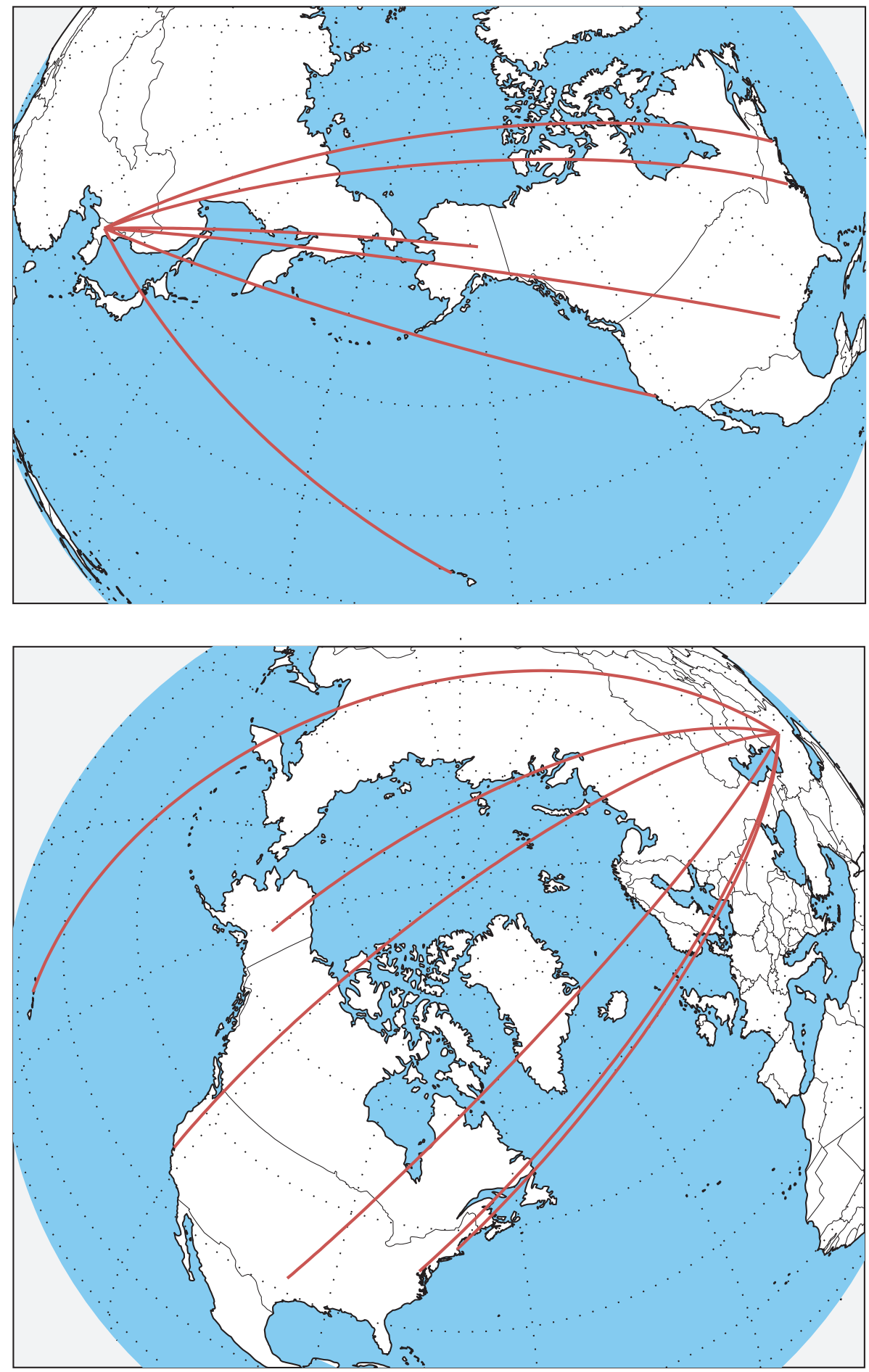

Figure 5.1. Great circles from North Korea (top) and Iraq or Iran (bottom) to Boston, Washington, D.C., Dallas, San Francisco, Fairbanks, and Honolulu. 
Table 5.2. Great-Circle Ranges and Azimuths to Various U.S. Cities ${ }^{a}$

\begin{tabular}{|c|c|c|c|c|c|c|}
\hline \multirow[b]{2}{*}{ City } & \multicolumn{2}{|c|}{ From North Korea } & \multicolumn{2}{|c|}{ From Iraq } & \multicolumn{2}{|c|}{ From Iran } \\
\hline & $\begin{array}{r}\text { Range } \\
(\mathrm{km})\end{array}$ & $\begin{array}{r}\text { Azimuth } \\
\text { (deg) }\end{array}$ & $\begin{array}{r}\text { Range } \\
(\mathrm{km})\end{array}$ & $\begin{array}{r}\text { Azimuth } \\
\text { (deg) }\end{array}$ & $\begin{array}{r}\text { Range } \\
(\mathrm{km})\end{array}$ & $\begin{array}{r}\text { Azimuth } \\
\text { (deg) }\end{array}$ \\
\hline Portland, Maine & 10,500 & 11 & 9,000 & 319 & 10,000 & 324 \\
\hline Boston & 10,700 & 13 & 9,400 & 319 & 10,200 & 324 \\
\hline Washington, D.C. & 10,900 & 18 & 10,300 & 319 & 10,800 & 325 \\
\hline Dallas & 10,800 & 36 & 12,000 & 328 & 12,300 & 336 \\
\hline Fairbanks, Alaska & 5,900 & 32 & 9,300 & 6 & 9,000 & 10 \\
\hline Seattle & 8,000 & 42 & 11,000 & 345 & 11,300 & 360 \\
\hline San Francisco & 8,900 & 49 & 12,100 & 351 & 12,300 & 360 \\
\hline Los Angeles & 9,400 & 51 & 12,400 & 340 & 12,900 & 355 \\
\hline Honolulu & 7,300 & 82 & 13,700 & 27 & 13,050 & 36 \\
\hline Spread of azimuths & & $\sim 40$ & & $\sim 40$ & & $\sim 40$ \\
\hline
\end{tabular}

${ }^{a}$ Ranges are approximate great-circle distances from the country listed to the U.S. city. Azimuths are relative to local north at the launch site. The precise distances and azimuths would depend on the assumed location of the launch site.

${ }^{b}$ Not including Alaska or Hawaii.

of intercept, we consider both ICBM models S1 and S2, as well as model L.

Threat missile range control We assume for this part of the analysis that all ICBM models would attack targets closer than their maximum range by one or a combination of three alternatives: (A) by shutting down the final stage early; (B) by trajectory-shaping, such as dog legs or lofting; and (C) by ejecting the payload on the fly during boost. In later chapters where the kill-vehicle requirements are discussed, we also consider that solidpropellant missiles could also reduce range by executing energy-management maneuvers. In the latter case, the final stage would continue burning until burnout (i.e., for $170 \mathrm{~s}$ ).

\subsubsection{Interceptor models, firing, and flight}

Interceptor models For this analysis we employed the model interceptors constructed in Chapter 16, focusing on the three interceptors listed in Table 5.3. Interceptor I-2 is small enough to be fired either from the existing Vertical Launch System of Aegis-class ships or from a large aircraft, but it has a relatively low burnout velocity. Interceptor I- 4 has a higher burnout velocity, but it is also much larger and heavier. Comparable in size to the Spartan interceptor deployed in North Dakota in the early- to mid-1970s, it is too large to be fired from an aircraft but could be fired from a ship. Interceptor I-5 has a high acceleration and a very high burnout velocity, but it is larger and heavier than most ICBMs (its launch weight is more than twice that of the U.S. Minuteman III, and it is 25 percent longer). It is too large to be launched from an aircraft or existing ships. We did not analyze the basing options for an $8-\mathrm{km} / \mathrm{s}$ interceptor. Such an interceptor would be more manageable than the $10-\mathrm{km} / \mathrm{s}$ interceptor, though still massive. In situations where the $10-\mathrm{km} / \mathrm{s}$ interceptor 
Table 5.3. Interceptors Considered ${ }^{a}$

\begin{tabular}{cccr}
\hline \hline & $\begin{array}{c}\mathrm{V}_{\text {bo }} \\
(\mathrm{km} / \mathrm{s})\end{array}$ & $\begin{array}{c}\text { Burn time } \\
(\text { seconds })\end{array}$ & $\begin{array}{r}\text { Mass } \\
(\mathrm{kg})\end{array}$ \\
\hline $\mathrm{I}-2$ & 5 & 47 & 2,300 \\
$\mathrm{I}-4$ & 6.5 & 40 & 16,900 \\
$\mathrm{I}-5$ & 10 & 45 & 65,600 \\
\hline
\end{tabular}

${ }^{a}$ For further details, see Chapter 16.

would provide more than adequate battlespace, ${ }^{5}$ using a smaller interceptor could naturally be considered.

For simplicity, we analyze one-on-one engagements: a single potentially threatening rocket by a single interceptor.

In this chapter we assume that if an interceptor is able to reach the target missile, its kill vehicle will home on and disable or destroy the missile. The kill-vehicle performance that would be needed is considered in Chapters 11 to 14 .

In analyzing the effective range of an interceptor when fired against a specific ICBM, we assume the defense has knowledge of the trajectory that will be flown by the ICBM. This sidesteps the problems involved in tracking, homing on, and hitting the target booster. The sensor and kill-vehicle performance needed to carry out these tasks is analyzed in Chapters 10 and 12 and in Appendix C. This assumption is valid as long as sufficiently frequent and precise data on the position of the target are available to the interceptor during its flyout and as long as the kill vehicle is sized to handle the large uncertainty remaining after interceptor boost.

\subsubsection{Margins for uncertainties}

In reality, the United States cannot know in advance the intended target of each missile or the precise trajectory each has been programmed to fly. It also may not know the precise launcher locations.

These uncertainties, together with tracking uncertainties, the variability of boost trajectories, and maneuvers during flight make it more difficult for the defense to predict the intercept point for a particular missile. Therefore, when estimating the basing requirement, the defense must include a margin of safety large enough to ensure that its interceptors can get to the attacking missile at the desired time, despite these uncertainties. The uncertainties also make it more difficult for interceptors to home on and hit attacking missiles; this problem is analyzed in Chapter 12 and Appendix C.

Firing solution The defense is able to predict only roughly where and when intercept might be achieved at the time it must launch an interceptor. Therefore, the interceptor must be capable of receiving continued tracking data while it is in its own boost phase. It must also be capable of altering its trajectory during flyout as additional information about the behavior of the target becomes available. The boost burntime for our candidate interceptors

\footnotetext{
${ }^{5}$ As used here, battlespace is the volume in space and time within which a defense system can engage the target successfully.
} 
is optimized at 40 to $45 \mathrm{~s}$ after the interceptor is committed. After interceptor booster burn out, the kill vehicle must be capable of overcoming the remaining uncertainties.

Decision time We refer to the time interval between the moment an acceptable firing solution is first obtained and the moment an interceptor is fired as the decision time. At best, the decision time could be essentially zero, with the system firing an interceptor the moment a firing solution is available. However, more time is likely to be required, given the complexity of a boost-phase intercept system and the consequences of firing an interceptor. If the decision time is increased, the flyout time available for the interceptor is decreased, with a corresponding decrease in the reach of a given interceptor and the size of the area where interceptors could be based. On the other hand, waiting another $30 \mathrm{~s}$, for example, before committing the interceptor would allow observing a staging event from most solidpropellant ICBMs. This delay in turn could help in typing the missile and potentially avoiding having to fire two interceptors to bracket the large differences between liquid- and solid-propellant threats. Therefore, in our geographic analysis, we present two cases - a decision time of zero and a decision time of $30 \mathrm{~s}$ - to show the sensitivity of the engagement to this parameter.

Available decision time Any robust defense system seeks to have sufficient time margin or battlespace to permit replacement in case of an interceptor launch failure, provide additional decision time, or even a second-shot opportunity. We refer to the time interval between the moment an acceptable firing solution has first been obtained and the last moment an interceptor can be fired and still achieve intercept soon enough to protect the defended area as the available decision time. We use the available decision time as a figure of merit for boost-phase intercept systems intended to defend against ICBMs launched from the three countries of concern, estimating it for the liquid- and solid-propellant ICBM models and the three interceptor models we consider.

\subsection{Analysis of Options for Basing Interceptors}

As noted earlier, the missile must be intercepted before it has achieved the velocity needed to carry its munitions to the defended area. Here we show how to apply the methodology developed in Chapter 4 to actual geographic situations. We first show how to determine the last safe intercept time for a given ICBM trajectory and then use this time to determine the interceptor ground range - the maximum distance an interceptor can travel to reach the missile in time for a successful engagement. We then show how the results for a single trajectory can be combined to determine the basing area to cover a range of launch sites, targets, and trajectories.

\subsubsection{Determining the latest time to intercept safely}

The acceleration of an ICBM's final stage increases as it approaches burnout, causing the payload impact range to lengthen rapidly but idiosyncratically for the different models of ICBM. This phenomenon is illustrated by Figure 5.2, which shows the payload impact range for the three model ICBMs as a function of the time before burnout at which the thrust is terminated. In this plot, the liquid-propellant ICBM model $\mathrm{L}$ is on the boost segment of its maximum-range trajectory, while the solid-propellant ICBM models S1 and S2 are either on the boost segments of their maximum-range trajectories or on lofted trajectories having 


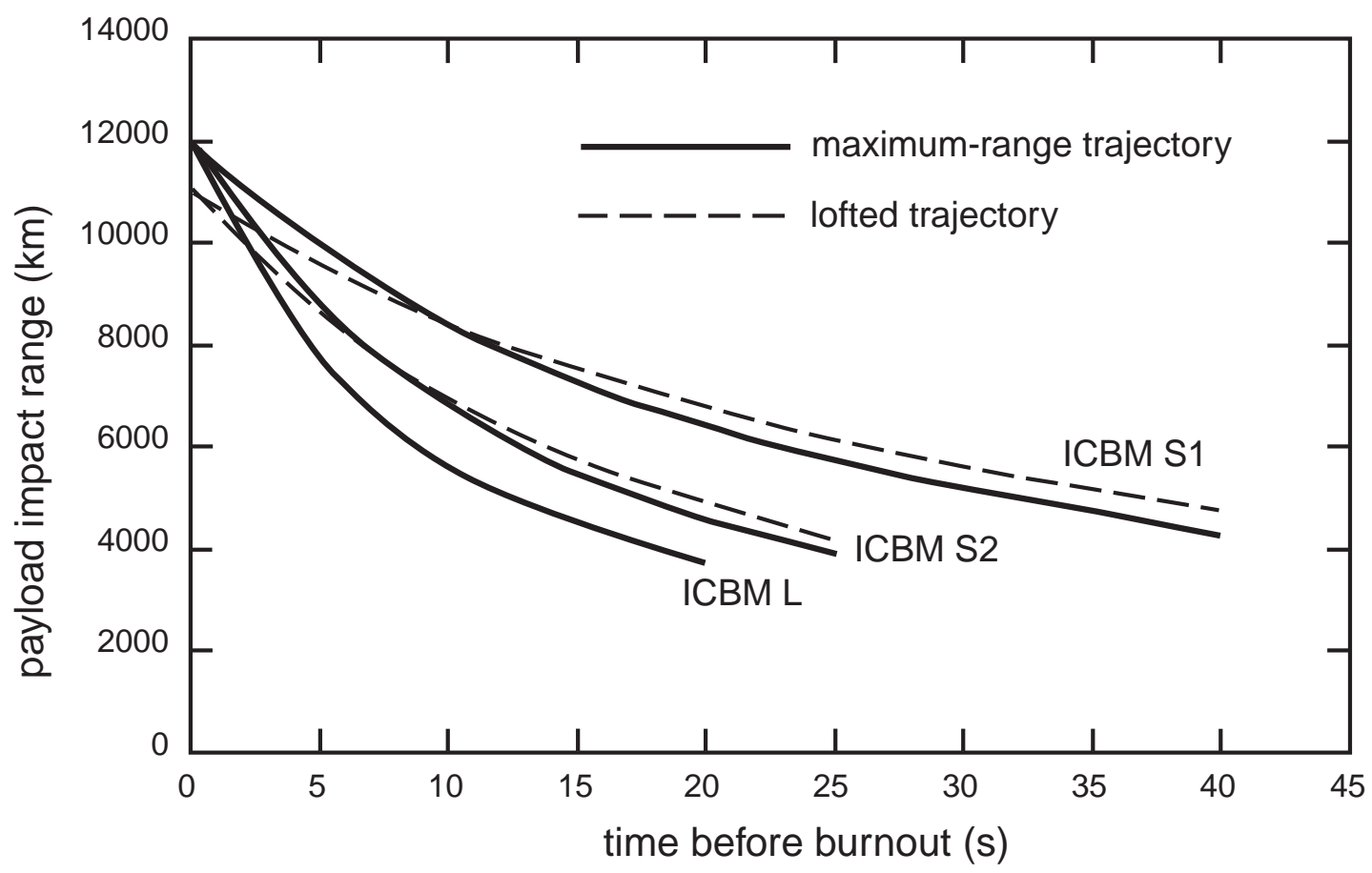

Figure 5.2. Payload range of the missiles modeled in this study as a function of the number of seconds before burnout that their thrust is terminated. The full burn range is $12,000 \mathrm{~km}$. The full burn range for lofted trajectories is $11,000 \mathrm{~km}$ (shown for the solid-propellant missiles). Full burn for ICBM L is $240 \mathrm{~s}$; full burn for ICBMs S1 and S2 is $170 \mathrm{~s}$.

Table 5.4. Range and Velocity for Early Thrust Termination ${ }^{a}$

\begin{tabular}{|c|c|c|c|c|c|c|c|c|c|}
\hline \multirow[b]{2}{*}{$\begin{array}{l}\Delta t \\
(\mathrm{~s})\end{array}$} & \multicolumn{3}{|c|}{ ICBM S1, Solid } & \multicolumn{3}{|c|}{ ICBM S2, Solid } & \multicolumn{3}{|c|}{ ICBM L, Liquid } \\
\hline & $\begin{array}{r}\text { Range } \\
(\mathrm{km})\end{array}$ & $\begin{array}{r}V_{f} \\
(\mathrm{~km} / \mathrm{s}) \\
\end{array}$ & $\begin{array}{r}V_{d} \\
(\mathrm{~km} / \mathrm{s}) \\
\end{array}$ & $\begin{array}{r}\text { Range } \\
(\mathrm{km})\end{array}$ & $\begin{array}{r}V_{f} \\
(\mathrm{~km} / \mathrm{s}) \\
\end{array}$ & $\begin{array}{r}V_{d} \\
(\mathrm{~km} / \mathrm{s}) \\
\end{array}$ & $\begin{array}{r}\text { Range } \\
(\mathrm{km})\end{array}$ & $\begin{array}{c}V_{f} \\
(\mathrm{~km} / \mathrm{s}) \\
\end{array}$ & $\begin{array}{r}V_{d} \\
(\mathrm{~km} / \mathrm{s}) \\
\end{array}$ \\
\hline 0 & 12,000 & 7.20 & 0 & 12,000 & 7.20 & 0 & 12,000 & 7.25 & 0 \\
\hline 5 & 9,980 & 6.98 & 0.22 & 8,780 & 6.72 & 0.48 & 7,740 & 6.71 & 0.54 \\
\hline 10 & 8,450 & 6.73 & 0.47 & 6,800 & 6.31 & 0.89 & 6,982 & 6.26 & 0.99 \\
\hline 15 & 7,310 & 6.51 & 0.59 & 5,400 & 5.94 & 1.26 & 4,500 & 5.87 & 1.38 \\
\hline 20 & 6,430 & 6.30 & 0.90 & 4,580 & 5.62 & 1.58 & 3,730 & 5.53 & 1.72 \\
\hline 25 & 5,730 & 6.12 & 1.08 & 3,900 & 5.32 & 1.88 & & & \\
\hline 30 & 5,160 & 5.94 & 1.26 & & & & & & \\
\hline 40 & 4,290 & 5.63 & 1.67 & & & & & & \\
\hline
\end{tabular}

${ }^{a} \Delta t$ is the time before normal burnout, when the thrust of the final stage is terminated by shutting down its rocket motor (ICBM L), blowing thrust-termination ports (ICBMs S1 and S2), or collision with a kill vehicle. The ICBMs are assumed to be flying their maximum-range trajectories. ICBMs S1 and S2 would normally burn out after $170 \mathrm{~s}$, whereas ICBM L would normally burn out after $240 \mathrm{~s}$. $V_{f}$ is the velocity of the final stage at thrust termination; $V_{d} \equiv$ $V_{b o}-V_{f}$ is the velocity decrement relative to the normal burnout velocity $V_{b o}$ caused by early thrust termination. 
a normal impact range of $11,000 \mathrm{~km}$. Table 5.4 lists the payload ranges for the ICBMs as a function of the time before burnout $\Delta t$, at which the thrust is terminated. Also listed are the velocity $V_{f}$ when the thrust is terminated and the velocity decrement $V_{d}$ relative to the normal burnout velocity $V_{b o}$ of the final stage. This velocity decrement is potentially available for executing a dog-leg or other maneuver, as described in Section 9.1.4. Note that the last $10 \mathrm{~s}$ of final-stage burn increase the impact range of the payloads of ICBMs L, S1, and $\mathrm{S} 2$ by $6,000 \mathrm{~km}, 3,500 \mathrm{~km}$ and $5,000 \mathrm{~km}$, respectively.

Using results presented in Figure 5.2 and Table 5.4, we computed the impact range contours corresponding to intercepts at 5-second intervals for each model ICBM. These contours are shown for ICBMs fired from North Korea toward the United States in Figure 5.3 and later in the chapter for missiles launched from Iran and Iraq.

\subsubsection{Determining required interceptor performance}

Figure 5.4 shows that the maximum feasible standoff distance $D$ between the interceptor base and the missile launch site is greatest for the planar case. The feasible interceptor basing area for a particular target missile, target-missile trajectory, and interceptor under consideration is the shaded circular area centered on the ground projection $\mathrm{P}$ of the intercept point IP; its radius is equal to the interceptor ground range for the engagement.

It is helpful to plot interceptor ground range (IGR) and threat or target ground range (TGR) vs. time after target launch as shown in Figure 5.5. The 1000-km standoff range used in this example is a nominal value that happens to be about the right value to get the desired IGR for solid-propellant missile launches from North Korea to the U.S. East Coast, where a minimum IGR of about $550 \mathrm{~km}$ is needed. The plot can be adjusted for any scenario or candidate interceptor-threat combination to get the IGR desired for the selected intercept time along the threat trajectory. In this display, it is easier to see the consequences of changing the decision time or interceptor flyout characteristics. By shifting the threat curve to the left, the maximum possible interceptor ground range can be estimated if the intercept is constrained to occur at any earlier time.

These plots can be used to determine which interceptor can be used in various situations. For example, consider the engagement of the liquid-propellant ICBM model L by the 10$\mathrm{km} / \mathrm{s}$ interceptor shown in the top panel of Figure 5.5. The dark solid continuous curve rising from the right at a ground range of $1,000 \mathrm{~km}$ is the ICBM time history, $(1,000 \mathrm{~km}-$ TGR). The light dotted curve rising from the left is the IGR time history of the $10-\mathrm{km} / \mathrm{s}$ interceptor when fired with zero decision time. It intersects the ICBM trajectory at $171 \mathrm{~s}$, when the interceptor's ground range is $775 \mathrm{~km}$. For this engagement, the target missile's ground range at the intercept point is $(1,000-775) \mathrm{km}$, or $225 \mathrm{~km}$.

Now suppose the defense requires $30 \mathrm{~s}$ after a firing solution is first obtained to decide to fire at the missile. We can make a good estimate of the intercept point for a 30 -second decision time by shifting the interceptor curves for zero decision time (the light dotted curve in the top panel of Fig. 5.5) upward by $30 \mathrm{~s}$. The result for the $10-\mathrm{km} / \mathrm{sec}$ interceptor is the dark dotted curve rising from the left, which begins at $95 \mathrm{~s}$ and intersects the ICBM trajectory at $196 \mathrm{~s}$, when the interceptor's ground range is $690 \mathrm{~km}$. The target missile's ground range at the intercept point is $310 \mathrm{~km}$.

Intercept times and interceptor and target missile ground ranges can be estimated for other firing times in the same way. As another example, note that the $6.5-\mathrm{km} / \mathrm{s}$ interceptor would intercept the liquid-propellant ICBM model at a ground range of about $550 \mathrm{~km}$, at $225 \mathrm{~s}$, or $130 \mathrm{~s}[(225-30) \mathrm{s}$ decision time $-65 \mathrm{~s}$ firing-solution time $]$ after the interceptor was 

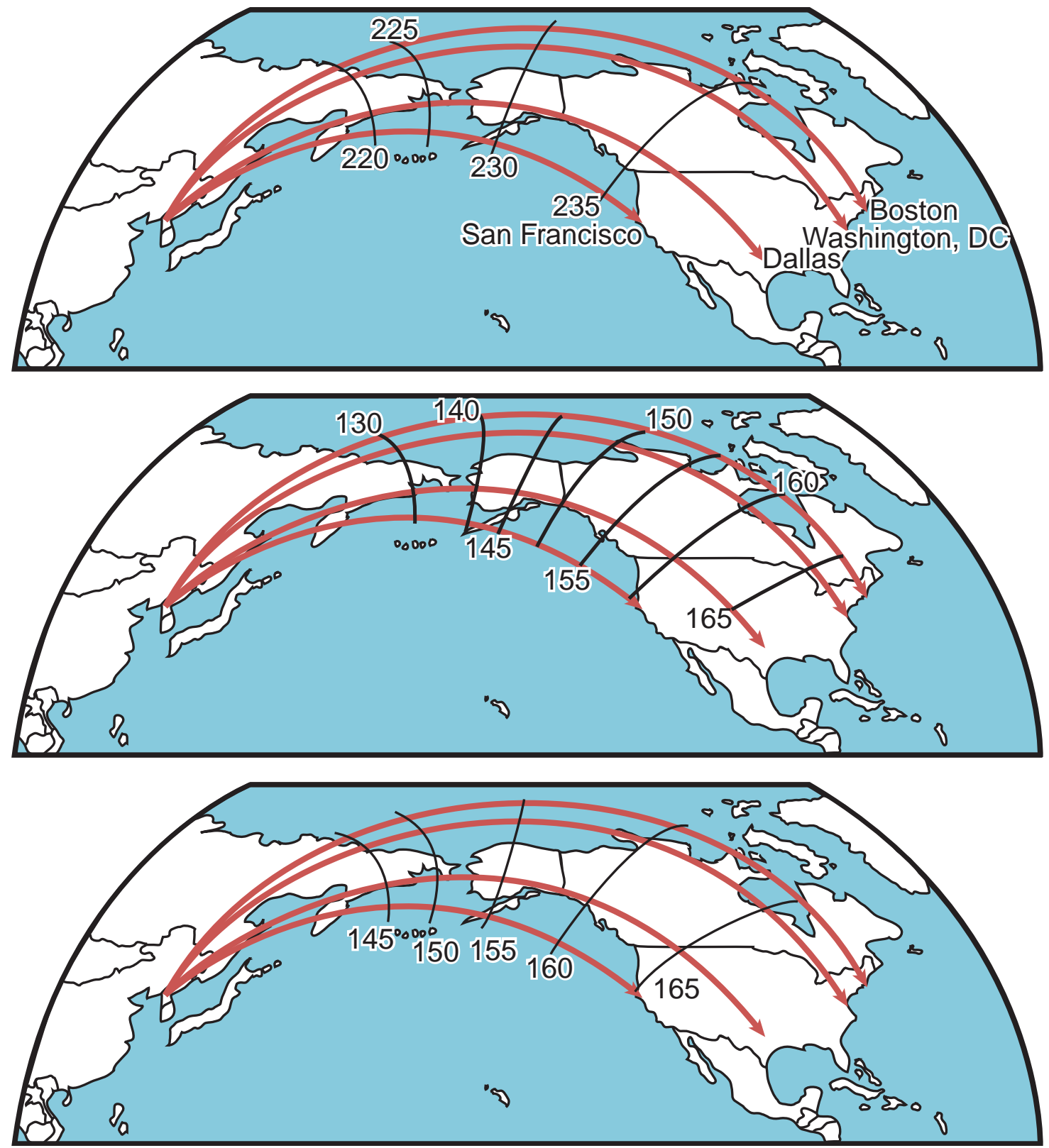

Figure 5.3. Ground tracks of illustrative trajectories of the liquid-propellant ICBM model L (top) and the solid-propellant ICBM models S1 (middle) and S2 (bottom) from North Korea to four cities in North America. These trajectories were computed neglecting Earth's rotation, the mid-section of the actual trajectories would be shifted somewhat farther north. The contours that cross the ground tracks show where the munitions of the missiles would fall if their final stages burned normally until the time in seconds after launch that their thrust was terminated, as indicated by the numbers. For comparison, the full burn times of the liquid- and solid-propellant ICBMs would be 240 and $170 \mathrm{~s}$, respectively. 


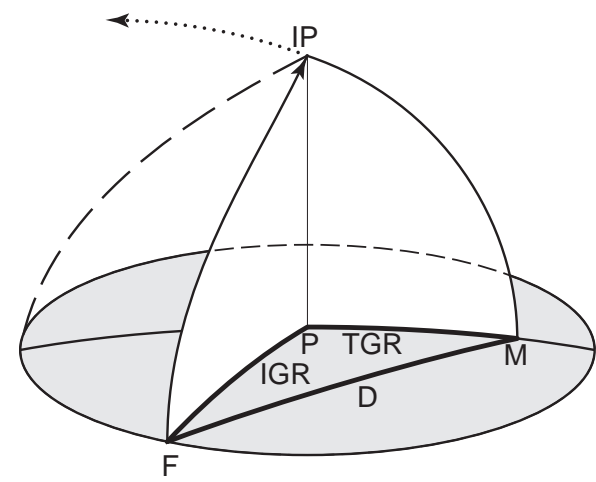

M target missile launch point

$F \quad$ interceptor firing point

IP intercept point

$P$ ground intercept point

TGR target missile ground range

IGR interceptor ground range

D standoff distance

Figure 5.4. Planar missile and interceptor trajectories and the feasible interceptor basing area for an idealized boost-phase engagement.

fired. This intercept would occur $15 \mathrm{~s}$ before the missile's final stage burnout. However, with this decision time, the $6.5-\mathrm{km} / \mathrm{s}$ interceptor would be unable to reach either of the solid-propellant ICBM models before their final stages burned out (see the bottom panel of Fig. 5.5), making it unable to defend the United States. If fired with zero decision time, the $6.5 \mathrm{~km} / \mathrm{s}$ interceptor could intercept ICBM S1 at a ground range of about $550 \mathrm{~km}$ a few seconds before burnout but would be unable to reach ICBM S2 before burnout and could not reach ICBM S1 if it were on a slightly lofted trajectory.

To intercept a solid-propellant ICBM and allow $30 \mathrm{~s}$ of decision time, the $6.5-\mathrm{km} / \mathrm{s}$ interceptor would have to be based closer to the threat trajectory plane and fired on a different trajectory.

\subsubsection{Determining interceptor basing areas}

For each geographical situation considered in this chapter, we determined the last safe intercept time for a given ICBM, trajectory, and defended area. We then computed the corresponding IGR and TGR for an intercept at that time with an algorithm equivalent to the graphical method described above. These results were then used to determine feasible basing areas by the method we now describe.

The interceptor basing area is the area within which interceptors of a particular type can be launched to intercept in the required time an ICBM flying out on a specific trajectory. This circular area has a radius equal to the interceptor's ground range at the intercept point and centered on the point on the ground directly beneath the intercept point, shown as the circular shaded area in Figures 5.4 and 5.6-a.

Defending against missiles launched on different azimuths Defending against missiles on either of two azimuths by interceptors at a single location results in the almond-shaped area shown in Figure 5.6-b. ${ }^{6}$ If locating interceptors within that area is precluded by geographic or operational considerations, that range of azimuths can be covered only by interceptors located in two different places, one anywhere in the left-hand circle in Figure 5.6-b, and the other anywhere in the right-hand circle. Such positioning is possible in some geographic locations, but not others.

\footnotetext{
${ }^{6}$ The shaded area is symmetric in the figure because the IGRs for trajectories on the two azimuths are assumed to be identical. However, this would not generally be the case. If additional azimuths between the two extremes are to be defended, the bottom of the almond would be rounded off or possibly indented.
} 

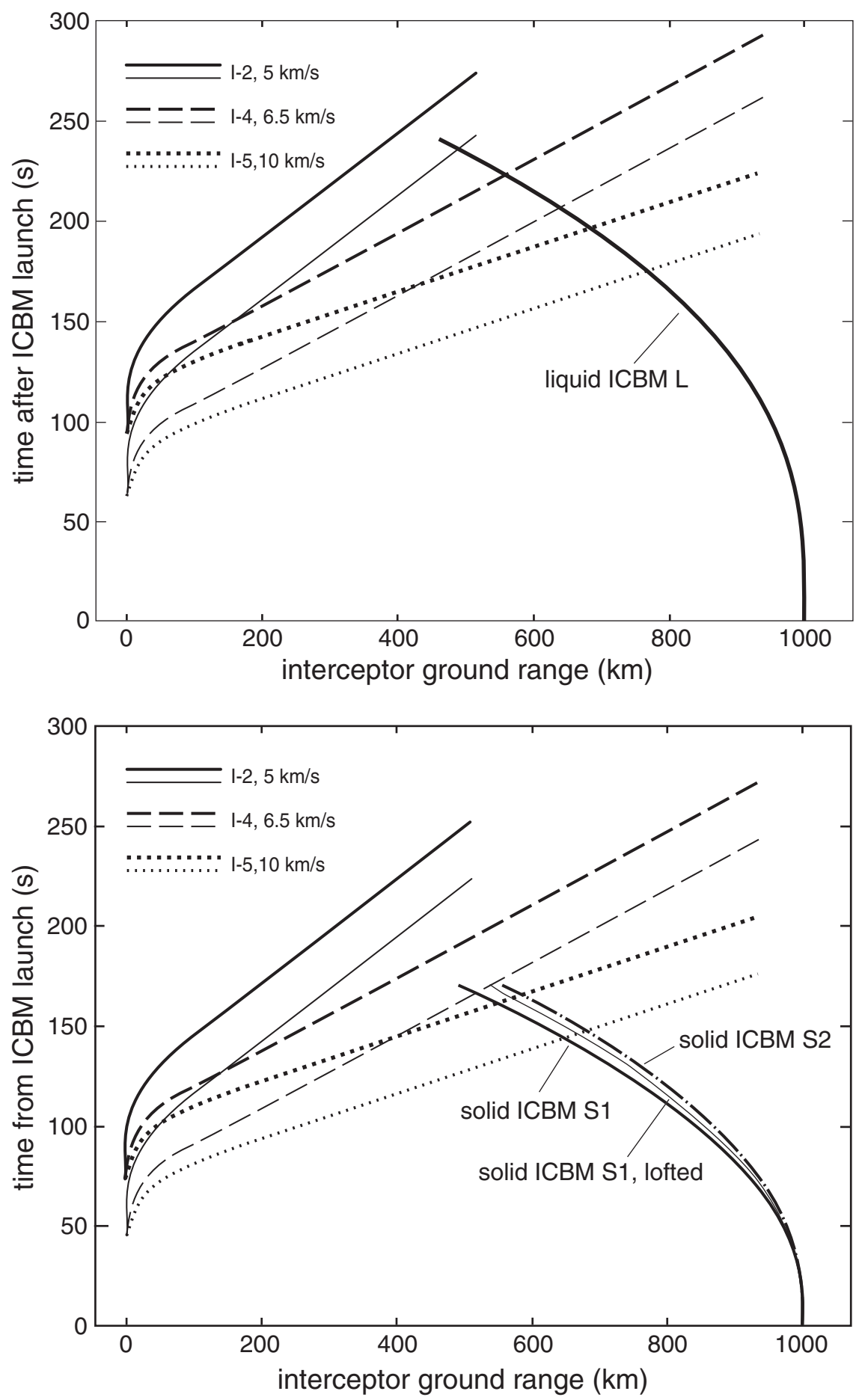

Figure 5.5. Plots of ground range vs. time for the liquid-propellant ICBM model with candidate interceptors (top panel) and the two solid-propellant ICBM models with the same interceptors (bottom panel), at a representative standoff distance of $1000 \mathrm{~km}$. Light curves: zero decision time; dark curves: 30 -second decision time. The results can be generalized to other standoff distances and intercept times. 


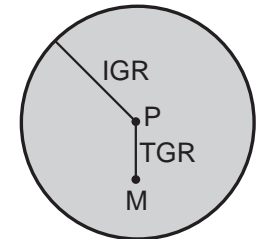

C

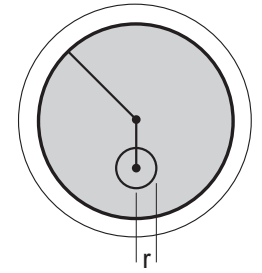

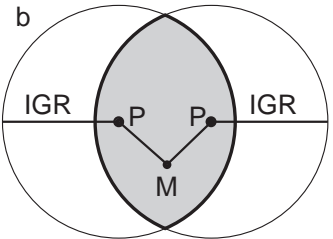

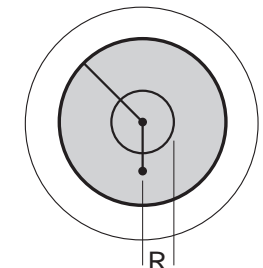

Figure 5.6. a) Ideal interceptor basing geometry; b) area for defending against missiles launched on two azimuths with a single interceptor base; c) reduction in area due to uncertainty $r$ in location of missile base; d) reduction in area due to normal uncertainties in missile flyouts and possible tracking uncertainty.

Reductions to interceptor basing areas The actual interceptor basing areas would be smaller than those we show in this chapter for zero decision time, which assume knowledge of the ICBM trajectories. As noted earlier, the defense may not know the locations of all ICBM launch sites or the performance characteristics of the ICBMs that could be launched from each site, and almost certainly would not know the intended target of each missile or the exact trajectory it had been programmed to fly. To ensure that its boost-phase defense system would be effective, the defender would have to compensate for lack of knowledge and uncertainty by placing the system's interceptors close enough to all possible intercept points to be certain that the system could intercept ICBMs of any plausible type launched from any possible launch site flying out on any possible threatening trajectory before their munitions could reach the United States. Figure 5.6-c and -d illustrate how the defense would be affected by those uncertainties. If the launch point is uncertain by distance $r$, the radius of the interceptor basing area is also reduced by $r$ (Fig. 5.6-c). In addition to target launcher location, uncertainties in the intercept point caused by normal variations in missile flyouts and possible tracking uncertainty result in a total uncertainty of $R$. Thus the radius of the interceptor basing area must be reduced by $R$ (Fig. 5.6-d).

\subsubsection{Determining the available decision time}

For each geographic scenario we considered, we first determined the boundaries of the feasible interceptor basing area for zero decision time and for a 30-s decision time. In general, the possible locations for interceptor bases do not lie on either of these boundaries. If a location lay between the boundaries, then the available decision time would be somewhere between zero and $30 \mathrm{~s}$. If the location lay inside the 30-s boundary, the available decision time would be greater than $30 \mathrm{~s}$. Using the basing area map for the geographic scenario of interest, the available decision time for each interceptor basing location of interest was estimated from its position with respect to the zero and 30-s decision-time boundaries, using linear interpolation. In doing this, we rounded the available decision time to the nearest $5 \mathrm{~s}$. 


\subsection{Basing Analysis Conclusions}

Defending the entire United States We find in Sections 5.4, 5.5, and 5.6 following this summary that defending the United States against liquid-propellant ICBMs launched from countries such as North Korea, Iraq, and Iran to be technically feasible. Defending against the liquid-propellant missiles with more than $30 \mathrm{~s}$ of decision time appears practical against launches from North Korea and Iraq although not from Iran. Furthermore, defending the United States against solid-propellant ICBMs launched from Iran is unlikely to be practical, when all factors are considered. Even defense against solid-propellant ICBMs launched from North Korea would require interceptors having fly-out velocity in excess of $8 \mathrm{~km} / \mathrm{s}$. To achieve a decision time of $30 \mathrm{~s}$ would require using $10-\mathrm{km} / \mathrm{s}$ interceptors, which we judge to be a limiting and perhaps impractical case in achievable speed and acceleration. All things considered, we conclude that defending against typical solid-propellant ICBMs with burn times of $170-180 \mathrm{~s}$ is unlikely to be practical.

The analysis of boost-phase engagement fundamentals in Chapter 4 shows that the short duration of ICBM boost phases limits the maximum interceptor standoff distance (the distance from the ICBM launch site to the interceptor base) to less than approximately $1,000 \mathrm{~km}$, for typical ICBMs and interceptor speeds. Consequently, a boost-phase intercept defense system that employs terrestrial-based interceptors generally would not be effective against ICBMs launched from the interiors of countries having dimensions greater than $1,000 \mathrm{~km}$. Unless violation of the adversary's sovereign airspace by airplanes carrying interceptors were to be contemplated, airborne interceptors also would not be effective against missiles launched from such countries, which include Russia and China. ${ }^{7}$ A system using terrestrial-based interceptor rockets would be most effective against missiles launched from small countries that border international waters or that have neighbors willing to host interceptors.

The available decision times for intercepting missiles from North Korea, Iraq, and Iran to defend all 50 states are summarized in Table 5.5, based on the results of analyses presented in Sections 5.4, 5.5 and 5.6. Whether an intercept is feasible requires making a judgment as to whether the decision time is adequate.

Defending only the 48 contiguous states Defense of only the 48 contiguous states, or of only the major cities, is not significantly easier than defending the entire United States.

Shortfall Unless the warhead can be confidently destroyed in a boost phase intercept, which seems improbable, we know of no way to adequately control where the warheads or munitions of an intercepted threat missile would land, other than short of the defended area.

Warhead kill The ability to destroy the warhead or munitions would not extend the time in which the defense must achieve an intercept, though it would eliminate concerns about live munitions falling to Earth.

Defense against sea-launched medium-range ballistic missiles Theater ballistic missiles fired from ships at sea present a potential threat to the United States. A boost-phase

\footnotetext{
${ }^{7}$ In contrast to terrestrial-based interceptors, space-based interceptors could, in principle, provide global coverage. The advantages and disadvantages of space-based interceptors are analyzed in Chapter 6 .
} 
Table 5.5. Available Decision Times for Defending All 50 States $^{a}$

\begin{tabular}{|c|c|c|c|}
\hline $\mathrm{ICBM}^{b}$ & Interceptor & Time $(\mathrm{s})^{c}$ & Comments \\
\hline \multicolumn{4}{|c|}{ N. Korea ${ }^{d}$} \\
\hline Liquid & $\mathrm{I}-2(5-\mathrm{km} / \mathrm{s})$ & 0 & For interceptors in the Sea of Japan \\
\hline$" 1$ & $\mathrm{I}-4(6.5-\mathrm{km} / \mathrm{s})$ & 40 & $"$ \\
\hline$"$ & $"$ & 50 & For interceptors near Vladivostok \\
\hline$"$ & $\mathrm{I}-5(10-\mathrm{km} / \mathrm{s})$ & $>100$ & Interceptors this fast are not required \\
\hline Solid & $\mathrm{I}-2(5-\mathrm{km} / \mathrm{s})$ & - & Intercept not possible \\
\hline$"$ & $\mathrm{I}-4(6.5-\mathrm{km} / \mathrm{s})$ & $\sim 0$ & For interceptors in the Sea of Japan \\
\hline$"$ & $\mathrm{I}-5(10-\mathrm{km} / \mathrm{s})$ & 30 & For thrust termination or warhead deployment at $137 \mathrm{~s}^{e}$ \\
\hline \multicolumn{4}{|l|}{$\operatorname{Iraq}^{f}$} \\
\hline Liquid & $\mathrm{I}-2(5-\mathrm{km} / \mathrm{s})$ & - & Intercept not possible \\
\hline$"$ & $\mathrm{I}-4(6.5-\mathrm{km} / \mathrm{s})$ & 40 & Provides some flexibility in basing \\
\hline$"$ & $\mathrm{I}-5(10-\mathrm{km} / \mathrm{s})$ & 100 & Interceptors this fast are not required \\
\hline Solid & $\mathrm{I}-2(5-\mathrm{km} / \mathrm{s})$ & - & Intercept not possible \\
\hline$"$ & $\mathrm{I}-4(6.5-\mathrm{km} / \mathrm{s})$ & - & Intercept not possible \\
\hline$"$ & $\mathrm{I}-5(10-\mathrm{km} / \mathrm{s})$ & 30 & For interceptors in extreme southeastern Turkey \\
\hline$"$ & $"$ & 10 & For interceptors in the Persian Gulf \\
\hline \multicolumn{4}{|l|}{$\operatorname{Iran}^{g}$} \\
\hline \multirow[t]{3}{*}{ Liquid } & $\mathrm{I}-2(5-\mathrm{km} / \mathrm{s})$ & - & Intercept not possible \\
\hline & $\mathrm{I}-4(6.5-\mathrm{km} / \mathrm{s})$ & - & Possible only from unconventional bases \\
\hline & $\mathrm{I}-5(10-\mathrm{km} / \mathrm{s})$ & 15 & For interceptors in the Persian Gulf \\
\hline \multirow[t]{3}{*}{ Solid } & $\mathrm{I}-2(5-\mathrm{km} / \mathrm{s})$ & - & Intercept not possible \\
\hline & $\mathrm{I}-4(6.5-\mathrm{km} / \mathrm{s})$ & - & Intercept not possible \\
\hline & $\mathrm{I}-5(10-\mathrm{km} / \mathrm{s})$ & - & Intercept not possible \\
\hline
\end{tabular}

${ }^{a}$ Assumes that missiles are launched from the location that would be most challenging for the defense and that interceptors are stationed in or over accessible international waters or the territory of traditional allies and at least $100 \mathrm{~km}$ from the borders of potentially hostile countries. Defense of the United States by systems with interceptors based in other, unconventional locations and options for partial defense of the United States are discussed in Sections 5.4, 5.5, and 5.6.

${ }^{b}$ The liquid-propellant ICBM is model L; the solid-propellant ICBM is model S1.

${ }^{c}$ This is the time interval between the moment a firing solution has first been obtained and the last moment an interceptor can be fired and still intercept the ICBM early enough to defend all 50 states. Uncertainties in ICBM launch times, performance characteristics, and trajectories have been ignored; they would reduce the available decision time.

${ }^{d}$ For details, see Section 5.4.

${ }^{e}$ Based on impact on Fairbanks, Alaska.

${ }^{f}$ For details see Section 5.5. Potential basing sites considered here are in Turkey and the Persian Gulf.

${ }^{g}$ For details, see Section 5.6. Potential basing sites considered here are in Turkey and the Persian Gulf. For a discussion of boost-phase defense options using interceptors stationed in unconventional locations, such as in or over the Caspian Sea, Turkmenistan, and other countries to the east and north of Iran, see Section 5.6. 


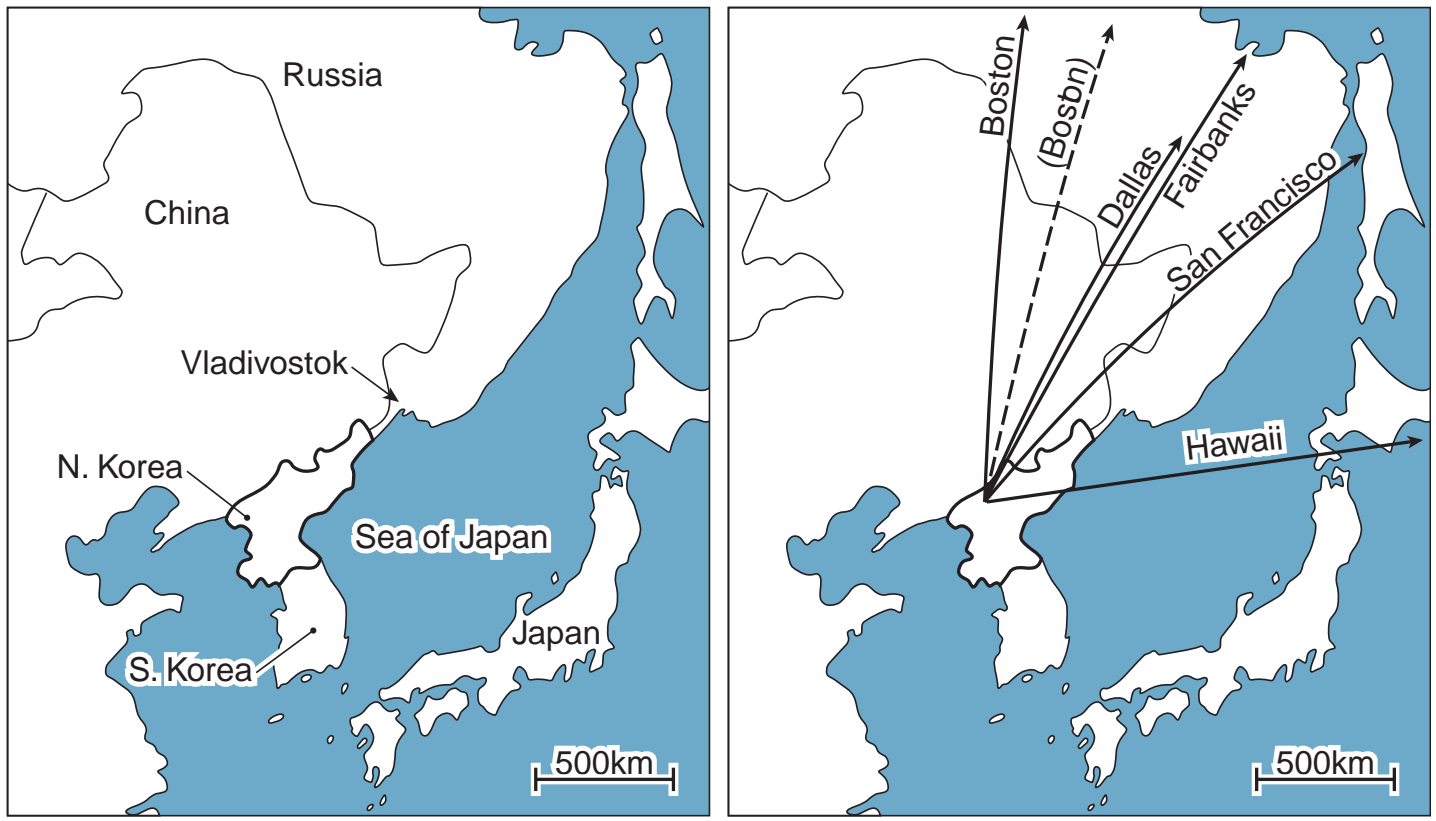

Figure 5.7. (left) North Korea and adjacent countries. (right) Azimuths for missiles launched from North Korea to targets in the United States. These differ from great circle azimuths because of the effect of the Earth's rotation. The great circle azimuth for Boston is shown dashed to illustrate the magnitude of this effect.

defense appears readily achievable by an interceptor similar to a Standard Missile 2, fired from a ship within a distance of about $40 \mathrm{~km}$ of the threat launch platform.

In the following three sections, we estimate the decision time available to boost-phase defense systems that use the three model interceptors we consider to intercept ICBMs launched by the three countries of concern.

\subsection{Defending Against ICBMs Launched from North Korea}

Defending against ICBMs launched from North Korea would be easier than defending against ICBMs launched from other countries of current concern, because North Korea is relatively small and borders seas that could be accessible to ship-based interceptors. Figure 5.7 illustrates the Korean peninsula and its immediate surroundings and also the azimuths of ICBM trajectories to targets spanning the United States. Figure 5.3 shows the ground tracks (the projection on the ground vertically below the position of the missile) of trajectories of liquid-propellant ICBM model L and solid-propellant ICBM models S1 and S2 from North Korea to Boston, Washington, D.C., Dallas, and San Francisco. ${ }^{8}$ The defense of Hawaii is a special case that will be examined separately in Section 5.4.1.

The U.S. city closest to North Korea is Fairbanks, Alaska (Table 5.2). In general, the closer the target the more difficult the intercept, a close target permits a relatively

\footnotetext{
${ }^{8}$ These maps, and the similar maps below that show the ground tracks of ICBM trajectories from Iraq to North America, are in the orthophanic or Robinson projection. The scale is uniform with longitude but varies with latitude; it is true along the $38^{\circ}$ parallels. This projection is not equal-area, conformal, or equidistant, but is considered to "look right" for world maps and is used by Rand McNally, the National Geographic Society, and others. The trajectories are great circles; taking Earth's rotation into account would shift the central regions of the tracks slightly to the North.
} 


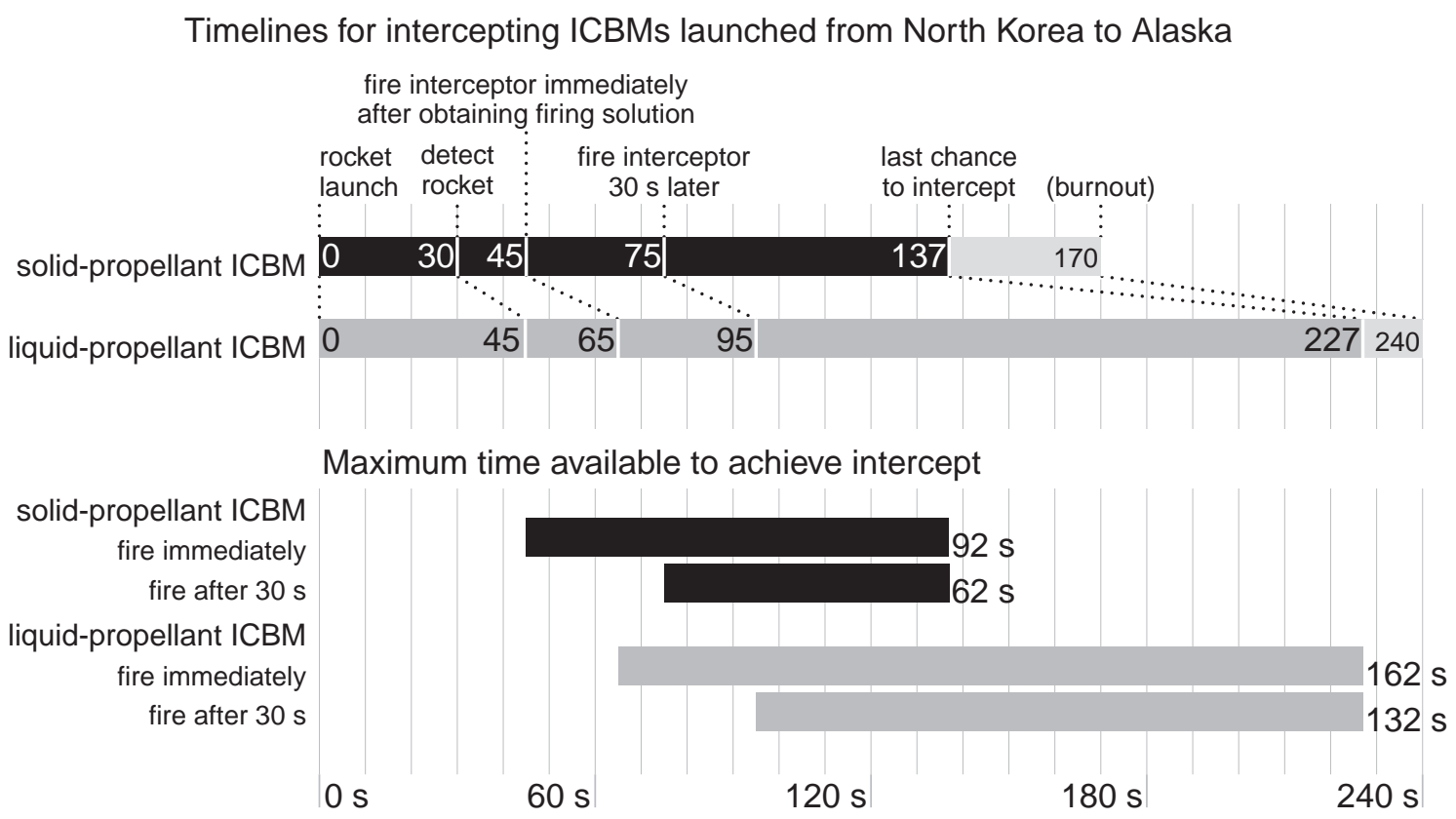

Figure 5.8. Timelines for engaging liquid-propellant ICBM model L and solid-propellant ICBM model S1 when launched from North Korea toward the middle of the United States on a trajectory over Alaska.

short burn time, thus limiting the flyout distance of the interceptor. However, the ease or difficulty of any particular intercept also depends on local geography. In the case of missiles launched from North Korea, it will be shown that missiles to the East Coast of the United States, which require almost the maximum burn time, can be more difficult to defend than the Alaska trajectory, because their boost-phase trajectories lie far to the west of the Sea of Japan and require relatively long IGRs. For the same reason, the northwest of the United States is relatively easy to defend, in spite of the relatively short range, because the boost-phase trajectories of these missiles lie close to the Sea of Japan. Consequently, we shall consider two baseline cases: defense against missiles headed toward Fairbanks (or the center of the United States) on a trajectory toward Alaska, and missiles headed to the East Coast, on a trajectory toward Boston.

From Figure 5.3, we take the latest time to safely intercept a missile on the Alaska trajectory to be $227 \mathrm{~s}$ for the liquid-propellant missile ICBM L, and $137 \mathrm{~s}$ for the solidpropellant ICBM S1. (Requirements for defense against ICBM model S2 are less stringent than S1 and will not be analyzed separately.) The timelines for the Alaska trajectory are illustrated in Figure 5.8. The timelines for the Boston trajectory with zero and 30-second delays are illustrated in Figure 5.3-239 s for the liquid-propellant ICBMs and $167 \mathrm{~s}$ for the solid-propellant ICBMs.

The intercept times and the corresponding TGRs are shown in Table 5.6. The TGRs were obtained from the trajectory calculations for the missiles (Appendix B). For each of the intercept times determined above, we calculated interceptor ground ranges (IGRs) for interceptors I-2, I-4, and I-5 for zero decision time and 30-second decision time, using the methods described in Section 5.2. These IGRs are listed in Table 5.7.

Using the values of target missile and interceptor ground ranges from Tables 5.6 and 5.7, 
Table 5.6. Intercept Times ${ }^{a}$ and ICBM Ground Ranges from North Korea

\begin{tabular}{llll}
\hline \hline & Fairbanks & Boston & Seattle \\
\hline ICBM model $\mathrm{L}^{b}$ intercept time(s) & 227 & 239 & 235 \\
TGR $(\mathrm{km})$ & 460 & 539 & 500 \\
ICBM model S1 ${ }^{b}$ intercept time(s) & 137 & 167 & 158 \\
TGR $(\mathrm{km})$ & 320 & 492 & 430 \\
\hline
\end{tabular}

${ }^{a}$ Times are latest time for safe intercept to target shown.

${ }^{b}$ Properties of the missiles are described in Chapter 15 .

we constructed interceptor basing areas for defense against the liquid- and solid-propellant ICBMs and plotted them for the two baseline trajectories (Fairbanks and Boston). The results are shown in Figures 5.9 and 5.10. These figures provide the key information for understanding the options for defending the continental United States from ICBMs launched from North Korea. The ground tracks for each trajectory are shown in the figures; the last chance to intercept the missile is indicated by an " $\mathrm{x}$ " on each ground track. The interceptor must be based inside the shaded circle for it to reach the missile with at least $30 \mathrm{~s}$ of decision time. It must be based on the dashed circle to reach the missile in the limiting case of zero

Table 5.7. Ground Ranges of Interceptors Fired Against ICBMs Launched from North Korea to the United States ${ }^{a}$

\begin{tabular}{cccrrr}
\hline \hline \multirow{2}{*}{$\begin{array}{c}\text { ICBM } \\
\text { Model }\end{array}$} & \multirow{2}{*}{$\begin{array}{c}\text { Decision } \\
\text { Time }(\mathrm{s})\end{array}$} & $\begin{array}{c}\text { Interceptor } \\
\text { Model }^{b}\end{array}$ & \multicolumn{3}{c}{ Nominal Range IGR } \\
\cline { 4 - 6 } & & Fairbanks & Boston & Seattle \\
\hline Liquid $^{b}$ & 0 & I-2 & 530 & 570 & 570 \\
& 0 & I-4 & 740 & 800 & 750 \\
& 0 & I-5 & 1,220 & 1,330 & 1250 \\
& 30 & I-2 & 410 & 460 & 450 \\
& 30 & I-4 & 570 & 640 & 600 \\
Solid $1^{b}$ & 30 & I-5 & 950 & 1,060 & 1000 \\
& 0 & I-2 & 260 & 380 & 350 \\
& 0 & I-4 & 350 & 530 & 470 \\
& 0 & I-5 & 590 & 880 & 800 \\
& 30 & I-2 & 140 & 270 & 230 \\
& 30 & I-4 & 190 & 370 & 300 \\
& 30 & I-5 & 310 & 600 & 530 \\
\hline
\end{tabular}

${ }^{a}$ The latest times to intercept safely and the corresponding TGRs are listed in Table 5.6

${ }^{b}$ The basic characteristics of these model interceptors are listed in Table 5.3.

${ }^{c}$ Values obtained from Fig. 16.5 for I2, and Fig. 4.9 for I4 and I5. The minimum required ground range can be seen in Figs. 5.9 and 5.10 . 
decision time.

\section{Defense against liquid-propellant ICBM model L}

Figure 5.9 (bottom) reveals that the most difficult trajectory to defend against for ICBM L is the trajectory to the northeastern United States - the Boston trajectory. To defend this trajectory, the $5-\mathrm{km} / \mathrm{s}$ interceptor would have to be fired with zero decision time. However, this interceptor could defend the Alaska trajectory with about $40 \mathrm{~s}$ of decision time and still remain based $100 \mathrm{~km}$ off the coast. The $5-\mathrm{km} / \mathrm{s}$ interceptor could be deployed by the Navy's Aegis system, based on ships near the coast. A $5-\mathrm{km} / \mathrm{s}$ interceptor could also be launched from aircraft, but its operational area would be subject to essentially the same restrictions as the surface-based $5-\mathrm{km} / \mathrm{s}$ interceptor.

The $6.5-\mathrm{km} / \mathrm{s}$ interceptor could provide about $40 \mathrm{~s}$ of decision time, if based at sea. If it were ground-based at Vladivostok, the decision time would be about $60 \mathrm{~s}$.

\section{Defense against solid-propellant ICBM model S1}

Defense against the solid-propellant missile ICBM model S1 is considerably more challenging than for the liquid-propellant missile. Figure 5.10 reveals that, for the solid-propellant missile, the most challenging trajectory is to Boston. The $5-\mathrm{km} / \mathrm{s}$ interceptor (not shown) could not be used. The lower-left panel of Figure 5.10 reveals that even the $6.5-\mathrm{km} / \mathrm{s}$ interceptor could be used to defend Boston only if it were fired very close to the coast with zero decision time. The giant $10-\mathrm{km} / \mathrm{s}$ interceptor (Fig. 5.10, lower right) could be used with about $30 \mathrm{~s}$ of decision time. Deploying such a large missile at sea could present a challenge, but it might be possible to land-base it in Vladivostok, which would permit the decision time to be increased by about five seconds.

\subsubsection{Defending Hawaii}

Hawaii lies $7300 \mathrm{~km}$ from North Korea at an azimuth of $82^{\circ}$. For the solid-propellant missile $\mathrm{S} 1$, the TGR at latest intercept is $435 \mathrm{~km}$, putting the intercept point well off the coast. The intercept point would lie in the Sea of Japan at a location that is well suited for a boost-phase defense. Figure 5.11 shows that even for the most stressing case - attack by a solid-propellant missile - a defense would be possible with the $5-\mathrm{km} / \mathrm{s}$ interceptor fired from a ship or plane.

\subsubsection{Defending against missiles from North Korea with multiple ground sites}

For the liquid-propellant missile, a single $6.5-\mathrm{km} / \mathrm{s}$ interceptor could defend both the continental United States and Hawaii (See Figs. 5.9 and 5.11). For the solid-propellant missile, defense of the continental states would require the capability of the $10-\mathrm{km} / \mathrm{s}$ interceptor. This same interceptor easily defends the Hawaii trajectory, though a smaller missile might provide operational advantages. Nevertheless, employing a second basing site would not offer any essential advantage for a boost-phase defense system to defend against missiles from North Korea. 

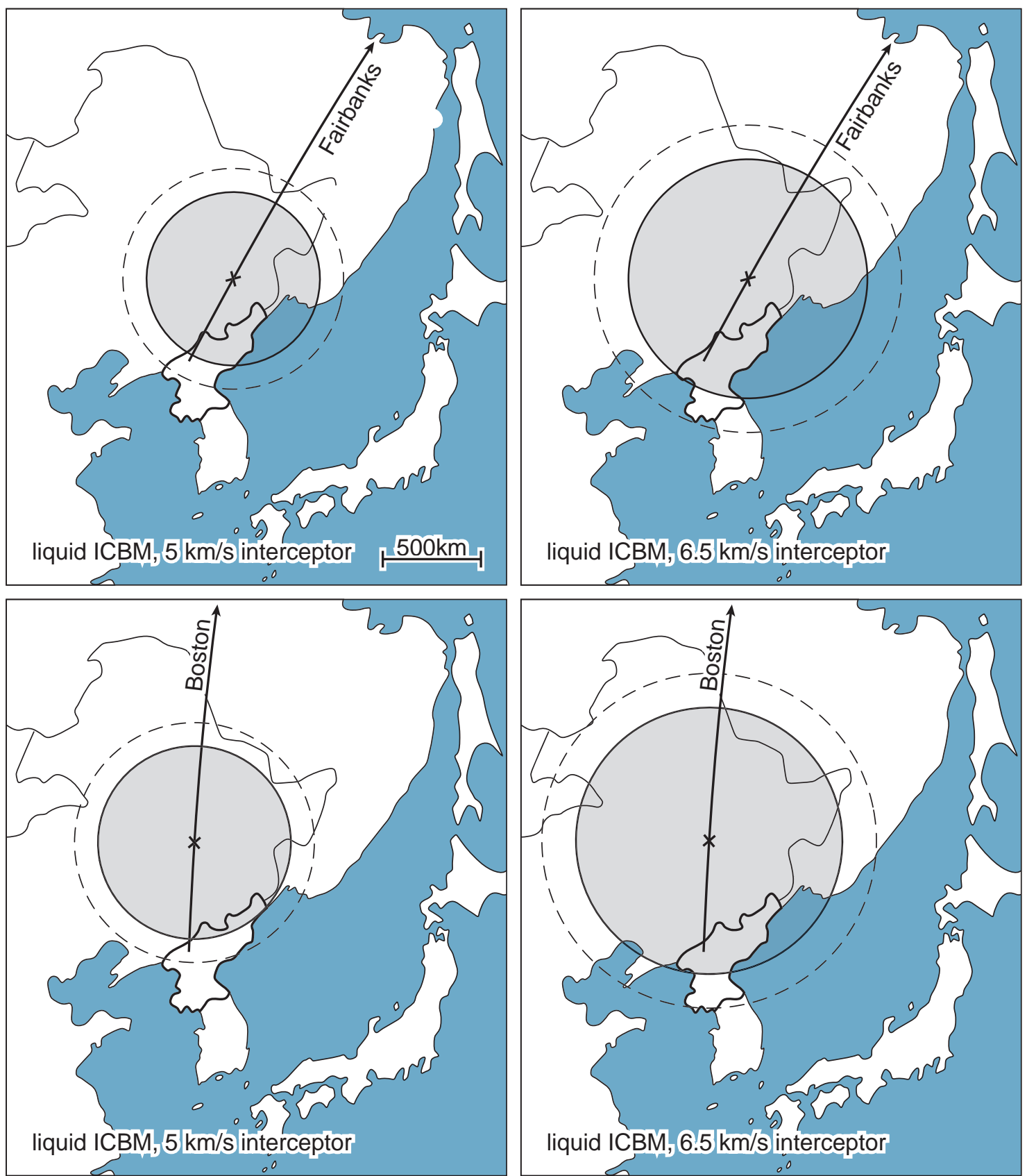

Figure 5.9. Interceptor basing areas for defending the contiguous United States against the liquidpropellant missile ICBM model L launched from North Korea. The position of the missile at the last chance it can be intercepted successfully is indicated by the " $\mathrm{x}$ " on the trajectory line. The dashed outer circles are the areas within which the interceptors must be based for the limiting case in which they are fired the instant a firing solution is constructed. The shaded inner circles are the areas within which the interceptors must be based if they are fired with a 30-second decision time. Top: trajectory toward Fairbanks, Alaska. (left) The model 5-km/s interceptor. (right) The $6.5-\mathrm{km} / \mathrm{s}$ interceptor. Bottom: same, but for a trajectory to Boston. 

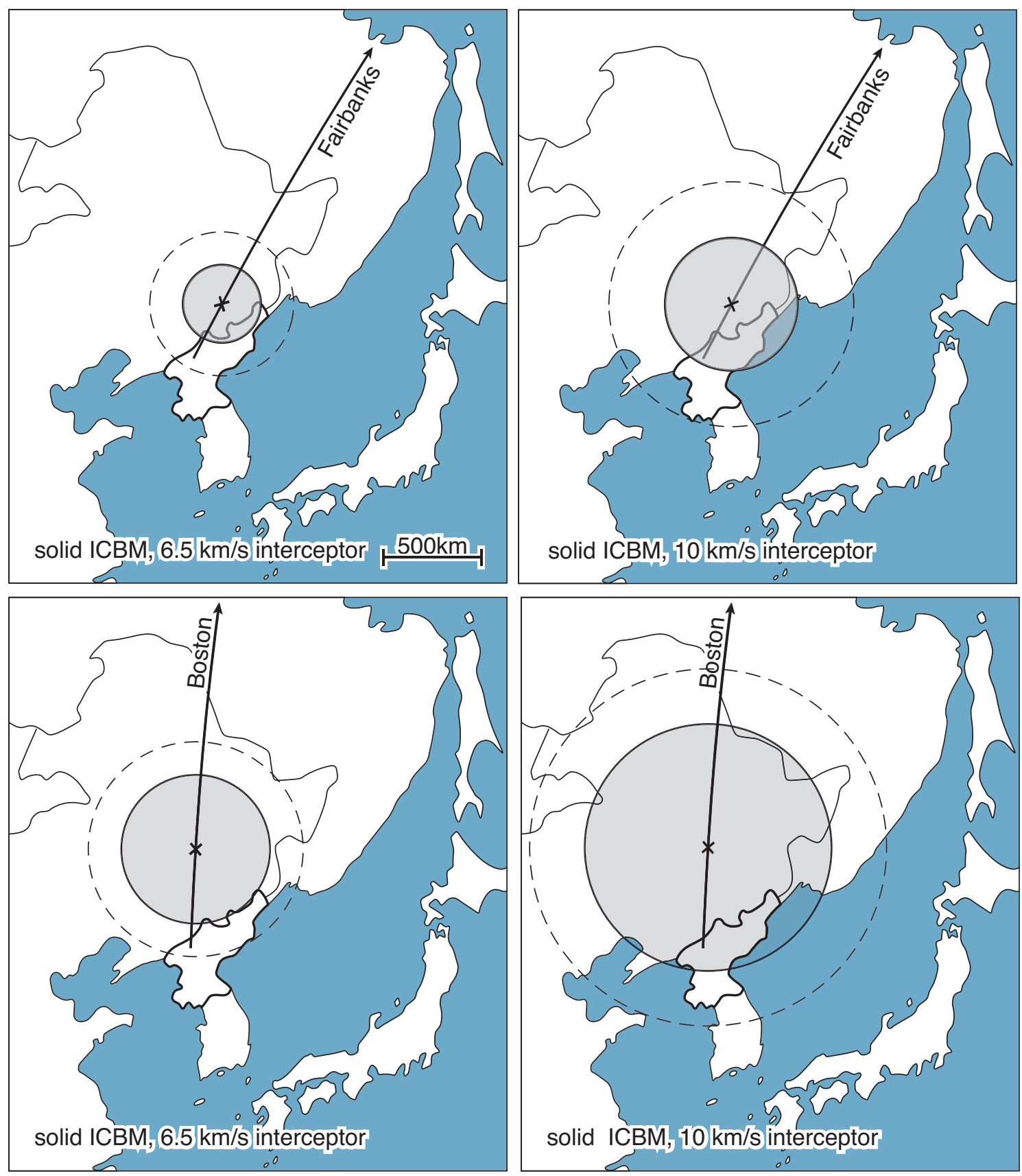

Figure 5.10. Interceptor basing areas for defending the contiguous United States against the solidpropellant missile ICBM model S1 launched from North Korea. The position of the missile at the last chance it can be intercepted is indicated by the " $\mathrm{x}$ " on the trajectory line. The dashed outer circles are the areas within which the interceptors must be based for the limiting case in which they are fired the instant a firing solution is constructed. The shaded inner circles are the areas within which the interceptors must be based if they are fired with a 30-second decision time. Top: trajectory toward Fairbanks, Alaska. (left) The model $5-\mathrm{km} / \mathrm{s}$ interceptor. (right) The $6.5-\mathrm{km} / \mathrm{s}$ interceptor. Bottom: same as above but for a trajectory to Boston. 

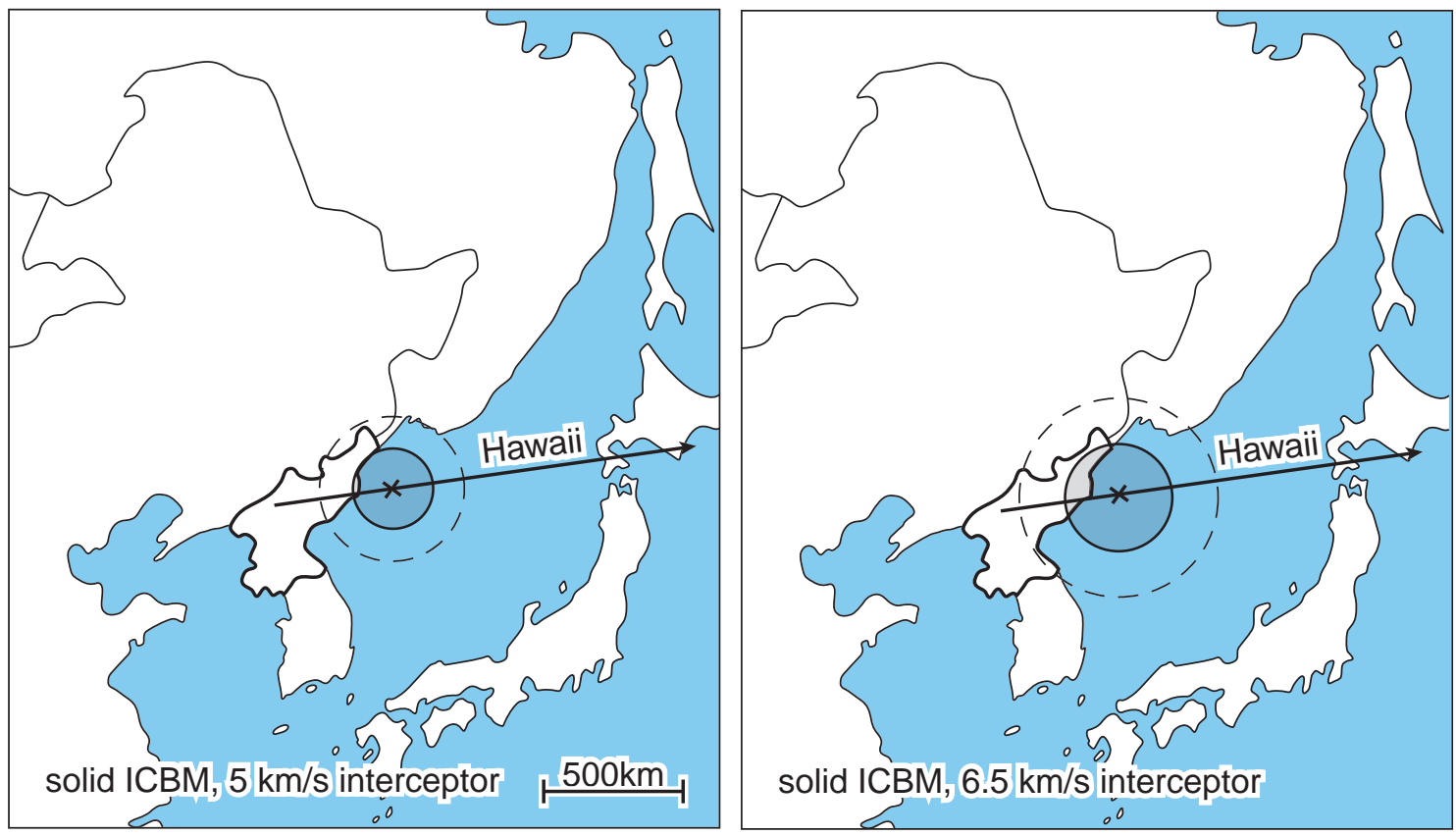

Figure 5.11. Basing areas for defending Hawaii from the solid-propellant missile model S1 launched by North Korea. (left) $5 \mathrm{~km} / \mathrm{s}$ interceptor. (right) $6.5 \mathrm{~km} / \mathrm{s}$ interceptor.

\subsubsection{Possibility of an interceptor being mistaken as a threat}

Interceptors having a velocity of $5 \mathrm{~km} / \mathrm{s}$ or more would have sufficient speed and range to be mistaken for a threat. The interceptor trajectories from North Korea are generally to the northwest and head across China to Russia. Thus, an interceptor fired in defense runs the risk of triggering retaliatory action by China or Russia.

\subsection{Defending Against ICBMs Launched from Iraq}

We turn now to the defense of ICBMs launched from Iraq and Iran. These nations are much larger than North Korea, and the considerations for siting have little in common with those for North Korea. Figure 5.12 shows how Iraq and Iran are situated with respect to nearby countries and bodies of water.

\subsubsection{Defending the contiguous states}

The latest time that the defense could safely intercept missiles from Iraq to the contiguous states can be found from Figure 5.13, which shows the ground tracks of trajectories of ICBM models L, S1, and S2 from Iraq to Washington, D.C., Dallas, and San Francisco.

As before, the numbered contours that cross the ground tracks show where the missiles' munitions would fall if their final stages terminated at the time indicated after launch. The liquid-propellant ICBM L would have to be intercepted no later than $237 \mathrm{~s}$ after launch, $3 \mathrm{~s}$ before final stage burnout, at which point its ground range would be $520 \mathrm{~km}$. The solid-propellant ICBM S1 would have to be intercepted no later than $161 \mathrm{~s}$ after launch, $8 \mathrm{~s}$ before burnout, at which point its ground range would be $460 \mathrm{~km}$. The IGRs for these intercept times are displayed in Table 5.8. For a 30-second decision time, the IGRs for 


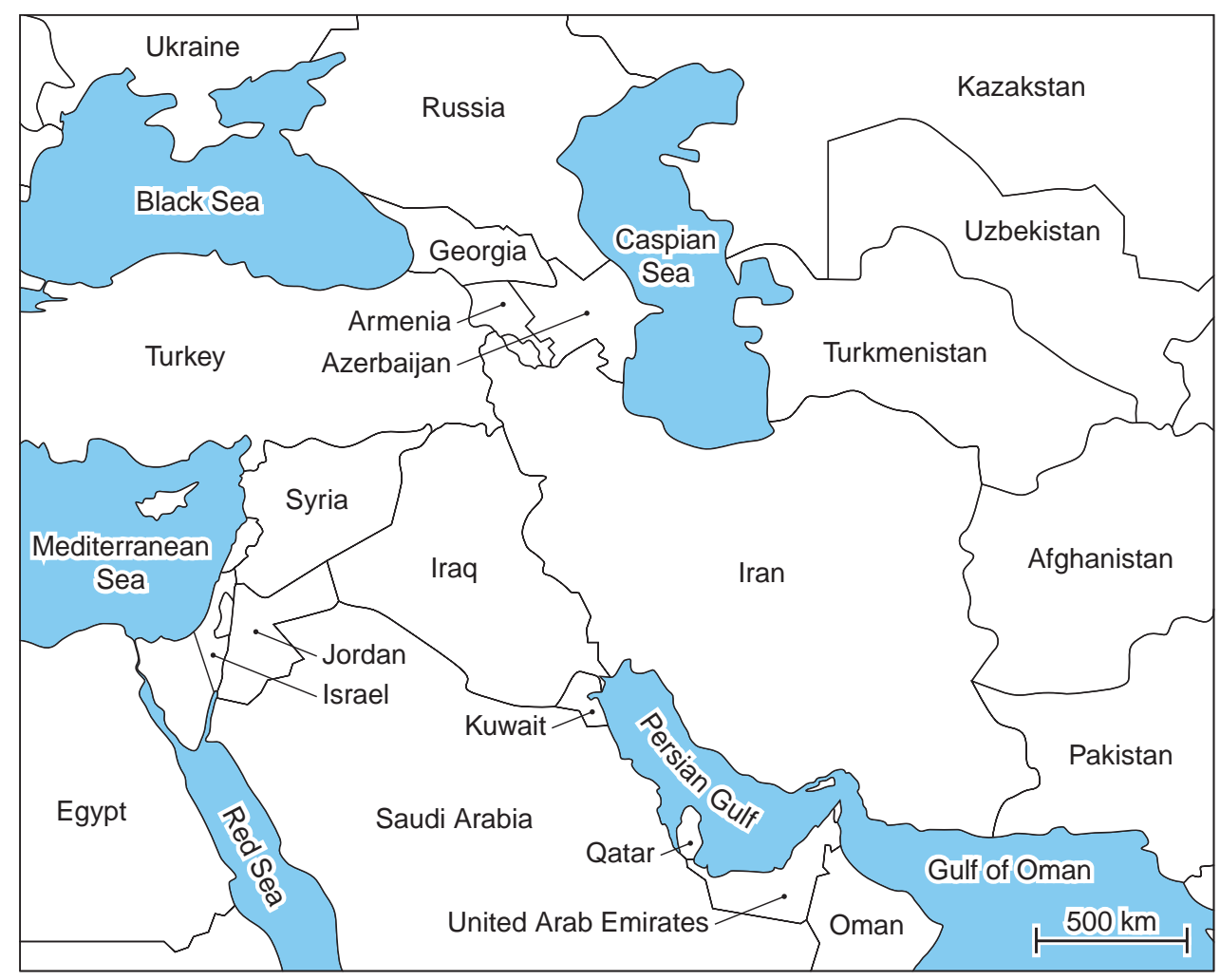

Figure 5.12. Map showing Iraq and Iran and nearby countries and bodies of water.

intercepting ICBM model L vary from about $450 \mathrm{~km}$ to about $1050 \mathrm{~km}$; for intercepting ICBM model S1, they vary from about $250 \mathrm{~km}$ to about $550 \mathrm{~km}$.

The possibilities for defending the continental United States are shown in Figure 5.14, which displays the initial portions of planar trajectories from southeastern Iraq to Washington, D.C. (or Boston) and San Francisco; the last safe intercept points on the Washington, D.C. and San Francisco trajectories; and the feasible basing areas for four ICBM-interceptor combinations and two decision times. The ICBM launch site chosen is one of the most challenging for a boost-phase defense. The interceptor basing areas are almond-shaped, because they indicate where a single interceptor base must be located to cover all possible trajectories. Defending with interceptors based at more than one location is discussed below.

\section{Defense against liquid-propellant ICBM L}

The top left-hand panel of Figure 5.14 shows that a boost-phase intercept system based in southeastern Turkey employing $5-\mathrm{km} / \mathrm{s}$ interceptors could defend the contiguous 48 states from attack by Iraq with a liquid-propellant ICBM like model L, with a decision time of $30 \mathrm{~s}$. However, the interceptors would have to be based closer than $100 \mathrm{~km}$ from the Iraqi border. The top right-hand panel shows that the $6.5-\mathrm{km} / \mathrm{s}$ interceptors would provide up to $60 \mathrm{~s}$ of decision time and would allow the interceptors to be based comfortably away from Iraq. 

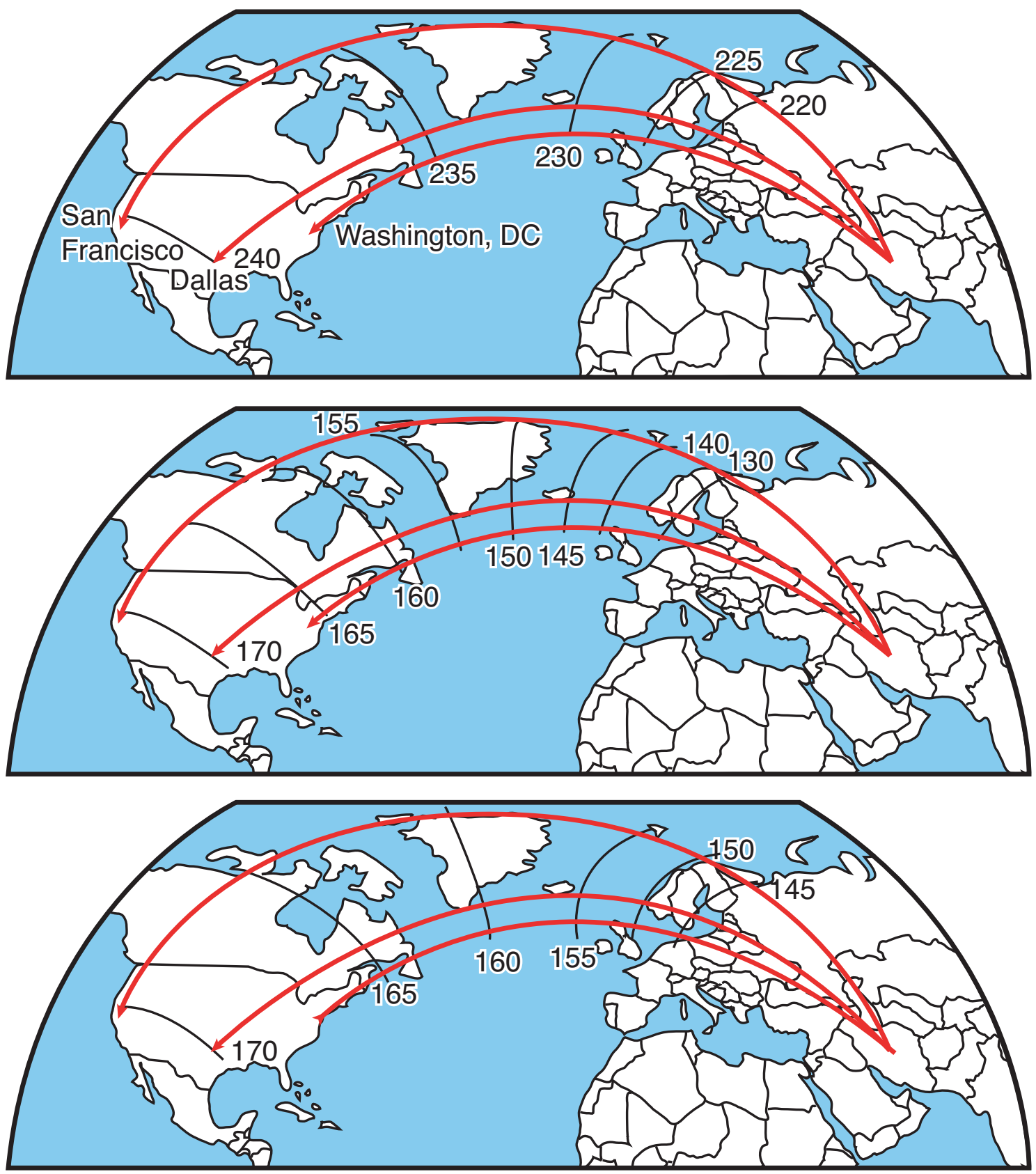

Figure 5.13. Ground tracks of illustrative trajectories of the liquid-propellant ICBM model L (top) and the solid-propellant ICBM models S1 (middle) and S2 (bottom) from Iraq to San Francisco, Dallas, and Washington, D.C. These trajectories were computed neglecting Earth's rotation. The effect of Earth rotation would shift their mid sections slightly further south. The contours that cross the ground tracks show where the munitions of the missiles would fall if their final stages burned normally until the time in seconds after launch that their thrust was terminated, as indicated by the numbers. For comparison, the full burn times of the liquid- and solid-propellant ICBMs would be 240 and $170 \mathrm{~s}$, respectively. 
Table 5.8. Ground Ranges of Interceptors Fired Against ICBMs Launched from Iraq or Iran to the United States

\begin{tabular}{cccc}
\hline \hline $\begin{array}{c}\text { ICBM } \\
\text { Model }\end{array}$ & $\begin{array}{c}\text { Decision } \\
\text { Time (s) }\end{array}$ & $\begin{array}{c}\text { Interceptor } \\
\text { Model }^{a}\end{array}$ & $\begin{array}{c}\text { Nominal } \\
\text { Range }^{d}(\mathrm{~km})\end{array}$ \\
\hline Liquid $^{b}$ & 0 & I-2 & 570 \\
& 0 & I-4 & 790 \\
& 0 & I-5 & 1,310 \\
& 30 & I-2 & 450 \\
& 30 & I-4 & 630 \\
Solid $1^{c}$ & 0 & I-5 & 1,040 \\
& 0 & I-2 & 350 \\
& 0 & I-4 & 490 \\
& 30 & I-5 & 800 \\
& 30 & I-4 & 230 \\
& 30 & I-5 & 310 \\
\hline
\end{tabular}

${ }^{a}$ The basic characteristics of these model interceptors are listed in Table 5.3.

${ }^{b}$ The latest time to intercept safely liquid-propellant ICBM model $\mathrm{L}$ is $237 \mathrm{~s}$ after launch.

${ }^{c}$ The latest time to intercept safely solid-propellant ICBM model S1 is $161 \mathrm{~s}$ after launch.

${ }^{d}$ The required ground ranges can be seen in Figs. 5.14 and 5.15 .

\section{Defense against solid-propellant ICBM S1}

The lower left panel of Figure 5.14 shows that defending the United States from attack by solid-propellant ICBMs like model S1 launched from Iraq with the $6.5-\mathrm{km} / \mathrm{s}$ interceptor would have a decision time of zero. The very large $10-\mathrm{km} / \mathrm{s}$ interceptor, based in the southeastern corner of Turkey near the Iraqi border, could cover all potential launch sites with a decision time of about $30 \mathrm{~s}$.

\subsubsection{Defending Alaska and Hawaii}

The range from Iraq to Fairbanks is about the same as its range to Boston, and the target ground ranges would be similar. The Fairbanks azimuth is east of the azimuth to San Francisco. Hawaii's azimuth is even further to the east. Thus, if Hawaii can be defended, so can Alaska. Consequently, we turn our attention to the defense of Hawaii.

The range to Hawaii-13,700 km - is larger than the range of the ICBMs we have studied, and so we can only roughly estimate what might be possible. Hawaii's great circle azimuth is $27^{\circ}$, or $36^{\circ}$ east of the San Francisco azimuth. The stress of defending this trajectory is somewhat reduced by the effect of the Earth's rotation on the trajectory. We have determined the rotating Earth azimuth to be approximately $15^{\circ}$ west of the great circle azimuth of $27^{\circ}$, or $12^{\circ}$. 

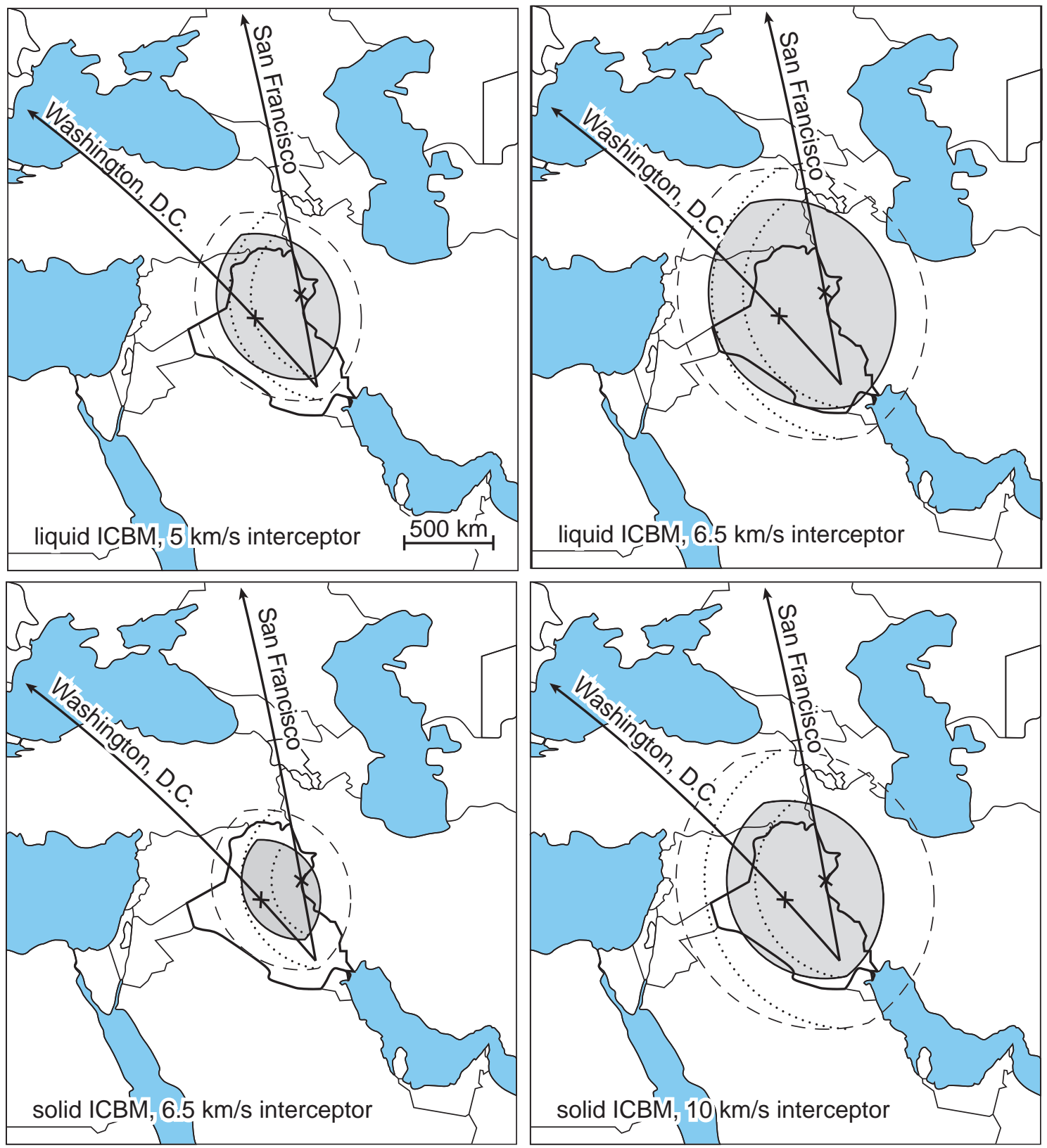

Figure 5.14. Interceptor basing areas for defending the contiguous United States against ICBMs launched from Iraq. The liquid-propellant ICBM is model L (above); the solid-propellant is model S1 (below). Shown are the ground tracks of planar trajectories to Washington, D.C., Dallas, and San Francisco. The last chance to intercept missiles to Washington, D.C. and San Francisco is shown by an " $\mathrm{x}$ " on their trajectories. (The trajectories neglect Earth's rotation, which would rotate them slightly counterclockwise.) Feasible basing areas for 30-second decision time are shaded, and for zero decision time are outlined by dashed curves. In the bottom panels, the ICBM launch site has been taken to be further south than in the top panels, where it is more challenging for the defense. The dotted curves show the western boundaries of the reduced basing areas if Alaska and Hawaii are included in the defense. 
By inspecting the basing areas whose western borders are limited by the dotted curves in Figure 5.14, it can be seen that, for a liquid-propellant missile, both Alaska and Hawaii might be defended by a $5-\mathrm{km} / \mathrm{s}$ interceptor sited in extreme southeast Turkey. However, for sites at least $100 \mathrm{~km}$ from the border with Iraq, the decision time would be no more than about $20 \mathrm{~s}$. The $6.5-\mathrm{km} / \mathrm{s}$ interceptor could provide about $45 \mathrm{~s}$ of decision time. For the solid-propellant missile, the $10-\mathrm{km} / \mathrm{s}$ interceptor, based in southeast Turkey, would be required and could provide about $30 \mathrm{~s}$ of decision time.

\subsubsection{Defending against missiles from Iraq with multiple interceptor sites}

The discussion of defending against missiles from Iraq has been limited until now to interceptors based at a single site. We now examine how the results might change if a second site were employed.

The defense of the entire United States against the liquid-propellant missile by a single $5-\mathrm{km} / \mathrm{s}$ interceptor in Turkey would require a site very close to the borders of Iraq and Iran. A second site could potentially ease the tight time constraints and vulnerability of the defense by permitting the interceptor base to be moved further from these borders. The possibilities are shown in Figure 5.15 (left), which displays the extreme azimuths for defending all of the United States: from Washington, D.C. to Honolulu (which also covers Alaska). It is evident that the second site must be in the Caspian Sea close to the coast of Iran, a less attractive alternative than carrying out the defense with a $6.5-\mathrm{km} / \mathrm{s}$ interceptor at a single site.

Figure 5.15 (right) shows the possibilities for defending against the solid-propellant ICBM S1 from Iraq using $6.5-\mathrm{km} / \mathrm{s}$ interceptors based at two sites. However, the only sites to the east would be in Iran, and could not be employed. Thus, there would be no alternative to using a faster interceptor, for instance the $10-\mathrm{km} / \mathrm{s}$ interceptor I-5, and there would be no advantage to employing a second site.

\subsection{Defending Against ICBMs Launched from Iran}

The large size of Iran poses a serious challenge for a boost-phase defense system. Potential basing sites for defending against ICBMs launched by Iran that are easily accessible include the Persian Gulf and Turkey. We describe such locations as "conventional", for they are in accessible international waters or within the borders of traditional allies. For purposes of completeness, however, we have also considered sites that are attractive geographically but problematic because of operational or political concerns. These include the land-locked Caspian Sea. Other sites would be in Turkmenistan, Kazakhstan, Uzbekistan, Afghanistan, or Azerbaijan. We describe such sites as "unconventional".

The trajectories from Iran to U.S. targets are similar to those from Iraq, and the ranges and intercept times from Iran are taken to be the same as for Iraq (see Table 5.8).

\subsubsection{Defending the contiguous states}

Figure 5.16 shows the initial portions of planar trajectories from central Iran to Washington, D.C. and San Francisco; the last safe intercept points for defense on the Washington, D.C., and San Francisco trajectories; and the feasible basing areas for four ICBM-interceptor combinations and two decision times.

The most desirable locations for a defense are in the Caspian Sea or Turkmenistan. Because the Caspian is land-locked, it is questionable whether such a site is practical. For 

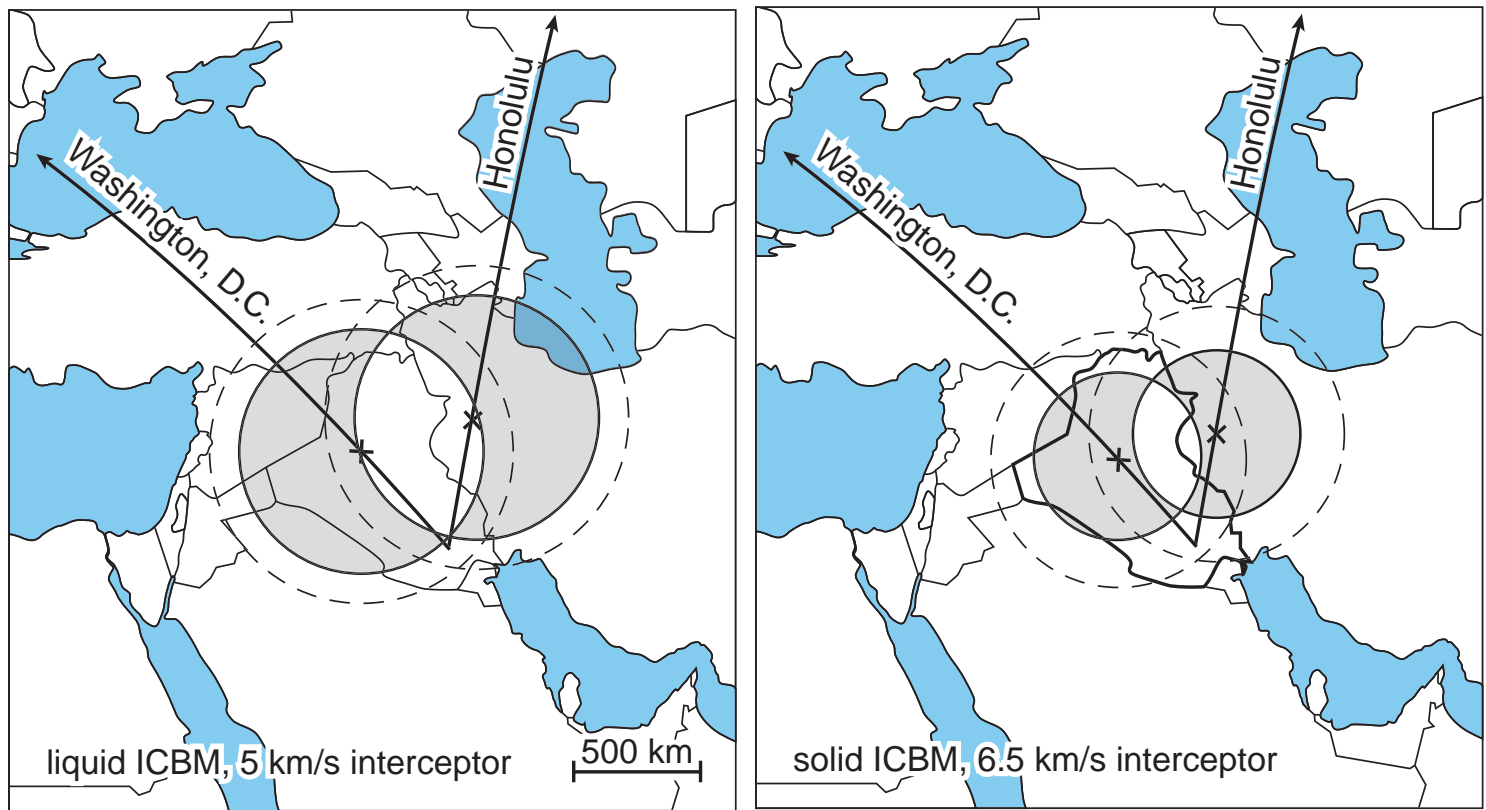

Figure 5.15. Defending all of the United States against missiles launched from Iraq, using interceptors based at two sites.

political reasons, it is questionable whether basing interceptors within Turkmenistan would be practical. Because of the problematic nature of these locations, we shall include them in our basing analysis only in parenthetical comments.

Defense against liquid-propellant ICBM L The top left-hand panel of Figure 5.16 shows that, for $6.5-\mathrm{km} / \mathrm{s}$ interceptors and 30 -second decision times, the only options for defending the contiguous United States from attack by a liquid-propellant ICBM like model L would be the unconventional locations of the Caspian Sea or Turkmenistan. The top right-hand panel shows that the very large 10-km/s interceptor could be based in the Persian Gulf with a 30-second decision time. However, this location would be vulnerable because it could be defeated by ICBMs launched from areas further to the east. Basing in southeast Turkey would provide about $15 \mathrm{~s}$ of decision time with the $10-\mathrm{km} / \mathrm{s}$ interceptors. The interceptors could also be located in the Caspian Sea or Turkmenistan, or other unconventional locations such as Afghanistan or Azerbaijan. Armenia is a possibility for the interceptor launch location, but it would be ineffective if the ICBM launch site were moved slightly to the east. Basing in the southern tip of the Russian Caucasus would be similarly vulnerable.

Defense against solid-propellant ICBM S1 The bottom panels of Figure 5.16 show that the $6.5-\mathrm{km} / \mathrm{s}$ interceptor could not defend the United States from attack by a solidpropellant ICBM like model S1 launched from southern Iran. For the 10-km/s interceptor, the only basing options would be in unconventional locations: the southern end of the Caspian Sea or Turkmenistan. These basing areas would permit a decision time of up to $30 \mathrm{~s}$, though this could be reduced if Iran located its launch site further to the south. 

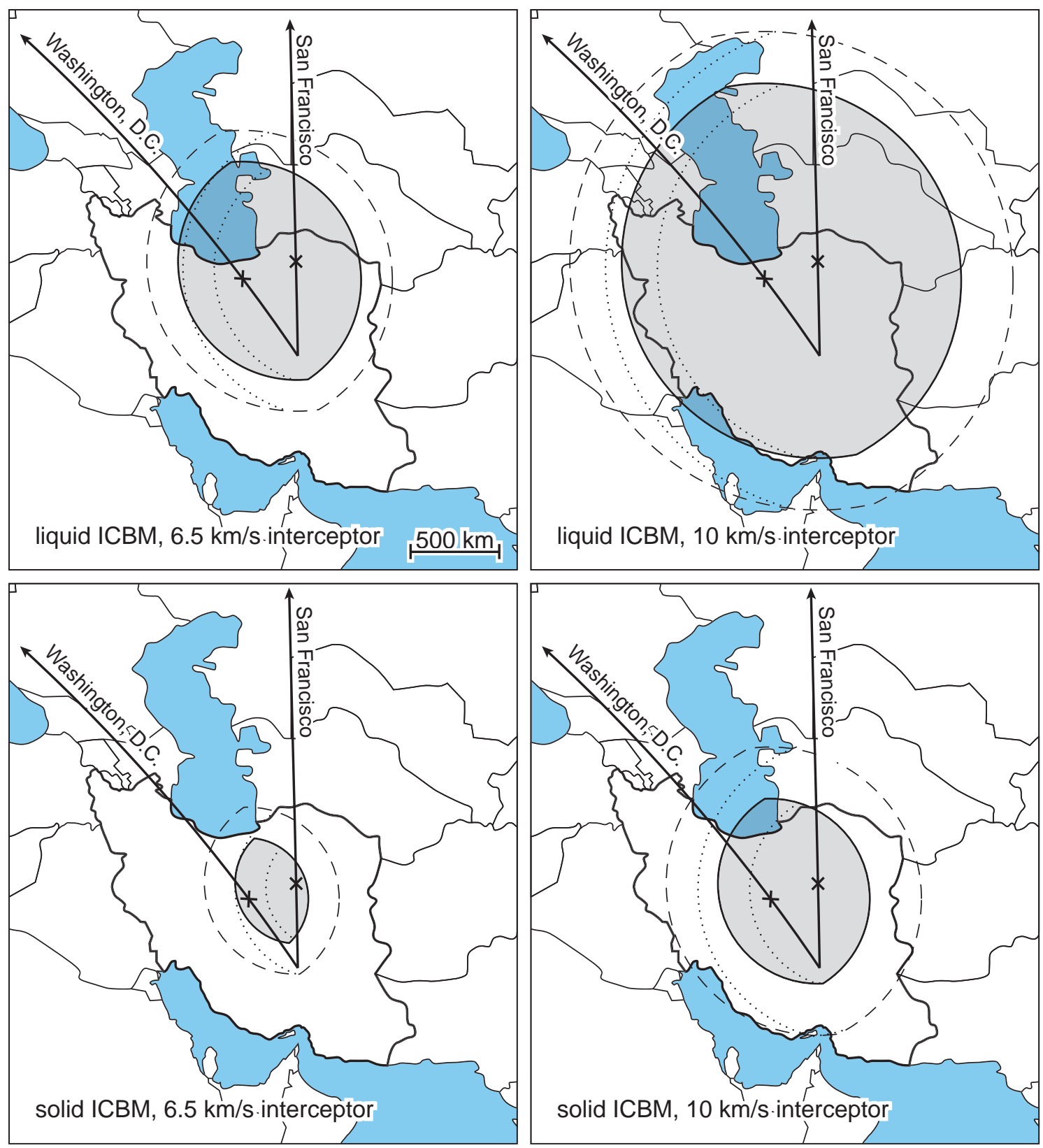

Figure 5.16. Interceptor basing areas for defending the contiguous United States against ICBMs launched from Iran. The liquid-propellant ICBM is model L (above); the solid-propellant is model S1 (below). Shown are the ground tracks of planar trajectories to Washington, D.C., Dallas, and San Francisco. The last chance to intercept missiles to Washington, D.C. and San Francisco is shown by an " $\mathrm{x}$ " on their trajectories. (The trajectories neglect Earth's rotation, which would rotate them slightly counterclockwise.) Feasible basing areas for 30-second decision time are shaded, and for zero decision time are outlined by dashed curves. In the bottom panels the ICBM launch site has been taken to be further south than in the top panels, where it is more challenging for the defense. The dotted curves show the western boundaries of the reduced basing areas when Alaska and Hawaii are included in the defense. 

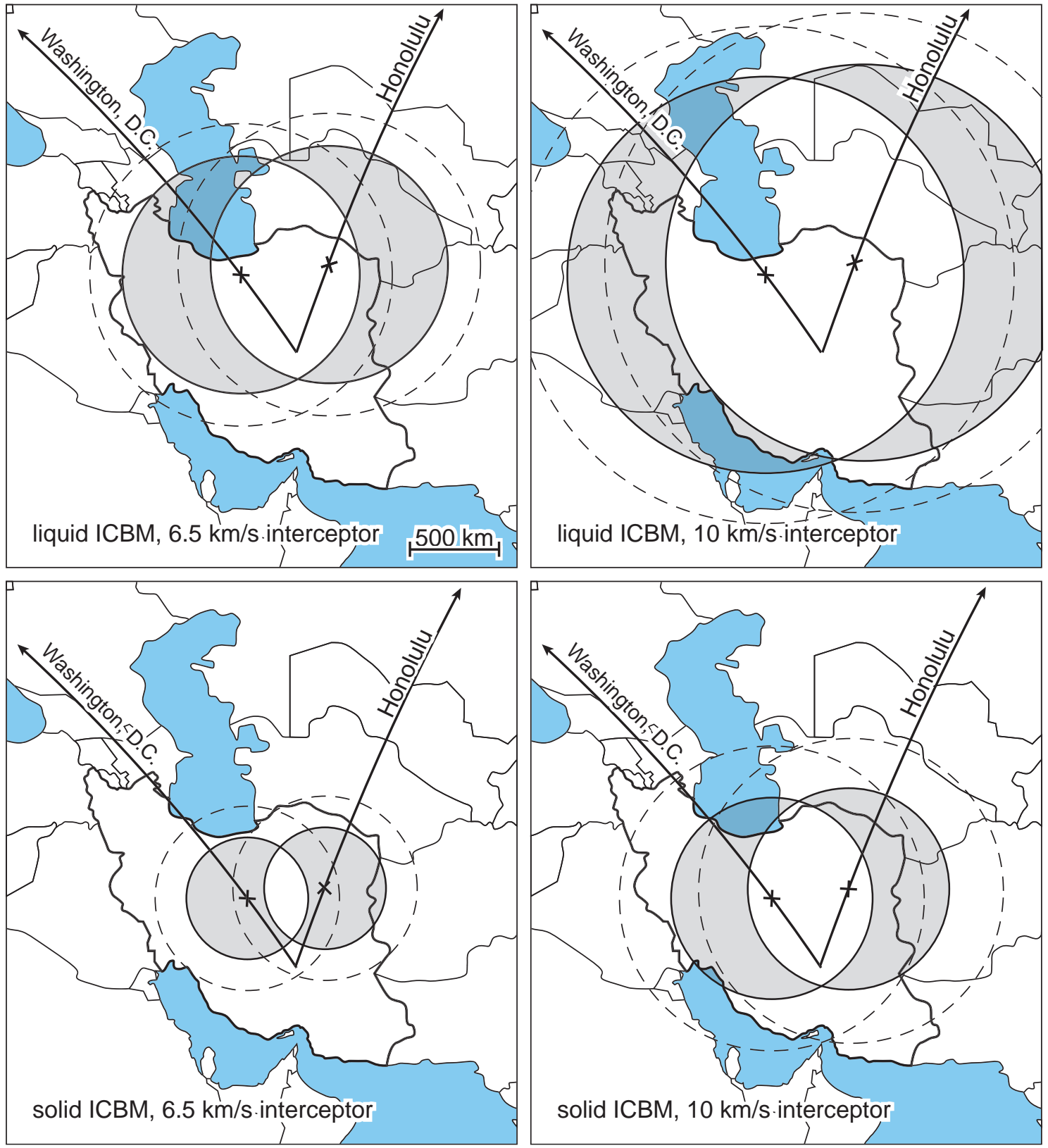

Figure 5.17. Defending all of the United States against missiles launched from Iran, using interceptors based at two sites.

\subsubsection{Defending Alaska and Hawaii}

The considerations for defending Alaska and Hawaii from Iran are similar to those discussed above for defense against Iraq. The great-circle range and azimuth from Iran to Hawaii are $13,050 \mathrm{~km}$ and $36^{\circ}$. We have taken the rotating Earth azimuth to be $21^{\circ}$.

By inspecting the basing areas whose western borders are limited by the dotted curves in Figure 5.16, it can be seen that basing in the Persian Gulf or southeast Turkey would no longer be options for the liquid-propellant missile. Defense against the solid-propellant missile appears not to be feasible. 


\subsubsection{Defending against missiles from Iran with multiple interceptor sites}

The preceding discussion has focused on finding a single site that could defend the entire United States. Using a second location could increase flexibility but would still require interceptors to be based in unconventional locations. Figure 5.17 shows the possibilities for defending all of the United States against the liquid- and solid-propellant missiles from Iran using two interceptor sites.

Defending against the liquid-propellant missile by the $6.5-\mathrm{km} / \mathrm{s}$ interceptor with a single site would require the site to be located in the Caspian Sea or Turkmenistan (Fig. 5.16, upper left). As Figure 5.17 (upper left) shows, the only new opportunity that would be offered by employing a second site would be the possibility of locating one site in Azerbaijan and a second site in Afghanistan. However, a single site located in Azerbaijan could provide a comparable defense

Figure 5.17 (upper right) reveals that employing a second site for the $10-\mathrm{km} / \mathrm{s}$ interceptor would permit one interceptor to be based in Turkey or the Persian Gulf, provided that the second could be based in Pakistan or one of the other countries to the east of Iran. However, a single interceptor base located in Afghanistan, Turkmenistan, Uzbekistan, Kazakhstan, or the Caspian Sea, would suffice.

Figure 5.17 (lower left) shows that for defense against a solid-propellant missile from Iran, a second site would not make it possible to employ the $6.5-\mathrm{km} / \mathrm{s}$ interceptor. In Figure 5.17 (lower right), it appears that by basing a 10-km/s interceptor in the Caspian Sea and a second one in Afghanistan or Turkmenistan, all 50 states could be defended. However, if the launching site for solid-propellant missiles destined for the East Coast (the Washington, D.C. trajectory) were moved about $200 \mathrm{~km}$ to the southeast, this defense would be precluded.

In summary, employing a second base would not permit defense of all 50 states from a solid-propellant missile launched from Iran.

\subsubsection{Possibility of an interceptor being mistaken as a threat}

Interceptors having a velocity of $5 \mathrm{~km} / \mathrm{s}$ or more would have sufficient speed and range to be mistaken for a threat. The interceptor trajectories from Iran could be to the north, over Russia, or to the southeast, towards Pakistan and India. An interceptor fired in defense runs the risk of inadvertently triggering a retaliatory action by these countries.

\subsection{Other Engagements Considered}

\subsubsection{Defending against sea-based medium-range ballistic missiles}

So far we have been concerned with the feasibility of defending the United States from attack by ICBMs. However, as discussed in Chapter 3, the U.S. intelligence community judges that an attack on the United States by medium-range ballistic missiles (MRBMs) launched from platforms such as ships, barges, or submarines positioned off the coasts of the United States is a more serious threat. Consequently, the Study Group also analyzed the requirements for a boost-phase intercept system that could defend the United States against such an attack using ship-based interceptors. In this particular scenario, the detection and tracking time is only a few seconds since the Aegis radar can see a tactical ballistic missile practically at launch. We believe the total track and engagement decision time could be as little as $10 \mathrm{~s}$, since any missile launch could be presumed to be hostile. 
Table 5.9. Model Medium-Range Ballistic Missiles $^{a}$

\begin{tabular}{lccr}
\hline \hline Model & $t_{b}(\mathrm{~s})$ & $V_{\text {bo }}(\mathrm{km} / \mathrm{s})$ & Range $(\mathrm{km})$ \\
\hline M1 & 65 & 2.2 & 600 \\
M2 & 95 & 3.2 & 1300 \\
\hline
\end{tabular}

${ }^{a} t_{b}$ is the total duration of the boost phase and $V_{\text {bo }}$ is the burnout velocity. For further details, see Chapter 15.

The Chinese M-9 missile, which is estimated to have a total burn time of about $65 \mathrm{~s}$ and a range of $600 \mathrm{~km}$, and the Shahab or No Dong missiles, which are estimated to have burn times of about $95 \mathrm{~s}$ and a range of $1,300 \mathrm{~km}$, were chosen as representative examples of missiles that could be launched against the United States from a sea-based platform. Their characteristics are summarized in Table 5.9. Models of these two missiles, denoted M1 and M2 respectively, were constructed as described in Chapter 15. As an example of a ship-based missile that could be used as an interceptor, we chose interceptor model I-1, which is similar to the U.S. Navy's existing Standard Missile 2 and is small enough to fit in the existing Vertical Launch System of Aegis-class destroyers and cruisers.

Figure 5.18 shows illustrative engagements of these MRBM models by interceptor I-1 located $40 \mathrm{~km}$ down range from the missile launch point. (We assume that the Aegis ship is trailing the suspected hostile ship and is close enough to detect the MRBM with its radar.) This interceptor uses aerodynamic forces to maneuver and consequently must intercept the target missile while it is still in the atmosphere. The two model MRBMs would have to be intercepted within about $45 \mathrm{~s}$ after they were launched, or they would be above the altitude where the interceptor could maneuver effectively. A detailed analysis of such engagements is outside of the scope of this study.

\subsubsection{Defending against a 300-second burn-time missile}

The possibilities for a boost-phase defense are obviously sensitive to missile burn time. For the reasons discussed in Chapter 3 and Chapter 15, we have chosen to base our analysis on a 240-second burn time liquid-propellant missile. However, since some prior work was based on a 300-second liquid-propellant missile it may be of interest to note approximately how the results would change for defense against such an ICBM.

As might be expected, the increased burn time of a 300-second missile would make the defense much easier. For any given basing area, the decision time would be increased by $60 \mathrm{~s}$. Or, for a given decision time, the basing area would be significantly increased. To a good approximation, the additional increment in ground range for the extra burn time can be estimated by taking the product of the increased time and the ground speed of the missile, which is somewhat less than its flyout speed because of its angle of inclination. Table 5.10 gives values for three interceptors.

For the $5-\mathrm{km} / \mathrm{s}$ interceptor, it can be seen from the lower left panel of Figure 5.9 that an increased range of $230 \mathrm{~km}$ would extend the basing area for North Korea far out to sea. For the other missiles, the decision time could be further increased. 


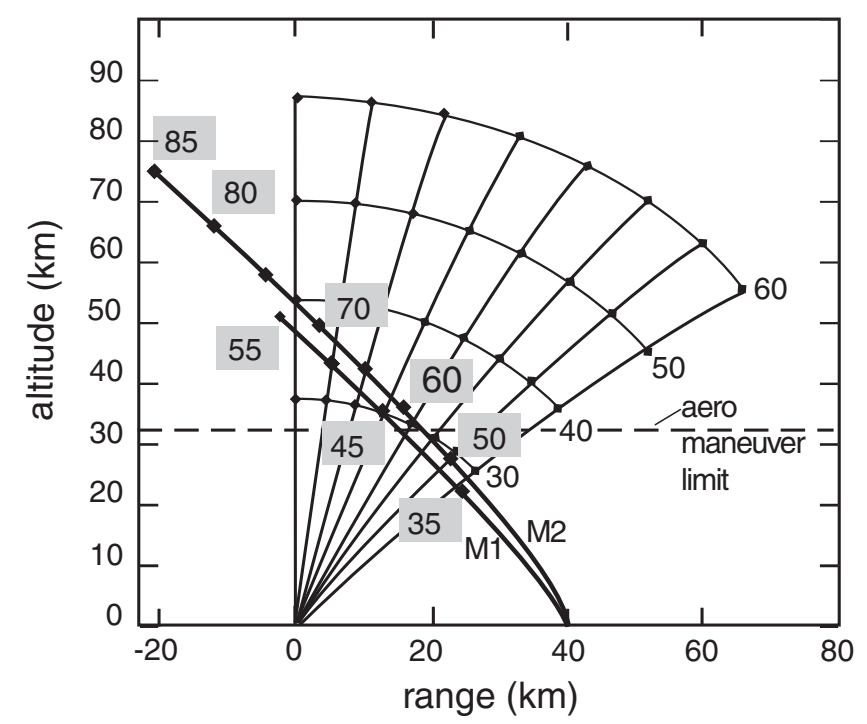

Figure 5.18. Illustrative planar engagements of medium-range ballistic missiles by interceptor I-1, which is similar to the U.S. Navy's Standard Missile 2, shown in the altitude-range plane. The interceptor standoff distance is $40 \mathrm{~km}$. The dark continuous curves rising from the right at a range of $40 \mathrm{~km}$ are the trajectories of MRBM models M1 and M2, which have total burn times of 65 and $95 \mathrm{~s}$, respectively. The dots along their trajectories show the missiles' positions at the time in seconds after launch as indicated by the number in the adjacent gray box. A fan of interceptor flyout trajectories rises from zero range. The interceptor was assumed to be fired $10 \mathrm{~s}$ after the missile was fired. The numbered contours that cross the interceptor trajectories show the positions of the interceptor at the indicated time in seconds after the MRBM was launched. The interceptor uses aerodynamic forces to maneuver; the dashed horizontal line shows the altitude above which it would be unable to do so.

\subsection{Avoiding Harming Other Countries}

The discussion in this chapter has so far focused on defending the United States against ICBMs launched by North Korea, Iraq, and Iran. We turn now to the question of what further steps would be needed to prevent possibly live munitions - nuclear, biological, or chemical - that survive an intercept from harming other countries. This is the problem of controlling shortfall. There would be no shortfall problem if the munitions could be reliably destroyed in the intercept. However, destroying the munitions is much more difficult than hitting the booster (see Chapter 13), and in this section we assume that the munitions have a good chance of surviving the intercept.

In general, controlling shortfall requires intercepting a missile within a narrow window in time: not too late for the munitions to fall on the United States (or on Canada, which we have not considered so far), and not so early that they fall on other countries. Ideally, the munitions would fall into the sea. Note that the munitions would almost never fall within the threat country itself.

All trajectories to the contiguous states from North Korea pass over Canada and Russia, so that these nations are potentially at risk. Trajectories from Iraq and Iran pass over Europe and Canada, putting many nations at risk. Trajectories from Iran and Iraq to Alaska and Hawaii pass over central Asia and Russia.

We discuss here the problem of timing an intercept so that it occurs within a given 
Table 5.10. Ground Speed and Increment in Range

\begin{tabular}{lccc}
\hline \hline Interceptor & $V_{\text {flyout }}(\mathrm{km} / \mathrm{s})$ & $V_{\text {ground }}(\mathrm{km} / \mathrm{s})^{a}$ & Increment in Range $(\mathrm{km})^{b}$ \\
\hline I-2 & 5.0 & 3.8 & 230 \\
I-4 & 6.5 & 5.5 & 350 \\
I-5 & 10 & 9.1 & 560 \\
\hline$a$ & The ground speed depends on the angle of inclination, which depends in turn \\
on the range and altitude of the intercept. The ground speeds are for intercepts \\
analyzed in this chapter. \\
${ }^{b}$ Increase in range for a booster with 300 s of burn time, which would provide an \\
additional 60 s relative to the 240-s liquid-propellant missile.
\end{tabular}

window and does not cause harm to other nations. We conclude that controlling shortfall to avoid damage to other countries would be difficult and perhaps even impossible.

\subsubsection{Controlling shortfall for North Korea and Iraq or Iran}

Assuming for the moment that the intercept could be timed precisely, let us examine what would be required to control shortfall in defending against missiles launched by North Korea and Iraq or Iran. Figures 5.3 and 5.13 provide a helpful orientation for this discussion. Of the three scenarios studied for defending the United States, North Korean launches pose the most difficult shortfall problem.

North Korea Managing shortfall for a missile from North Korea would require timing the intercept so that munitions do not fall on Canada or Russia. The situation varies with azimuth, and so we will discuss trajectories to the West Coast, interior, and East Coast of the United States.

U.S. West Coast Shortfall for these trajectories would generally cause the munitions to fall in the ocean, and so these need no further consideration.

Interior of the United States These trajectories generally pass over Alaska. We assume that the latest intercept time would prevent munitions from reaching Alaska. For such trajectories, Russia is highly vulnerable to shortfall. To avoid munitions from falling in Russia, they would have to be dropped into the sea. Figure 5.13 shows that the window for this scenario is extremely short, $10 \mathrm{~s}$ or less. Achieving such control would be problematic. It should be noted, however, that these trajectories cross over a great deal of wilderness area, and the window could be expanded somewhat if it were deemed acceptable.

U.S. East Coast Because the first landfall for East Coast trajectories is northern Canada, the major problem in controlling shortfall is to avoid hitting Canada. For the liquid-propellant missile on a trajectory to Washington, DC, the first landfall would occur at a burn time of about $233 \mathrm{~s}$. The basing areas described in Section 5.4 are for a burn time of $239 \mathrm{~s}$. Such a decrease in the intercept time would not alter the basing area enough to require abandoning the $6.5-\mathrm{km} / \mathrm{s}$ interceptor for defense.

The earliest intercept time is set by the requirement that the munitions not fall on 
Russia. The liquid-propellant missile leaves Russia territory after a burn time of about $220 \mathrm{~s}$, and accelerates for 10 to $15 \mathrm{~s}$ more before achieving a velocity that would carry it to Canada. Thus, the timing window for the liquid-propellant ICBM is about 10 to $15 \mathrm{~s}$.

The solid-propellant ICBM on the trajectory to Washington, D.C. would have to be intercepted at a burn time of $155 \mathrm{~s}$ to avoid hitting Canada. The intercept time used in Section 5.4 was $167 \mathrm{~s}$. The difference made by this 14 -second decrease in the radius of the basing area in Figure 5.10 (lower right) would be about $125 \mathrm{~km}$, thus making defense by the $10-\mathrm{km} / \mathrm{s}$ interceptor even more difficult.

Iraq and Iran The situation for these countries is straightforward. The decrease in intercept times to avoid hitting Canada is about $5 \mathrm{~s}$ for the liquid-propellant ICBM and $10 \mathrm{~s}$ for the solid-propellant ICBM. Such intercepts would significantly reduce the interceptor basing areas. Avoiding Greenland and Iceland would be difficult. Many of the trajectories pass over western Europe. The window for an intercept that avoids western Europe is about $10 \mathrm{~s}$ for the liquid-propellant ICBM, and $20 \mathrm{~s}$ for the solid-propellant ICBM.

In summary, the intercept time windows for safely controlling shortfall are only 10 to $20 \mathrm{~s}$ in duration - only slightly more than the uncertainties surrounding the threat trajectories. Controlling the time of intercept in the face of these uncertainties would be difficult.

\subsubsection{The problem of timing an intercept}

In an idealized situation, the rocket is known to be a threat missile and its properties and launching time are precisely known. Initially, however, there is some uncertainty in the missile's trajectory. The firing solution must allow for the possibility of intercepting the missile on a trajectory to any of the potential targets in the defended area, and it will generally call for launching the interceptor early enough to intercept the missile if it is on the most stressing trajectory.

As the interceptor flies out and the missile's trajectory becomes better known, it may turn out that the missile is not on the most stressing trajectory. Alternatively, the missile may undertake a maneuver, such as a dog-leg, that puts it on a new trajectory. To avoid shortfall, the intercept time must now be delayed. It might seem that delaying the intercept would make it easier, but that turns out not to be the case.

The best way to time an intercept precisely would be to choose the correct firing time and intercept point, but because of the uncertainties in the missile's trajectory, that is not possible; a second-best approach would be to compensate for the error in the firing time by controlling the interceptor's trajectory during its boost phase. (The acceleration of a solid-propellant interceptor cannot be controlled but it can be effectively reduced by trajectory maneuvers.) However, that control is possible only until the interceptor's boost it terminated, some 40 to $45 \mathrm{~s}$ after its launch. Because the threat missile continues to accelerate and maneuver after the interceptor boost has ended, a significant burden for controlling the exact time of intercept will be placed on the kill vehicle's guidance and divert systems.

Delaying the intercept time requires finding a guidance strategy for timing the intercept. Guidance algorithms, such as Proportional Navigation or Augmented Proportional Navigation (described in Chapter 12), are designed to assure that an intercept will occur, but the actual time of the intercept is not controlled. We have not examined this issue in detail, but our preliminary analysis suggests that the guidance laws generally used in the homing phase of a kill vehicle, which keep the line-of-sight to the missile constant, will in 
the best geometry generally compensate for about half of the variation and might in some cases actually worsen the problem of controlling shortfall. We conclude that a strategy for timing an intercept precisely is much more demanding than a strategy for merely assuring that it occurs. Significant analysis would be needed to understand this issue.

\subsection{Sensitivity to Other Assumptions}

So far in this chapter, we have examined the effect of varying ICBM types, interceptor types, and decision time on our results. We discuss here the implications of changing other assumptions: defending less than all 50 states, relying on existing early-warning systems, accounting for dog-leg maneuvers, hitting the warheads and not the boosters, and intercepting during the missile's ascent phase.

\subsubsection{Reducing the defended area}

As described at the beginning of this chapter, the stated policy of the current U.S. administration is the protection of all 50 states. However, the short time for boost-phase intercepts stresses every aspect of a boost-phase defense system, and reducing the goals of the defense could make it easier to deploy a system. Such a system might constitute the initial phase of a more extensive boost-phase system or an incomplete first layer of a layered defense. We discuss here whether a boost-phase system designed to defend only a portion of the United States, or some of the major cities, would be easier to deploy than a system that could defend the entire United States.

Defense of the contiguous states only For North Korea, an interceptor that could defend the 48 contiguous states against the liquid-propellant ICBM L could defend also Alaska and Hawaii. For the solid-propellant ICBM S1, Alaska is slightly more difficult to defend than the contiguous states because of the shorter burn time. However, the $10 \mathrm{~km} / \mathrm{s}$ interceptor would be needed in any case, and it could defend either trajectory. Thus, for missiles launched from North Korea, limiting the defense to the contiguous states would not offer an advantage.

For Iraq, Figure 5.14 shows that if the contiguous states can be defended, so can Alaska and Hawaii, though the interceptor site would have to be in extreme southeast Turkey. For Iran, however, Figure 5.16 shows that, although the contiguous states could be defended against the liquid-propellant missile by the large $10 \mathrm{~km} / \mathrm{s}$ interceptor located in the Persian Gulf (or possibly in southeast Turkey), the Hawaii trajectory could not be defended unless an unconventional site to the east of Iran were employed. Because the distance from Iran to Hawaii is longer than to any of the other states, Hawaii is an unlikely target. Consequently, defending the contiguous states cannot be regarded as significantly easier than defending the entire United States.

Defending only some major cities The major cities in the United States tend to be near the nation's borders. Furthermore, the intercept times for cities across the United States differ by only a few seconds. Thus, defending any of the major cities is equivalent to defending the contiguous states, as Figures 5.3 and 5.13 reveal.

Partial defense of the contiguous United States Finally, defending only those parts of the contiguous United States that were easiest to achieve might be considered. This could be 
done by attempting to intercept only those missiles with trajectories that take them within reach of interceptors, or by intercepting missiles too late to defend the closest targets but soon enough to defend targets farther away. A defense could use both approaches. An example of the first would be a defense only against North Korean ICBMs headed to the West Coast. Such an approach would be possible, but would leave the interior and the East Coast undefended. The second approach - arriving too late to defend the targets on the closest coast - could, in principle be useful against launches from the Middle East. In practice, however, no more than about $5 \mathrm{~s}$ of burn time would separate the closest targets from the farthest. Given that the uncertainties in the missile trajectories are likely to be larger than $5 \mathrm{~s}$, it would be difficult to plan on defending only a portion of the contiguous states by arriving late.

\subsubsection{Using the existing early-warning satellite system}

The timelines used in this chapter presume the ability to confirm a rocket launch by a system with the capability of the notional space-based system (NSBS) described in Chapter 10. The NSBS is intended to illustrate the capabilities that a modern, space-based missile early-warning and tracking system, such as the high-altitude Space-Based Infrared System (SBIRS-High) could have. The SBIRS-High, which is currently under development, is intended to replace the existing Defense Support Program (DSP) system.

We note here that if a system like SBIRS-High is not available and the early-warning system does not have the capabilities assumed for the NSBS, then any boost-phase intercept system would have to rely on the DSP for early warning and tracking of potentially threatening rockets. According to the analysis in Section 10.1.2, however, this system would need to be supplemented by a higher-sample-rate, off-board sensor, such as radar. Lacking such a sensor, the time required to construct a firing solution would be extended by a few tens of seconds, and the system's performance would be correspondingly diminished. The effect would be to require a faster interceptor for a given ground range. However, such an interceptor could be suited only for use against long-burning liquid-propellant ICBMs.

\subsubsection{Effect of dog-leg trajectories on basing}

In principle, a dog-leg maneuver might stress the defense by moving the last point where the missile could be successfully intercepted away from the interceptor's base or station. For instance, the liquid-propellant ICBM could execute a $10^{\circ}$ out-of-plane dog-leg on the NorthKorea-to-Boston trajectory that would shift the last point where it could be successfully intercepted $60 \mathrm{~km}$ to the west, away from the Sea of Japan. However, the additional time required to execute this dog-leg maneuver would allow the interceptor to fly farther. The net additional distance the $5-\mathrm{km} / \mathrm{s}$ interceptor I- 2 would have to travel would be only about $35 \mathrm{~km}$. The net additional distance for I-4 would be only about $25 \mathrm{~km}$, and for I-5, only about $6 \mathrm{~km}$.

For the solid-propellant missile S1, which has a lower-acceleration final stage than the liquid-propellant ICBM, a dog-leg would actually be counterproductive: the interceptor would be able to fly farther during the dog-leg than the intercept point would be displaced.

\subsubsection{Achieving warhead kill}

Our analysis assumes that the interceptor aims for and hits the final stage of the ICBM, not its warheads. Having the ability to home on and hit the missile's munitions while they 
are attached would have no effect on where a given interceptor could be based. It could, however, reduce the risk of a successful intercept causing live munitions to strike other countries.

To defend the United States, the final stage of the attacking missile must be disabled before its munitions have achieved the velocity needed to carry them to any part of the country. If the interceptor can disable the missile before this happens, having the ability to home on and hit the missile's munitions would provide no additional protection of the United States. If the interceptor cannot disable the missile before this happens, being able to destroy later any munitions that are still attached to the missile would not reliably defend the United States, because some munitions could already be separated from the missile. Once the missile's munitions have been deployed, destroying them would require the capabilities of a midcourse defense system, which are very different from those of a boost-phase defense system.

There would, however, be one real advantage to having the ability to destroy a missile's munitions while they are still attached: it would prevent an intercept that successfully protects the United States from causing possibly live munitions to strike other countries, including U.S. friends and neighbors. The difficulty of avoiding this with an interceptor that is not able to destroy the target missile's munitions is discussed in Section 5.8. The challenge of warhead kill is discussed in Chapter 13.

\subsubsection{Intercepting the missile during its ascent phase}

During a missile's ascent phase - the period shortly after the motor of the missile's final stage has burned out, or its thrust has been terminated - it might be possible to home on and hit the body of the final stage using active homing techniques, such as LIDAR (see Chapter 10). Although this would extend the time available to hit the missile's body, it would not be useful for the following reason.

As has been noted, a missile can deploy its munitions at any moment after it has achieved the velocity needed to carry them to the target area. The missile's munitions could be deployed while the missile is still in powered flight but would likely be deployed no later than immediately after the final stage has burned out or its thrust has been terminated. Homing on and hitting the body of the missile after it has deployed its munitions would not help the defense. Consequently, an interceptor's ability to home on a missile body after its boost phase has ended would be of no use.

\subsubsection{Defending by slower interceptors}

Others have suggested a possible role in boost-phase defense against ICBMs for slower interceptors such as interceptor model I-1, which is similar to the $1.7-\mathrm{km} / \mathrm{s}$ Standard Missile SM-2, or a $3-\mathrm{km} / \mathrm{s}$ interceptor similar to the Standard Missile SM-3 that is currently under development by the Navy. However, because we found the $5-\mathrm{km} / \mathrm{s}$ interceptor I- 2 to be only marginally useful in the most favorable engagement we considered-defending the United States against the liquid-propellant ICBM model L launched from North Korea-we judge that slower interceptors would not be useful in defending against ICBMs even if they have the ability to home on and hit a missile outside the atmosphere. 



\section{Chapter 6}

\section{Defending the United States Using Space-Based Interceptors}

\section{Contents}

6.1 Coverage by a Single Interceptor . . . . . . . . . . S105

6.2 Operational Altitudes for SBIs . . . . . . . . . . . S106

6.3 Calculating the Number of Interceptors for Earth Coverage S108

6.4 Sizing the SBI Satellite: Kill Vehicle, Interceptor, and Life Jacket . . . . . . . . . . . . . . . S110

6.4 .1 Sizing the kill vehicle . . . . . . . . . . . . . . . S111

6.4 .2 Sizing the interceptor . . . . . . . . . . . . . . . S111

6.4.3 Total mass of an SBI satellite . . . . . . . . . . . . . S112

6.4.4 The effects of divert velocity requirements on SBI mass . . . $\quad \mathrm{S} 112$

6.5 Optimizing System Mass-in-Orbit . . . . . . . . . . S114

6.6 The Effect of Decision and Intercept Time on System Mass 5116

6.7 The Effect of High-Acceleration Interceptors on System Mass S117

6.7.1 Divert penalty: the effect of booster burn time on mass . . . S S119

6.7.2 Acceleration penalty: the effect of acceleration on mass . . . S120

6.7 .3 Summary . . . . . . . . . . . . . . . S S122

6.8 Space-Launch Requirements for SBIs . . . . . . . . . S124

6.9 The Effect of Reducing Kill-Vehicle Mass . . . . . . . . S125

6.10 The Expense of Operating in Space ........... S126

6.11 Summary ............................. S127

Basing interceptors or lasers in space is sometimes suggested as a way to circumvent the geographic constraints that limit the applications of boost-phase defense systems that utilize terrestrial-based interceptors. The freedom to operate around the globe in space means that countries cannot use their size or inaccessibility to shield themselves from boost-phase defenses. This was one of the original concepts behind the Strategic Defense Initiative (SDI) program that President Reagan initiated in 1983, and the Brilliant Pebbles spacebased interceptor program of the early 1990s. A further attraction of a space-based system is that it would be on station continuously.

Space-based hit-to-kill interceptors (SBIs) raise many of the same technical issues as the terrestrial-based interceptors that have been discussed in preceding chapters. But operating 
in space also presents some unique challenges. In particular, because of the rotation of Earth and the orbital motion of the satellites, a large number of satellites (hundreds or thousands) would be needed to ensure that at least one satellite were always close enough to every potential boost-phase trajectory to intercept missiles during this phase of their flight. Also, the limited capacity of launchers and the high cost of placing mass in orbit (on the order of $\$ 10,000$ per pound to low-Earth orbit) puts a penalty on size and weight, pushing designers to make the interceptors as small and light as possible.

Space-based interceptors were not the central focus of this study, but given the renewed attention they are receiving, the Study Group applied the methodology and relevant results from its analysis of surface-based hit-to-kill boost-phase interceptors to illuminate some of the technical issues that would be involved in a space-based system. Specifically, we examined the performance that SBIs would require to defend the United States against the suite of target missiles that are presented in Chapter 15. We also examined the required sizes of kill vehicles and interceptors based on the analysis in Chapter 14, as well as likely engagement timelines based on the geographic coverage analysis presented in Chapter 5. Our approach was to illustrate the issues associated with SBIs by conducting a parametric analysis that highlighted the trade-offs that a system designer would face.

We approached the analysis by creating a "baseline" system that could intercept a single, solid-propellant ICBM $5 \mathrm{~s}$ before burnout with zero decision time. This baseline approach made for easy comparisons across parameters, but neglected some of the scenario-specific requirements that we applied to the ground-based interceptors in Chapter 5, specifically that the intercept had to occur earlier than $5 \mathrm{~s}$ before burnout to defend the United States and that some decision time might be required to provide operational margin. As a result, the baseline case is optimistic, underestimating perhaps by a factor of two or more the number of satellites that could be required for the more stressing scenarios.

For the baseline case, our analysis suggests that the flyout velocity of the interceptors should be about $4 \mathrm{~km} / \mathrm{s}$, and the flyout acceleration should be about $10 \mathrm{~g}$ to minimize the overall system mass. At that velocity, roughly 1,600 interceptor satellites at an altitude of $300 \mathrm{~km}$ would be required for a minimum of one (and an average of two) to be in position to intercept a single, solid-propellant ICBM launched from North Korea, Iraq, or Iran $5 \mathrm{~s}$ before burnout. The analysis presented in Chapter 12 indicates that, for the high closing velocities that occur when intercepting ICBMs from space, the kill vehicles should have a minimum divert velocity of $2.5 \mathrm{~km} / \mathrm{s}$, a total interceptor response lag of $0.1 \mathrm{~s}$ or less, and an acceleration capability of $15 \mathrm{~g}$ during the endgame. These minimum requirements result in a kill vehicle with a fully fueled mass of $136 \mathrm{~kg}$ and a total interceptor mass of $820 \mathrm{~kg}$. Altogether, this 1,600-SBI system would have a total mass of about 2,000 tonnes ( 1 tonne = $1000 \mathrm{~kg} \approx 1.1 \mathrm{ton})$. Deploying this system in orbit would require a five- to ten-fold increase in current U.S. space-launch capability.

The sizes and masses of constellations of SBIs could be significantly greater than the baseline case suggests if less optimistic assumptions were made. For example, defending the entire United States against ICBMs launched by Iran would require intercepts at least $12 \mathrm{~s}$ before burnout, which would drive up the number of interceptors required by 25 percent. Providing the system with $30 \mathrm{~s}$ of decision time would more than double the number of interceptors required.

Defending against a single liquid-propellant ICBM (as contrasted to a solid-propellant ICBM) would require less than half the number of interceptor satellites (roughly 700) required for the baseline case. However, a system designed to counter only liquid-propellant ICBMs runs the risk of becoming obsolete quickly, given the long time that it would take to 
deploy the system and the incentives that the development and deployment of that system would create for emerging missile states to build or procure solid-propellant missiles.

The estimates presented here for constellation size, kill-vehicle mass, and interceptor mass are significantly higher than those of other authors, even for the baseline case, because of our more conservative assumptions regarding a number of factors, including warning time, duration of ICBM boost phases, divert velocity and acceleration of the kill-vehicle, tankage mass, lifejacket mass, and the mass penalties associated with high flyout accelerations for the interceptor.

This chapter begins by calculating the coverage of a single interceptor, the likely operating altitudes for interceptor satellites, and the number of satellites required for Earth coverage. Next, it explores the factors that determine the sizes of the kill vehicles. Based on that analysis, it examines the optimum numbers of satellites and flyout velocities to minimize the total mass in orbit for the baseline case. Then it explores the effects of more realistic assumptions about decision time and required intercept times. Finally, the chapter explores strategies for reducing the mass of the kill vehicle, which is the key determinant of the total mass of the system.

The choice of threat missiles and the times for detecting a rocket launch and generating a firing solution are identical to those in Chapter 5. (These are described in Chapter 15 and Section 10.1, respectively.) However, in contrast to the analysis of boost-phase defense with terrestrial-based systems (Chapter 5), the kill-vehicle mass is of primary, not secondary, importance. The interplay between the mass and the range of the kill vehicle is central to the analysis of space-based boost-phase defense systems. Consequently, the material in Chapter 14 is essential to the analysis in this chapter.

\subsection{Coverage by a Single Interceptor}

For a space system in low Earth orbit, there is always a large absentee ratio; that is, most of the satellites are in the wrong place when they are needed, due to the physics of orbits and the rotation of Earth. The absentee ratio is governed by the coverage afforded by each satellite - the greater the coverage, the lower the absentee ratio. The coverage of an SBI is determined by the distance $r_{\text {flyout }}$ it can fly from the time when it is fired to the time it must intercept the target missile.

The interceptor is fired at time $t_{0}$. In general terms, the volume an interceptor can cover at a future time $t_{n}$ can be approximated by a sphere with a radius equal to its flyout range $r_{\text {flyout }}$, the distance it can fly in the interval $t_{n}-t_{0}$, centered on the position that it would have occupied at $t_{n}$ if it had not been fired from its storage or parking orbit. This is illustrated in Fig. 6.1. Assuming the intercept would occur at an altitude $h_{\text {intercept }}$, a given SBI could intercept a rocket that rises through this altitude anywhere within a circular area of radius

$$
R=\sqrt{r_{\text {flyout }}^{2}-\left(h_{\text {orbit }}-h_{\text {intercept }}\right)^{2}},
$$

where $h_{\text {orbit }}$ is the altitude of the satellite's orbit and $h_{\text {intercept }}$ is the altitude of the intercept point. For the times and distances of concern, the difference between the gravitational force felt by the interceptor on its new path and that on its former path is negligible, so that it may be assumed one is working in an inertial frame moving with the original orbital velocity of the interceptor.

The interceptor's flyout range depends on its acceleration profile and terminal velocity. Figure 6.2 shows this range as a function of time for interceptor burnout velocities of 2 , 


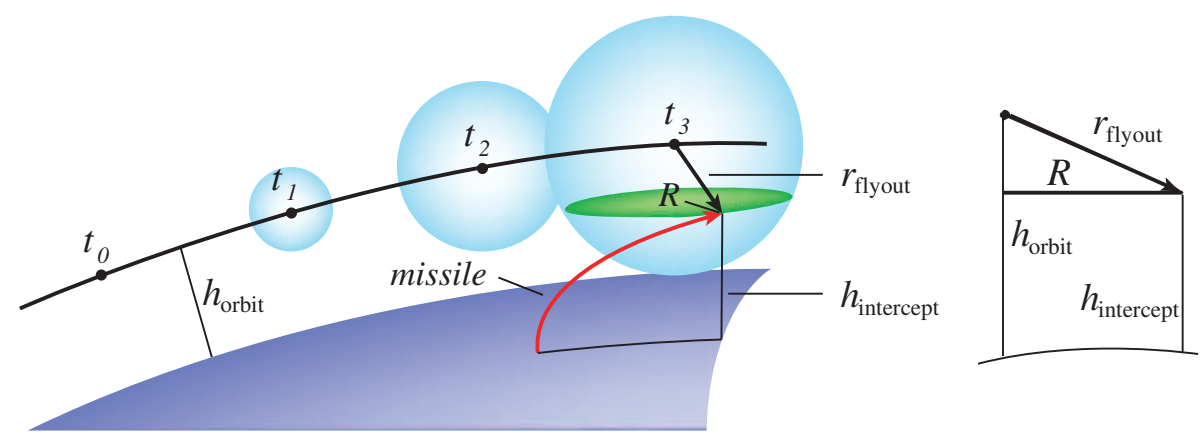

Figure 6.1. The coverage of a single interceptor at the height of an intercept is determined by the altitude $h_{\text {orbit }}$ of its orbit and its flyout range $r_{\text {flyout }}$, the distance it can travel in the time that is available to intercept the target. Here, the interceptor is fired at time $t_{0}$, and the intercept occurs at time $t_{3}$. The arrowheads are at the intercept point; $R$ is the intercept ground range.

4 , and $6 \mathrm{~km} / \mathrm{s}$, and mean accelerations of 6,8 , and $10 \mathrm{~g}$, assuming burn times of roughly $20 \mathrm{~s}$ to $100 \mathrm{~s}$. Both of these parameters are important for flyout times in the range of $100 \mathrm{~s}$ to $150 \mathrm{~s}$, which are typical for this application. In any case, the range will be measured in hundreds rather than thousands of kilometers, so a large number of interceptors will be needed. In addition, the interceptor will use some of its range in diving down from its parking altitude to the intercept altitude, and this effect must be considered in coverage calculations.

Shorter interceptor burn times (higher accelerations) were also considered but were found to have mass penalties that more than offset the potential benefit. The basis of this conclusion is discussed in Section 6.7.

\subsection{Operational Altitudes for SBls}

The optimal basing altitude for a system of SBIs would be a compromise between being high enough to minimize the effects of atmospheric drag and low enough to reach the intercept point in time. Generally, the desired intercept altitude would be lower than the storage altitude of a space interceptor. The target missile typically would burn out below an altitude of $200 \mathrm{~km}$, which is far too low for a long-lasting satellite, because at that altitude the atmosphere is dense enough that aerodynamic drag would quickly cause it to spiral into the lower atmosphere and burn up. To have a lifetime of many years without propulsion, a satellite must orbit at or above $500 \mathrm{~km}$. To orbit below $500 \mathrm{~km}$ for many years, a satellite would require a propulsion system with sufficient propellant to maintain the desired orbit.

The lifetime of a satellite at any altitude is a function of the ballistic coefficient, which is the ratio of the satellite's mass to the product of its drag coefficient and its cross-sectional area normal to its direction of motion through the atmosphere. The larger the ballistic coefficient, the less effect drag will have on the satellite. For a compact, pencil-shaped interceptor aligned with the velocity vector, the ballistic coefficient could be as high as $200 \mathrm{~kg} / \mathrm{m}^{2}$ (200-kg mass, $1-\mathrm{m}^{2}$ frontal area, and assuming a drag coefficient of unity).

Orbital lifetime is also a strong function of the solar cycle. At solar maximum, the upper atmosphere is distended and satellite lifetimes are diminished. We must consider this worst case, because solar maxima occur every 11 years and last for several years. At solar maximum, the lifetime of a $200-\mathrm{kg} / \mathrm{m}^{2}$ satellite without any propulsion is 3 years at 


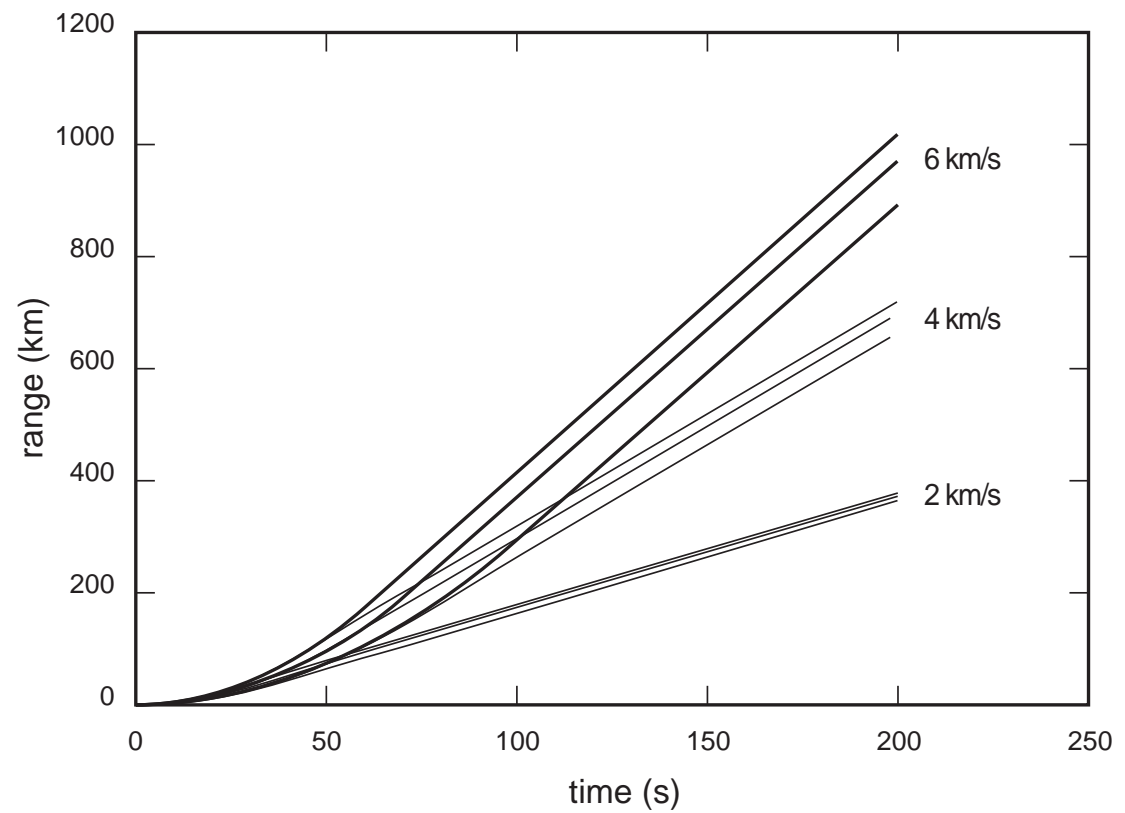

Figure 6.2. Flyout range $r_{\text {flyout }}$ as a function of time, for a range of accelerations and burnout velocities. In each group of interceptors having the same burnout velocity, the three trajectories are, from top to bottom, for accelerations of 10,8 , and $6 \mathrm{~g}$.

$500 \mathrm{~km}, 9$ months at $400 \mathrm{~km}, 50$ days at $300 \mathrm{~km}$, and 1-2 days at $200 \mathrm{~km}$ ([77, Fig. 8-4, p. 210], [78]).

Atmospheric drag can be overcome by thrust, however, provided by a rocket motor on the satellite. This is an ideal application for a low-thrust, high-specific-impulse $\left(I_{\mathrm{sp}}\right)$, ion engine. Given an atmospheric density at solar maximum of $6 \times 10^{-11} \mathrm{~kg} / \mathrm{m}^{3}$ at an altitude of $300 \mathrm{~km}\left[79\right.$, p. 261] and assuming an ion engine with an $\mathrm{I}_{s p}$ of $2000 \mathrm{~s}$, a satellite with a $1 \mathrm{~m}^{2}$ frontal area can maintain that altitude for 2.5 years at solar maximum by consuming $30 \mathrm{~kg}$ in ion engine fuel, thereby balancing the momentum lost to drag with the momentum provided by the engine. The mean thrust is very small, less than 1 millinewton. Maintaining altitude for 3 years is long enough to survive a solar maximum, and it is unlikely that an interceptor satellite would need to survive more than one maximum.

A benefit of these low altitudes is that the interceptor would not have to fly long distances to get down to the intercept altitude. Another advantage is that interceptors at these altitudes would not pose a space debris problem when they reach the end of their service lives; once their thrust were terminated, they would quickly re-enter the atmosphere. But there is a tradeoff between the advantages of operating at low altitudes and the weight penalty in thrusters and fuel needed to maintain orbit, which probably precludes basing below $300 \mathrm{~km}$ at solar maximum.

Basing at altitudes above $300 \mathrm{~km}$ would reduce the mass of the satellite by reducing the amount of fuel needed for the ion propulsion. But that may not reduce the total mass-inorbit - particularly for space-based interceptors designed to defend against solid-propellant ICBMs - due to the extra distance that the interceptor would have to fly to reach the intercept point and hence the extra propellant that it would need. 


\subsection{Calculating the Number of Interceptors for Earth Coverage}

There are several ways to estimate the number of SBIs that would be required so that at least one interceptor would be in position at all times to engage an ICBM. Perhaps the simplest approach is to assume that the satellites are fixed above Earth and uniformly distributed. In that case, the required number of interceptors is obtained by dividing the area to be defended by the area within reach of a single interceptor. If we are concerned with ICBMs launched from anywhere between $45^{\circ} \mathrm{N}$ latitude and $45^{\circ} \mathrm{S}$ latitude, the area of this spherical shell is:

$$
A_{\text {cover }}=\frac{\sqrt{2}}{2}\left[4 \pi\left(R_{\text {Earth }}+h_{\text {intercept }}\right)^{2}\right] .
$$

The radius of the area covered by one interceptor is given in Eq. 6.1. If the interceptors could be uniformly distributed around Earth, without overlap, a constellation of $N_{0}=$ $A_{\text {cover }} /\left(\pi a^{2}\right)$ interceptors would provide one interceptor to cover any launch.

In reality, a number of factors make $N_{0}$ a significant underestimate of the number of interceptors actually needed, because the nature of satellite orbits around a rotating Earth makes it difficult to provide uniform coverage from low-Earth orbit. For any given orbit, satellite coverage will be concentrated at the same latitude as the inclination of the orbit, leaving the equatorial region underpopulated, as discussed in [80]. (The inclination of an orbit is the angle that it makes with the equator.) Although that concentration would be beneficial if the defense needs only to intercept missiles from a very narrow latitude band, such as from North Korea, it makes complete coverage over a wide range of latitudes more difficult to achieve.

Since any holes in coverage are highly undesirable, steps must be taken to address this problem. For example, a more complex satellite constellation could be engineered having several rings of satellites that have different inclinations. This system must be engineered to provide continuous coverage without gaps. The solution does not lie in randomizing the satellite distribution, which would leave random gaps in coverage that could only be filled by deploying a large number of satellites. A fully engineered constellation, however, goes beyond the scope of our study.

Instead, we illustrate the approximate number of satellites that would be needed by studying the simpler case of a fixed-inclination constellation designed to defend against launches from anywhere on Earth between the $45^{\text {th }}$ parallels north and south. If we populate the constellation with $2 \times N_{0}$ interceptors, we obtain, on average, at least two interceptors within range of an intercept point at north latitudes between $34^{\circ}$ (most of Iran) to $45^{\circ}$ (North Korea). This coverage is sufficient to ensure that there would be virtually no gaps in coverage within that band of latitudes, although there is sometimes just one interceptor within range, and therefore no redundancy. That same number of interceptors $\left(2 N_{0}\right)$ could provide coverage down to $30^{\circ}$ North latitude and defend the United States against launches from anywhere in Iran, although the chance of a gap in coverage would increase to a few percent. This result comes from a satellite system simulation done by the Study Group that includes basic orbital and rotational effects. The satellites are in several orbital rings, but all are at an inclination of $45^{\circ}$. For a fixed number of satellites, the coverage is relatively insensitive to the exact partition between the number of satellites in a ring and the number of rings. There is a small advantage to having more satellites in each ring instead of more rings. The number of satellites would have to be doubled again to provide coverage down to the equator; in that case, a multiple-inclination design might be preferred. However, 


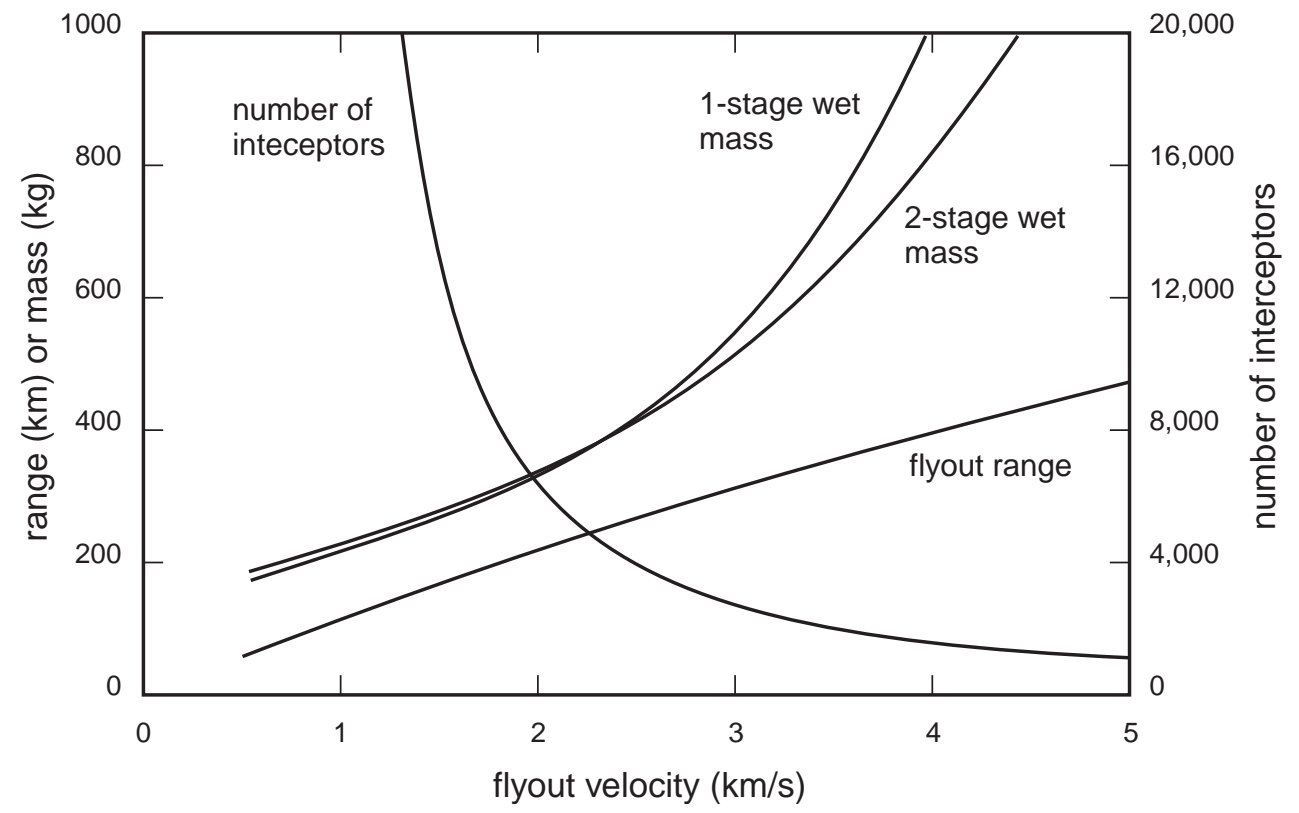

Figure 6.3. Range, mass, and required number of hypothesized space-based interceptors with a flyout acceleration of $10 \mathrm{~g}$ as a function of flyout velocity for a kill vehicle with a total divert velocity capability of $2.5 \mathrm{~km} / \mathrm{s}$. The mass does not include the garage or "life-jacket" that would be required to provide long-term orbital services, which we assume would increase the total mass of each satellite by roughly 50 percent. The required number of interceptors is for at least one interceptor (and an average of two) to be able to intercept a solid-propellant ICBM $5 \mathrm{~s}$ before burnout- the baseline case.

because most threats from the three countries of greatest concern to the United States would be intercepted within the approximately 30-degree to 45-degree North latitude band, we use this simple constellation design, populated with $2 N_{0}$ satellites, for a preliminary estimate of constellation size.

This number of interceptors, $2 N_{0}$, is shown as a function of flyout velocity in Fig. 6.3 for kill vehicles with $2.5 \mathrm{~km} / \mathrm{s}$ divert velocities against solid-propellant ICBMs like ICBM model S1. Our coverage calculation includes the additional range needed to fly down from the storage orbits at $300 \mathrm{~km}$ to intercepts at altitudes of $200 \mathrm{~km}$; an interceptor with a flyout range less than $100 \mathrm{~km}$ would be useless, because it could not even reach the intercept altitude.

The flyout range of an interceptor is determined by its flyout velocity and flyout time. The flyout velocity is a function of the interceptor design, as discussed in the previous section. The flyout time is determined by the ICBM, the detection and tracking time required of the space-based early-warning sensors, and the geography of the engagement. The Study Group constructed a baseline case for the purposes of illustration that assumes:

1. The target missile is a solid-propellant ICBM similar to model S1.

2. The interceptor is fired $15 \mathrm{~s}$ after the ICBM rises above $7 \mathrm{~km}$ (zero decision time), which is $45 \mathrm{~s}$ after the ICBM is launched.

3. The intercept occurs $5 \mathrm{~s}$ before the ICBM burns out. 
The first assumption is clearly optimistic because it does not take account of the effects of geography on the required intercept time. For example, defending Fairbanks, Alaska, from North Korea would require that ICBM S1 be intercepted no later than $137 \mathrm{~s}$ before burnout, or $28 \mathrm{~s}$ earlier than assumed in our baseline case (see Chapter 5). The second assumption - firing $15 \mathrm{~s}$ after the missile rises to $7 \mathrm{~km}$-is most certainly optimistic. It is the same as the zero-decision-time case discussed in Chapter 5. The final assumption-that the system is designed to counter solid-propellant ballistic missiles - is arguably pessimistic, because every state that has ICBMs today started with liquid-propellant missiles. If the system were designed to counter only liquid-propellant ICBMs, the interceptors would have considerably longer flyout times and the constellation size could be reduced significantly. Given the time that it will take to deploy an SBI system, however, such an assumption may be reasonable to avoid the risk of the system's becoming obsolete shortly after it is deployed (see Chapter 3).

The result of those three assumptions is that the SBIs would have $120 \mathrm{~s}$ of flyout time in the baseline case (an ICBM boost phase of $170 \mathrm{~s}$, an interceptor firing time of $45 \mathrm{~s}$, and an intercept that occurs $5 \mathrm{~s}$ before burnout). The rest of the analysis in this chapter is based on those assumptions (the baseline case) unless explicitly stated otherwise.

The downward-sloping curve in Fig. 6.3 indicates the number of interceptors that would be sufficient to cover any point in space and time in the region between approximately 30 and 45 degrees North latitude with an average of two interceptors and a minimum of one. For our baseline case of $v_{\text {flyout }} \approx 4 \mathrm{~km} / \mathrm{s}$, about 1600 orbiting interceptors would be needed.

This number would be inadequate, however, if the attacker launched several ICBMs nearly simultaneously from the same location; they would quickly deplete the one or two interceptors in the constellation that would be close enough to intercept them. Other factors that might drive up the size of the constellation are discussed in Section 6.6.

The numbers of interceptors that we estimate would be required is significantly higher than other authors have estimated because of the more conservative assumptions in our baseline case regarding warning and detection time, duration of the boost phase, and acceleration and flyout velocity of the interceptor $[81,82,83]$.

\subsection{Sizing the SBI Satellite: Kill Vehicle, Interceptor, and Life Jacket}

For a given performance, SBIs can be smaller than their terrestrial-based relatives because they are already at high altitude and are not subject to aerodynamic drag or gravity drop as they fly out to their targets. The penalty for overcoming drag and gravity has already been paid by the rocket that put them in orbit. Furthermore, the rocket nozzle on the interceptor can be tuned for a single external pressure (i.e., zero) for optimal performance, and no aerodynamic structure, such as a shroud, is needed.

On the other hand, a space-based interceptor would need life-support and stationkeeping systems during its multiyear lifetime on orbit that terrestrial-based interceptors do not. Life-support systems are needed to keep the satellite functioning. They include solar panels for power, communication links, and radiation shielding. Station-keeping systems include thrusters to maintain orbit and an attitude control system. They could be built into the interceptor itself, although it is more likely that they would be provided by an external structure, a "garage" or "life jacket," to reduce the mass that has to be accelerated from storage orbit to intercept. 
This section examines the sizing requirements for each of the three components for SBI satellites in turn: the kill vehicle, the interceptor rocket, and the life jacket.

\subsubsection{Sizing the kill vehicle}

Based on the analysis in Chapter 12, we estimate that the high closing velocities associated with intercepting ICBMs from space would require the kill vehicles to have a total divert velocity of $2.5 \mathrm{~km} / \mathrm{s}$, a total interceptor lag of less than less $0.1 \mathrm{~s}$, and an acceleration capability during the endgame of $15 \mathrm{~g}$. Using the same technology that we assumed for the terrestrial-based interceptors (based on technology that would be deployable within ten years or so), a kill vehicle that meets those requirements would have a wet mass of $136 \mathrm{~kg}$, as discussed in Chapter 14. (The wet mass of a kill vehicle is its inert or dry mass plus the mass of its propellant.) That kill-vehicle mass is based on current technology, extrapolated forward somewhat in the areas of sensors and avionics to achieve more capability and lower mass. Since kill-vehicle mass drives the size of each interceptor and the total mass of the constellation, several possible approaches for reducing kill-vehicle mass are explored in Section 6.9 .

It should be recognized that kill-vehicle mass is a strong function of the divert requirement, which we have estimated to first-order accuracy in this study. More agile ICBMs, which would drive up the divert requirement, would have a pronounced effect on the required kill-vehicle mass. For example, the thrust of a solid-propellant booster varies in an unpredictable way by as much as 10 percent as it burns, for a number of reasons. These variations are not knowable until after the interceptor booster has burned out. The killvehicle would have to compensate for this variation as it flies out, correcting the estimated time of the intercept by adjusting its closing velocity. These adjustments would require velocity corrections along the line of sight, in addition to the divert velocities calculated here. Liquid-propellant ICBMs would present the same problem; they could easily throttle their thrust up or down by 10 percent as a countermeasure.

\subsubsection{Sizing the interceptor}

The size of the interceptor is determined by the flyout speed and acceleration required of it and the mass of the kill vehicle that it must propel. Figure 6.3 shows the range and mass of the hypothesized interceptor with a $2.5 \mathrm{~km} / \mathrm{s}$ divert capability as a function of flyout velocity, which is controlled by fuel loading. Note that the masses in this figure are for the interceptors only and do not include the lifejacket. We consider one- and two- stage boosters for flyout (two and three stages total, counting the kill vehicle). For a singlestage rocket, our masses are based on the assumption that the tankage and motors total 16 percent of the propellant mass, plus an additional 5 percent of the payload. (The payload includes the wet kill vehicle for a one-stage flyout system; it includes the wet kill vehicle plus the flyout upper stage for the first stage of the two-stage flyout system). The $\mathrm{I}_{s p}$ of the flyout system is taken to be $300 \mathrm{~s}$. Those numbers are consistent with current practice for space-launched solid-propellant systems. The numbers for a two-stage rocket are based on the same assumption, plus the assumption that the staging time and the mass of interstage structures are negligible.

For simplicity, the calculations of flyout range assume that the interceptor has $120 \mathrm{~s}$ to reach the target, based on the 170-second duration boost phases of solid-propellant ICBMs S1 and S2, a 45-second delay between target and interceptor launch, and the arbitrary 
assumption that the interceptor must hit the target at least $5 \mathrm{~s}$ before burnout. This assumption turns out to be very optimistic because, in reality, the interceptor would have to hit the target earlier to defend the United States. It is more optimistic than the values assumed in our analysis of geographic scenarios in Chapter 5. More-realistic scenarios that take geography into account are discussed below in Section 6.6. If the interceptor could be fired earlier or the target missile burns longer, the coverage that could be provided by each interceptor would increase. Conversely, firing delays longer than the optimistic $45 \mathrm{~s}$ assumed here would decrease the coverage, as would any requirements to hit the booster sooner, before it could give its warheads or munitions the velocity needed to reach the United States.

According to Fig. 6.3, an interceptor that could propel the kill vehicle to a speed of $4 \mathrm{~km} / \mathrm{s}$ with an acceleration of $10 \mathrm{~g}$ would have a mass of $820 \mathrm{~kg}$, including the kill vehicle but not the lifejacket. As Fig. 6.3 indicates, there is an advantage to adding a second stage for flyout velocities that are greater than $3 \mathrm{~km} / \mathrm{s}$. The mass of a single-stage interceptor increases sharply for flyout speeds faster than $4 \mathrm{~km} / \mathrm{s}$. Beyond an optimum point (Fig. 6.5), the mass increase overwhelms the benefit of increasing range. The range improvement would begin to flatten out at higher speeds because the interceptor average acceleration has been limited to $10 \mathrm{~g}$. The 10-g acceleration implies a 30-50 s interceptor burn time, so most of the interceptor's energy is expended early, allowing no reserve for major course corrections late in its trajectory, such as a late re-classification of the target trajectory. If the interceptor has mistaken a liquid-propellant ICBM for a solid-propellant ICBM, it may overshoot and be unable to correct, which is similar to what may happen to surface-based interceptors (see Section 5.1.1).

\subsubsection{Total mass of an SBI satellite}

The interceptor mass is not the end of the story, however. The total weight of an SBI satellite also includes the "life jacket" or "garage" that provides the life-support and housekeeping services that are necessary for long-term operations in space. Those services include ion propulsion and propellant for maintaining orbit, radiation shielding, power, and other functions whose components are listed in Table 6.1. When all the subsystems listed in the table are added, the life jacket can have a mass nearly as large as the SBI itself. For the purposes of this analysis, we made what may be an optimistic assumption that the life jacket mass would be 50 percent of the mass of the interceptor.

\subsubsection{The effects of divert velocity requirements on SBI mass}

As discussed above, we use the $2.5 \mathrm{~km} / \mathrm{s}$ divert velocity requirement for the kill vehicle in our calculations, the assumption made in generating the two mass curves in Fig. 6.3. Other choices for divert velocities would affect the SBI mass significantly, although the divert velocity and acceleration requirements are determined primarily by the target missile and not the design of the SBI or constellation. Figure 6.4 shows the total mass for each SBI satellite (interceptor and life jacket) as a function of flyout and divert-velocity, assuming a two-stage interceptor. The $2.5 \mathrm{~km} / \mathrm{s}$ divert-velocity curve in this figure is the same as the two-stage mass curve in Fig. 6.3. Divert requirements could be greater than the $2.5 \mathrm{~km} / \mathrm{s}$ assumed in this chapter for two reasons: shorter interceptor burn times or higher closing velocities. Interceptor burn times could be significantly shortened if high acceleration motors are used, as some have proposed to either increase SBI reach or to get to a target sooner 
Table 6.1. Components of an SBI Life Jacket

\begin{tabular}{l}
\hline \hline Solar panel \\
Radiator \\
Momentum wheels and controller \\
Horizon/star tracker sensors \\
DC to DC converter \\
Battery \\
Hall-effect ion engine and controls \\
Structure and shielding \\
Attitude control system for momentum dump \\
Communication link and antenna \\
Miscellaneous \\
\hline
\end{tabular}

if geographic realities dictate. However, if the interceptor burn times are less than $40 \mathrm{~s}$, the kill-vehicle must respond to guidance uncertainties and unpredictable variations in the target's trajectory. (This issue is discussed in Section 6.7.) Second, the higher closing velocities that characterize SBI intercepts could increase kill-vehicle divert requirements relative to ground-based interceptors if the kill vehicle uses range-limited sensors for the endgame. In that case, the kill vehicle would have less time to respond to target maneuvers and would have to be able to divert farther.

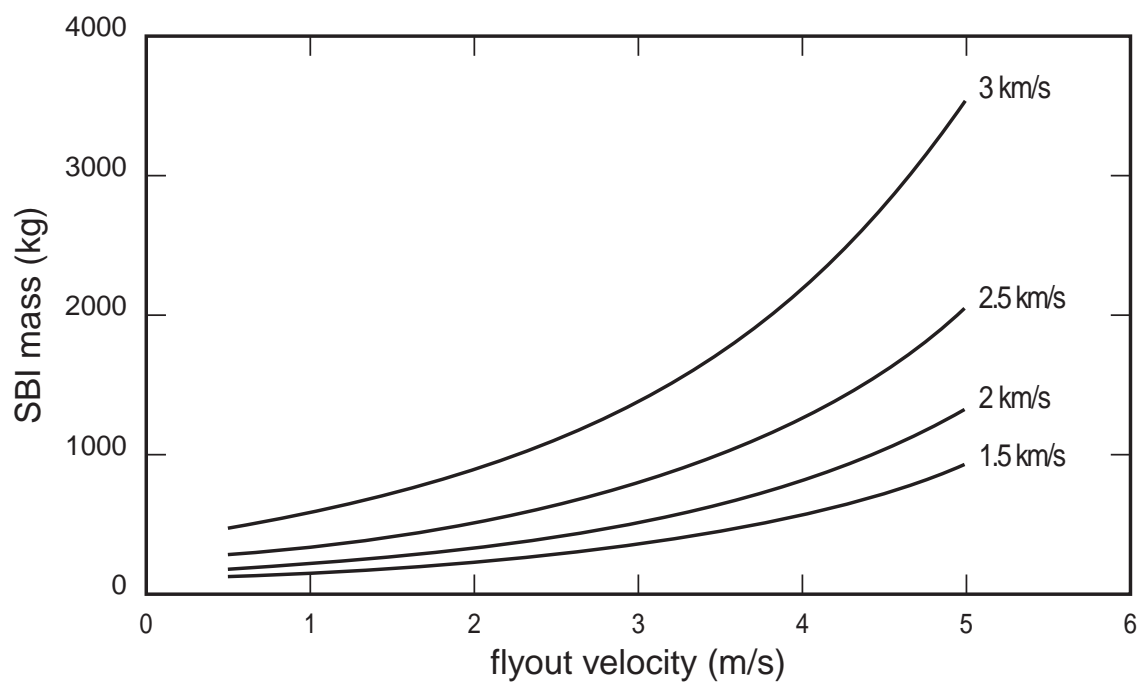

Figure 6.4. The effect of flyout and divert velocities on total mass of a single SBI with a two-stage booster. Total SBI mass includes the mass of the lifejacket, which we estimate to be 50 percent of the interceptor mass. The total divert velocity assumed for the kill vehicle in generating each curve is indicated next to it. 


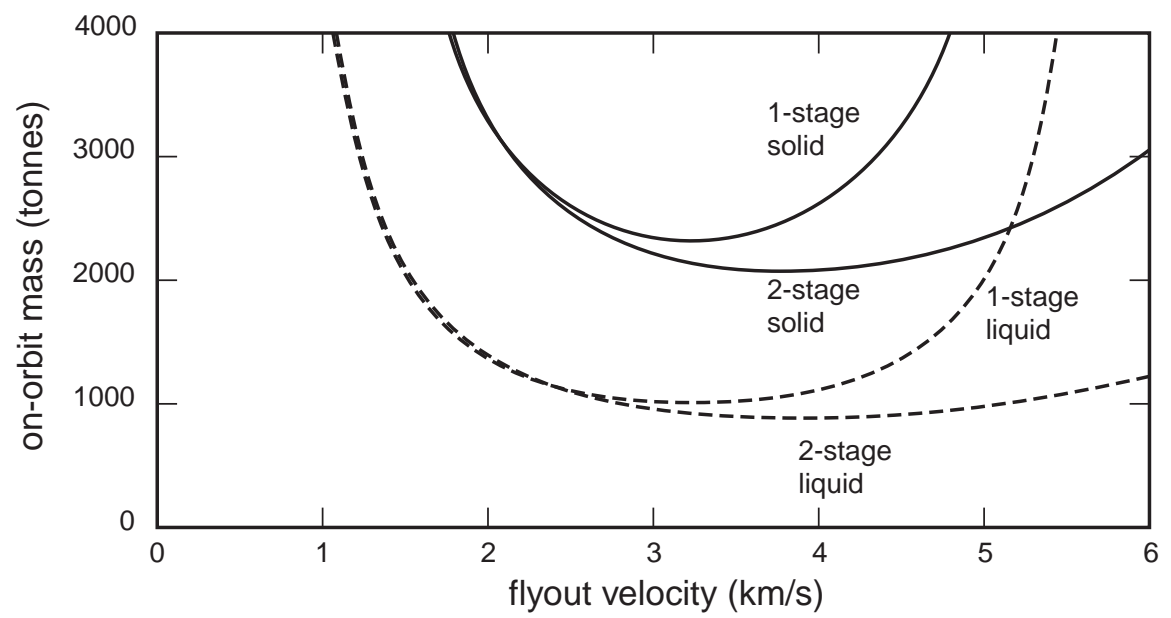

Figure 6.5. Total mass of an orbiting constellation for the baseline case, including lifejackets, for oneand two-stage SBIs against solid- and liquid-propellant missiles. The SBIs have $10-\mathrm{g}$ accelerations and carry kill vehicles capable of $2.5-\mathrm{km} / \mathrm{s}$ total divert velocity.

\subsection{Optimizing System Mass-in-Orbit}

The goal in optimizing a system of on-orbit interceptors is to provide adequate coverage with a minimum constellation mass. A tradeoff must be made between mass and range for each individual interceptor, based on the amount of fuel that is loaded into it. (The other significant factors that affect mass - kill-vehicle divert velocity and acceleration - are determined largely by the target missile.) The total system mass is very sensitive to the dry mass of the kill vehicle: a one kilogram increase in dry mass of the kill vehicle would increase the mass of the constellation by roughly ten tonnes.

To optimize the baseline system's on-orbit mass, we combined the coverage and interceptor mass results presented in Fig. 6.3 and Sections 6.3 and 6.4. Figure 6.5 shows the resulting on-orbit constellation mass for one- and two-stage interceptors against solid- and liquid-propellant missiles, assuming $136-\mathrm{kg}$ kill vehicles capable of accelerations of $10 \mathrm{~g}$, including the 50 percent allowance for the mass of the lifejacket. The system mass increases steeply at lower values of $v_{\text {flyout }}$, where most of the range is used for flying down from the parking orbit, and increases steeply at higher values of $v_{\text {flyout }}$ where the interceptor mass increases faster than the increase in coverage. Against solid-propellant ICBMs, the mass of the single-stage system has a shallow minimum between 2.5 to $3.5 \mathrm{~km} / \mathrm{s}$. For the two-stage system, the minimum mass is a little lower and stretches from 2.5 to $5 \mathrm{~km} / \mathrm{s}$. The mass savings appear to be worth the complication of incorporating a second stage for speeds greater than $3.5 \mathrm{~km} / \mathrm{s}$. Against the liquid-propellant missile, the masses on orbit are smaller and the minima are flatter and wider.

We selected a two-stage interceptor with a flyout velocity of $4 \mathrm{~km} / \mathrm{s}$ to minimize system mass for our baseline system, which assumes a $2.5-\mathrm{km} / \mathrm{s}$ divert kill vehicle capable of $15 \mathrm{~g}$ in the endgame and having total lag in the interceptor's response of less than $0.1 \mathrm{~s}$. With that flyout velocity, roughly 1,600 interceptor satellites at an altitude of $300 \mathrm{~km}$ would be required for two of them, on average, to be in position to intercept a single solid-propellant missile (like ICBM S1) launched from North Korea, Iraq, or Iran $5 \mathrm{~s}$ before its burnout (see Section 6.3). The total mass would be 2000 tonnes. The number of interceptors 


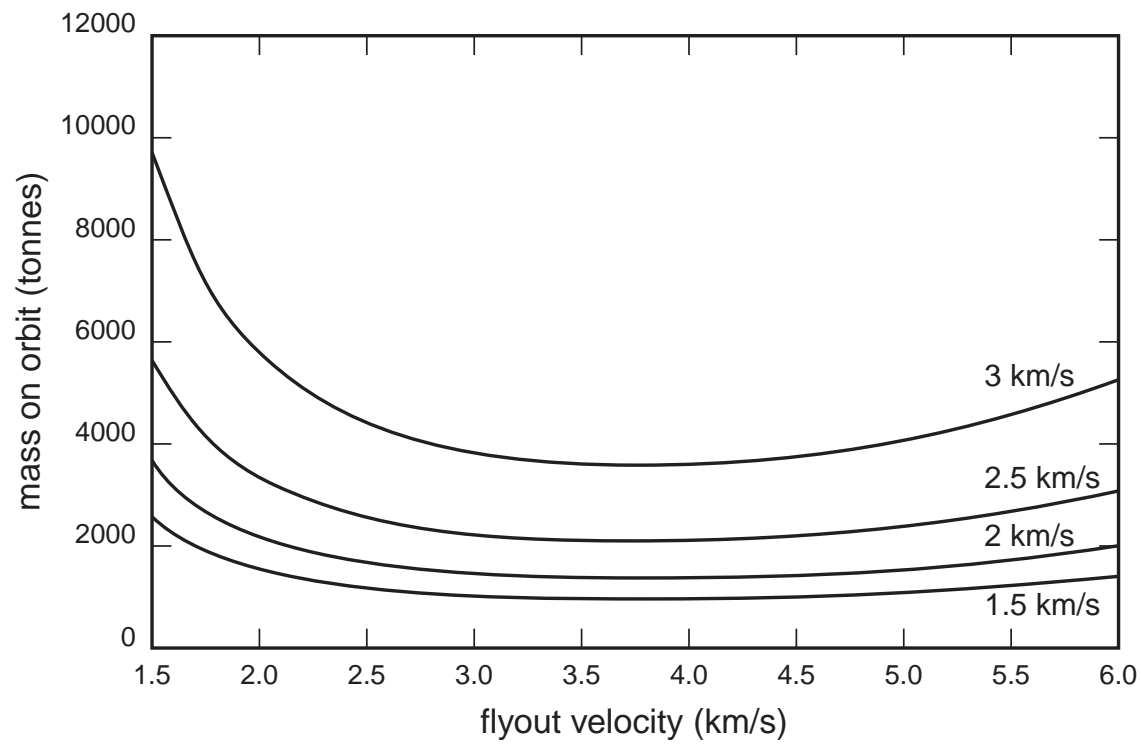

Figure 6.6. Total mass-in-orbit as a function of divert velocity and flyout velocity for a two-stage interceptor having an acceleration of $10 \mathrm{~g}$. Each curve is labeled by the assumed kill-vehicle divert velocity.

assumes that the interceptors could be fired $45 \mathrm{~s}$ after the target missile is launched (our zero-decision-time case).

If the firing of the interceptor were delayed by $30 \mathrm{~s}$ (our 30-second decision time case), the number of interceptors needed would increase, and the system mass would more than double (see Section 6.6). The mass of the constellations suggests that significant reductions in the mass of the SBI would be necessary to make such a system practical. Section 6.9 explores ways to reduce kill-vehicle mass.

If the system were designed to counter only liquid-propellant ICBMs, the interceptors would have considerably longer flyout times and the constellation size could be reduced significantly. As an example, if the flyout time were increased from $120 \mathrm{~s}$ to $170 \mathrm{~s}(240 \mathrm{~s}$ ICBM burn, interceptor launch $65 \mathrm{~s}$ after target missile launch, zero decision time, and an intercept $5 \mathrm{~s}$ before burnout), the on-orbit mass would drop by almost 60 percent to about 860 tonnes. This reduction would occur because the interceptors would have extra time to fly out to the target. Thus, only about 700 interceptors would be needed, even though the flyout velocity and size of the interceptor would remain the same. A more realistic decision time of $30 \mathrm{~s}$ would boost the on-orbit mass of a system designed to counter liquid-propellant missiles by about 60 percent, i.e., the total on-orbit mass would be 1,400 tonnes.

Changing the required total divert velocity would also affect the constellation mass significantly. Figure 6.6 shows the sensitivity of our results to the requirement that the kill vehicle have a total divert capability of $2.5 \mathrm{~km} / \mathrm{s}$. The constellation mass increases linearly with the kill-vehicle mass, increasing by nearly a factor of 2 for kill vehicles with $3 \mathrm{~km} / \mathrm{s}$ divert. The total constellation mass would drop by 30 percent if the divert requirement were lowered to $2 \mathrm{~km} / \mathrm{s}$ and by more than 50 percent if it were lowered to $1.5 \mathrm{~km} / \mathrm{s}$. The divert velocity requirements, however, are largely a function of the anticipated performance of the target missile and not the design of the SBI or the SBI constellation. Divert velocity requirements can also be driven by other design considerations (see Section 6.4.4). 
Table 6.2. Number of Interceptors as a Function of $v_{\text {flyout }}$, Decision Time, and Geography against Solid-Propellant ICBM S1 ${ }^{a}$

\begin{tabular}{|c|c|c|c|c|}
\hline \multirow[b]{2}{*}{$\begin{array}{c}v_{\text {flyout }} \\
(\mathrm{km} / \mathrm{s})\end{array}$} & \multicolumn{2}{|c|}{ Baseline case } & \multicolumn{2}{|c|}{ Defend U.S. from Iran } \\
\hline & $\begin{array}{c}\text { Zero Decision } \\
\text { time }\end{array}$ & $\begin{array}{l}\text { 30-second } \\
\text { delay }\end{array}$ & $\begin{array}{c}\text { Zero Decision } \\
\text { time }\end{array}$ & $\begin{array}{c}\text { 30-second } \\
\text { delay }\end{array}$ \\
\hline 3 & 2,800 & 6,100 & 3,700 & 12,400 \\
\hline 4 & 1,600 & 3,600 & 2,000 & 5,700 \\
\hline 5 & 1,100 & 2,600 & 1,400 & 3,700 \\
\hline $\begin{array}{l}{ }^{a} \text { The nun } \\
\text { function } \\
\text { baseline } \\
\text { case wher }\end{array}$ & $\begin{array}{l}\text { of SBIs requi } \\
\text { flyout velocity } \\
\text { e where the IC } \\
\text { it is intercepted }\end{array}$ & $\begin{array}{l}\text { ed to interce } \\
\text { nd decision } \\
\mathrm{M} \text { is interce } \\
10 \mathrm{~s} \text { before }\end{array}$ & $\begin{array}{l}\text { solid-propellant I } \\
\text { e for two differe } \\
\text { d } 5 \mathrm{~s} \text { before burr } \\
\text { nout, which is } \mathrm{w}\end{array}$ & $\begin{array}{l}\text { BM S1 as a } \\
\text { t cases: the } \\
\text { out, and the } \\
\text { at would be } \\
\text { by Iran. In } \\
\text { o }\end{array}$ \\
\hline
\end{tabular}

\subsection{The Effect of Decision and Intercept Time on System Mass}

As discussed above, the baseline system is optimistic for the solid-propellant case, particularly since it is built on the assumption that the interceptor has 120 -seconds flyout to the target. This time follows from two central assumptions: a 45-second firing time (the zero decision time case), and intercept occurs $5 \mathrm{~s}$ before burnout.

To illustrate the sensitivity of our results to the 45 -second firing time, we present the case in which the interceptor is launched $30 \mathrm{~s}$ later (the 30 -second decision time case). For the example considered in Fig. 6.3, a delay of $30 \mathrm{~s}$ would significantly increase the number of interceptors required. Table 6.2 illustrates the effect of changing the assumed decision time for three different flyout velocities. In the baseline case $(4 \mathrm{~km} / \mathrm{s}$ flyout velocity and $10 \mathrm{~g}$ ), the required number of interceptors would more than double, from 1,600 to 3,600 . With a flyout speed of $5 \mathrm{~km} / \mathrm{s}$, the increase in the required number of interceptors to reach the target missile after a 30-second delay is similar, more than double the zero-delay case.

The effect on constellation mass is the same for each flyout velocity because it is proportional to the number of satellites (see Table 6.3 ). The table also reveals that mass is minimized at flyout velocities around $4 \mathrm{~km} / \mathrm{s}$.

The required constellation size would also increase if more realistic geographic scenarios were considered, as they were for ground-based interceptors in Chapter 5. For example, defending the United States against a solid-propellant ICBM S1 launched from Iran would require the system to intercept the missile $160 \mathrm{~s}$ after launch, or $10 \mathrm{~s}$ before it burned out (see Chapter 5). This final intercept time is $5 \mathrm{~s}$ earlier than we have assumed in our baseline case for the SBI system and, although it represents only a small decrease in burn time, it would increase the number of interceptors and constellation mass required by 25 percent over the baseline case (see Tables 6.2 and 6.3).

If the two factors are combined and the interceptors must intercept a solid-propellant ICBM from Iran $160 \mathrm{~s}$ after launch and after a 30-second delay, the number of interceptors and constellation mass required would increase sharply, i.e., 250 percent higher than the baseline case.

Defending Alaska from North Korea could increase the size of the constellation even 
Table 6.3. Mass-in-Orbit as a Function of $v_{\text {flyout }}$, Decision Time, and Geography against Solid-Propellant ICBM S1 ${ }^{a}$

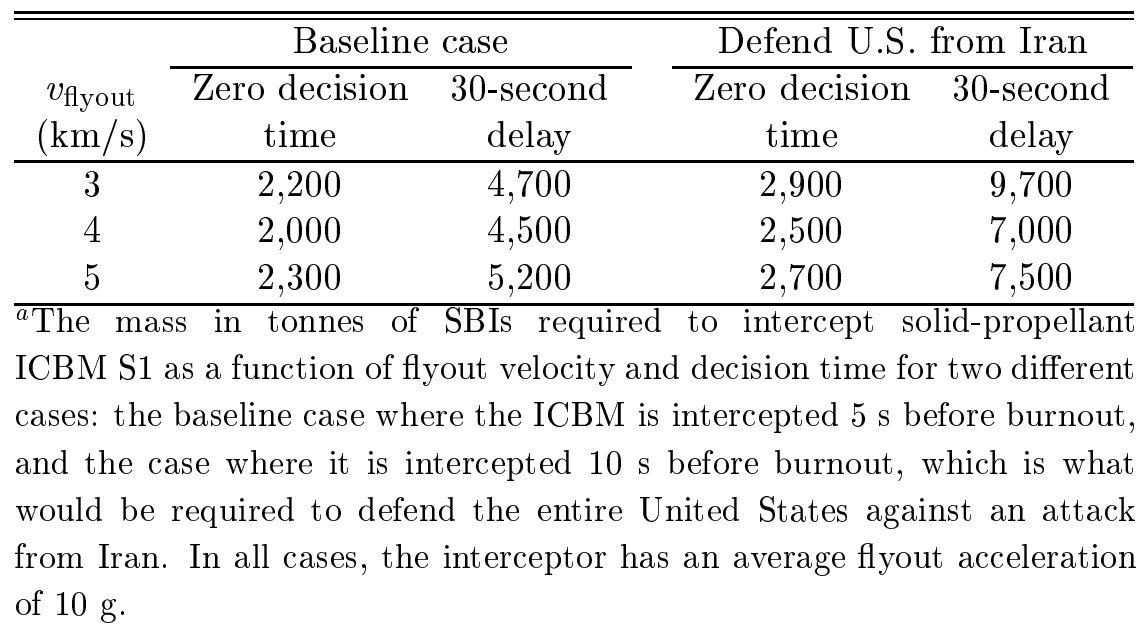

more than the Iranian case, because the intercept must occur before the missile reached sufficient speed to deliver the payload to its destination, as discussed in Chapter 5. Not only would the intercept have to occur more than $25 \mathrm{~s}$ earlier than the baseline case (more than doubling the number of interceptors required), but the interceptor would have to dive about $60 \mathrm{~km}$ lower to hit the ICBM in time, further increasing the number of interceptors required.

The effects of flyout velocity and decision time on the number of interceptors and the mass of the constellation are similar in the case of liquid-propellant ICBMs (see Tables 6.4 and 6.5). The biggest difference between liquid- and solid-propellant ICBMs is that the effect of delay is less pronounced for liquid-propellant missiles because they burn for a much longer time. Geographic constraints from more realistic scenarios are also less pronounced for liquid-propellant missiles, because they accelerate very rapidly at the end of their burn. In fact, there is no difference in the number of interceptors between the baseline system and defending against liquid-propellant missiles launched from Iran - both require intercept $5 \mathrm{~s}$ before burnout.

\subsection{The Effect of High-Acceleration Interceptors on System Mass}

Intuitively, one might expect that reducing the burn time of the SBI by increasing the acceleration of its booster would, for a fixed velocity, reduce the number of SBIs on orbit and, therefore, the total orbital mass of the system.

Although increasing acceleration would reduce the number of SBIs needed for coverage, two factors thwart the apparent mass savings: 1) a penalty related to divert velocity requirements that increases the propulsion requirements of the kill vehicle as burn time is shortened, and 2) a penalty related to acceleration that increases structural and propulsion system masses. For the storage altitude $(300 \mathrm{~km})$ and SBI flyout times used in this analysis (90 to $120 \mathrm{~s}$ ), these two factors would increase total orbital mass for interceptor burn times less than 30-40 s. In situations where the storage altitude is higher or the interceptor flyout time is less than we assumed, the minimum constellation mass would be achieved with veryhigh-acceleration interceptors. But the resulting constellation mass would be more massive 
Table 6.4. Number of Interceptors as a Function of $v_{\text {flyout }}$, Decision Time, and Geography against Liquid-Propellant ICBM L ${ }^{a}$

\begin{tabular}{cccccc}
\hline \hline & \multicolumn{2}{c}{ Baseline case } & & Defend U.S. against Iran \\
\cline { 2 - 3 } \cline { 5 - 6 }$v_{\text {flyout }}$ & Zero Decision & 30-second & & Zero Decision & 30 -second \\
$(\mathrm{km} / \mathrm{s})$ & time & delay & & time & delay \\
\hline 3 & 1,200 & 2,100 & & 1,200 & 2,100 \\
4 & 700 & 1,200 & & 700 & 1,200 \\
5 & 500 & 800 & & 500 & 800 \\
\hline
\end{tabular}

${ }^{a}$ The number of SBIs required to intercept liquid-propellant ICBM L as a function of flyout velocity and decision time for two different cases: the baseline case where the ICBM is intercepted $5 \mathrm{~s}$ before burnout, and the case where it is intercepted from Iran, which is also $5 \mathrm{~s}$ before burnout, to defend the entire United States against an attack. In all cases, the interceptor has an average flyout acceleration of $10 \mathrm{~g}$.

Table 6.5. Mass-in-Orbit as a Function of $v_{\text {flyout }}$, Decision Time, and Geography against Liquid-Propellant ICBM L ${ }^{a}$

\begin{tabular}{|c|c|c|c|c|}
\hline \multirow[b]{2}{*}{$\begin{array}{c}v_{\text {flyout }} \\
(\mathrm{km} / \mathrm{s})\end{array}$} & \multicolumn{2}{|c|}{ Baseline case } & \multicolumn{2}{|c|}{ "Defend U.S. against Iran } \\
\hline & $\begin{array}{c}\text { Zero decision } \\
\text { time }\end{array}$ & $\begin{array}{l}\text { 30-second } \\
\text { delay }\end{array}$ & $\begin{array}{c}\text { Zero decision } \\
\text { time }\end{array}$ & $\begin{array}{l}\text { 30-second } \\
\text { delay }\end{array}$ \\
\hline 3 & 1,000 & 1,700 & 1,000 & 1,700 \\
\hline 4 & 900 & 1,500 & 900 & 1,500 \\
\hline 5 & 1,000 & 1,600 & 1,000 & 1,600 \\
\hline
\end{tabular}

$\bar{a}$ The mass in tonnes of SBIs required to intercept liquid-propellant ICBM L as a function of flyout velocity and decision time for two different cases: the baseline case where the ICBM is intercepted $5 \mathrm{~s}$ before burnout, and the case where it is intercepted from Iran, which is also $5 \mathrm{~s}$ before burnout, to defend the entire United States against an attack. In all cases, the interceptor has an average flyout acceleration of $10 \mathrm{~g}$. 
than the baseline system, because high acceleration is not free.

In fact, if the two penalties were combined, they would add more to interceptor mass than the sum of their parts: the acceleration penalty would be amplified by the divert penalty, which would increase the mass of the kill vehicle and therefore the structural loads that must be handled by the booster stages. For simplicity we analyzed each penalty separately and did not estimate the combined effect. As a result, the magnitude of the effect of moving to higher accelerations is likely to be somewhat understated here.

For the analysis in this section, we took as the point of departure the baseline case described above - a two-stage interceptor having a flyout velocity of $4 \mathrm{~km} / \mathrm{s}$, an acceleration of $10 \mathrm{~g}$, and a burn time of nearly $40 \mathrm{~s}$ that carries a kill vehicle with a total divert velocity of $2.5 \mathrm{~km} / \mathrm{s}$. The total flight time of the interceptor is $120 \mathrm{~s}$.

\subsubsection{Divert penalty: the effect of booster burn time on mass}

The first mass penalty results from the inability of the guidance system on the kill vehicle to measure and predict the acceleration of the threat booster with precision. As long as the interceptor booster is burning, corrections for threat trajectory updates can be accommodated with negligible penalty. However, when the SBI booster burns out, any uncertainties that remain, along with the effects of unpredictable variations in threat acceleration after SBI burnout, must be corrected by the kill vehicle.

As described in Chapter 10, the limited tracking precision, even with an X-band radar, would result in significant uncertainties in the estimate of the threat acceleration. For the filters that we modeled, this uncertainty does not improve after about $15 \mathrm{~s}$ of tracking and remains on the order of $1 \mathrm{~g}$ (see Chapter 12). In addition, the unpredicted variations in the threat missile's thrust and drag add to the residual uncertainty and must be matched by kill-vehicle maneuvers after the interceptor booster burns out. Therefore, if the interceptor burns out in $20 \mathrm{~s}$ rather than $40 \mathrm{~s}$, the kill vehicle must match any unpredicted accelerations for 20 additional seconds. For a nominal $1-\mathrm{g}$ variation in acceleration that must be matched by the kill vehicle, an additional $200 \mathrm{~m} / \mathrm{s}$ in divert velocity must be delivered. This requires extra fuel which increases the size of the kill vehicle, in turn increasing the size of the interceptor that is required to reach the same flyout velocity.

The effect of varying interceptor burn time on interceptor mass, the number of interceptors, and total mass-in-orbit is illustrated in Fig. 6.7 for the $4 \mathrm{~km} / \mathrm{s}$, two-stage baseline case. Total flight time is assumed to be $120 \mathrm{~s}$ for the case of a solid-propellant missile. As expected, the number of interceptors required decreases and the mass of the interceptor increases as burn time gets shorter. While the number of SBIs required for coverage is reduced as SBI burn time is shortened, the increase in mass per SBI from this effect more than offsets the savings for burn times that are less than about $40 \mathrm{~s}$, as the top curve in Fig. 6.7 shows. For SBI burn times greater than $40 \mathrm{~s}$, the system mass also increases because the reduction in interceptor mass is more than offset by the need for more interceptors.

This effect can also be seen clearly in Fig. 6.8, which shows the total mass-in-orbit for a set of one- and two-stage interceptors. The lowest curve is for the $4 \mathrm{~km} / \mathrm{s}$, two-stage interceptor that we used as our baseline case. In this case, the mass-in-orbit is minimized for a burn time of about $40 \mathrm{~s}$, which corresponds to an average acceleration of about $10 \mathrm{~g}$. For single-stage interceptors, the mass-in-orbit is minimized for an interceptor having a flyout velocity of about $3.5 \mathrm{~km} / \mathrm{s}$ and a burn time of about $40 \mathrm{~s}$, which corresponds to an average acceleration of about $9 \mathrm{~g}$. Since the minimum is shallow for the assumed parameters, however, minor changes in the assumptions would cause substantial changes in the optimum 


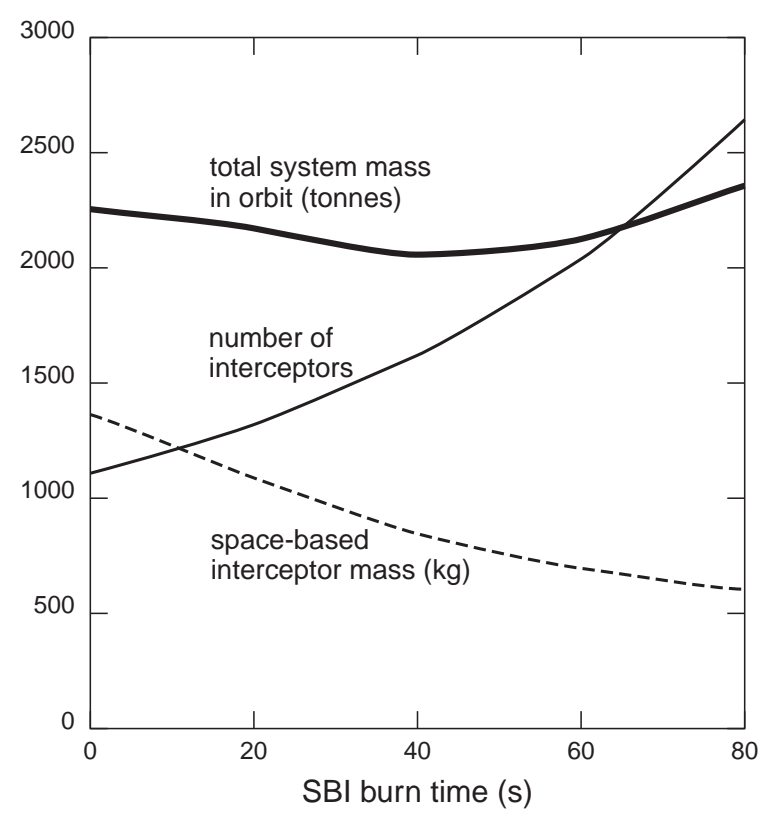

Figure 6.7. Variation of the number of interceptors, mass of an individual SBI, and on-orbit mass as a function of SBI burn time, including the estimated "divert penalty" for a two-stage SBI with a flyout velocity of $4 \mathrm{~km} / \mathrm{s}$.

acceleration but only minor changes in the mass-in-orbit.

\subsubsection{Acceleration penalty: the effect of acceleration on mass}

The second factor that works against higher acceleration is the mass penalty associated with the higher-thrust booster rocket and the inter-stage structure. There are three basic components to a booster's inert mass, whether it has a liquid- or solid-propellant motor.

- The mass of the inter-stage structure and release system which is a function of the vehicle mass forward of the inter-stage structure and the buckling loads caused by both axial and lateral acceleration.

- The dry or inert mass of tankage and the combustion chamber, or the rocket pressure vessel, which is a function of the propellant mass and the chamber pressure. For a given total impulse, greater thrust requires either a higher chamber pressure or a larger nozzle throat or some combination of the two. Higher chamber pressure means heavier chamber walls, which would be offset somewhat in the case of solid-propellant systems by a reduction in the thickness of the case insulator, which would be made possible by the shorter burn time.

- The mass of the thrust vector system and nozzle structural stiffness which is a function of the thrust or mass flow of the rocket. The increased mass flow for higher thrust requires a heavier nozzle and a more massive thrust-vector control mechanism.

To illustrate the combined effects of higher accelerations on these three components, we use an equation of the following form to describe these relationships. The inert or dry mass 


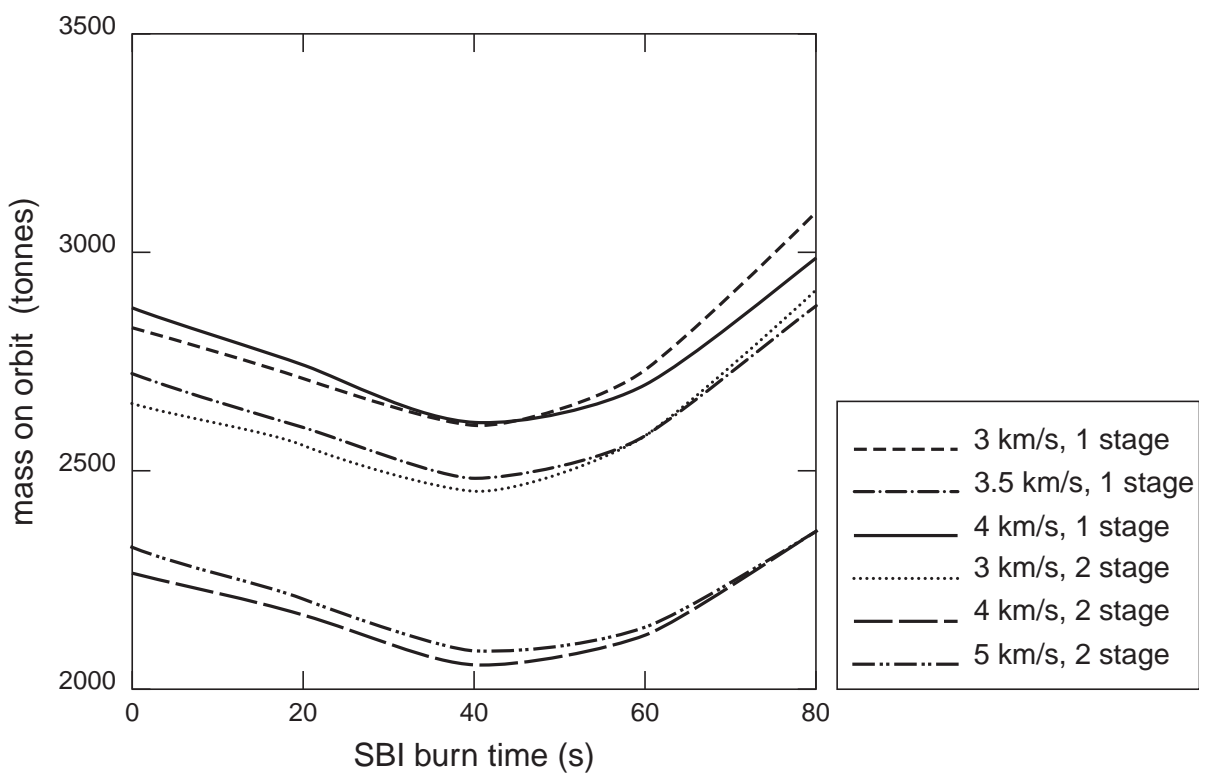

Figure 6.8. Mass-on-orbit for a family of one- and two-stage interceptors carrying the baseline kill vehicle as a function of SBI burn time, including the estimated "divert penalty."

of the stage, $M_{\text {inert }}$, is defined by $C_{1} \times f_{1}$ (upstage mass), which represents the inert mass of the inter-stage structure and separation hardware, plus $C_{2} \times f_{2}$ (propellant mass), which represents the mass of the basic motor case and fixed nozzle exit cone, plus $C_{3} \times f_{3}$ (thrust), which represents the movable nozzle joint, nozzle structural stiffening for the actuator loads, and thrust-vector control hardware.

Based on data for existing boosters, the best-fit linear relationship for a single-stage booster was found to be:

$$
M_{\text {inert }}=0.05 M_{\text {payload }}+0.087 M_{\text {propellant }}+0.0009 F,
$$

where $M_{\text {inert }}$ is the boost-stage dry mass in kilograms, $M_{\text {payload }}$ is the mass in kilograms of the payload forward of the inter-stage, $M_{\text {propellant }}$ is the mass of propellant in kilograms, and $F$ is the rocket thrust in newtons.

This equation can also be used for two-stage interceptors, where $M_{\text {payload }}$ for the first stage is the mass of the kill vehicle plus the mass of the second stage, and $M_{\text {payload }}$ for the second stage is the mass of the kill vehicle.

Figure 6.9 plots this equation for average accelerations of 6,10 , and $20 \mathrm{~g}$ for a singlestage interceptor and a kill-vehicle mass of $136 \mathrm{~kg}$, which corresponds to our baseline kill vehicle having a $2.5-\mathrm{km} / \mathrm{s}$ divert velocity capability. This figure also shows the kick-stage motor data that were used to generate the best-fit equation.

One can debate the coefficients of this equation, but while no data points from existing kick stages were found for the 20-g acceleration case, the equation fits both intuition and the available data points reasonably well. It should be generally valid for showing trends for either solid- or liquid-propellant stages and is used to illustrate how the mass of an SBI can be expected to vary with acceleration for a fixed flyout velocity.

Figure 6.10 illustrates the sensitivity of the SBI mass to thrust acceleration for a singlestage interceptor having a flyout velocity of $3.5 \mathrm{~km} / \mathrm{s}$. For a given flyout velocity, the 


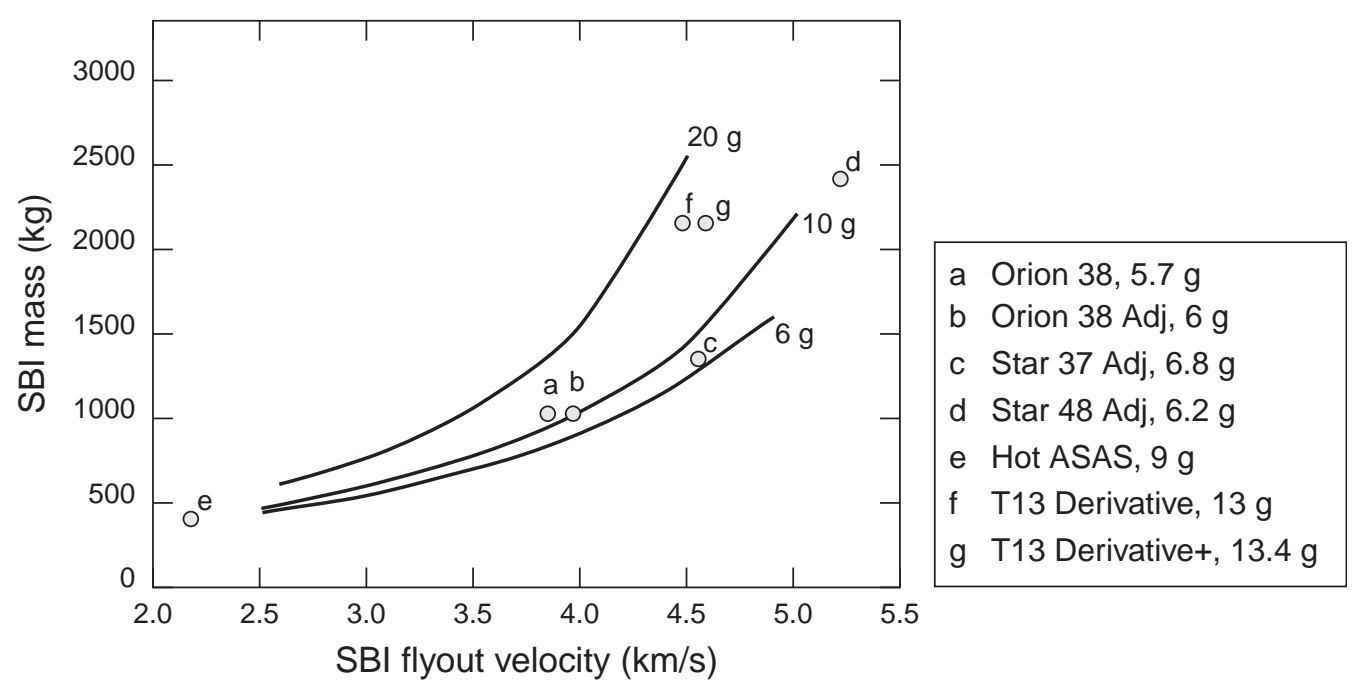

Figure 6.9. The variation of SBI mass as a function of flyout velocity for different accelerations of an SBI with a 136-kg kill vehicle. The curves represent the results of the best-fit equation for each acceleration. The data points used to generate the curves are also indicated. (Adj. = adjusted)

acceleration and SBI mass increase as the burn time is shortened. The figure also shows the number of satellites that would be required, and the resulting mass-in-orbit, as a function of burn time. Here, the minimum mass-in-orbit occurs for a burn time of about $30 \mathrm{~s}$, which corresponds to an average acceleration of about $12 \mathrm{~g}$. As in Fig. 6.7, the minimum is shallow for the assumed parameters; thus minor changes in the assumptions will cause substantial changes in the optimum acceleration but only minor changes in the mass-in-orbit.

Figure 6.11 shows the total mass-in-orbit for a set of one- and two-stage interceptors and accounting for the estimated acceleration penalty. The total mass-in-orbit is minimized at about the same value for either a one-stage, $3.5-\mathrm{km} / \mathrm{s}$ interceptor with a burn time of $30 \mathrm{~s}$ or the baseline two-stage, $4.0-\mathrm{km} / \mathrm{s}$ interceptor with a burn time of nearly $40 \mathrm{~s}$. Nevertheless, the minimum is shallow for the assumed parameters; minor changes in the assumptions will cause substantial changes in the optimum acceleration but only minor changes in the mass-in-orbit.

\subsubsection{Summary}

In summary, the mass-in-orbit of an SBI system can be optimized by selecting the appropriate burn time, flyout velocity, and number of stages. As burn times are shortened or lengthened from the optimal value, on-orbit mass will rise. This arises from two effects: a "divert penalty" where shorter burn times increase the number of course corrections that the kill vehicle would have to make on its own, and an "acceleration penalty" where the structural and rocket-motor masses increase. For simplicity, those penalties are treated separately here. If they were combined, the divert penalty would increase the acceleration penalty and result in a penalty that would be larger than the sum of the two parts.

The minimum value for a particular constellation is determined by the time that the SBI has to reach the target-its flyout time. The more flyout time that the interceptor has, the lower the optimal acceleration tends to be. In the set of cases examined here, flyout times are in the range of 90 to $120 \mathrm{~s}$. For an SBI system designed to intercept solid-propellant 


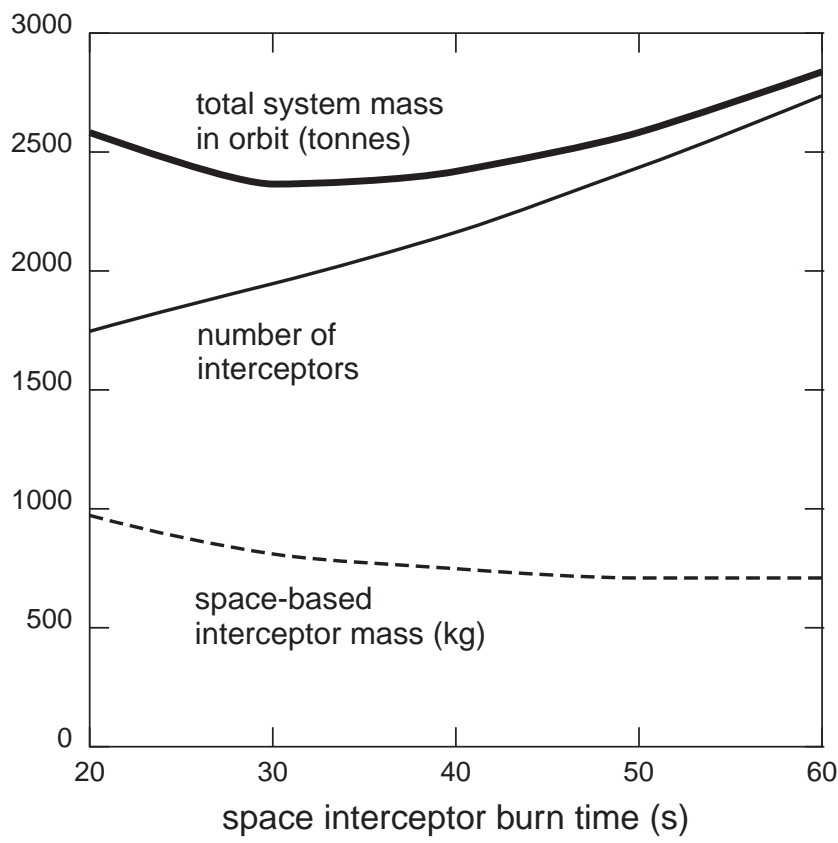

Figure 6.10. Variation of the required number of interceptors, the individual mass of an interceptor, and the mass-in-orbit for a one-stage, $3.5-\mathrm{km} / \mathrm{s}$ interceptor as a function of SBI burn time, including the estimated "acceleration penalty."

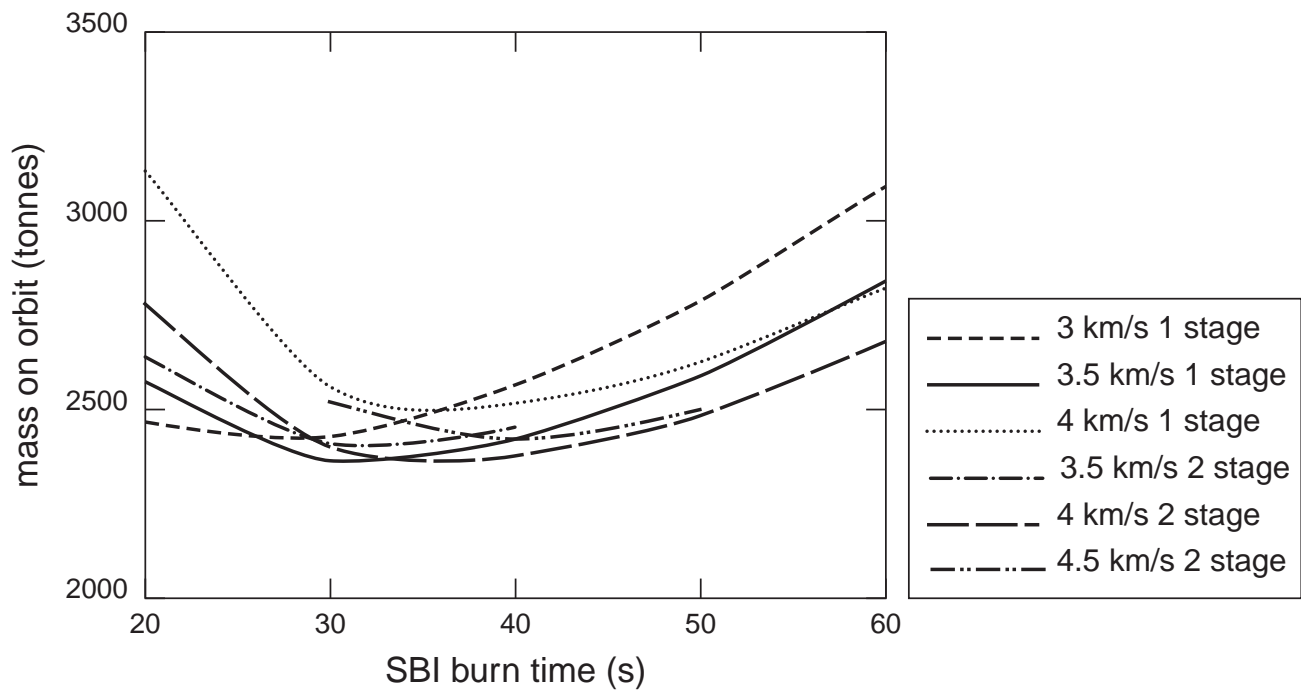

Figure 6.11. Mass-on-orbit for a family of one- and two-stage interceptors carrying the baseline kill vehicle as a function of SBI burn time, including the estimated "acceleration penalty." 
missiles, on-orbit mass is minimized when the burn time is $40 \mathrm{~s}$ and the flyout velocity is $4 \mathrm{~km} / \mathrm{s}$, which corresponds to an acceleration of about $10 \mathrm{~g}$.

\subsection{Space-Launch Requirements for SBIs}

Placing the 2,000-tonne mass of our baseline system into $300-\mathrm{km}$ altitude orbits at an inclination of $45^{\circ}$ would require roughly a five- to tenfold increase in the U.S. space-launch capacity (depending on how it is counted), if the constellation were deployed over a threeyear period. Measured in terms of mass placed into orbit, it would require a fivefold increase relative to the U.S. average mass deployed by medium- and heavy-lift rockets over the sixyear period from 1997 to $2002 .^{1}$ Measured in terms of the number of launches per year, deploying the baseline constellation in three years would require a five- to thirteenfold increase, depending on the type of launcher used. If the satellites were deployed on all medium and heavy launch vehicles but the Shuttle, they would have to be launched at five times the rate experienced over the past six years. If the satellites were deployed only on medium-launch vehicles, an eightfold increase would be necessary, and at least a tenfold increase over current rates would be required if the system were deployed on only expendable heavy-lift vehicles.

This is an enormous number of launches by contemporary or historical standards. In terms of specific launchers, deploying the baseline constellation over three years would require approximately 400 Delta II rockets, 250 Atlas II/Delta III/Atlas III rockets, or 100 Space Shuttles or Atlas V-Heavy/Delta IV-Heavy rockets [84, 85, 86]. If the cargo version of the Space Shuttle were ever built, with its nearly 70-tonne capacity, only about 30 launches would be needed to deploy the constellation [87, p. x]. To put these numbers in perspective, over the past five years the United States has launched an average of 16 unmanned medium-lift rockets, 3 heavy-lift rockets, and 5 Space Shuttles each year [84], illustrating why much of the focus of SBI research to date has been on reducing kill-vehicle mass, a subject that is taken up in the next section.

The number of launches needed each year to maintain the constellation would be fewer, but still high by current standards. The annual replenishment rate depends on the service lifetime of the satellites. If the service lifetime is approximately five to eight years, system maintenance would require about 30-50 Atlas II/Delta III/Atlas III launches per year on average, or 10-20 heavy expendable launch vehicles or shuttles. For economy, interceptors would need to be launched several at a time, which requires an adapter and dispenser and their associated mass. Once in orbit, the interceptors would be able to maneuver to distribute themselves within their orbital plane, but would have little flexibility to change the inclination of their orbits, as this would require a large velocity change and consume precious fuel, suggesting that at least a portion of the replenishment satellites would be delivered to orbit by medium-lift rockets.

This section has focused so far on the space-launch requirements for the baseline SBI

\footnotetext{
${ }^{1}$ This comparison is based on an estimate of the mass that each launcher could have placed into a circular orbit with altitude of $300 \mathrm{~km}$ and inclination of $45^{\circ}$ had it been used for that purpose (we refer to this as equivalent mass) and the average of the actual number of launches (successful or not) from 1997 through 2002 by each launcher type. The average annual equivalent mass deployed over that period was about 140 tonnes, or about 7 percent of the mass of the required constellation. This estimate excludes the contributions of the Space Shuttle, because a manned system is unlikely to be used to deploy an SBI system-the risks of manned space flight are too high for a mission that could be done effectively with expendable launchers. If the Space Shuttle were included, the average annual equivalent mass placed in orbit would be 250 tonnes, or about one-eighth of the total mass of the constellation.
} 
system, but for the reasons discussed in Section 6.3, those numbers are probably optimistic. The effect of less-optimistic assumptions about decision time and intercept times on constellation size and mass also have been examined (Section 6.6). Using those results (see Table 6.2), one can see that the launch requirements listed above could more than double (system mass increase from 2000 tonnes to 4500 tonnes) if the zero decision time assumption in the baseline case is relaxed. More realistic assumptions about the effects of geography on intercept times, such as those discussed in Section 6.6, could easily increase space-launch requirements by 50 percent or more. Those numbers could be understated by a factor of two or more if the constellation were sized to reflect more realistic assumptions about decision and intercept times.

\subsection{The Effect of Reducing Kill-Vehicle Mass}

The large number of launches required to deploy a constellation of the baseline interceptors highlights the strong incentives to reduce system mass. The impact of kill-vehicle mass on the total mass of the constellation is enormous, with a multiplier factor of more than 10,000 for the system considered here, thanks to the large number of interceptors needed for coverage and the exponential dependence of the mass of the interceptor on flyout velocity. As a result, each kilogram of additional kill-vehicle mass would require that roughly another 10 metric tonnes would have to be placed in orbit.

Since system mass scales linearly with the mass of the kill vehicle, new technologies that reduce the kill-vehicle mass can have a strong impact on the total system mass and cost. Our baseline estimate of kill-vehicle mass, approximately $140 \mathrm{~kg}$ wet for a divert capability of $2.5 \mathrm{~km} / \mathrm{s}$, is based on technology anticipated within the next 10 years, with some extrapolation in the area of sensors and avionics to more capability and lower masses (Chapter 14). With sufficient time and investment, however, it may be possible to reduce subsystem masses well-below our estimates, with the potential for reducing the kill-vehicle mass.

To explore the sensitivity of the baseline assumptions to improvements in technology, we examined three possible areas for improvement:

1. Further reductions in the mass of sensor, processing, and other electronics systems, driven by Moore's law and microtechnology. We consider the effect of a 50 percent decrease in the mass of these subsystems.

2. Reduction in the mass of the divert thrusters, which are scaled from current technology. There may be improvements in materials. There are also possible gains from the systems approach. Our baseline assumes a cruciform approach, with four substantial thrusters, each capable of accelerating the kill vehicle at $15 \mathrm{~g}$ in the endgame. If it is possible to keep the vehicle oriented so that only one thruster is needed to provide this acceleration in any direction, perhaps by imaging the target missile to monitor its thrust axis and anticipating its direction of possible acceleration, the other three thrusters might be made smaller. If they can be reduced to 25 percent - 33 percent of the size of the main thruster, the total divert thruster mass might be reduced by 50 percent.

3. Reduction in tankage mass. Our baseline estimate for the kill vehicle uses current state-of-the-art propellant tanks, with a mass equal to 20 percent of the mass stored in them. The tanks are pressurized to about 800 psi and have expulsion devices to 
Table 6.6. Effect of Possible Mass Reduction Strategies for Kill Vehicles ${ }^{a}$

\begin{tabular}{|c|c|c|c|c|c|}
\hline Option & Description & $\begin{array}{c}\mathrm{KV} \\
\text { wet mass } \\
(\mathrm{kg})\end{array}$ & $\begin{array}{c}\text { Mass of } \\
\text { inteceptor } \\
(\mathrm{kg})\end{array}$ & $\begin{array}{c}\text { Total } \\
\operatorname{mass}^{b} \\
\text { (tonnes) }\end{array}$ & $\begin{array}{r}\text { Reduction } \\
\text { (percent) }\end{array}$ \\
\hline Baseline & & 136 & 820 & 2,021 & n.a. \\
\hline Sensor & $\begin{array}{l}\text { Drop sensor and avionics } \\
\text { mass by } 50 \%\end{array}$ & 88 & 531 & 1,308 & 35 \\
\hline Rocket & $\begin{array}{l}\text { Drop divert motor mass } \\
\text { by } 50 \%\end{array}$ & 103 & 621 & 1,531 & 24 \\
\hline Tankage & $\begin{array}{l}\text { Drop tankage mass from } \\
20 \% \text { of propellant to } 10 \%\end{array}$ & 100 & 603 & 1,486 & 26 \\
\hline $\begin{array}{l}\text { Sensor \& } \\
\text { rocket } \\
\text { Sensor \& } \\
\text { rocket \& } \\
\text { tankage }\end{array}$ & $\begin{array}{l}\text { Combine sensor and } \\
\text { rocket improvements } \\
\text { Combine sensor, rocket, } \\
\text { and tankage } \\
\text { improvements }\end{array}$ & 66 & 398 & 981 & 51 \\
\hline
\end{tabular}

${ }^{a}$ Baseline assumes a KV wet mass of $136 \mathrm{~kg}$ for a $2.5-\mathrm{km} / \mathrm{s}$ divert and a $4-\mathrm{km} / \mathrm{s}$ flyout velocity and a $10-\mathrm{g}$ average flyout acceleration. Structure is assumed to scale at 10 percent of total KV mass.

${ }^{b}$ Total constellation mass.

force out the fuel and oxidizer as efficiently as possible. At a small scale, there are experimental miniaturized pumps that serve the function of a turbo pump on a large rocket engine. While more complex, this scheme would make possible lower-pressure tanks. We consider the possibility of reducing tank mass by 50 percent, from 20 percent of the propellant for our baseline system to 10 percent.

Table 6.6 shows the leverage that each of those technology improvements could provide, both individually and in combination. Clearly, if improved technologies could significantly reduce the kill-vehicle mass, they could lead to large reductions in the total mass-in-orbit. However, even if possible, the development and maturation of these technologies would require time and money. Moreover, cutting the mass of each of those three key subsystems by half (which would not be easy) would reduce the wet mass of the kill vehicle by only about 60 percent, from a kill-vehicle mass of $136 \mathrm{~kg}$ to about $52 \mathrm{~kg}$ in the $2.5-\mathrm{km} / \mathrm{s}$-divert case.

\subsection{The Expense of Operating in Space}

As discussed above, the required mass-in-orbit dominates the design considerations for any space system, largely because of the very high cost of deploying mass into orbit.

Despite a number of programs aimed at reducing the cost of space transportation, from the Space Shuttle to Orbital Science Corporation's Pegasus to NASA's failed X-33, launch cost is the single most important consideration for space systems and drives system costper-unit-mass far beyond comparable ground- and air-based systems. For example, heavy Delta II (Delta model 2920-H10) launches procured under NASA's MIDEX program for launch in 2007 , will cost of order $\$ 80 \mathrm{M}$ for delivery of $5,800 \mathrm{~kg}$ to a $300-\mathrm{km}, 28^{\circ}$-inclination 
orbit [88]. These figures yield a launch cost of $\$ 14,000$ per kilogram, or $\$ 14 \mathrm{M}$ per metric tonne. This is probably an optimistic figure, however; typical costs have been closer to $\$ 22,000$ per kilogram for low-Earth orbits [89, p. 20]. The interceptors would have to be packaged and launched several to a booster to realize this economy, as small launchers such as Orbital Science Corporation's Pegasus are several times more expensive per unit mass; their value is flexibility, not carrying capacity.

\subsection{Summary}

Space-based interceptors are a potentially attractive option for boost-phase intercept because they are not constrained by geography to being located close to the target missile. In addition, the practical limits on their accelerations and velocities are not constrained by the atmosphere and so, in theory, the interceptors could have longer reaches than surfaceand air-based interceptors.

Those advantages are offset, however, by a number of drawbacks. First, placing mass into orbit is very expensive, on the order of $\$ 22,000$ per kilogram for low-Earth orbits. This makes mass the dominant design criterion for space-based systems. For example, mass constraints limit the ability to exploit the lack of atmosphere to increase the reach of the interceptors. In fact, we found that the total mass-in-orbit was minimized when accelerations and flyout velocities were less than those assumed in almost all of our surfacebased interceptors. Second, the orbital motion of the satellites and the rotation of Earth result in requirements for very large numbers of satellites to ensure that at least one would be close enough to intercept a single missile before it achieved enough velocity to deliver its munitions to the United States. This coverage requirement, in turn, results in constellations with masses that are measured in many hundreds or thousands of tonnes.

In the baseline case that we examined in this chapter, we minimized total mass at 2,000 tonnes for a system that operated at an altitude of $300 \mathrm{~km}$, with 1,600 interceptors that would accelerate at an average of $10 \mathrm{~g}$, add a velocity of $4 \mathrm{~km} / \mathrm{s}$ to the kill vehicle, and have a mass of $820 \mathrm{~kg}$ exclusive of its lifejacket. The kill vehicle was assumed to have the same capabilities as the one used on the ground-based interceptors and had a mass of 136 $\mathrm{kg}$. This system would be capable of intercepting a single solid-propellant ICBM launched from North Korea, Iraq, or Iran $5 \mathrm{~s}$ before burnout with at least one (and an average of two) interceptors. This 2,000 tonnes is a huge mass to place into orbit and would require a five- to tenfold increase in the current launch capacity of the United States. Defending against only liquid-propellant ICBMs would cut the number of interceptors, mass-in-orbit, and launch requirements by almost 60 percent.

Nevertheless, the baseline case should be considered optimistic against solid-propellant missiles. If more realistic geographic scenarios are considered, ICBMs would have to be intercepted sooner than $5 \mathrm{~s}$ before burnout, and the number of interceptors and total system mass would increase. For example, the number of interceptors and total mass would increase by about 25 percent if the constellation were designed to defend the United States against Iran. More realistic decision times would also increase constellation size and mass; a 30second delay would more than double the number of satellites and mass-in-orbit. The system would also be larger if it were designed to defend against ICBMs that were launched nearly simultaneously from points on Earth that were separated by a few tens of kilometers or less. The effects of more realistic scenarios are less-pronounced against liquid-propellant ICBMs, because they burn longer and accelerate more rapidly at the end of their burns. 
The expense of placing mass in orbit creates significant incentives for reducing the mass of the kill vehicle, which drives the mass of the system. We found, however, that significant reductions would be possible only if the mass of all three major components of a kill vehicle were reduced. Even in that case, a technically challenging 50 percent reduction in all three components would reduce system mass by only about 60 percent.

Finally, we examined only the issues related to interceptor flyout requirements and mass. We did not examine the significant command and control issues related to controlling a system of 1000 or more semi-autonomous satellites.

\section{References for Chapter 6}

[77] James R. Wertz and Wiley J. Larson, eds., Space Mission Analysis and Design. (Microcosm Press, El Segundo), 3rd edition, 1999.

[78] Australian Space Weather Agency, Satellite Lifetimes and Solar Activity. Available at: http://www.ips.gov.au/Main.php?CatID=8\&SecID=1\&SecName=Space $\%$ 20Weather \&SubSecID $=3 \&$ SubSecName $=$ Space $\% 20$ Weather 20Effects\&LinkName=Satellite\%20Lifetimes\%20and\%20Solar\%20Activity.

[79] G. Schubert and R.L. Walterscheid, Allen's Astrophysical Quantities. (AIP Press, New York), 4th edition, 2000.

[80] N. Bloembergen et al., Rev. Mod. Phys. 59, S1 (1987).

[81] Lowell Wood, "From 'Smart Rocks' came 'Brilliant Pebbles'," Aerospace America, April 1990.

[82] Gregory Canavan and Edward Teller, "Strategic Defense for the 1990's," Nature 344, 699, 19 April 1990.

[83] Richard L. Garwin. "Brilliant Pebbles Won’t Do," Nature 346, 21 (Scientific Correspondence), 5 July 1990.

[84] Associate Administrator for Commercial Space Transportation, "Commercial Space Transportation: Year in Review, 1997-2002." Technical report, Department of Transportation, published Jan. 1998-Jan. 2003.

[85] Steven J. Isakowitz, Joseph P. Hopkins, and Joshua B. Hopkins, "International Reference Guide to Space Launch Systems." American Institute of Aeronautics and Astronautics, 3rd edition, December 1999.

[86] Federation of American Scientists Space Policy Project, "EELV: Evolved Expendable Launch Vehicle." Available at: http://www.fas.org/spp/military/program/launch/eelv.html.

[87] Office of Technology Assessment, "Launch Options for the Future: A Buyer's Guide." Technical Report OTA-ISC-383, U.S. Congress, Government Printing Office, Washington, D.C., July 1988. OTA-ISC-383.

[88] NASA MIDEX Library, "EELV Launch Services Information Summary." Available at: http://explorer.larc.nasa.gov/explorer/MIDEX_5_6_AO_ELV_Input_010515.pdf. 
[89] Liam Sarsfield, "The Cosmos on a Shoestring: Small Spacecraft of Space and Earth Science." Technical report, RAND Critical Technologies Institute, Santa Monica, California, 1998. MR-864-OSTP. 



\title{
Chapter 7
}

\section{Airborne Laser Engagement Fundamentals}

\author{
Contents
}

7.1 Differences Between Laser and Hit-to-Kill Intercepts . . . S131

7.2 ABL Engagement Geometry . . . . . . . . . . . S132

7.3 ABL Performance Parameters . . . . . . . . . . S132

\subsection{Differences Between Laser and Hit-to-Kill Intercepts}

Boost-phase intercept of missiles by directed energy and by kill vehicles both seek to disable the missile before its payload has reached a velocity great enough to reach a target being defended. The methods of disabling the missile are different. A kill vehicle directly strikes the missile, inflicting severe, almost-instantaneous mechanical damage. The Airborne Laser is an airborne system consisting of a high power laser with optical and tracking systems that permit it to focus the laser's energy on a missile. The resulting heating eventually causes mechanical failure of the missile, although the time required to absorb the requisite energy may be some seconds.

The damage mechanism itself will depend on the type of missile. Liquid propellant missiles are relatively thin-walled metal vessels containing the fuel, with a sturdy combustion chamber from which the burning fuel is exhausted. The fuel tanks are pressurized, and if the temperature of a portion of the tank is raised high enough, the metal softens and the tank ruptures. This breach will eventually cause the missile to fail, although the speed and details of the failure will depend on several variables, such as the construction materials and the internal pressure. Alternatively, the damage may come about because of the large column load on the fragile structure caused by the missile's acceleration. Heating the wall of the fuel tank could cause it to buckle. If it buckles, the center of mass will no longer be in line with the thrust, and the missile will quickly tumble and become ineffectual. In neither case is the heating required to melt the metal. Rather, it is only necessary to heat the metal to a temperature at which the structural integrity is compromised.

Solid-propellant missiles are of much sturdier construction. In this case, the motor is an integral part consisting of a thick wall, probably of a composite material, containing the solid propellant. Laser energy absorbed in the wall would damage it so that the side blows out, disabling the missile.

Because the construction of the target missile is unknown, we can only make reasonable estimates of the important parameters. The damage mechanism and estimates of the 
required fluence (energy density delivered to the vicinity of the missile) is discussed in Section 20.1. For present purposes we assume that a fluence of $32 \mathrm{MJ} / \mathrm{m}^{2}$ is required to disable liquid-propellant missiles and $240 \mathrm{MJ} / \mathrm{m}^{2}$ for solid-propellant missiles. These values include an estimate of the reflectivity of the missile and an average angle of incidence.

Another major difference between an intercept by the Airborne Laser and a kill vehicle is the transit time for delivering the blow. For the ABL, the propagation of the light takes only a few milliseconds, while a kill-vehicle interceptor may require several minutes from launch to impact. This time delay has important consequences in the choice of time at which an intercept by a kill vehicle may be attempted. In particular, the intercept is almost invariably most favorable at the latest possible intercept point because this gives the interceptor the most time to fly out. In contrast, the Airborne Laser can deliver its energy starting early in the missile's flight, considerably expanding the options for its location.

\subsection{ABL Engagement Geometry}

A major constraint on an ABL engagement comes from turbulence-induced inhomogeneities in the atmosphere, which cause small variations in the index of refraction. Over the very long propagation distances needed, even very small variations will prevent the beam being focused onto a target unless some form of correction is applied. Adaptive optics (AO) are used to sample the wavefront returning from an image on target and pre-distort the laser beam to undo the distortion of the atmosphere during the return trip. The ABL uses a sophisticated AO system for this purpose. The use of AO and its limitations are discussed in Chapter 19.

Obviously, the less air through which the beam must pass, the less effect its inhomogeneities will have on the focus of the beam. Since the ability of the AO to correct the laser beam is limited, it is advantageous for the aircraft carrying the ABL to fly at the highest possible altitude. The practical limit is approximately $12 \mathrm{~km}$, the altitude for which the ABL is designed. Similarly, the intercept should be attempted when the missile is at a high enough altitude to achieve good optical performance and maximum intercept range, i.e., above about $60 \mathrm{~km}$. See Chapter 21, in particular Section 21.5, for more details.

\subsection{ABL Performance Parameters}

We examined the performance of the ABL against two illustrative model missiles, the liquidpropellant missile ICBM L and the solid-propellant missile ICBM S2. Various parameters play a key role in determining the performance of the ABL. Among them are the beam power, the missile hardness, and the laser dwell time. In addition, because of the interplay between atmospheric turbulence and $\mathrm{AO}$ performance, the maximum effective range of the ABL depends on the altitude of the target. Missile characteristics, such as length, speed, and acceleration, also enter into the calculation, as discussed in Section 21.3.2. The maximum range varies with engagement altitude: it first increases with increasing altitude, then plateaus, for both classes of missiles. We use the plateau for the engagements discussed.

In summary, the following parameters have been used to characterize the ABL's performance. More information on these parameters is presented in the sections of this study given in parentheses.

- Beam power $=3 \mathrm{MW}(20.1,21.2)$.

- Engagement or laser dwell time $t_{e}=5$ or $20 \mathrm{~s}$; both scenarios are considered (21.5). 
- Atmospheric turbulence assumed for the ABL design $(19.3,19.7)$.

- AO performance $(19.4,19.5)$.

- Target hardness $F_{c}=32 \mathrm{MJ} / \mathrm{m}^{2}$ for ICBM L and $240 \mathrm{MJ} / \mathrm{m}^{2}$ for ICBM S2 (20.1).

- Optimum target altitude $h_{t}$ for engagement $\geq 60 \mathrm{~km}$. This altitude is reached by the threat missile much earlier than is useful for KV intercepts (21.5).

- Resulting maximum useful range $\approx 600 \mathrm{~km}$ for ICBM L and $\approx 300 \mathrm{~km}$ for ICBM S2 (21.5).

N.B. Because the exact laser power and the target hardness values are classified, we have had to make estimates based on the best-available public information. The engagement time is also classified. The 5-s dwell time is consistent with public literature on the Theater Ballistic Missile Defense, for which the ABL is designed; the $20 \mathrm{~s}$ time is our estimate of a maximum useful time. 



\title{
Chapter 8
}

\section{Defending the United States Using the Airborne Laser}

\author{
Contents \\ 8.1 Geometrical Considerations . . . . . . . . . . . . S136 \\ 8.2 Determining the Flying Area . . . . . . . . . . . S137 \\ 8.3 Defending Against Missiles Launched from North Korea . $\quad$ S137 \\ 8.4 Defending Against Missiles Launched from Iraq . . . . . . . S138 \\ 8.5 Defending Against Missiles Launched from Iran . . . . . . S S140

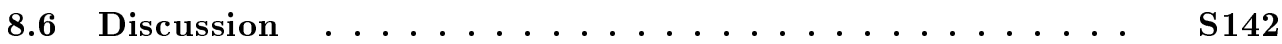 \\ 8.7 Controlling Shortfall _................ S143
}

The analysis of ABL boost-phase ICBM engagements shares a number of points in common with the analysis of kill-vehicle engagements described in Chapters 4 and 5, but there are also major differences. Points in common include the assumptions about missiles, trajectories, ICBM burnout or shutdown time, the times at which a missile is first detected and a firing solution is obtained, ${ }^{1}$ and geographic considerations.

However, in the case of a kill-vehicle engagement, the basing area is limited by the distance the interceptor can travel before the missile releases its warheads or submunitions. Interceptor speed and the decision time are critical factors. For the ABL, speed is not an issue, and the decision times available are relatively long; for an ABL, the critical parameter is range. Thus the ABL's flying area - the area within which the ABL must be stationed to disable the missile before its munitions or debris can reach the United States - is fundamentally different from a kill-vehicle's basing area.

The ABL can be fired any time from early in the ICBM's flight until shortly before the last safe intercept time. As summarized in Section 21.5.1, for a liquid-propellant ICBM the earliest time for a useful ABL engagement is $92 \mathrm{~s}$, although a firing solution could be available as early as $65 \mathrm{~s}$ after launch. For the solid-propellant ICBM, the firing solution is available at $45 \mathrm{~s}$ after launch, while the earliest firing time of interest is $82 \mathrm{~s}$.

Range is the dominating factor in an ABL engagement. If the ABL is within range, it can engage the ICBM at will. In this section we describe how to calculate the laser ground range (LGR) and how to use these results to determine the ABL flying area. In the next sections these results are used to analyze engagements with North Korea, Iraq, and Iran.

\footnotetext{
${ }^{1}$ In principle, the ABL's detection and tracking capabilities could provide earlier firing solutions. However, this would provide no important advantage, because the first useful firing time for the ABL occurs well-after a firing solution is available.
} 


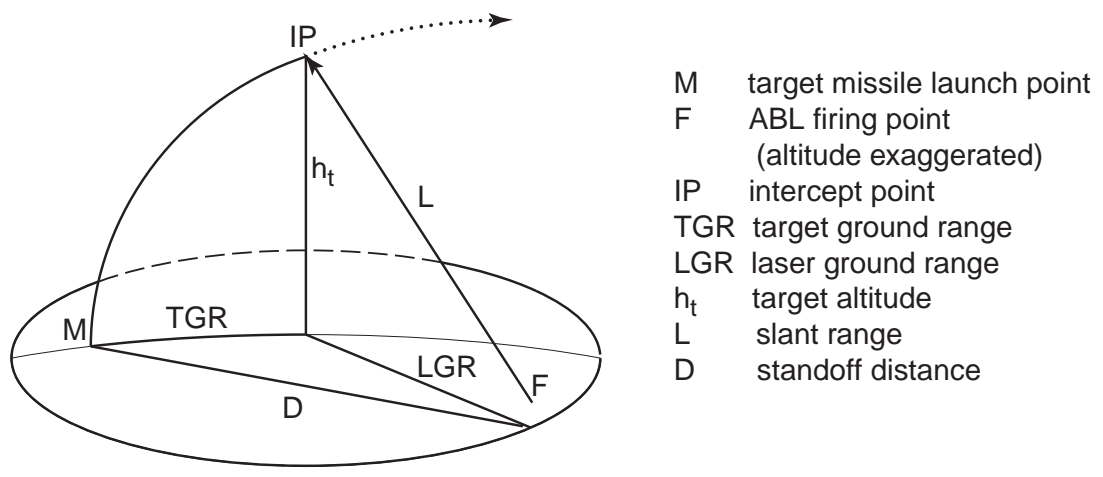

Figure 8.1. Geometry of an ABL intercept.

As explained above, the choice of threat missiles and the times for detecting a rocket launch and generating a firing solution are identical to those presented in Chapter 5 . The LGRs that are employed in this chapter are determined by the laser's performance (Chapter 18), beam propagation (Chapter 19), and the energy requirements for disabling a missile (Chapter 20). The process by which these results are integrated to determine the ground ranges is described in Chapter 21.

\subsection{Geometrical Considerations}

Geometry of an ABL intercept The geometrical quantities involved in an ABL engagement are illustrated in Fig. 8.1, which bears many similarities to the diagram for a kill-vehicle intercept (see Fig. 5.4). However, the kill-vehicle diagram is essentially static; the intercept point generally occurs shortly before the missile's burnout. For the ABL, the intercept can take place anywhere along the trajectory from early in its flight until the last safe intercept. Thus, the diagram evolves in time. Furthermore, the missile can move significantly while the ABL is firing.

For a kill-vehicle interceptor, the optimal time to intercept is generally the last possible moment before missile burnout (see Section 4.2). In such an intercept, target ground range (TGR) is the ground range at shutdown and the interceptor ground range (IGR) is the ground range of the interceptor in the flyout time available. The analog of a kill-vehicle interceptor ground range (IGR) is the ABL ground range, LGR, which varies throughout the engagement. As described in Section 21.5.2, for both liquid- and solid-propellant missiles the LGR first increases as the target ascends to higher altitude and then stays approximately constant throughout most of the time of interest.

ABL Ground range The first step in analyzing an ABL engagement is to choose a sequence of points along the trajectory of interest and determine LGR at each of these points. The range depends on the engagement altitude because of effects of atmospheric propagation that were previously discussed and also for reasons of geometry. ${ }^{2}$ Figures 21.12 (a) and (b)

\footnotetext{
${ }^{2}$ An important distinction must be made between the slant range $L$ from the ABL to the target that was used in the discussion of propagation and ground range of the ABL $L G R=\sqrt{L^{2}-h_{t}^{2}}$, where $h_{t}^{2}$ is the altitude. A high-altitude attack will use up some of the slant range, reducing the ground range available. There is a limit on the altitude angle to which the ABL may be directed, but an actual number is not available in the unclassified literature.
} 
show the maximum ABL range in planar geometry of an ABL engaging a liquid-propellant missile and a solid-propellant missile (ICBM S2) respectively. It displays altitude vs. range of the missiles, with arrows to the missile from the ABL laser beam at its altitude of $12 \mathrm{~km}$. (Fig. 21.12 is analogous to the engagement figures for kill-vehicle intercepts presented in Section 4.5.) These values of the ABL range are illustrative only, because of the uncertainties discussed in Sections 20.1 and 21.2.

For a liquid-propellant ICBM, the largest range is $L G R \approx 600 \mathrm{~km}$, which occurs for altitude $h_{t} \geq 90 \mathrm{~km}$. For a solid-propellant ICBM, the range for engaging is considerably more limited, because of the greater hardness of the booster. The maximum $L G R \approx 300 \mathrm{~km}$ occurs for $h_{t} \geq 105 \mathrm{~km}$. These values of the ABL range depend on the assumptions about the ABL performance that are described in Section 7.3.

\subsection{Determining the Flying Area}

An ABL engagement can occur from shortly after missile launch to missile burnout/shutdown. Consequently, the area within which the ABL must fly to engage the missile successfully is bounded by the LGR at each point along the trajectory. This area is called the ABL flying area. The procedure for calculating the ABL flying area for engaging a missile flying out on a given azimuth involves the following steps:

- The earliest times for starting the engagement are taken from the analysis in Section 21.5. The results are summarized in Fig. 21.9.

- The latest time for completing each engagement is taken from the analysis presented in Sections 5.4 to 5.6.

- A series of points at different times along the trajectory, from launch to burnout or shutdown, is selected. At each of these points, the altitude $\left(h_{t}\right)$ and ICBM ground range (TGR) are found from the trajectory of the missile.

- For each point, the maximum slant ranges $L_{\max }$ for engagements of $5 \mathrm{~s}$ and $20 \mathrm{~s}$, are found from the analysis in Section 21.5. The results are summarized in Figs. 21.8 and 21.7 , respectively.

- The laser ground range is found at each point using $L G R=\sqrt{L_{\max }^{2}-h_{t}^{2}}$.

- At each point, a flying circle having radius LGR is drawn.

- The boundary of the flying area is then generated by drawing a smooth curve tangential to the flying circles.

Flying areas generated by these steps are displayed in the next sections.

\subsection{Defending Against Missiles Launched from North Korea}

This discussion of ABL defense against ICBMs launched by North Korea is based on the threats described in Section 5.4. Flying areas will be displayed for defense against missiles on the most stressing trajectory from North Korea, the Boston trajectory to the East Coast. It is close to a full-range trajectory with shutdown or burnout at $239 \mathrm{~s}$ for ICBM L and $167 \mathrm{~s}$ for ICBM S2. For a kill-vehicle interceptor, the trajectory to Alaska is also stressing 
because of the short burn time. The ABL defense of this trajectory is also considered. The shutdown or burnout time is $232 \mathrm{~s}$ for ICBM L and $142 \mathrm{~s}$ for ICBM S2. Defense of Hawaii will be treated separately.

Flying area construction As the missile ascends the LGR grows. The basing area expands in all directions and moves out along the ground line of the trajectory. Because of the combination of these effects, for the liquid-propellant missile the initial boundary of the flying area is determined by the missile's position at the $130 \mathrm{~s}$ (after launch) for an engagement of $5 \mathrm{~s}$ duration, and $145 \mathrm{~s}$ for an engagement of $20 \mathrm{~s}$ duration. For the solid-propellant missile and a 20-second engagement, the corresponding times are $82 \mathrm{~s}$ for earliest start of engagement and $102 \mathrm{~s}$ for the boundary. Engagements of $5 \mathrm{~s}$ are too short to be useful against solid-propellant missiles.

The engagement must be completed before the ICBM reaches the last safe intercept time. Because its speed is greater then, the ICBM can travel a significant distance while the ABL is being fired. During a 20-second engagement, the missile travels more than $100 \mathrm{~km}$. Whether the limit of the flying area is set by the beginning or end of the engagement depends on whether the missile is moving toward or away from the ABL. For missiles from North Korea, the ABL attack is likely to be transverse, in which case the boundary is essentially stationary.

Figure 8.2 displays the ABL flying areas for liquid- and solid-propellant missiles launched from North Korea for 5- and 20-second engagements.

Liquid-propellant missile For the 5-second engagement time, the ABL flying area extends about $100 \mathrm{~km}$ into the Yellow Sea and the Sea of Japan.

For the 20-second engagement time, the ABL could be stationed several hundred kilometers from the coast, either over the Yellow Sea or the Sea of Japan. The latter is much more favorable, because the laser beam would have a more favorable angle of incidence on the missile.

Defense of the Alaska trajectory, Fig. 8.2, upper right, is much less constrained than the Boston trajectory. For the 5-second engagement, the ABL could be stationed away from the coast by almost $200 \mathrm{~km}$. For the 20 -second engagement, more than $300 \mathrm{~km}$ of sea room would be available.

Solid-propellant missile Defense against the solid-propellant missile by the ABL does not seem possible for the Boston trajectory, even for a 20-second engagement. For the Alaska trajectory, the ABL would be forced to fly within $100 \mathrm{~km}$ of the North Korean coast.

Defense of Hawaii The Hawaii trajectory passes almost due east over the Sea of Japan, which makes the missile a very favorable target for boost-phase intercept. An ABL aircraft stationed over the Sea of Japan could therefore defend Hawaii against solid-propellant as well as liquid-propellant missiles.

\subsection{Defending Against Missiles Launched from Iraq}

This discussion of ABL defense against ICBMs launched by Iraq is based on the threats described in Section 5.5. 

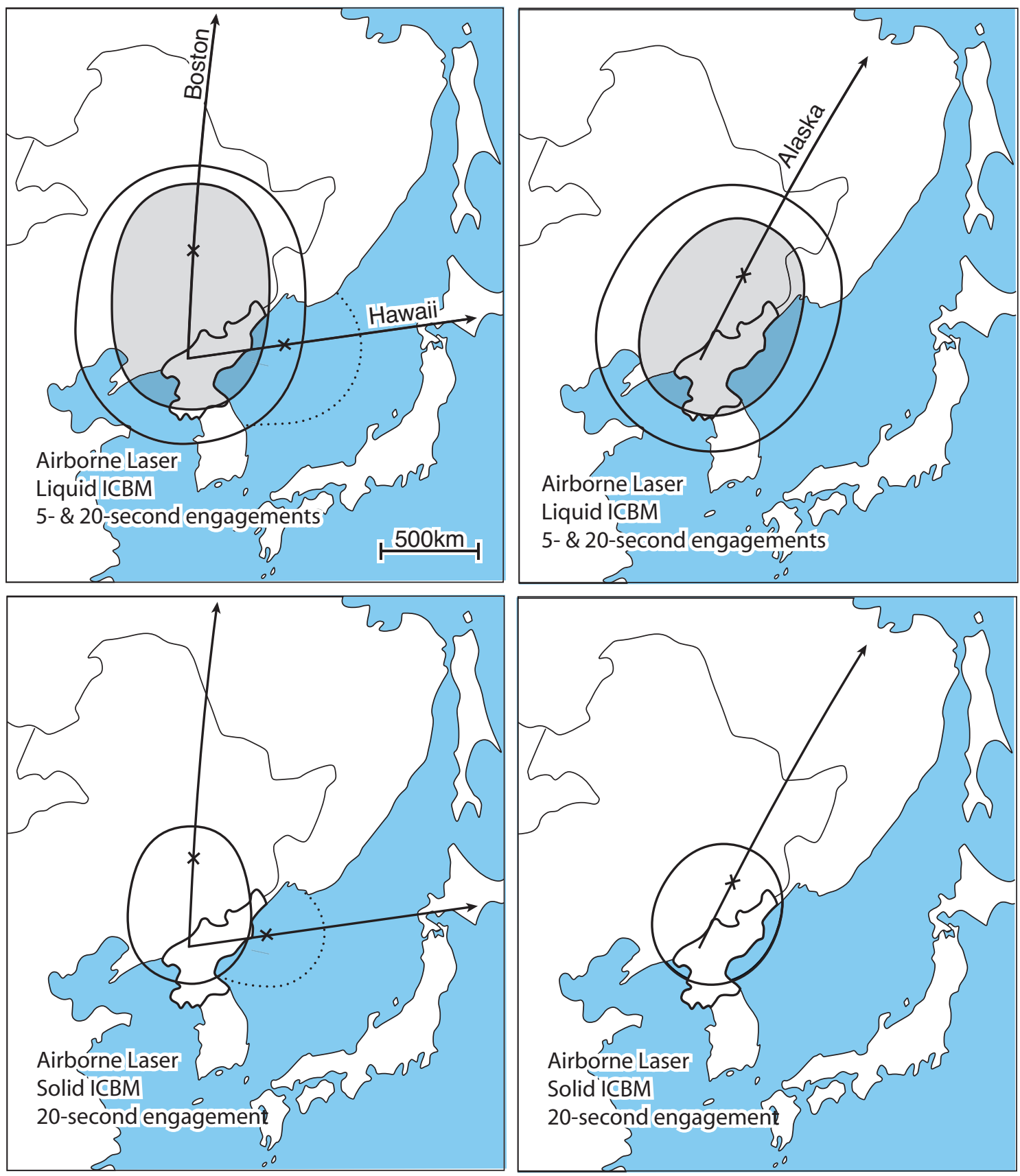

Figure 8.2. Illustrative flying areas of the ABL for the assumed performance capability: defense against missiles launched from North Korea to the United States. (Geographic areas are identified in Fig. 5.7.) The position of the missile at the last chance it can be intercepted successfully is indicated by the " $\mathrm{x}$ " on the trajectory line. The shaded region is the flying area for a 5 -second engagement; the outer curve is the boundary for a 20-second engagement. (left) Trajectory to the East Coast (Boston); (right) Trajectory toward Alaska. (top) Defense against liquid-propellant missile ICBM L. (bottom) Defense against the solid-propellant missile ICBM S2 (Only 20-second engagements are shown.) The dotted lines delimit the flying areas for defense of Hawaii for a 5second engagement against the liquid-propellant missile and a 20-second engagement against the solid-propellant missile. 
Flying area construction For defense against missiles from Iraq, the ABL could be stationed to the south, over the Persian Gulf, or northwards, over Turkey. For ABL defense from the south, the northernmost boundary at launch is the most stressing. Conversely, for defense from the north, the southernmost boundary of the last intercept is the most stressing. Note that this boundary moves northward by more than $100 \mathrm{~km}$ during a 20 -second engagement.

Liquid-propellant missile The ABL flying areas for defense by one or two ABLs against the liquid-propellant ICBM L launched from Iraq are shown in Fig. 8.3, top. These maps display the flying areas for defense of the East and West Coasts of the United States, i.e., defense of the contiguous states, and also defense of Hawaii and Alaska, for 5- and 20-second engagements.

For the 5-second engagement (top, left), there is no flying area, over friendly countries or international waters, for which either one or two ABLs could cover the range of azimuths.

For the 20-second engagement (top right), the flying area is significantly larger. A single ABL stationed in Saudi Arabia within about $200 \mathrm{~km}$ of the border with Iraq could defend the entire United States. The flying area extends into the Persian Gulf, but an ABL flying from the Gulf would be oriented toward the tail of the missile as it sped away, making an ABL defense from that point unfavorable. The flying area also extends into Turkey, though by less than $100 \mathrm{~km}$. The use of two ABLs would not significantly improve the possibilities for defense of the entire United States.

Solid-propellant missile Defense against the solid-propellant ICBM S2 launched from Iraq is illustrated in Fig. 8.4, left. There is no possibility for an ABL defense, even for the 20second engagement.

\subsection{Defending Against Missiles Launched from Iran}

This discussion of ABL defense against ICBMs launched by Iran is based on the threats described in Section 5.6. The range data for defense from Iran are the same as those for Iraq presented in the previous section.

Liquid-propellant missile For defense against the liquid-propellant ICBM L launched from Iran, Fig. 8.3, bottom, shows flying areas for defense of the contiguous states, and also Hawaii and Alaska, for 5- and 20-second engagements.

For a single ABL and a 5-second engagement, defense of the contiguous states might be possible, but the ABL but would have to be stationed within $200 \mathrm{~km}$ of the border of Iran, over the Caspian Sea or Turkmenistan. Two ABLs, one over the Caspian Sea, and one over Turkmenistan, within $200 \mathrm{~km}$ of the border, would be required to defend the entire United States.

For a 20-second engagement, the entire United States could be defended by a single ABL over Turkmenistan, though the flying room would be constrained to less than $200 \mathrm{~km}$. Employing two ABLs, one over the Caspian Sea and one over Turkmenistan, would provide a somewhat more robust defense, approximately $250 \mathrm{~km}$ from the Iranian border.

It should be noted that these defense possibilities could be diminished by moving the launching point further south, although such a deployment could make the ICBMs vulnerable to a kill-vehicle boost-phase intercept from the Persian Gulf (see Fig. 5.16). 

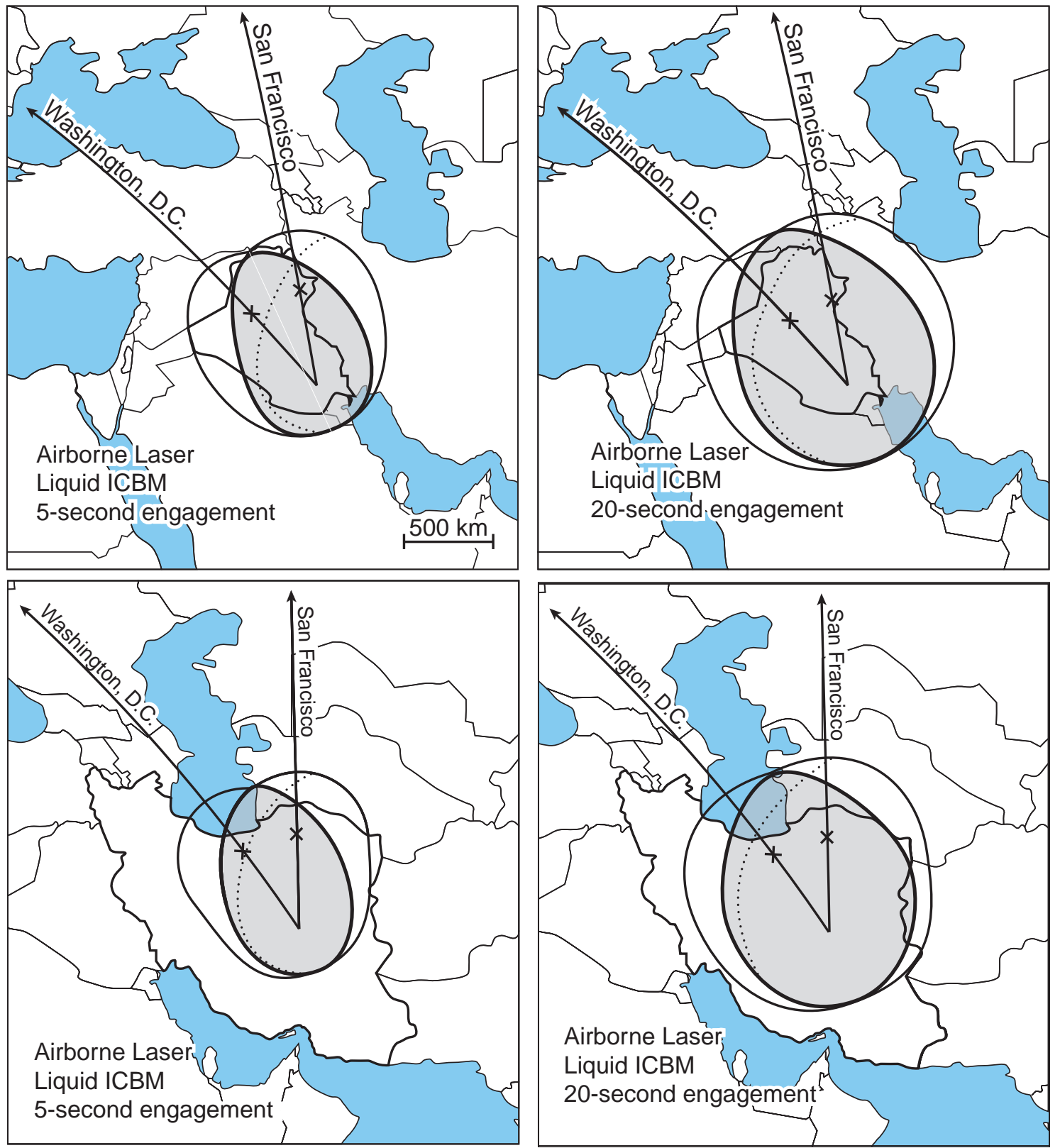

Figure 8.3. Illustrative flying areas of the ABL for the assumed performance capability: defense against liquid-propellant ICBMs launched from Iraq and Iran to the East and West Coasts of the United States. The dotted line indicates the boundary to the flying area for defense of Alaska and Hawaii, which are similar. (Geographic areas are identified in Fig. 5.12.) The position of the missile at the last chance it can be intercepted successfully is indicated by the " $\mathrm{x}$ " on the trajectory line. Iraq (top); Iran (bottom). Engagements of 5 seconds are shown on the left; 20-second engagements are on the right. Defense by a single ABL requires that it fly within the shaded area. Defense by two ABLs would require that one be in each of the oval shaped areas. 


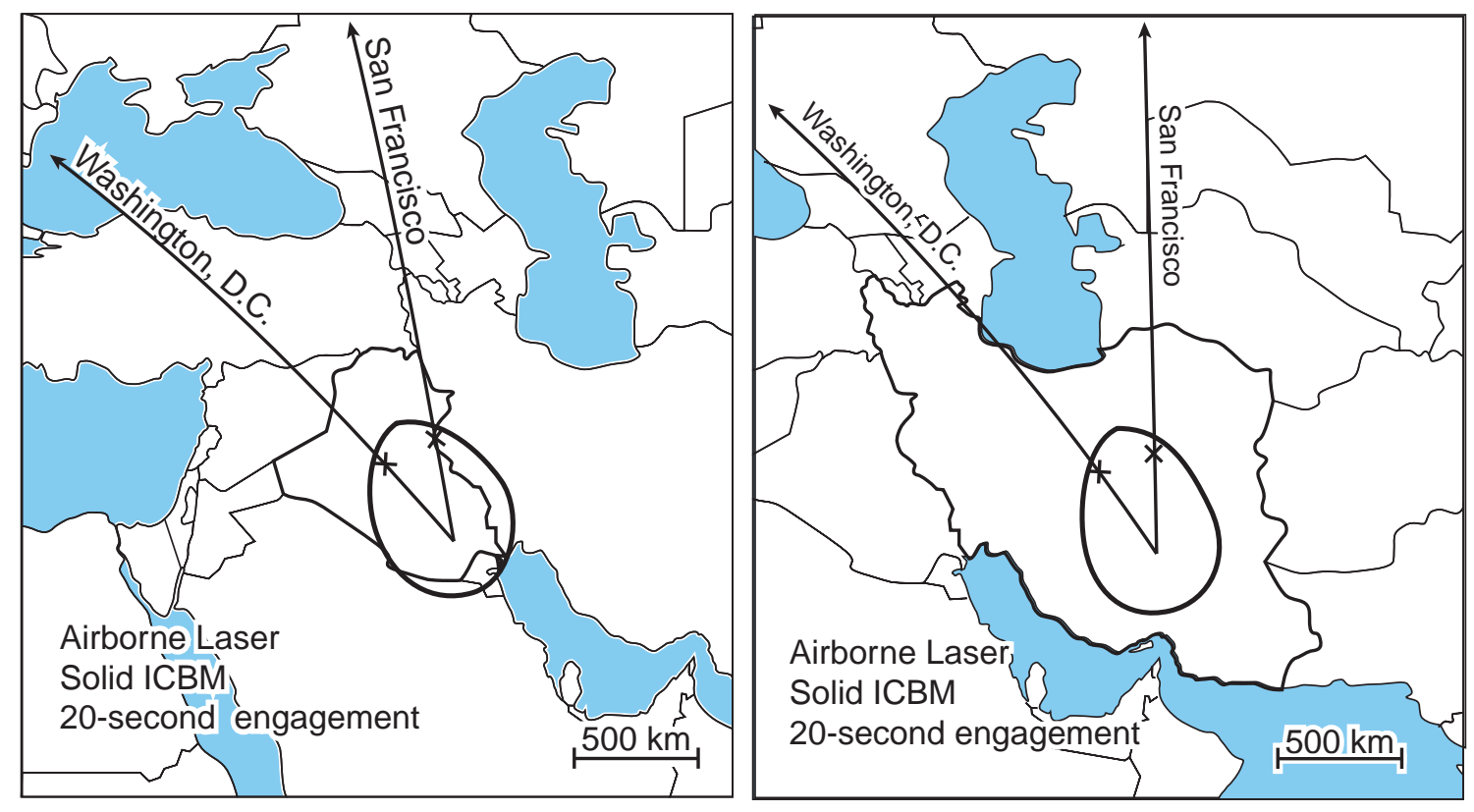

Figure 8.4. Illustrative flying areas of the ABL for the assumed performance capability: defense against a solid-propellant missile (ICBM S2) launched from Iraq (left) and Iran (right), to the East and West Coasts of the United States. The boundary for the Alaska and Hawaii trajectories is not illustrated. The " $\mathrm{x}$ " indicates the point where the boost-phase terminates.

Considering the somewhat constrained flying areas for defense against the liquid-propellant ICBM L from Iran, the probable need to employ ABLs in two unconventional locationsthe Caspian Sea and Turkmenistan - and the vulnerability of the defense to a shift of the launching area, defense against ICBM L launched from Iran should be regarded as problematical.

Solid-propellant missile The flying area for defense against a solid-propellant ICBM S2 launched from Iran is shown in Fig. 8.4, right. The flying area does not extend beyond Iran, precluding an ABL-based defense.

\subsection{Discussion}

The power, beam quality, and tracking capabilities of the ABL are crucial parameters for determining the ABL's effectiveness. Because the values assumed are based on estimates from only publicly available information, the analysis carries large uncertainties.

If the ABL achieves its postulated performance, it would be capable of defending the entire United States from liquid-propellant ICBM L launched by North Korea, assuming that the ABL could be deployed about $300 \mathrm{~km}$ from the border. For such a liquid-propellant missile launched by Iraq, defense of the entire United States is not possible, and partial defense would require basing the ABL over Saudi Arabia. For Iran, partial or full defense against the liquid-propellant ICBM L is problematical because of the tight constraints on the flying area and the need to station the ABL over the Caspian Sea and Turkmenistan.

Defense by the ABL against solid-propellant missiles from North Korea, Iraq, or Iran does not seem to be possible. 


\subsection{Controlling Shortfall}

To prevent a warhead or debris from an intercept from falling on populated areas of China, Russia, Europe, or the North American continent, the missile would have to be disabled within narrow time windows during the boost phase of the ICBM. The timing and width of these windows depend on the missile's launch site, type, and target. As shown in Chapter 5 (Fig. 5.3 [North Korea]) and [Iraq]) and Section 5.8, these windows are small. To prevent shortfall on Western Europe by a missile launched from the Middle East, the window is about $10 \mathrm{~s}$ for a liquid-propellant missile and $20 \mathrm{~s}$ for a solid-propellant missile. Launches from North Korea are a little more complex because of the trajectories. The window could be as small as $7 \mathrm{~s}$ for a liquid-propellant missile having Dallas as the target. Other trajectories are less stressful but still serious - $17 \mathrm{~s}$ to $39 \mathrm{~s}$. A San Francisco target is an exception, in which the ground track is over the Pacific Ocean.

While the ABL does not have a kill-vehicle's long travel times, it has other drawbacks with respect to controlling shortffall. These include uncertainties in the missile's hardness which cause the imprecision in when the missile can be disabled during an engagement which can last up to $20 \mathrm{~s}$. Consequently, timing the intercept to control shortfall would be difficult.

A further complication arises if several missiles are launched at once, because ABL engagements must be sequential. Even if an ABL could intercept one missile within the window, it is unlikely that a second missile could be intercepted in the window. Multiple ABLs could be deployed to defend against a moderate number of multiple launches, but we have not studied this scenario. 



\section{Chapter 9}

\section{Countermeasures to Boost-Phase Intercept}

\section{Contents}

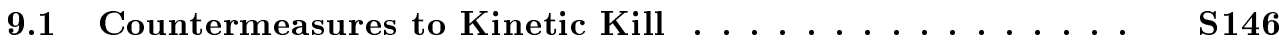

9.1.1 Solid-propellant threat ICBMs _... . . . . . . . . . . S146

9.1.2 Deploying payload during second- or third-stage boost . . . . S146

9.1 .3 Spoofing . . . . . . . . . . . . . . . . . . S147

9.1.4 Deliberate trajectory variations by the offense . . . . . . . . S148

9.1.5 Short burn boosters with multiple upper stages . . . . . . . . S148

9.1.6 Multiple missile launches . . . . . . . . . . . . . . . . . . S149

9.1.7 Masking the kill-vehicle aim point . . . . . . . . . . S S149

9.1.8 Attacking the interceptor system . . . . . . . . . . S150

9.1.9 Summary of kill-vehicle countermeasures . . . . . . . . . . S150

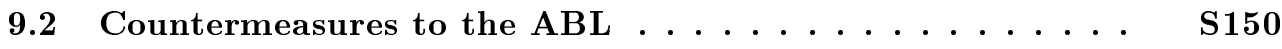

9.2.1 Vulnerability of airframe . . . . . . . . . . . S150

9.2 .2 Spreading the laser heat . . . . . . . . . . . . . . S151

9.2 .3 Ablative coating . . . . . . . . . . . . . . . . S151

9.2 .4 Optical properties . . . . . . . . . . . . . S152

9.2.5 Multiple missile launches . . . . . . . . . . . . . S152

9.2.6 Launch timing and cloud cover . . . . . . . . . . . . S152

In the debate about missile defense, the essential nature of military conflict-a series of moves that inevitably generate countermoves - is sometimes forgotten. Any missile defense system will necessarily lead to the creation of countermeasures that will in turn stimulate defense solutions to defeat them. In this continuum of move and countermove, the time to develop the response is a crucial factor. If an effective response to a vulnerability cannot be developed before the vulnerability is exploited, the ingenuity or sophistication of the response is irrelevant. There is no sound basis for assuming that either offense or defense has a time advantage in responding to the actions of the other. Discussions of countermeasures can become unrealistic if this constraint is not kept in mind.

We begin this discussion by treating countermeasures to intercepts by kill vehicles. Later we turn to countermeasures to intercepts by the ABL. 


\subsection{Countermeasures to Kinetic Kill}

Proposals for boost-phase defense against ICBMs are in large part motivated by the claim that it is not vulnerable to the countermeasures that are alleged to work against midcourse and terminal ballistic missile defense systems. Although this claim is essentially correct, there are nevertheless powerful countermeasures against boost-phase defense. The debates about midcourse countermeasures have sometimes become mired in arguments about what is feasible and what is not, particularly with respect to the question of discriminating between decoys and real targets. We attempt to avoid this kind of debate by considering only techniques that have actually been employed in operational systems over the last 40 years.

Missile defense schemes require the defender to implement a robust system that will not be obsolete before it is deployed. The goal is an architecture that is not brittle and fragile to the appearance of likely countermeasures. With that goal in mind, the following discussion presents some countermeasures that the Study Group believes deserve to be considered seriously and discusses their implications with respect to the implementation of a boost-phase intercept defense system. These countermeasures fall into three categories:

1. Compressing the available engagement time.

2. Creating multiple targets, real or false, that must be dealt with.

3. Exhausting the maneuver capability of the interceptor before it achieves a hit.

\subsubsection{Solid-propellant threat ICBMs}

As this report illustrates, the most effective and immediate countermeasure to boost-phase intercept system and one that challenges almost every aspect of such a defense is to reduce the time available to intercept the missile. As discussed in Chapter 3, solid-propellant technology is becoming widely available for purchase. Within the next $10-15$ years, potential adversaries may be expected to possess solid-propellant ICBMs that utilize at least the equivalent of late-1960s U.S. technology and have total burn times of 170-180 s, in contrast to the 260 to $300 \mathrm{~s}$ burn times of missiles based on the earliest liquid-propellant technology. The engagement analysis of Chapter 5 showed the effect of solid-propellant threats on the intercept requirements: the interceptor flyout velocities are nearly doubled, to orbital velocity or greater. Even faster solid-propellant missiles with even shorter boost phases are a feasible response to the deployment of a boost-phase defense system and would compress further the time available to complete the engagement.

Section 15.1 discusses solid-propellant ICBM S3 (see Table 15.1), a "fast-burn" variant of solid-propellant ICBM S1 that has first and second stages with burn times of $50 \mathrm{~s}$ each and a third stage with a burn time of $30 \mathrm{~s}$, for a maximum total boost time of $130 \mathrm{~s}$. Like the other ICBMs we modeled, the range of this fast-burn missile is $12,000 \mathrm{~km}$. Section 9.1.5 later in this chapter discusses a fast-burn solid-propellant ICBM like model S3 but with multiple third stages that would burn out $130 \mathrm{~s}$ after launch. A boost-phase defense against fast-burn ICBMs like either of these missiles would be practically impossible.

\subsubsection{Deploying payload during second- or third-stage boost}

A variety of payload deployment methods have been used on ICBMs over the years. One that was used on the Polaris A3 and the Russian SS-9 was deployment "on the fly" - 
while the booster was still accelerating. This deployment was used with multiple re-entry vehicles (RVs) to avoid the need for thrust termination and to create a separated RV impact pattern. While energy-wasting maneuvers, such as generalized energy-management steering (GEMS), may be used to reduce range, with "on-the-fly deployment" it is not necessary to do so. Therefore, the defense cannot count on booster burnout as an indicator of payload deployment. The implication is that the booster must be intercepted before its velocity yields a trajectory for the first RV that could strike the defended area.

\subsubsection{Spoofing}

Tracking sensors can be spoofed (i.e., fooled) using flares and jammers. During an interceptor's boost-phase, guidance information is provided by tracking data from off-board sources, including radar and space-based tracking systems.

Radar jamming denies range information. Because the boosting target is detectable to radar as soon as it rises above the horizon, a logical countermeasure would be to include in the final stage of the missile one or more barrage noise jammers. These devices would deny range information to the radar, forcing it to track in angle only and to rely on triangulation from another sensor to determine range for midcourse guidance. The electrical power required for radar jamming is analyzed in Section 10.2.8. The power, $10 \mathrm{~W}$, adds no significant burden to the missile launch, and more powerful jammers could be employed if required.

Disruption of the tracking data for any significant period, particularly if the target is maneuvering, results in increased acceleration and velocity requirements for the interceptor to correct its trajectory. Whether the kill vehicle is close enough to acquire the target with its suite of on-board sensors in the homing phase or is dependant on external tracking data earlier, the result would be significantly increased fuel usage. The disruption of tracking is particularly critical near the endgame, when it could cause the kill vehicle to exhaust its fuel supply or exceed its acceleration capability, thereby missing the target. The effects of tracking uncertainty and latency are discussed in detail in Chapter 12. The countermeasure described below employs thrusted decoys carrying radar jammers that would be deployed in a maneuver designed to obscure what the real target is doing, as shown in Fig. 9.1.

This boost-phase countermeasure employs thrusted, spin-stabilized, 20-kg decoys that are fired from rifled mortar tubes canted out perhaps $15^{\circ}$ from the missile's second- or thirdstage centerline and are mounted around the base of the warhead. They could be deployed simultaneously any time after the shroud was cast off. Immediately after the decoys were ejected, the missile would execute a dog-leg maneuver. Each decoy would contain:

- A low-power jammer having a thermal battery and a corner cube to reflect LIDAR energy, together weighing about $5 \mathrm{~kg}$.

- A small rocket motor to accelerate the decoy to match the acceleration of the booster for $20 \mathrm{~s}$, weighing about $10 \mathrm{~kg}$.

- A flare or plume-enhancement injectant formulated to mimic the optical signature of the booster rocket plume, weighing about $2 \mathrm{~kg}$.

At the same time that the decoys are deployed, the booster activates a rotating corner cube and jammer that are identical to those on the decoys. 


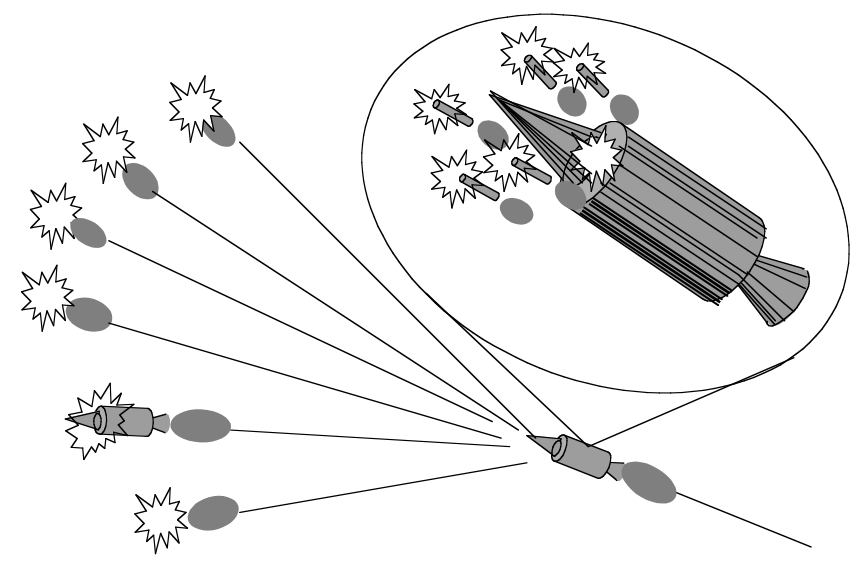

Figure 9.1. Illustration of concept of ejected decoys and jammers. These devices are deployed in conjunction with a dog-leg maneuver.

The launch tube, jammer and rotating corner cube are estimated to weigh a total of $20 \mathrm{~kg}$. Five such devices deployed on a missile having a 1000-kg payload, causes only an 11 percent loss in the useful payload. The principle of this countermeasure is to obfuscate the initiation of a significant maneuver by the deployment of multiple targets at a time when a delay in recognizing the change in booster acceleration could cause the interceptor to exceed its remaining acceleration and velocity-adding capability. This so-called "hard" deployment of decoy packages during boost has been successfully used by the United States since the $1960 \mathrm{~s}$.

\subsubsection{Deliberate trajectory variations by the offense}

With their advance knowledge of the intended target, the offense could program trajectory variations that would require the interceptor to consume additional fuel, limiting its effective range. These maneuvers might be trajectory-shaping maneuvers that are not specifically for evasion but for energy-management, as described in Chapter 15. They could also be specific maneuvers carried out at times that would be most likely to disrupt the interceptor guidance.

Examples of trajectory shaping are illustrated in Fig. 4.4, which shows how much variation can occur when the maneuvers are initiated when the shroud is ejected. These preplanned maneuvers can be employed in conjunction with the deployment of decoys. Energywasting maneuvers can be dramatic, as illustrated in Fig. 15.7. In extreme cases, the final stage may actually thrust back toward the launch point. Finally, cyclical maneuvers designed to disrupt the intercept can be used as discussed in Chapter 12 .

\subsubsection{Short burn boosters with multiple upper stages}

Figure 9.2 shows a countermeasure concept that employs multiple guided third stages. This deployment method could be an adjunct to other countermeasures, such as the shortburn booster. In the first- and second-stage solid-propellant missile, ICBM burn times 


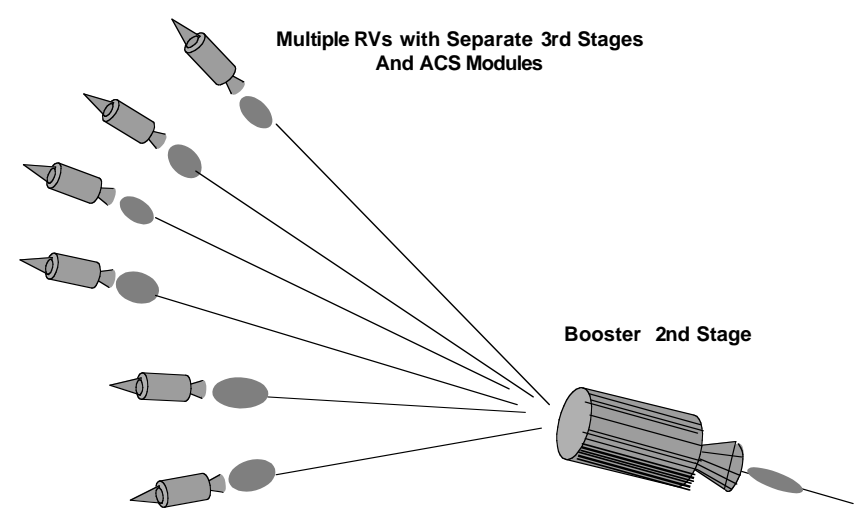

Figure 9.2. Illustration of concept of multiple third stages.

are reduced from $60-65 \mathrm{~s}$ each to $50 \mathrm{~s}$ each. When the second stage burns out at $100 \mathrm{~s}$, multiple RVs, each with its own guidance, attitude control and third-stage motor, are separated from the second stage simultaneously. Each can fly on its own guided trajectory toward widely separated targets. This early fractionation reduces the total available boostphase engagement time to less than $70 \mathrm{~s}$, which would make any single intercept virtually impossible. The defense would be forced to fire multiple interceptors before it knows if there are multiple upper stages.

The penalty to the offense for this approach is the requirement for multiple sets of guidance and control equipment and the volumetric inefficiency of multiple upper stages. Nevertheless, this approach is more efficient than the serial-deployment post-boost-vehicle approach currently used on multiple independently targeted re-entry vehicle (MIRV) systems. This type of multiple payload delivery was studied when those systems were devised in the mid-1960s. However, the mass and volume penalty for multiple guidance systems is minimal today, compared with the guidance cost and size constraints when those tradeoffs were first evaluated. It is likely that if non-nuclear boost-phase defense had been of concern, the parallel approach would have been taken even then. However, nuclear countermeasures were a major concern for boost-phase defense at the time, and the solution required radiation hardening of the missiles.

\subsubsection{Multiple missile launches}

If the attacker launched several missiles nearly simultaneously, it would complicate the problem for the boost phase-defense, especially for a space-based defense where only one or two SBIs are within range of the threat trajectories. This problem would be exacerbated if some of the launches were theater or tactical ballistic missiles (TBMs) used as decoys.

\subsubsection{Masking the kill-vehicle aim point}

If the goal of the intercept is to destroy the warhead (rather than to merely disable the booster), then a number of strategies can be employed to disguise its location. This will be discussed in Chapter 13. 


\subsubsection{Attacking the interceptor system}

A classic countermeasure to defense systems is "defense suppression", a physical attack to destroy the interceptor itself or to temporarily disable its detection and guidance sensors. The former category could include attacks by air or cruise missiles (e.g., widely available Silkworm-type missiles), attacks on land- or sea-based interceptor ships, and surface-to-air missiles (SAM) attacks on airborne interceptor platforms. Boost-phase defense, however based, is more at risk than other systems, because it must initiate its engagement no farther from the point of intercept than the interceptor can cover within 100-120 s.

\subsubsection{Summary of kill-vehicle countermeasures}

We have identified a number of countermeasures to boost-phase intercept of ICBM missiles. For the most part, they could employ actual techniques that were imbedded in U.S. and Russian systems in the 1960s and are in tactical countermeasure systems today. The most potent countermeasure would be a faster burning rocket. It is clear that the growing availability of solid-propellant rockets will make the job of a boost-phase intercept defense extremely challenging, or unfeasible.

\subsection{Countermeasures to the $\mathrm{ABL}$}

The previous discussion of countermeasures dealt with kinetic-kill vehicles. The following material discusses countermeasures to the ABL.

Some countermeasures to a kill-vehicle missile defense, such as those to confuse the detection of the ICBM launch or to shorten the time allowed for decision to fire the defensive weapon, will do little to degrade the effectiveness of an ABL defense, because the transit time for the laser beam is so short. However, attacking the ABL itself could be quite effective. The basing platform will be particularly vulnerable to SAMs, which have a range of $250-300 \mathrm{~km}$ or to threat fighters. Section 9.2.1 discusses this vulnerability.

Since damage to a missile by a laser depends on the energy density absorbed, an adversary may adopt strategies to limit energy absorption. Such methods are discussed in Sections 9.2.2-9.2.4. Because ABL engagements are sequential, launching multiple threat missiles together would stress the ABL's effectiveness, as discussed in Section 9.2.5. Finally, Section 9.2.6 discusses the possibility of timing missile launches to coincide with high cloud cover to hinder the ABL.

\subsubsection{Vulnerability of airframe}

A major weakness of the ABL is its vulnerability to attack by enemy aircraft or by SAMs. Escort fighters could defend the ABL against enemy aircraft; however, the very long times on station would make such defense difficult, unless absolute air superiority had been established or some warning mechanism could scramble fighters to respond to an attack on the ABL.

Siberian Airlines Flight 1812 was accidentally shot down on 4 October 2001 by a S-200 SAM missile at a range of about $250 \mathrm{~km}$. The maximum range of a S-200 is $250-300 \mathrm{~km}$ [90]. Countering SAMs would be very difficult [91]. Such missiles rely on totally passive means to acquire and track targets. They present no signature before launch, and after launch exhibit only an infrared exhaust plume. An ABL cannot outmaneuver a SAM. 
It has been suggested that the ABL could defend itself against aircraft or SAMs using its laser(s). Aircraft approaching from a $100^{\circ}$ to $120^{\circ}$ region (Section 21.1) at the rear of the ABL or at altitude above some unknown limit could not be engaged by the lasers. Otherwise, the lasers could be used, either for direct damage to the attacking aircraft with the high-energy laser (HEL) or by blinding the pilot with the target-illuminator laser (TILL). Scenarios for the ABL's use of its HEL to engage a SAM have not been studied, but they would be different from ICBM engagements because of the geometry, particularly in the low target altitude. Such use would place a demand on the fuel magazine needed for ICBM intercepts. The mechanism by which the laser could damage the nose of an oncoming SAM is different from those for damaging the wall of a missile booster. Another possible response would be to attempt to disable the SAM's infrared sensors, if it uses them. Either the TILL or the beacon-illuminating laser (BILL) could certainly damage a focal plane array if no filters are used to block the IR beams. However, if the SAM is guided by ground-based radar, there would be no sensors to disable. The S-200 is guided by ground radar, and it may have an active seeker for the final intercept. Most of the flight is under commands from the ground or a semi active seeker with illumination only from ground radar [92].

In any case, launching SAMs in the same time frame as the $\operatorname{ICBM}(\mathrm{s})$ would reduce the ABL's ability to respond to the ICBM threat.

\subsubsection{Spreading the laser heat}

By rolling the ICBM body, the laser energy could be distributed over a wide area, thereby increasing the energy required for the laser to damage the missile [93, p. 27, 127]. The threat missile's ability to execute this roll rate maneuver is determined by the configuration of its inertial reference package. If the gyros and accelerometers reside on a three-gimbalstabilized platform, the missile can be rolled without difficulty until the pitch-over angle reaches about $45^{\circ}$ from its launch vertical, provided the proper roll transformations are employed. If the instruments are a modern "strapped down" or body-mounted design, or if a four-gimbal- or attitude-stabilized platform were used, the roll rate could be maintained with no pitch constraint. Within the above constraints, roll rates of $20^{\circ}$ to $30^{\circ}$ per second can be executed without affecting the flight of the missile. Since several seconds of energy deposition are normally required for damage, this roll rate reduces the effectiveness of a continuous wave $(\mathrm{cw})$ laser by at least the ratio of the diameter of the beam spot width to the length of the stripe around the missile generated by the roll.

\subsubsection{Ablative coating}

With some sacrifice in payload capacity, it is possible to shroud the vulnerable parts of the ICBM with carbon-bearing or other material that must be ablated by the laser before the laser energy can be deposited in the missile body. Minuteman ICBMs had $0.6 \mathrm{~cm}(1 / 4$ ") of cork installed around the outside of the booster, not as a countermeasure, but as protection against atmospheric heating during ascent. Such a coating may have a substantial effect on hardening a booster. According to [93, p. 125] the heat of vaporization of carbon is about $32 \mathrm{~kJ} / \mathrm{g}$, and charred cork has very low reflectance. The density of cork is about $0.25 \mathrm{~g} / \mathrm{cm}^{3}$. Thus $0.6 \mathrm{~cm}$ of cork would require an incident fluence of about $5 \mathrm{~kJ} / \mathrm{cm}^{2} / 0.5=100 \mathrm{MJ} / \mathrm{m}^{2}$ (taking into account an average angle of incidence) to vaporize it and expose the missile body beneath it. Assuming that the material stays intact and does not flake off before this amount of energy is deposited, attacking a liquid-propellant booster having such a coating 
would require about 4 times the fluence shown in Table 20.2 for an unprotected missile, substantially changing an engagement. For comparison, this fluence would be about half the fluence required for a solid-propellant missile as shown in Table 20.2. Applying such a coating to a solid-propellant missile would increase the fluence requirement by only a factor of about 1.4, so the relative effect is less than that for liquid-propellant missiles.

Long engagement times caused by the use of ablative coatings or rolling the missile can substantially complicate the defense. The defense's response could be to reduce the slant range, with the concomitant penalty of coverage and increased vulnerability to attacking aircraft or SAMs.

\subsubsection{Optical properties}

The ABL target acquisition begins with broadband optical information on the plume, but it relies on the illumination of the target by the TILL to provide the hardbody hand over and an image for the tilt corrections. Similarly, the BILL image is essential for the adaptive optics corrections. If these functions are compromised, the quality of the beam's focus (Chapter 19) would be greatly degraded. The engagement strategy locates the nose of the missile and then places the BILL on the target to pursue the HEL attack. A specular reflecting cone, or a cylinder, which is a degenerate cone, is a very stealthy object for this kind of search. Apart from imperfections of diffuse reflection, a cone either reflects light at an angle given by the angle of the generatrix and the incident beam or absorbs it. Unless the generatrix is normal to the incident light, almost nothing will be seen. Whatever might be seen could be confusing to a system that is expecting a diffuse reflective surface. It is not known quantitatively what would be required to confuse the system, however. Aluminized Mylar has a diffuse reflectivity of the order of 0.1 at the wavelengths of interest [94]. Highly polished surfaces could achieve lower diffuse reflectivity. Any such surface would have to survive the ascent through the atmosphere and the associated heating.

\subsubsection{Multiple missile launches}

The simultaneous launch of multiple missiles could overwhelm an ABL platform, since its engagements are by necessity sequential. In such situations, the engagement time is critical in determining how many missiles could be engaged by a single platform. Multiple ABLs could conceivably be deployed to handle a modest number of multiple missile launches. For every aircraft on station around the clock, three to four aircraft must be deployed in the field, according to the present plan for the ABL. We have not examined the problems inherent to management of a fleet of aircraft on station.

\subsubsection{Launch timing and cloud cover}

As discussed in Section 22.2, cirrus cloud cover could effectively shield a threat missile from the ABL beam for a significant time. In such cases, coordinating the missiles launch with cloud cover could thwart the ABL's defensive action. Radar and LIDAR equipment to measure the cloud height [95] is certainly within reach of a country that could build or buy an ICBM. 


\section{References for Chapter 9}

[90] Almaz S-200 Angara/Vega (SA-5 'Gammon') low-to high-altitude surface-to-air missile system, Jane's Land-Based Air Defense 2002-2003 (2001).

[91] Scientific Advisory Board, http://www.sab.hq.af.mil/Archives/1995/NWV/de/direch2.pdf, 1995.

[92] Michael A. Dornheim, "Ukrainian Missile Exercises Likely Cause of Downed Tu-154," Aviation Week and Space Technology 155, 62, 15 October 2001.

[93] N. Bloembergen et al., Rev. Mod. Phys. 59, S1 (1987).

[94] D. M. Trotter and A. J. Sievers, Solar Energy Materials 3, 1980.

[95] D. A. Nahrstedt, "Cloud Modeling for Laser Weapon Propagation Analysis," SPIE 4034, 69 (2000). 



\section{Part B. Requirements to Hit an Accelerating Missile}





\title{
Chapter 10
}

\section{Detecting and Tracking Missiles in Powered Flight}

\author{
Contents
}

10.1 Space-Based Detection and Tracking f . . . . . . . S159

10.1.1 Plume emission spectra and luminosities _ . . . . . . . . S159

10.1.2 Capabilities of the DSP system _. . . . . . . . . S162

10.1.3 Capabilities of a modern space-based sensor system - the NSBS S166

10.1.4 Summary of space-based detection and tracking . . . . . . S S170

10.2 Radar Tracking . . . . . . . . . . . . . . . . S S171

10.2.1 The power-aperture product required for a search . . . . . S S171

10.2.2 Estimated radar cross sections for illustrative ICBMs $\ldots \ldots$ S172

10.2.3 Land-based radars . . . . . . . . . . . . . . . . S174

10.2.4 Shipboard radars . . . . . . . . . . . . . . S176

10.2.5 Airborne radars . . . . . . . . . . . . . . . S176

10.2.6 Sources of error in interceptor guidance using radars . . . . . S181

10.2.7 Error analysis for selected radar systems . . . . . . . . . S182

10.2.8 Countermeasures to radar tracking . . . . . . . . S S183

10.2.9 Forward-based radar . . . . . . . . . . . . . . . . . S185

10.3 Sensors on the Kill Vehicle . . . . . . . . . . . S S185

10.3.1 A notional infrared seeker on the kill vehicle . . . . . . . . S186

10.3.2 A notional LIDAR seeker on the kill vehicle . . . . . . . . S190

10.4 Applications of Missile Detection and Tracking . . . . . S 5196

Sensors would play essential roles in every phase of operation of a boost-phase antiballistic missile system. They are required to detect the launch of large rockets, to determine whether they are potentially threatening to the United States, and-if it were decided to attack them - to locate and track targets with sufficient precision to allow interceptors or lasers to hit and destroy them. This chapter is concerned with identifying sensors that could be used for these tasks and analyzing their likely performance. ${ }^{1}$ Some types of sensors could perform more than one of these tasks.

A wide variety of sensor types and basing locations could potentially be useful for boostphase intercept systems. Box 10.1 lists a number of possibilities, in the approximate order

\footnotetext{
${ }^{1}$ The analysis in this chapter provides signal-to-noise ratios, error matrices, and position uncertainties. Tracking filters that produce estimates of the target state from this information are described in Chapter 12 and Appendix C.
} 


\begin{tabular}{l} 
Box 10.1: Possible Detection and Tracking Sensors \\
\hline Space-based passive visible-light sensors \\
Space-based passive infrared (IR) sensors \\
Space-based radars \\
Airborne radars \\
Airborne electro-optical sensors \\
Ship-based radars \\
Land-based radars \\
On-board passive ultraviolet sensors \\
On-board passive short-wavelength IR and/or \\
medium-wavelength IR sensors \\
On-board passive long-wavelength IR sensors \\
On-board radar \\
LIDAR* with an on-board detector and \\
on-board laser illuminator \\
LIDAR with an on-board detector but \\
an off-board laser illuminator \\
LIDAR from other platforms \\
*light detection and ranging
\end{tabular}

in which they could be used to detect, identify, and track potentially threatening rockets. For systems that use interceptor missiles, the sensors used during the first phases of an engagement would likely be general-purpose sensors located far from the target, such as sensors on early-warning satellites. ${ }^{2}$ As the interceptor closed with the target, dedicated sensors that could provide more precise tracking and better discrimination would need to be employed, some of which would not be carried by the interceptor or kill vehicle. Such "off-board sensors" might include ground- or sea-based radars. Other sensors would have to be carried by the kill vehicle to perform their functions adequately. Such "on-board sensors" might include passive detectors that would simply image the target or active sensors that would also provide range information.

Given the limited time and resources available for the Study, we did not attempt an exhaustive analysis of these possibilities. Instead, we sought to identify a minimal set of sensors that would enable the various boost-phase systems considered to intercept the model ICBMs. ${ }^{3}$ For this purpose, we considered a notional system of space-based infrared (IR) sensors for launch detection and early tracking; land-, ship-, and air-based radars

\footnotetext{
${ }^{2}$ In contrast to systems that use interceptor missiles, the Airborne Laser is designed to function either autonomously, using only the sensors on the Airborne Laser aircraft to detect and track rockets, or as part of a larger system that would provide data from additional sensors; see Chapter 17.

${ }^{3}$ As discussed in Chapter 2, in analyzing boost-phase engagements we assumed the system would have available at any time only the information gathered by sensors up to that moment. The boost-phase intercept system's probability of success would depend critically on the types, number, and performance of the available sensors. In a real system, some redundancy would be desirable, to make the system more robust in the face of unexpected target behavior or characteristics, as well as failures of individual components.
} 
for tracking the target rocket after it has risen above the radar's horizon; imaging sensors on board the kill vehicle for tracking the rocket's plume as the engagement develops; and LIDAR (light detection and ranging) seekers on board the kill vehicle for imaging the rocket's hardbody and determining its range toward the end of the homing phase of the engagement and during the endgame.

Although system integration requirements would likely impose some constraints on any boost-phase tracking system, we focused only on physical limitations and confined our analysis to technologies that we judged would be available within the next decade. Spacebased visible-light sensors [96] or ultraviolet sensors on-board the kill vehicle [97] might have important advantages, but we did not analyze their possible contributions because there is insufficient information in the open literature to support such an analysis. We also did not consider on-board radar seekers because with current technology, a radar with the long-range capability needed would be substantially heavier than a LIDAR with this capability.

The model ICBMs described in Chapter 15 were used to estimate the plume luminosities and radar cross sections of various types of missiles. These ICBM models, the model interceptors described in Chapter 16, and the engagement trajectories described in Chapter 4 were used to determine the earliest times various types of sensors could be brought to bear on the target. The effects of sensor viewing angles were also examined.

The analysis presented in this chapter supports the simulations of illustrative engagements described in Chapters 4, 5, and Appendix C. The capabilities of the sensors that initially detect the launch of potentially threatening rockets and provide early information on their trajectories define the engagement timelines described in Chapters 2, 4, and 5. The capabilities of the sensors used to guide interceptors until their kill vehicles are launched and then guide the kill vehicles to the target are at the heart of the engagement analysis described in Chapter 12 and Appendix C.

We begin by analyzing the capabilities of current and prospective space-based sensors for detecting and tracking ICBM exhaust plumes. Next we consider the missile-tracking capabilities of a variety of land-based, ship-based, and airborne radars. We then analyze the likely near-term performance of passive IR and active LIDAR seekers on the kill vehicle. We conclude by summarizing our results.

\subsection{Space-Based Detection and Tracking}

The hot exhaust plumes of long-range ballistic missiles are powerful sources of IR radiation [98]. This radiation can be seen from space, and IR sensors in high orbits can provide broad-area coverage of Earth. These considerations make space-based IR sensing a very useful technique for detecting and tracking ICBMs during their boost phase. In this section we first summarize the phenomenology of ICBM exhaust plumes relevant to detecting and tracking them with space-based IR sensors. We then discuss the capabilities of the existing Defense Support Program (DSP) satellite system for supporting a boost-phase defense system and the capabilities that a modern follow-on system could have.

\subsubsection{Plume emission spectra and luminosities}

A rocket's bright exhaust plume is its principal signature. Exhaust plumes radiate strongly in the IR region of the electromagnetic spectrum, as illustrated by Fig. 10.1, which shows IR spectra of the emission from the exhaust plume of a large rocket burning hydrazine fuels 


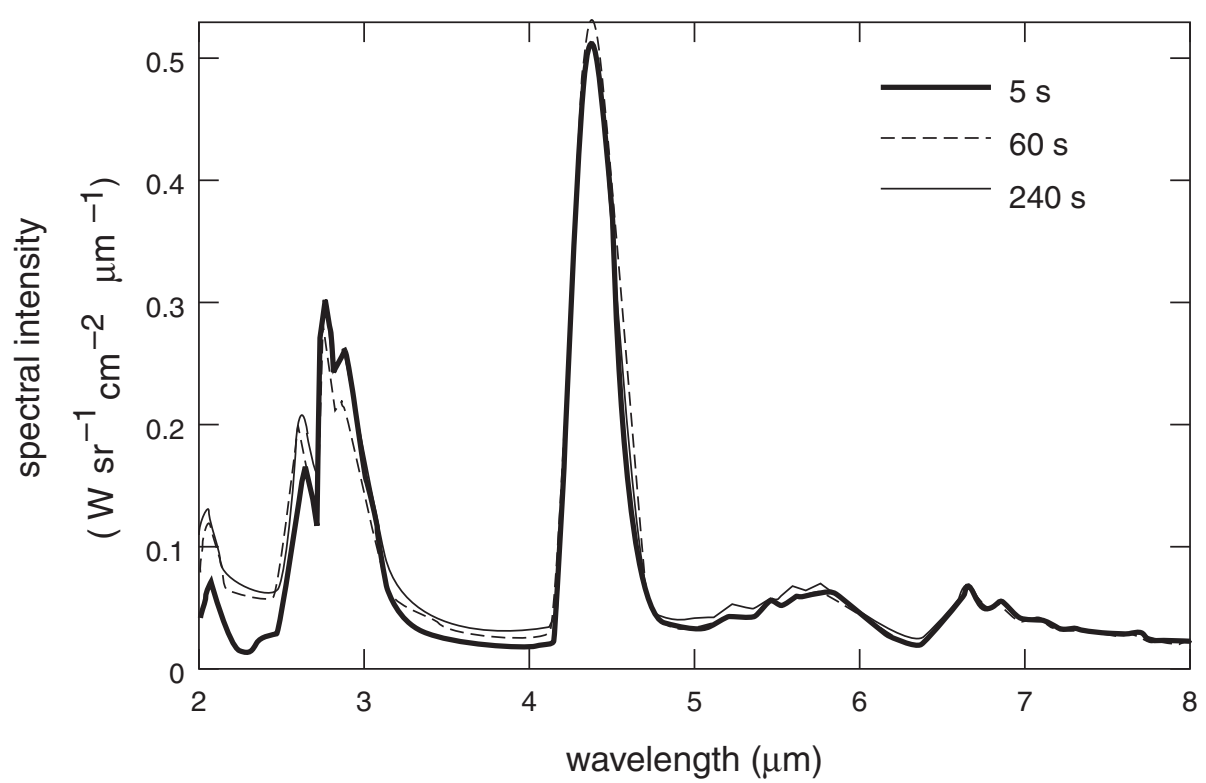

Figure 10.1. Illustrative spectra of the IR emission from the exhaust plume of a rocket burning hydrazine fuels in a ground test. The times indicated are the times after ignition. These spectra are similar to the spectrum of the so-called "intrinsic" or "vacuum" core. Except for rockets at extremely high altitudes, the spectrum of the intrinsic core seen from space would be altered because of absorption by the intervening atmosphere. Figure 4.11 from Ref. [98]. Used with permission. Copyright by The Aerospace Corporation.

in a ground test. Typically, a substantial fraction of the total luminosity ${ }^{4}$ is radiated in a short-wavelength IR (SWIR) peak at about $2.5-3.0 \mu \mathrm{m}$. A similar fraction is radiated in a medium-wavelength (MWIR) peak at about $4-5 \mu \mathrm{m}$. (As seen from space, this peak has a "notch" caused by absorption by cold atmospheric gases between 4.21 and $4.5 \mu \mathrm{m}$ ). These peaks in the spectrum are produced by the emission bands of combustion products, such as $\mathrm{H}_{2} \mathrm{O}$ and $\mathrm{CO}_{2}$. Spectra of the plumes produced by hydrazine-fueled rockets in flight would be qualitatively similar to the spectra shown, but the luminosity would depend on the size of the rocket and the stage of flight.

Rockets burning hydrocarbon fuels would produce carbon soot. Continuum emission from the soot would add to the peaks produced by molecular emission but would also increase the continuum emission between them. So would the continuum emission from the aluminum oxide particles in the plumes of solid-propellant rockets.

The properties of an ICBM's exhaust plume vary greatly as it rises through the atmosphere [98]. This variation affects the capabilities of space-based tracking systems. The characteristic variation with altitude of the IR luminosity of an ICBM exhaust plume is shown schematically in Fig. 10.2. ${ }^{5}$ The IR emission is initially caused by mixing of com-

\footnotetext{
${ }^{4}$ This report uses the radiation terminology standard in the physics literature: the power radiated in all directions and in all wavebands is called the luminosity; the power radiated in all directions within a prescribed bandpass is the in-band luminosity; the power per unit area and solid angle is the intensity; and the power per unit solid angle, area, and wavelength or frequency is the spectral intensity. In the literature concerned with rocket exhaust plume emission, the power per unit solid angle is the intensity; the power per unit solid angle and wavelength is the spectral intensity; the power per unit area and solid angle within a prescribed bandpass is the in-band radiance; and the power per unit area, solid angle, and wavelength is the radiance.

${ }^{5}$ See Figs. 14.3, 14.4, 14.19-14.23, and 5.2 of [98].
} 


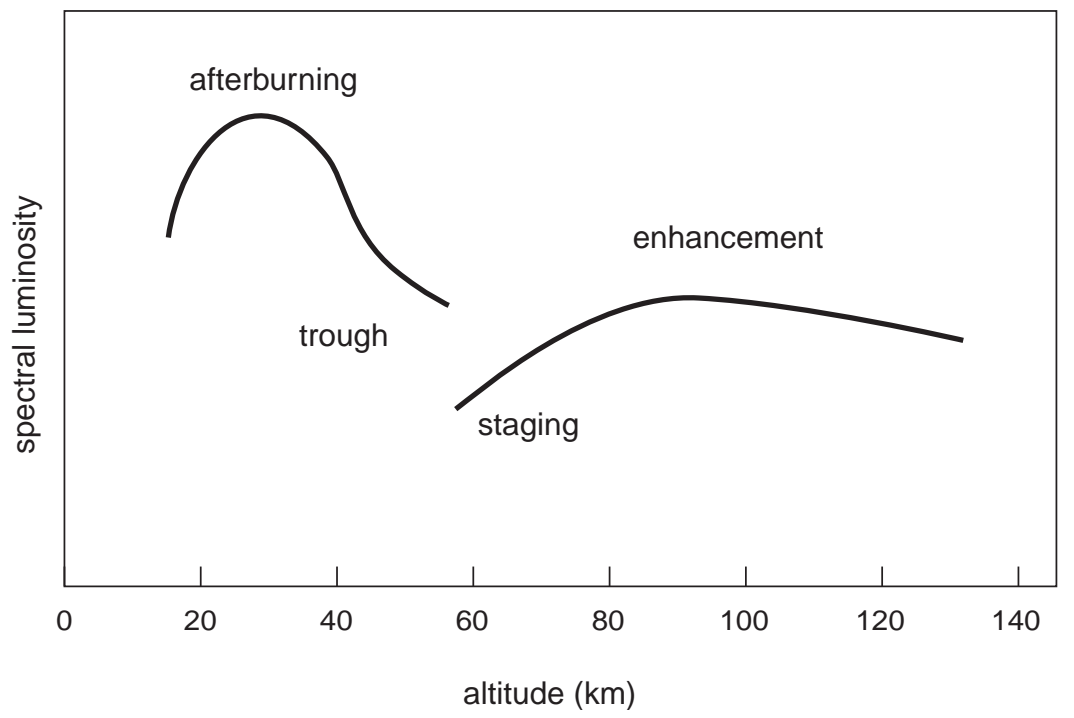

Figure 10.2. Sketch of the characteristic variation with altitude of the luminosity per unit solid angle of rocket exhaust plumes. The curve is intended to give only a qualitative impression of the typical dependence of the luminosity on altitude and does not represent the plume luminosity of any particular rocket. Note the peak at low altitudes, the "trough" at $\sim 50-70 \mathrm{~km}$, and the partial recovery at high altitudes. There is a break in the curve when the first stage stops burning and the second stage ignites.

bustion products, such as $\mathrm{H}_{2}$ and $\mathrm{CO}$, with atmospheric gases and their chemical reaction with atmospheric $\mathrm{O}_{2}$ ("afterburning"). As the rocket rises, the plume's apparent luminosity first increases as it expands in size and the atmospheric absorption between it and the space-based detector decreases. The luminosity of a rocket's plume peaks at an altitude of about $30 \mathrm{~km}$, where it can exceed $1 \mathrm{MW} \mathrm{sr}^{-1}$ for an ICBM.

As the rocket continues to rise and accelerate, the increasing expansion and cooling of its hot exhaust gases, their decreasing bulk velocity relative to the atmosphere, and the reduction in available oxygen causes afterburning to decline precipitously. As a result the plume's luminosity falls steeply to a minimum value at $\sim 50-70 \mathrm{~km}$ (the "trough region"). This decline is often exacerbated by burnout of the first stage, followed by ignition of the second stage, which typically has a much smaller thrust and hence produces a plume with a much smaller luminosity. The luminosity of the plume in the trough region is dominated by emission from the exhaust near the rocket nozzle, which depends on the pressure there and the nature of the particulates in the exhaust (e.g., soot particles in the exhaust from liquid-propellant rockets and $\mathrm{Al}_{2} \mathrm{O}_{3}$ particles in the exhaust from solid-propellant rockets).

As the rocket continues to accelerate, the bulk velocity of its exhaust gases relative to the atmosphere increases again, causing the plume's IR luminosity to increase again because of temperature recovery and resumption of chemical reactions ("enhancement"). The latter processes depend strongly on the velocity vs. altitude profile of the rocket. The IR luminosity of the plume continues to increase, but when the size scales of the processes causing this enhancement begin to exceed the dimensions of a sensor pixel, the plume's apparent luminosity again falls. At this point, the total luminosity of the plume is given approximately by summing the signals in the reporting detectors. Eventually the signals from the plume-atmosphere interaction fall below the noise-equivalent flux of the detectors. The 
ultimate minimum signal that remains comes from the altitude-invariant emission produced by the exhaust products as they expand into the low-pressure environment (the "intrinsic" or "vacuum" core).

Although a wide range of data is available on the plume emission of U.S. rockets, including both liquid- and solid-propellant missiles, to our knowledge there is no such database for the rockets of countries of concern. Moreover, none of these countries has yet tested an ICBM. Caution should therefore be exercised when attempting to characterize the plumes of hypothetical missiles that may pose a threat to the United States at some future date by extrapolating from past measurements of U.S. rockets. However, measurements of the exhaust plumes of some early U.S. rocket designs, such as the Titan, may be useful if countries of concern take a similar path in developing liquid-propellant ICBMs.

\subsubsection{Capabilities of the DSP system}

The DSP system has provided the United States with early warning of missile launches since 1970, using satellites in geosynchronous Earth orbit (GEO). A program is underway to replace the DSP system with a more modern satellite-based missile warning and tracking system. However, some authors (see, e.g., [97]) have argued that the DSP system would be adequate to support boost-phase intercept systems. Also, recent technical problems may delay deployment of the more modern system [99]. Consequently, in this section we analyze briefly the ability of the DSP system to serve as the launch detection and initial tracking system for a boost-phase intercept system. The capabilities that a modern space-based missile warning and tracking system could have are discussed in the next section.

DSP resolution The general characteristics of the DSP satellites and system are described in [100]. Each satellite has a rotating telescope with a linear array of 6000 detectors in the focal plane. The telescope of each satellite scans the whole disk of the Earth visible to it and a narrow annular region above the horizon once every $10 \mathrm{~s}$. The precision with which the DSP system can locate a missile's plume is determined by the footprints of the pixels in its satellites' sensor arrays. The number of detectors in each array suggests that the footprint of each pixel at Earth's surface is about $1 \mathrm{~km}$ per side. Typically at least three DSP satellites are in operation at any given time, at different longitudes around Earth's equator, so at least two can view a target. Stereo, three-dimensional tracking of missile plumes is possible if data from more than one DSP satellite are combined ("fused").

Although the DSP system cannot track an ICBM with the precision and sampling rate that would be required to guide a kill vehicle to hit the ICBM during its boost phase, the system can warn of rocket launches and could cue ground- and ship-based radars or sensors on board the kill vehicle to the position of a rising rocket. This early-warning capability would relieve the latter sensors from having to perform broad-area searches to acquire the rocket, allowing them to be made smaller and lighter.

Launch detection and initial tracking using DSP The earliest moment at which the launch of a large rocket could be confirmed and the direction of its flight estimated is of great importance for boost-phase intercept systems, because it is the earliest moment that interceptors could be fired. For the DSP system, early detection and tracking of a large rocket is limited by three factors: cloud cover, absorption of the radiation from the exhaust plume by water vapor in the atmosphere in the IR band to which the DSP sensors are tuned, and the low rate at which it scans Earth's disk. 


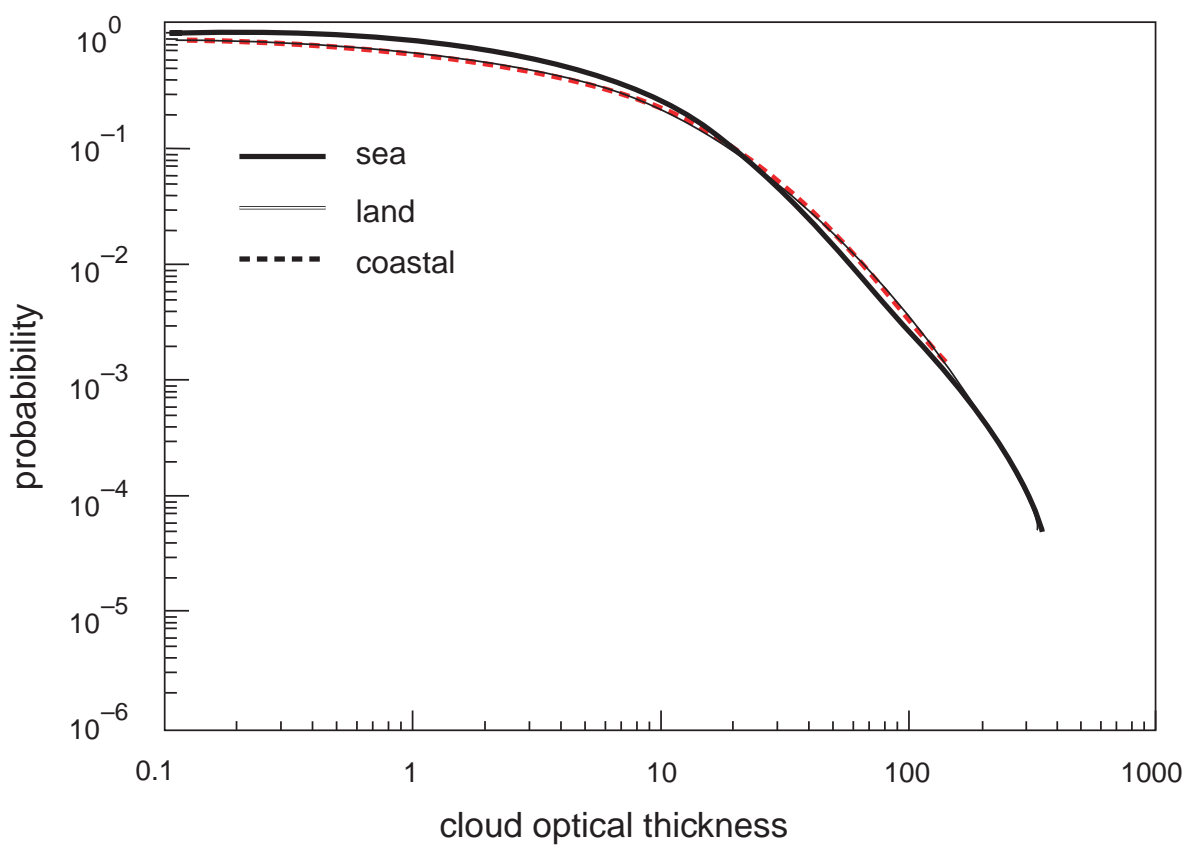

Figure 10.3. Probability that the optical path length of cloud cover over sea, land, and coastal areas at mid-latitudes exceeds a given value as a function of cloud optical thickness. The three probability distributions are almost indistinguishable on the scale of this plot. Figure 33 from Ref. [101]. Used with permission.

The probability that the cloud cover exceeds a given optical depth (the thickness of the cloud in units of the mean distance a photon travels before scattering) is shown in Fig. 10.3. There is a $\sim 20$ percent chance of clouds having an optical depth greater than 10 occurring at mid-latitudes at any given time. However, many clouds are low-lying, so the chance of seeing significantly below $10 \mathrm{~km}$ is high. The average cloud top height when clouds exist (about 50 percent of the time) is $4.7 \mathrm{~km}$ over land world-wide [102]. According to Rossow and Schiffer [103], "cloudiness on Earth can be described as a persistent ... background of optically thin clouds ..., together with a rare, highly variable component of precipitating clouds that are very sparse and intermittent in occurrence (total amount $<0.1$ )". While a detailed discussion of cloud climatology is beyond the scope of this study, we conclude that dense clouds are sufficiently rare above $7 \mathrm{~km}$ and that they would rarely be the main factor preventing DSP from detecting a large rocket by the time it reaches this altitude.

The SWIR passband of the DSP detectors is deliberately tuned to the strong 2.7$2.9 \mu \mathrm{m}$ absorption band of $\mathrm{H}_{2} \mathrm{O}$, shown in the central panel of Fig. 10.4, so that emission in this band from near Earth's surface is strongly attenuated by the water vapor in the atmosphere, reducing the background clutter that would otherwise interfere with detection and identification of rocket plumes. As shown in Fig. 10.5, the lower background clutter seen from space in this passband comes from the sunlight scattered by high-altitude clouds and bright solar glints from the oceans and other surface features, which are partially transmitted though the atmosphere and contribute to the background. Although the emission from the hot $\mathrm{H}_{2} \mathrm{O}$ and $\mathrm{CO}_{2}$ molecules in rocket exhaust plumes extends over a broader wavelength range than the absorption by the cold molecules in the atmosphere, the signal from exhaust plumes is severely attenuated (by design) in this passband, until the rockets rise above most 

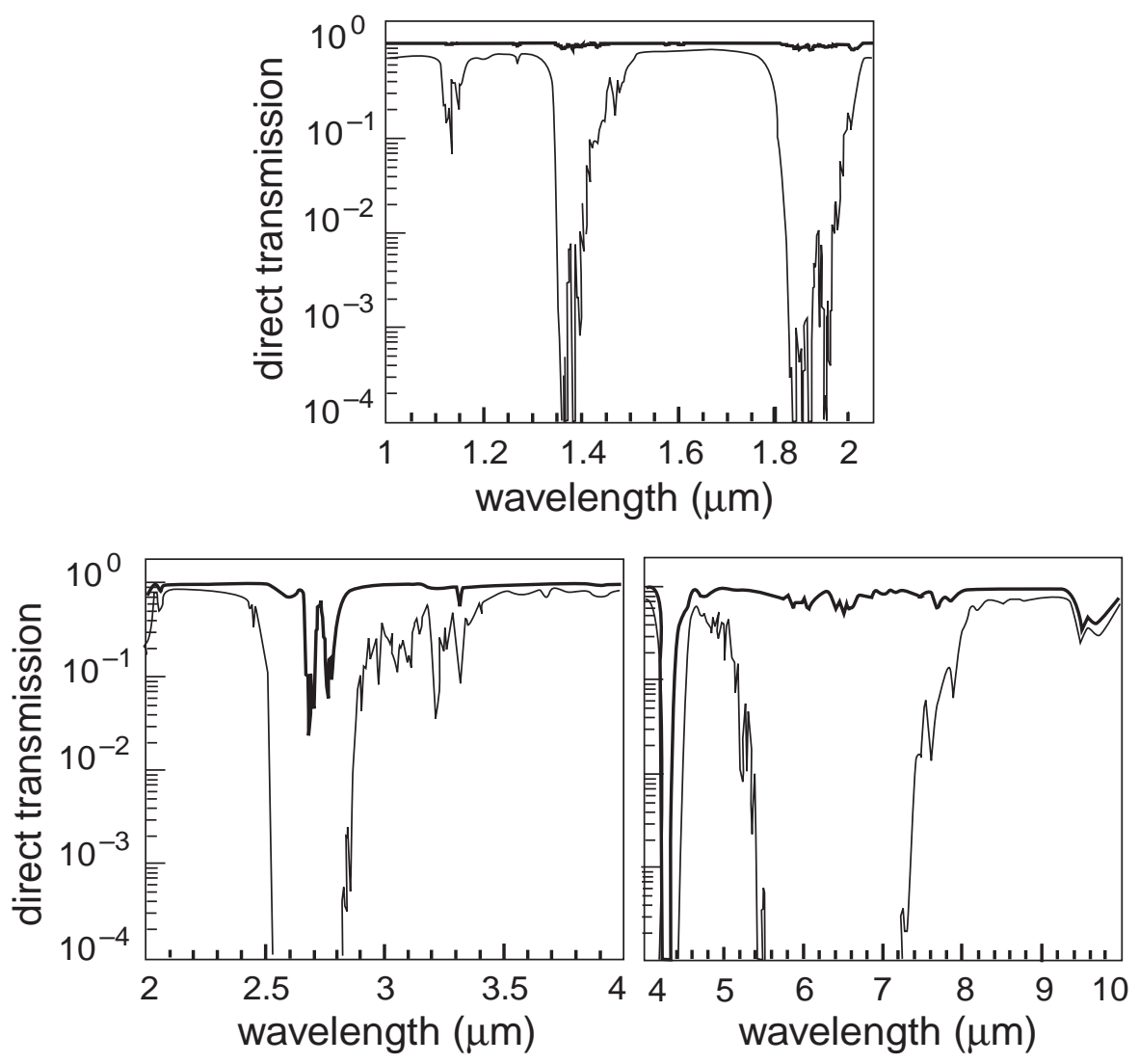

Figure 10.4. Transmission of Earth's atmosphere from ground to space (lower curve) and from 10$\mathrm{km}$ altitude to space (upper curve) as a function of wavelength in the 1-10 $\mu \mathrm{m}$ region for a typical mid-latitude atmosphere. Note the strong $\mathrm{H}_{2} \mathrm{O}$ absorption band at 2.7-2.9 $\mu \mathrm{m}$ and the windows at 2.2-2.4 and 3.5-4 $\mu \mathrm{m}$. Moderate resolution atmospheric transmission code (MODTRAN) calculation courtesy of William Clodius.

of the atmospheric water vapor. This attenuation occurs whether or not clouds are present. We estimate that the DSP system would be able to detect emission from the exhaust plume of a large rocket by the time the rocket reached an altitude of $10 \mathrm{~km} .{ }^{6}$

Unless the defense is certain a potentially threatening rocket is a relatively slowly accelerating liquid-propellant missile, it would have to treat it as a more rapidly accelerating solid-propellant missile to assure sufficient time for interceptors to reach it. Our solidpropellant ICBM models S1 and S2 reach an altitude of $10 \mathrm{~km}$ about $35 \mathrm{~s}$ after they are launched. At this point, they are also about $11 \mathrm{~km}$ downrange of the launch point. We estimate that three separate detections by a DSP sensor array would be needed to confirm, by its motion, that the SWIR source produced by either of these model ICBMs were the exhaust plume of a large rocket and to estimate its trajectory well enough to construct a firing solution. Thus, once the DSP system has detected a rocket exhaust plume, 30 more seconds, plus additional time for processing and communications, would be needed to construct a firing solution. We therefore estimate that the earliest time a firing solution could reliably be constructed using DSP data on solid-propellant ICBMs similar to the ones we

\footnotetext{
${ }^{6}$ By detection, we mean that the rocket's plume would create a bright pixel. Additional data would be needed to declare that the signal were coming from the exhaust plume of a large rocket
} 


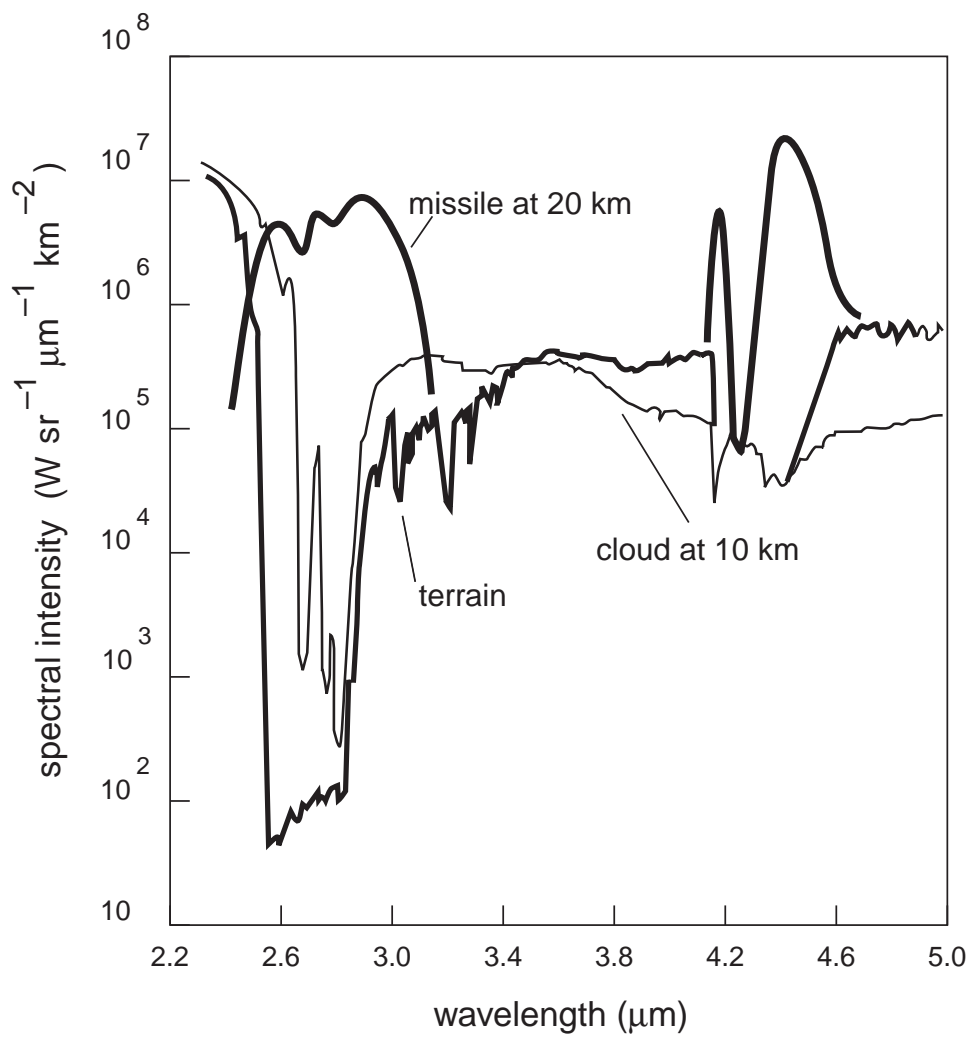

Figure 10.5. Comparison of the IR spectral luminosity of a representative missile plume at an altitude of $20 \mathrm{~km}$ with the equivalent intensity of Earth's atmosphere, clouds, and terrain in a $1 \mathrm{~km} \times 1 \mathrm{~km}$ pixel footprint, as viewed from space. After Fig. 3.8 in Ref. [98]. Used with permission. Copyright by The Aerospace Corporation.

modeled is $65 \mathrm{~s}$ after launch. A benefit of this delay is that it would give the defense a good chance of seeing first-stage burnout and second-stage ignition, if the target were indeed a solid-propellant ICBM.

Our liquid-propellant ICBM model reaches an altitude of $10 \mathrm{~km}$ about $50 \mathrm{~s}$ after it is launched. At this point it is only $5 \mathrm{~km}$ downrange and is moving much more slowly than the solid-propellant ICBMs are at this altitude. We therefore estimate that five or six separate detections would probably be needed to confirm that the SWIR source produced by ICBMs like this model is the exhaust plume of a large rocket and to estimate its trajectory well enough to construct a firing solution. We therefore estimate that the earliest time a firing solution could reliably be constructed using DSP data on liquid-propellant ICBMs like the one we modeled is $80 \mathrm{~s}$ after launch.

The analysis in the next section shows that a firing solution could probably be constructed considerably earlier using data from a modern space-based missile warning and tracking system.

Subsequent tracking using DSP Once interceptors are fired, they must be supplied updates on the developing trajectory of the target rocket and on their own flight toward the predicted intercept point. The tracking data supplied by the DSP system are likely to be inadequate for two reasons. First, the 1-km precision and $0.1-\mathrm{Hz}$ rate with which the DSP system samples the trajectories of the target and interceptor are probably inadequate to 
guide the interceptor efficiently. Second, the DSP sensors may not be able to see the rocket's exhaust plume with sufficient signal-to-noise ratio when the rocket is in the trough region. We have not carried out a detailed analysis of the effects of DSP's $0.1-\mathrm{Hz}$ sampling rate and of the trough, but we expect they would increase significantly the required velocity-change capability of the kill vehicle and its booster, which would in turn increase the weight of the kill vehicle and the booster. A "seeker" on the kill vehicle would not help, because it could not begin tracking the plume until the kill vehicle has reached the altitude $(\sim 100 \mathrm{~km})$ where atmospheric friction is sufficiently low that the seeker window could open and the seeker could begin operating (see Section 10.3.1). The latter points are illustrated by considering engagements of the three ICBM models presented in Chapter 15 by the $6.5-\mathrm{km} / \mathrm{s}$ and $10-\mathrm{km} / \mathrm{s}$ interceptors presented in Chapter 16 .

If the target is a rapidly-rising solid-propellant ICBM, like models S1 or S2, and the only available space-based tracking system is the DSP then, as discussed above, interceptors probably could not be fired until about $65 \mathrm{~s}$ after the ICBM were launched. These ICBMs would drop their first stages $60-65 \mathrm{~s}$ after launch, would enter the trough region at about $80 \mathrm{~s}$, and would emerge at about $95 \mathrm{~s}$. If the kill vehicle were lofted by either the $6.5-\mathrm{km} / \mathrm{s}$ or the $10-\mathrm{km} / \mathrm{s}$ interceptors and its seeker began operating at an altitude of $100 \mathrm{~km}$, the seeker would open about $65 \mathrm{~s}$ after the interceptors were launched, about $130 \mathrm{~s}$ after the ICBM launch. By this time, these ICBMs would have already passed through the trough region.

If instead the target is a slowly-rising liquid-propellant ICBM, like model L, interceptors probably could not be fired until about $80 \mathrm{~s}$ after the ICBM launch. This ICBM would enter the trough region about $115 \mathrm{~s}$ after launch, would drop its first stage at $120 \mathrm{~s}$, and would emerge from the trough at about $135 \mathrm{~s}$. If the kill vehicle were lofted by either the $6.5-\mathrm{km} / \mathrm{s}$ or the $10-\mathrm{km} / \mathrm{s}$ interceptors and its seeker began operating at an altitude of $100 \mathrm{~km}$, the seeker would open about $65 \mathrm{~s}$ after the interceptors were launched, or about $145 \mathrm{~s}$ after the ICBM launch. By this time, ICBM model L would also have passed through the trough region.

We conclude that although the DSP system could provide launch detection and initial tracking, its sampling rate would be too low to support efficient command guidance of boost-phase interceptors. The DSP system likely would have to be supplemented by an off-board sensor with a much higher sampling rate, such as a radar, to enable efficient command guidance.

\subsubsection{Capabilities of a modern space-based sensor system-the NSBS}

As noted in the previous section, the DSP system is expected to be replaced by a more modern satellite-based missile warning and tracking system. The system the United States is currently developing is called the Space-Based Infrared System-High (SBIRS-High). This system will consist of a constellation of satellites, with some in GEO and some in Molniya orbits, which are highly eccentric having the apogee (and hence long residence time) above the northern hemisphere of Earth. A system with the potential capabilities of SBIRS-High could make a critical contribution to the functioning any boost-phase intercept system. The first six satellites of this system are currently scheduled to be in place by 2008 [104], although recent technical problems may delay their deployment, as noted previously. Nevertheless, the initial complement of satellites is likely to be deployed within the next ten years. We therefore investigated the capabilities such a system could have.

Details of the anticipated performance of SBIRS-High are not available in the open 
literature. We therefore analyzed the potential performance of a generic space-based IR sensor system that uses current technology and could be fielded with a reasonable investment of resources and the contribution such a system could make to boost-phase intercept. For conciseness, we shall refer to this notional space-based system as the "NSBS".

NSBS sensors The NSBS we considered consists of a constellation of satellites in geosynchronous orbits having 30-cm-diameter aperture telescopes that image the visible disk of Earth and near-Earth space. The IR detectors would be cooled, but the optics need not be. The full-Earth focal plane is partially populated by arrays of IR detectors (see, e.g., Ref. [105]). Currently, $320 \times 256$ arrays of $\mathrm{HgCdTe}(\mathrm{MCT})$ detectors having frame rates of $30 \mathrm{~Hz}$ or greater are commercially available [106]. Quantum efficiencies are approximately 80 percent, well depths are approximately $10^{6}$ electrons, readout noise is $\sim 250$ electrons, and the pixel pitch is $30 \mu \mathrm{m}$.

Extrapolating forward in time, a $512 \times 512$ format $\sim 3 \times 10^{5}$-pixel array with $20-\mu \mathrm{m}$ pixel pitch should be available shortly [105]. We assume that the focal plane of our hypothetical space-based telescope is populated with 24 of these arrays, which together would cover approximately 1/24 of Earth's visible disk. By stepping to a fresh part of Earth's disk after each 33-ms frame, the whole disk could be scanned in less than $1 \mathrm{~s}$. Although the diffraction limit of the NSBS in the SWIR would be less than $0.5 \mathrm{~km}$ at Earth's surface, we choose pixels with a projected size of $1 \mathrm{~km} \times 1 \mathrm{~km}$ (at nadir) to obtain a manageable pixel number and data rate. To match a $20 \mu \mathrm{m} \times 20 \mu \mathrm{m}$ pixel to a $1 \mathrm{~km} \times 1 \mathrm{~km}$ footprint requires a final focal ratio of $f / 2.8$ for our 30 -cm aperture. ${ }^{7}$ This system would not be diffraction-limited.

NSBS sensitivity For a source with an SWIR luminosity of $6 \mathrm{~kW} \mathrm{sr}^{-1}$, comparable to the plume luminosity of a small rocket early in flight, the NSBS would collect $7 \times 10^{4}$ electrons during each 33-ms frame, in the pixel that is on the source, filling the well to less than 10 percent of its depth. The plume signal would compete with noise in the detector, the detector dark current, and thermal emission from the optics, baffles, and dewar window. The detector dark current decreases exponentially with decreasing temperature. Extrapolating from Fig. 2.3 of Ref. [106], we estimate that for an MCT detector having a cutoff at $3.0 \mu \mathrm{m}$ and a pixel pitch of $20 \mu \mathrm{m}$, the detector dark current can be reduced to $10^{4}$ counts per pixel per 33-ms frame by cooling the detector to $150 \mathrm{~K}$, which is possible using a thermoelectric cooler. We estimate the thermal background by assuming a 20 percent emissivity for the $f / 2.8$ optical path, which is conservative and includes some allowance for warm baffle edges that intrude into the otherwise cold optical path. This gives $2.6 \times 10^{4}$ electrons during each 33-ms frame. For such a detector, the plume signal exceeds the instrumental background and is more than 300 times the statistical (shot noise) variations in these backgrounds, which is 160 electrons per frame. When the weather is clear or only low clouds are present, the signal of a plume having a luminosity of $6 \mathrm{~kW} \mathrm{sr}^{-1}$ in the SWIR would greatly exceed the backgrounds themselves. The limiting sensitivity of the NSBS would therefore be set not by noise but by clutter in the SWIR background, especially from cirrus clouds at high altitudes.

\footnotetext{
${ }^{7}$ The performance of this system is limited by the number of detectors and the pixel format of each detector. By increasing these, we could approach a projected pixel size of $0.5 \mathrm{~km}(<0.25 \mathrm{~km} 1 \sigma$ position uncertainty) and an update rate of tens of hertz. However, we have limited our design to the stated capability to maintain a reasonable focal-plane-array size and data volume. Although the aperture could be increased beyond $30 \mathrm{~cm}$ in a medium-sized satellite, with a concomitant improvement in its diffraction limit, it would be difficult to build a detector array large enough to utilize this resolution over a significant part of Earth.
} 


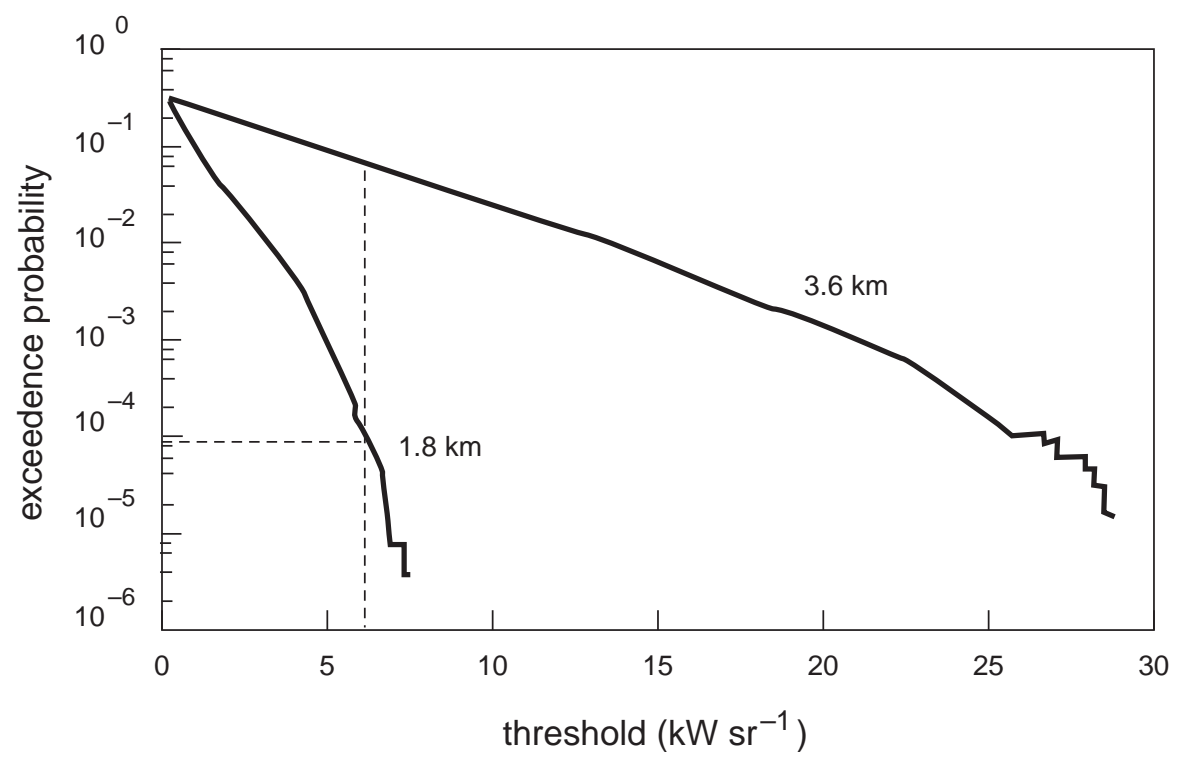

Figure 10.6. Single-pixel false alarm probability as a function of threshold luminosity for the IR scanner considered, when viewing the stressing background scene. The lower curve shows that the probability of exceeding the threshold when no signal is present is approximately $10^{-4}$ per pixel for a $6 \mathrm{~kW} \mathrm{sr}{ }^{-1}$ threshold and a $1.8 \mathrm{~km} \times 1.8 \mathrm{~km}$-pixel footprint. The upper curve shows that at this threshold, the false-alarm probability is about 1,000 times higher for a $3.6 \mathrm{~km} \times 3.6 \mathrm{~km}$ footprint. From Ref. [107]. Used with permission. Copyright by The Aerospace Corporation.

A global cloud model developed by the Aerospace Corporation [107] indicates that cirrus clouds above $10 \mathrm{~km}$ occur 20 percent to 30 percent of the time over northeast Asia in summer; they are not far from the top of the water vapor layer and hence appear as bright spots when viewed from space. These clouds produce a complex background, and the limiting sensitivity in the SWIR is therefore a complicated function of climatology, cloud structure, and atmospheric radiative transport. The expected sensitivity of the postulated NSBS cannot be determined by an analysis simple enough to be within the scope of our study. We therefore rely on the work of Lawrie and Lomheim [107], who have shown that for a given false-positive rate (the probability that any given pixel will yield a false alarm in one scan), the minimum detectable flux is a function of the size of the pixel footprint (see Fig. 10.6). For a $1.8 \times 1.8 \mathrm{~km}$ footprint, setting the threshold at $6 \mathrm{~kW} \mathrm{sr}^{-1}$ yields a singlepixel false-alarm rate of $10^{-4}$, which drops to $10^{-6}$ for a threshold of about $8 \mathrm{~kW} \mathrm{sr}^{-1}$. If the projected area of each pixel is increased by a factor of four, to $3.6 \times 3.6 \mathrm{~km}$, the luminosity threshold for a constant false alarm rate also increases by a factor of four. We conclude that the NSBS detectors, which have pixels with footprints of $1 \times 1 \mathrm{~km}$, could detect sources with luminosities as low as $6 \mathrm{~kW} \mathrm{sr}^{-1}$ with some margin of safety. We estimate that the $1 \sigma$ uncertainty in the position of the rocket would be less than $300 \mathrm{~m}(\sim 1 \mathrm{~km} / \sqrt{12})$ for a sampling rate of $1 \mathrm{~Hz}$. By using multiple satellites to view the target, it should be possible to determine its position in all three dimensions with this precision. The precision of the position measurements and the sampling rate could both be improved by adding a second, high-resolution slewing telescope to the system, but such a telescope would not be able to track multiple launches at widely separated locations. 
Launch detection and missile tracking using NSBS As noted previously, the earliest moment at which the launch of a large rocket could be confirmed and the direction of its flight estimated is of great importance for boost-phase intercept systems, because this is the earliest moment interceptors could be fired. If all its sensors were tuned to the $2.7-2.9-\mu \mathrm{m}$ $\mathrm{H}_{2} \mathrm{O}$ absorption band, the NSBS would give up the possibility of seeing the rocket sooner in favor of lower and more predictable backgrounds. There are windows in the IR, such as those at $2.2-2.4 \mu \mathrm{m}$ and 3.5-4 $\mu \mathrm{m}$ (see Fig. 10.4), in which a space-based sensor could see to the ground at the cost of introducing ground clutter as a new source of background. If the problem of detecting the plume of a large rocket in the presence of ground clutter can be solved, cloud cover would determine the lowest altitude at which missiles can be detected. By this, we mean thick (optical depths greater than a few) clouds that would strongly attenuate the signal of the rocket exhaust plume, not the optically thin but nevertheless bright clouds that cause spurious glints in the SWIR.

Consistent with the optimistic approach adopted in this study, we assume the problem of detecting the plume of a large rocket in the presence of ground clutter can be solved, and that cloud cover would then be the factor limiting early detection of large rockets. Based on the consideration of cloud cover presented in Section 10.1.2, we adopt $7 \mathrm{~km}$ as the altitude at which the NSBS would reliably detect radiation from the exhaust plume of a large rocket. ${ }^{8}$ Additional data would be needed to declare that the signal emanates from the exhaust plume of a large rocket, and still more data would be needed to estimate the rocket's trajectory precisely enough to permit construction of a firing solution for the interceptors.

We assumed that the azimuth of the rocket's current velocity is a better indicator of its future trajectory than the azimuth of its average velocity since launch. We then estimated in two ways the time required to gather enough data to construct a firing solution. First, we estimated that by the time the rocket plume had moved ten pixels from the point at which its IR signal was first detected, the current azimuth of its trajectory could be determined precisely enough to fire interceptors. Second, we used tuned polynomial tracking filters to estimate the precision with which the azimuth of the target could be estimated to within $7^{\circ}$ from target positions measured once a second with a $1 \sigma$ uncertainty of $<300 \mathrm{~m}$. Both approaches gave approximately the same results.

As noted previously, our solid-propellant ICBM models S1 and S2 require 30 s to reach an altitude of $7 \mathrm{~km}$. At that time, they are about $7 \mathrm{~km}$ downrange from the launch point. We believe that using a system like NSBS, the trajectories of ICBMs like these could be estimated with sufficient precision to construct a firing solution about $15 \mathrm{~s}$ later, when they have reached an altitude of $16 \mathrm{~km}$ and are about $20 \mathrm{~km}$ downrange. We therefore adopt $45 \mathrm{~s}$ after launch as the earliest time a firing solution could reliably be constructed. The first stages of ICBMs like these would still be burning at this time, so the defense would not have any staging events to help identify the type the rocket.

Our liquid-propellant ICBM model reaches an altitude of $7 \mathrm{~km}$ and is about $3 \mathrm{~km}$ downrange $45 \mathrm{~s}$ after it is launched. We estimate that using a system like NSBS, the trajectories of ICBMs like this one could be estimated with sufficient precision to construct a firing solution about $20 \mathrm{~s}$ later, when the missiles have reached an altitude of $16 \mathrm{~km}$ and are about $11 \mathrm{~km}$ downrange. We therefore adopt $65 \mathrm{~s}$ after launch as the earliest time a firing solution could reliably be constructed $-55 \mathrm{~s}$ before the first stage of our liquid-propellant

\footnotetext{
${ }^{8}$ We again emphasize that by "detection" we mean that the plume would produce a bright pixel. Further data would be needed to declare the existence of a large rocket in powered flight.
} 
ICBM model burns out.

Tracking rockets through the trough It is possible that low-luminosity plumes might be lost as they move into the trough region, where their luminosity could become as low as $\sim 3 \mathrm{~kW} \mathrm{sr}{ }^{-1}$ in the SWIR. This potential problem is ameliorated by the fact that a much larger per-pixel false alarm rate can be accepted once a missile's track has been established, because the number of pixels of interest drops from the number in the entire field to the few pixels where the rocket plume might reasonably next appear. Various signal-processing techniques, such as frame-to-frame subtraction, could help to achieve this sensitivity.

If a detailed analysis shows that there is a sensitivity problem for missiles within the trough region, the NSBS could employ a second telescope having a single high-resolution array that slews to track the rocket exhaust plume continuously. In this case, the system could provide position updates with a $30-\mathrm{Hz}$ or greater frame rate, and clutter could be reduced further by using the full diffraction resolution of the telescope. For a 30-cm slewing sensor, the $0.5-\mathrm{km}$ diffraction limit in the SWIR should reduce the clutter signal by a further factor of 4, compared with the postulated survey instrument on NSBS. This 30$\mathrm{cm}$ telescope would be mounted on the same platform as the surveillance system discussed above and independently pointed by a two-axis pointing system. Because the mass of such a telescope would increase at least with the square of its aperture, and its moment of inertia would increase at least with the cube of its aperture, if the size of such an instrument were increased significantly, its pointing motion could seriously perturb the attitude of the main spacecraft. We therefore selected $30 \mathrm{~cm}$ as a reasonable estimate of a feasible aperture.

\subsubsection{Summary of space-based detection and tracking}

From the analysis in this section, we reached the following conclusions.

DSP capabilities We conclude that the DSP could reliably detect rockets by the time they reach an altitude of $10 \mathrm{~km}$, which occurs $35 \mathrm{~s}$ after the launch of our solid-propellant ICBM models S1 and S2 and $52 \mathrm{~s}$ after the launch of our liquid-propellant ICBM model. We shall assume that a firing solution could be constructed using DSP data no earlier than $65 \mathrm{~s}$ after the launch of solid-propellant ICBMs like models S1 and S2 and $65 \mathrm{~s}$ after the launch of liquid-propellant ICBMs like model L. We emphasize that our assumption that interceptors could be fired this early represents a very optimistic bounding case.

Although the DSP system could locate the exhaust plume of an ICBM in three dimensions with a $1 \sigma$-uncertainty of $1 \mathrm{~km}$ once every $10 \mathrm{~s}$ until the rocket entered the trough region, this sampling rate would be too low to support efficient command guidance of boostphase interceptors. Consequently the DSP system likely would have to be supplemented by an off-board sensor with a much higher sampling rate, such as a radar, to enable efficient command guidance.

Modern space-based capabilities In contrast to DSP capabilities, we conclude that a modern space-based launch-detection and missile-tracking system could reliably detect rockets by the time they reach an altitude of $7 \mathrm{~km}$, which occurs $30 \mathrm{~s}$ after the launch of our solidpropellant ICBM models S1 and S2 and $45 \mathrm{~s}$ after the launch of our liquid-propellant ICBM model. We conclude further that a firing solution could be constructed using data from a modern space-based tracking system no earlier than $45 \mathrm{~s}$ after the launch of solid-propellant ICBMs (like models S1 and S2) and $65 \mathrm{~s}$ after the launch of liquid-propellant ICBMs (like 
model L). Again, our assumption that interceptors could be fired this early represents a very optimistic bounding case.

Also in contrast to DSP, a modern space-based launch-detection and missile-tracking system could locate the exhaust plumes of large rockets in three dimensions with a $1 \sigma$ uncertainty of $<300 \mathrm{~m}$ once a second, sufficient to support command guidance of interceptors during their flyout.

\subsection{Radar Tracking}

Ground-, sea-, or air-based radars could be used to supplement space-based IR sensors for detecting and tracking missiles in their boost phase, thus providing additional information and creating a more robust system. Radar can detect a missile as soon as it ascends above the radar's horizon and, like space-based IR sensors, can provide tracking information on both the missile and the interceptor. The radar's sensitivity and accuracy depend on the transmitted power, the cross section for reflection, the antenna properties, and propagation effects. In the following sections, we analyze the performance that radars would need to support boost-phase intercept for ICBMs and evaluate the ability of several currently operational radars to meet these requirements. We also consider the capabilities of enhanced versions of several existing systems. We assume that other sensors are used for target designation, so the radar does not have to perform a wide-area search.

\subsubsection{The power-aperture product required for a search}

For a radar station to detect the launch of a missile within a given volume, the product of its average radiated power $P_{\mathrm{av}}$ and the effective receiving aperture $A_{r}$ must satisfy the search radar equation

$$
P_{\mathrm{av}} A_{r}=\frac{4 \pi \Psi_{s} R_{m}^{4} k T_{s} D L_{s}}{t_{s} \sigma F^{4}}
$$

where $\Psi_{s}$ is the solid angle to be searched, $R_{m}$ is the maximum search range in meters, $k$ is Boltzmann's constant in $\mathrm{J} / \mathrm{K}, T_{s}$ is the system noise temperature in $\mathrm{K}, D$ is the detectability factor (the signal-to-noise power ratio required for detection), $L_{s}$ is the total search loss, $t_{s}$ is the search frame time in seconds, $\sigma$ is the radar cross section (RCS) of the target in $\mathrm{m}^{2}$, and $F$ is the pattern-propagation factor for the path. These quantities have the following interpretations.

Solid angle of search $\boldsymbol{\Psi}_{s}$. This solid angle is defined as the product of the azimuth sector width $A_{m}$ and the sines of the maximum and minimum elevation angles, $\theta_{m}$ and $\theta_{o}$, i.e., $\Psi_{s}=A_{m}\left(\sin \theta_{m}-\sin \theta_{0}\right)$. For a narrow beam above the horizon, $\Psi_{s} \approx A_{m} \theta_{e}$, where $\theta_{e}$ is the elevation beam width. We assume that the search frame time $t_{s}$ is short enough, and the detection probability $P_{d}$ high enough, that the target will be detected before it has passed through the elevation beam width.

Range $R_{m}$. In the case of detection early in the boost-phase, the range can be taken as the distance from the available radar site (often co-located with the interceptor battery) to the target launch site, since the range to the target will change only slightly between launch and the required time of detection. 
System noise temperature $T_{s}$. This temperature is set by the receiver noise figure and the contributions by the atmosphere and the surface, as seen by the antenna. A value of $500 \mathrm{~K}$ may be assumed for a low-noise receiver when the antenna beam grazes the surface.

Detectability factor $D$. This factor must be set to provide a sufficiently high cumulative probability of detection $P_{c}$ before the target passes through the beam. Typically, with a phased-array radar that can scan at an arbitrarily high rate, two scans will be made during the time of target passage. If the probability of detecting on a single scan is $P_{d}=0.9$, then $P_{c}=1-\left(1-P_{d}\right)^{2}=0.99$. This requires $D=20.9=+13.2 \mathrm{~dB}$ on a steady target. For fluctuating targets, a fluctuation loss $L_{f}$ must be included as a component of the search $\operatorname{loss} L_{s}$.

Search loss $L_{s}$. This factor will be evaluated for each radar case discussed below.

Search frame time $t_{s}$. This time will be determined by the vertical velocity profile of the target and the range, as will be discussed later.

Radar cross section of the target $\sigma$. The RCS of various ICBMs are discussed in the following section.

Pattern-propagation factor $F$. This factor depends on the surface and the beam width and elevation angle, but may be assumed unity for a narrow beam elevated to place its lower edge above the horizon.

\subsubsection{Estimated radar cross sections for illustrative ICBMs}

The countries of concern in this study have neither tested nor deployed any ICBMs. The detailed characteristics of the ICBMs they may deploy at some time in the future are therefore unknown. Consequently it is not possible to evaluate the radar requirements for a boost-phase defense system using detailed models. However, a review of available theory and data shows that, with rare exceptions, detection would occur when the aspect angle of the missile is some tens of degrees away from beam aspect. In these intermediate sectors, a missile's RCS depends on the shape of the nose cone and on small features in the missile's shape (e.g., the edges of the booster or engine structure, the presence or absence of fins or antennas, etc.), rather than on the regular scattering from cylindrical or conical surfaces.

We have chosen to estimate the sizes of the radar cross sections that may be encountered and illustrate possible variations with aspect using the RCS of the Chinese DF-5 and the U.S. Minuteman III missile. We have used the average RCS of these missiles at the relevant aspects to estimate the radar performance requirements needed to support boot-phase intercept systems. Outlines of these two ICBMs are shown in Fig. 10.7. The liquid-propellant DF-5 appears to have a slightly rounded tip on a conical nose cone, and a cylindrical twostage booster. The small irregularities along the missile will dominate the RCS in most of the forward hemisphere, whereas scattering from the motors will set the level in the tail hemisphere. A peak in the RCS will appear when the missile is viewed normal to its conical nose surface. The solid-propellant Minuteman III has an ogive nose cone attached to a cylindrical third stage that has the same radius as the second stage. A conical transition connects the second stage to the first stage, which has a larger radius. The ogive surface 

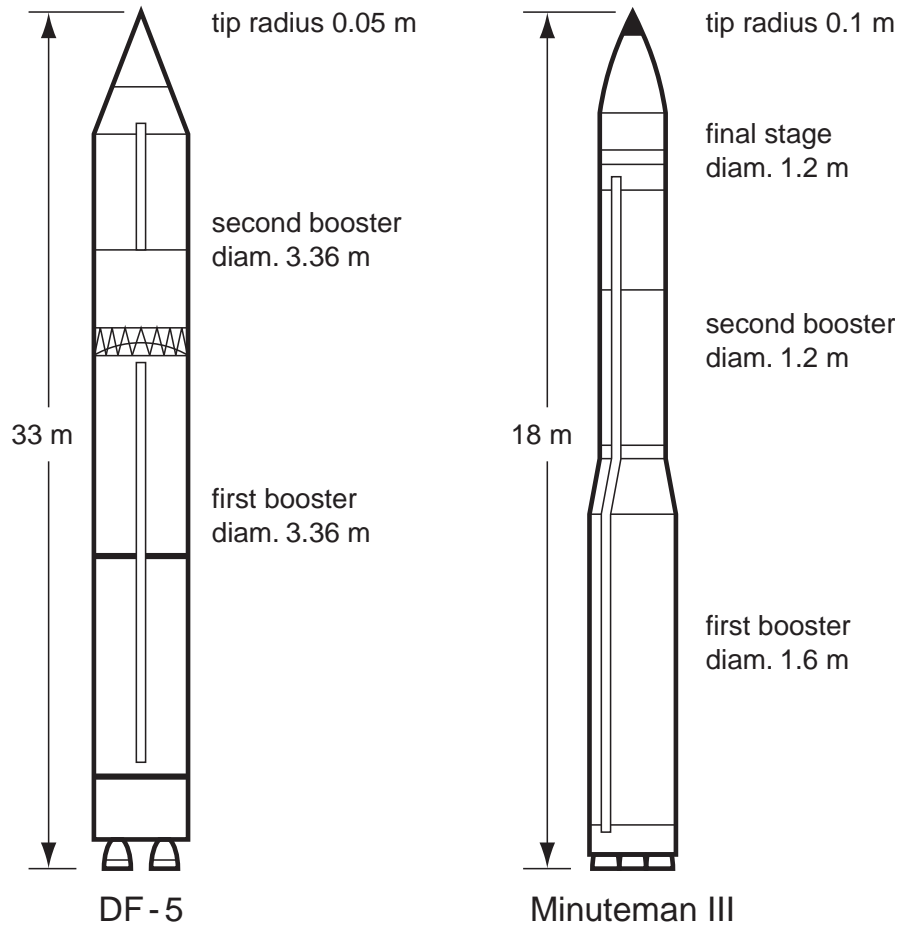

Figure 10.7. Outlines of the two ICBMs used to illustrate missile radar cross sections and analyze radar performance requirements. See [108, 109].

generates a plateau in the RCS just forward of the beam-aspect peak, tending to mask the peak caused by the conical transition section.

Our estimates of the average RCS of the DF-5 and Minuteman III for S-band radars are shown in Fig. 10.8, as a function of aspect angle (from nose aspect). In both cases, the RCS tends toward constant levels, subject to local fluctuations, when the missiles are viewed from the nose and tail hemispheres, which are determined by the nose cone shape and the irregularities visible to the radar. In general, the RCS is larger in the rear hemisphere, where the motor is necessarily exposed. In the nose hemisphere, the RCS during second-stage boost is just greater than $0.1 \mathrm{~m}^{2}$ for both targets. The RCS of the DF- 5 is dominated by irregular features, whereas the RCS of the Minuteman III is dominated by the rounded nose tip. Such details cannot be predicted in advance for future enemy missiles. Consequently, arbitrary assumptions must be made about the average RCS. We shall assume that the S-band RCS is generally between $0.1 \mathrm{~m}^{2}$ and $1 \mathrm{~m}^{2}$ in the nose hemisphere and between $1 \mathrm{~m}^{2}$ and $5 \mathrm{~m}^{2}$ in the tail hemisphere. In all cases, the RCS tends to decrease as stages are discarded.

The ability of a radar system to detect and track a missile during its boost phase depends on the missile's trajectory. The main purpose of the analysis in this chapter is to support the simulations of boost-phase engagements of the slowly rising liquid-propellant ICBM model L and the faster-rising solid-propellant ICBM models S1 and S2, described in Chapter 12 and Appendix C. The properties of the trajectories of these ICBMs that are important for searches by radars were computed using the flyout trajectories described in Chapter 15. Their average RCS at the relevant aspects was estimated as just described. The trajectory properties relevant to searches by land- and ship-based radars are listed in Table 10.1 . 

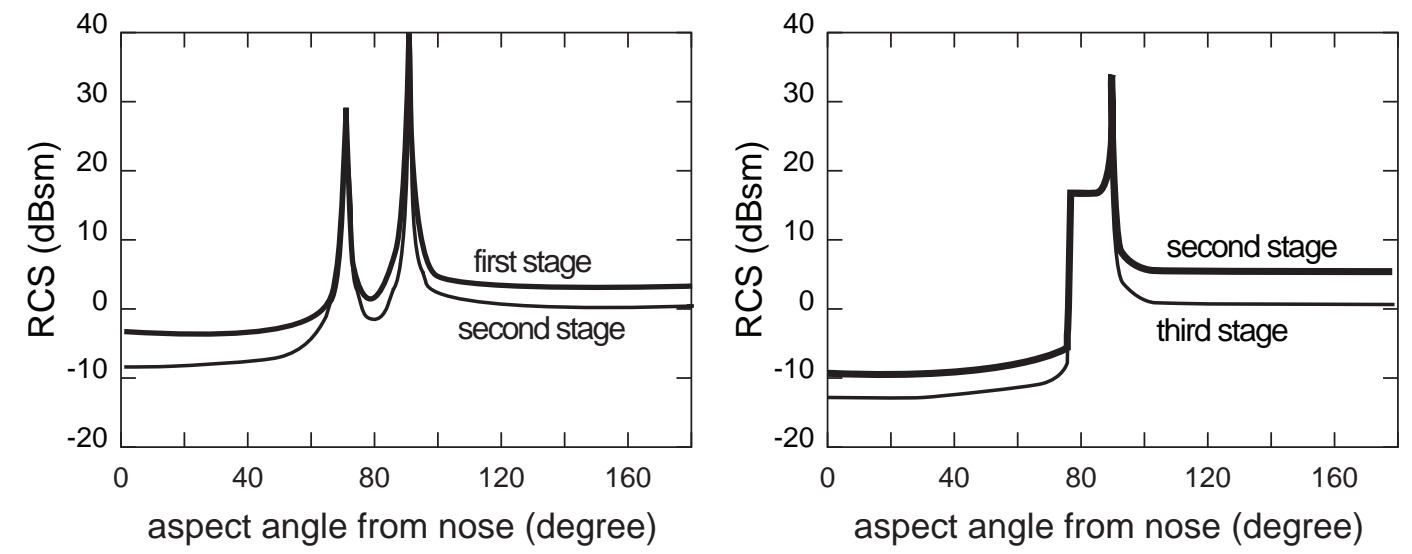

Figure 10.8. Estimated average S-band radar cross sections of the liquid-propellant ICBM model L (left) and the solid-propellant ICBM models S1 and S2 (right) as functions of aspect angle from the nose. (The scale is logarithmic: $\mathrm{dBsm}=10 \log _{10}\left(\operatorname{radar}\right.$ cross section $\left./ 1 \mathrm{~m}^{2}\right)$

Table 10.1. ICBM Trajectory Parameters for Land- and Ship-Based Radars

\begin{tabular}{lcc}
\hline \hline Parameter & Liquid & Solids \\
\hline Standoff distance from radar site to missile launch site $(\mathrm{km})$ & 800 & 1,000 \\
Time from launch to radar horizon $(\mathrm{s})$ & 106 & 87 \\
Maximum horizon range from radar $(\mathrm{km})$ & 747 & 887 \\
Vertical velocity at horizon $(\mathrm{km} / \mathrm{s})$ & 0.8 & 1.3 \\
Pitch angle at horizon (deg from horizontal) & 28 & 23 \\
Aspect angle at horizon (deg from nose) & 34 & 31 \\
Elevation rate at horizon $(\mathrm{rad} / \mathrm{s})$ & 0.0011 & 0.0015 \\
\hline
\end{tabular}

The elevation rates and scan times for the radars we considered were computed as described in Appendix $\mathrm{C}$ from the flyout trajectories described in Chapter 15. The curvature of Earth blocks distant ground- and ship-based radars from seeing missiles early enough to affect the decision to launch interceptors, but these radars could provide tracking data later in the missile's boost phase and hence could potentially contribute to command guidance of the interceptor during its boost phase and to the initial phase of the kill vehicle's flight. A forward-based phased-array airborne radar could provide launch detection as well as tracking data.

\subsubsection{Land-based radars}

The THAAD radar The THAAD multifunction X-band radar is designed for use in the Theater High-Altitude Area Defense (THAAD) system. This U.S. Army ground-based radar is an air-transportable system intended for both search and fire control. Its performance has been demonstrated over the past several years at White Sands Missile Range. It is analyzed here as the fire control element in a possible boost-phase missile defense system, where it would receive target designation data from an external source. This analysis is 
Table 10.2. Theater High-Altitude Area Defense (THAAD) Radar Characteristics ${ }^{a}$

\begin{tabular}{lr}
\hline \hline Parameter & Value \\
\hline Operating Frequency & $9.5 \mathrm{GHz}$ \\
Wavelength & $3.2 \mathrm{~cm}$ \\
Physical aperture area & $9.2 \mathrm{~m}^{2}$ \\
Effective aperture area & $6 \mathrm{~m}^{2}$ \\
Number of active elements & 25,344 \\
Receiving gain (with weighting) & 76,000 \\
Azimuth beam width & $0.6^{\circ}$ \\
Elevation beam width & $0.8^{\circ}$ \\
Scan sector & $90^{\circ}$ \\
Search solid angle (one line) & $0.011 \mathrm{sr}$ \\
Module peak power & $10 \mathrm{~W}$ \\
Module average power & $2.1 \mathrm{~W}$ \\
Total average power & $54 \mathrm{~kW}$ \\
Power-aperture product & $324 \mathrm{kWm}$ \\
Noise temperature & $500 \mathrm{~K}$ \\
Equipment loss (beam center) & $2.8 \mathrm{~dB}$ \\
Extra loss in search & $7.2 \mathrm{~dB}$ \\
Atmospheric loss & $b, c$ \\
Total search loss & $5.5 \mathrm{~dB}$ \\
\hline
\end{tabular}

${ }^{a}$ See $[110]$ and [111].

${ }^{b}$ At $0.5^{\circ}$ elevation for $90^{\circ}$ scan sector. Additional loss may apply to longer dwells in small sectors.

${ }^{c}$ For clear-air conditions.

based on open-source literature and open-source information briefed to the Study Group. The estimated performance characteristics of the THAAD radar are listed in Table 10.2.

As shown in Table 10.3, the THAAD radar, with a power-aperture product of $324 \mathrm{kWm}^{2}$, meets the requirement for detection at the horizon of the slower liquid-propellant ICBM

Table 10.3. THAAD Performance Requirements for ICBM Searches

\begin{tabular}{lcc}
\hline \hline Parameter & Liquid & Solids \\
\hline Target nose-aspect RCS at horizon $\left(\mathrm{m}^{2}\right)$ & 0.45 & 0.094 \\
Time in THAAD elevation beam width $(\mathrm{s})$ & 9.0 & 6.0 \\
Allowable search frame time for two scans on the targets $(\mathrm{s})$ & 4.5 & 3.0 \\
Required power-aperture product $\left(\mathrm{kW} \mathrm{m}^{2}\right)$ & 53 & 602 \\
\hline
\end{tabular}


model at a standoff distance of $800 \mathrm{~km}$ and is within $2.7 \mathrm{~dB}$ for detection of the faster solidpropellant ICBM models at a standoff distance of 1,000 km, under clear-air conditions.

An enhanced THAAD radar The THAAD radar is close to meeting the requirements for detecting and tracking the liquid- and solid-propellant ICBM models at standoff distances of $800-1,000 \mathrm{~km}$. It is therefore reasonable to postulate that a minor enhancement, either through use of higher module average power or a slightly larger aperture, would support horizon detection from a radar co-located with the maximum-range interceptor launcher. The enhanced radar would presumably remain air-transportable. Allowing for non-clear air conditions, e.g., intervening rain between the radar and the target, would require several more decibels. Such enhanced capability could be accomplished by a doubling of aperture area and number of modules which might require larger aircraft for transportation.

\subsubsection{Shipboard radars}

The AN/SPY-1B (Aegis) radar is a reference point for shipboard anti-ballistic missile $(\mathrm{ABM})$ radars. Its estimated performance characteristics are listed in Table 10.4. We estimated the performance needed to search for and track the liquid-propellant model ICBM at a standoff distance of $800 \mathrm{~km}$ from the ICBM launch site and the solid-propellant models at a standoff distance of $1,000 \mathrm{~km}$. The relevant ICBM trajectory parameters are listed in Table 10.1. The AN/SPY-1B performance requirements are listed in Table 10.5.

Assuming that the entire resources of the radar are available for a horizon search, the available $700-\mathrm{kW} \mathrm{m}^{2}$ power-aperture product of AN/SPY-1B, a multi-function radar of the U.S. Aegis system, is adequate to provide horizon detection at the maximum interceptor standoff distance for the slower liquid-propellant ICBM and for the faster solid-propellant ICBMs. To meet the requirement for the solid-propellant ICBMs and provide some margin for operational flexibility, an enhanced Aegis radar with a 3- to 6-dB increase in the poweraperture product could be used.

\subsubsection{Airborne radars}

The AWACS AN/APY-2 airborne radar The existing AWACS AN/APY-2 airborne Sband radar provides a baseline for considering launch detection and tracking of missiles by airborne radars. Table 10.6 lists the estimated performance characteristics of this radar, and Table 10.7 shows the requirements for detecting the liquid-propellant ICBM model at the horizon for a standoff distance from the aircraft to the missile launch site of $800 \mathrm{~km}$ and the solid-propellant ICBM models for a standoff distance of $1,000 \mathrm{~km}$. The RCS seen by AWACS differs slightly from that by the Aegis because the missile attitude and aspect angle differ for the horizon as seen from the elevated radar.

The AWACS scans a full $360^{\circ}$ in azimuth and has an elevation beam width of $5^{\circ}$, making its search solid angle 13 times greater than that of the AN/SPY-1B, even if the latter were to scan a $90^{\circ}$ sector. Consequently, the power-aperture requirements for detection at the horizon at the maximum interceptor standoff distance are unreasonable. We therefore computed the performance requirements for a more realistic standoff distance of $300 \mathrm{~km}$ from the aircraft to the target missile's launch site. The radar would provide coverage to the surface and would therefore see ground clutter, including moving surface vehicles. Thus a Doppler rejection notch would have to be applied to reject targets moving at radial velocities less than about $40 \mathrm{~m} / \mathrm{s}$. The resulting delays in target detection and the minimum altitudes 
Table 10.4. AN/SPY-1B Radar Characteristics ${ }^{a}$

\begin{tabular}{lr}
\hline \hline Parameter & Value \\
\hline Operating Frequency & $3.3 \mathrm{GHz}$ \\
Wavelength & $9.1 \mathrm{~cm}$ \\
Physical aperture area & $12 \mathrm{~m}^{2}$ \\
Effective aperture area & $12 \mathrm{~m}^{2}$ \\
Number of active elements & 5,600 \\
Receiving gain (with weighting) & 14,000 \\
Azimuth beam width & $1.6^{\circ}$ \\
Elevation beam width & $1.5^{\circ}$ \\
Scan sector & $90^{\circ}$ \\
Search solid angle (one line) & $0.020 \mathrm{sr}$ \\
Total average power & $58 \mathrm{~kW}$ \\
Power-aperture product & $700 \mathrm{k} \mathrm{m}{ }^{2}$ \\
Noise temperature & \\
Equipment loss (beam center) & $500 \mathrm{~K}$ \\
Extra loss in search & $2.8 \mathrm{~dB}$ \\
Atmospheric loss ${ }^{b, c}$ & $7.2 \mathrm{~dB}$ \\
Total search loss ${ }^{b}$ & $3.2 \mathrm{~dB}$ \\
\hline${ }^{a}$ See [112]. & $13.2 \mathrm{~dB}$ \\
${ }^{b}$ At 0.5 elevation. & \\
${ }^{c}$ For clear-air conditions. &
\end{tabular}

Table 10.5. AN/SPY-1B Performance Requirements for ICBM Searches

\begin{tabular}{lcc}
\hline \hline Parameter & Liquid & Solids \\
\hline Target nose-aspect RCS at horizon $\left(\mathrm{m}^{2}\right)$ & 0.48 & 0.1 \\
Time in Aegis elevation beam width $(\mathrm{s})$ & 16 & 11 \\
Allowable search frame time for two scans on the targets $(\mathrm{s})$ & 8 & 5.5 \\
Required power-aperture produce $\left(\mathrm{kW} \mathrm{m}^{2}\right)$ & 58 & 708 \\
\hline
\end{tabular}

for detection would be as shown in Table 10.8. Additional delay averaging $t_{s} / 2=6 \mathrm{~s}$ would result from the slow scan rate, but detection could be early enough to provide the initial warning, and subsequent tracking in range and azimuth could support an interceptor launch decision. Only coarse elevation data would be available from this radar. Consequently, it could not support command guidance of the interceptor or kill vehicle. The radar's performance is more than $10 \mathrm{~dB}$ below the minimum performance to detect and track the rapidly accelerating solid-propellant ICBM models. 
Table 10.6. AN/APY-2 Radar Characteristics

\begin{tabular}{|c|c|}
\hline Parameter & Value \\
\hline Operating Frequency & $3.3 \mathrm{GHz}$ \\
\hline Wavelength & $9.1 \mathrm{~cm}$ \\
\hline Physical aperture area & $6 \mathrm{~m}^{2}$ \\
\hline Effective aperture area & $3.3 \mathrm{~m}^{2}$ \\
\hline Receiving gain (with weighting) & 5,000 \\
\hline Azimuth beam width & $1.0^{\circ}$ \\
\hline Elevation beam width & $5.0^{\circ}$ \\
\hline Scan sector & $360^{\circ}$ \\
\hline Search solid angle (one line) & $0.55 \mathrm{sr}$ \\
\hline Total average power & $30 \mathrm{~kW}$ \\
\hline Power-aperture product & $100 \mathrm{~kW} \mathrm{~m}^{2}$ \\
\hline Noise temperature $^{a}$ & $650 \mathrm{~K}$ \\
\hline Equipment loss (beam center) & $9.0 \mathrm{~dB}$ \\
\hline Extra loss in search & $5.0 \mathrm{~dB}$ \\
\hline Atmospheric $\operatorname{loss}^{a, b}$ & $3.2 \mathrm{~dB}$ \\
\hline Total search loss ${ }^{a}$ & $17.2 \mathrm{~dB}$ \\
\hline
\end{tabular}

${ }^{a}$ For $0.5^{\circ}$ elevation.

${ }^{b}$ For clear-air conditions.

Table 10.7. AN/APY-2 Performance Requirements for ICBM Searches

\begin{tabular}{lcc}
\hline \hline Parameter & Liquid & Solids \\
\hline Standoff distance $(\mathrm{km})$ & 800 & 1,000 \\
Target nose-aspect RCS at horizon $\left(\mathrm{m}^{2}\right)$ & 0.59 & 0.1 \\
Elevation rate at horizon $(\mathrm{rad} / \mathrm{s})$ & 0.0005 & 0.0009 \\
Time in AWACS elevation beam width $(\mathrm{s})$ & 63 & 48 \\
Search frame time (fixed) (s) & 12 & 12 \\
Required power-aperture product $\left(\mathrm{kW} \mathrm{m}^{2}\right)$ & 25,000 & 480,000 \\
\hline
\end{tabular}

A phased-array AWACS radar The performance characteristics of a postulated S-band phased-array AWACS radar are shown in Table 10.9. The major advantages of the array are the larger aperture, the ability to hold the scan sector within $90^{\circ} \times 1.6^{\circ}$, to perform the scan at any required rate, and to provide subsequent tracking data in all three coordinates. The required power-aperture products for the two ICBM models as they rise above the horizon at their maximum standoff distances are shown in Table 10.10. The performance needed to detect and track the slower-rising liquid-propellant ICBM at a standoff distance of $800 \mathrm{~km}$ is well within the postulated $1,100 \mathrm{~kW} \mathrm{~m}^{2}$. Even at a standoff distance of $1,400 \mathrm{~km}$, the radar would still have a 3 -dB performance margin.

The postulated phased-array AWACS would be capable of detecting the liquid-propellant 
Table 10.8. AN/APY-2 Performance Requirements for Searches at $300 \mathrm{~km}$

\begin{tabular}{lcc}
\hline \hline Parameter & Liquid & Solid \\
\hline Time from launch to minimum radial velocity $(\mathrm{s})$ & 18 & 4 \\
Target altitude for minimum radial velocity $(\mathrm{km})$ & 1.1 & 0.1 \\
Vertical velocity at that altitude $(\mathrm{km} / \mathrm{s})$ & 0.11 & 0.05 \\
Pitch angle at that altitude $($ deg from nose) & 72 & 45 \\
Aspect angle at that altitude $(\operatorname{deg}$ from nose) & 72 & 43 \\
Target nose-aspect RCS at horizon $\left(\mathrm{m}^{2}\right)$ & 1.6 & 0.1 \\
Time in AWACS elevation beam width $(\mathrm{s})$ & 87 & 60 \\
Search frame time (fixed) (s) & 12 & 12 \\
Required power-aperture product $\left(\mathrm{kW} \mathrm{m}^{2}\right)$ & 77 & 2000 \\
\hline
\end{tabular}

Table 10.9. A Phased-Array AWACS Radar

\begin{tabular}{|c|c|}
\hline Parameter & Value \\
\hline Operating Frequency & $3.3 \mathrm{GHz}$ \\
\hline Wavelength & $9.1 \mathrm{~cm}$ \\
\hline Physical aperture area & $35 \mathrm{~m}^{2}$ \\
\hline Effective aperture area & $21 \mathrm{~m}^{2}$ \\
\hline Number of active elements & 10,000 \\
\hline Receiving gain (with weighting) & 32,000 \\
\hline Azimuth beam width & $0.57^{\circ}$ \\
\hline Elevation beam width & $1.6^{\circ}$ \\
\hline Scan sector & $90^{\circ}$ \\
\hline Search solid angle (one line) & $0.022 \mathrm{sr}$ \\
\hline Module peak power & $10 \mathrm{~W}$ \\
\hline Module average power & $2.1 \mathrm{~W}$ \\
\hline Total average power & $50 \mathrm{~kW}$ \\
\hline Power-aperture product & $1100 \mathrm{~kW} \mathrm{~m}{ }^{2}$ \\
\hline Noise temperature $^{a}$ & $650 \mathrm{~K}$ \\
\hline Equipment loss (beam center) & $2.8 \mathrm{~dB}$ \\
\hline Extra loss in search & $7.0 \mathrm{~dB}$ \\
\hline Atmospheric loss ${ }^{a, b}$ & $3.2 \mathrm{~dB}$ \\
\hline Total search loss ${ }^{a}$ & $13.0 \mathrm{~dB}$ \\
\hline
\end{tabular}

${ }^{a}$ At $0.5^{\circ}$ elevation for $90^{\circ}$ scan sector. Additional loss may apply to longer dwells in small sector.

${ }^{b}$ For clear-air conditions.

ICBM model L from an aircraft at a standoff distance of $800 \mathrm{~km}$, and the solid-propellant ICBM models S1 and S2 at a standoff distance of $1,000 \mathrm{~km}$. It is therefore worthwhile to 
Table 10.10. Phased-Array AWACS Performance Requirements for ICBM Searches

\begin{tabular}{lcc}
\hline \hline Parameter & Liquid & Solids \\
\hline Target nose-aspect RCS at horizon $\left(\mathrm{m}^{2}\right)$ & 0.59 & 0.1 \\
Elevation rate at horizon $(\mathrm{rad} / \mathrm{s})$ & 0.0005 & 0.0009 \\
Time in AWACS elevation beam width $(\mathrm{s})$ & 37 & 20 \\
Allowable search frame time for two scans on the targets $(\mathrm{s})$ & 18.5 & 10 \\
Required power-aperture product $\left(\mathrm{kW} \mathrm{m}^{2}\right)$ & 22 & 793 \\
\hline
\end{tabular}

determine the times after target launch and the altitudes at which these detections would occur.

- Liquid-propellant ICBM

At a standoff distance of $800 \mathrm{~km}$, detection would become possible when the liquidpropellant ICBM model reached an altitude of $11 \mathrm{~km}, 55 \mathrm{~s}$ after launch. The phasedarray AWACS radar therefore could not contribute to warning of the launch of this missile if an IR sensor system like the NSBS described in Section 10.1.3 were available, because the NSBS would provide a launch warning about $50 \mathrm{~s}$ after the launch. If, however, only the DSP satellites were available, the phased-array AWACS could provide the launch warning, because the DSP system likely could not do so until 10-20 s later.

The phased-array AWACS radar could make only a marginal contribution to deciding whether to fire interceptors, if the firing doctrine were to fire as soon as a firing solution could be constructed and if a system like the NSBS were available. A firing solution could probably be constructed using only NSBS data about $65 \mathrm{~s}$ after the missile were launched. If, however, only the DSP satellites were available or the firing doctrine was to wait to fire interceptors until some time after a firing solution were first obtained, the phased-array AWACS radar could contribute to the decision to fire.

At standoff distances shorter than $800 \mathrm{~km}$, the phased-array AWACS radar could by itself provide launch detection and the tracking data needed to decide whether to launch an interceptor.

- Solid-propellant ICBMs

At a standoff distance of $1,000 \mathrm{~km}$, detection would become possible when the solidpropellant ICBM models reached an altitude of $29 \mathrm{~km}, 55 \mathrm{~s}$ after launch. The phasedarray AWACS radar therefore could not contribute to warning of the launch of these missiles if an IR sensor system like the NSBS, described in Section 10.1.3, were available, because the NSBS would provide a launch warning about $35 \mathrm{~s}$ after the launch. Even the DSP satellites would likely be able to provide a launch warning by about $50 \mathrm{~s}$ after launch.

The phased-array AWACS radar could not contribute significantly to deciding whether to fire interceptors, if the firing doctrine were to fire as soon as a firing solution could be constructed and a system like the NSBS were available. A firing solution could 
probably be constructed using only NSBS data about $45 \mathrm{~s}$ after solid-propellant missiles like these were launched. If, however, only the DSP satellites were available or the firing doctrine was to wait to fire interceptors until some time after a firing solution were first obtained, the phased-array AWACS radar could potentially contribute to the decision to fire. If the phased-array AWACS radar could be stationed somewhat closer to the missile launch site, it could contribute to the decision whether to fire interceptors. At standoff distances of $750 \mathrm{~km}$ or less, detection would occur early enough to support an interceptor launch decision.

The above considerations of performance of a phased-array AWACS system are based on a postulated system. However such a system is not likely to be available within the ten-year time horizon of this study and hence we do not consider such a system in our findings.

\subsubsection{Sources of error in interceptor guidance using radars}

Radar tracking errors Radar tracking can be initiated after the target has been detected by the radar operating in a search mode, or after it has been detected by an external sensor if the target is above the radar horizon. In the latter case, a tracking radar or phased array need scan only a small solid angle to acquire the target, which extends the acquisition range beyond the range that would apply if a larger sector had to be searched. For command guidance of an interceptor in an engagement in which both the target and the interceptor are tracked by the radar, many error components can be neglected because they are correlated between the two tracks, and hence will not affect their relative position and the ability of the radar to support target acquisition by the on-board seeker. The remaining errors to be estimated are as follows.

Thermal noise For an adequate single-pulse signal-to-noise ratio $(S / N>4)$ this error is

$$
\sigma_{\theta t}=\frac{\theta_{3}}{k_{m} \sqrt{2(S / N) n}},
$$

where $\theta_{3}$ is the half-power beam width, $k_{m} \approx 1.5$ is the monopulse error slope constant, and $n$ is the number of pulses integrated in the tracking loop time constant. Errors for data points separated by more than this constant will be uncorrelated.

Fluctuating target noise This additional noise component results from the use of singlepulse normalization of the monopulse error signal on a fluctuating target [113, p. 412]. It can be approximated by the relationship

$$
\sigma_{\theta f}=\frac{\theta_{3}}{2(S / N+5)^{1.5}}
$$

Errors for data points separated by more than the time constant will be uncorrelated. There may be signal processing techniques that would permit this error component to be reduced, especially if the tracking of a single target is allocated most of the radar's resources (average power and time), as has been assumed here. However, if the resources allocated to tracking a given target are less than about 50 percent of the total, the errors calculated here are optimistic. 
Phase quantizing noise The digital control of phase shifters with $m$-bit quantization introduces an angle error [114, p. 197]

$$
\sigma_{\theta q}=\frac{1.12 \theta_{3}}{2^{m} \sqrt{n T}}
$$

where $T$ is the number of phase-shifting elements in the array. The single-pulse variance of errors uncorrelated at each new beam-steering command is reduced by $n$, the number of beam positions during the tracking-loop time constant.

Multipath In elevation tracking of a target at angle $\theta_{t}$ within a few beam widths of the horizon, surface reflections introduce a multipath error [113, p. 526], which can be approximated by

$$
\sigma_{\theta m}=\frac{0.02 \theta_{e}}{\left(\theta_{t} / \theta_{e}\right)^{2}}, \quad \theta_{t}>\theta_{e} / 4
$$

where $\theta_{e}$ is the half-power beam width in elevation. Below $\theta_{e} / 4$, the track becomes indeterminate and no specific error can be defined. This error will be correlated over periods measured in seconds, and hence cannot be reduced by smoothing over intervals useful in guidance applications.

Tropospheric refraction A bias error in elevation due to tropospheric refraction can be corrected using a measured value of refractivity at the radar site. A residual error of 5 percent of the original bias can be expected, approximated by [113, p. 306]

$$
\sigma_{\theta_{p}}=N_{s} \cot \theta_{t}\left(\frac{h_{t}}{h_{t}+11}\right) \operatorname{rad} \approx 0.313 \cot \theta_{t}\left(\frac{h_{t}}{h_{t}+11}\right) \mathrm{mr}
$$

where $N_{s}$ is the surface refractivity in $N$-units and $h_{t}$ is the target altitude in $\mathrm{km}$.

Tropospheric fluctuation Random inhomogeneities in the troposphere will cause slow fluctuations in the measured angle of arrival in both coordinates, approximated by [113, p. 369]

$$
\sigma_{\mathrm{off}}=\frac{0.44 \times 10^{-3} \sqrt{R_{a}}}{h_{a}^{1 / 4}},
$$

where $R_{a}$ is the length of the tropospheric path and $h_{a}$ is the vertical extent of the antenna aperture, both measured in meters.

These six error components are uncorrelated; the total elevation error is the square root of the sum of squares of these errors. Four of these components will similarly add to determine the total azimuth error.

\subsubsection{Error analysis for selected radar systems}

THAAD Using the THAAD radar parameters given in Tables 10.1 and 10.2, the angle tracking errors for the target liquid-propellant and solid-propellant ICBM models have been calculated with the results shown in Fig. 10.9. The increased error for the liquid-propellant ICBM model at $t=120 \mathrm{~s}$ is caused by the reduction in its RCS when its first stage is discarded. 

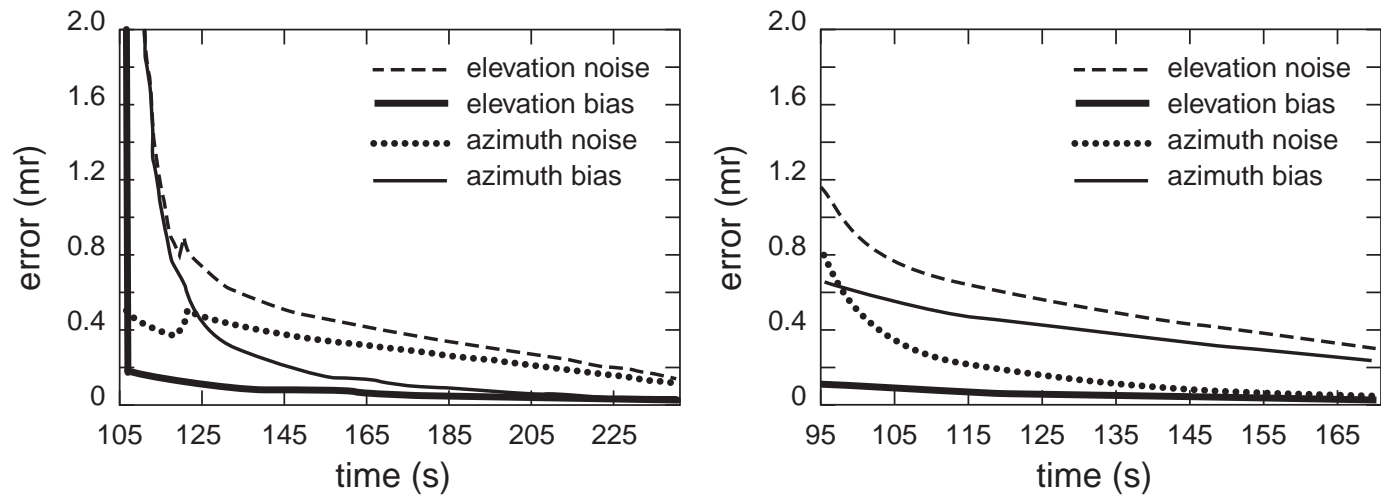

Figure 10.9. THAAD tracking errors vs. time for the liquid-propellant ICBM model (left) and the solid-propellant ICBM models (right).
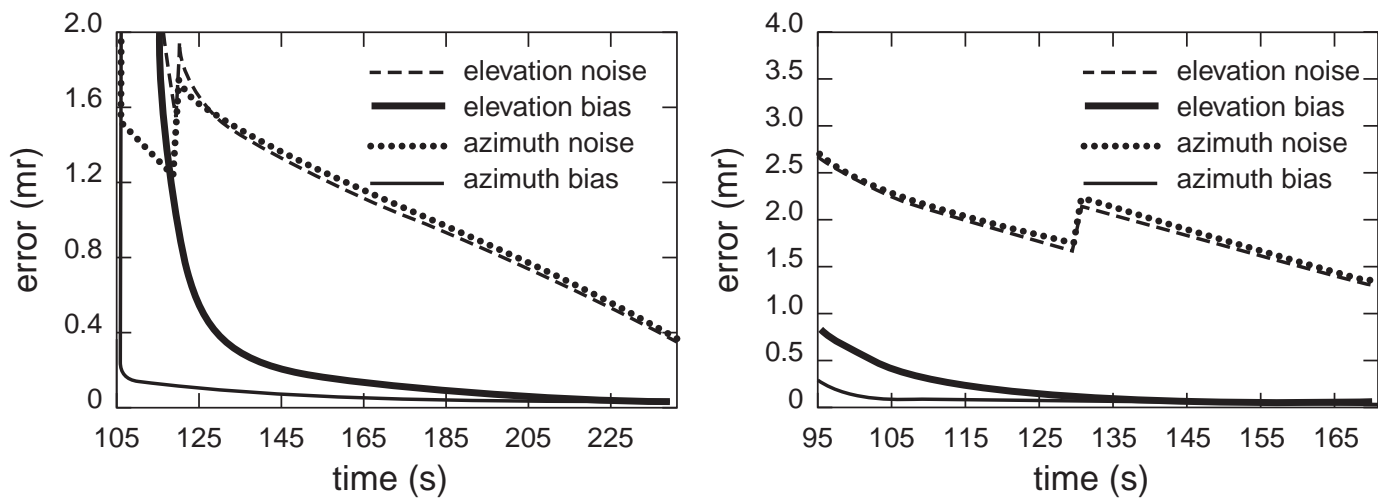

Figure 10.10. Aegis tracking errors vs. time for the liquid-propellant ICBM model (left) and the solid-propellant ICBM models (right).

AN/SPY-1B Using the AN/SPY-1B parameters given in Tables 10.4 and 10.5, the angle tracking errors for the liquid- and solid-propellant ICBM models were computed for a 1second tracking loop time constant, with the results shown in Fig. 10.10.

AWACS Phased array radar Using the parameters given in Tables 10.9 and 10.10 , the angle tracking errors for the slow-burn and fast-burn targets have been calculated, also for 1-second tracking loop time constant, with the results shown in Fig. 10.11. In all cases, the large initial noise errors are caused by the low signal-to-noise ratio entering into Eqs. 10.2 and 10.3, whereas the large initial elevation bias are caused by multipath propagation and the uncorrected portion of tropospheric refraction.

\subsubsection{Countermeasures to radar tracking}

All of the ICBMs considered here would be detectable to any of the radars discussed when the missiles rise above the horizon; hence a logical countermeasure would be to include in the final stage of the missile one or more barrage noise jammers. Such jammers would deny range information to the radar, forcing it to track in angle only and to rely on triangulation 

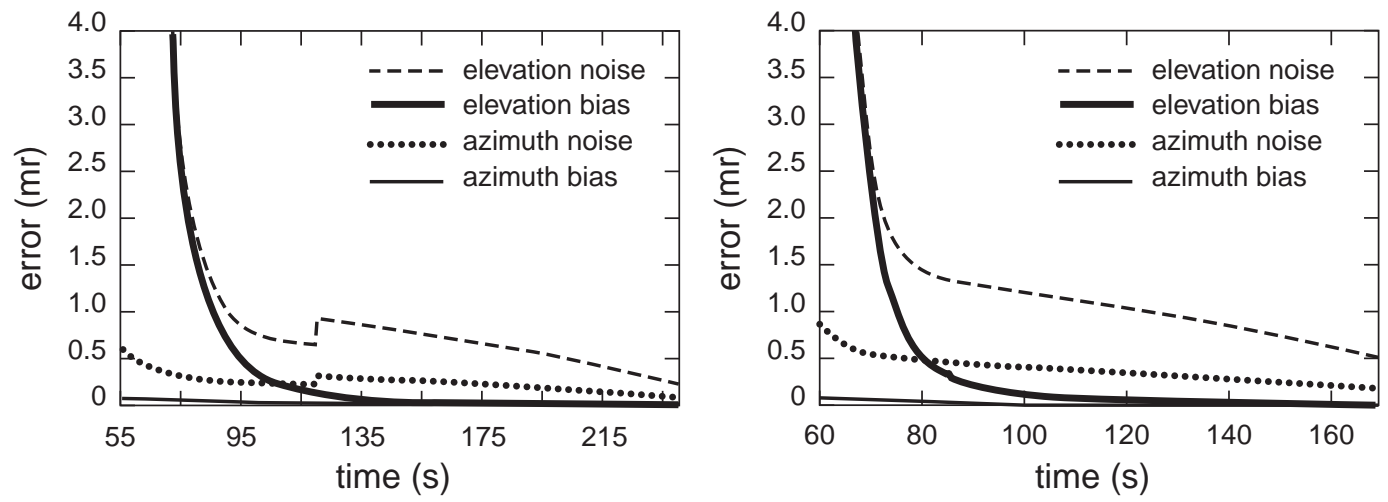

Figure 10.11. AWACS phased array radar tracking errors vs. time for the liquid-propellant ICBM model (left) and the solid-propellant ICBM models (right).

with another sensor to determine the range for command guidance of the interceptor during its flyout.

The intent of the barrage jammer is to raise the noise level in the radar receiver high enough to prevent detection of the echo signal. Since the required signal-to-noise ratio for radar detection is normally about a factor of 100 , i.e., $+20 \mathrm{~dB}$, introduction of broadband noise jamming at a level $20 \mathrm{~dB}$ above the receiver noise would be sufficient to prevent detection of the echo signal. The equation for jamming power density in the radar receiver is

$$
J_{0}=\left(P_{j} G_{j} / B_{j}\right) \times\left(A_{r} / 4 \pi R_{j}^{2}\right)
$$

where $P_{j}$ is the jammer power, $G_{j}$ is the jammer antenna gain toward the radar, $B_{j}$ is the jammer noise bandwidth, $A_{r}$ is the radar receiving aperture, and $R_{j}$ is the range of the jammer from the radar. It is assumed that the radar beam is pointed directly toward the jammer, and losses are neglected.

Consider a small jammer package with the following characteristics: $P_{j}=10 \mathrm{~W} ; \quad G_{j}=$ $10 ;$ and $B_{j}=300 \mathrm{MHz}$.

When operating against the Aegis AN/SPY-1B, with $A_{r}=9 \mathrm{~m}^{2}$ from a range $R_{j}=$ $740 \mathrm{~km}$, the jamming density would be $J_{0}=4.36 \times 10^{-19} \mathrm{~W} / \mathrm{Hz}=-183.5 \mathrm{~dB}(\mathrm{~W} / \mathrm{Hz})$ compared with a noise level $N_{0}=k T_{s}=\left(1.38 \times 10^{-23} \mathrm{~W} / \mathrm{Hz} \mathrm{K}\right) \times 500 \mathrm{~K}=6.9 \times 10^{-21} \mathrm{~W} / \mathrm{Hz}=$ $-201.6 \mathrm{~dB}(\mathrm{~W} / \mathrm{Hz})$. The jamming-to-noise ratio for this case is a factor of 63 , i.e., $+18 \mathrm{~dB}$, preventing echo detection.

If a greater margin of performance were considered necessary, the low jammer power of $10 \mathrm{~W}$ could readily be increased without placing a burden on the jammer installation. The 10-dB antenna gain could be obtained with a simple cavity antenna, flush-mounted on the surface of the final stage and covering a sector $90^{\circ}$ in azimuth by $45^{\circ}$ in elevation. This tactic would obviate the necessity of knowing the radar's location, while the use of the $300-\mathrm{MHz}$ barrage centered on the known frequency band of the Aegis radar would eliminate the need for any special intelligence about the radar's frequency or waveform. Since the antenna aperture of the Aegis radar is well known, such a jammer could be designed with high confidence. 


\subsubsection{Forward-based radar}

All of the radar systems discussed in Sections 10.2.3-10.2.5 have significant limitations unless they can be placed within a range of $300 \mathrm{~km}$ from all potential ICBM launch sites. Because Iran has a large land area, it could launch missiles from sites many hundreds of kilometers inside its borders. Even radars positioned close to Irans borders would be blocked from seeing missiles launched from such sites by the horizon until well after they could be detected and tracked by space-based detection and tracking systems, diminishing the radars usefulness. In contrast, North Korea has a relatively small land area and borders international waters. We therefore focused on the possible contribution that radars deployed near North Korea could contribute to early detection and tracking of missiles. The closest that radars could be stationed to all possible launch sites in North Korea and be consistent with the requirement that they be at least $100 \mathrm{~km}$ from hostile territory is about $300 \mathrm{~km}$. Examination of tables in Sections 10.2.3-10.2.5, plus consideration of noise and multipath effects, shows that radars at this distance would not significantly advance the time at which an ICBM launch could be tracked reliably relative to tracking by a space-based system.

\subsection{Sensors on the Kill Vehicle}

Using passive ultraviolet (UV), short-wavelength infrared (SWIR), or medium-wavelength infrared (MWIR) sensors on the kill vehicle to track the exhaust plume of the target missile could supplement tracking by space-based sensors during the initial fly-out of the interceptor and kill vehicle and could supplement or perhaps even replace radar tracking, once the kill vehicle has reached sufficient altitude for these sensors to operate (see [97, 115]). The IR emission of a rocket exhaust plume is more easily detected by a sensor on the kill vehicle than by the same sensor on a satellite in GEO because even at the largest standoff distance of interest $(1,000 \mathrm{~km})$, the kill vehicle is still 40 times closer to the target than a satellite in GEO. The IR flux at the kill vehicle is therefore 1600 times greater than at the satellite.

As it closes on its target, the kill vehicle must shift from homing on the plume to homing on the rocket body (or the warhead, if the intent is to destroy the warhead). This shift is called the plume-to-hardbody handover problem. For ICBMs launched by the states of concern for this study, knowledge of the characteristics of the ICBM and of the phenomenology of its plume may well be inadequate to allow the kill vehicle to home reliably on the rocket body using only images of the plume to determine the correct aim point. One alternative would be to use long-wavelength infrared (LWIR) sensors on board the kill vehicle to detect and image the rocket body's thermal emission. The radiation would have to be detected in the presence of the background LWIR emission from the plume. Another alternative would be to use active sensing by the kill vehicle to locate the rocket body. Possibilities include an optical illuminator, as suggested by Postol [116, 117], or a LIDAR system. An advantage of LIDAR is that it would provide range as well as angle information.

The approach we took in analyzing engagements for this study was to analyze a minimal set of sensors that could reasonably be expected to support successful intercepts by the boost-phase intercept systems that we considered, rather than to attempt an analysis of all possibilities. We first discuss the general phenomenology of rocket exhaust plumes at the high altitudes relevant for kill-vehicle homing and the structure of high-altitude plume emission in the MWIR and SWIR. To illustrate the potential contribution of passive sensing by detectors on the kill vehicle, we analyze a system that uses an SWIR seeker cued initially 
by a space-based launch warning and tracking system like DSP or a follow-on system like the NSBS described in Section 10.1. We show that an on-board SWIR seeker could measure the brightness distribution of the plume with a precision adequate to allow handover to another, shorter-range system designed to resolve and track the rocket hardbody from a range of $100 \mathrm{~km}$ until intercept.

Medium-wavelength infrared sensors may have advantages over SWIR sensors. In particular, at high altitudes the intensity of the MWIR emission diminishes uniformly from the nozzle exit plane, facilitating location of the rocket body. Ultraviolet sensors may also have advantages (see Chapter 12 of Ref. [98]), but the performance of UV sensors is more difficult to analyze using only open-source information. We have chosen to consider an SWIR sensor on the kill vehicle because such a sensor is simpler and, as we show below, appears adequate to allow an on-board LIDAR to locate the rocket body. In practice, a suite of UV, MWIR, and SWIR sensors would probably be more robust, especially if the kill vehicle must confront a variety of plume spectra and structures with little advance information.

To illustrate the potential contribution of active sensing by the kill vehicle, we analyze a LIDAR system. In contrast to a passive imaging system or a laser illuminator, a LIDAR could in principle provide precise range information, allowing the rocket body to be tracked in three dimensions, which would have important advantages during the endgame of the intercept. As shown in Chapter 12, having range information is crucial for success during the endgame.

\subsubsection{A notional infrared seeker on the kill vehicle}

We consider now detection and tracking of the plume by a cooled SWIR sensor on-board the kill vehicle. We assume the sensor will be covered during launch and the attempt to acquire the target's plume will begin once the interceptor reaches a sufficient altitude that the seeker's window can be opened and it can begin operating. (The atmosphere must be thin enough so that aerodynamic heating and the resulting radiation will not overwhelm the sensor and its cooling system.) A seeker of the type we have analyzed could begin operating when the kill vehicle reaches an altitude of about $100 \mathrm{~km}$.

Plume structure at high altitudes As noted in Section 10.1, the total luminosity of a rocket exhaust plume first increases as the rocket exits the trough region and then decreases steadily as the rocket continues to rise, falling to an almost constant "intrinsic" or "vacuum" level at very high altitudes. Although exhaust plumes broaden at high altitudes, they have a bright central region extending downstream from the nozzle. This core component dominates the emission in the MWIR, is strongest at the exit of the rocket nozzle, and decreases uniformly away from it. This phenomenology could be used to locate the rocket body using an MWIR sensor. For ICBM second stages, the MWIR luminosity of the core is $1 \mathrm{~kW} / \mathrm{sr}$ even for a rocket with a thrust of $10^{4} \mathrm{~N}$, similar to those used in ICBM second stages. Radiative de-excitation of $\mathrm{CO}_{2}$ rotational levels contributes a major part of the core emission in the MWIR. The rotational temperature of the $\mathrm{CO}_{2}$ is low, and consequently the spectral distribution of the $\mathrm{CO}_{2}$ emission is quite narrow, facilitating rejection of non-core background using a sensor with a bandpass tuned to this band.

The SWIR luminosity of exhaust plumes comes both from the core region and from $\mathrm{H}_{2} \mathrm{O}$ emission in the enhancement region where the hot plume gases mix and react with atmospheric oxygen. Hence the SWIR emission comes from a broader region and is strongest downstream from the rocket nozzle, as evident in Fig. 10.12, which shows the measured spa- 


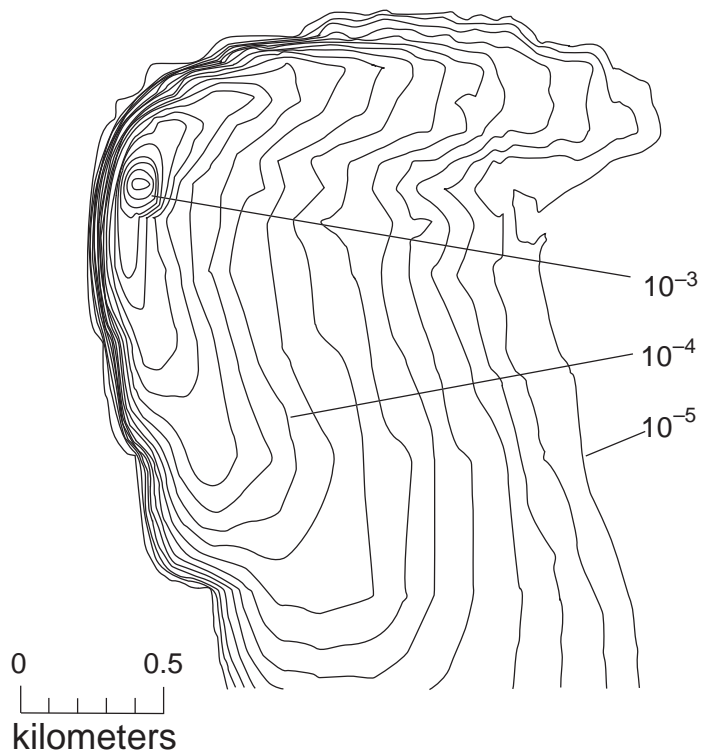

Figure 10.12. Contours of constant SWIR spectral radiance $\left(\mathrm{Wcm}^{-2} \mathrm{ster}^{-1} \mu \mathrm{m}^{-1}\right)$ in the exhaust plume of a Titan IIIC at an altitude of $110 \mathrm{~km}$, flying with a slight angle of attack, and viewed at an aspect of about $130^{\circ}$ from the nose with a fairly coarse $(\sim 100 \mathrm{~m})$ spatial resolution. The general spatial structure is more or less typical of exhaust plumes at high altitude. The peak in the intensity is about $100 \mathrm{~m}$ behind the rocket nozzle. Figure 14.8 of Ref. [98]. See also Figs. 14.19-14.23 of Ref. [98]. Used with permission. Copyright by The Aerospace Corporation.

tial structure of the exhaust plume of a Titan IIIC on a space-launch trajectory at an altitude of about $110 \mathrm{~km}$. (See also the computed contours shown in Fig. 5.9 of Ref. [98].) Although the location of the rocket nozzle is not precisely known, the strongest emission appears to come from a region with a spatial extent of about $100 \mathrm{~m}$ some 100-200 m downstream from the nozzle. The spectral luminosity of the hot gas within the $10^{-3} \mathrm{~W} \mathrm{~cm}^{-2} \mathrm{sr}^{-1} \mu \mathrm{m}^{-1}$ spectral intensity contour appears to be about $300 \mathrm{~kW} \mathrm{sr}^{-1} \mu \mathrm{m}^{-1}$.

In practice, the structure and luminosity of the plume depends strongly on the missile's thrust and angle of attack, the nozzle expansion ratio, and the type of propellant used. For large (thrust $\sim 10^{5} \mathrm{~N}$ ) liquid-propellant rockets, the spectral luminosity of the intrinsic core at $2.7 \mu \mathrm{m}$ is typically $\sim 10^{4} \mathrm{~W} \mathrm{sr}^{-1} \mu \mathrm{m}^{-1}$, comparable to the spectral luminosity at $4.3 \mu \mathrm{m}$ (see Fig. 5.22 of Ref. [98]). For smaller (thrust $\sim 10^{4} \mathrm{~N}$ ) liquid-propellant rocket motors, similar to those used in ICBM second stages, the spectral luminosity of the core at $2.7 \mu \mathrm{m}$ is typically $\sim 10^{2} \mathrm{~W} \mathrm{sr}^{-1} \mu \mathrm{m}^{-1}$, about one tenth the spectral luminosity at $4.3 \mu \mathrm{m}$. The situation is complicated further, because the brightest emission sometimes comes from the shockwave in front of the rocket.

Although imaging the plume in the MWIR has some advantages, we chose to analyze an SWIR sensor because it is simpler than an MWIR sensor and appears adequate to allow a LIDAR on the kill vehicle to locate the rocket body. For the purposes of this analysis, we assumed the SWIR luminosity of the gas within $50 \mathrm{~m}$ of the brightness peak is $1 \mathrm{~kW} \mathrm{sr}-1$.

A notional SWIR seeker The key enabling technology for an SWIR seeker on the kill vehicle is IR sensor arrays of the type discussed in Section 10.1.2 in connection with our notional space-based IR missile detection and tracking system. Here we adopt the same 
$512 \times 512$ format $\mathrm{HgCdTe}(\mathrm{MCT})$ arrays discussed there. Frames could be read out at video rates $(30 \mathrm{~Hz})$ or faster. We assume a quantum efficiency of approximately 80 percent, well depths of about $10^{6}$ electrons, readout noise of about 250 electrons, and a $20-\mu \mathrm{m}$ pixel pitch. As discussed further below, the dark count can be made negligible by cooling the detector.

In the present analysis we concentrate on the 2.7-3.0- $\mu \mathrm{m}_{2} \mathrm{O}$ emission band in the SWIR and postulate a telescope with a 10-cm-diameter aperture, which would have a diffraction limit of about $3 \times 10^{-5} \mathrm{rad}$, equivalent to $20 \mathrm{~m}$ at a range of $700 \mathrm{~km}$. We assume the optical path has a throughput of 80 percent and the detector has a quantum efficiency of 70 percent. With a focal ratio of $f / 7$, the detector pitch is well matched to the diffraction limit. Each pixel then corresponds to $20 \mathrm{~m}$ at $700 \mathrm{~km}$. With a $512 \times 512$ array, the field of view would be almost $10 \mathrm{~km}$ at that range. Since we expect cuing from the space-based IR alert system with a positional precision better than $1 \mathrm{~km}$, the IR sensor on the kill vehicle should be able to find the target's plume immediately, with no need to search for it in a scanning mode.

Plume signal and backgrounds The signal that would be produced in our hypothetical on-board seeker by an ICBM plume is substantial. At a range of $700 \mathrm{~km}$, the signal produced by a luminosity of $1 \mathrm{~kW} \mathrm{sr}^{-1}$ is $\sim 10^{8}$ electrons per second. Even when the rocket is in the trough, the SWIR luminosity of its exhaust plume would exceed $1 \mathrm{~kW} \mathrm{sr}^{-1}$ and could therefore be detected easily by the on-board SWIR sensor described above. As discussed earlier, the diameter of the peak in the plume emission is expected to be about 100-200 m; hence, at a range of $700 \mathrm{~km}$ most of the signal is spread over a $4 \times 4$ pixel patch of the focal plane array. For a $30-\mathrm{Hz}$ frame rate, the signal in each pixel would be about $2 \times 10^{5}$ electrons per frame, which is a substantial fraction of the well depth but will not overfill the well. The shot noise in the signal is larger than the readout noise of the detector. As the range decreases, the flux in each pixel will remain constant, but the plume will spread over an ever larger number of pixels.

The background would include the detector dark current and thermal emission from the optics, baffles, and dewar window. The dark count varies as $\exp (-1 / T)$ and could be suppressed to an acceptable level by cooling the detector. Extrapolating from Fig. 2.3 of Ref. [106] and assuming a 3- $\mu \mathrm{m}$ cutoff and $2-\mu \mathrm{m}$ pixel pitch, we estimate that the dark current could be reduced to $10^{4}$ counts per pixel per 33-ms frame by cooling the detector to $150 \mathrm{~K}$. Thermal emission from a room temperature object, such as the interceptor, is small in the $2.7-3.0-\mu \mathrm{m}$ band; only about $7 \times 10^{-5}$ of the total emission from a $300-\mathrm{K}$ blackbody is within this band. Assuming a 20 percent emissivity for the optical train outside the dewar, which is conservative and makes some allowance for baffle edges that can be seen by the detector, the thermal background would contribute $4 \times 10^{3}$ electrons per $33-\mathrm{ms}$ frame per pixel, 50 times less than the plume signal at $700 \mathrm{~km}$.

Another possible source of background is emission from Earth, including scattered sunlight and thermal emission. When viewed from high altitudes, both are small in the 2.7$3.0 \mu \mathrm{m}$ water-vapor absorption band. Scattered sunlight is minimal because the atmosphere is strongly absorbing in this band, whereas thermal emission is suppressed because the only emission visible at high altitudes is that from regions where the air temperature is much less than $300 \mathrm{~K}$. The strongest backgrounds are from cloud tops, which have a smaller absorption path to space, and bright solar glints from the oceans. Both backgrounds contribute $\sim 10^{-5} \mathrm{~W} \mathrm{~cm}^{-2} \mathrm{sr}^{-1}$. At high altitudes, a ground- or sea-based interceptor would usually 
be looking upward or at least horizontally at the target's plume, which would then appear against the dark background of space. Emission from Earth is obviously not directly relevant for such an engagement geometry, although some small fraction of it could be scattered into the optical path. However, even in the worst case of an interceptor that finds itself looking downward at the target against a background of cloud tops, the background would be less than $2 \times 10^{4}$ electrons per 33-ms frame, 10 times less than the signal produced by the plume per pixel. The shot noise contributed by variations in the background would be smaller still.

When the seeker is looking away from the Earth's disk, there is a possibility that sunlight could enter the optical path, causing a lens flare. However, unless the Sun is directly behind the target missile, the missile's plume would produce enough signal in the seeker that it should be able to detect the plume against such a flare.

We conclude that even for a plume with an SWIR luminosity as low as $1 \mathrm{~kW} \mathrm{sr}^{-1}$, the signal in our hypothetical kill-vehicle seeker would be well above anticipated backgrounds and clearly detectable. The sensor array, and hence the field of view, could be made large enough that the seeker would be able to acquire the plume using cuing information provided by the space-based IR or surface-based radar missile detection and tracking systems discussed previously in this chapter.

Locating the rocket body A successful intercept requires that the kill vehicle home on the missile body or warhead as it approaches the missile. The precision with which the position of the rocket body could be located by an on-board passive IR sensor is determined by the shape and structure of the plume and what advance knowledge the defense has of the relationship of the rocket body to this structure. Various algorithms have been developed to determine the position of the rocket body using measurements of plume structure. Generally one looks for the rocket at the narrower end of the plume. The diameter of the brightest part of the plume is less than $100 \mathrm{~m}$ in the SWIR, even at high altitudes (see [98, Fig. 7.10]). Hence we expect that on-board passive IR sensing could determine the projected position of the rocket body to within a region $100 \mathrm{~m}$ in diameter, and we have therefore assumed that such sensing could provide in-flight target updates during the flyout of the interceptor and kill vehicle with an uncertainty $(1 \sigma)$ of $25 \mathrm{~m}$. Another sensor, such as an LWIR passive imager or a LIDAR system, would be required to allow the kill vehicle to home on the missile body or warhead at close range.

Summary As soon as the interceptor emerges from the atmosphere and the cover of the seeker is opened, the on-board passive IR sensor should be able to acquire the exhaust plume as a high signal-to-background, multiple-pixel source. As the kill vehicle closes on the target, the plume would expand in the sensor's field of view, but even at $100 \mathrm{~km}$, the shortest range at which the passive IR sensor would be required to track the plume, the plume's bright central peak would still fit within the sensor's field of view. We judge that the position of the rocket body could be estimated to within $100 \mathrm{~m}$. At this point, tracking could be handed over to a shorter-range hardbody detector, such as an LWIR passive imager or a LIDAR. In the following section we describe a notional LIDAR system that could perform this task. It has a 100-m field of view and could therefore find the rocket body in the field specified by the long-range passive IR sensor just described. If necessary, the LIDAR's field of regard could be expanded by having it search an area on the sky that is $2 \times 2$ or $3 \times 3$ times its field of view. This expanded search probably would not be necessary, because the 
positional uncertainty of less than $100 \mathrm{~m}$ provided by the passive SWIR seeker should allow the LIDAR to be pointed accurately enough that the rocket body would be well within its field of view when the kill vehicle is $100 \mathrm{~km}$ from the missile.

We conclude from this analysis that a passive SWIR sensor on the kill vehicle could detect and track the plume from a range of at least $700 \mathrm{~km}$ and estimate the location of the hard body to within $100 \mathrm{~m}(1 \sigma)$ when it is $100 \mathrm{~km}$ from the missile.

\subsubsection{A notional LIDAR seeker on the kill vehicle}

As it closes on the target, the kill vehicle must shift from homing on the plume to homing on the rocket body. In contrast to the plume, the rocket body is relatively cool and produces only dim thermal emission that is difficult to see in the vicinity of the very bright emission produced by the hot plume. One way to circumvent this problem is to use a laser illuminator and an active optical sensor, as suggested by Postol [116, 117]. Alternatively, a LIDAR could provide range as well as angle information, allowing the rocket body to be tracked in three dimensions. This approach would have important advantages during the endgame of the intercept, when the "time to go" is an important consideration in guiding and controlling the kill vehicle to achieve intercept.

A possible LIDAR system Using LIDAR to track a rocket body or warhead when it is near or within the rocket's exhaust plume is not a well understood or documented technique. The performance that could be achieved depends on a variety of factors, including whether the rocket uses liquid or solid propellants and the particulate content of the plume (soot and alumina particles). In the present analysis, we ignore these potentially important issues and examine the best performance that could be achieved by a notional LIDAR system for tracking the rocket hard body.

Unlike radar, a LIDAR system is almost always photon-statistics limited. The count rate $d N / d t$ returned from a LIDAR target is approximately

$$
d N / d t \approx\left(P_{\text {laser }} / h \nu\right)\left(\alpha A_{\text {target }} / A_{\text {illum }}\right)\left(\epsilon A_{\text {collect }} / \pi R^{2}\right) \eta
$$

where the precise count rate depends on the angular distribution of the light scattered from the surface of the target, $P_{\text {laser }}$ is the radiated power of the laser, $A_{\text {target }}$ is the cross-sectional area of the illuminated target, $\alpha$ is the target's albedo, $A_{\text {illum }}$ is the cross-sectional area of the outgoing laser beam at the range of the target, $R$ is the range from the LIDAR to the target, $\epsilon$ and $A_{\text {collect }}$ are the efficiency and area of the collecting optics, and $\eta$ is the quantum efficiency of the detector (see Fig. 10.13). The range from the illuminating source to the target enters only via the area $A_{\text {illum }}$ of the illuminating spot. If the size of this spot can be adjusted to keep $A_{\text {target }} / A_{\text {illum }}$ approximately constant, the return from the target will scale as $R^{-2}$ rather than as $R^{-4}$, in contrast to the scaling usually encountered with radar systems. Creating a narrow laser beam and adjusting its width is not difficult in principle, because of the short wavelength of the radiation and the correspondingly small diffraction angle.

The available laser power is typically modest, because the wallplug efficiency of spacequalified lasers is small. Table 10.11 lists the efficiencies and output powers of some recently flown or planned space laser systems. These low efficiencies mean that for a small interceptor, the output laser power is likely to be no more than tens of watts. One way to overcome this obstacle is to use a high-efficiency ( $\sim 50$-percent) laser diode, rather than a 


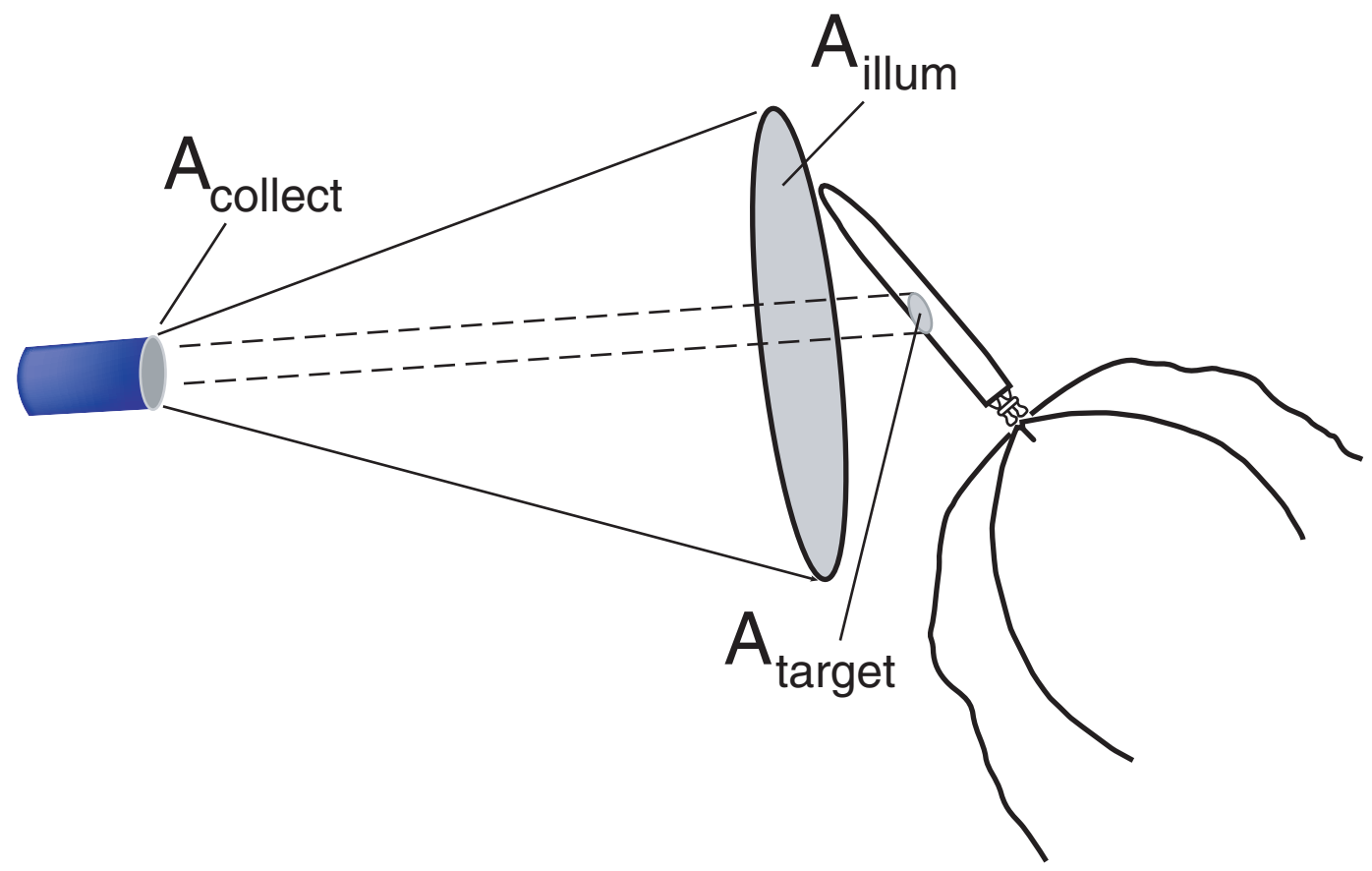

Figure 10.13. The geometry involved in detecting and tracking a rocket body using a LIDAR system on the kill vehicle.

Table 10.11. Characteristics of Space Lasers

\begin{tabular}{lr}
\hline \hline Laser & Efficiency \\
\hline Mars Observer Laser Altimeter $(1.06 \mu \mathrm{m}, 40 \mathrm{~mJ}, 10 \mathrm{pps})[120,121]$ & $3 \%$ \\
Vegetation Canopy Laser $(1.06 \mu \mathrm{m}, 15 \mathrm{~mJ}, 10-240 \mathrm{pps})[122]$ & $6 \%$ \\
Fibertek proposal for improved 1.06- $\mu \mathrm{m}$ laser $[123]$ & $10 \%$ \\
$\mathrm{Nd}: \mathrm{YAG} \mathrm{slab}^{a}(1.06 \mu \mathrm{m}, 808-\mathrm{nm}$ diode pump, 100 W) & $6 \%$ \\
Yb fiber $^{a}(1.03-1.10 \mu \mathrm{m}, 100 \mathrm{~W})$ & $6 \%-8 \%$ \\
\hline
\end{tabular}

${ }^{a}$ S. Cameron, Sandia National Laboratory, private communication.

low-duty-cycle pulsed laser. However, the ranging system would then have to demodulate a time-coded illumination pattern, rather than a simpler low-duty-cycle pulse train, because laser diodes are efficient only when used in a quasi-cw mode. If a modulated signal can be used, output powers of up to hundreds of watts may be possible. High-efficiency laser diodes have been developed with output in the 808-nm region (see, e.g., Ref. [118, 119]).

A photon-counting system is preferred because the very limited number of photons available necessitates high detection efficiency and the need to preserve the time structure of the return signal to correlate it with the transmitted signal. One option would be an intensified micro-channel plate with a photon-counting readout. The readout could be a multi-anode array [124] or a centroiding anode [125]. The detector efficiency would be determined by the photocathode [126, Fig. 4.33]. The time resolution could be a few nanoseconds or less. Detector resolutions of $1 \mathrm{k} \times 1 \mathrm{k}$ are possible using non-pixelized, centroiding readout systems. 
Another option would be to use an array of avalanche photodiodes (APDs) [127]. Vasile et al. [128] describe a $6 \times 14$ array with a readout time resolution of about $0.25 \mathrm{~ns}$. These devices must be reset after a pixel triggers on a photon. Passive quenching schemes allow each pixel to count at rates of several tens of kilohertz; active schemes can be much faster. Hamamatsu [124] offer an existing $4 \times 8$ array. Quantum efficiencies are those characteristic of the silicon material and, like charge-coupled devices (CCDs), are high; the quantum efficiency of the Hamamatsu device is greater than 70 percent from 400 to $900 \mathrm{~nm}$ and peaks at 85 percent. Larger APD arrays are under development.

We base our analysis on the "Ballistic Missile Defense" laser, of unknown efficiency, advertised by Fibertek, Inc. [123]. This laser has an output of $355 \mathrm{~mJ}$ per pulse with a $100-\mathrm{Hz}$ repetition rate at the $1.06-\mu \mathrm{m}$ fundamental of Nd:YAG and is converted efficiently to $230 \mathrm{~mJ}$ of $0.532-\mu \mathrm{m}$ frequency-doubled radiation at the same repetition rate. The net signal is similar for the two options, as the photon loss from $0.532 \mu \mathrm{m}$ to $1.06 \mu \mathrm{m}$ is made up by the better response of photoemissive detectors at the shorter wavelength.

Table 10.12. Return Signal from a Notional LIDAR ${ }^{a}$

\begin{tabular}{lr}
\hline \hline Parameter & Value \\
\hline Optics efficiency $\epsilon$ & $70 \%$ \\
Detector quantum efficiency $\eta^{b}$ & $30 \%$ \\
Laser spot diameter & $100 \mathrm{~m}$ \\
Assumed rocket length & $10 \mathrm{~m}$ \\
Assumed rocket diameter & $1 \mathrm{~m}$ \\
Receiver aperture $A_{\text {collect }}$ & $\frac{\pi}{4}(15 \mathrm{~cm})^{2}$ \\
Target albedo $\alpha$ & $0.1^{c}$ \\
Count rate at $1,000 \mathrm{~km}$ & $9.23 \mathrm{~s}^{-1}$ \\
Required pointing accuracy at $1,000 \mathrm{~km}^{d}$ & $20 \mu \mathrm{rad}$ \\
Count rate at $100 \mathrm{~km}$ & $923 \mathrm{~s}^{-1}$ \\
Required pointing accuracy at $100 \mathrm{~km}^{d}$ & $200 \mu \mathrm{rad}$ \\
\hline
\end{tabular}

${ }^{a}$ With 23 watts average power at $0.532 \mu \mathrm{m}$.

${ }^{b}$ Photoelectric, Generation III photocathode.

${ }^{c}$ Typical of space debris.

${ }^{d} 20 \%$ of the beam.

Basic performance Several conclusions are evident from Table 10.12. Photons are at a premium, and the count rate can disappear altogether. At a range of $1,000 \mathrm{~km}$, there is essentially no usable signal if a $10-\mathrm{Hz}$ update rate is required. Also, the return signal is a strong function of the illuminating beam size. There is simply too little power to illuminate a large volume of space. Instead, the LIDAR must be cued onto the hardbody or warhead, either by an on-board IR system such as that described in the previous section or an external radar or IR sensor. For a laser illumination pattern that is only $100 \mathrm{~m}$ across at a range of $100 \mathrm{~km}\left(10^{-3} \mathrm{rad}\right)$, the laser must be pointed with a precision that is a small fraction (typically 20\%) of the size of the illuminated spot. However, at ranges of $100 \mathrm{~km}$ or less there appears to be a robust signal at a $10-\mathrm{Hz}$ frame rate, with some range information 
available out to $200-300 \mathrm{~km}$ at a reduced update rate. By optimizing the system, it might be possible to use it robustly at these ranges.

As a countermeasure, the adversary could either paint the missile black or make it highly reflective. However, "black" coatings still have a finite reflectivity. Note that the dazzlingly bright moon has an albedo of just 7\%, which is similar to charcoal. Flat black "lampblack" paint typically reflects 3-4\%, while fragile optical coatings such as Parson's Black still reflect about $1.2 \%$ in the red and near infrared [129]. These countermeasures would degrade the count rate of the LIDAR proportionately, but would not preclude detection and location of the target. Because of this possibility, we do not count on achieving ranges greater than $100 \mathrm{~km}$ in our engagement simulations, which are described in Chapter 12 and Appendix C.

Given a return signal, the LIDAR should be able to locate the hardbody in all three coordinates to a precision $\Delta x \propto N^{-1 / 2}$, where $N$ is the number of counts detected in an update time. (This result assumes that the image is spatially resolved.) The error terms we are averaging out include (1) the digitization error inherent to a pixelized detector, (2) the optical blur of the collection optics, limited at best to the diffraction blur of the aperture, and (3) the random distribution of return photons along the rocket body. If we assume a large format detector, such as the remote ultra-low light imaging (RULLI) delay-line instrument developed at Los Alamos [125], the digitization error could be made negligible. We can bound the contribution of the third error term by ignoring all edges and boundaries that contribute high spatial frequency information and considering only statistical variations in the centroid of the return photons. In this case,

$$
\Delta x=(1 / N)\left[(\Theta R / 2.35)^{2}+L^{2} / 12\right]^{1 / 2},
$$

where $L$ is the extent of the hardbody along the axis in question and $\Theta$ is the diffraction blur (FWHM) of the collecting optics. For the system described in Table 10.12, the variance in the centroid of the target is dominated by the geometrical size of the target (the second term on the right-hand side of Eq. [10.10]) rather than the diffraction blur of the system. In this geometric case, the precision with which the centroid can be determined is limited largely by photon statistics and varies from $3 \mathrm{~cm} \times 0.3 \mathrm{~cm}$ at a range of $10 \mathrm{~km}$ to $30 \mathrm{~cm}$ $\times 3.4 \mathrm{~cm}$ at $100 \mathrm{~km}$. Here we have assumed that the radius of the illuminating spot can be held fixed at $100 \mathrm{~m}$, independent of the range; otherwise, the photon statistics would improve even more steeply as the range is reduced.

More information could be obtained from the edges of the target, which may be essential if there are perturbations, such as non-uniformity of the illumination or the missile albedo, specular reflections, or countermeasures that provide spurious returns, such as corner cubes or laser beacons. By imaging the target, some of these problems could be eliminated. With a $15-\mathrm{cm}$ aperture, the diffraction limit for $0.532-\mu \mathrm{m}$ illumination at $100-\mathrm{km}$ range is $0.35 \mathrm{~m}$. This diffraction resolution could be adequately sampled throughout a 100-m illumination area, given a detector with a format of $512 \times 512$ or more pixels.

We can estimate the information contained in the edges by considering a simple rectangular target. The uncertainty in determining the location of the edge along a given axis is approximately the distance between the edge and the detected photon that is closest to the edge. The spacing of the closest photon is approximately $L / N$ and decreases linearly with the photon count rate detected. In the case described in Table 10.12, it should be possible to determine the edge to within approximately $0.1 \mathrm{~m}$ and $0.01 \mathrm{~m}$, respectively, for the long and short axes. While this estimate is admittedly rough, it suggests that edge detection would indeed provide more precise information than would the centroid of the 
overall return. Therefore, even if the centroid were perturbed by systematic effects, the position errors that we have estimated are safe upper limits.

Sophisticated signal processing may be required to reference the individual detected photons to the moving frame of the target. However, this is not ruled out in principle, because the LIDAR detector records the four-dimensional $(x, y, z$, and $t$ ) location of each photon returned, with a very high precision in $t$.

Discussion The counting statistics of a LIDAR system like the one discussed here could be improved in several ways. The illuminating spot could be tightened, perhaps by using feedback from the target location obtained in the initial detection. The laser power and-or the detection aperture could be increased. This might be difficult for a monostatic sensor on a lightweight kill vehicle, but might be possible for a bistatic LIDAR system in which the laser is moved to a separate, larger platform. Keeping the laser beam tight enough to illuminate only the target from a great distance, such as from geosynchronous orbit, would require only a modest optic; generating the absolute pointing information needed to guide the beam would be a more difficult problem. In any case, it appears unlikely that the range of a LIDAR system based on near-term technology could be extended to 1,000 $\mathrm{km}$.

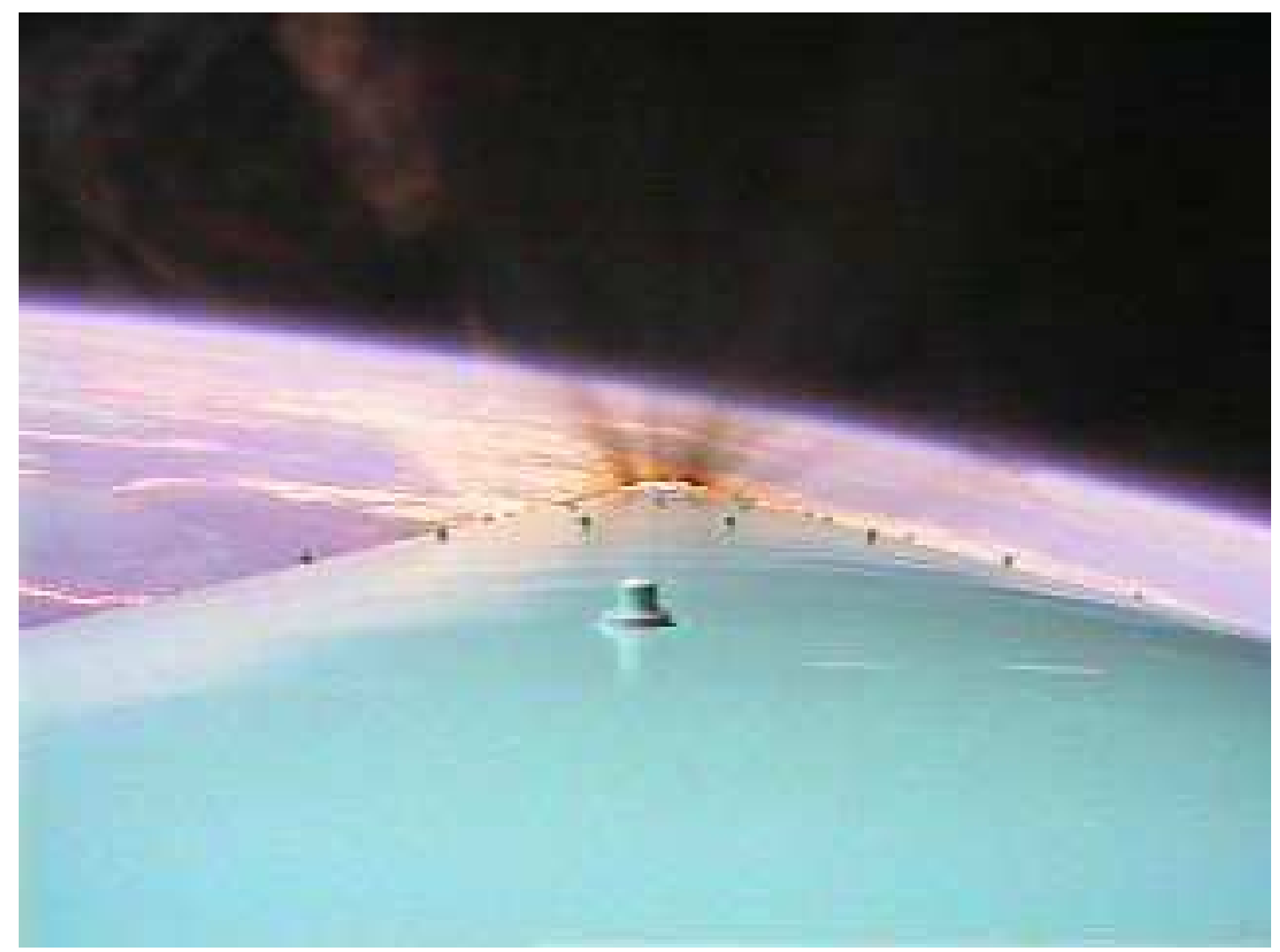

Figure 10.14. The plume from a liquid-propellant Delta launcher. Earth is clearly visible. It would be transparent to LIDAR interrogation. Courtesy NASA/JPL-Caltech.

An important issue is whether a LIDAR would fail to penetrate to the target because of scattering or absorption of the plume. This is not an issue for liquid-propellant rockets and illumination in the visible, as can be seen in Fig. 10.14, which shows the high-altitude plume 


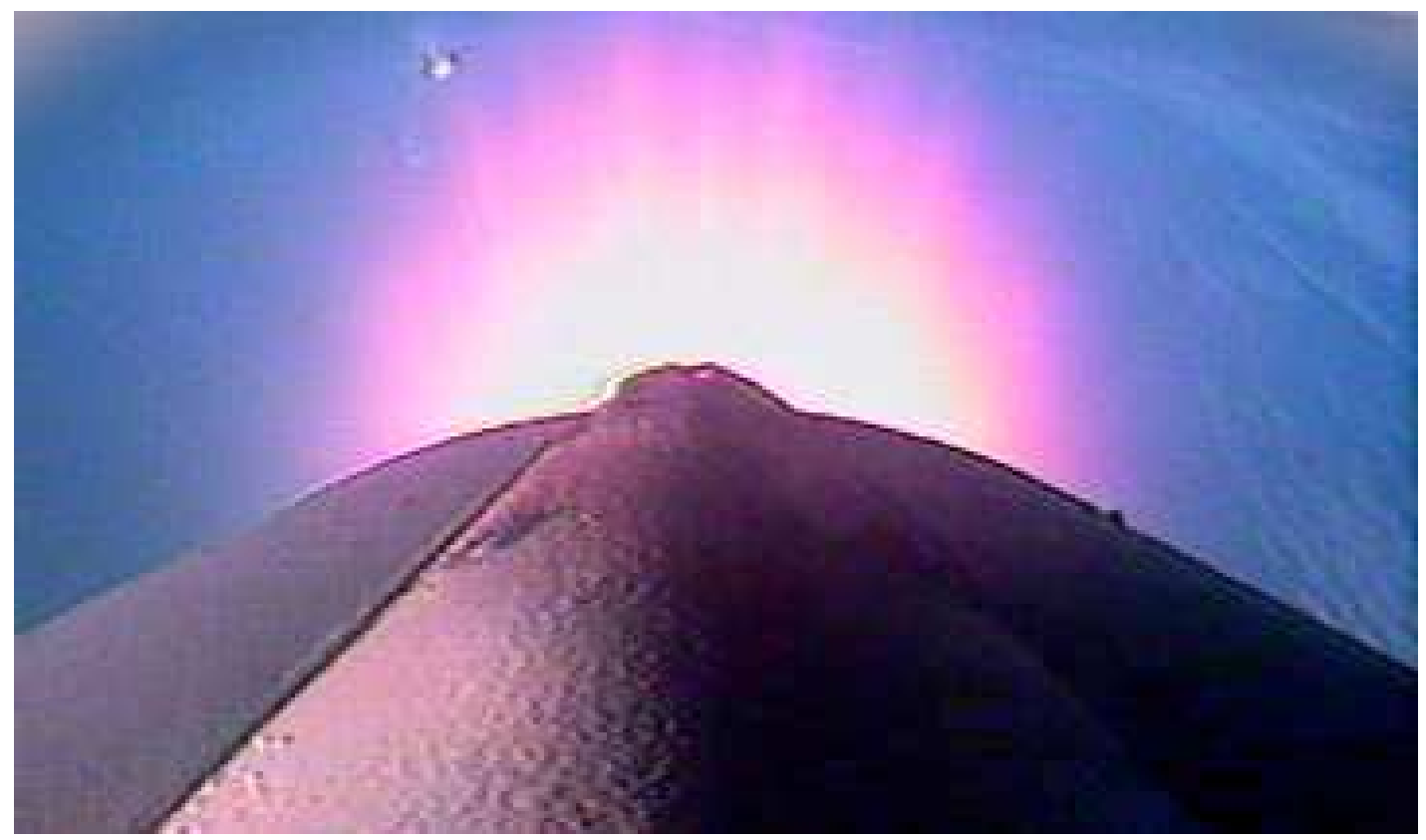

Figure 10.15. The plume from a solid-propellant Pegasus rocket, just after first-stage separation. The plume might be sufficiently opaque to have some effect on LIDAR interrogation. Courtesy of Orbital Sciences Corporation.

produced by the Delta Launcher during launch of the Mars Odyssey mission. Earth can be seen clearly through the plume in this photo taken by a camera on board the spacecraft.

In contrast, the plume produced by a solid-propellant rocket, like that shown in Fig. 10.15, might interfere with the operation of a LIDAR. Alumina particles in the plume would not only produce the bright background radiation seen in Fig. 10.15, they could also absorb the interrogating light beam. To understand better the possible magnitude of this effect, we perform a simplified analysis.

In this analysis, we take the parameters of the third-stage motor of our solid-propellant ICBM model S2 (propellant mass $900 \mathrm{~kg}$, burn time $40 \mathrm{~s}$, nozzle velocity $2.7 \mathrm{~km} / \mathrm{s}$ ) and assume that the alumina particle properties are the same as reported in Ref. [130] for the Space Shuttle's solid-rocket motors. For those motors, 7.6 percent of the fuel mass appears as alumina particles, which can be modeled as an equal mix (by mass) of particles with radii of $3.08 \mu \mathrm{m}, 2.18 \mu \mathrm{m}, 1.70 \mu \mathrm{m}, 1.26 \mu \mathrm{m}$, and $0.78 \mu \mathrm{m}$, with densities ranging from 1.80 to $2.84 \mathrm{~g} / \mathrm{cm}^{3}$, respectively. For a $0.532-\mu \mathrm{m}$ interrogator, these particles are significantly larger than the laser wavelength, and we therefore approximate their attenuation cross sections by their geometric cross sections. For a given mass flux, the attenuation depends on the angular spread of the alumina exhaust. For a full-width divergence of 30 degrees, the optical depth looking directly up the plume would be 0.36 . Although significant, this attenuation is small enough that laser detection of the hardbody at any aspect angle is still assured. Of course, most of the time the LIDAR's line of sight would lie entirely outside the alumina plume, and absorption by the plume would be negligible. 
Summary Based on these results we conclude that a LIDAR system with a $10-\mathrm{Hz}$ update rate on board the kill vehicle could detect and track the rocket body as soon as the kill vehicle were within $100 \mathrm{~km}$ of the target booster and could locate the position of an aim point on the target within $0.5 \mathrm{~m}(1 \sigma)$.

\subsection{Applications of Missile Detection and Tracking}

Based on the analysis in Section 10.1, we shall make the following assumptions in constructing illustrative engagement timelines (see Chs. 2, 4 and 5) and simulating engagements (Chapter 12 and Appendix C).

Capabilities of DSP Based on the analysis described in Section 10.1, we shall assume that the DSP satellite system could:

- Reliably detect large rockets by the time they reach an altitude of $10 \mathrm{~km}$, which occurs $35 \mathrm{~s}$ after the launch of our solid-propellant ICBM models S1 and S2 and $52 \mathrm{~s}$ after the launch of our liquid-propellant ICBM model.

- Enable construction of a firing solution $65 \mathrm{~s}$ after the launch of solid-propellant ICBM models S1 and S2 and $80 \mathrm{~s}$ after the launch of our liquid-propellant ICBM model. We emphasize that our assumption that interceptors could be fired this early represents a very optimistic bounding case.

- Locate the exhaust plumes of large rockets in three dimensions to within a $1 \mathrm{~km}$ pixel once every $10 \mathrm{~s}$ until the rockets enter the trough region. The DSP sampling rate is too low to enable efficient command guidance. Thus DSP likely would have to be supplemented by a much higher sample rate off-board sensor, such as a radar, to enable efficient command guidance of the interceptors, unless the limited capability for defending against only the long-burning liquid-propellant ICBMs is the objective.

Capabilities of a modern space-based system Based on the analysis described in Section 10.1, we shall assume that a modern space-based launch detection and tracking system with the potential capabilities of SBIRS-High could:

- Reliably detect large rockets by the time they reach an altitude of $7 \mathrm{~km}$, which occurs $30 \mathrm{~s}$ after launch for solid-propellant ICBM models S1 and S2 and $45 \mathrm{~s}$ after launch for our liquid-propellant ICBM model.

- Enable construction of a firing solution $45 \mathrm{~s}$ after launch for solid-propellant ICBM models S1 and S2 and 65 s after launch for our liquid-propellant ICBM model. Again, our assumption that interceptors could be fired this early represents a very optimistic bounding case.

- Locate the exhaust plumes of large rockets in three dimensions with a $1 \sigma$-uncertainty of $<300 \mathrm{~m}$ once per second, which would be adequate to support command guidance of interceptors during their flyout.

In Chapter 5, we showed that the earlier launch warning and more precise missile tracking that could be provided by a system like SBIRS-High would be critical to the effectiveness of any boost-phase intercept system. 
Missile and trajectory typing Prompt typing of missiles and their trajectories is a complex issue that depends on the range of possible rockets and missiles that could be launched from a given site and many other factors, including what prior knowledge the defense has of the ICBM and space-launch systems available to the countries of concern and whether the intelligence assets available to the defense provide information that a missile attack or space launch is about to occur in advance of the launch.

Substantially more tracking data, and hence more time, would generally be needed to type a rocket than to estimate its initial trajectory, especially if - as the Rumsfeld Commission [131] and recent reports by the U.S. Intelligence Community $[132,133,134,135,136]$ has argued - the missile being launched may have been tested only a few times or even not at all. With the space-based IR systems discussed above, the defense would know only the time at which it is able to confirm the existence of a large rocket in powered flight, not when the rocket was launched, so the time it took the rocket to reach the altitude at which it is first detected would not be available to help characterize the rocket.

The most primitive type of trajectory for injecting a satellite into orbit is direct ascent with coasting. In this approach, the boost phase of the space launch is similar to the boost phase of a ballistic missile [137]. The payload then coasts until the apex of its ballistic trajectory is reached, at which point upper stages are ignited to provide the additional impulse needed for orbital insertion. This approach was used by the United States to launch its Vanguard and Explorer satellites, and it might well be used by a country with an immature rocket program to launch its first satellites.

Given the wide variety of trajectories that are possible for space launches, TBM launches, and ICBM attacks, determining with confidence that a large rocket is an attacking ICBM and not a TBM or a rocket launching a satellite would take so long that waiting to fire interceptors would make it impossible to intercept an ICBM. Hence interceptors would have to be fired whenever a large rocket in powered flight is detected, without waiting until the nature of the rocket or its trajectory could be established.

Radar detection and tracking Based on the analysis described in Section 10.2, we conclude that:

- The sea-based, land-based, and airborne radar systems expected to be available within the ten-year time horizon of this study would not be able to detect and track liquid- or solid-propellant ICBMs like models L, S1, and S2 much earlier than a modern space-based infrared detection and tracking system unless they could be positioned within $300 \mathrm{~km}$ of the missile launch site. Such positioning would not be possible for all potential launch sites within North Korea or Iran unless radar systems were based closer than $100 \mathrm{~km}$ to hostile territory, contrary to the ground rules of this study.

- The AN/APY-2 (AWACS) radar could detect and identify as a missile a liquidpropellant ICBM like model L from a standoff distance of approximately $300 \mathrm{~km}$ early enough to provide initial warning of a missile launch and subsequent tracking in range and azimuth adequate to support a decision to fire interceptors. But it could not support command guidance of interceptors during the first $20 \mathrm{~s}$ of their flight, when the ICBM would be too low to be tracked reliably by space-based infrared sensors, because the AWACS radar could not provide sufficiently accurate elevation data. Even at this small standoff distance, the performance of the AWACS radar 
would be inadequate to support firing interceptors against solid-propellant ICBMs like models $\mathrm{S} 1$ and $\mathrm{S} 2$.

- The AN/SPY-1B (Aegis) radars could support boost-phase intercept from the maximum interceptor standoff distances considered $(800 \mathrm{~km}$ for the liquid-propellant ICBM model and $1000 \mathrm{~km}$ for the solid-propellant ICBM models). However, detection occurs too late to support initial warning of an ICBM launch or to contribute to the decision to fire interceptors. At these standoff distances, the Aegis radar could support command guidance of interceptors during their flyout. Aegis radar tracking errors exceed $2 \mathrm{mr}$ at elevation angles less than 1.5 above the horizon and approach about $0.4 \mathrm{mr}$ ( $1 \mathrm{mr}$ for the solid-propellant target) as the target nears the intercept point.

- The existing THAAD radar could support ICBM target detection at the 800-km maximum interceptor standoff distance considered in Chapter 4 for the liquid-propellant ICBM model and (marginally) at the 1000-km maximum standoff distance considered for the solid-propellant ICBM models. However, detection occurs too late to support initial warning of an ICBM launch or to contribute to the decision to fire interceptors. A THAAD radar at these standoff distances could support command guidance of interceptors during their flyout. THAAD radar tracking errors exceed $1 \mathrm{mr}$ at elevation angles less than 1 degree above the horizon but approach or drop below $0.2 \mathrm{mr}(0.4 \mathrm{mr}$ for the solid-propellant ICBM models) as the target nears the intercept point.

- The phased-array AWACS radar postulated in Section 10.2 could detect ICBMs at standoff distances greater than $800 \mathrm{~km}$ and provide initial warning of an ICBM launch, data to support the decision whether to fire interceptors, and command guidance of interceptors during their flyout. The tracking errors would exceed 1.5 $\mathrm{mr}$ for elevation angles less than 2 degrees but would decline to about $0.3 \mathrm{mr}(0.5$ $\mathrm{mr}$ for the solid-propellant ICBM models) as the target nears the intercept point.

All the radars discussed in this chapter could achieve earlier detections and provide the initial warning of an ICBM launch if they could be sited within $300 \mathrm{~km}$ of potential missile launch sites.

Kill vehicle seekers Based on the analysis of kill vehicle seekers described in Section 10.3, we conclude that:

- A passive SWIR seeker on the kill vehicle could detect and track the missile plume at a range of $700 \mathrm{~km}$ and could estimate the location of the rocket hardbody to within $100 \mathrm{~m}(1 \sigma)$ at a range of $100 \mathrm{~km}$.

- A LIDAR seeker on-board the kill vehicle could detect and track the hardbody as soon as it is within $100 \mathrm{~km}$ of the target with a $10-\mathrm{Hz}$ update rate and could locate the position of the aim point on the target within $0.5 \mathrm{~m}(1 \sigma)$.

Plume-to-hardbody handover The analysis in this chapter of passive SWIR tracking of the rocket's exhaust plume and LIDAR tracking of the rocket's hardbody by sensor systems on-board the kill vehicle is intended to illustrate how this might be done. Our analysis 
shows that technologies are available that could in principle satisfying the tracking requirements needed for the kill vehicle to track and hit an ICBM in powered flight (Chapter 12). We emphasize, however, that realistic modeling, testing, and evaluation as well as more extensive analysis would be needed to show that handing over from tracking the plume to tracking the hardbody with the precision required can be achieved reliably for the range of engagement geometries and operational conditions that a kill vehicle could encounter in an actual engagement. In addition to SWIR and LIDAR sensors, MWIR, optical, and other sensors may need to be considered.

\section{References for Chapter 10}

[96] Frank Clark, "Space Based Detection and Tracking." Briefing Presented to the APS Study Group, July 2001.

[97] Richard L. Garwin, "Boost-phase intercept: A better alternative." Arms Control Today 30(7), 8-11 September 2000.

[98] Frederick S. Simmons, Rocket Exhaust Plume Phenomenology. (Aerospace Press, El Segundo, California), 2000.

[99] Philip E. Coyle, "Missile Defense Testing." Statement Prepared for the House Government Reform Committee: National Security, Veterans Affairs, and International Relations Subcommittee, June 11, 2002.

[100] John Kidd and Holly Caldwell, "Defense Support Program: Support to a changing world," Proceedings of the AIAA Space Programs and Technologies Conference, March 24-27 1992, Huntsville, Alabama (1992).

[101] Keith L. Brower, "Statistical cloud coverage as a function of cloud optical thickness." Technical Report SAND98-1694, Sandia National Laboratory, 1998.

[102] Junhong Wang, William B. Rossow, and Yuanchow Zhang, "Cloud vertical structure and its variations from a 20-yr global rawinsonde dataset," J. Climate 13, 3041 (2000).

[103] William B. Rossow and Robert A. Schiffer, "Advances in understanding clouds from ISCCP," Bull. Am. Meteor. Soc. 80, 2261 (1999).

[104] SBIRS Program Office, 2001. http://www.losangeles.af.mil/SMC/MT/sbirsbrf/sbirsbrf.ppt.

[105] Alain Manissadjian et al., "Sofradir infrared detector products: The past and the future." Proc. SPIE 4130, 480 (2000).

[106] Philippe Chorier and Philippe Tribolet, "High performance HgCdTe SWIR detector development at Sofradir." Proc. SPIE 4369, 698 (2001).

[107] D. G. Lawrie and T. S. Lonheim, "Space-based systems for missile surveillance." Crosslink, Winter 2000/2001, 45 (2001).

[108] T. A. Postol, "A preliminary analysis of boost- phase missile defense concepts for protecting the US from postulated rogue-state ICBMs," undated. 
[109] D. Wilkening, "Boost-phase ballistic missile defense." Presentation to APS Study Group, 15 July 2001.

[110] M. Sarcione et al., "The design development and testing of the THAAD (Theater High Altitude Area Defense) solid state phased array (formerly ground based radar)." IEEE Int. Symp. Phased Arrays, 1996. Boston.

[111] A. Sessler et al., "Countermeasures: A Technical Evaluation of the Operational Effectiveness of the Planned US National Missile Defense System," Union of Concerned Scientists, April 2000.

[112] G. Lewis, "Assessing the ABM Treaty Compliance of the U.S. Navy's Upper Tier Missile Defense System,", 7th International Summer Symposium on Science and World Affairs, Kiev, Ukraine, September, 1995.

[113] D. K. Barton, Modern Radar System Analysis. (Artech House, Norwood MA), 1988.

[114] D. K. Barton and H. R. Ward, Handbook of Radar Measurement. (Prentice Hall and Artech House), 1969 and 1984.

[115] Richard L. Garwin, "Ballistic missile threats to the United States, missile defense concepts, and technical issues." Briefing presented to the APS Study Group on Boost-Phase Systems for NMD, July 2001.

[116] T. A. Postol, "Boost-phase missile defense concepts for protecting the U.S. from postulated rogue-state ICBMs." Technical report, MIT Security Studies Program, February 2001.

[117] T. A. Postol, "Boost-phase missile defense concepts and issues." Briefing presented to the APS Study Group on Boost-Phase Systems for NMD, July 2001.

[118] J. Sebastian et al., "High-power 810-nm GaAsP-AlGaAs diode lasers with narrow beam divergence," IEEE J. Quantum Electron. 7, 334 (2001).

[119] H. Wenzel et al., "High power diode lasers with small vertical beam divergence emitting at 808 nm," Electron. Lett. 37, 1024 (2001).

[120] Luis Ramos-Izquierdo, Jack L. Bufton, and Patricia Hayes, "Optical system design and integration of the Mars Observer Laser Altimeter," Appl. Opt. 33(3), 307 (1994).

[121] M. T. Zuber et al., "The Mars Observer Laser Altimeter investigation," J. Geophys. Res. 97(E5), 7781 (1992).

[122] Ralph Dubayah, "VCL: The Vegetation Canopy Lidar Mission." Available at: http://essp.gsfc.nasa.gov/vcl/.

[123] Fibertek Corporation, http://www.fibertek.com/Products/ballistic_missile_defens.htm.

[124] Hamamatsu Corporation, 2001. "4 Pixel × 8 Pixel APD Array Optimized for Short Wavelength Light (S8550)." Available at: http://sfa.hamamatsu.com/ . 
[125] M. H. Baron and W. C. Priedhorsky, "Crossed delay line detector for ground and space based applications," Proc. SPIE 2006, 188 (1993).

[126] William D. Rogatto, ed., Infrared and Electro-Optical Systems Handbook: Volume 3, Electro-Optical Components. (SPIE Optical Engineering Press Bellingham, Washington) 1993.

[127] S. Vasile et al., "Photon detection with high gain avalanche photodiode arrays," IEEE Trans. Nucl. Sci. 45, 720 (1998).

[128] S. Vasile et al., "High gain avalanche photodiode arrays for DIRC applications," IEEE Trans. Nucl. Sci. 46, 848 (1999).

[129] William L. Wolfe, Optical Materials. Number 3 in Electro-Optical Components.(SPIE Optical Engineering Press), 1993.

[130] Edward J. Beiting, "Solid rocket motor exhaust model for alumina particles in the stratosphere," J. Spacecraft \& Rockets 34, 303 (1997).

[131] Donald Rumsfeld et al., "Executive Summary of the Report of the Commission to Assess The Ballistic Missile Threat to the United States." Available at: http://www.fas.org/irp/threat/bm-threat.htm, July 1998.

[132] National Intelligence Council, "Foreign Missile Developments and the Ballistic Missile Threat to the United States Through 2015, Unclassified Summary of a National Intelligence Estimate." Available at:

http://www.cia.gov/cia/publications/nie/nie99msl.html\#rtoc7, September 1999.

[133] National Intelligence Council, "Foreign Missile Developments and the Ballistic Missile Threat to the United States Through 2015, Unclassified Summary of a National Intelligence Estimate." Available at: http://www.cia.gov/nic/pubs/, December 2001.

[134] Robert D. Walpole, "The ballistic missile threat to the United States." Speech by the National Intelligence Officer for Strategic and Nuclear Programs at the Carnegie Endowment for International Peace. Available at: http://www.cia.gov/cia/public_affairs/speeches/1998/walpole_speech_091798.html, 17 September 1998.

[135] Robert D. Walpole, "The ballistic missile threat to the United States." Statement for the Record by the National Intelligence Officer for Strategic and Nuclear Programs to the International Security, Proliferation, and Federal Services Subcommittee of the Governmental Affairs Committee, U.S. Senate. Available at: http://www.cia.gov/cia/public_affairs/speeches/archives/2000/nio_speech_020900.html, 9 February 2000.

[136] Robert D. Walpole, "The ballistic missile threat to the United States." Testimony by the National Intelligence Officer for Strategic and Nuclear Programs before the International Security, Proliferation, and Federal Services Subcommittee of the Governmental Affairs Committee, U.S. Senate. Available at: http://govt-aff.senate.gov/031102witness.htm, 11 February 2002. 
[137] Heinz Herman Koelle, ed., Handbook of Astronautical Engineering. (McGraw-Hill Book Company, Inc.) 1961. 


\section{Chapter 11}

\section{Kill Vehicles for Boost-Phase Defense}

\section{Contents}

11.1 Background: The Evolution of Kill Vehicles . . . . . . . S203

11.2 Three Kill-Vehicle Configurations . . . . . . . . . . S204

11.3 Endoatmospheric Kill Vehicles . . . . . . . . . . . S208

11.4 Kill Mechanisms Other than Body-to-Body Hit . . . . . S208

11.5 Key Requirements for Boost-Phase Kinetic Kill Above the At-

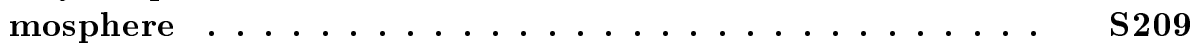

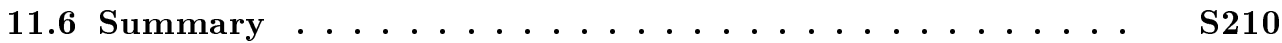

The kill vehicle is the final stage of the interceptor. It must supply enough propulsive divert capability to correct any remaining errors after its booster has burned out and to track the remaining unpredictable acceleration of the target. Initially, the kill vehicle is guided by signals from either radar or IR trackers or both, but its on-board passive and active sensors take increasing control as it approaches the target, and it operates autonomously. Thus, the kill vehicle has to meet stringent demands for propulsion, sensing, and control. It must also meet stringent demands on mass because of the large multiplicative factor that the kill-vehicle mass has on the overall size of the interceptor missile.

This chapter provides background on kill-vehicle design concepts. It reviews the evolution of kinetic energy kill vehicles, describes the three basic types of kill vehicles and their use in boost-phase intercept systems, and introduces lethality mechanisms. Finally the killvehicle requirements for boost-phase intercept are discussed. The kill-vehicle concepts and requirements presented here are used in Chapters 12 and 14, which illustrate the effect of those requirements on kill-vehicle size. Lethality measures are discussed in Chapter 13.

\subsection{Background: The Evolution of Kill Vehicles}

Homing stages of interceptors having accuracy sufficient to achieve a collision have evolved during the last 30 years. Key technology milestones were the initial development of small efficient IR focal-plane arrays for mid- and long-wavelength detection, lightweight telescopes having cooled optics, miniaturized cryogenic cooling units to reduce the internal thermal noise of these devices, small inertial measuring units, and small but powerful signal and data processing devices. Examples of direct-hit exoatmospheric kill vehicles are the Homing Overlay Experiment (HOE) [138], HIT (a spinning kill vehicle used in the 1970s and 1980s), Miniature Homing Vehicle (MHV), Exoatmospheric Reentry Intercept System (ERIS) [139], 
the Light Exo-Atmospheric Projectile (LEAP), and the Exoatmospheric Kill Vehicle (EKV) currently being tested for midcourse national missile defense (NMD).

Following closely on the development of the new exoatmospheric intercept technologies, similar capabilities were developed for intercept within the atmosphere. Major developments include window materials for mid-wave IR sensors, small radio frequency (RF) seekers, and innovative vehicle control methods. Examples of endoatmospheric interceptors include the Flexible Light Weight Agile Guided Experiment (FLAGE) and the Extended Range Interceptor (ERINT), which is now the Patriot PAC 3 missile.

In addition, hybrid methods have been developed that are capable of intercept both in the upper atmosphere and exoatmospherically. These were primarily designed to work against theater ballistics missiles. Examples include: High-Endoatmospheric Defense Interceptor (HEDI), the Army's Theater High-Altitude Area Defense (THAAD), and the Advanced Interceptor Technology (AIT) interceptor. The first two of these have flown in flight tests; the last one has not.

With one notable exception, the engagement of boosters capable of striking the United States necessarily takes place above the atmosphere, during the second or third stage of boosted flight. The exception is the case of medium-range ballistic missiles (MRBMs) launched from off shore near the coasts of the United States, which burn out before leaving the atmosphere. Interceptors that could disable ICBMs during their boost phase using direct impact - regardless of how they are based - are likely to use the same general type of kill vehicle configurations that are being developed for midcourse defense, though with some notable differences that will be described in the following discussion.

Several key elements are essential for the functioning of the homing kill vehicle for midcourse or boost-phase intercept:

- An on-board sensor that can acquire and track a target at a range that allows the kill vehicle enough action time to handle the remaining uncertainties in the predicted intercept point.

- An on-board inertial measurement capability to provide information on the kill vehicle's location with respect to the target, before target acquisition.

- An on-board processing capability to process the information from both of these sensing subsystems, as well as data communicated from off-board sources, and convert them into commanded actions.

- An on-board receiving communication link and, if possible, also a transmitting link.

- Enough propulsion capability to respond to the commanded actions needed to drive the threat trajectory prediction uncertainty volume to zero in less than the time remaining to closest approach. This includes rocket engines, attitude control thrusters, propellant, tanks for the propellant and the means to expel the fuel at a pressure compatible with the rocket engines.

- The batteries, power supplies, wiring, and structure to support all of the foregoing elements.

\subsection{Three Kill-Vehicle Configurations}

Three exoatmospheric hit-to-kill vehicle configurations have been developed and successfully demonstrated for midcourse and high terminal intercept during the last 30 years. All three 
use some form of proportional navigation (see Fig. 11.1 and the discussion in Section 12.2), which works on the principle of adding velocity normal to the line of sight to drive its rate of change toward zero. As sailors and aviators know, if their boat or aircraft is closing on an object whose bearing is not changing, a collision is imminent. In the case of an intercept, the closing velocity is initially determined by the motion of the target and the booster that launched the homing stage (i.e., the kill vehicle) on a nominal collision course.

During the homing phase, the kill vehicle can employ a variety of types of thrust control: proportional or simple on-off thrusters, pulse-width modulated on-off thrusters, multiple fixed-pulse solid rockets, or, in the extreme, even a continuous burning thruster.

Single axial thruster: Sine Alpha steering One kill vehicle configuration is the single axial thrusting system in which the sensor is gimbaled. During divert maneuvers, the sensor's line of sight (LOS) is initially oriented perpendicular to the vehicle thrust axis. Overshooting would require the homing vehicle to rotate $180^{\circ}$ about the line of sight to reverse the direction of thrust, but this can be avoided by employing a dead band in the thrust command. A variation of this configuration is to turn the vehicle to thrust along the line of sight toward the target while maintaining the seeker stable on the target with the gimbal torquers. Pitch and yaw are then used to generate acceleration normal to the line of sight.

This type of endgame steering derives from air-to-air missiles and is known as Sine Alpha steering. An advantage of this technique is that it can be accomplished with a single large axial thruster for both divert and homing, yet it can track a target that accelerates in any direction. The sensor is always slaved to the target line of sight through the gimbal system. A disadvantage is that once the vehicle turns to accelerate along the line of sight, the energy remaining is consumed primarily to increase the closing velocity, which is wasteful.

In the case of midcourse intercept, the energy penalty of Sine Alpha steering is not as severe as might be expected because the maneuver begins at a point with a fixed time to the intercept, and the acceleration of the target is determined primarily by gravity. Thus the amount of propellant needed during the remainder of the engagement is the same, no matter what the target does. The first midcourse hit-to-kill intercept was done by

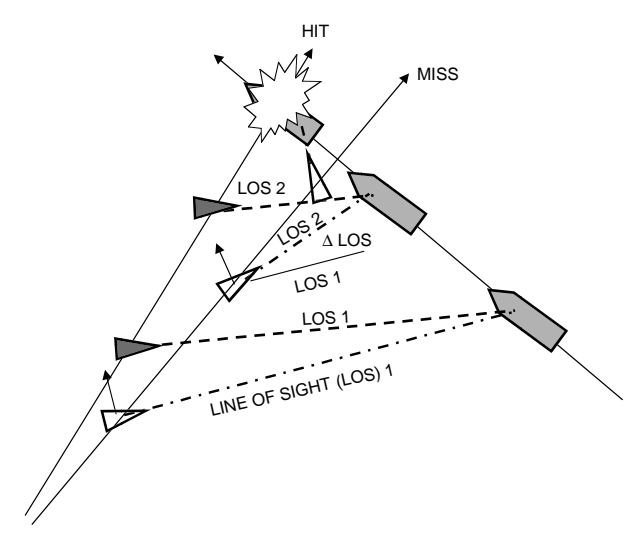

Figure 11.1. Line-of-sight behavior in intercepts: illustration of the principle of proportional navigation. 


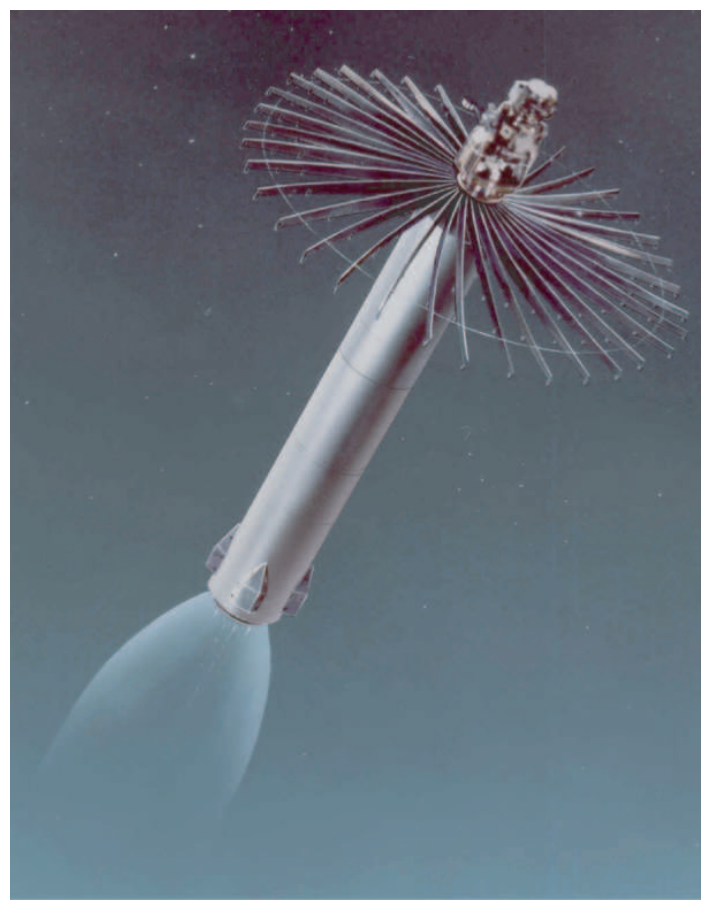

Figure 11.2. Homing Overlay Experiment [138]

the Homing Overlay Experiment (HOE) in 1984 (see Fig. 11.2). For boost-phase defense, however, the target acceleration is unpredictable, and large interceptor accelerations in any direction may be required. Because the response time of the kill vehicle is constrained by the time that it takes to turn the vehicle, Sine Alpha steering is not desirable for boost-phase defense.

Figure 11.3 illustrates a possible design of an exoatmospheric kill vehicle.

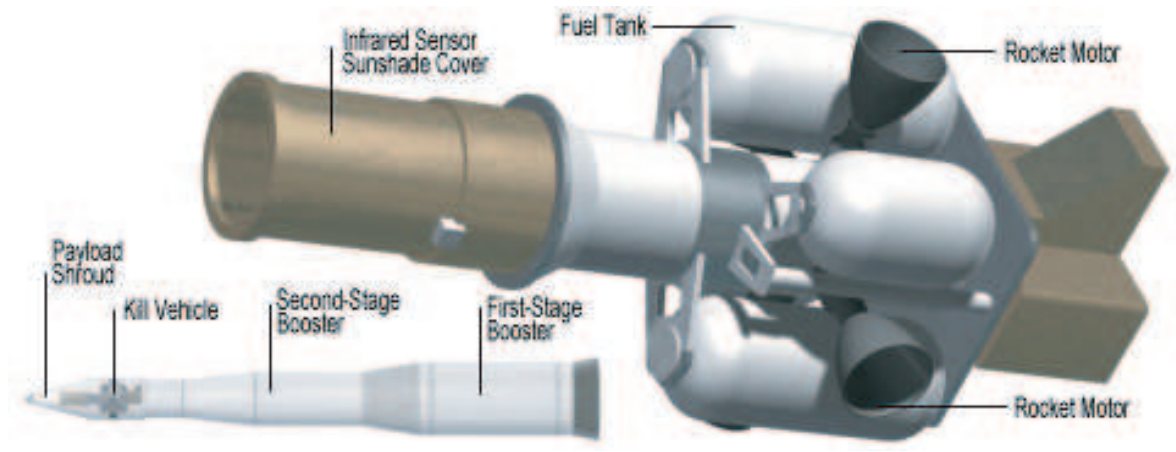

Figure 11.3. Kill-vehicle designed by Raytheon for use in a midcourse intercept system. Inset: Interceptor rocket designed by Boeing. (Illustration by John MacNeill.)

Cruciform configuration To minimize size and weight, the second and more popular approach for midcourse defense is the cruciform divert thruster configuration. In this approach, the body-mounted sensor has a line of sight along the vehicle's roll axis. Four divert thrusters are mounted perpendicular to the roll axis of the kill vehicle in a plane at the center of gravity so that they form an ' $\mathrm{X}$ '. The thrusters are used to maneuver normal 
to the line of sight. Small thrusters mounted on the axis at the end opposite the sensor provide pitch, yaw, and roll attitude control. This configuration requires that the expendable weights are both distributed and maintained so that the center of gravity of the kill vehicle does not move as propulsion fuel is expended.

The Exoatmospheric Reentry Intercept System (ERIS) shown in Fig. 11.4 first successfully demonstrated a midcourse intercept with the cruciform configuration in 1991, using a fully fueled kill vehicle weighing about $150 \mathrm{~kg}$. (The mass of the kill vehicle when it is fully loaded with fuel is sometimes referred to as the wet mass.) More recently, smaller versions of this configuration have been used by LEAP and the EKV currently being developed for midcourse missile defense.

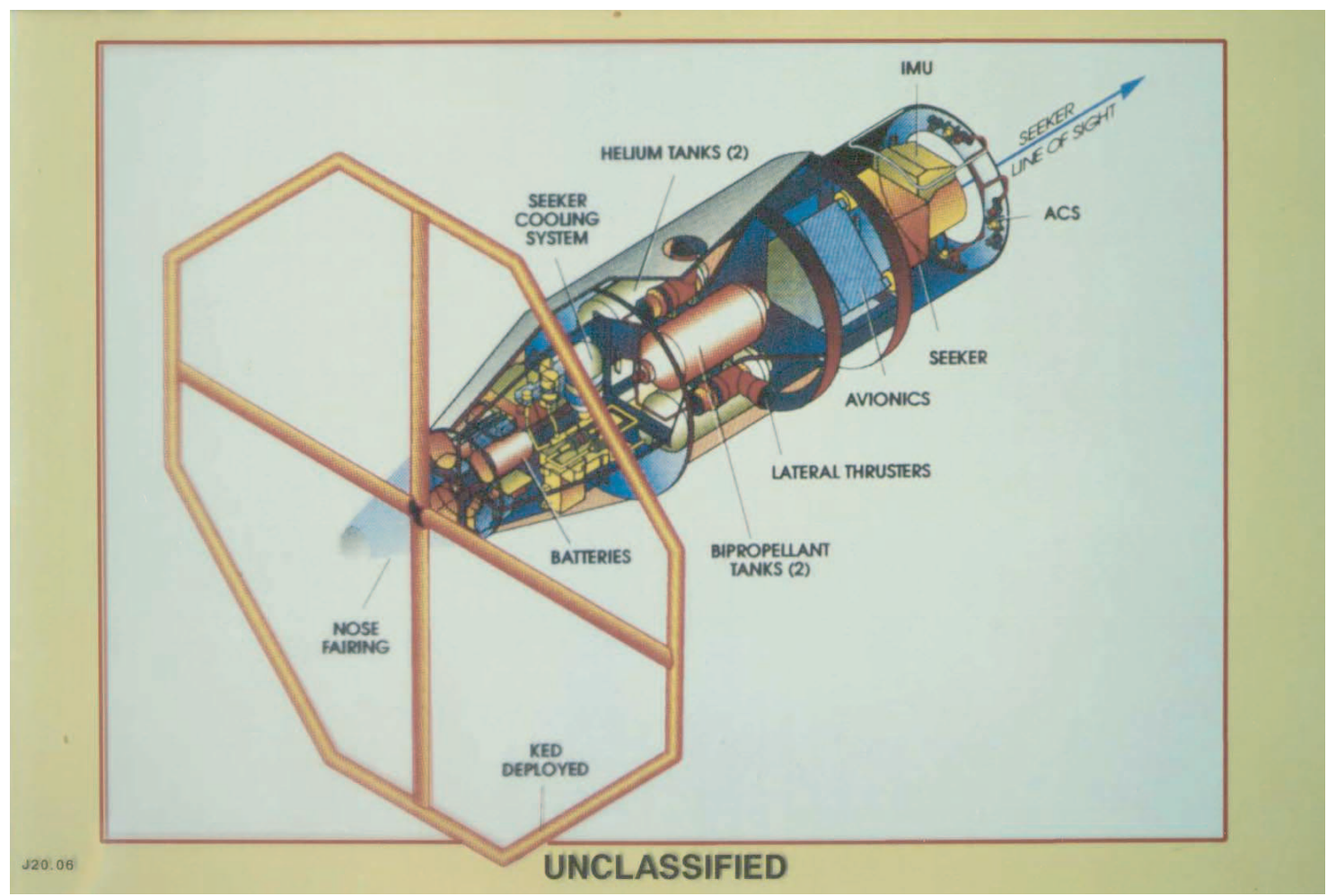

Figure 11.4. Artist's conception of a lethality enhancement device on the ERIS experiment. Courtesy of LDM Associates.

In our view, the cruciform configuration (or some variant) is likely to be the preferred kill-vehicle design approach for boost-phase defense because the predicted intercept point is constantly moving around because of the tracking noise and unanticipated target maneuvers that characterize the boost phase. To cope with such a target, acceleration must be applied quickly to the kill vehicle in any direction normal to the line of sight to correct for its rapid changes. Potential target maneuvers will drive the acceleration required of the kill vehicle in the endgame to almost an order of magnitude greater than that needed for midcourse intercept. 
The spinning kill vehicle The third configuration, originally called "HIT," is a spinning kill vehicle - elegantly simple in principle but constrained by its simplicity. It was popular in the 1970s and early 1980s and was flown in the USAF's air-launched anti-satellite (ASAT) weapon demonstration. This configuration, oriented and spun up prior to its deployment, used a body-mounted sensor having S-shaped line detectors that scanned the conical field of view at a rate determined by the spin velocity. Tiny solid-pulse motors around the periphery were fired at the time needed to impart a fixed $\Delta V$ pulse in the desired direction to reduce the LOS rate. Because this concept had inherently limited discrimination and divert ability, it lost favor as inertial measurement units shrank in size and weight, allowing the cruciform configuration, with its inherent versatility, to be as small.

\subsection{Endoatmospheric Kill Vehicles}

High endoatmospheric kill vehicles have followed the same general configuration trends as exoatmospheric kill vehicles but, because they operate in Earth's atmosphere, they require aerodynamic controls and a shape and thermal protection similar to those for a re-entry vehicle. Although designed for a much lower-velocity and dynamic pressure regime than ballistic missile intercept, the AIM 9 Sidewinder provides an example of the single axial thrust configuration that flies along the line of sight using steering similar to Sine Alpha.

The THAAD and Advanced Interceptor Technology (AIT) kill vehicles are designed for both high endo- and exoatmospheric intercepts. These kill vehicles use the cruciform configuration for divert thrusters. The thrusters are ported through a composite heat shield that provides a neutrally stable aerodynamic configuration. When in the atmosphere, small attitude-control thrusters are used to generate trim angle of attack for aerodynamic divert, and also to maintain the desired orientation during flight outside the atmosphere. The divert thrusters are used both inside and outside the atmosphere. The homing sensor looks out a window in the side of the fore-body and is steerable over limited angles. The vehicle is rolled to point its seeker in the plane described by the velocity vectors of the interceptor and the target. Limited roll-and-yaw angular freedom of the line of sight with respect to the vehicle is provided by movable mirrors within the optics of the sensor itself or by gimbaling the sensor.

\subsection{Kill Mechanisms Other than Body-to-Body Hit}

A number of other concepts for destroying the target have been developed over the years (see Chapter 13). Some of them, such as the Israeli Arrow missile, use a fragmentation warhead to relieve the endgame guidance and acceleration requirements. This approach is an outgrowth of anti-aircraft defense and is driven by the belief that it would be easier than a direct hit, particularly under the high dynamic pressure environment within the atmosphere. Fragmentation warheads typically eject their fragments in a radial pattern normal to the interceptor's roll axis. The disadvantage is that their weight is significant. Also, they usually require predictive fusing - typically a radar or optical double-cone fuse.

Other options generally known as "kill enhancement devices" have been developed for exoatmospheric kill vehicles. The basic concept is to increase the cross section of the kill vehicle around the seeker with a lightweight array of masses to handle any small misseswithout the need for fusing. The HOE kill vehicle shown in Fig. 11.2 had such an array based on unfurlable antenna technology that was deployed several seconds before intercept. The ERIS kill vehicle shown in Fig. 11.4 used a similar but much lighter device, employing 
an inflatable tubular frame that supported a thin membrane. It was also deployed based on estimated time-to-go.

Equipped with kill-enhancement mechanisms, both of these interceptors achieved bodyto-body hits during their successful flight tests. ERIS also incorporated the capability for selecting the aim point on the target. As a result, the confidence in direct hit-to-kill was increased, but no fight data about the lethality of those devices was obtained.

Lethality can also be enhanced in some applications by supplementing a hit-to-kill kill vehicle with deployable rods to increase the lethal radius. The issue of lethality for boostphase intercept is discussed further in Chapter 13.

\subsection{Key Requirements for Boost-Phase Kinetic Kill Above the Atmosphere}

Boost-phase intercept imposes requirements on the kill vehicle that significantly exceed those for midcourse intercept for two principal reasons. First, the intercept must occur on a much tighter timeline, constrained on one end by when the threat is detected and in track and on the other end by when the threat booster reaches the velocity required for its munitions' reaching targets being defended. Consequently, closing velocities for boost-phase defense are typically 40-50 percent higher than for midcourse defense. Second, the target is accelerating, which makes predicting the intercept point difficult. Prediction is further complicated because the acceleration changes rapidly, and there is uncertainty about its magnitude and direction.

The net result of those requirements is that the kill vehicles for boost-phase defense must have higher accelerations, more total divert velocity, and faster response times than those that have been designed or built for midcourse, all of which increase kill-vehicle weight. The demands of boost-phase intercept also require different guidance laws than are usually applied to the midcourse guidance problem. These issues are examined in detail in Chapter 12.

Midcourse intercept with proportional navigation In both midcourse and boost-phase intercepts, the kill vehicle is boosted into a nominal trajectory that will intercept the target at the desired intercept point, if events occur in accordance with the predictive model. In midcourse intercepts, this prediction is straightforward because the only forces acting on the target are gravity and whatever limited maneuver capability exists on the payload. The nominal intercept point can be predicted accurately based on current or recent observation of position and velocity. By and large, the amount of propellant in the kill vehicle is determined by the uncertainty that exists at the time of hand-off from external sensors that tracked the target, and the time to go before point of closest approach (POCA). The guidance scheme used is one of the many variants of proportional navigation, and accurate knowledge of the time to go is not an important issue in the accuracy of a hit. The rangeto-go estimate at any time may simply be the difference between the estimated target state vector provided to the kill vehicle and the vehicle's knowledge of its own state vector, based on its inertial measurement system or external tracking data.

Boost-phase intercept with augmented proportional navigation By comparison, during the boost phase, the target is accelerating because of propulsive thrust as well as gravity. The acceleration varies because the thrust varies with the burn rate, and the mass varies 
with the fuel consumed. The proportional navigation approach to midcourse intercept must be modified to take into account the estimated future acceleration of the target. This approach is called augmented proportional navigation and is discussed by Zarchan [140]. He points out that the predictive term does not have to be very accurate; it is possible to use merely the last measured acceleration for the future estimate because the noise in the tracking requires a large $\Delta V$ capability in any case. Augmented proportional navigation is modified somewhat and applied to the boost-phase problem in Chapter 12 .

Once out of the atmosphere, significant unpredictable target maneuvering can occur. Consequently, the propellant that is required on board the kill vehicle is a function not only of the uncertainty in the estimate of the target trajectory at any time, but also of the maneuver variations from the projected future trajectory. To a good approximation, any variation in acceleration from the prediction must be matched one-for-one by the kill-vehicle propulsion system. Except for cyclical tracking noise, any sensed acceleration must either be paid for at that time or paid for later at a much higher price. The difficulty, of course, is discerning which is noise and which is a real change in acceleration.

If the kill vehicle has an acceleration advantage, it might be argued that the augmented navigation gain should be chosen to cause the kill vehicle to "get out ahead" of target maneuvers, with the potential for saving fuel by allowing the target to "catch up" later. If the target feints or reverses field, however, the propulsive requirements to recover are greater than if the kill vehicle simply matched target's acceleration changes.

Chapter 10 analyzed the tracking accuracy and Chapter 12 will analyze in depth guidance and control issues for boost-phase hit-to-kill vehicles. Both chapters provide the basis for the sizing decisions made in Chapter 14.

\subsection{Summary}

The United States has a 30-year history of designing and developing hit-to-kill interceptors for midcourse defense. However, none have been built or tested for boost-phase intercept. Intercepting missiles in their boost phases places additional requirements on the kill vehicle, particularly on its ability to respond to rapid changes in the acceleration of the target. Those additional requirements tend to increase the weight of the kill vehicle and the size of the interceptor. We conclude that interceptor accelerations in any direction will be required. In consequence, because the response time of the kill vehicle would be constrained by the time that it takes to turn the vehicle, Sine Alpha steering is not desirable for exoatmospheric boost-phase defense.

Chapter 12 examines the requirements on the kill vehicle for hitting a boosting target. Chapter 14 then analyzes the effect that those requirements would have on the size of the kill vehicle, which affect the final sizes of the surface-based interceptors presented in Chapter 16 and space-based interceptors presented in Chapter 6 .

\section{References for Chapter 11}

[138] HOE, Ballistic Missile Defense, GAO/NSAID-94-219, July 1994.

[139] “Test Firing Downs ICBM", Jane's Defense Weekly, February 1991.

[140] P. Zarchan, "Tactical and Strategic Missile Guidance," Vol. 176, AIAA Progress in Astronautics and Aeronautics (1997). 


\section{Chapter 12}

\section{Hitting the Target}

\section{Contents}

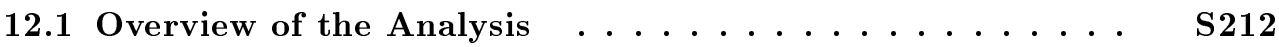

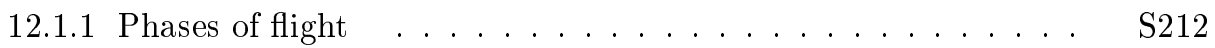

12.1.2 Requirements for intercept . . . . . . . . . . . . S214

12.1.3 Methodology . . . . . . . . . . . . . . . . . S214

12.2 Guidance Laws . . . . . . . . . . . . . . S216

12.2.1 Proportional navigation . . . . . . . . . . . . . . S217

12.2.2 Augmented proportional navigation . . . . . . . . . S220

12.2.3 Predictive guidance . . . . . . . . . . . . . . . . . . . S221

12.2.4 The guidance scheme used here . . . . . . . . . . . S221

12.3 Required Velocity Change Capability . . . . . . . . . S222

12.3.1 Engagement and tracking model . . . . . . . . . . . . . . . . S222

12.3.2 Estimating $\Delta V_{\text {tot }}$ requirements $\ldots \ldots \ldots \ldots \ldots$ S225

12.3.3 Summary of $\Delta V_{\text {tot }}$ requirements $\ldots \ldots \ldots \ldots \ldots \ldots$

12.4 The Endgame $\ldots \ldots \ldots \ldots \ldots \ldots \ldots \ldots$ S229

12.4.1 Endgame model . . . . . . . . . . . . . . . S230

12.4.2 Lunge maneuver . . . . . . . . . . . . . . . . S S231

12.4 .3 Jinking maneuver . . . . . . . . . . . . . . . . S233

12.4.4 Summary of endgame requirements . . . . . . . . . S234

12.5 Summary of Requirements for Hitting the Target . . . . S235

12.5.1 $\Delta V_{\text {tot }}$ requirements $\ldots \ldots \ldots \ldots \ldots \ldots \ldots \ldots \ldots$

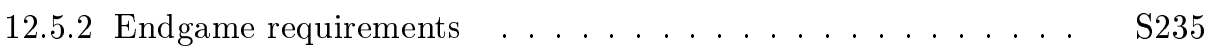

This chapter continues the second half of our analysis of boost-phase engagements using kinetic-energy weapons (Chapter 2). In the first half, which ended with Chapter 5, we explored the way in which the characteristics of the target missile and the performance of the interceptor determine the areas in which interceptors could be based and the missile launch area that a single interceptor battery could cover. For this exploration, we simply assumed that the performance of the off-board and on-board tracking sensors and the kill vehicle were adequate to achieve an intercept. In the second half of our analysis of boostphase engagements using kinetic-energy weapons, we investigate the sensor and kill-vehicle performance that would be needed to ensure that engagements have a high probability of being successful, despite the unpredictably of the target acceleration and maneuvering. 
The required performance of the sensors and kill vehicles was studied by first modeling the errors expected in tracking large rockets using space-based IR sensors, surface- and air-based radars, and passive IR and active LIDAR sensors on the kill vehicle, based on the analysis of these sensors in Chapter 10. Using these error models, a variety of engagements were simulated numerically, to explore the dependence of the required total kill-vehicle velocity change on the precision of the off-board missile-tracking sensors and the dependence of the final miss distance on the precision of the on-board homing sensors, the closing velocity, and the kill vehicle's performance. The model of the kill vehicle included the guidance algorithm used, the latency in the kill-vehicle response to the accelerations commanded by the guidance system, and its maximum acceleration. The effects of illustrative trajectory-shaping and evasive maneuvers by the target missile were studied. Performance requirements for the off-board sensors and the kill vehicle were estimated by requiring the miss distance, defined as the closest approach of the kill vehicle to the aimpoint on the target rocket, to be small enough to ensure a collision of the kill vehicle with the rocket body.

Our analysis of the required performance of the sensors, interceptor, and kill vehicle is described here and in Appendix C. The results of this analysis are used in Chapter 14 to assess how soon the needed technology is likely to be available, to determine the required mass of the kill vehicle, and to compute the masses and dimensions of the booster stacks that would be needed to accelerate the kill vehicle to the burnout velocities assumed in Chapters 4 and 5 .

Section 12.1 provides an overview of how we determined the required performance of the sensors, interceptor, and kill vehicle. It outlines the three phases of kill-vehicle flight, the key requirements for success in each phase, and the methodology we followed in our analysis. Section 12.2 describes several standard interceptor guidance laws, their advantages and disadvantages for intercepting a missile in powered flight, and the hybrid guidance scheme that we used. In Section 12.3, we describe how we estimated the total velocity-change capability the kill vehicle must have to reach the endgame of the engagement. Finally, in Section 12.4, we discuss the endgame, how we modeled it, the two illustrative evasive maneuvers we considered (lunges and jinking), and our estimates of the sensor and killvehicle performance required to achieve a miss distance of $\leq 0.5 \mathrm{~m}$ with high probability.

\subsection{Overview of the Analysis}

Before going into the details of the quantitative analysis, we first review the principal phases of the flight of the interceptor and kill vehicle, summarize qualitatively the performance requirements for achieving intercept, and describe the methodology used in the analysis. The acronyms used in this chapter are defined in Box 12.1 .

\subsubsection{Phases of flight}

The flight of the interceptor and kill vehicle may be divided into four phases (Chapter 2): (1) interceptor boost, (2) kill-vehicle divert, (3) kill-vehicle homing, and (4) the endgame. As a help in understanding the material in this chapter, we briefly review the characteristics of these phases. ${ }^{1}$

\footnotetext{
${ }^{1}$ Different definitions of the various phases are used by different authors; see for example [141, 142].
} 


\begin{tabular}{|ll|}
\hline Box & $12.1:$ Acronyms Used in This Chapter \\
\hline APN & Augmented proportional navigation \\
IP & Intercept point (actual) \\
LOS & Line of sight \\
PG & Predictive guidance \\
PN & Proportional navigation \\
ZEM & Zero effort miss \\
\hline
\end{tabular}

Interceptor boost Before an interceptor can be launched, the target missile must be detected, identified as a potentially threatening rocket, and tracked long enough to determine its gross characteristics and its general direction of flight (the azimuth of its trajectory would probably have to be determined to within about $10^{\circ}$ ). Space-based sensors would be used unless ground-, sea-, or air-based radars or other sensors could be positioned close enough to the missile launch site that their sightlines to the missile early in its flight would not be blocked by the curvature of Earth.

Once a sufficiently accurate target track has been constructed, which would likely take 10-15 s after detection using the planned SBIRS-High early warning system, or longer using a less capable system, a firing solution is computed and used to initialize the interceptor. ${ }^{2}$ As part of this process, the interceptor is told the predicted intercept point and an estimate of the path required to place the kill vehicle on a trajectory to this point so that it arrives simultaneously with the target.

During its powered flight, the interceptor receives frequent updates on its position relative to the target from off-board tracking sensors, such as space-based detectors or surfacebased radars, that are tracking the target. During this period the interceptor can correct its heading and flight path angle via changes in its orientation. As long as these changes are small, they do not significantly reduce the distance and velocity at which the final stage of the interceptor burns out. When the interceptor's final stage burns out, the kill vehicle is on a ballistic trajectory that takes it to the intercept point predicted just before burnout.

Initial divert In this phase, the kill vehicle relies on off-board tracking sensors. To efficiently correct for prediction errors and target maneuvers that occurred during its initial coast, the kill vehicle starts making guidance corrections as soon as its shroud is ejected and its propulsion system can begin to operate. The velocity change required to make these corrections is called the divert velocity. We include this velocity change in the total velocity change $\Delta V_{\text {tot }}$ made by the kill vehicle. ${ }^{3}$

Homing Assuming that the engagement develops as the defense intends, the kill vehicle will eventually approach the target close enough to acquire and track it with more precise

\footnotetext{
${ }^{2}$ When clouds are present, we assume the target will not be detected until it rises above the clouds; see Chapter 10.

${ }^{3}$ In this report we use the term divert velocity to refer to the integral of the absolute magnitude of the kill-vehicle's acceleration from the time its propulsion system begins to operate until it begins to use its own sensors for guidance. We use the term cumulative velocity change, denoted $\Delta V(t)$, to refer to the integral of the absolute magnitude of the kill-vehicle's acceleration from the time its propulsion system begins to operate until time $t$. The total velocity-change capability $\Delta V_{\text {tot }}$ that the kill vehicle must have to achieve an intercept is an important performance requirement.
} 
on-board sensors. The homing phase begins when the kill vehicle begins to rely on its onboard sensors. During this phase, the kill vehicle continues to make guidance corrections to compensate for sensor errors and maneuvers by the target, although not necessarily using the same algorithm at all times. We include the additional velocity changes made during this phase in the total velocity change $\Delta V_{\text {tot }}$ made by the kill vehicle.

Endgame The final few seconds of the homing phase is called the endgame. During this phase, the success of the attempted intercept depends more critically on the responsiveness and maximum acceleration of the kill vehicle, as well as on the continued availability of sufficiently precise and timely information on the target's changing trajectory.

\subsubsection{Requirements for intercept}

The performance required of the kill vehicle to hit the target depends on the phase of its flight.

Initial divert and homing The kill vehicle must have sufficiently precise information on the time evolution of the target's current and probable future state (position, velocity, and acceleration) and enough total velocity-change capability $\left(\Delta V_{\text {tot }}\right)$ and acceleration to reach a "basket" in position and velocity space small enough to begin the endgame.

Endgame The kill vehicle must have sufficiently precise and timely information on the target's current and probable future state and sufficient responsiveness and acceleration to hit the target.

Consideration of the initial divert and homing phases leads to a relationship between the precision and sampling rates of the off-board and on-board sensors used to track the target during these phases and the total velocity change capability $\Delta V_{\text {tot }}$ needed by the kill vehicle. Consideration of the endgame leads to requirements on the tracking precision and update rate provided by the kill vehicle's seeker, the accuracy and latency of the tracking and guidance systems, the dynamical responsiveness of the kill vehicle, and the kill vehicle's maximum acceleration. These factors determine the kill vehicle's design and minimum weight, which in turn determines the size and weight of the interceptor needed to boost the kill vehicle to the required velocity.

\subsubsection{Methodology}

As noted in Chapter 2, the standard approach for determining the performance requirements for a complex system such as a boost-phase intercept system is to develop detailed computer models that simulate all the processes involved, including measurement errors. By running many simulations having different parameter choices, the behavior of the system can be characterized statistically. Then, given a goal, the system performance that would be needed to achieve the goal can be determined. The Study Group lacked the time and resources needed to carry out such a comprehensive analysis of boost-phase intercept. Instead, the Group simulated several representative engagements in some detail. The results of these simulations were then used to study the sensitivity of the outcome to the performance of various components and to estimate the performance that would be needed to achieve a high probability of intercept. The simulations discussed in this report are listed in Table 12.1. 
Table 12.1. ICBM Engagements Simulated in the Study

\begin{tabular}{lcl}
\hline \hline Location in the Report & ICBM Model & Interceptor $\left(V_{b o}\right)$ \\
\hline This chapter & S2 & I-4 $(6.5 \mathrm{~km} / \mathrm{s})$ \\
Appendix C, Section C.2 & $\mathrm{L}$ & $\mathrm{I}-2(5 \mathrm{~km} / \mathrm{s})$ \\
Appendix C, Section C.1 & $\mathrm{S} 1$ & $\mathrm{I}-5(10 \mathrm{~km} / \mathrm{s})$ \\
\hline
\end{tabular}

This chapter describes our investigation of the initial divert, homing, and endgame phases of the kill vehicle's flight for engagements of the solid-propellant ICBM model S2 by the $6.5-\mathrm{km} / \mathrm{s}$ interceptor model I- 4 . One purpose of these simulations was to explore the dependence of the outcome of the engagement on the precision of the off-board and on-board tracking sensors, the latency in the kill vehicle's information about the target missile, the guidance algorithm used, the kill vehicle's dynamical response, and the kill vehicle's maximum acceleration. Another purpose was to evaluate the effects of several ICBM maneuvers that would stress the capabilities of the kill vehicle. We expect the kill vehicle performance that would be needed to intercept the liquid-propellant ICBM model or other solid-propellant ICBMs would be similar to the performance needed in the engagement we studied. One of the most important performance characteristics of a kill vehicle is the total change $\Delta V_{\text {tot }}$ that it can make in its velocity.

The target maneuvers we investigated are reasonable for an attacking ICBM and would not be particularly difficult to implement. The maneuvers considered during the initial divert and homing phases were a sudden increase or decrease in the target missile's angle of attack ${ }^{4}$ by $15^{\circ}$ and a switchback maneuver in which the target switched from a positive to a negative angle of attack. Maneuvers like these might be performed either to shape the missile's trajectory or to try to evade an anticipated kill vehicle. The maneuvers considered during the endgame were a single 8 g lunge executed during the last few seconds before the predicted intercept time and a sinusoidal modulation of the acceleration that would produce a fishtail-like motion of the missile during the last few seconds before the predicted intercept. An ICBM would execute these maneuvers only as an attempt to evade the kill vehicle.

We find that the distribution of miss distances is generally dominated by systematic errors, making it easy to bound the miss distance with high confidence. After exploring the problem, we chose to deem the performance of a kill vehicle adequate if it missed its aim point by $0.5 \mathrm{~m}$ or less in all the simulations we performed. For comparison, the final stages of the model ICBMs considered in the present Study have radii ranging from $1.5 \mathrm{~m}$ for the liquid-propellant model to $0.75 \mathrm{~m}$ for the two solid-propellant models. We have generally taken an optimistic view of what can be achieved and have neglected some sources of error that would increase the required performance of the kill vehicle. The performance requirements reported here are therefore minimum requirements.

Appendix $\mathrm{C}$ describes our investigation of the flyout of the interceptor and the initial di-

\footnotetext{
${ }^{4}$ In this report, a rocket's angle of attack is defined as the angle between its centerline and its velocity vector. It is positive if the projection of the centerline is above the projection of the velocity vector. Except during short time intervals when a rocket is being rotated, its thrust vector is parallel to its centerline. Hence the direction of motion of a rocket that is maintaining a nonzero angle of attack is changing (see Appendix B).
} 
vert and homing phases of kill-vehicle flight for an engagement of the liquid-propellant ICBM model L by the $5-\mathrm{km} / \mathrm{s}$ interceptor model I-2 and an engagement of solid-propellant ICBM model S1 by the $10-\mathrm{km} / \mathrm{s}$ interceptor model I- 5 . These simulations were run to evaluate the performance of the interceptor and kill vehicle if operated under command guidance, using tracking data supplied by the notional space-based IR sensor system and the surface-based radar described in Chapter 10. Various tracking filters were explored. These simulations showed that appropriately designed Kalman filters could provide adequate precision and maintain tracking through staging events.

The results described here and in Appendix $\mathrm{C}$ are for planar engagements. We do not expect the $\Delta V_{\text {tot }}$ required to be significantly less for out-of-plane engagements than for planar engagements. It could be more, because of the greater changes in the acceleration of the target perpendicular to the line of sight that are possible for out-of-plane engagements. Preliminary Monte Carlo calculations of the $\Delta V_{\text {tot }}$ required for both planar and out-of-plane engagements without target maneuvers do not show much difference [143].

We did not consider the additional demands that would be placed on the kill vehicle if the defense is required to time the intercept to occur within the narrow window needed to avoid causing possibly live warheads to fall on third countries (see Chapter 5). This problem is beyond the scope of the Study. Nor did we consider the effects of the countermeasures described in Chapter 9 that might delay the kill vehicle's awareness of a target maneuver, thereby increasing the velocity change capability it must have.

\subsection{Guidance Laws}

At any given time, even with complete and perfect current information, the defense can predict the future trajectory of the target missile only approximately, because the defense does not know what the target will do in the future. A guidance scheme is therefore needed. Such a scheme uses updated information on the position and motion of the target to issue instructions to the interceptor's propulsion and steering mechanisms that will steer the interceptor toward the target $^{5}$. The guidance scheme used affects the sensor precision, the $\Delta V_{\text {tot }}$, and the maximum acceleration required to achieve a given miss distance. Here we discuss the behavior and limitations of several different guidance schemes when applied to the present problem ${ }^{6}$.

We first discuss the effectiveness of three commonly used algorithms for guiding an interceptor attempting to hit an accelerating target. These algorithms are proportional navigation, augmented proportional navigation, and predictive guidance. Each has numerous variants, but their main features are distinct. Next we discuss the applicability of these algorithms to the ICBM intercept problem. For our simulations, we adopted the hybrid scheme outlined at the end of this section.

Several basic concepts are important for understanding the following discussion. The line of sight (LOS) is the imaginary line connecting the interceptor and the target. The $L O S$ angle $\lambda$ is the angle between the LOS and a fixed reference direction. The length of the LOS (the instantaneous separation between the interceptor and the target) is the range to the target or simply the range, denoted $R_{t}$. The closing velocity $V_{c}$ between the interceptor

\footnotetext{
${ }^{5}$ For conciseness, in this section we use 'interceptor' to refer either to the interceptor missile with the kill vehicle attached or to the kill vehicle after it separates from the interceptor, whichever is relevant.

${ }^{6}$ For a tutorial on the guidance and control issues involved in ballistic missile defense, see [142]. The problem of estimating and predicting ballistic missile trajectories using data from sensors is discussed in [144]. For detailed analyses of the problem of missile guidance and control, see [141, 145].
} 


\begin{tabular}{|ll|}
\hline \multicolumn{2}{|c|}{ Box $12.2:$ Symbols Used in This Chapter } \\
\hline$\lambda$ & Angle of the line of sight (LOS) \\
$\dot{\lambda}$ & LOS rate (actual) \\
$\hat{\lambda}$ & LOS rate (estimated) \\
$X_{t}$ & Target position (actual) \\
$\hat{X}_{t}$ & Target position (estimated) \\
$V_{t}$ & Target velocity (actual) \\
$\hat{V}_{t}$ & Target velocity (estimated) \\
$a_{t}$ & Target acceleration (actual) \\
$\hat{a}_{t}$ & Target acceleration (estimated) \\
$X_{i}$ & Interceptor position (actual) \\
$V_{i}$ & Interceptor velocity (actual) \\
$a_{i}$ & Interceptor acceleration (actual) \\
$a_{c}$ & Interceptor acceleration (commanded) \\
$V_{c}$ & Interceptor-target closing velocity \\
$\hat{V}_{c}$ & Estimated closing velocity \\
$N^{\prime}$ & Proportional navigation guidance gain \\
$t_{\mathrm{go}}$ & Time to go to closest approach \\
$t_{\mathrm{go}, 0}$ & Initial time to go \\
$\Delta V_{t}$ & Cumulative target velocity change \\
$\Delta V_{i}$ & Cumulative interceptor velocity change \\
& \\
\hline
\end{tabular}

and the target is $-\dot{R}_{t}$. The miss distance $d_{m}$ characterizes the accuracy of the intercept. It is the closest approach of the interceptor to the aimpoint on the target. The time to go $t_{\mathrm{go}}$ is time remaining until closest approach. The most important symbols used in this chapter are defined in Box 12.2.

\subsubsection{Proportional navigation}

In proportional navigation (PN), the basic guidance signal is the time derivative $\dot{\lambda}$, which is called the line-of-sight rate. Knowledge of the range and the time to go are not required. Thus, PN can be implemented using an angle-only sensor on board the kill vehicle, which is a great advantage in many applications.

The commanded acceleration $a_{c}$ of the kill vehicle is normal to the LOS and proportional to both $\dot{\lambda}$ and $V_{c}$, with negative feedback to drive the rate to zero [141, pp. 27-28]. If the closing velocity is positive, i.e., the interceptor is approaching the target, an unchanging LOS angle implies that the kill vehicle is on a collision course with the target. The intercept time (or the time of closest approach if the kill vehicle misses the target) is not controlled and is largely determined by the initial separation between the kill vehicle and the target and their initial velocities.

Although the PN guidance law was not derived with target acceleration as a fundamental consideration, with perfect information, a constant closing velocity, and unlimited interceptor acceleration and velocity change capability, PN will drive the miss distance 
caused by an initial heading error or a constant target acceleration to zero. For an initial heading error and-or a constant target acceleration normal to the LOS, the time history of the zero-effect miss, $(\mathrm{ZEM})^{7}$, the commanded acceleration $a_{c}$, and the cumulative divert velocity $\Delta V$ can all be expressed in closed form. For the constant target acceleration case, the equations for the commanded acceleration and the cumulative velocity change are

$$
\frac{a_{c}}{a_{t}}=\frac{N^{\prime}}{N^{\prime}-2}\left[1-\left(\frac{t_{\mathrm{go}}}{t_{\mathrm{go}, 0}}\right)^{N^{\prime}-2}\right]
$$

and

$$
\Delta V_{i, P N}=\frac{N^{\prime}}{N^{\prime}-1} \Delta V_{t}
$$

The symbols are defined in Box 12.2. For this case, the velocity change capability needed by the interceptor exceeds the total velocity change of the target. For example, for $N^{\prime}=4$ the ratio is $4 / 3$.

For target accelerations and heading errors small enough that the guidance system is operating in the linear regime, $\mathrm{PN}$ will always drive the miss distance to zero at the time of intercept. This is true not only for a constant acceleration, but also for a time-varying one, as may be seen by approximating a time-varying acceleration by a sequence of constant accelerations. If the target does not accelerate, and the terminal controller is linear with a performance index quadratic in the state variables and the control effort, $\mathrm{PN}$ with $N^{\prime}=3$ can be shown to be the optimal linear control law [146, pp. 154-155].

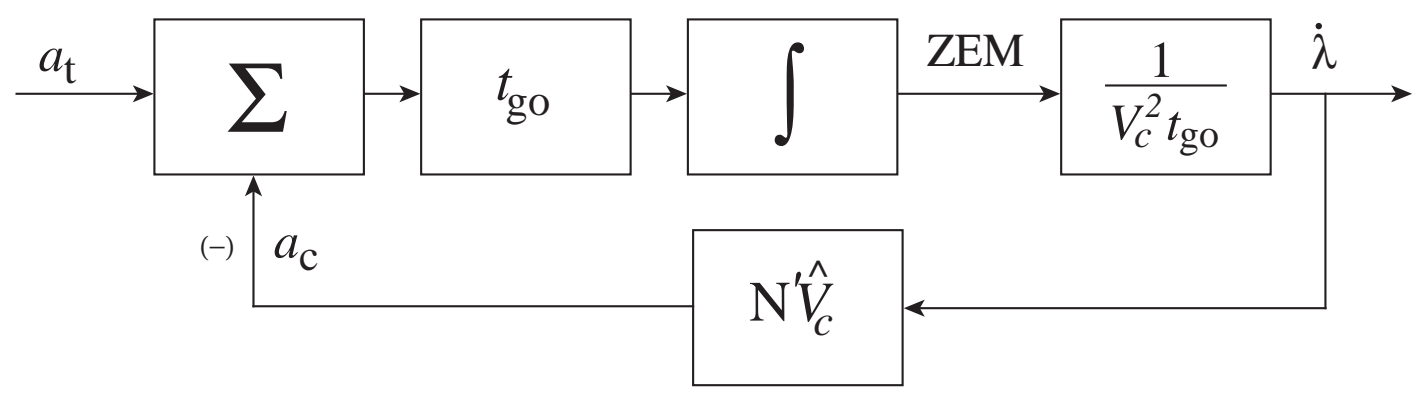

Figure 12.1. Block diagram of an idealized proportional navigation scheme for a two-dimensional engagement, formulated in terms of the zero effort miss (ZEM). See Box 12.2 for definitions of the symbols used.

Figure 12.1 shows a block diagram of an idealized PN scheme for a two-dimensional engagement. The scheme is idealized in the sense that sensor error is not included $(\hat{\dot{\lambda}}$ is equated to $\dot{\lambda}$ ), and the interceptor's actual acceleration is equated to the acceleration commanded by the guidance system, i.e., control system lags and the dynamics of the kill vehicle have been neglected. Proportional navigation can be expressed in various mathematically equivalent forms, depending on the dynamical variables used. The form shown here is formulated in terms of the ZEM; note that in this formulation the equation for the ZEM is first-order in time. Only the LOS rate and an estimate of the closing velocity are required to compute the commanded acceleration $a_{c}$ of the kill vehicle. ${ }^{8}$

\footnotetext{
${ }^{7}$ The zero-effort miss is the distance by which the interceptor would miss the target if the target continued on its present course with its present speed and the interceptor made no further corrective maneuvers.

${ }^{8}$ Although $t_{\text {go }}$ appears explicitly in Fig. 12.1, it is not needed to implement the guidance scheme and enters only through the LOS rate; see [141].
} 
The loop gain is $t_{\text {go }} \times\left(1 / V_{c} t_{\text {go }}^{2}\right) \times N^{\prime} \hat{V}_{c}=\left(N^{\prime} / t_{\text {go }}\right)\left(\hat{V}_{c} / V_{c}\right)$, where $N^{\prime}$ is the proportional navigation guidance gain, sometimes called the effective navigation ratio, and $\hat{V}_{c}$ is the closing velocity estimated by the guidance system. The closed-loop performance is relatively insensitive to small changes in the loop gain, provided it is about 3 or more divided by the time to go in seconds. Because the loop gain is proportional to the ratio of the estimated closing velocity to the true closing velocity, errors of 10 percent or so in the estimated closing velocity have little effect. Hence, estimates of the closing velocity based on simulated engagements or a priori knowledge are often used.

Numerical simulations using finite-difference implementations of the equations represented by the block diagram in Fig. 12.1 reproduce the closed-form results for constant target acceleration mentioned above but can also be used to calculate the commanded response produced by arbitrary target accelerations. With appropriate modifications, such simulations can be used to evaluate the effects of limits to the interceptor's ability to accelerate and lags in its response.

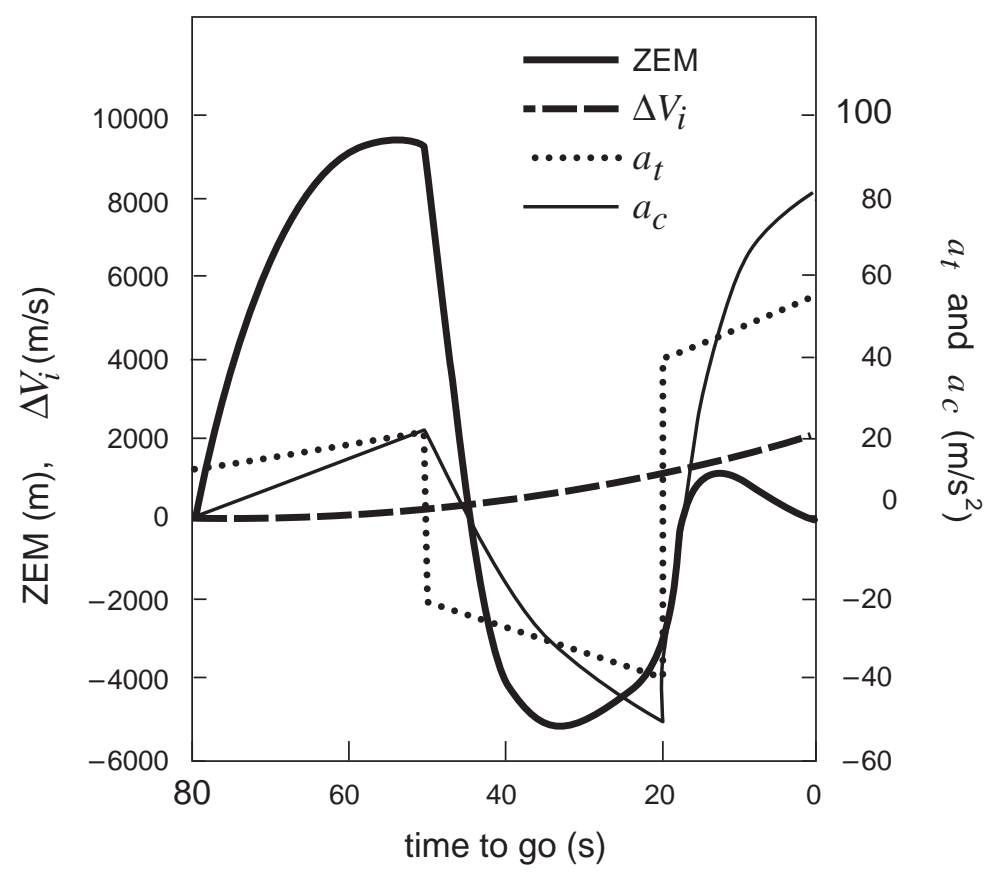

Figure 12.2. Example of the time evolution of an engagement in which a kill vehicle using proportional navigation with a gain of 6 attempts to hit a target with a time-varying acceleration. Shown are the time histories of the zero-effort miss in meters (left-hand scale), the target's acceleration $a_{t}$ and the interceptor's commanded acceleration $a_{c}$ normal to the line of sight in $\mathrm{m} / \mathrm{s}^{2}$ (right-hand scale), and the cumulative commanded velocity change $\Delta V$ of the interceptor in $\mathrm{m} / \mathrm{s}$ (left-hand scale). In this example, the kill vehicle hits the target at the end of the engagement, as indicated by the vanishing of the zero-effort miss. By then, $\Delta V$ has grown to $2.2 \mathrm{~km} / \mathrm{s}$.

Figure 12.2 shows the simulated behavior of a kill vehicle using the idealized PN scheme as it attempts to intercept a target that is accelerating in a complicated way. In spite of the complex response, the ZEM is driven to zero with no time to go, implying a direct hit. In this example, the cumulative velocity change computed by integrating the absolute value of the acceleration is $2.2 \mathrm{~km} / \mathrm{s}$. For comparison, the integral of the absolute value of target acceleration is $2.3 \mathrm{~km} / \mathrm{s}$. Depending on the target acceleration history, the kill-vehicle cumulative velocity may be either more or less than the integral of the absolute value of 
the target acceleration. For a high value of the $\mathrm{PN}$ gain, the kill vehicle and target total velocities tend to be nearly equal, as in this example.

To improve the performance of the guidance system when confronting an accelerating target, an augmented proportional guidance law can be considered.

\subsubsection{Augmented proportional navigation}

For a target with a constant acceleration, augmented proportional navigation (APN) feeds the target acceleration forward to the interceptor with a gain of $N^{\prime} / 2$, as shown in the block diagram in Fig. 12.3 [141, p. 145]. For constant target acceleration, the closed-form equations for the commanded acceleration and the cumulative velocity change are

$$
\frac{a_{c}}{a_{t}}=\frac{N^{\prime}}{2}\left(\frac{t_{\mathrm{go}}}{t_{\mathrm{go}, 0}}\right)^{N^{\prime}-2}
$$

and

$$
\Delta V_{i, A P N}=\frac{N^{\prime}}{2\left(N^{\prime}-1\right)} \Delta V_{t}
$$

The symbols are defined in Box 12.2 .

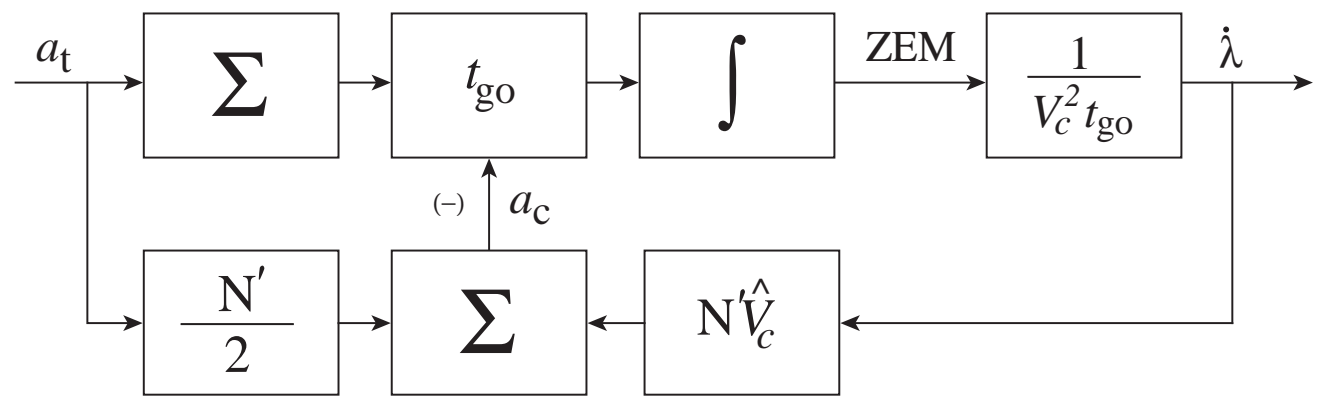

Figure 12.3. Block diagram of an idealized augmented proportional navigation scheme. See Box 12.2 for definitions of the symbols used.

In contrast to PN, for APN the total change in the interceptor's velocity can actually be less than the total change in the target's velocity. For example, for $N^{\prime}=4$, the ratio is $2 / 3$. For this or any value of $N^{\prime}$, the total change in the interceptor's velocity using APN is half of that for PN. To achieve this reduction, the guidance system initially commands an acceleration greater than the target's acceleration - in essence inflating the ZEM. To derive a significant benefit from using APN requires some knowledge of the target's acceleration. In general, the target's acceleration cannot be estimated accurately enough using only angle measurements made by imaging sensors on the interceptor; usually range information is used. The range information could be supplied either by off-board passive sensors or by on-board active sensors.

Even in the noise-free case, it is not always possible to assure that the interceptor's total velocity change will be less than the target's total velocity change. For example, if the target executes certain switching maneuvers, APN can cause the total change in the interceptor's velocity to exceed the total change in the target's velocity. However, if the interceptor can match the target's acceleration normal to the LOS, the interceptor and target velocity changes will be equal. Acceleration matching can therefore minimize the maximum total 
velocity change required if maneuvers are unpredictable and arbitrary. As an aside, in a two-sided "game-theoretic optimal intercept," zero-miss distance can be guaranteed if the pursuer is more maneuverable than the target. However, when the relative maneuverability approaches unity, the APN guidance law approaches PN guidance with an infinite gain, which forces acceleration matching [147, pp. 2-3].

\subsubsection{Predictive guidance}

Predictive guidance (PG) assumes that the guidance system has some knowledge of the future motion of the target and uses this knowledge to help predict the intercept point. PG is particularly effective if the target's acceleration is constant, but it can also be useful if the target's acceleration is changing, provided the interceptor has some knowledge of the target's future acceleration profile. For example, a missile trajectory template or the trajectory of a model of the missile with some constraints on steering can be used. For the boost-phase intercept problem, predictive guidance would involve predicting the target missile's trajectory and using this information to compute a predicted intercept point. The interceptor's nominal trajectory would also be projected forward and the ZEM calculated. At each corrective step, the interceptor would be steered to null the component of the ZEM normal to the LOS. For reasonably well-characterized ballistic targets and targets with relatively small unpredictable maneuvers, PG can be highly effective [148].

For targets that make larger unpredictable maneuvers, such as maneuvering re-entry vehicles, a manageable set of predictions can be considered, particularly when there are constraints on the target missile, such as reaching a specific target area. However, all prediction schemes are challenged when - as in the boost-phase intercept problem - the area to be defended is large, the intended target is unknown, knowledge of the performance characteristics of the target missile may be poor, and the target intentionally maneuvers.

\subsubsection{The guidance scheme used here}

Because the behavior of an ICBM in powered flight is difficult to predict, we decided to make minimal a priori assumptions about the characteristics and behavior of the target. The target's acceleration, velocity, and position are estimated initially from measurements by off-board sensors (see Chapter 10). The interceptor's state was assumed to be known perfectly, relative to other uncertainties in the problem. The interceptor was guided using PN, augmented by matching, the target's acceleration normal to the LOS. A PN gain of 3 and an APN gain of 2 were used. A block diagram of this scheme is shown in Fig. 12.4.

This "hybrid" scheme is motivated by the discussion of APN and acceleration matching in Section 12.2.2. The APN gain of 2 produces acceleration matching and the PN gain of 3 provides a relatively low-gain feedback correction for errors. Some variations were studied, but none that we considered appeared to perform as well overall. Since this exploration was by no means comprehensive, we cannot preclude the possibility that a significantly superior guidance scheme could be found.

An important point is that the final boost stages of ICBMs produce significant axial accelerations. Hence the simple classical equations for PN and APN, which assume a constant closing velocity, do not strictly apply. For the equations to be accurate, the additional velocity that is gained by the target before the intercept must be much less than the closing velocity [149]. Generally this does not occur until the endgame.

Setting aside these issues, the hybrid PN/APN guidance scheme shown in Fig. 12.4 was 


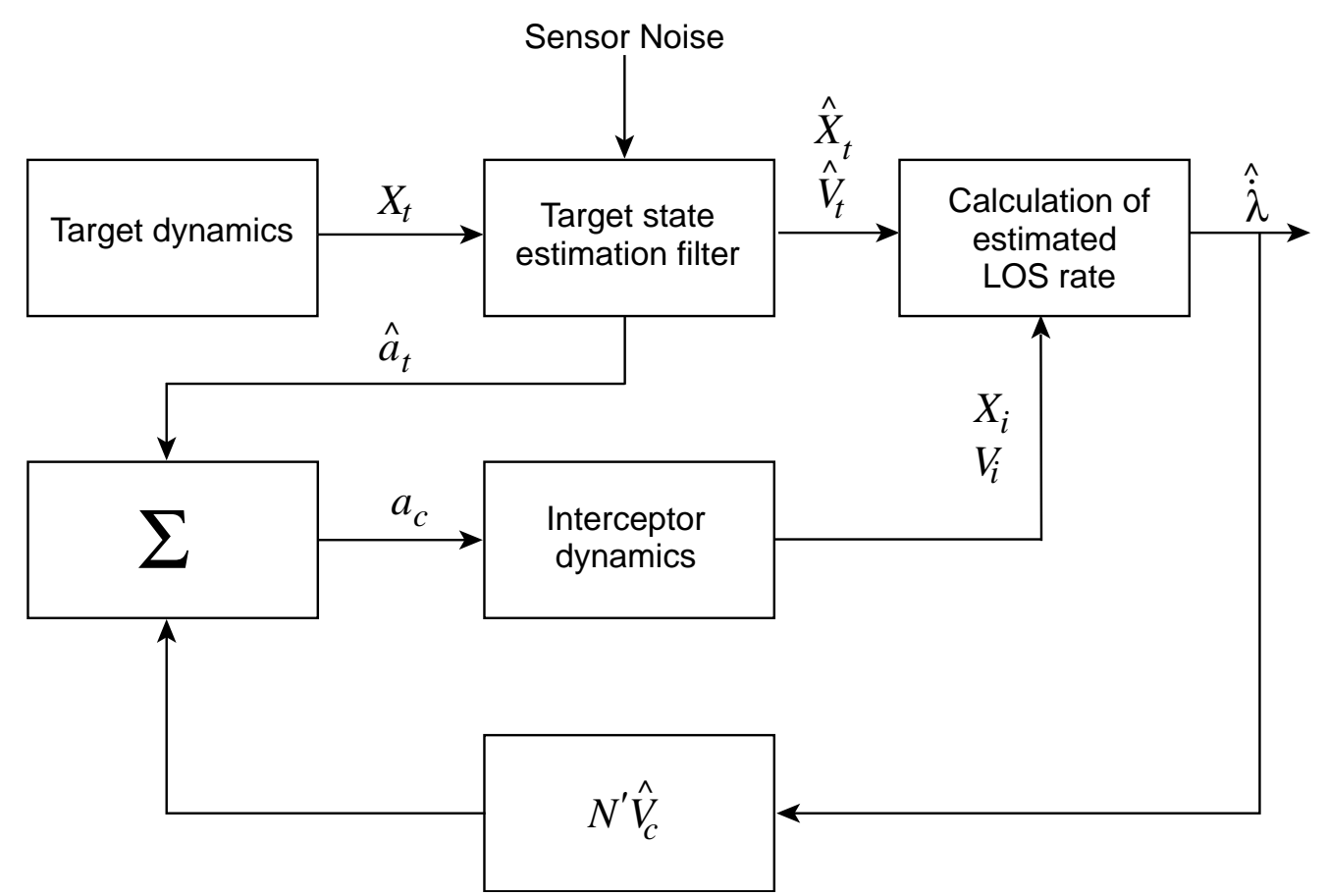

Figure 12.4. Block diagram of the guidance scheme used in simulating the engagements discussed in this chapter, except in the endgame, where a different scheme was used. The variables estimated by the tracking and guidance algorithm are indicated by carets. See Box 12.2 for definitions of the symbols used.

used to simulate a variety of engagements in which the target accelerated and maneuvered as an ICBM would be expected to do. The sensor errors, the latency in processing target information, the kill vehicle's dynamical behavior, and the lag in its response to guidance commands were modeled in the simulations, as described in the relevant sections below. Each simulation yielded the kill vehicle's trajectory, acceleration profile, and total velocity change. The simulations were used to explore the kill-vehicle performance needed to drive the miss distance down to a level (a fraction of a kilometer) at which the endgame could be started. In the endgame, a different guidance law and a finer resolution were used to compute miss distances with the centimeter precision that is necessary to determine whether the kill vehicle successfully hits the target.

\subsection{Required Velocity Change Capability}

This section summarizes the results of the simulations used to estimate the total velocitychange capability $\Delta V_{\text {tot }}$ that the kill vehicle would need to have a high probability of intercepting a target ICBM. The approach used in the Study is illustrated by the following illustrative engagement.

\subsubsection{Engagement and tracking model}

Engagement model Most of our exploration of the $\Delta V_{\text {tot }}$ needed for intercept considered planar engagements of solid-propellant ICBM model S2, which has a 170-second boost phase, by a kill vehicle boosted and deployed by interceptor model I-4, which has a 40- 
second boost phase and a burnout velocity of $6.5 \mathrm{~km} / \mathrm{s} .{ }^{9}$ Unless otherwise noted, all times given are relative to the time the target ICBM was launched.

In the engagements simulated here, the interceptor was fired at $45 \mathrm{~s}$ from a standoff distance such that it would intercept the target ICBM at about $155 \mathrm{~s}, 15 \mathrm{~s}$ before the ICBM's final stage would have burned out. The interceptor was assumed to have a heading error of no more than $20 \mathrm{~km}$ when it burned out at $85 \mathrm{~s}$. The kill vehicle's shroud was ejected when the interceptor reached an altitude of $85 \mathrm{~km}$. At this altitude, which the interceptor reached at $100 \mathrm{~s}$, the dynamic pressure is low enough for the kill vehicle to begin to operate. Once deployed, the kill vehicle was assumed to begin steering itself using measurements of the target's position provided initially by off-board sensors and later by on-board sensors. The closing velocity for this engagement is about $10 \mathrm{~km} / \mathrm{s}^{10}$

Tracking sensors As described in Chapter 10, the launch of a large rocket would first be detected by space-based IR sensors, unless surface- or air-based radars could be positioned close enough to the missile launch site that their sightlines to the missile early in its flight would not be blocked by the curvature of Earth. The precision of the information on the target's position that could be provided by modern space-based IR sensors was estimated to be about $300 \mathrm{~m}(1 \sigma)$ in all three directions with a $1-\mathrm{Hz}$ rate. This level of accuracy corresponds to a uniform distribution of errors over a pixel with a $1 \mathrm{~km} \times 1 \mathrm{~km}$ footprint on Earth's surface (see Section 10.1.3).

Once it has risen high enough, the target rocket could also be tracked by distant surfaceor air-based radars (see Chapter 10). Although a radar could measure the target rocket's range much more precisely than a space-based IR system, if the rocket were above the radar's horizon and the radar were not being jammed (but see Chapter 9), there are engagement geometries for which any of the three directions could be critical. We estimate that radars could measure the position of target ICBMs with an uncertainty of $300 \mathrm{~m}$ in each direction.

The plume of a large rocket could be detected by a passive IR sensor on board the kill vehicle as soon as the kill vehicle reached sufficient altitude for such a sensor to open and operate (Chapter 10). Measurements of the plume by such a sensor could in principle be used to determine the position of the rocket body to within about $100 \mathrm{~m}$ in the two directions normal to the LOS at a frame rate of $30 \mathrm{~Hz}$ or even greater, provided the plume and its relationship to the position of the rocket body are understood well enough for the relevant engagement geometry. However, a passive IR sensor would not be able to provide range information precise enough to be useful.

When the kill vehicle is close enough to the target, active on-board sensing techniques can provide information on the position of the target in all three dimensions. For example, the on-board LIDAR seeker analyzed in Chapter 10 can provide precise measurements of the range and increasingly precise measurements of the position of the rocket body normal to the LOS at a frame rate of $1 \mathrm{~Hz}$ or even greater, once the range were $250 \mathrm{~km}$ or less. Although

\footnotetext{
${ }^{9}$ As noted at the beginning of this chapter, Appendix $\mathrm{C}$ also describes simulated engagements of liquidpropellant ICBM model L by the $5-\mathrm{km} / \mathrm{s}$ interceptor I-2 and engagements of the solid-propellant ICBM model $\mathrm{S} 1$ by the $10-\mathrm{km} / \mathrm{s}$ interceptor I- 5 .

${ }^{10}$ The closing velocity for these same ICBM and interceptor models would be less for non-planar engagements, e.g., about $7 \mathrm{~km} / \mathrm{s}$ for an interceptor trajectory nearly normal to the plane of the ICBM trajectory. We focused on engagements in which the trajectories of the target and interceptor are essentially planar and in approximately the same plane because this is the simplest case. As explained in Section 12.1.3, we do not expect the required velocity change capability to be qualitatively different for non-planar engagements.
} 
the LIDAR frame rate could be increased as the target range decreases, we assumed a $1-\mathrm{Hz}$ rate until the endgame.

Sensor data In studying the $\Delta V_{\text {tot }}$ required to intercept an ICBM, we used target-position update rates and uncertainties representative of the performance of the off-board and onboard sensors discussed above. Off-board measurements of the target's position were assumed to be provided by modern space-based IR sensors on two early-warning satellites operating in stereo mode. The uncertainty in the position of the target provided by such space-based sensors would be independent of the target's range from the kill vehicle. After some initial exploration of the implications of other values, most of the simulations were carried out assuming that space-based IR sensors would measure the position of the target at a $1-\mathrm{Hz}$ rate with an uncertainty of about $300 \mathrm{~m}(1 \sigma)$ in all three directions from the time the kill vehicle began operating until the range to the target became less than $250 \mathrm{~km}$. ${ }^{11}$ We assumed that once the kill vehicle had closed to within $250 \mathrm{~km}$ of the target, a LIDAR on board the kill vehicle would measure the position of the target with an uncertainty of about $30 \mathrm{~m}(1 \sigma)$ in all three directions at a frame rate of $1 \mathrm{~Hz}$. For simplicity, we assumed that the uncertainties in the position measurements made by the LIDAR were also independent of the range to the target, except in the endgame (see below).

Estimating the target's state Estimating the relevant aspects of the target's state (in this case its position, velocity, and acceleration) could be done using a Kalman filter ${ }^{12}$ or in several other ways. Our simplifying assumption that the uncertainties in the measured positions of the target rocket are independent of the rocket's range from the kill vehicle makes the uncertainties independent of time. We therefore used fixed-length, second-order polynomial filters [151], tuned to the measurement error, sampling rate, and target dynamics.

For simplicity, we used only the data from the off-board sensor from the time the kill vehicle began operating $55 \mathrm{~s}$ after the interceptor was fired (100 s after the target missile was launched) until the range to the target became less than $250 \mathrm{~km}, 85 \mathrm{~s}$ after the interceptor was fired (130 s after the target missile was launched). ${ }^{13}$ A filter length of $15 \mathrm{~s}$ was used for these data. Once the range to the target became less than $250 \mathrm{~km}$, we used only the data from the sensor onboard the kill vehicle. A filter length of $6 \mathrm{~s}$ was used for these data.

The estimates of the target's state supplied by the filters are uncertain both because of measurement noise and because of deviations of the target's acceleration from the constant value assumed by the polynomial filters. The $1 \sigma$ uncertainties in estimates of the target's position, velocity, and acceleration induced by measurement noise are listed in Table 12.2. The uncertainties caused by the deviations of the target from a constant acceleration profile are roughly the same size. For example, a constant jerk of $1 \mathrm{~m} / \mathrm{s}^{3}$ introduces an $8-\mathrm{m} / \mathrm{s}^{2}$ error in the estimated acceleration of the target for measurements with $300-\mathrm{m}$ resolution and a $3-\mathrm{m} / \mathrm{s}^{2}$ error for measurements with $30-\mathrm{m}$ resolution.

\footnotetext{
${ }^{11}$ If a follow-on space-based IR sensor system is not available and DSP data had to be used, the update rate would be $0.1 \mathrm{~Hz}$, which would increase the $\Delta V_{\text {tot }}$ required for intercept. As noted earlier, radars could be used to measure the position of the target rocket with precisions and update rates similar to those provided by modern space-based IR sensors if they could be located close enough to the ICBM's launch point.

${ }^{12}$ For an introduction to Kalman filtering, see [150].

${ }^{13}$ Estimation of the state of the target by fusing and filtering off-board data from modern space-based IR sensors and a surface-based radar is illustrated in Appendix C.
} 
Table 12.2. Uncertainties in Estimates of the State of the Target $\mathrm{ICBM}^{a}$

\begin{tabular}{lcc}
\hline \hline Uncertainty $(1 \sigma)$ & Off-Board Sensors $^{b}$ & On-Board Sensor $^{c}$ \\
\hline Position uncertainty $(\mathrm{m})$ & 200 & 25 \\
Velocity uncertainty $(\mathrm{m} / \mathrm{s})$ & 60 & 20 \\
Acceleration uncertainty $\left(\mathrm{m} / \mathrm{s}^{2}\right)$ & 8 & 6 \\
\hline
\end{tabular}

${ }^{a}$ Induced by errors in the measured position of the target during the flight of the kill vehicle. ${ }^{b}$ Representative of modern IR sensors on two early-warning satellites operating in stereo mode and providing measurements of the target's position with a $300-\mathrm{m}(1 \sigma)$ uncertainty in all three directions at a $1-\mathrm{Hz}$ update rate.

${ }^{c}$ Representative of a LIDAR seeker providing measurements of the target's position with a $30-\mathrm{m}(1 \sigma)$ uncertainty in all three directions at a $1-\mathrm{Hz}$ frame rate. The endgame was treated separately.

\subsubsection{Estimating $\Delta V_{\text {tot }}$ requirements}

Figure 12.5 shows the actual acceleration profile of the target missile during its boost phase and the acceleration estimates provided by the filter used in the simulations. ${ }^{14}$ In the left panel, the sensor noise has been set to zero to show the basic effect of the polynomial filter. The two staging events are clearly evident. The sensor noise makes these events less obvious, as the right panel shows. The effect of the noise diminishes only modestly at $130 \mathrm{~s}$, when the guidance algorithm starts using the far more accurate on-board sensor data, because at this point the filter length is reduced from $15 \mathrm{~s}$ to $6 \mathrm{~s}$ to make it more responsive to target maneuvers.
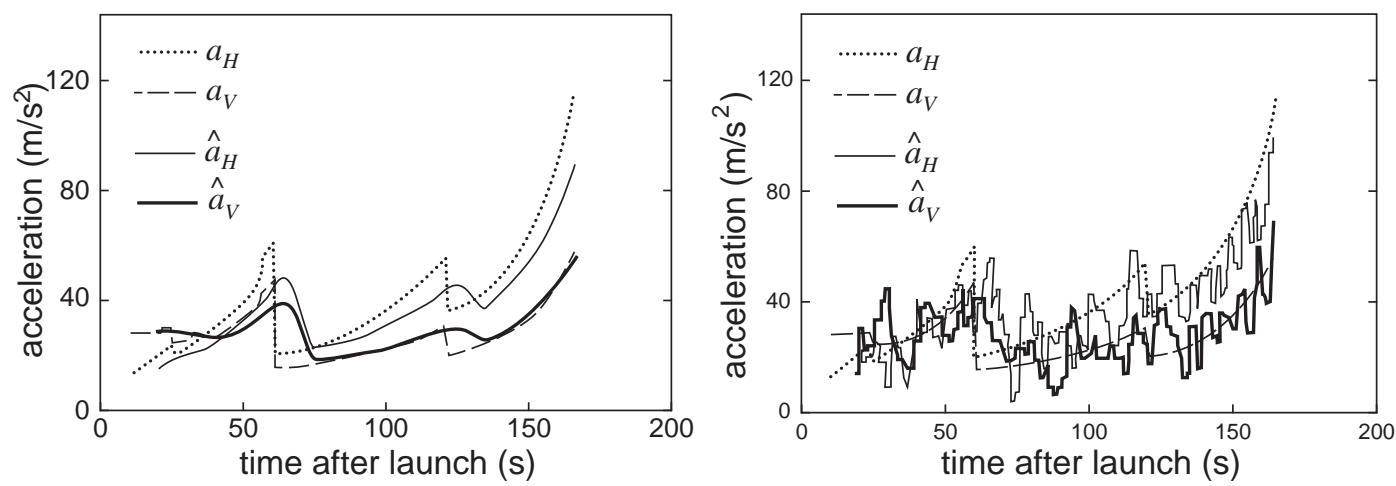

Figure 12.5. Illustration of estimates of target acceleration using polynomial filters, with the sensor noise turned off (left) and with sensor noise included (right). Shown are the horizontal and vertical accelerations $a_{H}$ and $a_{V}$ and the corresponding estimates $\hat{a}_{H}$ and $\hat{a}_{V}$ provided by the filter. The acceleration drops sharply at each of the two staging events which occur at $\sim 60 \mathrm{~s}$ and $120 \mathrm{~s}$. After each event, the acceleration estimates deviate from the actual acceleration but recover after a time comparable to the filter length ( $15 \mathrm{~s}$ before $130 \mathrm{~s}$ and $6 \mathrm{~s}$ afterward). The sensor noise was assumed to be $300 \mathrm{~m}(1 \sigma)$ before $130 \mathrm{~s}$ and $30 \mathrm{~m}$ afterward.

\footnotetext{
${ }^{14}$ Reducing the delay in estimating the change in target acceleration after staging events or step target maneuvers is a subject of active research. Somewhat superior results are cited in [152]; we did not explore these methods to determine their generality and robustness.
} 
To determine the kill-vehicle $\Delta V_{\text {tot }}$ required to assure intercept, Monte Carlo runs of 100 samples each were performed for a range of off-board sensor resolutions to determine the average $\Delta V_{\text {tot }}$ and the 90 th-percentile point in the distribution of the required $\Delta V_{\text {tot }}$ first, by the nominal target dynamics and sensor noise, and second, by these and evasive maneuvers by the target. Two simple maneuvers were considered: a sudden increase in the angle of attack of the target missile by $15^{\circ}$ or a sudden decrease by the same angle. Maneuvers like these could be executed either to adjust the trajectory of the ICBM or to attempt to exhaust the velocity-change capability of the kill vehicle.

In order to investigate the most stressing consequences of such maneuvers, we assumed they were initiated at $86 \mathrm{~s}$. This timing is disadvantageous for the defense because the kill vehicle is unable to maneuver from the time the final stage of the interceptor burns out at $86 \mathrm{~s}$ until its shroud is ejected at $100 \mathrm{~s}$. By changing its angle of attack at $86 \mathrm{~s}$, the target missile will follow a trajectory different from the one expected by the defense when the interceptor burned out. Because the kill vehicle is temporarily unable to maneuver, it cannot compensate immediately for the change in the target's trajectory and therefore continues in what is now the wrong direction. Once the kill vehicle can maneuver, which in this situation is 14 seconds after the change in target trajectory, it now has a larger possible heading error to correct for than in the case of no evasive maneuver. Furthermore, until the ICBM burns out, the target can continue to accelerate in the new direction, or make further evasive changes in its direction of acceleration; the $\Delta V$ required to have high confidence of compensating for these later changes is the dominant factor in the total $\Delta V$ budget.

Figure 12.6 shows the trajectories of the target ICBM and interceptor in the rangealtitude plane for a simulated engagement in which the second stage of the ICBM increases its angle of attack by $15^{\circ}$ when the final boost stage of the interceptor burns out at $86 \mathrm{~s}$. The kill vehicle begins to steer toward the ICBM's second stage at $100 \mathrm{~s}$. In this simulation, the kill vehicle's closest approach to the ICBM's final stage occurs at about 157 s. In Fig. 12.6 the missile and kill vehicle trajectories have been extended to $160 \mathrm{~s}$. On the scale shown, the crossing of the target and kill vehicle trajectories and their slight upward curvatures near the crossing are clearly evident.
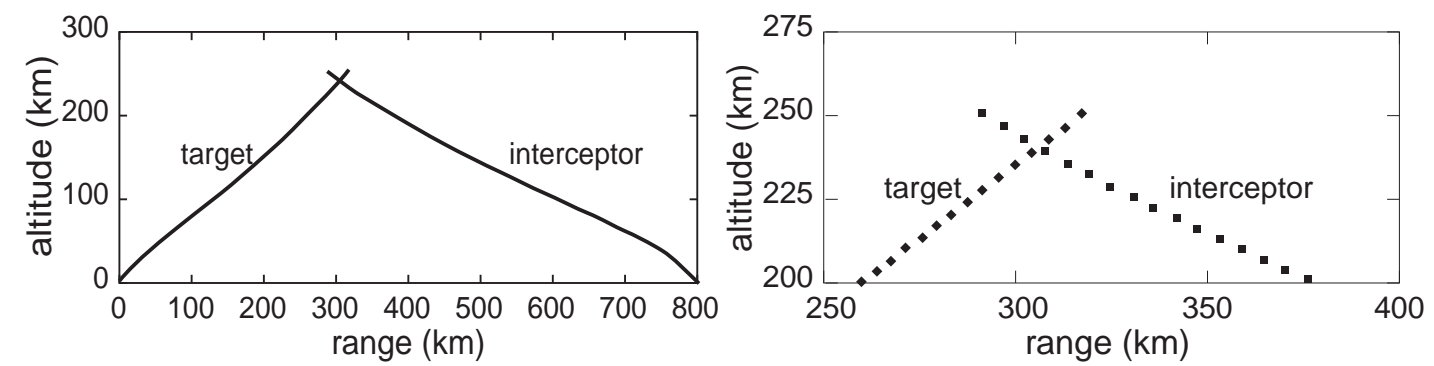

Figure 12.6. Left: Overview of the engagement of ICBM model S2 by interceptor model I-4 analyzed in the text, showing the trajectories of the ICBM (left) and interceptor (right) in the range-altitude plane. In this engagement the interceptor is launched $45 \mathrm{~s}$ after the ICBM, rises to get above the densest part of the atmosphere, and then pitches over toward the anticipated intercept point. It burns out $86 \mathrm{~s}$ after the launch of the ICBM. When the interceptor burns out, the second stage of the ICBM increases its angle of attack by $15^{\circ}$. The kill vehicle begins to steer toward the ICBM at $100 \mathrm{~s}$ and its trajectory intersects that of the ICBM at $157 \mathrm{~s}$. Right panel: Detail of the final $100 \mathrm{~km}$ of the engagement, showing that the intercept was successful. The dots along each trajectory show the positions of the target and interceptor at 1-second intervals. Both trajectories have been extended to $160 \mathrm{~s}$. Note that there are exactly three dots on each trajectory after they cross. 
Additional simulations were performed to study the velocity-change capability required to deal with "switchback" maneuvers by the target, in which it suddenly changes its angle of attack from a positive value to a negative value or vice versa. Using simulations, we investigated the dependence of the velocity change capability required to compensate for such maneuvers as a function of the time the maneuver was performed. Finally, we investigated the dependence of the required velocity-change capability on the closing velocity between the kill vehicle and the target.

Various aspects of the kill vehicle's behavior during the simulated engagements are shown in the next few figures to give some insight into the behavior of the guidance scheme and the effects of the filter dynamics and measurement noise.
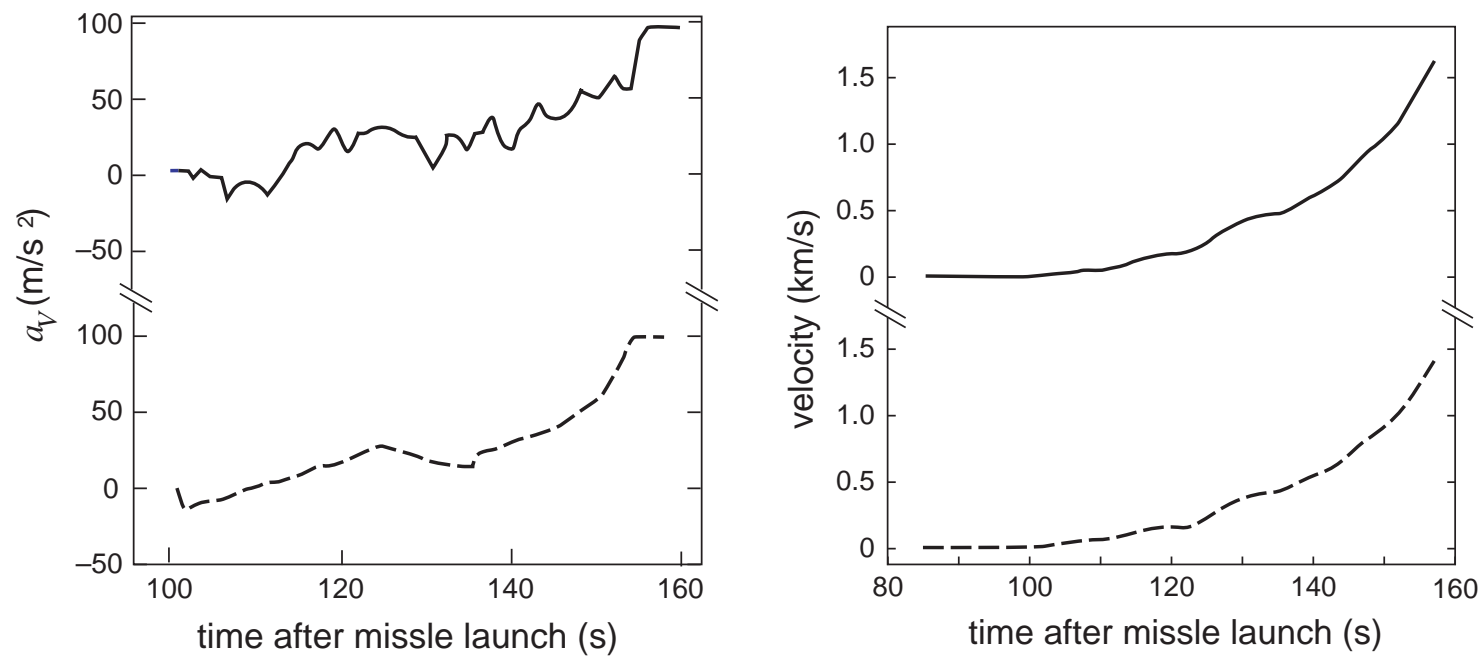

Figure 12.7. Left-hand panel: Vertical (dominant) component $a_{V}$ of the kill vehicle's acceleration with (top curve) and without (bottom curve) sensor noise included. Although the two curves show the same overall trends, it is clear that the sensor noise induces significant fluctuations in the acceleration commanded by the guidance scheme. Right-hand panel: Cumulative kill-vehicle velocity change with (top curve) and without (bottom curve) sensor noise included, as a function of time since the launch of the target missile. The sensor noise increases the required velocity change capability by about $150 \mathrm{~m} / \mathrm{s}$.

The left-hand panel of Fig. 12.7 shows the vertical component of the kill vehicle's acceleration when the sensor noise listed in Table 12.2 is included (top curve) and when it is not (bottom curve). The vertical component of the acceleration is the dominant component because the LOS to the target is nearly horizontal. Although the two acceleration curves have the same overall trend, the sensor noise creates significant fluctuations in the acceleration commanded by the guidance scheme, increasing the cumulative velocity change that is needed.

The increase in the cumulative velocity change during the engagement caused by the sensor noise is shown in the right-hand panel of Fig. 12.7. Noise at the level assumed increases the $\Delta V_{\text {tot }}$ required by about $150 \mathrm{~m} / \mathrm{s}$ relative to the noise-free case. If the missile did not maneuver and the changes in its acceleration were modest, the acceleration sign reversals induced by the sensor noise would make a significant contribution to the small $\Delta V_{\text {tot }}$ required. If, however, the missile is expected to execute maneuvers like the angleof-attack changes discussed earlier, the kill vehicle must have a much larger $\Delta V_{\text {tot }}$ to reach 
the endgame, as shown below.

To determine the $\Delta V_{\text {tot }}$ required to assure intercept, Monte Carlo runs of 100 samples each were performed for a range of off-board sensor resolutions to determine the average $\Delta V_{\text {tot }}$ required and the 90 th-percentile point in the distribution of the required $\Delta V_{\text {tot }}$ produced by sensor measurement errors for two illustrative evasive maneuvers by the target. Figure 12.8 shows the 90 th percentile $\Delta V_{\text {tot }}$ as a function of the resolution of the off-board sensor, for no target maneuvers and for an increase or a decrease in the missile's angle of attack by $15^{\circ}$ at $86 \mathrm{~s}$.

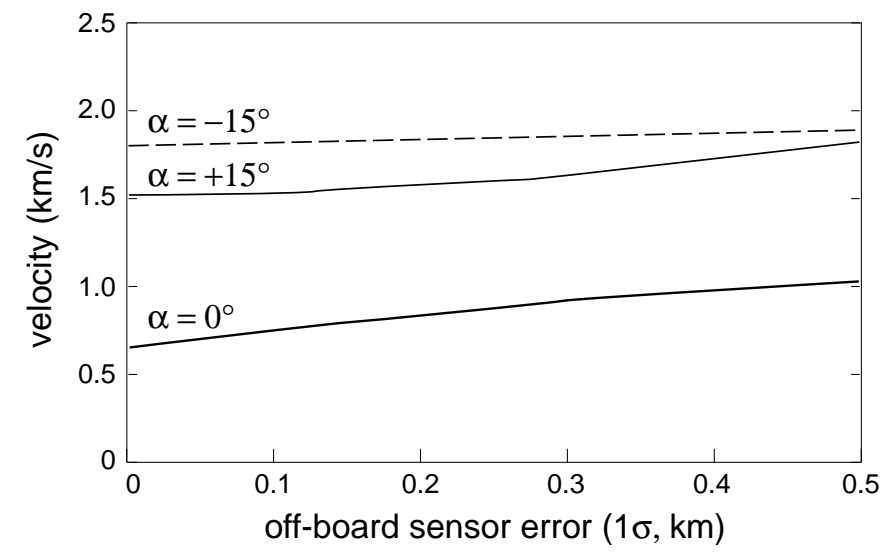

Figure 12.8. Total kill-vehicle velocity change required to reach the endgame as a function of the uncertainty in the measurements of the target's position, when the target does not maneuver (the curve labeled $\alpha=0^{\circ}$ ) and when the target makes the two angle-of-attack maneuvers (the curves labeled $\alpha=-15^{\circ}$ and $\alpha=+15^{\circ}$ ).

Figure 12.8 shows that the $\Delta V_{\text {tot }}$ required is fairly insensitive to the uncertainty in the measured position of the target for uncertainties less than about $500 \mathrm{~m}$. When the target does not maneuver, an increase in the sensor error from zero to $500 \mathrm{~m}$ increases the $\Delta V_{\text {tot }}$ required from about $0.6 \mathrm{~km} / \mathrm{s}$ to about $1 \mathrm{~km} / \mathrm{s}$. If, however, the target makes either of the angle-of- attack maneuvers described above, the $\Delta V_{\text {tot }}$ required increases to $1.8-2.0 \mathrm{~km} / \mathrm{s}$. Simulations showed that a switchback maneuver near the worst possible time increased the required kill-vehicle $\Delta V_{\text {tot }}$ by less than 5 percent. To be robust, a boost-phase intercept system would have to be able to successfully complete the intercept attempt in the face of these and other feasible maneuvers.

The bulk of the kill-vehicle $\Delta V$ analysis was for a kill vehicle launched by an interceptor having a burnout velocity of $6.5 \mathrm{~km} / \mathrm{s}$ against an ICBM target. The closing velocity for this engagement is about $10 \mathrm{~km} / \mathrm{s}$. Figure 12.8 shows that for engagements having closing velocities of this order, the $\Delta V_{\text {tot }}$ required is at least $2 \mathrm{~km} / \mathrm{s}$, if a small margin is included to allow the kill vehicle to correct initial aiming errors.

We also performed a small number of simulations of engagements having the same geometry but higher closing velocities, up to $16 \mathrm{~km} / \mathrm{s}$. These simulations suggest that the $\Delta V_{\text {tot }}$ required would be slightly greater for engagements in which the closing velocity is significantly higher than $10 \mathrm{~km} / \mathrm{s}$. We estimate that for kill vehicles launched by surfacebased interceptors having burnout velocities between $6.5 \mathrm{~km} / \mathrm{s}$ and $10 \mathrm{~km} / \mathrm{s}$, the $\Delta V_{\text {tot }}$ required would be between $2 \mathrm{~km} / \mathrm{s}$ and $2.5 \mathrm{~km} / \mathrm{s}$. For space-based interceptors, which could have closing velocities in excess of $15 \mathrm{~km} / \mathrm{s}$, the $\Delta V_{\text {tot }}$ required could be as high as $2.5 \mathrm{~km} / \mathrm{s}$.

These estimates of the total $\Delta V$ required for engagements at high closing velocities are 
based on a small number of simulations. Further analytical work or controlled numerical comparisons would be required to draw definitive conclusions about the effect of the closing velocity on the total $\Delta V$ required. However, the following preliminary discussion may be useful (see also [142, p. 109]).

For simplicity, suppose that the time at which the engagement is initiated is determined by external factors unrelated to the interceptor's burnout velocity or its standoff distance. For the simple case of PN or APN guidance, a constant-velocity, inverse-trajectory engagement $^{15}$, and noise-free measurements of the LOS rate and the target's acceleration, the total $\Delta V$ required is independent of the closing velocity. The effect of the closing velocity on the LOS rate is cancelled by its effect on the feedback gain (assuming the estimate of the closing velocity is reasonably accurate). The target acceleration normal to the LOS is also independent of the closing velocity. However, when sensor measurement errors are included, these errors may depend on the range to the target. If so, they will have a different time history. For example, an on-board passive IR sensor or laser ranger having a fixed maximum range would be able to begin operation only at a later time if the closing velocity is higher. Thus the influence of filter dynamics lags and the noise-induced estimation errors will be greater during a portion of the kill vehicle's flight, which will increase the total $\Delta V$ required. Also, for space-based interceptors (and even for higher velocity surface-based interceptors) the engagement geometry would be different, thus changing the component of the target's acceleration normal to the LOS, which will also create differences in the total $\Delta V$ required.

\subsubsection{Summary of $\Delta V_{\text {tot }}$ requirements}

The results presented in this section show that increasing the precision of the off-board or on-board sensors would not significantly reduce the total $\Delta V$ capability that the kill vehicle must have. The reason is that the largest contributions to the total $\Delta V$ requirement come from the circumstances that (1) the defensive system is unlikely to have full knowledge of the characteristics of ICBMs deployed (perhaps with little or no testing) by countries with emerging missile programs; (2) the intended target of such an ICBM is unlikely to be known; and (3) an ICBM is an accelerating, maneuvering target having an inherently unpredictable trajectory.

Our finding that the kill vehicle must have a total $\Delta V$ capability of at least $2.0-2.5 \mathrm{~km} / \mathrm{s}$ to reach the endgame is the most significant result obtained from our engagement simulations, because this requirement is one of the most important factors that determine the minimum size and mass of the kill vehicle.

\subsection{The Endgame}

As discussed in the introduction to this chapter, the purpose of our endgame analysis was to estimate the seeker, guidance and control system, and propulsion system performance that would be needed to assure that the kill vehicle would have a high probability of hitting the target missile. The major guidance requirement is sufficiently precise and timely information on the target's state and, if possible, some estimate of its likely future behavior. In addition, the kill vehicle must have sufficient acceleration and acceleration responsiveness to maneuver to hit the target. This section examines these requirements quantitatively.

\footnotetext{
${ }^{15} \mathrm{An}$ inverse-trajectory engagement is an engagement in which the velocity vector of the interceptor is antiparallel to the velocity vector of the target.
} 
The greatest challenge during the endgame is coping with maneuvers by the target. As discussed earlier, the target may maneuver to manage its energy, shape its trajectory, or for other reasons, even if it is not attempting to evade a defense. If the attacker knows there is a defensive system, the missile may be programmed to perform maneuvers designed to cause the kill vehicle to miss. The maneuvers considered here are thought to be reasonable and likely to be within the capabilities of ICBMs that could be deployed by countries that have emerging missile programs, but they would not be performed if the attacker thought there was no chance of an intercept attempt. We took the ability to cope with these maneuvers to be a requirement for any boost-phase system, because they would not be difficult to implement. Moreover, although the particular maneuvers considered here were studied as examples of maneuvers intended to evade the kill vehicle, coping with energy-management maneuvers could make similar demands on the performance of the kill vehicle.

Because of the limited time and resources available for this study, we studied only two types of maneuvers during the endgame. Following Zarchan [141, p. 104], we considered (1) a sudden "lunge" maneuver executed within the last $5 \mathrm{~s}$ before the predicted time of intercept and (2) a continuous "jinking" maneuver during the last $5 \mathrm{~s}$. For a lunge to be effective, the missile must execute the maneuver near the worst possible moment for the defense. If the attacking missile lunges too early or too late, the lunge will not increase the miss distance significantly. For a jinking maneuver, the challenge is to achieve sufficient amplitude and bandwidth.

The kill-vehicle performance required to cope with these maneuvers was explored by a series of endgame simulations. Before reporting the results, we first describe the model of the endgame used and the parameter values adopted. We then discuss some illustrative examples. Finally, we summarize our results. The analysis assumes that by the end of the initial divert and homing phases of the engagement, the kill vehicle is within a small enough "basket" in position and velocity space to begin the endgame.

Lacking specific dimensions of either the target missile or the kill vehicle, we adopted the goal of keeping the miss distance to $0.5 \mathrm{~m}$ or less in all ten Monte Carlo runs performed. A 0.2-m-diameter kill vehicle that is able to aim at the centerline of 1-m-diameter booster and has a high probability of a miss distance of $0.5 \mathrm{~m}$ or less would almost certainly collide with the missile. ${ }^{16}$

\subsubsection{Endgame model}

To estimate the likely miss distance in the face of various target maneuvers, we used a one-dimensional model of the endgame (only target accelerations normal to the LOS were considered). The model has the following features:

- A PN guidance law was used for the kill vehicle. Various navigation gain factors were explored.

- The latency in the kill vehicle's information about the target's current state and its predictions of the target's future state was modeled using a second-order Kalman filter to estimate the ZEM and LOS angle from measurements of the LOS angle with sensor noise included. The amplitude of the sensor noise was assumed to be constant.

\footnotetext{
${ }^{16}$ For comparison, the final stages of the model ICBMs we considered have diameters ranging from $3 \mathrm{~m}$ for the liquid-propellant model to $1.5 \mathrm{~m}$ for solid-propellant models.
} 
- The delay in the kill vehicle's dynamical response was modeled as a fifth-order lag with five equal time constants. ${ }^{17}$

- The dynamics of the engagement were modeled as a second-order process with the ZEM and the LOS to the target as the two state variables.

- The maximum acceleration that could be provided by the kill vehicle's propulsion system was modeled by imposing a fixed upper limit on the acceleration that could be commanded by the guidance system.

The following assumptions were made in designing the Kalman filter. The LOS angle is measured at a rate of $100 \mathrm{~Hz}$ with a standard deviation of $100 / \sqrt{12} \mu \mathrm{rad}$ (the standard deviation of a zero-mean uniform distribution having a width of $100 \mu \mathrm{rad}$ ). The target's range as a function of time is known precisely, ${ }^{18}$ allowing distances normal to the LOS to be computed directly from the measured LOS angle. For LOS-angle measurements having a constant uncertainty, the response time of the Kalman filter becomes shorter as the time to go decreases. The time to go was computed using the distance to the target and the estimated closing velocity. The standard deviation of the unpredictable target acceleration was assumed to be $2 \mathrm{~g}$ with a correlation time of $1 \mathrm{~s}$. These assumptions determine the Kalman filter gains. In simulations of the endgame, we computed the response of this filter to the deterministic inputs caused by the two types of target maneuvers studied.

A series of simulations like the ones described here showed that a 15-g acceleration is adequate to assure a miss distance of $0.5 \mathrm{~m}$ or less for closing velocities less than or about $14 \mathrm{~km} / \mathrm{s}$. An acceleration of $15 \mathrm{~g}$ was thought to be reasonable for a boost-phase kill vehicle. A few exploratory simulations of engagements with the higher closing velocities that would be encountered in engagements of ICBMs by very fast, surface-based interceptors or by space-based interceptors indicated that accelerations of 17-18 g may be needed to ensure intercept at the higher closing velocities produced by these interceptors. More simulations would be needed to establish the acceleration requirements for such high closing velocities. The acceleration of the kill vehicle was limited to $15 \mathrm{~g}$ or less for the endgame simulations described here.

\subsubsection{Lunge maneuver}

As an example of a lunge maneuver, consider an $8 \mathrm{~g}$ step in the acceleration of the target missile normal to the LOS initiated sometime during the last $5 \mathrm{~s}$ before the predicted time of the intercept. This is the largest acceleration that the final stages of the model ICBMs considered in the Study could reasonably produce $15 \mathrm{~s}$ before they burn out. The target missile might make a smaller step in its acceleration as an energy-management maneuver, but a step this large would be made only as an evasive maneuver.

In our simulations of the endgame for this maneuver, we used proportional navigation with a guidance gain of 6 applied to the estimated LOS rate derived from the estimated

\footnotetext{
${ }^{17}$ Low-order transfer functions for interceptor lag can be cancelled out if the parameters are known and there are no saturation effects. However, actual interceptor dynamics are higher-order, and the effect on miss is considerably greater for the same overall lag. Cancellation is problematic for higher-order dynamic lags in the presence of saturation effects and parameter uncertainties. To be conservative, we assumed a high-order lag model and did not attempt to cancel out or phase compensate its effects.

${ }^{18}$ As shown in Chapter 10, a LIDAR on board the kill vehicle could in principle measure the range with high precision.
} 
ZEM. The total lag in the response of the interceptor to acceleration commands was set to $0.1 \mathrm{~s}$ in the simulations described here. This relatively high gain reduces the maximum kill-vehicle acceleration demands. Although a high gain also tends to increase the effects of the sensor noise, in the endgames analyzed the noise was low enough relative to the other factors to be acceptable.
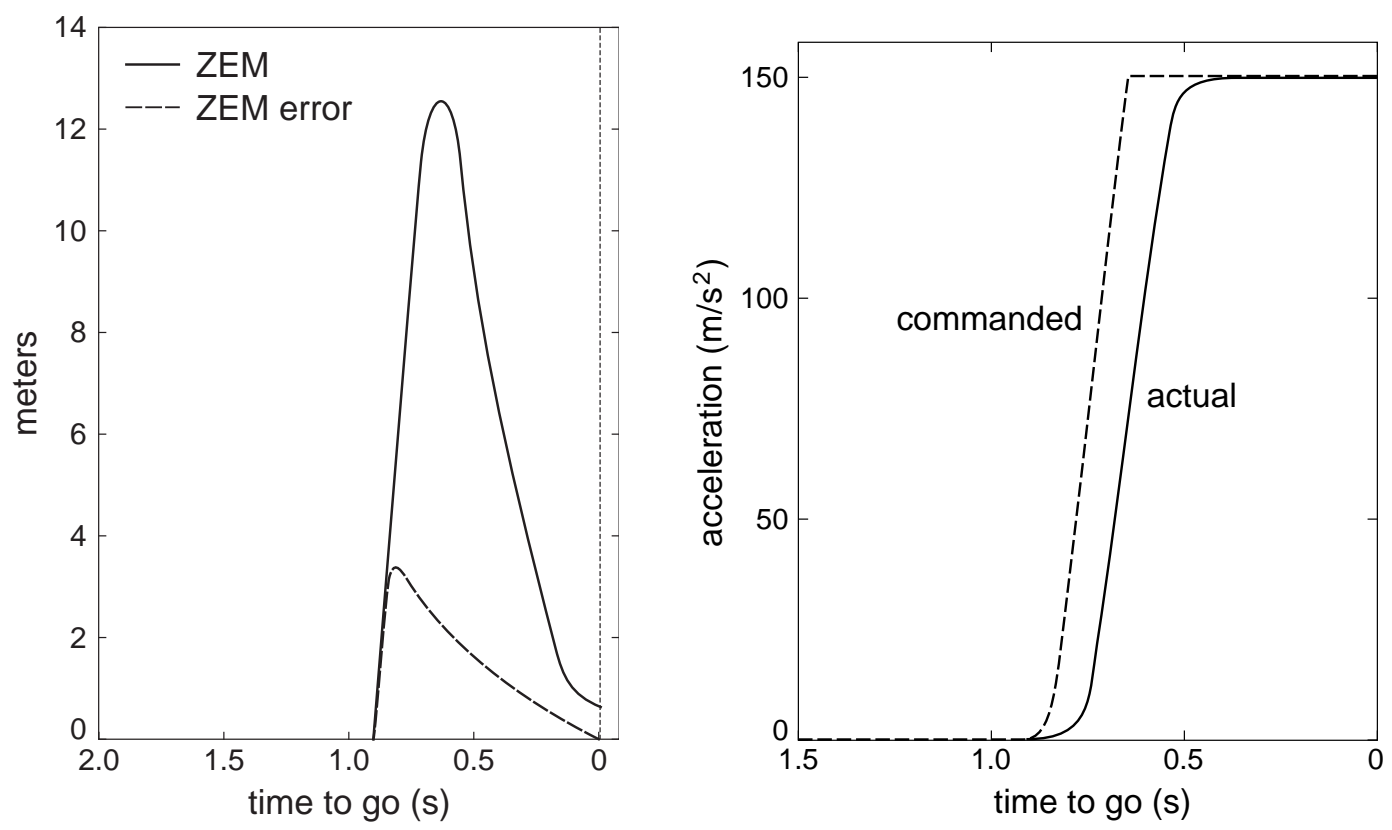

Figure 12.9. Guidance signal and kill-vehicle response to an 8-g lunge maneuver by the target missile $0.9 \mathrm{~s}$ before the predicted intercept time, in the absence of sensor noise. Left panel: ZEM and ZEM error, defined as the difference between the ZEM and the estimated ZEM, as a function of the estimated time to go, which decreases from left to right. Right panel: Commanded and actual kill vehicle accelerations as a function of the estimated time to go, showing the effect of the 100-ms lag in the response of the kill vehicle.

Figure 12.9 shows a simulated endgame beginning $5 \mathrm{~s}$ before the predicted intercept time. The sensor noise has been set to zero so the filter and kill-vehicle acceleration responses can be seen clearly. The ZEM and ZEM estimation error were set to zero at the beginning of the endgame and remain at zero (for the noise-free case) until the target executes an $8 \mathrm{~g}$ step maneuver with $0.9 \mathrm{~s}$ to go. The left-hand panel shows the evolution of the ZEM and the ZEM error (defined as the difference between the ZEM and the estimated ZEM) during the endgame. The lag of the estimated ZEM caused by the filter response is very apparent. Although it is a few hundredths of a second initially, it rapidly decreases as the kill vehicle approaches the target. In this simulation, the kill vehicle missed its aimpoint by about $0.7 \mathrm{~m}$, as shown by the $0.7 \mathrm{~m}$ ZEM at zero time to go. As the kill vehicle approaches the target, the ZEM error decreases almost to zero, showing that the miss was not a result of the final error in estimating the ZEM.

In contrast to the filter lag, which decreases rapidly as the kill vehicle approaches the target, the lag in the kill vehicle's dynamical response to acceleration commands is independent of the time to go. The effects of the lags and the limited acceleration of the kill vehicle can be seen clearly in the right-hand panel of Fig. 12.9. The filter and kill-vehicle dynamical lags and the kill vehicle's finite maximum acceleration all contributed to the $0.7 \mathrm{~m}$ miss.

Figure 12.10 shows how the noise-free miss distance caused by an 8-g lunge of the target 


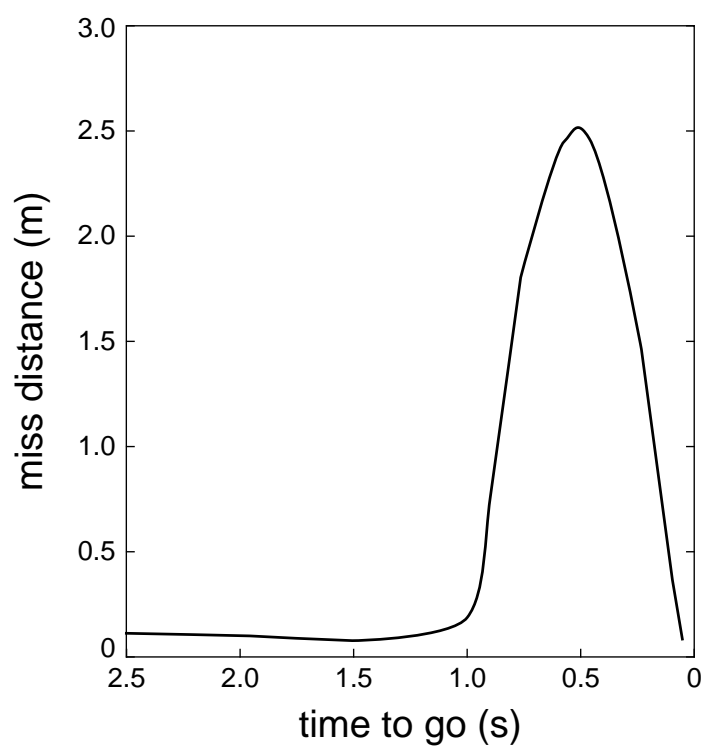

Figure 12.10. The miss distance produced by the 8 -g lunge maneuver by the target missile as a function of the time that the missile executes the maneuver, neglecting sensor noise. If the maneuver is made with more than $1 \mathrm{~s}$ to go, it has a negligible effect on the miss distance, whereas if it is made in the last $0.8 \mathrm{~s}$, the kill vehicle will miss the target.

missile depends on the time at which the missile executes the maneuver. If the lunge occurs more than a second before the time of intercept, the noise-free miss distance is only $0.19 \mathrm{~m}$. The miss distance becomes unacceptable only if the lunge occurs when the time to go is less than $0.8 \mathrm{~s}$. It is unlikely that an attacker could time the maneuver this precisely, because it would be difficult to know the defender's planned engagement time to better than a few seconds. If the magnitude of the lunge is less than $8 \mathrm{~g}$, the window of vulnerability would be narrower still.

Including the sensor noise does not alter this conclusion. The simulation shown in Fig. 12.11 is the same as that shown in Fig. 12.9, except that the lunge occurs with $1 \mathrm{~s}$ to go and the sensor noise is included. The effects of the sensor noise are pronounced during the first few seconds of the simulation but diminish rapidly as the kill vehicle approaches the target. In this simulation, the kill vehicle missed the aimpoint by $0.21 \mathrm{~m}$, hardly different from the noise-free miss distance of 0.19 meters for an 8-g lunge with $1 \mathrm{~s}$ to go. The conclusion that the effect of sensor noise is small in this case is further supported by Fig. 12.11, which shows a bar chart of the miss distances obtained in ten simulations of the 8 -g lunge with $1 \mathrm{~s}$ to go, with sensor noise included. All the miss distances are between 0.15 and 0.25 meters.

\subsubsection{Jinking maneuver}

In contrast to a lunge, which is made at a single instant, a jinking maneuver is a periodic maneuver executed over an extended time interval that is intended to span the period during which an intercept attempt could occur. The goal is to create kill-vehicle maneuverability requirements that periodically exceed the performance of the kill vehicle, thereby creating a sequence of intervals during which the kill vehicle would be unable to close on the target with sufficient precision to hit it. 

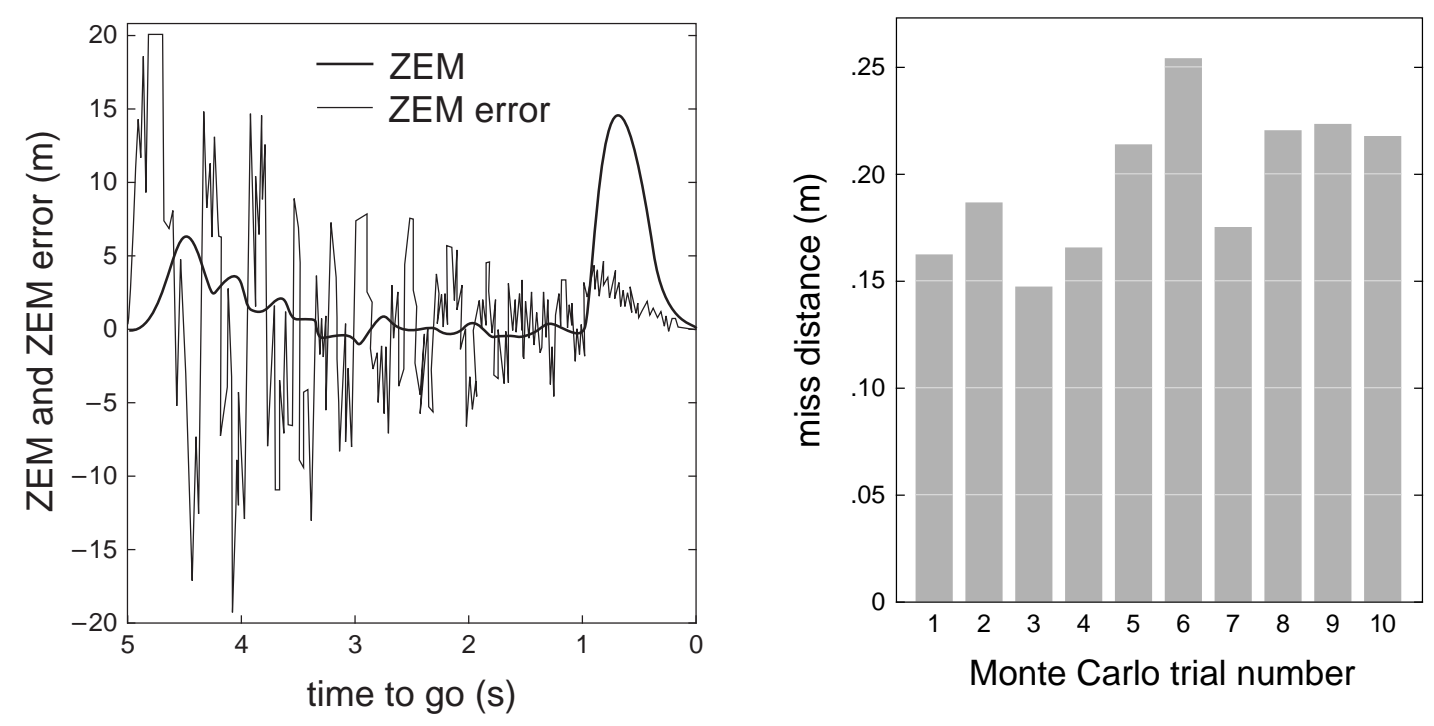

Figure 12.11. Simulation of the endgame for an 8-g lunge by the target missile one second before the predicted intercept time with sensor noise included. Left panel: ZEM and ZEM error as a function of the time to go, which decreases from left to right. Right panel: Bar chart of the miss distances for 10 simulations of the endgame, showing the variation in the miss distance produced by the noise in the LOS angle measurements.

Zarchan [141, p. 106] considers both square-wave and sinusoidal target accelerations. On average, the miss distances are similar for acceleration waveforms of the same amplitude, although the miss distances for the square wave are a little greater than for the sinusoidal wave. A square-wave acceleration would be more difficult for the missile to achieve. We therefore considered sinusoidal target accelerations in our study of the effects of jinking maneuvers. The other assumptions and model parameters were the same as for the analysis of lunges.

Figure 12.12 shows the acceleration of a target missile performing a 1-Hz, 2-g jinking maneuver and the response of the kill vehicle, with and without sensor noise. In the absence of sensor noise, the error made in estimating the ZEM decreases steadily during the $5 \mathrm{~s}$ before the predicted intercept time. With no time to go, the ZEM error is less than $0.01 \mathrm{~m}$. However, this reduction occurs so late that the lag in the response of the kill vehicle and the limits on its ability to accelerate prevent it from reducing the miss distance to such a small value. Instead, the final miss distance is $0.36 \mathrm{~m}$. Simulations of jinking maneuvers having different frequencies showed that the miss distance grows with increasing frequency and that the highest frequency for which the miss distance is less than $0.5 \mathrm{~m}$ is about $1 \mathrm{~Hz}$. For a $1.3-\mathrm{Hz}$ jinking maneuver, the miss distance is $0.8 \mathrm{~m}$.

As with a target lunge, sensor noise of the magnitude assumed in the simulations has only a small effect on the response of the kill vehicle to jinking by the target during the endgame. Figure 12.12 shows that the behavior of the ZEM and ZEM error with sensor noise is very similar to the behavior with no noise. In this example, the sensor noise increased the final miss distance by about 20 percent, to 0.44 meters.

\subsubsection{Summary of endgame requirements}

For the lunge and jinking evasion maneuvers that were investigated, a kill vehicle that has a maximum acceleration of $15 \mathrm{~g}$ and a response lag of $0.1 \mathrm{~s}$ or less is adequate to achieve a 

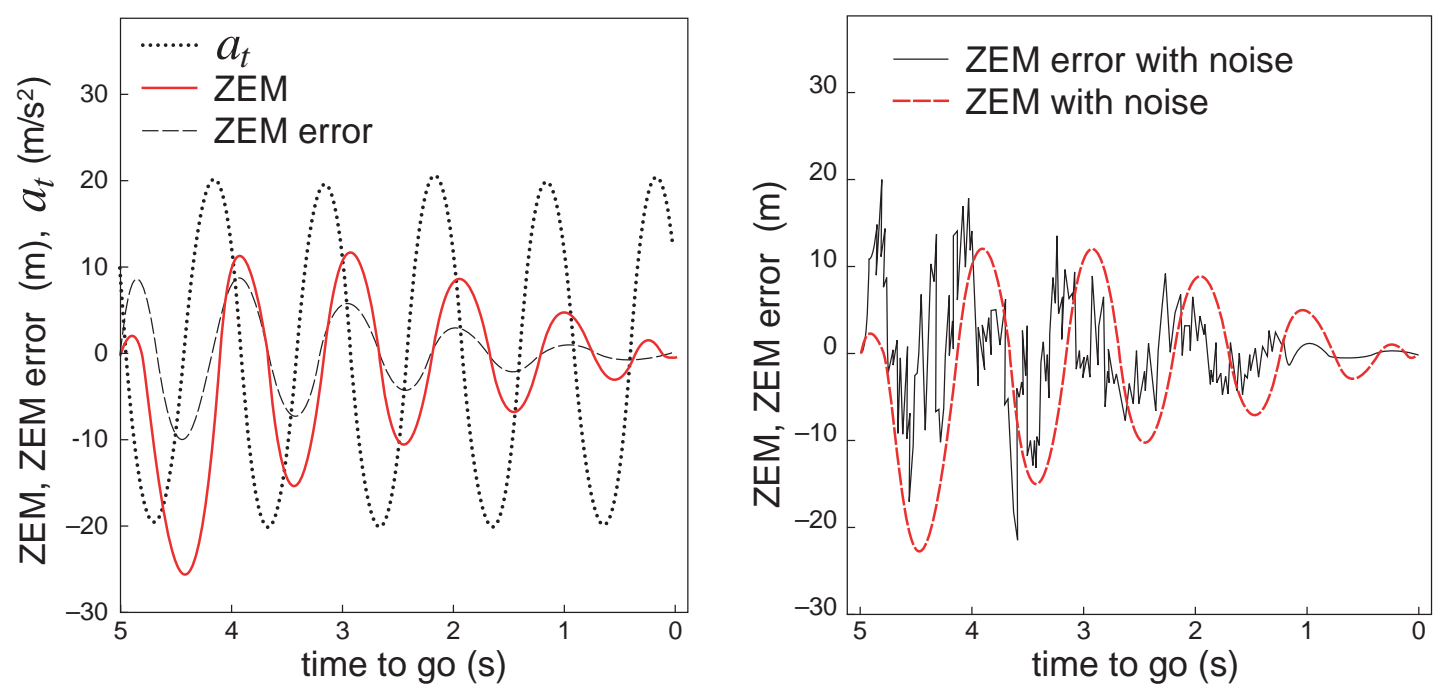

Figure 12.12. Target acceleration and response of the kill vehicle to a $1-\mathrm{Hz}, 2 \mathrm{~g}$ target jinking maneuver without (left) and with (right) the sensor noise included. Shown are the target's acceleration $a_{t}$ in $\mathrm{m} / \mathrm{s}^{2}$ and the ZEM and ZEM error in meters as a function of the time to go.

miss distance of no more than $0.5 \mathrm{~m}$ with high probability. This conclusion assumes that the terminal sensor errors are no greater than the assumed uniform distribution of $100 \mu \mathrm{rad}$ and that there are highly accurate range measurements from the LIDAR.

\subsection{Summary of Requirements for Hitting the Target}

The major results obtained in the preceding analysis can be divided into the total velocity change capability that is required and the performance requirements for the endgame.

\subsection{1 $\Delta V_{\text {tot }}$ requirements}

The major result of the simulations described in this chapter-the total kill-vehicle $\Delta V$ required to achieve an intercept-is summarized in Fig. 12.8. For closing velocities of about $10 \mathrm{~km} / \mathrm{s}$, our results show that a total $\Delta V$ of at least $2 \mathrm{~km} / \mathrm{s}$ is required. For engagements at the higher closing velocities that would be produced by very fast surface-based interceptors, we estimate that the required total $\Delta V$ would be between $2 \mathrm{~km} / \mathrm{s}$ and $2.5 \mathrm{~km} / \mathrm{s}$. For spacebased interceptors, which would have closing velocities of $15 \mathrm{~km} / \mathrm{s}$ or more, the required total $\Delta V$ could be as high as $2.5 \mathrm{~km} / \mathrm{s}$.

\subsubsection{Endgame requirements}

To hit the target within about $0.5 \mathrm{~m}$ of the intended aim point requires a kill vehicle with a maximum acceleration of at least $15 \mathrm{~g}$, a total kill-vehicle response lag of $0.10 \mathrm{~s}$ or less, and a seeker having an angular measurement error of $30 \mu \mathrm{rad}$ or less $(1 \sigma)$ and a 100- $\mathrm{Hz}$ update rate.

These requirements are presented as adequate for only the guidance laws that were adopted for the particular problem. For example, APN might reduce somewhat our $15 \mathrm{~g}$ acceleration result, but its response to switchback maneuvers and jinking maneuvers could be counterproductive and would have to be studied. Moreover, results in [153, Table 1] show 
very little advantage for APN vs. PN. Only optimal guidance or phase lead estimators show large improvements (and this was for a known single-time-constant lag). As explained in footnote 16, we conservatively assumed that our five-time-constant lag could not be phase compensated. Reducing the kill-vehicle response lag to, say, $0.05 \mathrm{~s}$ could help somewhat, but such a requirement could be technologically stressing in itself. Moreover, our kill vehicle with its $15 \mathrm{~g}$ endgame capability has only about a $7 \mathrm{~g}$ acceleration capability early in its operation, when it is much heavier. This capability approximately matches the required acceleration capability at that time. Unexpected maneuvers are inherent in ballistic missile flight and are even more likely to occur with target missiles that may have been tested only once, if at all. Therefore, a robust guidance system is highly desirable.

We emphasize that these requirements do not reflect the additional demands that would likely be placed on the kill vehicle if a decision is made to attempt to time the intercept within the narrow window that would be required to avoid causing possibly live warheads to fall on third countries. Analyzing this issue (either technically or politically) is outside the scope of the current Study. The analysis presented here also did not consider the effects of countermeasures (Chapter 9) that would delay detection of an unexpected acceleration, thereby increasing the $\Delta V$ requirement.

\section{References for Chapter 12}

[141] P. Zarchan, "Tactical and Strategic Missile Guidance," Vol. 176, AIAA Progress in Astronautics and Aeronautics (1997).

[142] P. Zarchan, "Ballistic Missile Defense Guidance and Control Issues," Science \& Global Security 8, 99 (1998).

[143] P. Zarchan, "Guidance and Control: Boost Phase Intercept Tradeoffs." Briefing presented to the APS Study Group on Boost-Phase Defense, October 2001.

[144] Jeffrey A. Isaacson and David R. Vaughan, "Estimation and Prediction of Ballistic Missile Trajectories." Technical Report MR-737-AF, ISBN 0-8330-2376-4, RAND, Santa Monica, CA, 1996.

[145] N. A. Shneydor, Missile Guidance and Pursuit: Kinematics, Dynamics and Control. Horwood Engineering Science (Horwood Publishing, Chichester) 1998.

[146] A. E. Bryson and Y.C. Ho, Applied Optimal Control. (Blaisdell, Waltham, MA), 1969.

[147] J. Z. Ben-Asher and Y. Isaac, "Advances in Missile Guidance Theory," Vol. 180, AIAA Progress in Astronautics and Aeronautics (1998).

[148] K. L. Zondervan et al., "Airborne Fire Control for Boost Phase Intercept." National Fire Control Symposium, 1994.

[149] J. P. Janus, "Homing Guidance." Technical Report A-62-1732.3-68, Aerospace Corporation, 1962.

[150] P. Zarchan, "Fundamentals of Kalman Filtering: A Practical Approach," Vol. 190, AIAA Progress in Astronautics and Aeronautics (2000).

[151] P. B. Liebelt, An Introduction to Optimal Estimation, (Addison Wesley) 1967. 
[152] T. Shima, Y. Oshman, and J. Shinar, "Efficient Multiple Model Adaptive Estimation in Ballistic Missile Interception Scenarios," J. Guidance, Control, and Dynamics 25(4), 667 (2002).

[153] I. A. Shkolnikov et al., "Sliding Mode Observors Versus Kalman Filter in the Homing Loop." Unclassified report U7034 prepared for the U.S. Army SMDC under contract number DASG60-94-C-0045. 



\title{
Chapter 13
}

\section{Disabling the Target}

\author{
Contents
}

13.1 Damage Caused by Collision with a Booster . . . . . . . S240

13.2 Warhead Destruction ................. S241

13.2.1 Types of warheads . . . . . . . . . . . . . . . . S241

13.2.2 Technical challenges for destroying the warhead _..... . S241

13.3 Effects of Incomplete Warhead Kill on Other Layers of a De-

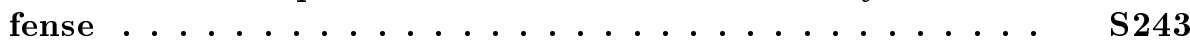

13.4 Enhancing the Lethality of the Kill Vehicle . . . . . . . S243

13.5 Summary of Conclusions . . . . . . . . . . . S244

13.5.1 Booster destruction . . . . . . . . . . . . . . S244

13.5.2 Warhead destruction ............... . . S245

There are two possible targets in a boost-phase intercept. The first is the threat missile booster. Disabling the booster so that its thrust terminates is the primary goal for an intercept. All the possibilities for defending the United States discussed in Chapter 5 are based on the premise that the intercept causes the missile's thrust to cease at the moment of impact.

A second possible target is the warhead itself, which is much smaller than the missile (the relative sizes can be seen in Fig. 16.6). Destroying the warhead- "warhead kill" - is not essential for defending the United States-hitting the booster is sufficient. However, warhead kill would be a great advantage with respect to the problem of not harming other nations by causing live munitions to fall on them. As discussed in Section 5.8, timing an intercept to prevent munitions from falling on other countries presents a formidable problem and may not be possible. If warhead kill could be assured, this problem would be eliminated.

This chapter examines the assumption about an intercept more carefully and points out the large gaps in our understanding of the entire problem of lethality. In short, it finds that if a kill vehicle hits a booster, the energy of the collision is so great that the booster would likely be disabled or destroyed, although the thrust may not be terminated instantly in all cases. However, destroying the booster may not disable or destroy the warhead, which is only loosely coupled. Therefore a live warhead from a missile that has been "successfully" intercepted may well detonate when or before it hits the ground.

This chapter also finds that destroying a warhead can be made even more difficult because the "sweet spot" or vulnerable area that the kill vehicle must hit to ensure destruction may be quite small, particularly in the case of submunitions. Moreover, the warhead may 
be difficult to find if its location on the missile is obscured by a large shroud. This chapter discusses the cases where it might be useful to destroy the warhead and some techniques for increasing the lethality of the kill vehicle against warheads.

Overall, our ability to predict how effectively a boost-phase intercept (BPI) kill vehicle will disable a booster or warhead is seriously limited by the absence of data from actual tests. No one has tried to intercept a booster in space before, nor has anyone demonstrated in flight the lethality of a kill vehicle against a warhead with submunitions or a warhead that has been hidden. These issues would need to be explored through a robust testing program. The dearth of flight test data must be addressed before informed decisions can be made about the effectiveness of boost-phase intercept systems.

\subsection{Damage Caused by Collision with a Booster}

The kinetic destruction of kill-vehicle-to-solid-body hit has been demonstrated in several flight tests, starting with the Homing Overlay Experiment(HOE)in 1984 [154] and continuing through three successful tests of the National Missile Defense midcourse system in the last few years. In those tests, a kill vehicle hit a solid body simulating a unitary warhead, and the energy of the collision pulverized both objects. The same basic physics that governed kill vehicle collision with a solid body govern the collision of a kill-vehicle and a booster.

As discussed in Chapter 12, sensor and guidance and control technology that will be available within the next decade are sufficient for a BPI kill vehicle to achieve a miss distance $^{1}$ against an accelerating target of $0.5 \mathrm{~m}(\mathrm{rms})$, to be compared with typical threat missile diameters of a meter or more (see Fig. 16.6). Thus, hitting a point on the missile that will disable the powered rocket is possible in principle.

During the impact of a kinetic-energy kill vehicle with a target, the energy of the collision is deposited in the target and kill vehicle primarily in the form of hydrodynamic impulse. This causes failure of typical missile structures by impulse loading and thermal energy that manifests itself in vaporizing material from target and kill vehicle $[155,156]$. In the process, radiant energy is released across a broad spectrum. A deposition of approximately $5 \mathrm{~kJ} / \mathrm{cm}^{2}$ on impact is considered to be a typical damage threshold. A modest kill-vehicle payload mass of $5 \mathrm{~kg}$ that strikes at a closing velocity of $4 \mathrm{~km} / \mathrm{s}$ would deliver 40 MJ. Even if this were distributed over an area of $1 \mathrm{~m}$ diameter, it would be enough to destroy the target.

In reality, for reasons described in Chapter 12, the contemplated systems have killvehicle masses of perhaps $50 \mathrm{~kg}$ at the time of intercept and closing velocities of $10 \mathrm{~km} / \mathrm{s}$ or greater. At such velocities, the collision would generate kinetic energy almost 60 times the nominal damage threshold. However, such extra energy might indeed be required to effect warhead kill, especially in the case of warheads carrying submunitions.

Nevertheless, the results of a collision between an interceptor kill vehicle and a booster are not well understood. Unlike the unitary warhead, the missile structure is not solid but has empty pockets, particularly liquid-propelled missiles. Although the energy calculations suggest that there would be more than enough energy to destroy the booster, the specific details of the collision may matter and differ from the idealized scenario enough that thrust would not be terminated completely or immediately. Uncertainty in when thrust would be terminated could require intercepting the missile sooner to prevent it from reaching the United States. It would also seriously complicate strategies for precisely timing the intercept

\footnotetext{
${ }^{1}$ Defined as the closest approach of the kill vehicle to its aimpoint on the target rocket.
} 
to avoid having the warhead from the missile damage other countries. There is little, if any, flight-test data today that would help answer these questions.

Damaging the warhead by striking the booster Despite the energy released by the collision of the kill vehicle with the booster, it is not clear that the warhead would be disabled or destroyed or that such damage would be observable. Warheads are usually coupled loosely to their missiles, often with a few explosive bolts or squibs. Long experience with missile tests and space launches, where the booster exploded or was intentionally destroyed by the range safety officer, suggest that a violent explosion of the booster may not damage the warhead. Therefore, destruction of the booster does not mean destruction of the warhead.

Moreover, it would be difficult, if not impossible, in many cases to verify that the warhead has been disabled by this indirect mechanism. Up close, it may look fine from the outside even to the expert eye, and less-than-catastrophic damage would almost certainly be invisible to the long-range sensors that are tracking it for the defense. In sum, booster kill is an unreliable means for destroying the warhead. If destroying the warhead is required, a direct attack on it would produce better results.

\subsection{Warhead Destruction}

As discussed in Chapter 5, the ability to destroy the warhead does not change the timeline for a successful intercept: as long as the missile's propulsion is terminated in time to prevent it from entering a ballistic trajectory that would carry it to the United States, it is not essential that the warhead be destroyed. Thus, if one regards boost-phase intercept solely from the point of view of defending the United States, warhead kill is not a fundamental issue: whether or not the warhead is destroyed, if the intercept is successful no munitions would reach the United States.

Nevertheless, there are reasons for attempting to destroy the warhead, primarily to reduce the risk that a boost-phase intercept by the United States could pose to other nations. As discussed in Chapter 5, it may be unacceptable for the United States to deploy a boostphase intercept system that could cause a live nuclear warhead or chemical or biological submunitions to strike another nation. Chapter 5 presents strategies for preventing debris from falling on other nations that involve timing the intercept in a narrow time window ( $5 \mathrm{~s}$ to $10 \mathrm{~s}$ ) so that the warhead would fall in the ocean, but that goal is challenging and in some situations might not be achievable, given that the basic timing uncertainties in missile's trajectory are of the same order. The ability to destroy an attacking missile's munitions with high probability could alleviate those concerns.

\subsubsection{Types of warheads}

A warhead can comprise a nuclear weapon, unitary loads of chemical or biological agents, or dozens to hundreds of bomblets containing chemical or biological agents. A missile can also carry several large unitary warheads. The warheads and submunitions could be deployed from the booster within seconds of burnout, or even before burnout.

\subsubsection{Technical challenges for destroying the warhead}

The physics of high-speed collisions and the results of flight tests of midcourse interceptors show that if a unitary warhead is hit, it is likely to be destroyed. To succeed in boost 
phase, however, the defense would have to accomplish four tasks: find the warhead, hit the warhead, destroy any submunitions, and verify destruction.

Finding the warhead First, the defense must find the warhead on the missile. This task is usually quite straightforward - the warhead is attached to the leading end of the missile. But as Fig. 16.6 shows, the warhead's exact location may not be obvious. For structural and mass distribution reasons, the threat missile designer would prefer to affix the warhead to the forward end of the final stage motor. However, the designer could include a shroud that disguises that location by a meter or more. For the relatively small kill vehicle with a diameter of $50 \mathrm{~cm}$ or less described in Chapter 14, missing the warhead by a meter would cause the intercept to fail. If the missile is delivering a payload of submunitions, the designer could distribute them around the outside of the missile body. A number of techniques for enhancing the kill vehicle's radius of destruction will be discussed below.

Hitting the warhead Aiming the kill vehicle to destroy the warhead is similar to aiming to destroy the missile, except that the sweet spot (optimal impact area) for destroying the warhead can be much smaller than the area for disabling the booster. Thus, greater accuracy would be needed in the kill vehicle (miss distances of $10 \mathrm{~cm}$ to $20 \mathrm{~cm}$ rather than $50 \mathrm{~cm}$ ), which places more stringent demands on the homing algorithms, responsiveness of the divert and attitude control system of the kill vehicle, and acceleration capability.

Attempting to destroy the warhead introduces additional challenges. Even assuming that the properties of the missile are known, which is unlikely to be the case, the sweet spot for a warhead's destruction would depend upon its specific design. Dealing with this problem would increase the complexity of the logic and processing required for a boost-phase kill vehicle, compared with one designed to hit the booster.

Destroying submunitions The challenge of destroying the warhead is greater if it is an assembly of lightly packed submunitions. Such an assembly is not a homogeneous structure and, consequently, the transmission of the hydrodynamic shock that is so effective in hardbody-to-hardbody collisions becomes less efficient. This result has been shown quantitatively in many experiments [156]. There has been progress in understanding these processes and simulating them quantitatively, particularly at the lower closing velocities of a few kilometers per second characteristic of theater ballistic missile engagements. However, this technical issue needs more exploration.

Verifying warhead destruction Unless the intercept results in obliteration of the warhead, it will be difficult to verify that the intercept has been successful and that live warheads will not land in other countries. Verifying the destruction of submunitions would be even more difficult. Verifying the destruction of the warhead or submunitions is an important issue for a layered defense. To ensure that warheads do not land on other countries, a midcourse defense could engage any warheads that survive the boost-phase layer. To avoid wasting interceptors, the midcourse system must know which warheads have been destroyed. Moreover, the midcourse system must be able to handle the unpredictable debris that the boost-phase layer may create (see the next section). 


\subsection{Effects of Incomplete Warhead Kill on Other Layers of a Defense}

Boost-phase defenses cannot be viewed in isolation from other layers of the defense system, should they exist. For example, the demands placed on a boost-phase defense would be less severe if a midcourse system were able to engage warheads that survived boost-phase engagement. Similarly, the boost-phase layer can reduce the demands on the midcourse defense if it can destroy missiles or damage them before they can deploy countermeasures against the midcourse defense. But, as previously mentioned, collisions in the boost phase could present problems for other layers of the defense. A midcourse system would face a greater challenge in identifying the warhead if it were surrounded by debris created during the collision with a boost-phase interceptor, or if the collision caused the warhead to tumble or spin in ways that the midcourse defense had not anticipated. If the boost-phase-intercept resulted in a target that included the warhead still attached to part of the booster, the midcourse system might have trouble identifying and destroying the warhead, particularly if the tankage--warhead assembly were tumbling.

These considerations do not imply that the goal of destroying the warhead rather than the booster is not worthwhile, but that the complexities that a boost-phase system might create for other layers must be carefully examined and accounted for in the design of each of the layers.

\subsection{Enhancing the Lethality of the Kill Vehicle}

Because of the potential difficulties in finding the warhead and hitting the sweet spot with a standard kill vehicle, it may be desirable to significantly increase the radius of destruction of the kinetic kill vehicle. A primary advantage of increasing lethality is that doing so could improve the chances of verifying destruction (particularly of submunitions) and thereby reduce the load on the midcourse layer (if there is one). One approach, employed in the Homing Overlay Experiment [154], is an umbrella-like device to enhance lethality (see Fig. 11.2). A similar idea was used in the Exo-atmospheric Reentry Vehicle Interceptor System (ERIS) experiment shown in Fig. 13.1 [157, 158].

In the ERIS test, a film of some plastic material, such as Mylar was stretched on a frame that was inflated shortly before impact. In the cases of interest here, the diameter of such a structure might be approximately two meters. For a Mylar film 0.67-mm-thick moving at a closing velocity of $10 \mathrm{~km} / \mathrm{s}$, the kinetic energy delivered per square centimeter of target surface is about $5 \mathrm{~kJ}$, sufficient to cause severe damage in its own right as noted above. In both of these cases, however, destruction was achieved by direct body-to-body impact, so the effectiveness of lethality enhancements in flight tests remains to be demonstrated.

Other lethality enhancement approaches have been proposed to increase lethal radius or lethality against submunitions, including the release of many rods from the kill vehicle prior to impact [156]. While these might be effective against a target in ballistic flight where the trajectory is predictable, the situation in boost phase is made much more stressful by the maneuvering target, where the fusing problem is complicated. In fact, deploying any mechanical device in a kill vehicle that could be undergoing transverse accelerations of as much as $15 \mathrm{~g}$ up to the time of impact has yet to be demonstrated.

The mass of any lethality enhancement device would also be a critical factor in the design of the kill vehicle. Because the kill vehicle must be able to maneuver rapidly and undergo large acceleration to the moment of impact, its mass is a critical design parameter. The 


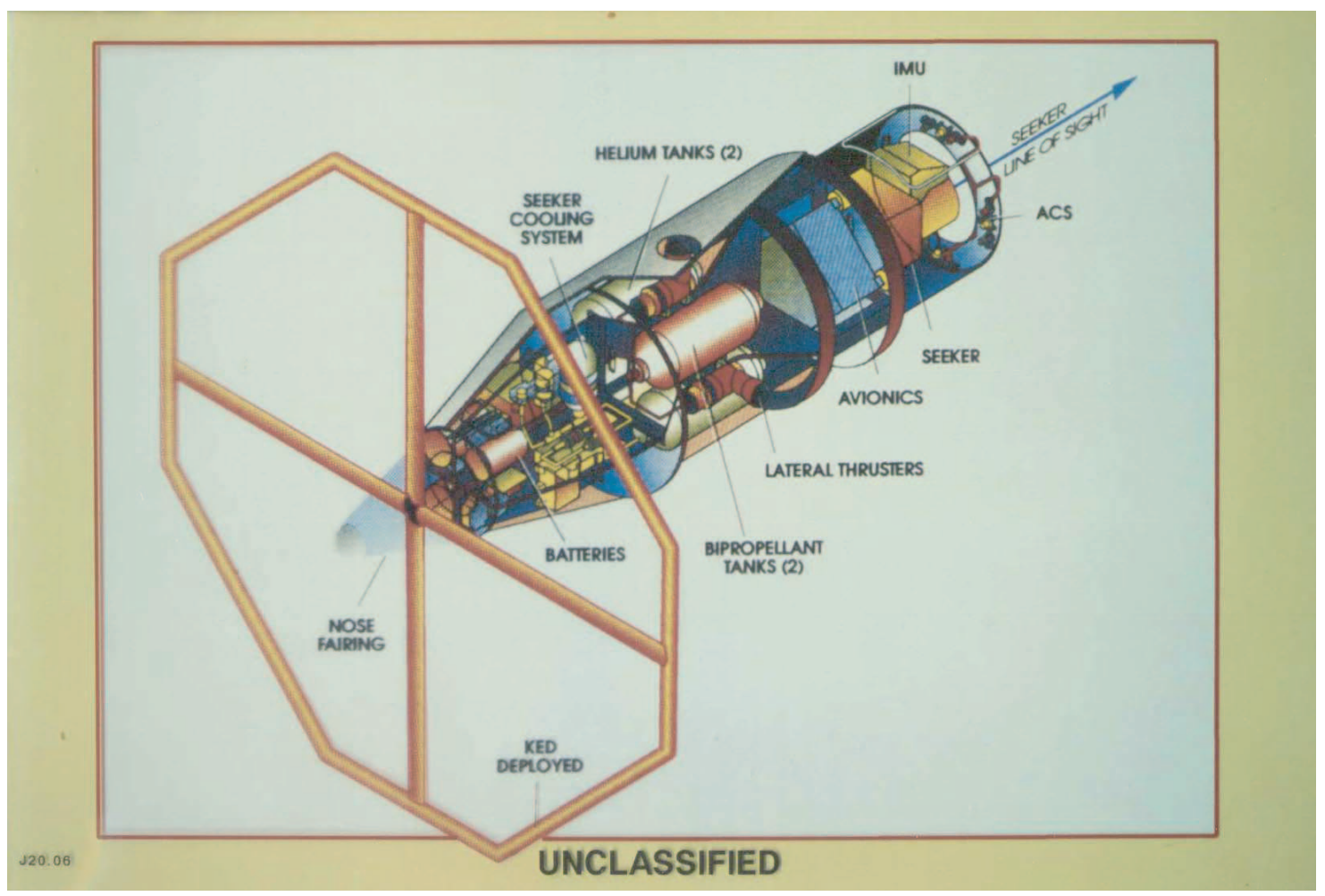

Figure 13.1. Artist's conception of a lethality enhancement device on the ERIS experiment. (Courtesy of LDM Associates)

additional mass of a lethality enhancement device could significantly increase the overall size of the interceptor. Thus, the tradeoff between the mass of a lethality-enhancement device against the performance of the kill vehicle would need to be carefully evaluated, particularly in deployment modes where size or mass is constrained, such as space-based interceptors or missiles deployed on aircraft or in the Aegis vertical launch system on ships.

\subsection{Summary of Conclusions}

We draw the following conclusions about destroying the booster and the warhead.

\subsubsection{Booster destruction}

1. The impact of a kill vehicle with a booster would have more than enough energy to disable or destroy the booster and terminate thrust, although perhaps not immediately, depending on the design of the missile and the specifics of the intercept.

2. Destruction of the booster does not assure that the warhead has been disabled or destroyed, because it is usually loosely coupled to the missile. 


\subsubsection{Warhead destruction}

1. Capability for destroying the warhead (in contrast to merely terminating the thrust of the target missile's booster) does not provide additional time for the intercept to take place to defend the nation. In either case, the missile must be intercepted before it first achieves the range to reach the United States. (In terms of its trajectory, the intercept must occur before the missile reaches the intercept points described in Chapter 5.) Thus, the ability to destroy the warhead does not simplify the problem of achieving a successful intercept.

2. As discussed in Section 5.8, to avoid causing live munitions to fall on other nations, the target missile's thrust must be terminated in a very narrow time window. Our assessment is that, in the face of the probable uncertainties in a boost-phase engagement, in many situations it would be extremely difficult, if possible at all, to achieve this timing. In the absence of such a timing capability, high confidence of warhead destruction would be needed to ensure that achieving an intercept would not cause harm to other nations.

3. If boost-phase intercept is one tier of a layered defense system, then the destruction of a warhead in the boost-phase would have to be confirmed with high reliability to avoid unnecessary firing of additional defenders in subsequent layers. This need becomes even more urgent if several missiles are launched in a short time window, which is a likely scenario. Confirming warhead destruction in the boost-phase with high reliability appears to be a challenging problem because of the difficulty of identifying the warhead fragments among the debris of the intercept.

4. If warhead destruction in the boost-phase cannot be confirmed with high reliability, in a layered defense, intercepting the warhead in midcourse could be made more difficult by the problem of detecting it among the debris of the intercept, which would follow the same general trajectory. Consequently, unless a reliable warhead kill assessment is possible and the midcourse defense is robust enough to handle unpredictable targets clusters and target behavior, intercept during boost phase may in some cases be detrimental to the midcourse defense.

5. We conclude that because of the wide variability of predictive models of booster kill and submunition payloads for ICBM engagement conditions, the only reliable way to determine the lethality of kinetic-energy interceptors in boost-phase defense against different types of missiles and warheads would be to conduct actual intercepts as part of a development program.

\section{References for Chapter 13}

[154] HOE, Ballistic Missile Defense, GAO/NSAID-94-219, July 1994.

[155] N. Bloembergen et al., Rev. Mod. Phys. 59, S1 (1987).

[156] R. M. Lloyd, "Physics of direct hit and near miss warhead technology." Vol. 194, AIAA Progress in Astronautics and Aeronautics (2001).

[157] “Test Firing Downs ICBM", Jane’s Defense Weekly, February 1991. 
[158] ERIS, "Big Bang Greets Slimmed Down Star Wars," New Scientist, February 1991. 


\title{
Chapter 14
}

\section{Size of the Kill Vehicle}

\author{
Contents
}

14.1 Performance Requirements for Boost-Phase Kill Vehicles . $\quad$ S248

14.2 Natural and Induced Environmental Considerations . . . S248

14.3 Design Concept for a Notional Kill Vehicle . . . . . . . S S249

14.4 Final Kill-Vehicle Sizing . . . . . . . . . . . . . S249

14.4.1 Surface-based baseline kill vehicle . . . . . . . . . . . S250

14.4.2 Space-based kill vehicle . . . . . . . . . . . . . . S254

14.4.3 Kill-vehicle kick stages . . . . . . . . . . . . . S S254

The size and mass of the kill vehicle have a profound effect on the size of the interceptor. In the case of volume or weight limited interceptors deployed from aircraft or Vertical Lunch System (VLS) tubes on ships, the kill-vehicle mass determines interceptor flyout speed. The flyout speed in turn determines how close the interceptor must be based to the intercept point to reach the ICBM in time to disable it. In the case of space-based interceptors, the kill-vehicle mass is critical because it determines the amount of interceptor and support system mass that must initially be placed into orbit. Since deploying mass into orbit is very expensive (more than $\$ 22,000 / \mathrm{kg}$ ), mass-in-orbit is a critical parameter for space-based systems.

This chapter develops models of a notional kinetic-energy kill vehicle for BPI that are based on extrapolating technologies that are well into development and likely to be mature enough to be included in a BPI system that is deployed within a decade. Using the killvehicle performance criteria that are developed in Chapter 12, the model kill vehicles are sized to illustrate the approximate masses of BPI kill vehicles for different divert requirements. The results of this analysis are used to size the interceptors presented in Chapter 16 and the space-based interceptors discussed in Chapter 6 .

The philosophy behind the sizing model was to use technologies that either exist today or are reasonable extensions of current technologies. Care was taken to include all of the components that would be necessary for a kill vehicle to function (not just the central components). Care was also taken to adjust the key propulsion components (nozzles, thrusters, and tanks) to meet the large divert velocities and accelerations required for a boost-phase kill vehicle. As such, the estimates of kill-vehicle mass for BPI developed here are significantly greater than some have estimated previously, $[159,160]$ although less than would be possible with technologies that could be deployed today. 


\subsection{Performance Requirements for Boost-Phase Kill Vehicles}

The flight of the kill vehicle may be thought of in three distinct phases after it separates from its booster:

- The initial divert phase, when the kill vehicle is maneuvering based on information about the target's behavior from external (off-board) sensors, before its own sensors have acquired the target.

- The homing phase, which begins when the kill vehicle's on-board sensors have acquired and designated the target to be hit and the kill vehicle no longer depends on external data.

- The endgame, the last few seconds of the homing phase when the maneuvering limits, time lags, and sensor accuracy of the kill vehicle determine the miss distance.

Each of the three phases establishes requirements for the kill vehicle and affects its configuration. According to the analysis done in Chapter 12, the first two phases generate the requirements for total divert impulse and the minimum acceleration on the kill vehicle. Those requirements are driven by uncertainties created by sensor noise and target maneuvers. For the closing velocities that characterize surface-based interceptors engaging ICBMs, the required total change in velocity is $2 \mathrm{~km} / \mathrm{s}$ (see Section 12.4). For the higher closing velocities associated with space-based interceptors, the required total change in velocity is expected to be at least $2.5 \mathrm{~km} / \mathrm{s}$.

The total velocity change that is required of a kill vehicle affects its design in two ways: it drives the amount of propellant that is needed and thus the size of the propellant tanks that are required to store that propellant.

The final phase, the endgame, determines the requirements for maximum acceleration and the system response characteristics. Section 12.4 estimates that to have a high probability of achieving a miss distance of $50 \mathrm{~cm}$ or less against an ICBM, the BPI kill vehicle would need to accelerate by as much as $15 \mathrm{~g}$ with a response lag of $0.1 \mathrm{~s}$ or less during the endgame (the last $10 \mathrm{~s}$ or so before intercept). In our view, a 0.1 -s response lag should be achievable with modern guidance and control systems and thrusters, and would not have a significant effect on kill vehicle size. However, meeting the high acceleration requirement in the last $10 \mathrm{~s}$ would have a significant effect on the size of the four divert thrusters - they must be large enough to provide the needed impulse when the kill vehicle has about 15 percent of its propellant remaining in the tank. The larger divert thrusters increase the dry weight of the kill vehicle, which, in turn increases the amount of propellant required. When the divert thrusters are sized to provide $15 \mathrm{~g}$ during the endgame, they can also provide about $8 \mathrm{~g}$ with a full load of propellant, which meets the early minimum acceleration requirement to keep up with target maneuvers.

Together, these requirements establish the inputs for the sizing analysis that follows.

\subsection{Natural and Induced Environmental Considerations}

In addition to the shock, vibration, and acceleration environmental requirements associated with powered flight, the kill-vehicle design must take other factors into account. First, consideration must be given to sun and Earth shine and their effects on the design of the optical systems. Careful off-axis rejection is required in the design. Depending on the time of day, there are exclusion regions for engagements where the kill vehicle's homing sensor cannot be 
pointed. We did not attempt to estimate those environmental considerations explicitly, but they must be considered in designing a real kill vehicle and in actual engagement geometries.

Nuclear detonation One effect that we did take into account in our kill-vehicle design was the radiation environment that would be created by the detonation of a nuclear weapon in space. Such an event could happen either as a countermeasure or if the warhead were designed to detonate if it were hit by an interceptor.

A nuclear weapon detonated anywhere in Earth's magnetic field would result in electrons becoming trapped, oscillating from pole to pole along the magnetic field lines. The electrons would quickly equilibrate around Earth and persist long after the burst [161]. Both spaceand terrestrial-based exoatmospheric interceptors operate at altitudes where the density of electrons would be significant. (Intercepts would occur at 150-200 km altitudes for surface- or space-based kill vehicles, and the SBIs would probably be maintained at orbital altitudes between 300-500 km.) A kill vehicle would therefore be bombarded by high-speed electrons that would be captured by its metal structure, emitting gamma photons in the process. Detector arrays designed to measure very-low-energy photon flux would see these high-energy events as a huge spike, with a recovery time that depends on the material and volume of the detectors. If proper care is taken in design, the effect does not cause permanent damage. However, it would create repetitive upset events which, depending on the recovery time, could be frequent enough to prevent the detector array from getting useful data. To prevent this from being a potential countermeasure, high $\mathrm{Z}$ shielding around the focal plane is recommended to reduce the gamma flux. An arbitrary $1 \mathrm{~kg}$ of tungsten has been provided for in the kill-vehicle sizing model used in this study.

\subsection{Design Concept for a Notional Kill Vehicle}

To examine sizing issues, the Study Group created a notional kill vehicle. It includes five major components: the sensor suite, divert and altitude control system (DACS), avionics, the basic structure, and propellant and propellant storage vessels (tankage). Other components include a battery, an inertial measurement unit (IMU), ordnance initiation, and radiation shielding for the focal plane array.

The sensor suite includes passive IR and visible sensors to track the target and its plume during initial divert and homing, and a LIDAR to provide high-quality range, velocity, and spatial measurements during homing and the endgame (see Chapter 10). As discussed in Chapter 11, the divert system uses the cruciform configuration for the thrusters: four equally sized thrusters arranged in an ' $\mathrm{X}$ ' perpendicular to the roll axis of the vehicle. Each thruster must be large enough to provide the maximum acceleration required of the kill vehicle during the endgame. Figure 14.1 shows the notional kill vehicle design that was used to size the structure and tank configuration needed to handle the $50 \mathrm{~g}$ boost acceleration and kill vehicle dynamic requirements.

\subsection{Final Kill-Vehicle Sizing}

The sizing model for kill vehicles used in this study is based on analysis from several different sources. Table 14.1 shows the weight breakdowns for four different BPI kill vehicles capable of divert velocities of $2 \mathrm{~km} / \mathrm{s}$ and accelerations of $15 \mathrm{~g}$ - the requirements that were estimated for intercepting an ICBM target with a $6.5-\mathrm{km} / \mathrm{s}$ interceptor (closing velocities of $10-14 \mathrm{~km} / \mathrm{s}$ ). All four of the kill vehicles use the cruciform configuration for the divert 


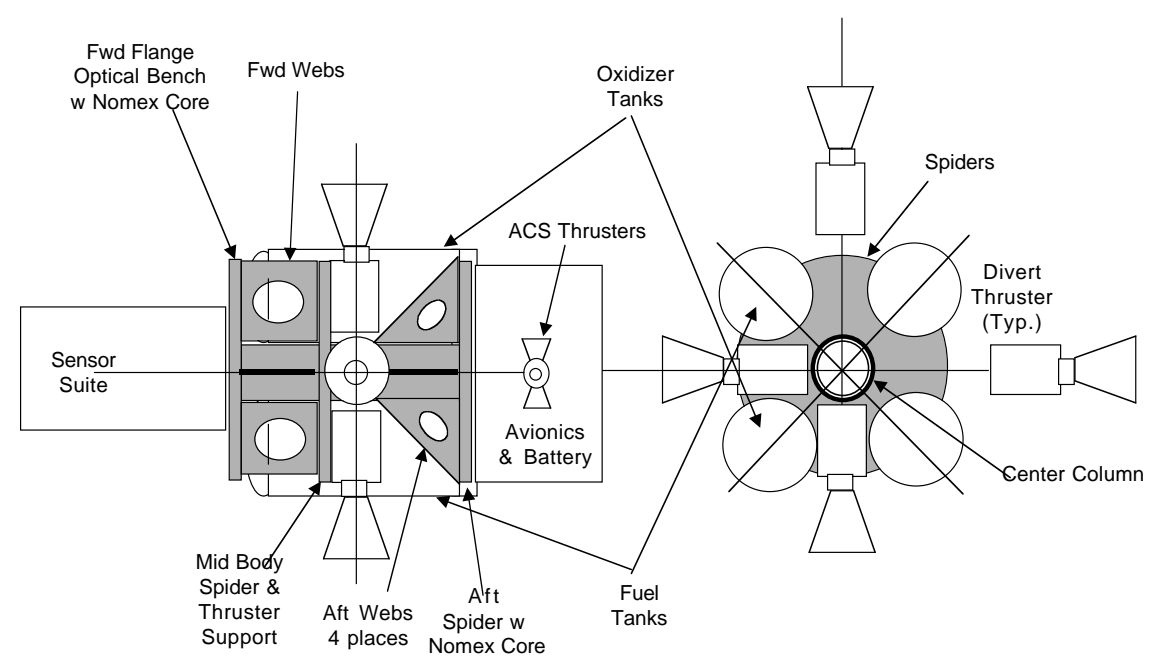

Figure 14.1. Assumed KV structure. The shaded areas indicate the Kevlar/epoxy frame.

thrusters. The first column lists the components that we believe would be required to build a working BPI kill vehicle. The second column shows the mass breakdown of a notional kill vehicle that could be built using components from existing components or well-established technologies.

The third column shows the weight breakdowns for a light weight, notional space-based design for the Advanced Technology Kill Vehicle (ATKV) [160] from a Lawrence Livermore National Laboratory presentation to the panel. This design would reduce weight from current technology by using miniaturized components that were flight-tested on the Clementine technology demonstration satellite. It also includes some prototype components that are still in development, but we assume that given sufficient funding, development could be completed with sufficient for deployment within about a decade. The ATKV remains largely a conceptual design at this point; it has not been built or flight-tested.

\subsubsection{Surface-based baseline kill vehicle}

From the weight estimates for the current technology kill vehicle and ATKV (which, with some exceptions, were found to be reasonably consistent if modest improvements to avionics packaging technology are assumed), it is possible to project component sizes including sensors, avionics, batteries, and comparable structural weight that make up the non-varying part of the dry weight of the kill vehicle. When combined with the necessary propellant to generate $2 \mathrm{~km} / \mathrm{s}$ of divert thrust, the result is the "surface-based baseline kill vehicle" shown in the fourth column, which was used in sizing the interceptors modeled in Chapter 16.

The surface-based baseline kill vehicle is patterned largely on the notional ATKV design, but it has been adjusted in several important ways to reflect our judgment about what would be needed to operate in the challenging boost-phase environment. It differs from the ATKV 
Table 14.1. Properties of a surface-based kill vehicle. Total divert: $2 \mathrm{~km} / \mathrm{s}$.

\begin{tabular}{|c|c|c|c|c|c|}
\hline KV Segment or Subassy & $\begin{array}{c}\text { Existing } \\
\text { Technology } \\
\mathrm{KV} \\
(\mathrm{kg}) \\
\end{array}$ & $\begin{array}{l}\text { LLNL } \\
\text { Space- } \\
\text { Based } \\
\text { ATKV } \\
(\mathrm{kg}) \\
\end{array}$ & \begin{tabular}{|c|}
$\begin{array}{c}\text { Surface- } \\
\text { Based } \\
\text { Baseline KV } \\
(\mathrm{kg})\end{array}$ \\
\end{tabular} & $\begin{array}{l}\text { Space- } \\
\text { Based } \\
\text { Baseline } \\
\mathrm{KV} \\
(\mathrm{kg}) \\
\end{array}$ & Notes \\
\hline $\begin{array}{l}\text { Divert and Attitude Control System (DACS) } \\
\text { Pressure regulator } \\
\text { Divert Thrusters } \\
\text { ACS Trusters } \\
\text { Valve drivers } \\
\text { Manifold } \\
\quad \text { Subtotal, DACS }\end{array}$ & $\begin{array}{l}\text { Included } \\
17.15 \\
1.50 \\
\text { included } \\
\text { included } \\
\mathbf{1 8 . 6 5}\end{array}$ & $\begin{array}{l}2.00 \\
3.00 \\
\text { included } \\
\text { included } \\
\text { included } \\
\mathbf{5 . 0 0}\end{array}$ & $\begin{array}{c}0.50 \\
10.09 \\
1.01 \\
\text { included } \\
\text { included } \\
\mathbf{1 1 . 6 0}\end{array}$ & $\begin{array}{r}0.50 \\
9.77 \\
0.98 \\
\text { included } \\
\text { included } \\
\mathbf{1 1 . 2 4}\end{array}$ & $\begin{array}{l}\mathrm{KV} \text { sized for closing velocities of } 10-14 \mathrm{~km} / \mathrm{sec} \text {. Total time of KV } \\
\text { operation } 120 \text { sec. Assumes } 4 \text { divert thrusters in cruciform } \\
\text { configuration, sized to deliver } 15 \mathrm{~g} \text { in last } 10 \mathrm{sec} \text {. LLNL example } \\
\text { uses fixed thruster mass regardless of divert requirements; baseline } \\
\mathrm{KV} \text { corrects for this by adjusting divert thruster mass for } 15 \mathrm{~g} \\
\text { using Wilkening scaling factors. ACS impulse assumed to be } 5 \% \text { of } \\
\text { divert impulse. }\end{array}$ \\
\hline $\begin{array}{l}\text { Seeker (less IMU) } \\
\text { Contingency for shielding for focal plane } \\
\text { array } \\
\text { IMU (Inertial measurement unit) } \\
\text { Avionics }\end{array}$ & $\begin{array}{c}1.50 \\
11.50\end{array}$ & $\begin{array}{l}1.00 \\
8.00\end{array}$ & $\begin{array}{l}7.00 \\
1.00 \\
1.00 \\
8.00\end{array}$ & $\begin{array}{l}7.00 \\
1.00 \\
1.00 \\
8.00\end{array}$ & $\begin{array}{l}\text { Includes LIDAR and passive IR and visible sensors. Used Clementine } \\
\text { sensor suite masses } \\
\text { See Natural and Induced Environment section } \\
\text { Avionics include: guidance/control computer,tactical } \\
\text { communications transponder, KV electronic safe arm, FTS antenna } \\
\text { (non-tactical), FTS battery, command destruct receiver, signal } \\
\text { conditioner/submux, X-band antennas, X-band transmit module, } \\
\text { power divider/hybrid coupler, J-box, control module, logic module, } \\
\text { tactical signal and power distribution }\end{array}$ \\
\hline $\begin{array}{l}\text { Separation system } \\
\text { Ordnance inititiate lines } \\
\text { KV primary battery } \\
\text { KV basic structure \& installation hardware } \\
\text { Subtotal, KV dry weight less tankage }\end{array}$ & $\begin{array}{l}0.50 \\
0.25 \\
1.90 \\
\underline{5.00} \\
\mathbf{4 4 . 2 0}\end{array}$ & 22.80 & $\begin{array}{r}0.50 \\
0.25 \\
1.80 \\
\\
\underline{3.50} \\
\mathbf{3 4 . 6 5}\end{array}$ & $\frac{3.50}{33.54}$ & $\begin{array}{l}\text { Separation system and ordnance initiation lines not include on space } \\
\text { based KV systems } \\
\text { Estimate based on other programs } \\
\text { Kevlar epoxy composite structure used for hign axial and lateral } \\
\text { accelerations;propellant tanks used as load carrying structure }\end{array}$ \\
\hline $\begin{array}{l}\text { Propellant } \\
\text { Useful Fuel and Oxidizer } \\
\text { ACS \& press fraction of useful fuel } \\
\text { ACS \& pressurization fuel } 5 \% \\
\text { Unusable Propellant fraction } \\
\text { Unusable Propellant }\end{array}$ & $\begin{array}{l}60.10 \\
5.0 \% \\
3.01 \\
1.80\end{array}$ & $\begin{array}{c}27.10 \\
\mathrm{n} / \mathrm{a} \\
\mathrm{n} / \mathrm{a}\end{array}$ & $\begin{array}{l}47.00 \\
5.0 \% \\
2.35 \\
3.0 \% \\
1.41\end{array}$ & $\begin{array}{l}45.50 \\
5.0 \% \\
2.28 \\
3.0 \% \\
1.37\end{array}$ & $\begin{array}{l}\text { Useful propellant needed to produce a total } \Delta \mathrm{V} \text { of } 2000 \mathrm{~m} / \mathrm{sec} \\
\text { Added ACS fuel @ } 5 \% \text { of divert } \\
\text { Propellant trapped in system assumed to be } 3 \% \text { of total }\end{array}$ \\
\hline Subtotal, Propellant & 63.11 & 27.10 & 49.35 & 47.78 & $\begin{array}{l}\text { Total propellant includes useful propellant and ACS propellant, of } \\
\text { which the amount shown as unusable is trapped in the system. }\end{array}$ \\
\hline Tankage & 12.62 & 3.12 & 9.68 & 9.37 & $\begin{array}{l}\text { To calculate tankage mass, LLNL uses } 0.115^{*} \text { propellant mass, which } \\
\text { is based on a pumped DACS and lower pressure tanks. We assume } \\
\text { conventional high pressure tanks with mass } 0.2^{*} \text { propellant mass }\end{array}$ \\
\hline Total, KV wet & 119.93 & 53.02 & 93.68 & 90.69 & $\begin{array}{l}\text { Total KV wet mass includes the dry mass, propellant mass, and } \\
\text { tankage mass }\end{array}$ \\
\hline $\begin{array}{l}\Delta V \text { check } \\
\text { Isp of propellent (sec) } \\
\text { Isp (effective) after ACS \& press. } \\
\Delta \mathrm{V} \text { from the rocket equation (check) } \\
\Delta \mathrm{V} \text { desired (input) }\end{array}$ & $\begin{array}{c}300 \\
285 \\
1999 \\
2000 \\
\end{array}$ & $\begin{array}{r}300 \\
285 \\
1999 \\
2000 \\
\end{array}$ & $\begin{array}{r}300 \\
285 \\
2002 \\
2000 \\
\end{array}$ & $\begin{array}{r}300 \\
285 \\
2002 \\
2000 \\
\end{array}$ & To account for ACS/pressure fuel used but not effective for thrust \\
\hline $\begin{array}{l}\text { Endgame acceleration calculation } \\
\text { KV Mass with } 15 \% \text { fuel remaining } \\
\text { Thrust for } 15 \mathrm{~g} \text { 's @ } 15 \% \text { fuel load(Newtons) } \\
\text { G's at full fuel load }\end{array}$ & $\begin{array}{l}66.29 \\
9744 \\
8.29\end{array}$ & $\begin{array}{l}29.98 \\
4407 \\
8.48\end{array}$ & $\begin{array}{l}51.73 \\
7604 \\
8.28 \\
\end{array}$ & $\begin{array}{l}50.08 \\
7362 \\
8.28 \\
\end{array}$ & Used to estimate thruster size \\
\hline
\end{tabular}

design primarily in its treatment of the divert and altitude control systems (DACS) and the mass of the tankage. One of the principal sources that we used for those adjustments was a series of physics- and historically based scaling relationships developed by Dean Wilkening in a study that examines airborne BPI options for both tactical and ICBM engagements and validates those scaling relationships with hardware examples [162].

Six adjustments were made to the fixed dry weights estimated for the ATKV:

- The size of the divert thrusters was scaled using Wilkening's scaling relationships for the thrust levels needed to produce $15 \mathrm{~g}$ accelerations in the last $10 \mathrm{~s}$ of the endgame (assumed here to be when 15 percent of the propellant remains), as described in Chapter 12.

- A 20 percent ratio of tank weight to propellant weight was used to reflect current state-of-the-art pressurization, instead of 11.5 percent in the ATKV design. The ATKV's smaller ratio reflects a pumped approach to feeding propellant to the 
thrusters, which, if successful, would reduce the requirement to pressurize the fuel and result in simpler, more lightweight tanks. We used the conventional approach because the sizes required for the kill vehicles considered here for BPI are much larger than the very small prototype systems that have been demonstrated to date for the pumped system. The 20 percent ratio used here was cross-checked using Wilkening's scaling relationships.

- So that the kill vehicle could operate at high axial and lateral loads, $3.5 \mathrm{~kg}$ of composite structure was added. The fuel tanks were also used as load-carrying structure.

- Shielding $(1 \mathrm{~kg})$ for the sensor focal plane assembly was added to reduce the flux of trapped electron-induced radiation noise from a nuclear detonation.

- Weight was added to the terrestrial-based kill vehicle for separation hardware and ordnance that remains with the kill vehicle after separation from the booster. It is not required for the space-based kill vehicle because that hardware is included in the mass fraction of the space-based interceptor boost stage.

Using those estimates for dry mass, we determined the wet mass of the kill vehicle by calculating, via the rocket equation, the propellant needed to provide enough divert thrust, with an allowance of 3 percent for unusable propellant. The propellant used for the altitude control system (ACS), which consumes propellant but does not contribute to divert thrust, was accounted for by using a slightly reduced ISP (285 s) (ISP is the specific impulse of the propellant) in the rocket equation instead of the actual ISP of the fuel (300 s). This 5 -percent reduction in effective ISP is consistent with the design assumption that the ACS impulse required would be 5 percent of the divert impulse.

For the surface-based baseline kill vehicle to be capable of a $2-\mathrm{km} / \mathrm{s}$ divert, our model yields a total wet mass of about $90 \mathrm{~kg}$ (see Table 14.1). If the divert-thrust requirement is increased to $2.5 \mathrm{~km} / \mathrm{s}$, the wet mass of the surface-based kill vehicle increases to $140 \mathrm{~kg}$ (see Table 14.2).

The wet masses of these kill vehicles are greater than the Exoatmospheric Kill Vehicle (EKV) planned for the midcourse system. That kill vehicle is reported to have a wet mass of about $55 \mathrm{~kg}$ [161], compared to the $90-\mathrm{kg}$ wet mass of the BPI kill vehicle having a $2-\mathrm{km} / \mathrm{s}$ divert capability. The dry mass of the EKV is actually greater than for the BPI kill vehicles estimated here. With $9-14 \mathrm{~kg}$ of propellant [163], the dry mass of the EKV is about $40 \mathrm{~kg}$, compared with $35 \mathrm{~kg}$ for the BPI kill vehicle. The difference is in propellant, with the BPI kill vehicle requiring significantly more propellant $(47 \mathrm{~kg})$ to generate $2 \mathrm{~km} / \mathrm{s}$ of divert propulsion. By comparison, the EKV divert requirement is well below $1 \mathrm{~km} / \mathrm{s}$.

Using the relationships from the tables and adjusting divert thruster size to maintain 15-g maneuver capability with 15 percent of fuel remaining, vehicles were sized for four total $\Delta$ V cases: $1500-, 2000-, 2500-$, and $3000-\mathrm{m} / \mathrm{s}$ capability. Table 14.3 shows the results for surface-based kill vehicles. The limited Monte Carlo analysis conducted during the Study indicated that a 90 percent probable total divert $\Delta \mathrm{V}$ requirement for closing velocities of $10 \mathrm{~km} / \mathrm{s}$ is about $2 \mathrm{~km} / \mathrm{s}$ (see Section 12.3). At the $16-\mathrm{km} / \mathrm{s}$ closing velocities that would be encountered by space-based interceptors against ICBMs, the divert requirement may be $2.5 \mathrm{~km} / \mathrm{s}$ or higher. 
Table 14.2. Properties of terrestrial- and space-based kill vehicles. Total divert: $2.5 \mathrm{~km} / \mathrm{s}$.

\begin{tabular}{|c|c|c|c|c|c|}
\hline KV Segment or Subassy & $\begin{array}{c}\text { Existing } \\
\text { Technology } \\
\mathrm{KV} \\
(\mathrm{kg}) \\
\end{array}$ & $\begin{array}{l}\text { LLNL } \\
\text { Space- } \\
\text { Based } \\
\text { ATKV } \\
(\mathrm{kg}) \\
\end{array}$ & $\begin{array}{c}\begin{array}{c}\text { Surface- } \\
\text { Based } \\
\text { Baseline KV } \\
(\mathrm{kg})\end{array} \\
\end{array}$ & $\begin{array}{l}\text { Space- } \\
\text { Based } \\
\text { Baseline } \\
\mathrm{KV} \\
(\mathrm{kg}) \\
\end{array}$ & Notes \\
\hline $\begin{array}{l}\text { Divert and Attitude Control System (DACS) } \\
\text { Pressure regulator } \\
\text { Divert Thrusters } \\
\text { ACS Trusters } \\
\text { Valve drivers } \\
\text { Manifold } \\
\quad \text { Subtotal, DACS }\end{array}$ & $\begin{array}{l}\text { Included } \\
17.15 \\
1.50 \\
\text { included } \\
\text { included } \\
\mathbf{1 8 . 6 5}\end{array}$ & $\begin{array}{l}2.00 \\
3.00 \\
\text { included } \\
\text { included } \\
\text { included } \\
\mathbf{5 . 0 0}\end{array}$ & $\begin{array}{c}0.50 \\
13.17 \\
1.32 \\
\text { included } \\
\text { included } \\
\mathbf{1 4 . 9 9}\end{array}$ & $\begin{array}{c}0.50 \\
12.75 \\
1.28 \\
\text { included } \\
\text { included } \\
\mathbf{1 4 . 5 3}\end{array}$ & $\begin{array}{l}\text { KV sized for closing velocities of } 10-14 \mathrm{~km} / \mathrm{sec} \text {. Total time of KV } \\
\text { operation } 120 \text { sec. Assumes } 4 \text { divert thrusters in cruciform } \\
\text { configuration, sized to deliver } 15 \mathrm{~g} \text { in last } 10 \mathrm{sec} \text {. LLNL example } \\
\text { uses fixed thruster mass regardless of divert requirements; baseline } \\
\mathrm{KV} \text { corrects for this by adjusting divert thruster mass for } 15 \mathrm{~g} \\
\text { using Wilkening scaling factors. ACS impulse assumed to be } 5 \% \text { of } \\
\text { divert impulse. }\end{array}$ \\
\hline $\begin{array}{l}\text { Seeker (less IMU) } \\
\text { Contingency for shielding for focal plane } \\
\text { array } \\
\text { IMU (Inertial measurement unit) } \\
\text { Avionics }\end{array}$ & $\begin{array}{c}1.50 \\
11.50\end{array}$ & $\begin{array}{l}1.00 \\
8.00\end{array}$ & $\begin{array}{l}7.00 \\
1.00 \\
1.00 \\
8.00\end{array}$ & $\begin{array}{l}7.00 \\
1.00 \\
1.00 \\
8.00\end{array}$ & $\begin{array}{l}\text { Includes LIDAR and passive IR and visible sensors. Used Clementine } \\
\text { sensor suite masses } \\
\text { See Natural and Induced Environment section } \\
\text { Avionics include: guidance/control computer,tactical } \\
\text { communications transponder, KV electronic safe arm, FTS antenna } \\
\text { (non-tactical), FTS battery, command destruct receiver, signal } \\
\text { conditioner/submux, X-band antennas, X-band transmit module, } \\
\text { power divider/hybrid coupler, J-box, control module, logic module, } \\
\text { tactical signal and power distribution }\end{array}$ \\
\hline $\begin{array}{l}\text { Separation system } \\
\text { Ordnance inititiate lines } \\
\mathrm{KV} \text { primary battery } \\
\mathrm{KV} \text { basic structure \& installation hardware } \\
\text { Subtotal, KV dry weight less tankage }\end{array}$ & $\begin{array}{r}0.50 \\
0.25 \\
1.90 \\
\underline{5.00} \\
\mathbf{4 4 . 2 0}\end{array}$ & $\overline{22.80}$ & $\begin{array}{r}0.50 \\
0.25 \\
1.80 \\
\\
\underline{3.50} \\
\mathbf{3 8 . 0 4}\end{array}$ & $\frac{3.50}{36.83}$ & $\begin{array}{l}\text { Separation system and ordnance initiation lines not include on space } \\
\text { based KV systems } \\
\text { Estimate based on other programs } \\
\text { Kevlar epoxy composite structure used for hign axial and lateral } \\
\text { accelerations;propellant tanks used as load carrying structure }\end{array}$ \\
\hline $\begin{array}{l}\text { Propellant } \\
\text { Useful Fuel and Oxidizer } \\
\text { ACS \& press fraction of useful fuel } \\
\text { ACS \& pressurization fuel } 5 \% \\
\text { Unusable Propellant fraction } \\
\text { Unusable Propellant }\end{array}$ & $\begin{array}{l}95.00 \\
5.0 \% \\
4.75 \\
2.85 \\
\end{array}$ & $\begin{array}{c}39.60 \\
\mathrm{n} / \mathrm{a} \\
\mathrm{n} / \mathrm{a}\end{array}$ & $\begin{array}{l}81.20 \\
5.0 \% \\
4.06 \\
3.0 \% \\
2.44 \\
\end{array}$ & $\begin{array}{l}78.60 \\
5.0 \% \\
3.93 \\
3.0 \% \\
2.36 \\
\end{array}$ & $\begin{array}{l}\text { Useful propellant needed to produce a total } \Delta \mathrm{V} \text { of } 2500 \mathrm{~m} / \mathrm{sec} \\
\text { Added ACS fuel @ } 5 \% \text { of divert } \\
\text { Propellant trapped in system assumed to be } 3 \% \text { of total }\end{array}$ \\
\hline Subtotal, Total Propellant & 99.75 & 39.60 & 85.26 & 82.53 & $\begin{array}{l}\text { Total propellant includes useful propellant and ACS propellant, of } \\
\text { which the amount shown as unusable is trapped in the system. }\end{array}$ \\
\hline Tankage & 163.90 & 66.95 & $\begin{array}{r}16.73 \\
\mathbf{1 4 0 . 0 3}\end{array}$ & 16.19 & $\begin{array}{l}\text { To calculate tankage mass, LLNL uses } 0.115^{\star} \text { propellant mass, which } \\
\text { is based on a pumped DACS and lower pressure tanks. We assume } \\
\text { conventional high pressure tanks with mass } 0.2^{\star} \text { propellant mass at } \\
\text { completion } \\
\text { Total KV wet mass includes the dry mass, propellant mass, and } \\
\text { tankage mass }\end{array}$ \\
\hline $\begin{array}{l}\Delta V \text { check } \\
\text { Isp of propellent (sec) } \\
\text { Isp (effective) after ACS \& press. } \\
\Delta \mathrm{V} \text { from the rocket equation (check) } \\
\Delta \mathrm{V} \text { desired (input) } \\
\end{array}$ & $\begin{array}{r}300 \\
285 \\
2499 \\
2500 \\
\end{array}$ & $\begin{array}{r}300 \\
285 \\
2500 \\
2500 \\
\end{array}$ & $\begin{array}{r}300 \\
285 \\
2500 \\
2500 \\
\end{array}$ & $\begin{array}{r}300 \\
285 \\
2500 \\
2500 \\
\end{array}$ & To account for ACS/pressure fuel used but not effective for thrust \\
\hline $\begin{array}{l}\text { Endgame acceleration calculation } \\
\text { KV Mass with } 15 \% \text { fuel remaining } \\
\text { Thrust for } 15 \mathrm{~g} \text { 's @ } 15 \% \text { fuel load(Newtons) } \\
\text { G's at full fuel load }\end{array}$ & $\begin{array}{c}79.11 \\
11630 \\
7.24\end{array}$ & $\begin{array}{c}33.29 \\
4894 \\
7.46\end{array}$ & $\begin{array}{l}67.56 \\
9931 \\
7.24 \\
\end{array}$ & $\begin{array}{c}65.40 \\
9614 \\
7.24\end{array}$ & Used to estimate thruster size \\
\hline
\end{tabular}

Table 14.3. Surface-based kill vehicles.

\begin{tabular}{cc}
\hline \hline $\begin{array}{c}\text { Divert } \Delta \mathrm{V} \\
(\mathrm{m} / \mathrm{s})\end{array}$ & $\begin{array}{c}\text { Kill vehicle } \\
\text { wet mass }(\mathrm{kg})\end{array}$ \\
\hline 1500 & 67 \\
2000 & 94 \\
2500 & 140 \\
3000 & 240 \\
\hline
\end{tabular}




\subsubsection{Space-based kill vehicle}

In addition to estimating the mass of a surface-based kill vehicle, we estimated the mass of a space-based kill vehicle (see column 5 of Table 14.2), which is a little lighter because it does not include separation hardware. In addition to the kill vehicle mass indicated in the table, the space-based kill vehicle would be mounted on a booster that provides its initial flyout velocity, and the whole interceptor would be housed in an orbital garage or lifejacket. The lifejacket would protect the interceptor from the space environment and provide essential station-keeping and life-support functions that would allow the interceptor to remain in space for years. In addition to the protective structure and shielding, the life jacket would include solar panels, a battery, and a dc-to-dc converter to collect and store energy; radiators to cool the satellite; momentum wheels, controllers, and horizon- and star-tracker sensors for station keeping; an ACS for dumping momentum from the wheels; a Hall effect ion engine and controls for maintaining altitude; a communications link and antenna; and other miscellaneous equipment. The size of the space-based interceptors and lifejackets is discussed further in Chapter 6 .

\subsubsection{Kill-vehicle kick stages}

In sizing our kill vehicles, we considered only single-stage kill vehicles. Two-stage kill vehicles have been suggested to reduce the over all mass of both midcourse and boost-phase kinetic kill vehicles. Adding a stage would entail the addition of a "kick" motor or stage to the kill vehicle to perform a post boost initial divert using either external tracking data or on-board sensor data taken before the kick motor is fired. There are several reasons to consider this approach. For example, it might allow the adaptation of an existing kill vehicle design by providing additional divert capability without resizing existing tankage and thrusters. For a new design, a kick stage could be used for the initial large divert to remove the significant target trajectory error that will inevitably accrue during interceptor boost. The kick stage would be jettisoned before continuing kill-vehicle operations.

The advantage of this approach would be lower kill-vehicle tankage and thruster sizes to meet the endgame requirements presented in Chapter 12. The advantages must be weighed against the added mass and complexity of the additional interstage structure, separation mechanism, and thrust vector control components. This trade-off may be favorable for midcourse interceptors, where once the kill-stage sensors have established a target trajectory, the future target motion is very predictable, and a 'blind' divert can be used.

For boost-phase defense, however, the target continues to accelerate unpredictably. Unless the kill vehicle's on-board sensor is gimbaled or has an internal steering mirror having more than $90^{\circ}$ of angular freedom with its associated weight penalty, an axial-thrust kick stage will not allow the kill vehicle to look at or track the target during kick-stage burn. The kill vehicle must then rely on external track data during that burn, which introduces larger acceleration uncertainties that must then be removed by the final kill vehicle stage. Whether we gimbal the sensor or rely on external track data, we incur a weight penalty that erodes the gain that accrues from a kick stage; we therefore did not include a kick stage in our baseline design. 


\section{References for Chapter 14}

[159] Gregory Canavan, "Space-Based Boost Phase Defenses." Briefing Presented to the APS Study Group, December 2001.

[160] Arno G. Ledebuhr and Larry C. Ng, "Space-Based Hit-to-kill Boost Phase Intercept: System analysis and Performance Trades." Presentation to APS Boost-Phase Intercept Study, September 2001.

[161] John Parmentola, "High Altitude Nuclear Detonations Against Low Earth Orbit Satellites." Defense Threat Reduction Agency, April 2000.

[162] Dean Wilkening, Private communication.

[163] Ballistic Missile Defense Organization. EKV Fact Sheet, 1998. 



\section{Part C. Supporting Analysis for Hit-to-Kill Engagements}





\section{Chapter 15}

\section{Ballistic Missile and Trajectory Models}

\section{Contents}

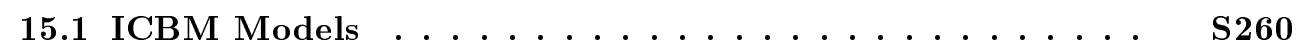

15.2 ICBM Trajectories $\ldots \ldots \ldots \ldots \ldots$ S264

15.2.1 Maximum-range and lofted trajectories . . . . . . . . S S267

15.2 .2 Other trajectories . . . . . . . . . . . . . . S269

15.3 Models of Medium-Range Ballistic Missiles . . . . . . . S274

Any analytical study of the potential feasibility and performance of various BPI systems must begin by developing computer models of the various types of ballistic missiles that these systems might face. Of greatest concern is the possible eventual deployment of intercontinental ballistic missiles (ICBMs) by countries that have ballistic-missile development programs but currently do not have friendly relations with the United States. None of these countries are believed to have tested or deployed long-range missiles that could threaten the territory of the United States, and the likelihood, timing, and character of any future ICBM threat from these countries is uncertain (see Chapter 3). Development and deployment of a BPI missile defense system would be a major investment and would probably take a decade or more to complete. The Study Group therefore considered a spectrum of ICBMs that might be acquired or developed and deployed by countries of concern during the next 10-15 years, including ICBMs that might be developed or acquired and fielded in response to prospective or actual deployment of BPI missile defense system by the United States.

Current estimates by the U.S. intelligence community (see Chapter 3) suggest that the first ICBMs that might be deployed by countries of concern would be liquid-propellant missiles. Although the first ICBMs deployed would probably have shorter ranges, it is estimated that missiles with ranges of $12,000 \mathrm{~km}$ could be deployed within the next decade or so. Deployment of solid-propellant ICBMs would be a natural response to a boostphase missile defense system, because solid-propellant ICBMs burn out sooner than liquidpropellant ICBMs, reducing the time available to intercept them. The U.S. intelligence community estimates that countries of concern could deploy solid-propellant ICBMs within the next decade, if they were able to acquire solid-propellant missiles or technology from countries with more advanced missile programs. The intelligence community and others have cited numerous examples of the transfer of solid-propellant technology to countries of concern. ${ }^{1}$

\footnotetext{
${ }^{1}$ Chapter 3 describes the characteristics of different types of ballistic missiles and discusses current as-
} 
The studies of BPI that have been published previously in the open literature $[164,165]$ considered primarily models of liquid-propellant ICBMs with very long boost phases, such as the Titan II, which had a total boost time of about 330 s [166, pp. 456-458]. Such a long boost phase reduces significantly the performance requirements for a boost-phase missile defense system. Liquid-propellant ICBM-technology now available to countries of concern typically have total boost times of $250 \mathrm{~s}$ or less, and solid-propellant ICBMs based on 1960s technology could be expected to have total boost times of 170 to $180 \mathrm{~s}$. For these reasons the Study Group developed computer models of solid-propellant ICBMs based on 40-year-old, first-generation solid-propellant technology, as well as liquid-propellant ICBMs similar to those first deployed by the Soviets in the early 1960s and by the Chinese in the early 1980s, to explore the potential ability of BPI systems to defend against a spectrum of possible missiles. For the missiles that were modeled in detail, the Study Group characterized their stage-by-stage properties, burn times, the sequence of events during their flight, and their possible trajectories.

To reduce the number of ICBMs constructed and engagements analyzed, the Study Group focused on ICBMs with ranges of $12,000 \mathrm{~km}$ (6,400 nautical miles). The Study Group also constructed two medium-range ballistic missile (MRBM) models to investigate the possible relevance of BPI of shorter-range missiles launched from platforms positioned off the coasts of the United States. The basic properties of these missiles are listed in Tables 15.1 and 15.2. The model ballistic missiles and trajectories described in this chapter are used throughout the rest of the Study. The meanings of the most important symbols used in this chapter are listed in Box 15.1. Some are defined below. Definitions and discussions of all of them may be found in Appendix B.

\subsection{ICBM Models}

The Study Group constructed three primary ICBM models: one liquid-propellant and two solid-propellant missiles. All have maximum final velocities of about $7.2 \mathrm{~km} / \mathrm{s}$ and maximum ranges of about $12,000 \mathrm{~km}$ (6,400 nautical miles). The liquid-propellant model is based on the Chinese DF-5 missile and has a maximum total boost time of $240 \mathrm{~s}$. The two solid-propellant models are typical of first-generation U.S. and Soviet long-range ICBMs. As single-warhead designs, they are based on solid propulsion technology that is 40 years old. Both have maximum total boost times of $170 \mathrm{~s}$, but they have different staging ratios, to test the sensitivity of defense performance requirements to differences in ICBM acceleration profiles. The Study Group also constructed a fast-burn solid-propellant ICBM having a maximum total boost time of $130 \mathrm{~s}$ and a maximum range of $12,000 \mathrm{~km}$, to explore the impact of fast-burn ICBMs on defense performance requirements. The ICBM models used are based on generic but plausible performance characteristics drawn from the open literature (see, e.g., $[167,168]$ ). Where details about foreign missiles were unavailable, the models were based on older U.S. technology.

The mass properties and performance characteristics of ICBM models L, S1, and S2 are listed in Tables 15.3-15.5. The meanings of and symbols for the relevant masses are as follows. The mass fraction of a given stage is $m_{p} /\left(m_{p}+m_{\text {inert }}\right)$, where $m_{\text {inert }}$ is the inert mass of the stage and $m_{p}$ is the mass of the propellant that will be consumed during that stage of boost. The inert mass of a stage includes its propulsion hardware, fuel tanks, thrust vector controls, associated power supplies and interstage structure, as well as the

sessments of potential ballistic missile threats to the territory of the United States. 
Table 15.1. Model ICBMs Considered in the Study ${ }^{a}$

\begin{tabular}{cccl}
\hline \hline Name & Propellant & ${\text { Boost Time }(\mathrm{s})^{b}}$ & Remarks \\
\hline L & Liquid & 240 & Intermediate between the SS-11 and DF-5 \\
L2 & Liquid & 300 & Similar to the U.S. Titan II ICBM \\
S1 & Solid & 170 & Based on 40-year-old, first-generation technology \\
S2 & Solid & 170 & Based on 40-year-old, first-generation technology \\
S3 & Solid & 130 & Illustrates the challenge of a fast-burn ICBM \\
\hline
\end{tabular}

${ }^{a}$ Liquid-propellant ICBMs have two stages; solid-propellant ICBMs have three stages. All models have nominal ranges of 12,000 km.

${ }^{b}$ Total duration of the boost phase.

${ }^{c}$ Large, heavy missile; considered but not analyzed in detail.

Table 15.2. Single-Stage Model MRBMs Considered in the Study

\begin{tabular}{rccrl}
\hline \hline Name & Propellant & ${\text { Boost Time }(\mathrm{s})^{a}}$ & Range $(\mathrm{km})$ & Remarks \\
\hline M1 & Liquid & 95 & 1300 & Similar to the No Dong \\
M2 & Solid & 65 & 600 & Similar to the Chinese M-9 \& Shahab-3
\end{tabular}

${ }^{a}$ Total duration of the boost phase.

mass of any propellant that is unburned when the stage shuts down or burns out. Once a given stage has shut down or burned out, its inert mass is dropped, leaving the initial mass of the subsequent stage. The initial mass $m_{\text {initial }}$ of a stage includes the mass that will be discarded, $m_{\text {inert }}$, its final mass $m_{\text {final }}$, the mass of the shroud $m_{\text {shroud }}$ if it is ejected while that stage is in powered flight, and the mass of the propellant that will be consumed during that stage of boost, i.e., $m_{\text {initial }}=m_{\text {inert }}+m_{\text {final }}+m_{\text {shroud }}+m_{p}$. The final mass of a given stage includes its inert mass and the initial mass of the subsequent stage. The final mass of the final stage includes its inert mass, guidance and navigation equipment,

\begin{tabular}{|ll|}
\hline & Box 15.1: Symbols Used in This Chapter \\
\hline$\gamma$ & Flight-path angle relative to local horizontal \\
$I_{\mathrm{sp}}$ & Specific impulse of the propellant \\
$I_{t}$ & Impulse of the propellant \\
$m_{\text {initial }}$ & Initial mass of the missile stage \\
$m_{\text {final }}$ & Mass of the missile stage at burnout \\
$m_{\text {inert }}$ & Inert mass of the missile stage \\
$\Delta V$ & Total velocity change \\
$m_{p}$ & Mass of the propellant in the missile stage \\
\hline
\end{tabular}


Table 15.3. Characteristics of Liquid-Propellant ICBM L ${ }^{a}$

\begin{tabular}{llrrr}
\hline \hline Attribute & Units $^{b}$ & Stage 2 & Stage 1 & Totals \\
\hline Diameter & meters & 3.00 & 3.00 & \\
Mass fraction & - & 0.90 & 0.90 & \\
$m_{\text {initial }}{ }^{2}$ & tonne & 41.5 & 201 & 201 \\
$m_{\text {final }}$ & tonne & 6.39 & 57.4 & \\
$m_{\text {inert }}$ & tonne & 3.86 & 15.9 & \\
$m_{p}$ & tonne & 35 & 143 & \\
$I_{\text {sp }}$ & seconds & 282 & 275 & \\
Thrust (average) & $\mathrm{kN}$ & 810 & 3226 & \\
Burn time & seconds & 120 & 120 & 240 \\
$I_{t}$ & $\mathrm{MN}-\mathrm{s}$ & 96 & 385 & \\
$\Delta V$ added (ideal) & $\mathrm{km} / \mathrm{s}$ & 5.16 & 3.38 & 8.54 \\
$\Delta V$ added (actual) & $\mathrm{km} / \mathrm{s}$ & 4.78 & 2.48 & 7.26 \\
\hline
\end{tabular}

${ }^{a}$ Nominal range: $12,000 \mathrm{~km}$; payload mass $m_{\text {payload }}=2,545 \mathrm{~kg}$; shroud mass $m_{\text {shroud }}=66 \mathrm{~kg}$; shroud eject occurs at $146 \mathrm{~s}, 26 \mathrm{~s}$ into second-stage burn.

${ }^{b}$ Units: $\mathrm{MN}-\mathrm{s}=$ Meganewton-seconds, $\mathrm{kN}=$ kilonewtons, 1 tonne $=1,000 \mathrm{~kg}$.

${ }^{c}$ Cumulative from left to right.

Table 15.4. Characteristics of Solid-Propellant ICBM S1 ${ }^{a}$

\begin{tabular}{|c|c|c|c|c|c|}
\hline Attribute & 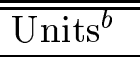 & Stage 3 & $\overline{\text { Stage } 2}$ & Stage 1 & Totals \\
\hline Diameter & meters & 1.50 & 1.85 & 1.85 & \\
\hline Mass fraction & & 0.90 & 0.90 & 0.90 & \\
\hline$m_{\text {initial }^{c}}$ & tonne & 1.93 & 8.81 & 41.1 & 41.1 \\
\hline$m_{\text {final }}$ & tonne & 1.02 & 2.61 & 12.0 & \\
\hline$m_{\text {inert }}$ & tonne & 0.10 & 0.68 & 3.2 & \\
\hline$m_{p}$ & tonne & 0.91 & 6.12 & 29.0 & \\
\hline$I_{\mathrm{sp}}$ & seconds & 277 & 275 & 265 & \\
\hline Thrust (average) & $\mathrm{kN}$ & 62 & 254 & 1160 & \\
\hline Burn time ${ }^{c}$ & seconds & 40 & 65 & 65 & 170 \\
\hline$I_{t}$ & MN-s & 2.4 & 16.5 & 75.4 & \\
\hline$\Delta V$ added (ideal) ${ }^{c}$ & $\mathrm{~km} / \mathrm{s}$ & 1.73 & 3.27 & 3.21 & 8.21 \\
\hline$\Delta V$ added (actual) ${ }^{c}$ & $\mathrm{~km} / \mathrm{s}$ & 1.60 & 2.96 & 2.64 & 7.20 \\
\hline
\end{tabular}

${ }^{a}$ Nominal range: $12,000 \mathrm{~km}$; payload mass $m_{p}=918 \mathrm{~kg}$; shroud mass $m_{\text {shroud }}=66 \mathrm{~kg}$; shroud eject occurs at $102 \mathrm{~s}, 28 \mathrm{~s}$ before the second stage burns out.

${ }^{b}$ Units: MN-s = Meganewton-seconds, $\mathrm{kN}=$ kilonewtons, 1 tonne $=1,000 \mathrm{~kg}$.

${ }^{c}$ Cumulative from left to right. 
Table 15.5. Characteristics of Solid-Propellant ICBM S2 ${ }^{a}$

\begin{tabular}{llrrrr}
\hline \hline Attribute & Units $^{b}$ & Stage 3 & Stage 2 & Stage 1 & Totals \\
\hline Diameter & meters & 1.50 & 1.50 & 1.50 & \\
Mass fraction & & 0.90 & 0.90 & 0.90 & \\
$m_{\text {initial }}{ }^{2}$ & tonne & 4.37 & 12.80 & 43.1 & 43.1 \\
$m_{\text {final }}$ & tonne & 1.37 & 5.21 & 15.8 & \\
$m_{\text {inert }}$ & tonne & 0.33 & 0.84 & 3.0 & \\
$m_{p}$ & tonne & 3.00 & 7.55 & 27.3 & \\
$I_{\text {sp }}$ & seconds & 277 & 275 & 265 & \\
Thrust (average) & $\mathrm{kN}$ & 163 & 340 & 1183 & \\
Burn time & seconds & 50 & 60 & 60 & 170 \\
Total impulse & $\mathrm{MN}-\mathrm{s}$ & 8.1 & 20.3 & 70.7 & \\
$\Delta V$ added (ideal) & $\mathrm{km} / \mathrm{s}$ & 3.15 & 2.42 & 2.61 & 8.17 \\
$\Delta V$ added (actual) & $\mathrm{km} / \mathrm{s}$ & 2.97 & 2.15 & 2.11 & 7.22 \\
\hline
\end{tabular}

${ }^{a}$ Nominal range: $12,000 \mathrm{~km}$; payload mass $m_{p}=1,040 \mathrm{~kg}$; shroud mass $m_{\text {shroud }}=51 \mathrm{~kg}$; shroud eject occurs at $103 \mathrm{~s}, 17 \mathrm{~s}$ before second stage burns out.

${ }^{b}$ Units: $\mathrm{MN}-\mathrm{s}=$ Meganewton-seconds, $\mathrm{kN}=$ kilonewtons, 1 tonne $=1,000 \mathrm{~kg}$.

${ }^{c}$ Cumulative from left to right.

and the mass $m_{\text {payload }}$ of the payload. The total impulse $I_{t}$ is the time integral of the thrust $T$ and is therefore equal to the product of the total burn time and the time-averaged thrust. The specific impulse $I_{\mathrm{sp}}=T / \dot{m}_{p} g_{s}$, where $\dot{m}_{p}$ is the propellant flow rate and $g_{s}$ is the acceleration of gravity at sea level, is a standard measure of the performance of a propulsion system. For further discussion of these quantities, see Appendix B and [168].

We turn now to a brief description of the principal ICBM models considered in the Study.

\section{Liquid-propellant ICBM model L}

The ICBM model L is based roughly on the large Chinese DF-5 missile, which is a twostage, liquid-propellant missile having a range that is reported to be between $12,000 \mathrm{~km}$ and $13,000 \mathrm{~km}$ [167] and a maximum total burn time estimated to be between $230 \mathrm{~s}$ and $255 \mathrm{~s}[166,169]$. A missile similar to the DF-5 was constructed because such a missile could be built using technology that is more than 20 years old - old enough to be accessible to countries that are currently developing their first long-range missiles. Model L is a twostage, 12,000-km-range missile with first- and second-stage burn times of $120 \mathrm{~s}$ each. The physical characteristics of this model are listed in Table 15.3.

It has been reported (see Chapter 3 ) that the Taepo Dong 2 missile currently under development by North Korea will have a range of about $6000 \mathrm{~km}$ and burn for about $200 \mathrm{~s}$. Increasing its range by adding a third stage or making the first two stages larger would increase its burn time to something close to that of the DF-5. 


\section{Solid-propellant ICBM model S1}

The ICBM model S1 has three stages and a maximum total boost time of $170 \mathrm{~s}$. The first two stages burn for $65 \mathrm{~s}$ each, whereas the third stage burns for $40 \mathrm{~s}$. Staging ratios were not optimized. The vehicle is assumed to have a payload shroud that is ejected when the dynamic pressure becomes less than $0.5 \mathrm{psf}$ (pounds per square foot), which occurs at $102 \mathrm{~s}$ after launch for its maximum-range trajectory. The physical characteristics of this model are listed in Table 15.4 .

\section{Solid-propellant ICBM model S2}

Different staging ratios can have a profound effect on the performance requirements for a boost-phase defense. The ICBM model S2 was created after it was noticed that the acceleration curve of the final stage of the DF-5-type ICBM model L becomes very steep as it approaches burnout, making its range highly sensitive to the time at which its thrust is terminated. In contrast, ICBM model S1 has a relatively low-acceleration third stage and its range is therefore much less sensitive to the time at which its thrust is terminated. To ensure that the results of the Study were not biased by a particular choice of booster-staging ratios, ICBM model S2 was designed so that its range would depend more sensitively on the time at which its thrust was terminated during third-stage burn.

Like ICBM model S1, model S2 is a three-stage missile having a maximum total boost time of $170 \mathrm{~s}$ and a maximum velocity of $7.2 \mathrm{~km} / \mathrm{s}$, but it has different staging ratios. Its first and second stages burn for $60 \mathrm{~s}$ each, whereas its third stage burns for $50 \mathrm{~s}$. Staging ratios were not optimized. The vehicle is assumed to have a payload shroud that is ejected when the dynamic pressure becomes less than $0.5 \mathrm{psf}$, which occurs at $103 \mathrm{~s}$ for its maximum-range trajectory. The physical characteristics of ICBM model S2 are listed in Table 15.5.

\section{Solid-propellant ICBM model S3}

In addition to the three ICBM models described above, a variant of solid-propellant ICBM S1 having reduced burn times for each stage was used as a variation to examine the effect of a short-burn missile, often suggested as a countermeasure to boost-phase intercept. The first- and second-stage burn times were reduced from $65 \mathrm{~s}$ to $50 \mathrm{~s}$ each, and the third-stage burn time was reduced from $40 \mathrm{~s}$ to $30 \mathrm{~s}$, for a maximum total boost time of $130 \mathrm{~s}$. Like the other ICBMs that we modeled, the range of this fast-burn missile is $12,000 \mathrm{~km}$. Its implications are discussed in Chapter 9.

\subsection{ICBM Trajectories}

\section{The significance of trajectory variations}

To be successful, a BPI system that uses interceptor missiles must perform two basic tasks. First, it must be able to get interceptors to attacking missiles soon enough to prevent them from giving their munitions velocities that would carry them to the United States. This task is analyzed in Chapters 4 and 5. Second, once they are close to the target missile, the system's interceptors must be able to maneuver to hit and disable or destroy it. This task is analyzed in Chapters 12 and 13. One of the most important factors determining the system performance required to achieve these tasks is the spectrum of possible boost-phase trajectories that attacking missiles may fly. The defense may not know in advance the 
type and performance characteristics of the missiles available to the attacker and almost certainly will not know in advance the trajectories attacking missiles are going to follow. Consequently, the defensive system must be capable of reaching and hitting in time any missiles available to the attacker, flying on any trajectories of which they are capable. ${ }^{2}$

The spectrum of possible attacking missile trajectories defines the potential intercept volume, which is the locus in time and space of the intercept points that the defense system's interceptors might have to reach to prevent the attacker's missiles from giving munitions a velocity that would carry them to the United States. In order to determine the potential intercept volume, the defense must have at least some information about the types and performance characteristics of all the missiles that might be available to the attacker.

Countries of concern for this study may or may not have tested the missiles used in an attack (see Chapter 3). Consequently, the defense may not know in advance the relevant characteristics of all the ICBMs that may be launched against the United States by a given country, or even whether they use liquid or solid propellants. Such uncertainties increase the potential intercept volume the defense must be able to cover.

In addition to the uncertainties introduced by the defense's lack of complete information on the characteristics of potentially threatening missiles, further uncertainties are introduced by the defense's lack of knowledge of the particular trajectory a given attacking missile has been programmed to fly. A missile attacking a target with a range significantly less than its maximum range could fly any of a broad spectrum of boost-phase trajectories. Even if the missile's maximum range is not much greater than the range to the target, the variety of possible trajectories is significant.

The trajectories flown may be designed to attack a particular target or to evade the defense. Examples include trajectories that are lofted or depressed relative to the maximumrange trajectory, trajectories that have single or multiple vertical or lateral deviations such as dog-legs, and trajectories that include energy-expending maneuvers to hit a particular target $^{3}$. All such possibilities must be considered in determining the intercept volume the defense must cover.

The spectrum of boost-phase trajectories that attacking missiles may fly is also important in determining the minimum kill-vehicle performance required to have a high probability of hitting them. Because the defense does not know which of the many possible boost-phase trajectories a given attacking missile has been programmed to fly, it must observe the position of the missile as a function of time, estimate its state (position, velocity, and acceleration), use a guidance law that predicts, either explicitly or implicitly,

\footnotetext{
${ }^{2}$ One of the ways in which this study differs from studies published previously in the open literature is that it considers a full spectrum of feasible trajectories and maneuvers and does not assume that the defense knows in advance the trajectory the attacking missile will follow.

${ }^{3}$ Every missile has a so-called "maximum-range" trajectory, which is the trajectory for which the impact point of the payload is farthest from the launch site. The shape of a missile's maximum range trajectory depends on the missile's shape, structure, acceleration profile, and payload mass (see Appendix B). For tactical reasons, an attacker may program a missile to fly a trajectory that is higher ("lofted") or lower ("depressed") than the missile's maximum range. As shown below, the range penalty for deviating from the maximum-range trajectory by a modest amount is small. "Dog-legs" are maneuvers in which the missile starts out in one direction and then veers off in another, making it difficult for the defense to anticipate the intended missile trajectory. The missile may maneuver in the vertical direction, remaining in the plane of its previous trajectory, in the horizontal direction, or in an arbitrary direction. Energy-expending maneuvers reduce the final velocity of the missile by causing the missile to make excursions around the trajectory to the target. Solid-propellant missiles in powered flight can and sometimes do rotate and fly nearly nose-backward at very high altitude where atmospheric friction is unimportant, to enable them to burn all their propellant and still hit a target at less than their maximum range.
} 
the future position of the missile, and guide the kill vehicle so that it hits the missile. The performance of the kill vehicle's sensors, guidance and control system, and propulsion system that is required to have a high probability of hitting the missile depends on the spectrum of trajectories and maneuvers the missile may execute. ${ }^{4}$ Vertical or lateral deviations may be programmed into the missile's flight to obfuscate the missile's flight path and intended target, to evade the kill vehicle, or simply to manage the missile's propulsive energy. Energy-management maneuvers could include in-plane and out-of-plane dog-legs or the generalized energy management maneuvers (GEMS) that are used on modern U.S. solid-propellant missiles to avoid having to terminate the thrust of the solid rocket motor. ${ }^{5}$ Evasive maneuvers could include dog-legs, lunges, or jinking maneuvers. (These are described in Chapter 12). They could be employed alone or in concert with decoys to exhaust the kill vehicle's propellant or increase the probability that it will miss its target. Most of these maneuvers, such as lofting or dog-legs, could be executed by any missile, whether liquid- or solid-propellant. Other maneuvers, such as GEMS, could be executed only by solid-propellant missiles.

To understand the implications of boost-phase trajectory variations for the performance of BPI systems, we investigated a variety of trajectories and maneuvers.

\section{Trajectories used in the Study}

The trajectories used in the Study were computed as described in Appendix B. Several computer programs were used to compute missile and interceptor trajectories. They were validated by comparing their results for selected cases and by checking selected runs against the results given by a six-degree-of-freedom computer code at Sandia National Laboratories that has been carefully validated over years of use in rocket design. Most of our analysis is based on calculations of planar missile and interceptor trajectories over a spherical, nonrotating Earth. These can be used to analyze out-of-plane engagements (engagements in which the missile and interceptor trajectory planes do not coincide), as shown in Chapter 4. In addition to lofted and depressed trajectories, we also simulated a variety of planar missile trajectories produced by changing the missile's flight path angle after the payload shroud was ejected. A few non-planar, dog-leg trajectories were also simulated. These calculations confirmed that the effect on a missile's range of moderate out-of-plane maneuvers, such as dog-legs, can be estimated by analyzing similar in-plane maneuvers. We also simulated a generalized energy-management (GEMS) maneuver. The results of these simulations are described in this chapter. In addition, we simulated separately a variety of smaller-scale in-plane maneuvers as part of our analysis of the homing phase of boost-phase engagements. These maneuvers included trajectory changes produced by a sudden increase or decrease in the missile's angle of attack, switchback maneuvers in which the target switched from a positive to a negative angle of attack, sudden lunges, and fishtail-like jinking maneuvers intended to evade the kill vehicle. The results of these simulations are described in Chapter 12 and Appendix C.

\footnotetext{
${ }^{4}$ We note that even if the defense knew exactly the characteristics of the attacking missiles and the trajectories they have been programmed to fly, the intercept points would still be uncertain because the actual trajectories flown by the missiles would be affected by unpredictable variations in propellant formulation, winds aloft, and other factors.

${ }^{5}$ Terminating the thrust of a solid-propellant rocket before all the propellant is expended is challenging; it involves blowing a hole in the missile case, which can be difficult to control. By comparison, liquid-propellant rocket motors can easily be throttled back by reducing the flow of propellant to the combustion chamber.
} 


\subsubsection{Maximum-range and lofted trajectories}

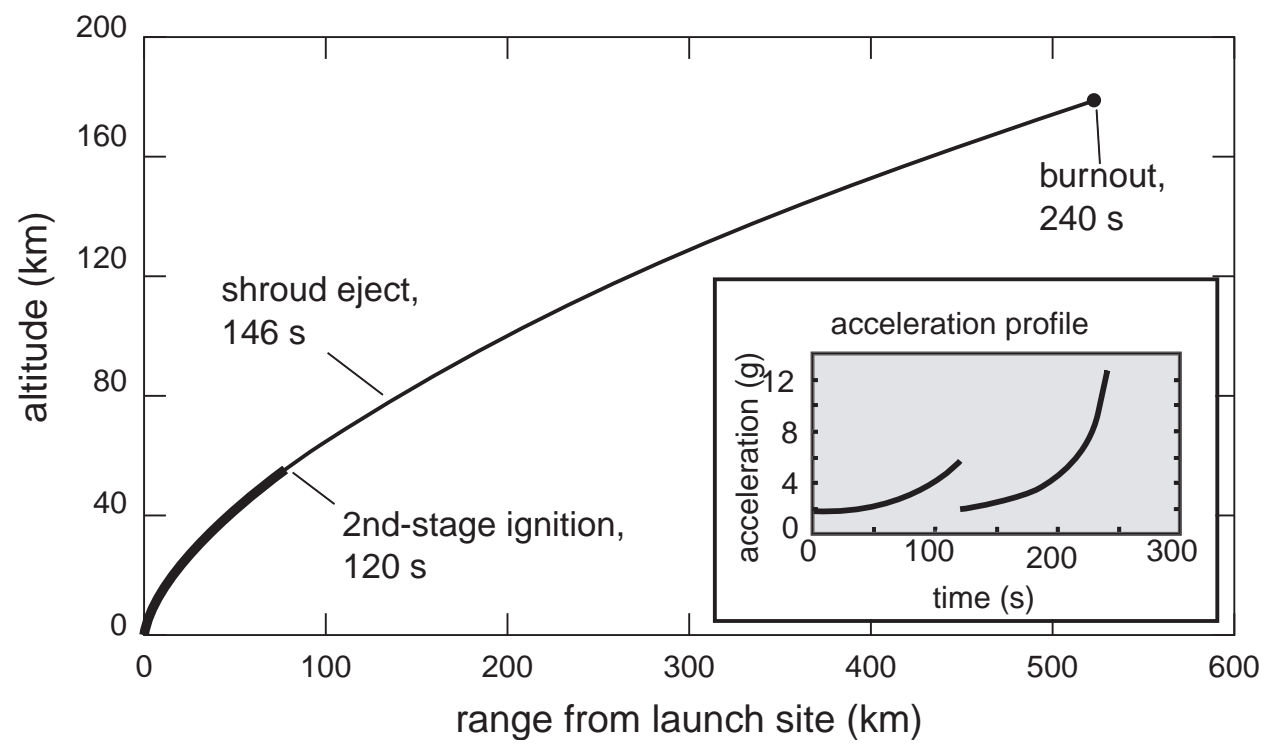

Figure 15.1. Maximum-range boost-phase trajectory of liquid-propellant ICBM model L in the altitude-range plane. The first- and second-stage boost-phase trajectories are shown respectively by the heavy and light lines. Inset: Acceleration profiles of the two stages. The acceleration of the second stage reaches $12 \mathrm{~g}$ before it burns out.

The nominal maximum-range trajectory of liquid-propellant ICBM model L and the acceleration time history of its two stages are shown in Fig. 15.1. The trajectories during first- and second-stage boost are indicated by the heavy and light lines, respectively. The second stage ignites $120 \mathrm{~s}$ after launch, the payload shroud is ejected at $146 \mathrm{~s}$, and the second stage burns out at $240 \mathrm{~s}$. Because of the low flight-path angle of the missile's maximumrange trajectory and the high acceleration of its second stage during the last half of its burn, differences of a few seconds in the time at which the second stage burns out or has its thrust terminated and small changes in its flight-path angle have a very large effect on the missile's range. For example, terminating the thrust of the second stage $15 \mathrm{~s}$ before normal burnout would reduce the missile's range by more than 50 percent. Lofting the missile's trajectory would reduce this time sensitivity slightly.

The nominal maximum-range trajectory of solid-propellant ICBM model S1 and the acceleration time history of its three stages are shown in Fig. 15.2. Also shown is the lofted trajectory produced by increasing the initial flight-path angle (measured relative to the local horizontal) from $19^{\circ}$ to $27^{\circ}$. The trajectories during first-, second- and third-stage boost are indicated by the heavy, light, and dotted lines, respectively. The second stage ignites $65 \mathrm{~s}$ after launch, and the shroud is ejected at $102 \mathrm{~s}$. The third stage ignites at $130 \mathrm{~s}$ and burns out at $170 \mathrm{~s}$.

The nominal maximum-range trajectory of solid-propellant ICBM model S2 and the acceleration time history of its three stages are shown in Fig. 15.3. As in Fig. 15.2, the lofted trajectory was produced by increasing the initial flight path angle from $19^{\circ}$ to $27^{\circ}$. The trajectories during first-, second-, and third-stage boost are indicated by the heavy, light, and dotted lines, respectively. For this model, the second stage ignites at $60 \mathrm{~s}$ and the shroud is ejected at $103 \mathrm{~s}$. The third stage ignites at $120 \mathrm{~s}$ and burns out at $170 \mathrm{~s}$.

Figure 15.4 shows missile altitude as a function of range for the maximum-range boost- 


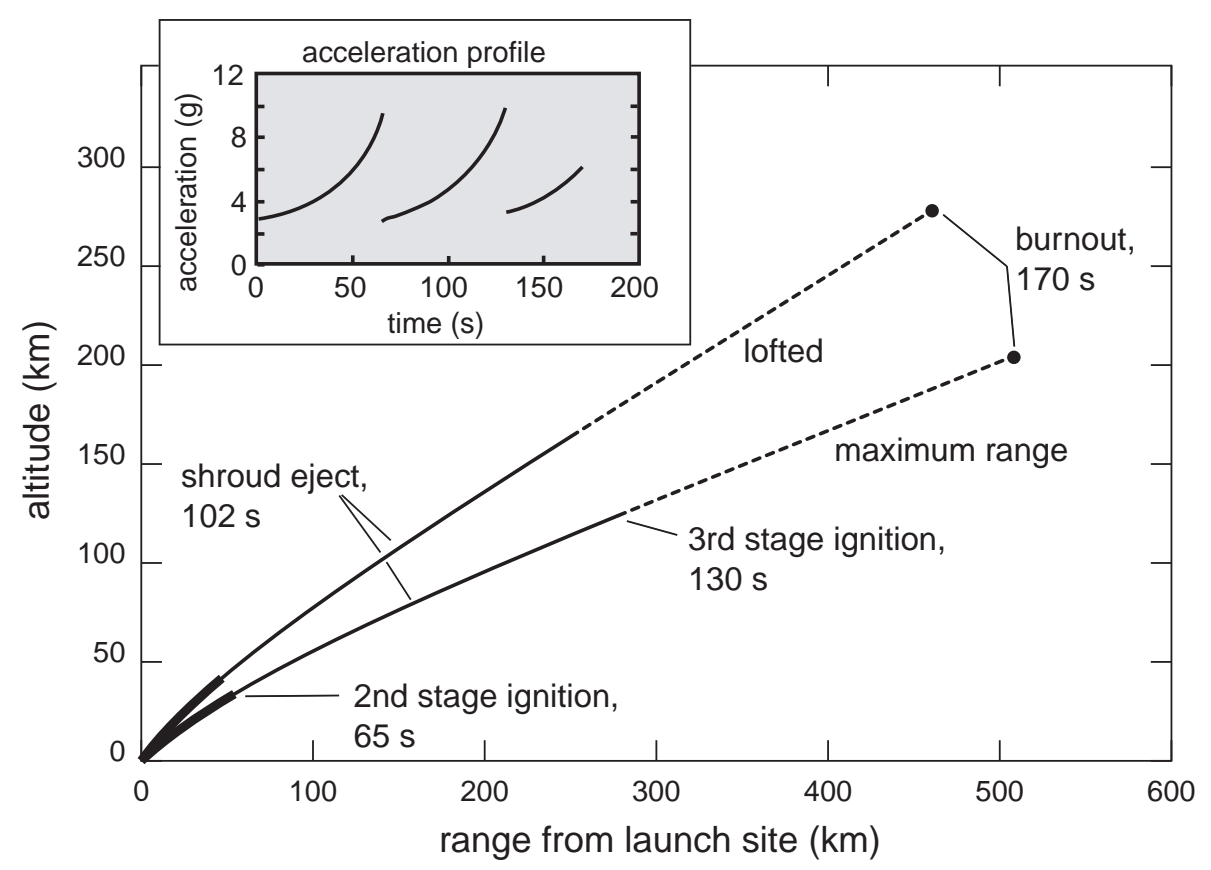

Figure 15.2. Maximum-range and lofted boost-phase trajectories of solid-propellant ICBM model S1 in the altitude-range plane. The trajectories during first-, second- and third-stage boost are indicated by the heavy, light, and dotted lines, respectively. The flight-path angle measured from the local horizontal is $19^{\circ}$ for the maximum-range trajectory and $27^{\circ}$ for the lofted trajectory. Inset: Acceleration profiles of the three stages.

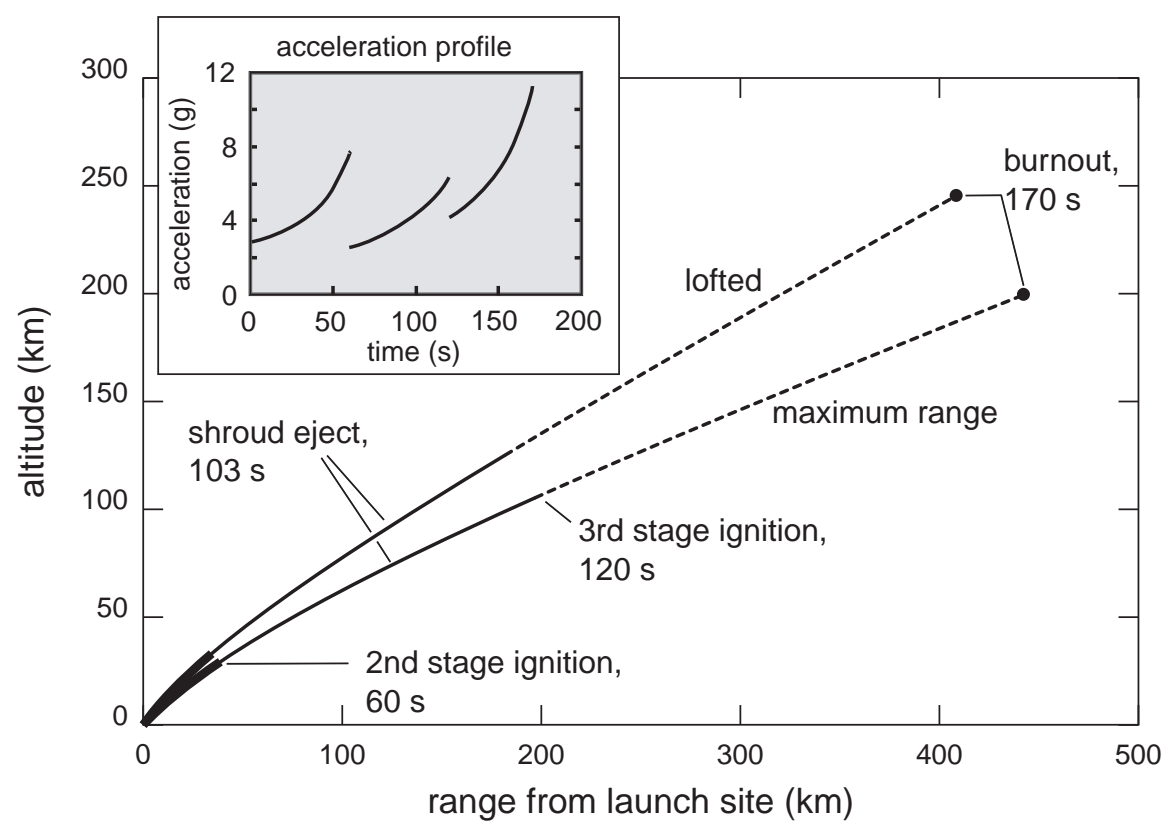

Figure 15.3. Maximum-range and lofted boost-phase trajectories of solid-propellant ICBM model S2 plotted in the altitude-range plane. As in Fig. 15.2, the trajectories during first-, second-, and thirdstage boost are indicated by the heavy, light, and dotted lines, respectively. The flight-path angle measured from the local horizontal is $19^{\circ}$ for the maximum-range trajectory and $27^{\circ}$ for the lofted trajectory. Inset: Acceleration profiles of the three stages. 


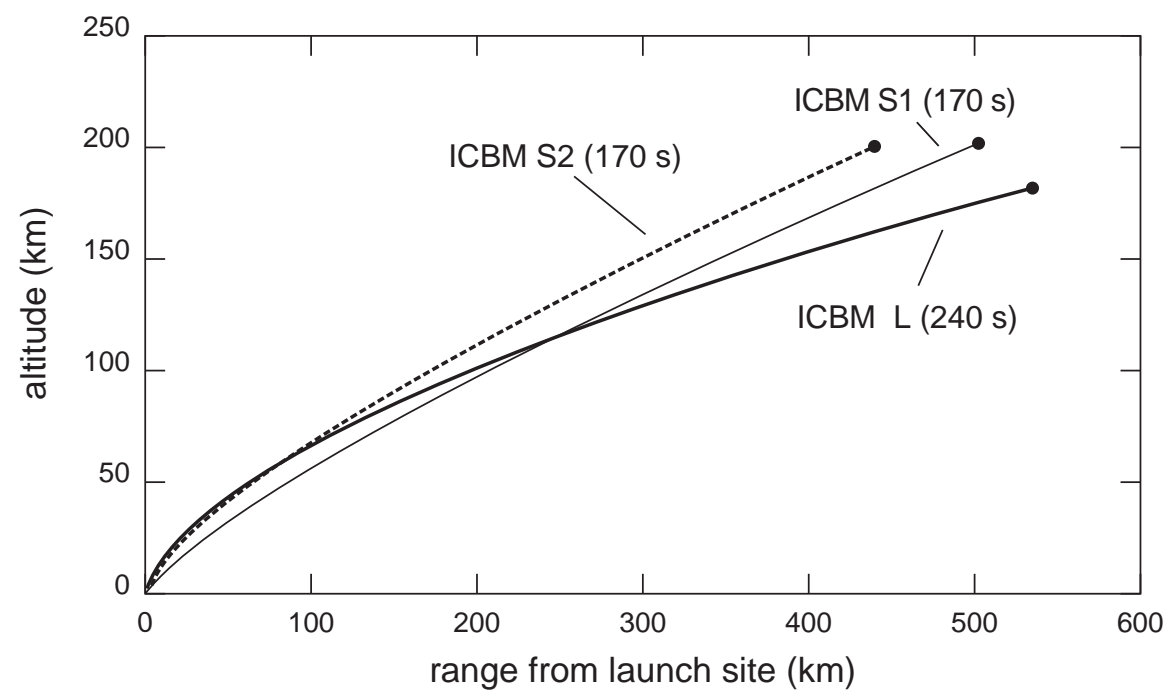

Figure 15.4. Comparison of the missile's altitude as a function of its range for the maximum-range boost-phase trajectories of the three primary ICBM models. (The numbers in parentheses indicate burn times.)

phase trajectories of the three primary ICBM models. Because of its lower acceleration and longer burn time, a liquid-propellant ICBM similar to model L experiences more gravity drop than a solid-propellant missile with the same range. As a result, its altitude during second-stage flight is lower and its entire trajectory is flatter (the flight-path angle of ICBM model $\mathrm{L}$ is $11.5^{\circ}$ at burnout, whereas the flight path angles of solid-propellant models S1 and $\mathrm{S} 2$ are $19^{\circ}$ at burnout).

Figure 15.5 shows elapsed time as a function of range for the maximum-range boostphase trajectories of the three primary ICBM models. The solid-propellant ICBMs burn out closer to their launch site and much more quickly than the liquid-propellant ICBM. This makes them more difficult to intercept during their boost phase and drives a BPI system to use faster interceptors and base them closer to potential ICBM launch sites.

\subsubsection{Other trajectories}

An almost infinite variety of maneuvers is possible for an ICBM attacking a target that is at a range less than the missile's maximum range. In choosing which maneuvers to consider, we used practical considerations to circumscribe the possibilities. First, with the exception of GEMS maneuvers and dog-leg maneuvers of ICBMs launched from North Korea against targets in Alaska, no maneuvers were considered that would reduce the ICBM's range by more than $1500 \mathrm{~km}$ from its maximum. Second, no planned maneuvers were considered that would increase the structural loading or heating of the missile. Accordingly, all major maneuvers were initiated only after the shroud was ejected, which was assumed to be done once the dynamic pressure fell below 0.5 psf. Finally, limits were placed on transient or oscillatory accelerations by assuming an outer control loop corner frequency of $2 \mathrm{~Hz}$ or less and a thrust-vector-control corner frequency of about $20 \mathrm{~Hz}$.

Trajectory-shaping maneuvers An ICBM attacking a target that is closer than its maximum range can be programmed to follow a wide variety of trajectories, especially after it has climbed above the atmosphere. The range penalty for small deviations from the missile's 


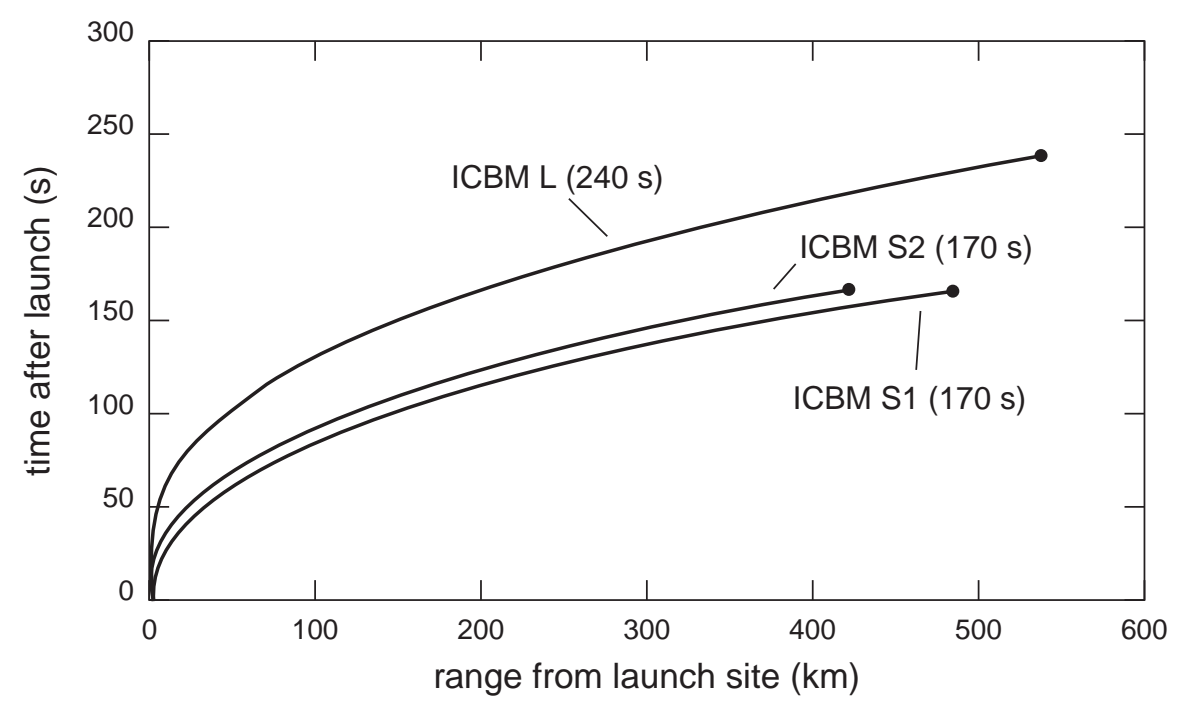

Figure 15.5. Comparison of time after launch as a function of range for the maximum-range boostphase trajectories of the three primary ICBM models. (The numbers in parentheses indicate burn times.)

maximum-range trajectory is second-order in the deviation and is therefore relatively small. Figure 15.6 illustrates the variety of planar trajectories that are possible for solid-propellant ICBM model S1 by lofting it slightly at launch or by changing its flight-path angle after shroud eject. Trajectory variations like these are also possible for liquid-propellant ICBM model L and solid-propellant ICBM model S2. The cases shown are as follows:

1. The nominal maximum-range trajectory for ICBM model S1. The missile was launched with an initial flight-path angle of $81.5^{\circ}$ and conducted a gravity turn ${ }^{6}$ from launch through shroud eject, which occurred during second-stage flight, $102 \mathrm{~s}$ after the missile was launched.

2. The trajectory produced by lofting the missile so that its flight-path angle at burnout is $27.5^{\circ}$. The range of the missile (when all of its propulsive energy is used) is shortened by $1,000 \mathrm{~km}$, relative to its nominal maximum range.

3. The trajectory produced by launching the missile as in Case 1 but increasing its angle of attack by $20^{\circ}$ from shroud eject through second-stage burnout.

4. The trajectory produced by launching the missile as in Case 1 but increasing its angle of attack by $10^{\circ}$ from shroud eject through third-stage burnout.

5. The trajectory produced by launching the missile as in Case 1 but decreasing its angle of attack by $15^{\circ}$ from shroud eject through second-stage burnout.

None of these trajectory variations reduce the missile's range by more than $1,500 \mathrm{~km}$ (12 percent). Out-of-plane deviations by similar distances reduce the range by a similar amount, as confirmed by computing several out-of-plane trajectories. Thus, the missile can

\footnotetext{
${ }^{6}$ If the thrust vector of a rocket having a flight-path angle less than $90^{\circ}$ is kept aligned with its velocity vector, gravity will cause its flight path angle to decrease gradually, i.e., the missile will gradually turn toward horizontal flight; this effect is called a "gravity turn."
} 


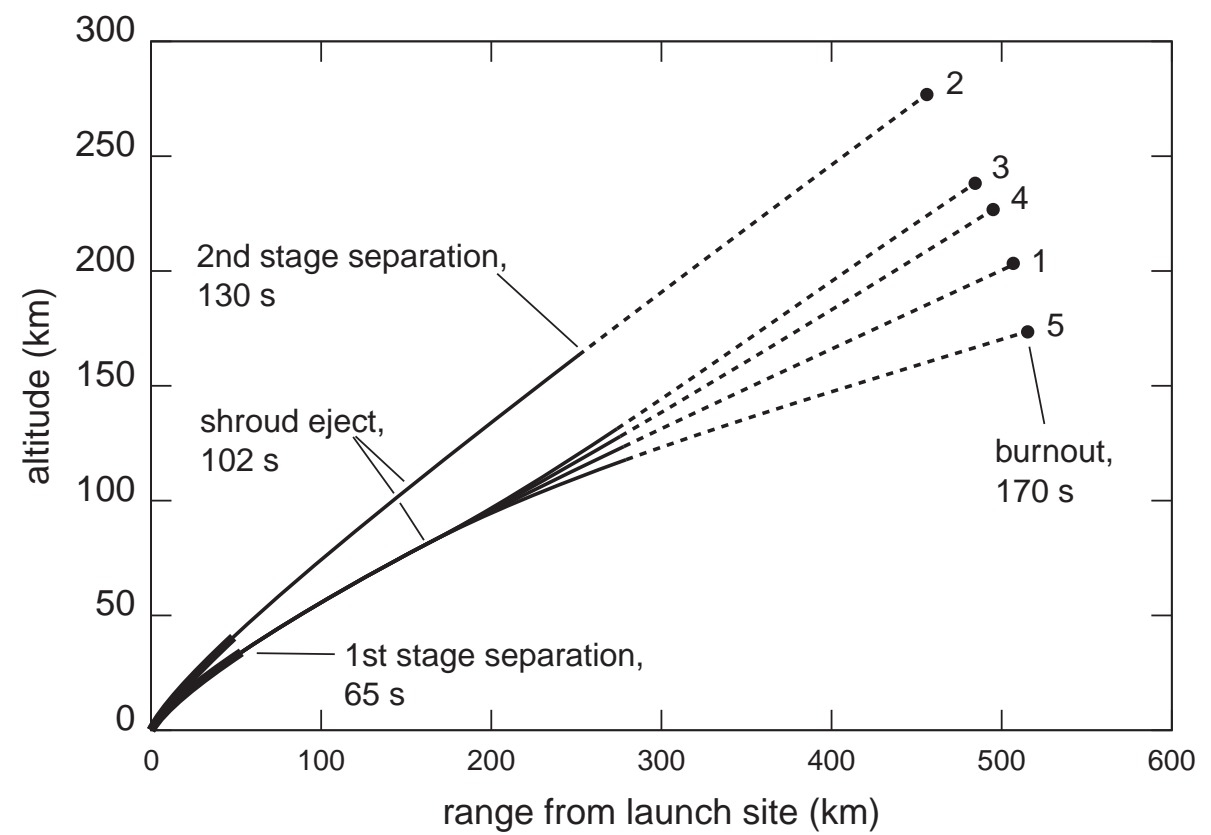

Figure 15.6. Examples of the variety of planar trajectories that are possible for solid-propellant ICBM model S1 by lofting it slightly at launch or by changing its flight path angle after shroud eject. (The numbers are keys for the text.) The large-scale trajectory variations shown reduce the range of the missile by at most $12 \%$ of its maximum range. Out-of-plane deviations by similar distances reduce the range by a similar amount. Trajectory variations like these are also possible for liquid-propellant ICBM model L and solid-propellant ICBM model S2, with similar range penalties. The volume in space that these trajectories span illustrates the volume that interceptors must be able to cover to ensure that a boost-phase intercept is possible.

easily vary its azimuth by $\pm 10^{\circ}$ before cut-off with little loss of range. Trajectory-shaping maneuvers like these are routinely performed by some U.S. missiles.

Figure 15.6 shows the large-scale trajectory variations that are possible for a given range penalty span an approximately conical volume in space centered on the maximum-range trajectory. The diameter of the cone at the missile's burnout point can be $120-150 \mathrm{~km}$ if a 10 percent reduction in the missile's range is acceptable, or as large as $250 \mathrm{~km}$ if a 50 percent reduction in the missile's range is acceptable. The latter would still allow any of the model missiles used in the Study to reach targets anywhere in Alaska from launch sites in North Korea. The kill vehicles of a BPI system must be able to reach points anywhere in this volume within the time available to ensure that an intercept is possible (to actually achieve intercept, the kill vehicle must then hit the missile).

Energy-management maneuvers Generalized energy-management steering (GEMS) maneuvers may be performed late in a solid-propellant ICBM's boost phase in lieu of thrust termination to reduce the missile's effective range, allowing it to hit a target closer than its maximum range. Figure 15.7 shows an example of a GEMS maneuver performed to reduce the impact range of ICBM model S2, which has a third stage similar to that of the Minuteman I. In such a maneuver, the centerline of the missile may deviate from its flight path by up to $120^{\circ}$. The need to cope with maneuvers like this, which are quite typical for solid-propellant missiles that have no thrust termination, increases significantly the performance requirements for the guidance and control systems, and also the propulsion systems, 


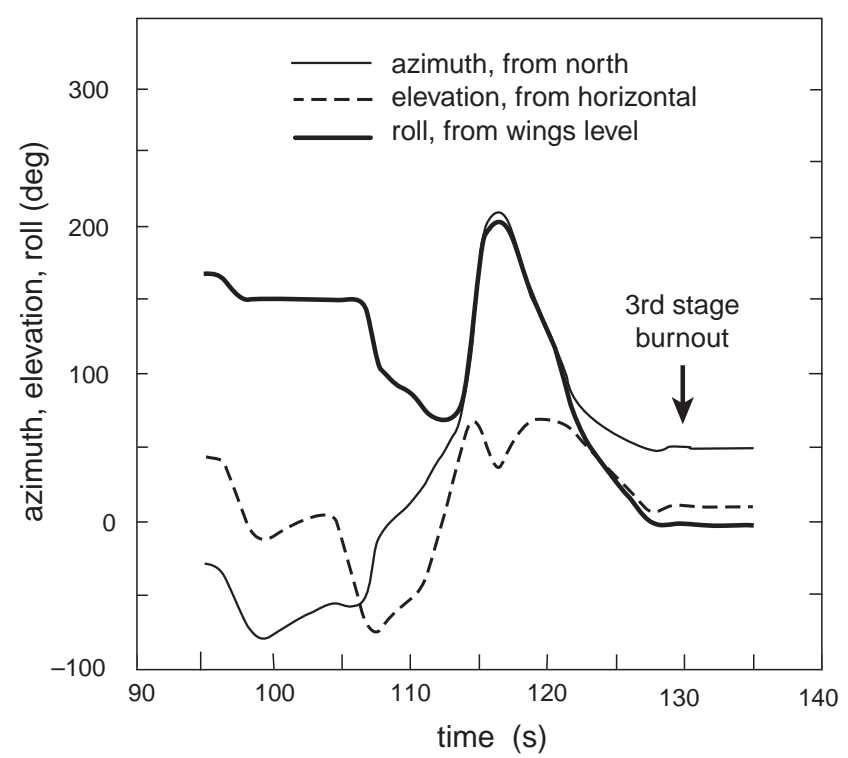

Figure 15.7. Illustrative GEMS maneuver for solid-propellant ICBM S2 third stage.
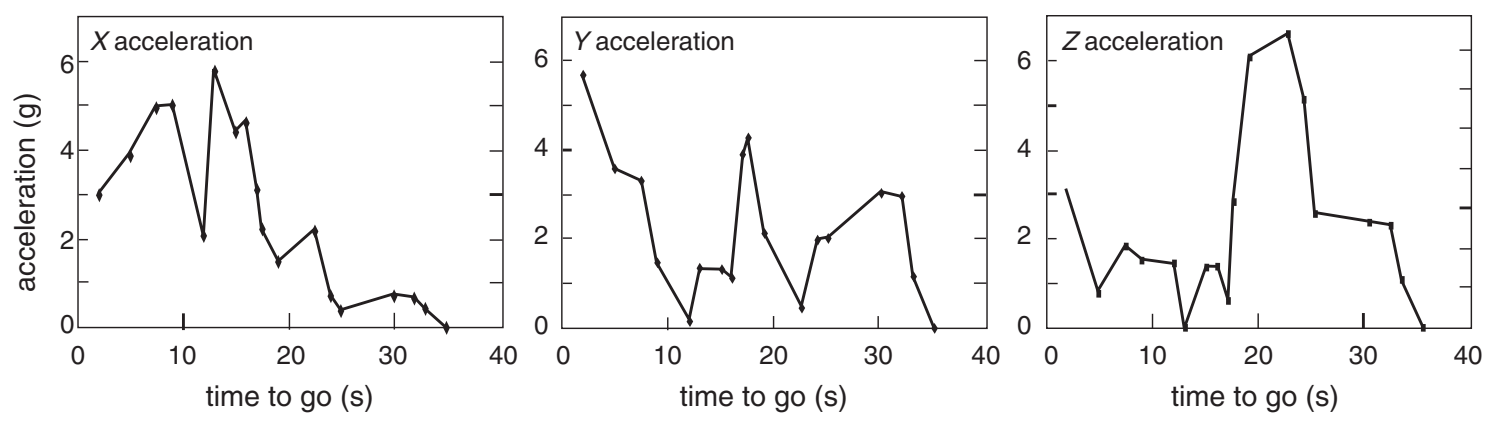

Figure 15.8. Components of the acceleration of the third stage of ICBM model S2 along the $x, y$, and $z$ axes during an illustrative GEMS maneuver.

of the kill vehicle.

During a GEMS maneuver, the time-varying thrust vector can be assumed to be approximately coaxial with the centerline of the vehicle. The vehicle's attitude is defined by its Euler angles, which are shown as functions of time in Fig. 15.7 for an illustrative GEMS maneuver. We express the thrust vector, which varies in both magnitude and direction, by specifying its Cartesian components as functions of time in the reference frame of the missile at launch. We choose the $z$ axis in the vertical direction, the $x$ axis along the projection of the firing direction into the horizontal plane, and the $y$ axis perpendicular to the $x-z$ (firing) plane. For the example shown, the firing direction is about $25^{\circ}$ east of north. Using the thrust acceleration of the third-stage of ICBM model S2 to fix the magnitude of the missile's acceleration and the Euler angles shown in Fig. 15.7 to determine its components along the $x, y$, and $z$ axes, we obtain the results shown in Fig. 15.8. This GEMS maneuver reduced the velocity of the third stage of ICBM S2 along its flight path by $970 \mathrm{~m} / \mathrm{s}$, decreasing its impact range from $12,000 \mathrm{~km}$ to $6,280 \mathrm{~km}$. The latter is the range from North Korea to just south of the southern boundary of Alaska, so a GEMS maneuver similar to this one would be appropriate for an ICBM launched from North Korea against targets in Alaska. 
Since the defense does not know the ICBM's intended target or how its maneuver will unfold, to be sure of hitting the ICBM, the kill vehicle must match or exceed the ICBM's unpredicted acceleration normal to the line-of-sight from the interceptor to the ICBM at every moment. ${ }^{7}$ Hence the interceptor must expend propellant, regardless of the direction of the ICBM's acceleration. To be sure of hitting the ICBM, the kill vehicle must change its velocity by an amount equal to the integral of the absolute magnitude of the ICBM's unanticipated acceleration normal to the line-of-sight.

For the GEMS maneuver shown in Figs. 15.7 and 15.8, an interceptor approaching the ICBM from a direction approximately perpendicular to the ICBM's trajectory plane would have to be able to change its velocity by about $1,200 \mathrm{~m} / \mathrm{s}$ in order to be certain of hitting the missile, assuming that the state of the target ICBM can be determined instantly and exactly and that the kill vehicle can change its acceleration instantly to match that of the ICBM. In reality, errors and lags in estimating the state of the target and the latency in the kill vehicle's dynamical response must also be considered in determining the required performance of the kill vehicle.

Implications for boost-phase intercept systems The results presented in this section illustrate the large unpredictability in performance of an attacking ICBM that increases the potential intercept volume that the defensive system must be prepared to cover. Figures 15.4 and 15.5 show that the burnout altitude and range of liquid-propellant ICBM L differ from those of solid-propellant ICBM S2 by more than $50 \mathrm{~km}$ and more than $100 \mathrm{~km}$, respectively. Even the burnout ranges of the two solid-propellant ICBM models differ by more than $50 \mathrm{~km}$. The boost phases of the solid-propellant missiles are $70 \mathrm{~s}$ shorter than the boost phase of the liquid-propellant missile.

The trajectory variations and maneuvers that were simulated show that even if the performance characteristics of the attacking ICBM are known precisely, the defense's lack of knowledge of the trajectory the missile has been programmed to fly increases the required performance of the interceptor and kill vehicle because they occur after the interceptor has been fired and has completed its boost. Figure 15.6 shows that the large scale trajectory variations possible for a 10-percent reduction in the missile's range fill an approximately conical volume having a diameter at the burnout point of $120-150 \mathrm{~km}$; for a 50-percent reduction in the missile's range, the diameter can be as large as $250 \mathrm{~km}$. The latter reduction in range would still allow any of the model missiles used in the Study to attack targets anywhere in Alaska from launch sites in North Korea.

The trajectory variations shown in Fig. 15.6 underscore another important point about the design of a BPI system: there is a downside to using interceptors having very short boost times or committing them early. As long as the interceptor is in its boost phase, it can compensate for deviations of the ICBM's trajectory from the trajectory expected when the interceptor was fired with little additional expenditure of propellant, provided the interceptor receives nearly continuous updates about the target missile's position from offboard sensors, such as space-based IR detectors or radars. However, once the interceptor's final boost stage burns out, which occurs $85-105$ s after the launch of the target ICBM for an interceptor with a 40-s boost phase fired 45-65 s after the ICBM was launched, the kill vehicle must use its own propulsion to compensate for any unpredicted maneuvers by the ICBM.

\footnotetext{
${ }^{7}$ As discussed in Chapter 12, acceleration-matching minimizes the total velocity change the kill vehicle must be able to achieve if the target's maneuvers are unpredictable and arbitrary.
} 
For example, if the ICBM initiates a $15^{\circ}$ out-of-plane dog-leg beginning immediately after it ejects its shroud and continuing until its third stage has burned out, the kill vehicle would have to change its velocity by about $840 \mathrm{~m} / \mathrm{s}$ to compensate, assuming that the kill vehicle has perfect knowledge of the target's acceleration without any time lag. In reality, the total velocity change required will be larger, because of random and systematic errors and delays in estimating the state (position, velocity, and acceleration) of the target and latency in the dynamical response of the kill vehicle to commanded accelerations.

Our simulation of a GEMS maneuver showed that the kill vehicle would have to be able to change its velocity by about $1,200 \mathrm{~m} / \mathrm{s}$ to be certain of hitting the missile, assuming that the state of the target ICBM can be determined instantly and exactly and that the kill vehicle can change its acceleration instantly to match that of the ICBM. As with the dog-leg maneuver, the total velocity change required will be larger, because of the errors and delays in estimating the target's state and latency in the dynamical response of the kill vehicle to commanded accelerations.

We emphasize that the ICBM trajectory variations and maneuvers discussed here are merely examples of a broad spectrum of possible maneuvers. An ICBM could execute any one of these maneuvers or a combination of them, but could not execute more than one of the extreme examples without reducing its impact range to significantly less than $6,000 \mathrm{~km}$.

The kill-vehicle performance required to intercept an ICBM executing maneuvers like these is analyzed quantitatively in Chapter 12. The resulting performance requirements are then used in Chapter 14 to determine the required sizes and masses of kill vehicles for boost-phase intercept.

\subsection{Models of Medium-Range Ballistic Missiles}

To explore the issues associated with BPI of shorter-range missiles launched off the coast of the United States, the Study Group constructed two models of medium-range theater ballistic missiles:

1. A 1300-km, single-stage, liquid-propellant missile having a 95-second burn time, having characteristics similar to those that have been reported [170] for Shahab-3, No Dong, and Ghauri missiles. We denote this model M1.

2. A $600-\mathrm{km}$, single-stage, solid-propellant missile having a 65 -second burn time, having characteristics roughly similar to the solid-propellant Chinese M-9. We denote this model M2.

The maximum-range trajectories of these models are shown in Fig. 15.9 and their physical characteristics are summarized in Tables 15.6 and 15.7. 


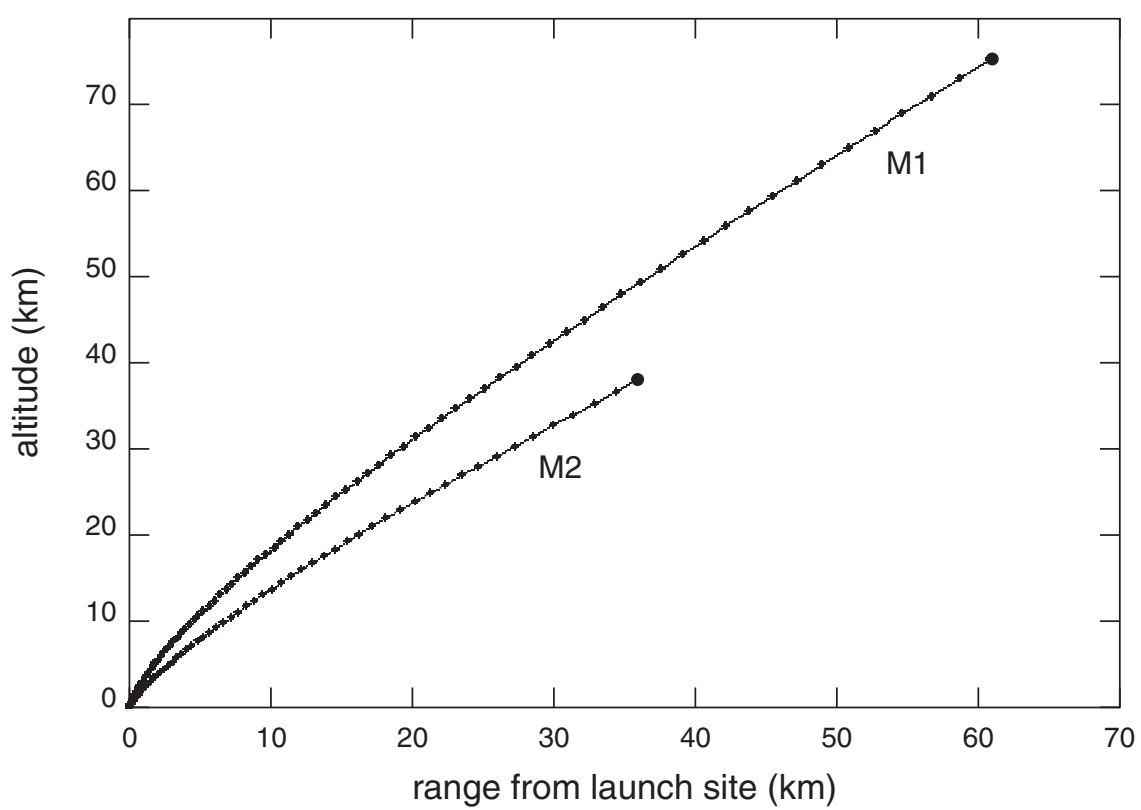

Figure 15.9. Maximum-range trajectories of the two medium-range missile models listed in Tables 15.6 and 15.7. M1 has a range of $1,300 \mathrm{~km}$ and burn time of $95 \mathrm{~s}$; M2 has a range of $600 \mathrm{~km}$ and a burn time of $65 \mathrm{~s}$.

Table 15.6. Characteristics of 1300-km M1 ${ }^{a}$

\begin{tabular}{llr}
\hline \hline Attribute & Units & Stage 1 \\
\hline Mass fraction & & 0.85 \\
$I_{s p}$ & $\mathrm{~s}$ & 265 \\
$I_{t}$ & MN-s & 35.5 \\
$W_{p}$ & tonne & 13.6 \\
$m_{\text {inert }}$ & tonne & 2.4 \\
$m_{\text {initial }}$ & tonne & 17.1 \\
$m_{\text {final }}$ & tonne & 3.4 \\
$\Delta V$ added (ideal) & $\mathrm{km} / \mathrm{s}$ & 4.17 \\
$\Delta V$ added (actual) & $\mathrm{km} / \mathrm{s}$ & 3.22 \\
Burn time & $\mathrm{s}$ & 95 \\
Thrust (average) & $\mathrm{kN}$ & 374 \\
Diameter & $\mathrm{m}$ & 1.85 \\
\hline
\end{tabular}

${ }^{a}$ Payload: $975 \mathrm{~kg}$; shroud mass: $66 \mathrm{~kg}$; ejected after burnout. 
Table 15.7. Characteristics of $600-\mathrm{km} \mathrm{M}^{a}$

\begin{tabular}{llr}
\hline \hline Attribute & Units & Stage 1 \\
\hline Mass fraction & & 0.85 \\
$I_{s p}$ & $\mathrm{~s}$ & 265 \\
$I_{t}$ & $\mathrm{MN}-\mathrm{s}$ & 17.7 \\
$W_{p}$ & tonne & 6.8 \\
$m_{\text {inert }}$ & tonne & 1.2 \\
$m_{\text {initial }}$ & tonne & 9.1 \\
$m_{\text {final }}$ & tonne & 2.26 \\
$\Delta V$ added (ideal) & $\mathrm{km} / \mathrm{s}$ & 2.84 \\
$\Delta V$ added (actual) & $\mathrm{km} / \mathrm{s}$ & 2.20 \\
Burn time & $\mathrm{s}$ & 65 \\
Thrust (average) & $\mathrm{kN}$ & 273 \\
Diameter & $\mathrm{m}$ & 1.0 \\
\hline
\end{tabular}

${ }^{a}$ Payload: 2,176 kg; shroud mass: $66 \mathrm{~kg}$; ejected after burnout.

\section{References for Chapter 15}

[164] Richard L. Garwin, "Boost-phase intercept: A better alternative," Arms Control Today 30(7), 8 (2000).

[165] Theodore A. Postol, "A preliminary analysis of a Russian-U.S. boost-phase defense to defend Russia and the U.S. from postulated rogue-state ICBMs: A potential option for Russian-U.S. cooperation in missile defense." Technical report, Carnegie Endowment for International Peace, October 1999.

[166] Steven J. Isakowitz, Joseph P. Hopkins, and Joshua B. Hopkins, International Reference Guide to Space Launch Systems. American Institute of Aeronautics and Astronautics, 3rd edition, December 1999.

[167] Jane's Strategic Weapons Systems (JSWS) 36, 12 July 2001. Article: CSS-4 (DF-5).

[168] George P. Sutton and Oscar Biblarz, Rocket Propulsion Elements. (John Wiley \& Sons, Inc.), 7th edition, 2001.

[169] Dean Wilkening, "Boost-Phase Missile Defense." Briefing Presented to the APS Study Group, July 2001.

[170] David Wright, "Ballistic missile systems." Briefing Presented to the APS Study Group, July 2001. 


\title{
Chapter 16
}

\section{Terrestrial-Based Interceptor Models}

\author{
Contents
}

16.1 Analytical Approach . . . . . . . . . . . . S277

16.2 Models of Unconstrained Interceptors _ . . . . . . . S S279

16.3 Models of Constrained Interceptors . . . . . . . . . $\quad$ S282

16.4 Summary of Interceptor Models . . . . . . . . . . . S284

16.5 Interceptor Basing Options . . . . . . . . . . . S285

16.5.1 Land basing . . . . . . . . . . . . . . S286

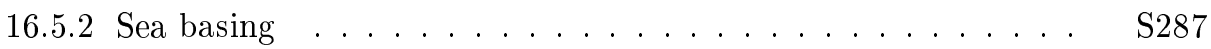

16.5.3 Air basing . . . . . . . . . . . . . . . . . . S288

16.5.4 Deployment for defense against SRBMs and MRBMs .... S S288

No interceptors suitable for use in a boost-phase defense exist today. Consequently, to analyze boost-phase engagements, the Study Group had to create computer models of interceptors. Two sets of interceptors were created: interceptors in the first set were unconstrained in size, whereas those in the second set were constrained to fit an existing launch system. The interceptors were chosen to span the range of performance relevant to the BPI problem and to explore a variety of issues, including the ability of interceptors having different flyout velocities and accelerations to engage the model target missiles in the suite developed in Chapter 15.

The model interceptors developed here are employed throughout Part A of this report and in the engagement simulations in Appendix C. The interceptor's trajectories were computed as described in Appendix B.

\subsection{Analytical Approach}

A set of notional dynamic models for interceptors were developed to define plausible flyout characteristics for a variety of scenarios of interest. As a first step, a rough estimate of the interceptor performance required to reach the target during its boost phase may be obtained using a kinematic equation for $R_{i}$, the distance the interceptor must fly to reach a desired nominal intercept point on the boost-phase trajectory of the target missile, namely

$$
R_{i}=\frac{1}{2} a t_{b}^{2}+V_{b o}\left(t_{i}-t_{b}\right)
$$

where $a$ is the nominal mean acceleration of the interceptor, $t_{b}$ is the total duration of the interceptor's boost phase, $V_{b o}$ is the interceptor's burnout velocity, and $t_{i}$ is the desired 
intercept time measured from time at which the interceptor was launched. The time available to intercept the target missile is the burn time the target missile needs to achieve the velocity required to deliver its payload to the nearest defended area, less the interval from time the missile was launched to the time the interceptor was fired.

Equation (16.1) presumes the interceptor's acceleration is constant during its boost phase, whereas the actual acceleration of an interceptor varies continuously during its flyout because of variations in its thrust, the decreasing mass of the propellant, staging events, the changing dynamic pressure, and trajectory shaping, as well as the effect of gravity. Therefore, to compute an interceptor's trajectory accurately, the time history of the interceptor's flight to the intercept point must be carefully modeled. To account for these complex factors, notional interceptors were initially defined using rough estimates of the required kill-vehicle mass and then flying them out on a variety of trajectories through a standard atmosphere and the central gravitational field of a spherical model Earth. These initial studies were used to establish the "flyout fan" or field of fire needed to engage the target ICBMs during their boost-phases. Like the target missile trajectories used in the Study, the interceptor trajectories were validated by comparing sample trajectories with trajectories computed by Sandia National Laboratories using codes that have been carefully validated during years of use. The results of the Sandia simulations matched the Study Group's results very closely.

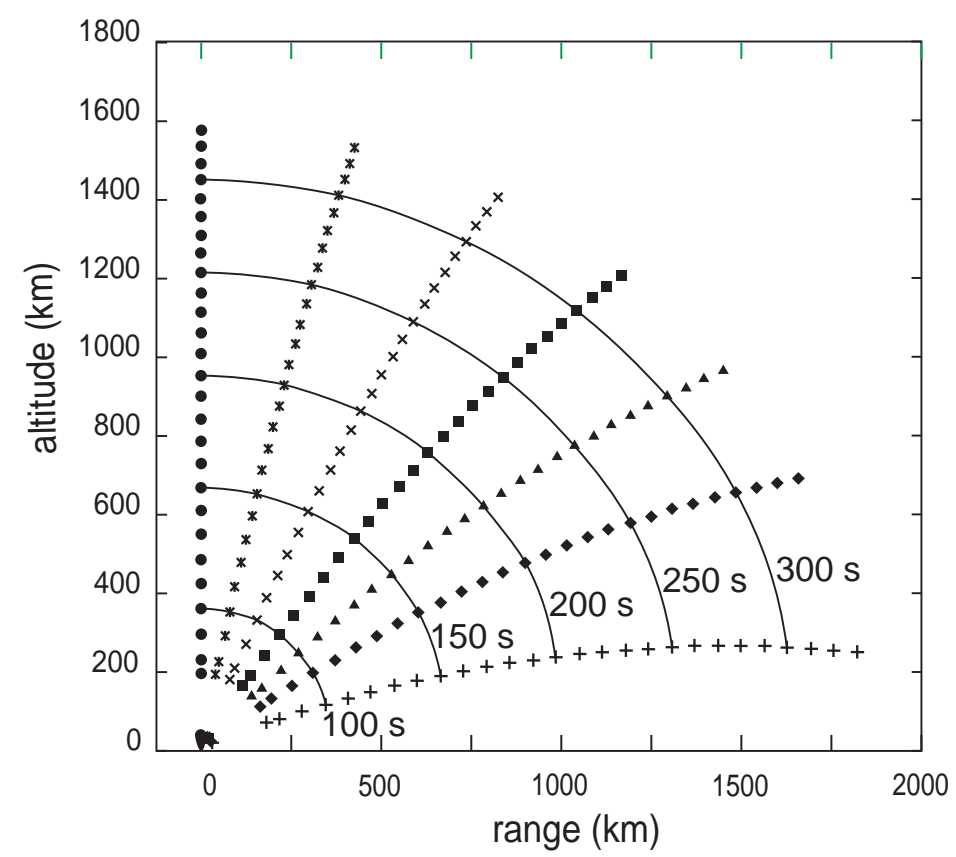

Figure 16.1. Fan of planar flyout trajectories computed for the notional $6.7-\mathrm{km} / \mathrm{s}$ interceptor I-3, which has a 75 -second boost phase.

Figure 16.1 illustrates one set of trajectories that evolved from the analysis of a two-stage interceptor having a $6.7-\mathrm{km} / \mathrm{s}$ vertical burnout velocity $\left(V_{b o}\right)$ and a total burn time of $75 \mathrm{~s}$. The radial lines are specific flyout trajectories, and the circumferential curves are contours of constant time after the time of interceptor launch. This interceptor also has a burnout velocity of about $6.4 \mathrm{~km} / \mathrm{s}$ on typical shallow intercept trajectories. It was chosen to compare with results of prior analyses using an interceptor of similar performance [171, 172]. The physical and performance characteristics of this interceptor are summarized in Table 16.1. 
Table 16.1. Characteristics of the $6.7-\mathrm{km} / \mathrm{s}$ Interceptor I- $3^{a}$

\begin{tabular}{llrrr}
\hline \hline Attribute & Units $^{b}$ & Stage 2 & Stage 1 & Totals \\
\hline Mass fraction & & 0.85 & 0.85 & \\
$I_{s p}$ & $\mathrm{~s}$ & 270 & 265 & \\
$I_{t}$ & $\mathrm{MN} \cdot \mathrm{s}$ & 4.82 & 27.3 & \\
$W_{p}$ & tonne & 1.82 & 10.5 & \\
$m_{\text {inert }}$ & tonne & 0.32 & 1.85 & \\
$m_{\text {initial }}$ & tonne & 2.25 & 14.60 & 14.60 \\
$m_{\text {final }}$ & tonne & 0.43 & 4.10 & \\
$\Delta V$ added (ideal) & $\mathrm{km} / \mathrm{s}$ & 4.37 & 3.31 & 7.67 \\
$\Delta V$ added (actual) & $\mathrm{km} / \mathrm{s}$ & 4.05 & 2.61 & 6.66 \\
Burn time & $\mathrm{s}$ & 40 & 35 & 75 \\
Thrust (average) & $\mathrm{kN}$ & 121 & 781 & \\
Diameter(average) & $\mathrm{m}$ & 1.0 & 1.2 & \\
\hline
\end{tabular}

${ }^{a}$ Kill vehicle mass $91 \mathrm{~kg}$; shroud mass $22 \mathrm{~kg}$; eject after burnout.

${ }^{b}$ Units: $\mathrm{MN} \cdot \mathrm{s}=$ Meganewton $\mathrm{s}, \mathrm{kN}=$ kilonewton; tonne $=1000 \mathrm{~kg}$.

${ }^{c}$ Cumulative from left to right.

The initial interceptor models were constructed without regard to size or launch platform constraints, a reasonable assumption for ground-based interceptors or interceptors based on large ships. Interceptors having different burn times and burnout velocities $V_{b o}$ were considered to understand the implications of burn time, flyout velocity, and acceleration profiles for the BPI problem. The first step in that process was to size several interceptors with an initial set of mass properties, stage configuration, thrust, and aerodynamic characteristics, to meet a range of performance criteria. The interceptors were then used to analyze engagements and establish kill-vehicle sizing requirements. The kill vehicle masses that resulted from that analysis (Chapter 14) were then used to develop the final mass properties of the interceptors.

\subsection{Models of Unconstrained Interceptors}

The unconstrained interceptor models had burnout velocities ranging from $6.5 \mathrm{~km} / \mathrm{s}$ to $10 \mathrm{~km} / \mathrm{s}$ (vertical). Specifically, these interceptors included:

1. Interceptor I-3: a $6.7-\mathrm{km} / \mathrm{s}$, two-stage booster having a 75 -second burn time and a large kill vehicle capable of a total velocity change $(\Delta \mathrm{V})$ of $2 \mathrm{~km} / \mathrm{s}$ for divert and homing. ${ }^{1}$

2. Interceptor I-4: a $6.5-\mathrm{km} / \mathrm{s}$, three-stage booster having a 40 -second burn time and the same kill vehicle as I-3. This model represents a missile that burns out very quickly.

3. Interceptor I-5: a 10-km/s, three-stage booster having a 45 -second burn time and a larger kill vehicle having a divert velocity of $2.5 \mathrm{~km} / \mathrm{s}$ to account for the higher closing velocities associated with this faster interceptor.

\footnotetext{
${ }^{1}$ Throughout the Report we refer to the integral of the absolute magnitude of the kill vehicle's acceleration from the time it is deployed until the end of the engagement as the total velocity change $\Delta V$ (see Chapter 12).
} 


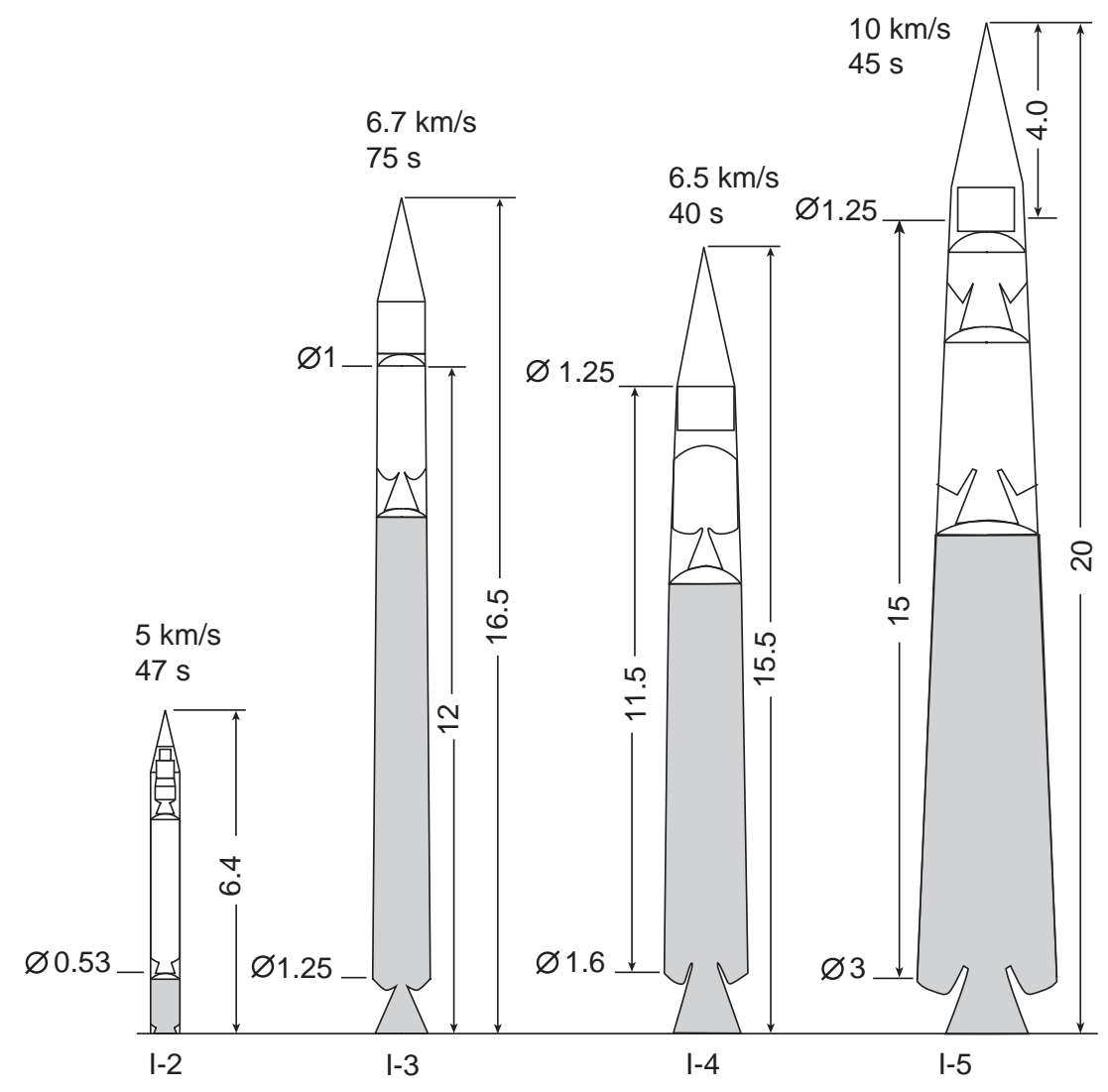

Figure 16.2. Notional profiles of the interceptor models used in the Study; the dimensions are in meters. These drawings are intended only to represent the approximate physical sizes of the interceptors that were modeled; they do not represent actual missiles. Interceptor models I-2, I-4, and I-5 were used in the analysis.

The notional dimensions of the three unconstrained interceptor models are shown in Fig. 16.2.

The capability of an interceptor is sometimes described by the ideal velocity from the rocket equation, but this does not account for aerodynamic drag effects or gravity and can overstate actual performance by 20 percent or more. In this study, we chose to define interceptors by their burnout velocity when fired vertically from the surface of Earth through the atmosphere, a better standard for comparing the actual fly-out performance of different interceptors.

In the early phase of the engagement analysis, it was found that the 75 -second acceleration time of the $6.7-\mathrm{km} / \mathrm{s}$ interceptor I-3 limited its range to such an extent that it was not useful in most engagements, particularly against solid-propellant ICBMs. The $6.5-\mathrm{km} / \mathrm{s}$ interceptor I-4, which has a boost time of $40 \mathrm{~s}$, was found to be much more effective than interceptor I-3. The basic physical and performance characteristics of interceptor I-4 are listed in Table 16.2. Figure 16.3 shows a flyout fan of planar trajectories and the acceleration profile calculated for interceptor I-4.

Engagement analyses done by the Study Group indicated that in some cases very fast interceptors were needed to reach the target in time. Those cases either required large standoff distances for geographic reasons or required early intercepts and short engagement times. The fastest interceptor considered in this study was the $10-\mathrm{km} / \mathrm{s}$ interceptor I-5, 
Table 16.2. Characteristics of the $6.5-\mathrm{km} / \mathrm{s}$ Interceptor I- $4^{a}$

\begin{tabular}{llrrr}
\hline \hline Attribute & Units $^{b}$ & Stage 2 & Stage 1 & Totals \\
\hline Mass fraction & & 0.83 & 0.83 & \\
$I_{s p}$ & $\mathrm{~s}$ & 280 & 270 & \\
$I_{t}$ & $\mathrm{MN} \cdot \mathrm{s}$ & 3.5 & 33.8 & \\
$W_{p}$ & tonne & 1.28 & 12.73 & \\
$m_{\text {inert }}$ & tonne & 0.26 & 2.55 & \\
$m_{\text {initial }}$ & tonne & 1.63 & 16.91 & 16.91 \\
$m_{\text {final }}$ & tonne & 0.36 & 4.18 & \\
$\Delta V$ added (ideal) & $\mathrm{km} / \mathrm{s}$ & 4.19 & 3.71 & 7.90 \\
$\Delta V$ added (actual) & $\mathrm{km} / \mathrm{s}$ & 3.56 & 2.94 & 6.50 \\
Burn time & $\mathrm{s}$ & 15 & 25 & 40 \\
Thrust (average) & $\mathrm{kN}$ & 234 & 1350 & \\
Diameter (average) & $\mathrm{m}$ & 1.2 & 1.4 & \\
\hline
\end{tabular}

${ }^{a}$ Kill vehicle mass $91 \mathrm{~kg}$; shroud mass $9.1 \mathrm{~kg}$; eject after burnout.

${ }^{b}$ Units: $\mathrm{MN} \cdot \mathrm{s}=$ Meganewton $\mathrm{s}, \mathrm{kN}=$ kilonewton; tonne $=1000 \mathrm{~kg}$.

${ }^{c}$ Cumulative from left to right.
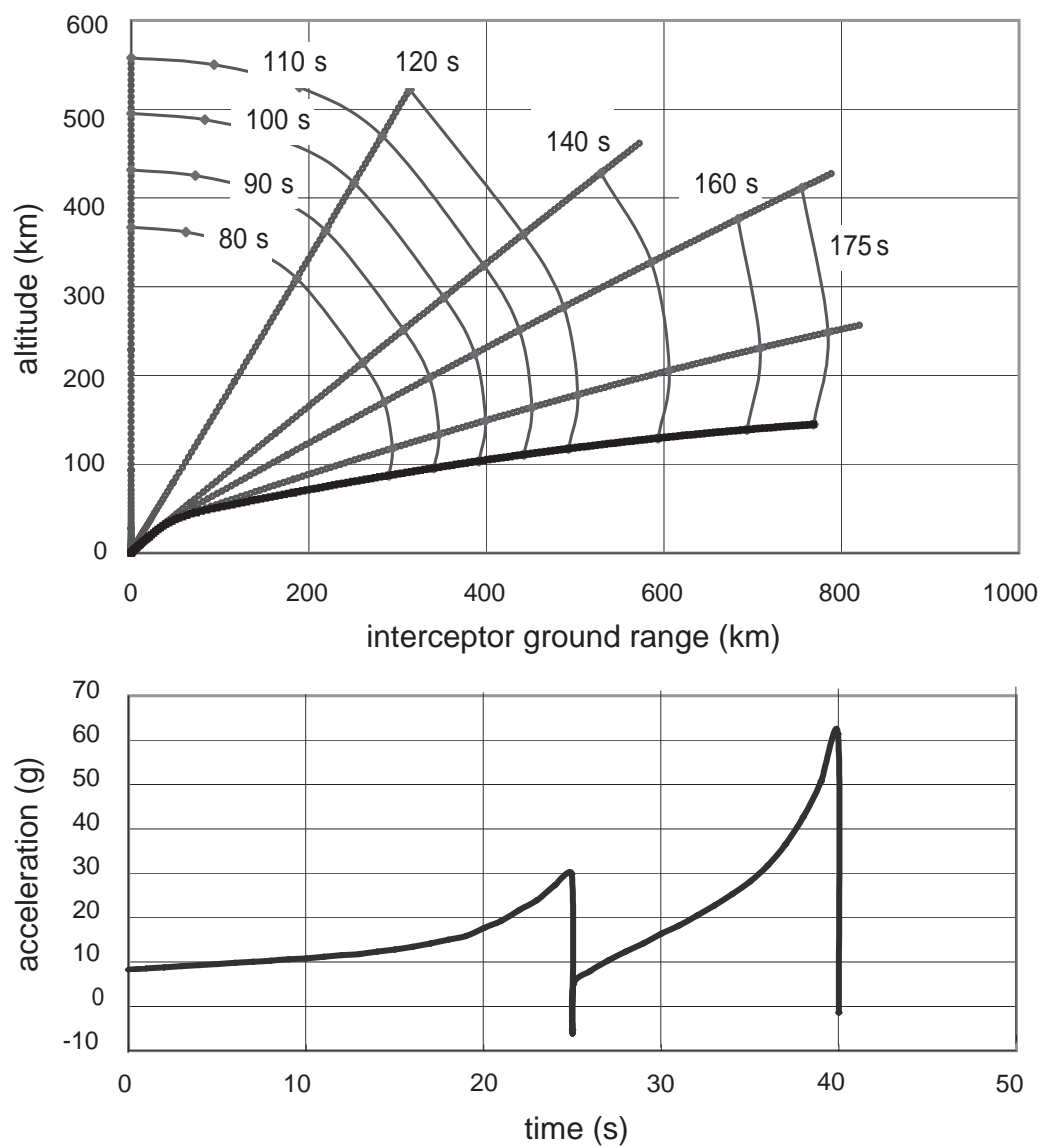

Figure 16.3. A flyout fan of planar trajectories and the acceleration profile computed for the $6.5-\mathrm{km} / \mathrm{s}$ interceptor I-4. 
which has a 45-second burn time. The basic physical and performance characteristics of this interceptor are listed in Table 16.3. Figure 16.4 shows a flyout fan of planar trajectories and the acceleration profile calculated for interceptor I-5.

Table 16.3. Characteristics of the $10-\mathrm{km} / \mathrm{s}$ Interceptor I- $5^{a}$

\begin{tabular}{llrrrr}
\hline \hline Attribute & Units $^{b}$ & Stage 3 & Stage 2 & Stage 1 & Totals \\
\hline Mass fraction & & 0.90 & 0.85 & 0.85 & \\
$I_{s p}$ & $\mathrm{~s}$ & 280 & 280 & 270 & \\
$I_{t}$ & $\mathrm{MN} \cdot \mathrm{s}$ & 1.9 & 13.7 & 132.6 & \\
$W_{p}$ & tonne & 0.68 & 5.00 & 50.0 & \\
$m_{\text {inert }}$ & tonne & 0.08 & 0.88 & 8.80 & \\
$m_{\text {initial }}$ & tonne & 0.92 & 6.80 & 65.60 & 65.60 \\
$m_{\text {final }}$ & tonne & 0.24 & 1.80 & 15.60 & \\
$\Delta V$ added (ideal) & $\mathrm{km} / \mathrm{s}$ & 3.71 & 3.65 & 3.81 & 11.18 \\
$\Delta V$ added (actual) & $\mathrm{km} / \mathrm{s}$ & 3.38 & 3.24 & 3.38 & 10.00 \\
Burn time & $\mathrm{s}$ & 15 & 15 & 15 & 45 \\
Thrust (average) & $\mathrm{kN}$ & 125 & 917 & 8840 & \\
Diameter (average) & $\mathrm{m}$ & 1.32 & 1.50 & 2.20 & \\
\hline
\end{tabular}

${ }^{a}$ Kill vehicle mass $136 \mathrm{~kg}$. Shroud mass $27 \mathrm{~kg}$; eject after burnout.

${ }^{b}$ Units: $\mathrm{MN} \cdot \mathrm{s}=$ Meganewton $\mathrm{s}, \mathrm{kN}=$ kilonewton, tonne $=1000 \mathrm{~kg}$.

${ }^{c}$ Cumulative from left to right.

Although building such a fast interceptor may be possible within the next 10 years, it would be technically challenging. The interceptor would have a mass of 66 tonnes, which is as heavy as an ICBM, and yet must reach a speed 50 percent faster than an ICBM in only one-quarter of the time. Such a missile has never been built.

Any rocket moving faster than about $7 \mathrm{~km} / \mathrm{s}$ would have the range of an ICBM and could therefore be mistaken for an ICBM. Interceptor I-2 is slower than this, and there would be no danger of such a mistake. However, Interceptor I-4 $(6.5 \mathrm{~km} / \mathrm{s})$ and I-5 (10 km/s) could be mistaken for an ICBM. The interceptor trajectories from North Korea are generally to the northwest, and head across China to Russia. Thus, an interceptor fired in defense runs the risk of inadvertently triggering a retaliatory action by another country. This scenario is of particular concern for defense of missiles from North Korea, where the interceptors would be on trajectories headed for China and Russia. While boost-phase interceptors fly a flat trajectory, even a 4000-km-range would be a threat to most of eastern Russia and all of China, so even a $5-\mathrm{km} / \mathrm{s}$ interceptor could be a problem.

\subsection{Models of Constrained Interceptors}

The Study Group also developed models of three interceptors constrained in volume and-or mass so that they could be based on ships or aircraft. Those models include:

1. Interceptor I-1: A $1.7-\mathrm{km} / \mathrm{s}$, two-stage interceptor having a dual-thrust second stage that is similar to the existing Standard Missile SM-2, currently deployed on U. S. Navy Aegis cruisers and destroyers. While much too slow to intercept ICBMs, this interceptor could be used to intercept theater-range missiles launched from ships off U.S. coasts, provided that the Aegis ship could be located close to the missile launch 

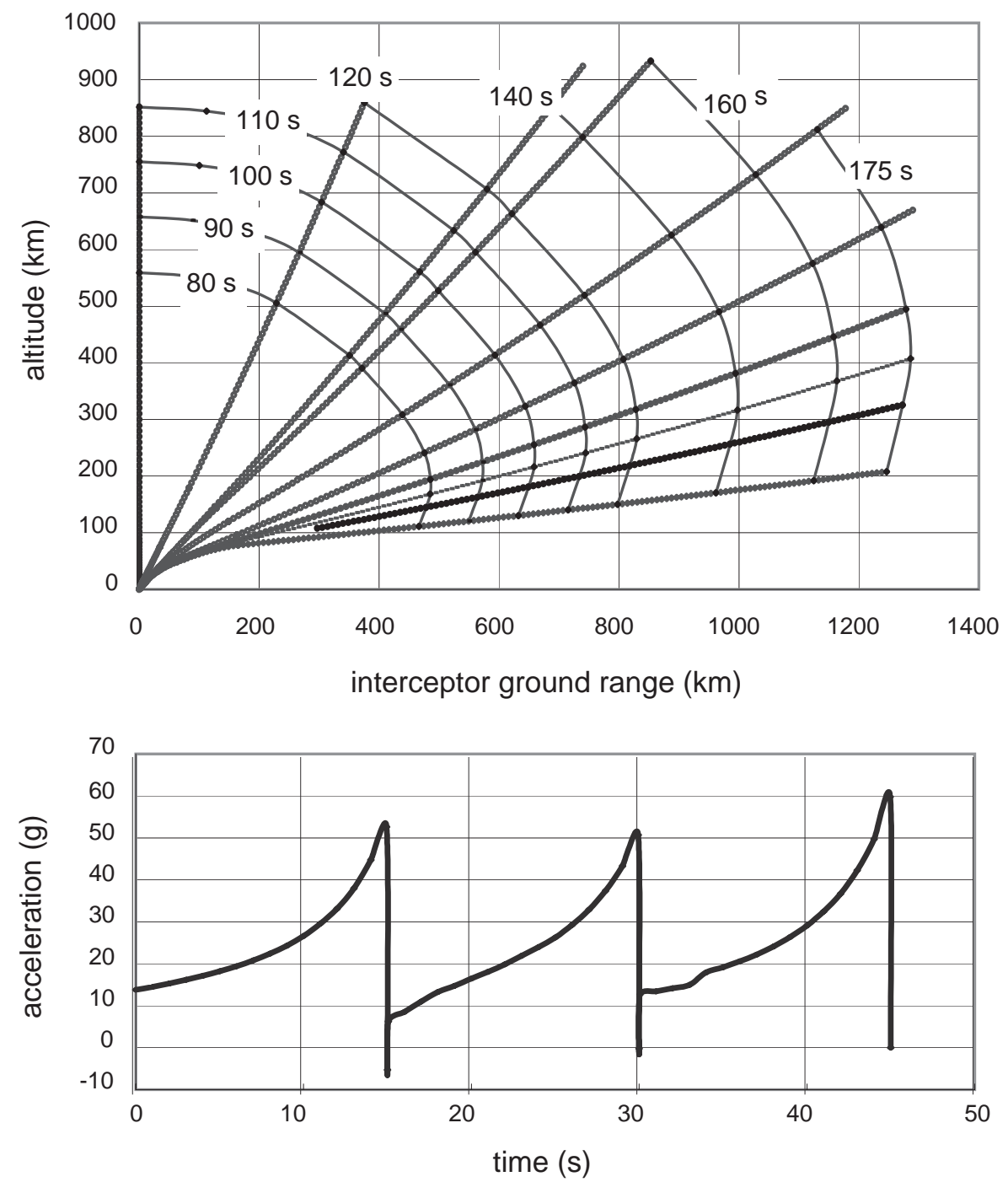

Figure 16.4. A flyout fan of planar trajectories and the acceleration profile computed for the $10-\mathrm{km} / \mathrm{s}$ interceptor I-5.

platform. There would be no kill vehicle on this interceptor; instead, it would use the blast-fragmentation warhead on the Standard Missile SM-2.

2. Interceptor I-2: A 5-km/s, three-stage interceptor having a 21-inch diameter that would fill the entire volume of the vertical-launch-system (VLS) tubes in Aegis ships. It would carry the same $2-\mathrm{km} / \mathrm{s}$ kill vehicle as interceptors I-3 and I- 4 . This missile would have a launch mass of about $2,200 \mathrm{~kg}$. Note that it would be a new missile and not a modification of the existing Standard Missile SM-2, currently deployed on U.S. Navy cruisers and destroyers.

3. An air-launched interceptor constrained for bomber carriage with a gross weight of about $2,000 \mathrm{~kg}$, similar to the size and weight of an Air-Launched Cruise Missile (ALCM) that is carried by B-52H bombers [173]. This interceptor would carry the same $2-\mathrm{km} / \mathrm{s}$ kill vehicle as interceptor I-2. Due to time constraints, we did not develop a detailed model of this missile. However, it would be only slightly smaller than the $5-\mathrm{km} / \mathrm{s}$ VLS model (I-2) and would have about the same capabilities (the 
smaller size would be compensated by its being launched at an altitude of about $13 \mathrm{~km}$ where the atmosphere is thinner and the drag is less). Therefore, we used I-2 as a surrogate for the range capabilities of the ALCM-sized interceptor.

Interceptor I-2 was an important interceptor in our analysis because it could be deployed in a VLS tube on Aegis ships - a basing mode commonly suggested for BPI. In our view, an interceptor similar to I-2 would be the most capable interceptor that could be deployed on an Aegis ship without making significant modifications to the ship. The basic physical and performance characteristics of this interceptor are listed in Table 16.4. Figure 16.5 shows a flyout fan of planar trajectories and the acceleration profile calculated for interceptor I- 2 .

Table 16.4. Characteristics of the VLS $5-\mathrm{km} / \mathrm{s}$ Interceptor I- $2^{a}$

\begin{tabular}{llrrrr}
\hline \hline Attribute & Units $^{b}$ & Stage 3 & Stage 2 & Stage 1 & Totals \\
\hline Mass fraction & & 0.80 & 0.90 & 0.67 & \\
$I_{s p}$ & $\mathrm{~s}$ & 270 & 265 & 256 & \\
$I_{t}$ & $\mathrm{MN} \cdot \mathrm{s}$ & 0.24 & 3.21 & 1.14 & \\
$W_{p}$ & tonne & 0.091 & 1.23 & 0.45 & \\
$m_{\text {inert }}$ & tonne & 0.023 & 0.137 & 0.224 & \\
$m_{\text {initial }}$ & tonne & 0.21 & 1.58 & 2.26 & 2.26 \\
$m_{\text {final }}$ & tonne & 0.11 & 0.35 & 1.81 & \\
$\Delta V$ added (ideal) & $\mathrm{km} / \mathrm{s}$ & 1.56 & 3.98 & 0.57 & 6.10 \\
$\Delta V$ added (actual) & $\mathrm{km} / \mathrm{s}$ & 1.22 & 3.32 & 0.49 & 5.02 \\
Burn time & $\mathrm{S}$ & 20 & 20 & 6.5 & 46.5 \\
Thrust (average) & $\mathrm{kN}$ & 12 & 160 & 176 & \\
Diameter & $\mathrm{m}$ & 0.53 & 0.53 & 0.53 & \\
\hline
\end{tabular}

${ }^{a}$ Kill vehicle mass $91 \mathrm{~kg}$; shroud mass $9.1 \mathrm{~kg}$; eject after burnout.

${ }^{b}$ Units: MN. $\mathrm{s}=$ Meganewton $\mathrm{s}, \mathrm{kN}=$ kilonewton, tonne $=1000 \mathrm{~kg}$.

${ }^{c}$ Cumulative from left to right.

\subsection{Summary of Interceptor Models}

The five interceptors described in this chapter are central to the analysis in the remainder of this Report. Table 16.5 compares them. Most of the analysis in the Report focuses on interceptor models I-2, I-4, and I-5. Interceptor I-3, which has a 75-second burn time, accelerates too slowly to be very useful against ICBMs. Interceptor I-1 could be used only to defend the United States against medium-range ballistic missiles (MRBMs) launched from forward-based platforms such as ships. An airborne interceptor would have a performance similar to interceptor I-2, which is approximately the size of an ALCM.

Table 16.5. Interceptor Models Used in the Study

\begin{tabular}{ccccc}
\hline \hline Model & $\begin{array}{c}\text { Burnout } \\
\text { Velocity }(\mathrm{km} / \mathrm{s})\end{array}$ & Boost Time (s) & $\begin{array}{c}\text { Liftoff Mass } \\
\text { (tonne) }\end{array}$ & $\begin{array}{c}\text { Number of } \\
\text { Stages }\end{array}$ \\
\hline I-1 & 1.7 & 47 & 1.57 & 2 \\
I-2 & 5.0 & 47 & 2.26 & 3 \\
I-3 & 6.7 & 75 & 14.60 & 2 \\
I-4 & 6.5 & 40 & 16.91 & 2 \\
I-5 & 10.0 & 45 & 65.60 & 3 \\
\hline
\end{tabular}

Note: Burnout velocity is that for a vertical trajectory. 

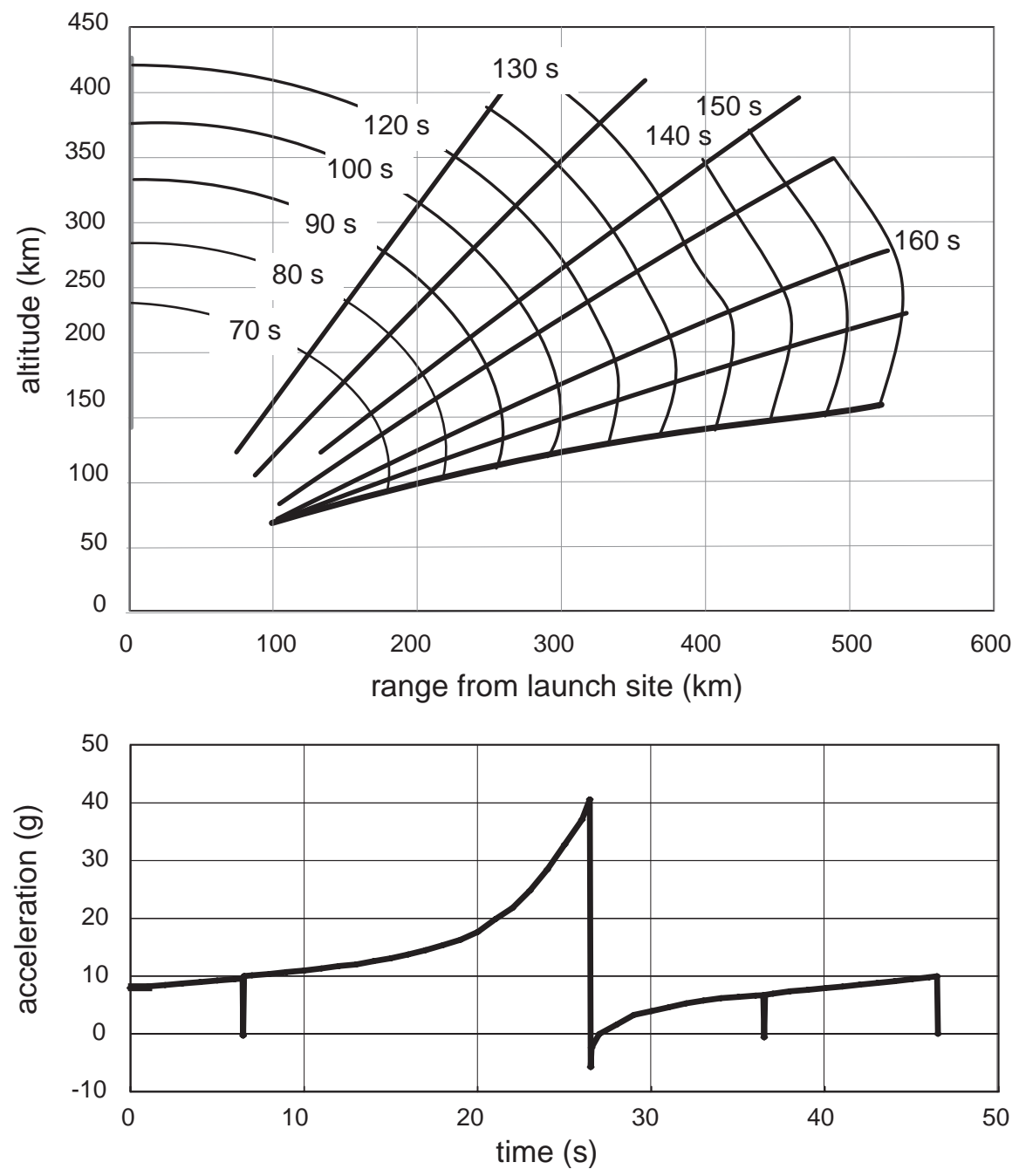

Figure 16.5. A flyout fan of planar trajectories and the acceleration profile computed for the $5-\mathrm{km} / \mathrm{s}$ interceptor I-2.

Figure 16.6 compares the sizes of the five model interceptors to other missiles that have been built in the past. Notice that the faster $(6.5-\mathrm{km} / \mathrm{s}$ to $10-\mathrm{km} / \mathrm{s})$ interceptors are comparable in size to most ICBMs, or even larger. Interceptors I-2 and I-3 are about half the weight of the Minuteman III; I-5 is twice as heavy as the Minuteman III and about 10 percent heavier than the Trident II. All are heavier than the Spartan interceptor used in the ground-based antiballistic missile (ABM) system the United States deployed near Grand Forks, North Dakota in the early 1970s.

\subsection{Interceptor Basing Options}

We conclude our discussion of interceptors for boost-phase defense by commenting briefly on some of the options for deploying them. These include basing on land, on ships at sea, and on aircraft. Basing interceptors in space would involve quite different considerations; discussion of this option is therefore given in Chapter 6. The discussion here focuses chiefly 


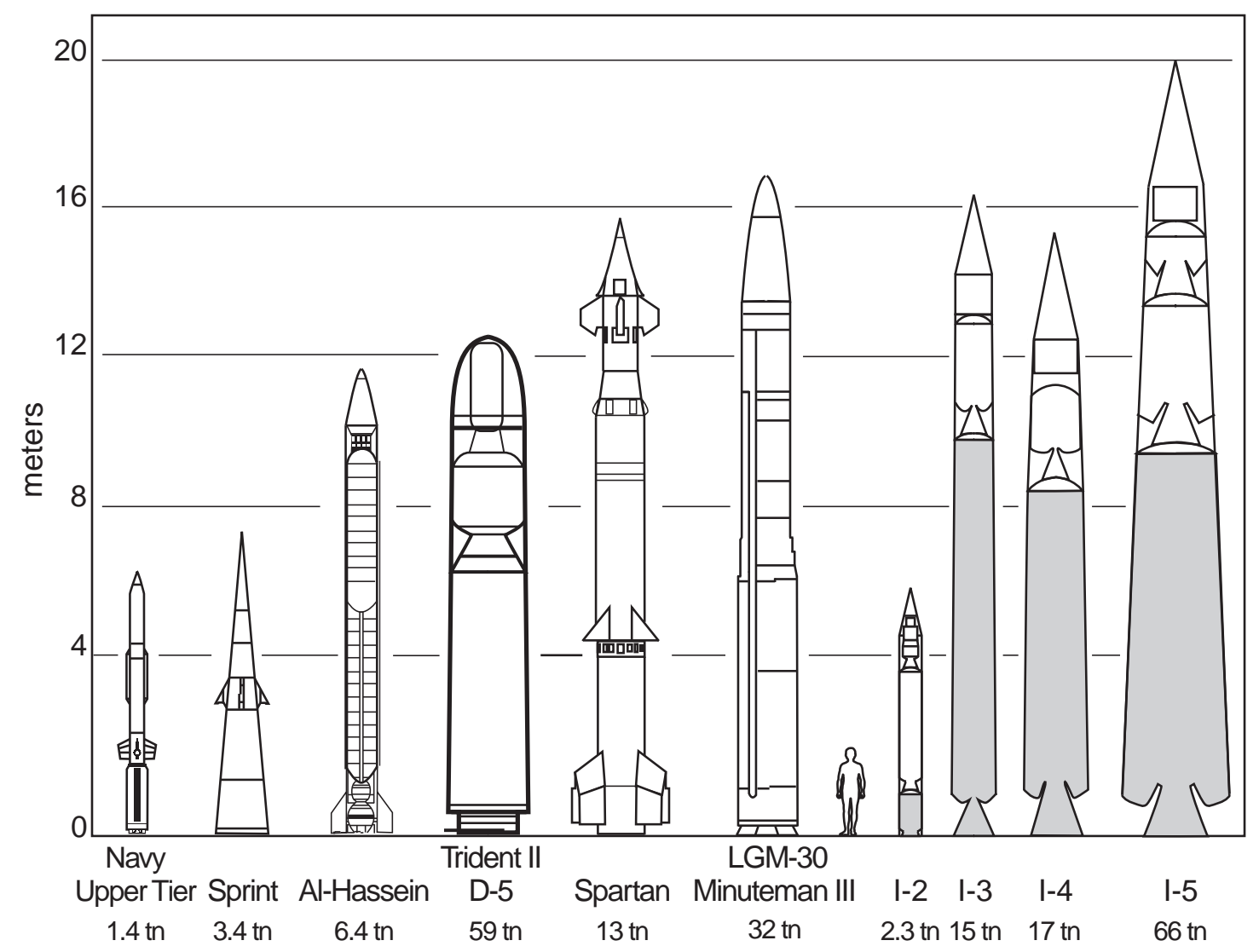

Figure 16.6. Comparison of model interceptors with various existing missiles. The liftoff mass of each interceptor or missile in tonnes $(1 \mathrm{tn}=1000 \mathrm{~kg})$ is listed beneath the name of the interceptor or missile.

on interceptors for defending against ICBMs, but we discuss briefly some deployment options for interceptors intended to defend the United States against MRBMs launched from platforms off the coasts of the United States.

Although command, control, and communications are vitally important, they are outside the scope of the Study and are not discussed.

\subsubsection{Land basing}

It can be assumed that $6.5-\mathrm{km} / \mathrm{s}$ interceptors like I-4 could be air-transported and deployed with field army units. Transporter-erector-launcher vehicles for land-basing of missiles in this class should be feasible and should not require advance construction of special sites or launching pads. Interceptor I-5 may be road-transportable, but its mass is 66 tonnes (73 tons), more than twice the mass of a Minuteman III, and its diameter is three $\mathrm{m}$. It could not be transported readily by air and might require fixed launch pads or silos. (See Fig. 16.2). 


\subsubsection{Sea basing}

Basing boost-phase interceptors on sea-going vessels would offer some potential advantages over land-based systems, but would also impose some limitations. An archetype for a seabased boost-phase system is the Aegis system, which is currently deployed on many cruisers and destroyers in the U.S. fleet. These ships have the normal complement of combat systems, including systems for launching Tomahawk cruise missiles as well as Standard Missiles, which are designed primarily to defend the fleet against unmanned air-breathing threats (often called cruise missiles). The Standard Missile SM-2 block 4A was designed to engage short-range tactical ballistic missiles (SRBMs) reentering the atmosphere as well as cruise missiles. However, its production was recently cancelled. Another variant, the SM-3, is in the early stages of prototype development and is intended to provide "theater-wide" defense against MRBMs.

The Aegis VLS can launch only missiles having diameters of 21 inches or less. Our analysis indicates that given a capable kill vehicle with the necessary on-board sensors, acceleration, and $\Delta \mathrm{V}$ capability, an interceptor like model I-2, which has a burnout velocity of $5 \mathrm{~km} / \mathrm{sec}$, is the largest that could be launched using the VLS. It is possible the system could be modified to take a slightly larger, and hence faster, interceptor.

The Aegis system contains three additional organic components: a capable, large, phased-array radar (the SPY-1), which is closely integrated into the ship's superstructure; a weapon battle-management and fire-control system capable of engaging multiple targets simultaneously; and the communications suite required for tying into a larger, integrated defense structure within the theater of combat.

The $6.5-\mathrm{km} / \mathrm{s}$ interceptor I-4 would be the size of a Polaris A3 missile, too large to be launched by the VLS. Hence, if this interceptor were to be deployed on ships, a new launching system must be developed.

There is some practical limit to the size of an interceptor that could be carried aboard ships other than Aegis platforms. With steam-eject launch, missiles such as the 74-inchdiameter, 70,000-lb. Poseidon C3 missile were launched from tubes on the USS Observation Island in the late 1960s. Trident submarines carry 24 missiles weighing more than 120,000 lbs. each and having a diameter of nearly 90 inches. For purposes of comparison, the model I- $5-10 \mathrm{~km} / \mathrm{s}$ interceptor has a diameter of 127 inches and weighs 144,000 lbs. (see Fig. 16.2). To accommodate such a missile, a ship larger than the existing Aegis would be needed - perhaps a new ship or a converted cargo ship - as well as a new launch system.

The primary benefits of using submarines as the interceptor launchers are that they would be relatively difficult to detect and could launch their interceptors in very poor weather and sea conditions. However, a wide-band communication link between the radar and the ship launching the interceptor would be essential. A submarine would therefore have to have an exposed wide-band antenna, which would compromise its stealthiness.

The advantages of sea basing include the ability to move interceptors to critical locations almost anywhere in the world when a conflict arises. In addition, such a defensive system could remain on-station at such locations indefinitely, taking advantage of the crew infrastructure, replenishment, and protection of the host vessel and its associated battle group. 


\subsubsection{Air basing}

Previous studies of airborne BPIs have assumed that interceptors having burnout velocities of $4 \mathrm{~km} / \mathrm{s}$ to $5 \mathrm{~km} / \mathrm{s}$ could be based on aircraft, such as the B-52 [174, 175, 176]. At the same time this study has been underway, Dean Wilkening at Stanford University has been specifically studying airborne boost-phase solutions and briefed our Study Group on his interim results [177].

There are three issues that must be recognized with air-based ICBM BPIs-endurance, payload limits, and self defense. In similar air operations involving maintaining one aircraft continuously on station, typically five to six aircraft plus some tankers are required. Hence the operational cost of maintaining an air-based defense is quite high. In planning an airbased BPI system, the vulnerability of interceptor-carrying aircraft to long-range surfaceto-air missiles (SAMS) would have to be considered.

The SA-5 (Russian S-200) SAM system has been operational since the mid-1960s, has been exported to hostile nations, and remains operational today. It can intercept large aircraft operating at high altitudes out to distances of $200 \mathrm{~km}$ or more from the SAM site. More modern SA-12 (Russian S-300V) SAMs have comparable range capability coupled with mobility that would permit surprise deployments. There is a possible electronic countermeasure (ECM) defense against these long-range SAMs, but the risk involved in stationing large aircraft within $200 \mathrm{~km}$ of hostile territory would probably prevent such operation for long periods. In addition, the vulnerability of such aircraft to air-to-air missiles delivered by enemy fighters would require continuous protection by U.S. combat air patrols or long-range SAMs based within 50 to $100 \mathrm{~km}$ of the BPI aircraft, negating most of the potential basing advantage. Interceptors having velocities in excess of $5 \mathrm{~km} / \mathrm{s}$ appear to be too large to be deployed on aircraft.

While having a radar at altitudes of 10-13 $\mathrm{km}$ offers some viewing advantages, the size of the radar required to acquire these targets at ranges of $800 \mathrm{~km}$ is prohibitive for most aircraft.

For these reasons, and the fact that another study was focusing on that approach, we did not explore air-based solutions explicitly, but rather treated it generically.

\subsubsection{Deployment for defense against SRBMs and MRBMs}

If there is an ideal application of boost-phase defense in the near term, it is defending against SRBMs or MRBMs launched from vessels positioned off U.S. coasts ${ }^{2}$. Engagements of a typical SRBM and a typical MRBM launched from ships at sea are analyzed in Section 5.7.1. There it is shown that the SRBMs and MRBMs could be engaged by interceptor I-1, which is similar to the U.S. Navy's existing SM-2. This interceptor would have to be stationed within approximately $40 \mathrm{~km}$ of the vessel launching the ballistic missile.

During the Cold War, the U.S. Navy maintained a large and effective wide-area surveillance of surface vessels as well as submarines in U.S. coastal waters. The assets used for this purpose, such as the P-3 Orion patrol aircraft and the U.S. Underwater Sound Surveillance System (SOSUS), still exist. Suspect or unidentified vessels could be shadowed by Aegis ships, which might be able to intercept SRBMs or MRBMs during their boost phase, before they could deploy submunitions. It is unlikely that any uncoordinated preparation for or actual launch of a rocket from waters near the U.S. could have a peaceful purpose. It is also

\footnotetext{
${ }^{2}$ Medium-range ballistic missiles encompass the missiles that, in a military context, are sometimes called "theater" or "tactical" ballistic missiles.
} 
unlikely that such a launch would involve many ships. Such launches could therefore be engaged immediately with pre-delegated authority, thus avoiding three of the most serious challenges facing most BPI systems-being close enough to the target launch site to be able to intercept the missile during its boost phase, having the decision time required between the time a potentially threatening rocket is detected to the time interceptors must be fired, and avoiding shortfall of the missile's munitions on friendly territory. With adequate surveillance, it can be assumed that an Aegis ship could be stationed in-shore of the suspect ship.

\section{References for Chapter 16}

[171] Richard L. Garwin, "Boost-phase intercept: A better alternative." Arms Control Today 30(7), 8 (2000).

[172] Theodore A. Postol, "A preliminary analysis of a Russian-U.S. boost-phase defense to defend Russia and the U.S. from postulated rogue-state ICBMs: A potential option for Russian-U.S. cooperation in missile defense." Technical report, Carnegie Endowment for International Peace, October 1999.

[173] Jane's Strategic Weapons Systems (JSWS), 36, December 2001. Article: AGM-86 ALCM/CALCM.

[174] D. C. Johannsen et al., "Airborne Interceptor Program." Proceedings of the 4th Annual Technology Readiness Conference (BMDO/AIAA), 1995.

[175] D. R. Vaughan, J. A. Isaacson, and J. S. Kvitky, "Airbore Intercept: Boost- and Ascent-Phase Options and Issues," RAND, 1996.

[176] Dean Wilkening, "Boost-Phase Missile Defense." Briefing Presented to the APS Study Group, July 2001.

[177] Dean Wilkening, "Airborne Boost-Phase Ballistic Missile Defense," Science and Global Security, Vol. 12:2-3, to be published. 

Part D. Supporting Analysis for Airborne Laser Engagements 



\section{Chapter 17}

\section{Overview of the Airborne Laser Analysis}

Chapter 7 in Part A described the fundamentals of ABL engagements and Chapter 8 presented conclusions on the coverage the ABL could offer for boost-phase ICBM defense against North Korea, Iraq, and Iran. We turn now to the technical considerations involved in employing the ABL in a boost-phase defense system.

Basing lasers on satellites was an option discussed some years ago in the context of the Strategic Defense Initiative (SDI), but this technology is not considered to be available within our time frame. The only laser technology that could reasonably be hoped to be ready for deployment within the 10-year time frame of this report is the ABL, which is currently being developed. The high-power laser of the ABL would be carried by a modified Boeing 747. As a consequence of launching the laser beam from an aircraft in the atmosphere, the propagation of the beam through the atmosphere is a major issue.

As was shown in Chapter 5, the time available for a kill-vehicle intercept is very short, especially if the 30-s decision time is imposed before launching the interceptors. This compressed time line forces the defense into using very high speed interceptors to reach the target in time. The ultimate extension of this trend is travel at the speed of light, which a laser beam does.

The ABL is designed from the beginning to be a boost-phase-defense weapon, although its design mission is for use against theater missiles having relatively short range, compared with the long range of ICBMs. Nevertheless, the same principles apply for theater missile defense as for boost-phase defense against ICBMs. We shall see that because of the higher burnout altitude of ICBMs compared with theater missiles, intercepting ICBMs with a laser is in some ways easier than intercepting theater missiles.

We have adopted the ABL hardware as best we can determine, subject to the limits of classification, as the basis of our analysis. No major modifications are considered. As a consequence of classification issues, some important parameters are unknown, such as the laser power and the amount of energy required to be delivered to disable a missile. We make what we consider to be reasonable estimates and show the sensitivity to changes in these estimates. In cases of doubt, we have taken an optimistic view and given the ABL the benefit of the doubt. We compute the range from the ABL to the missile at which the ABL could be effective against both liquid- and solid-propellant boosters. As expected, the "speed-of-light" weapon is insensitive to the launch delay decision time that stressed the kill-vehicle performance, as shown in Section 5.1.2. Nevertheless, the effective range is not as great as one would desire, but it is comparable to that of the various kill vehicles, depending on the circumstances.

Our discussion of the ABL starts with Chapter 18, which provides an overview of the ABL hardware, the airframe, the lasers on board, and the assumed performance. Chap- 
ter 19 describes the issues related to propagating the laser beam long distances through the atmosphere and focusing it on the target. Doing this requires a sophisticated adaptive optics (AO) system to compensate for the atmospheric distortions, and this is described in Section 19.4. Although the ABL has not yet been field-tested, small-scale experiments have been done to validate the specifications and the performance of the AO system. These are described in Section 19.5, and the results are used as the basis for the expected ABL performance used in our calculations. Section 19.7 summarizes the limits of AO parameters and engagement time to which all calculations are subject.

The damage mechanism for a kill vehicle is simple: The physical impact deposits a very large amount of energy on the target in a very short time (less than a millisecond). If impact is achieved, severe damage to the target is assured. Delivery of sufficient energy to the target by a laser, however, is more complex. A time measured in seconds is required, and the amount of energy delivered to the target required to assure destruction depends on many things, such as the different mechanical and optical absorption properties of liquidvs. solid-propellant boosters. Solid-propellant boosters require much more energy density (fluence) delivered by the laser. Section 20.1 describes how we estimated the fluence required. Section 20.2 discusses the problem of assessing whether and when an engagement is successful.

Section 21.1 describes the sequence of events of a laser attack, from the initial observation of the target through the delivery of the high-energy laser (HEL) beam onto the target. The optical engagement itself is complex, oddly enough because of the finite speed of light. The AO system needed to focus the beam onto the target after traversal of a long air path depends on receiving an image of the target at the ABL, and then returning the HEL along the same air path (ideally) to the missile. During this round trip time for light over hundreds of $\mathrm{km}$ (a few milliseconds) the target, moving at several $\mathrm{km} / \mathrm{s}$, will have moved a few meters. Hitting the target at the selected spot imposes serious constraints on the engagement, as described in Sections 21.3 and 21.4.

The results of the analysis in terms of the effective range of the ABL against liquid- and solid-propellant missiles are given in Section 21.5. This analysis folds in the target flight profile (altitude, speed, and acceleration vs. time) with the missile hardness (fluence required for destruction), the laser power, and the performance and limitations of the AO system to compute the maximum range vs. intercept altitude. The altitude is a key parameter, because it plays a major role in the amount of atmospheric turbulence with which the ABL must contend. Figure 21.7 shows this relationship for both kinds of ICBMs. In broad terms, we estimate that liquid-propellant missiles could be intercepted at distances up to about $600 \mathrm{~km}$ from the ABL, but solid-propellant missiles could be intercepted only at about $300 \mathrm{~km}$.

Because there are substantial uncertainties in basic parameters, such as the laser power, the beam quality, the atmospheric turbulence, the minimum fluence required to disable a booster, and the effect of aim point on AO performance, we did a sensitivity analysis for these variables, which is presented in 21.5.2. We find that our conclusions on useful range are robust with respect to reasonable changes. For example, decreasing the laser power by a factor of 2 or, alternatively, doubling the fluence required, reduces the effective range by only $15-20$ percent. It is important to note that this sensitivity analysis applies to the ICBM intercepts we studied; it may not apply to the TBM case for which the ABL is designed.

Chapter 22 describes how the ABL might be deployed in the field. Section 22.2 discusses possible problems caused by high-altitude cirrus clouds, which could disrupt the laser beam. Section 22.3 briefly treats the application of the ABL to the defense of the United States from 
missiles launched from ships off its coasts. This discussion is analogous to the discussion of Section 5.7.1 for kill vehicles.

All the analysis was done without taking into account countermeasures that might be taken to reduce the effectiveness of the ABL. One effective countermeasure would be to destroy the whole airframe with aircraft or surface-to-air missiles (SAMs), as is discussed in Section 9.2.1. Other measures that reduce the energy density absorbed by the target are discussed in Sections 9.2.2-9.2.4. Each ABL airframe can, of necessity, engage only one target at a time, and multiple targets must be engaged sequentially. As a consequence, multiple ABLs may be needed to defend against multiple simultaneous missile launches, as discussed in Section 9.2.5. The defense would require three or four ABLs to be deployed in the region for every ABL on station around the clock.

Finally, Appendix D provides reference material and additional detail for topics treated in Chapters 18-22. This material is provided for the reader wishing more detailed information, particularly with respect to AO systems. 



\title{
Chapter 18
}

\section{Technology of the Airborne Laser}

\author{
Contents
}

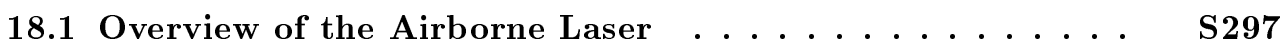

18.2 Description of COIL . . . . . . . . . . . . . S299

18.3 Chronology of the Airborne Laser . . . . . . . . . S S300

18.4 Airborne Laser Performance . . . . . . . . . . . S301

This chapter describes the ABL hardware as it has been designed for use in theater missile defense. The subsequent chapters use the same hardware without modification as a basis to examine the ABL's capability for use against ICBMs. Here we give a general description of the airframe, the lasers, other major systems that it carries, and some of their parameters.

The ABL will use a high-power chemical oxygen-iodine laser (COIL) as a directedenergy-beam weapon to disable missiles during their boost phase. The COIL laser belongs to a class of lasers that are driven by exothermic chemical reactions. The chemistry and typical operating parameters of a COIL are described in Section 18.2. The laser beam energy delivered during a several-second engagement onto a spot on the missile will cause heating and a reduction in material strength of the missile's "skin." Because of internal fuel tank pressure and structural stress in the missile during boost-phase acceleration, the missile skin may then rupture, resulting in destruction of the missile or termination of acceleration.

The atmosphere in which the COIL operates is a nonuniform medium, and its turbulence would normally seriously degrade the focus of the laser beam as it travels the long distance through the atmosphere, much like the distortion of images seen close to the surface of a long, hot, paved road. The ABL uses an advanced system of adaptive optics (AO) to measure the distortion and then adjust the high-energy-laser (HEL) beam to focus it on the target. To do this, two other lasers are used to illuminate the target, whose image is then transmitted to the ABL for this correction.

\subsection{Overview of the Airborne Laser}

The ABL uses a modified 747-400 freighter aircraft cruising at an altitude of about $12 \mathrm{~km}$ as the platform for a complex system of optical devices that will target threat missiles with laser beams focused to tens of centimeters at distances up to several hundred kilometers (see Fig. 18.1). The ABL is to have a capability of firing tens of shots against threat missiles 
and an extended operation period (based on refueling). It could be deployed singly to a threat area or in concert with other theater defense resources [178].

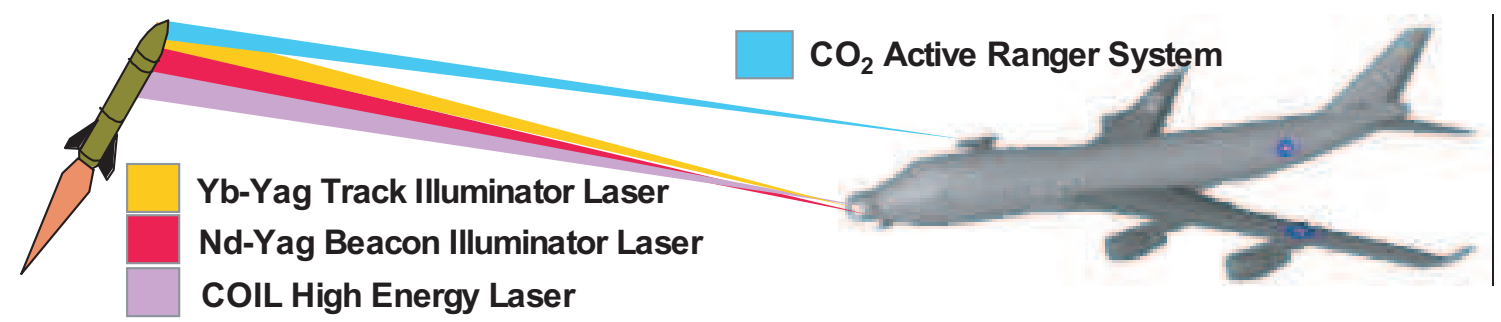

Figure 18.1. Schematic drawing of ABL engagement, showing the four laser beams onto the target. From top to bottom these are: (a) the $\mathrm{CO}_{2}$ laser for range, (b) the Yb-YAG track illuminator laser (TILL), (c) the Nd-YAG beacon illuminator laser (BILL), and (d) the COIL high-energy laser (HEL). Reproduced with permission from [183].

There are four major sub-systems on the aircraft:

- The cw (continuous wave) high-power COIL laser weapon.

- The beam control assembly, which aligns and transports multiple laser beams through the aircraft and provides AO systems to compensate for atmospheric distortion of the laser beams.

- The battle management and communications, command, control, and intelligence system, which provides engagement and fire control.

- The nose-mounted, gimbaled turret assembly, which can be rotated to aim sensors and laser beams in horizontal and vertical planes, so as to locate, track, and target missiles.

The initial Program Definition and Risk Reduction phase of the ABL program includes 6 COIL modules in series in the aircraft, while the Engineering and Manufacturing Development phase plans for 14 laser modules. Multiple-gain modules are used to provide higher output power than conventional COIL systems.

While operation of the ABL COIL with both extremely high output power and good beam quality is crucial to the ABL's ability to deliver significant energy to the surface of a missile at relevant target distances, we have not found any reference to measured beam power and beam quality of the ABL COIL in the available literature. Lethality-based requirements for COIL power and beam quality are discussed in subsequent chapters.

Additional high-power lasers in the ABL are used for missile tracking and AO compensation for atmospheric distortion. A $\mathrm{kW}$-power-level Yb-YAG tracking illuminator laser (TILL) provides illumination of the tip of the threat missile; return light from this sharpedged feature is used for a tracking sensor and the first stage (tilt) of AO beam corrections. A kW-power-level Nd-YAG beacon illuminating laser (BILL) provides an illuminated spot on the missile; return light from this spot is used in higher-order (phase) AO corrections of atmospheric distortion for the cw COIL laser. Both the TILL and BILL are pulsed at $5 \mathrm{kHz}$.

The beam-control system and transport of the three main laser beams within the aircraft is based on a common-path-common-mode approach. A shared set of optical components 
and a counter-propagating alignment laser are used for alignment, targeting, focusing, and beam compensation, as well as sensing and control functions. This shared-aperture design offers advantages in tracking, jitter, and alignment control. Deformable and rapid-steering mirrors provide high-bandwidth $(500-\mathrm{Hz})$ beam clean-up and atmospheric compensation and pointing. Significant demands on mirror and coating quality to handle high power loading [179] appear to be achievable. Alignment and pointing at the level of 100 nanorad is sought to keep the COIL focused on the missile. This is a challenging requirement, since a 747 aircraft frame can experience significant relative motion front-to-rear during flight. An active alignment system is planned [180].

The ABL may be initially cued to a missile launch by IR search and track sensors located on the airplane, or by satellite or radar detection systems. An active ranging system uses a laser illuminator mounted on top of the airplane to provide early position coordinates of the missile. Subsequent engagement and fire control steps, including IR-based coarse and fine plume tracking, placement of the TILL, BILL, and COIL laser beams on the missile, and AO compensation of atmospheric distortions are discussed in Chapter 21.

The nose of the aircraft holds the approximately $6,000-\mathrm{kg}$ turret assembly that carries a 1.7-m-diameter window [181] and a 1.5-m-diameter focusing telescope mirror. The gimbaled mirror assembly points the three, nearly co-propagating laser beams toward the missile in a hemispherical field-of-regard, which is centered on the aircraft flight path and which extends back towards the wings. The turret window transmits the TILL, BILL, and COIL lasers, as well as the images of the missile plume and the optical signals for the AO compensation system. The window must be constructed to minimize air turbulence and distortion for the optical signals.

These are design goals for the HEL:

- Beam power of several megawatts. The actual power specification is classified, but we assume $3 \mathrm{MW}$ for the purposes of this report.

- Beam quality: 1.2 times diffraction limit.

\subsection{Description of COIL}

The COIL was first demonstrated at the Air Force Weapons Laboratory in 1977. Chemical oxygen-iodine lasers are currently being developed in several countries for a range of applications, including industrial uses, for reasons which include their high $\mathrm{cw}$ power at relatively low cost and suitable wavelength for fiber optics and interaction with materials. An example of demonstrated high-power $(30 \mathrm{~kW})$, was the RotoCOIL, which had an aperture of about $40 \mathrm{~cm}^{2}$ and a gain length of $54 \mathrm{~cm} \mathrm{[182].}$

The primary chemical reactions in the ABL COIL are:

$$
\begin{gathered}
\mathrm{Cl}_{2}(\mathrm{~g})+\mathrm{H}_{2} \mathrm{O}_{2}(\mathrm{l})+2 \mathrm{KOH}(\mathrm{l}) \rightarrow \mathrm{O}_{2}\left(\mathrm{a}^{1} \Delta\right)+2 \mathrm{KCl}+2 \mathrm{H}_{2} \mathrm{O} \\
\mathrm{O}_{2}\left(\mathrm{a}^{1} \Delta\right)+\mathrm{I}_{2} \rightarrow \mathrm{O}_{2}\left(\mathrm{X}^{3} \Sigma\right)+\mathrm{I}_{2}(\mathrm{v}) \\
\mathrm{O}_{2}\left(\mathrm{a}^{1} \Delta\right)+\mathrm{I}_{2}(\mathrm{v}) \rightarrow \mathrm{O}_{2}\left(\mathrm{X}^{3} \Sigma\right)+2 \mathrm{I}\left({ }^{2} \mathrm{P}_{3 / 2}\right) \\
\left.\mathrm{O}_{2}\left(\mathrm{a}^{1} \Delta\right)+\mathrm{I}\left({ }^{2} \mathrm{P}_{3 / 2}\right) \rightarrow \mathrm{O}_{2}\left(\mathrm{X}^{3} \Sigma\right)+\mathrm{I}^{2} \mathrm{P}_{1 / 2}\right) \\
\left.\mathrm{I}\left({ }^{2} \mathrm{P}_{1 / 2}\right)+\mathrm{h} \nu \rightarrow \mathrm{I}^{2} \mathrm{P}_{3 / 2}\right)+2 \mathrm{~h} \nu \quad(\lambda=1.315 \mu \mathrm{m})
\end{gathered}
$$

Reaction 18.1 involves a multi-step process for the production of excited-state $\mathrm{O}_{2}\left(\mathrm{a}^{1} \Delta\right)$. In an ABL-COIL gain module, basic hydrogen-peroxide BHP, a mixture of hydrogen peroxide, 
potassium hydroxide, ${ }^{1}$ and water, is sprayed through holes in an injector plate, forming a droplet field [183]. A helium-chlorine gas mixture is forced through this droplet field, and the resulting reactions yield $\mathrm{O}_{2}\left(\mathrm{a}^{1} \Delta\right)$ and chlorine as dissolved salt. Remaining BHP is recycled, water vapor is removed, and waste heat and gases are exhausted beneath the airplane through a titanium exhaust system. Excited $\mathrm{O}_{2}\left(\mathrm{a}^{1} \Delta\right)$ is relatively long-lived in the absence of collisions, and it is transported to the next reaction region by helium gas flow.

The He- $\mathrm{O}_{2}\left(\mathrm{a}^{1} \Delta\right)$ mixture is accelerated through a supersonic nozzle and $\mathrm{I}_{2}$ molecules are injected into the flow. In Eqs. 18.2-18.3, excited $\mathrm{O}_{2}\left(\mathrm{a}^{1} \Delta\right)$ dissociates an iodine molecule, forming atoms in the ground state through a multi-step collision process. It is estimated that several $\mathrm{O}_{2}\left(\mathrm{a}^{1} \Delta\right)$ excited-state molecules are effectively removed in the dissociation of an $\mathrm{I}_{2}$ molecule, a process that probably proceeds through vibrationally excited $\mathrm{I}_{2}(\mathrm{v})$.

In Eq. 18.4, the ground-state iodine atoms are excited to the spin-orbit split state $\mathrm{I}\left({ }^{2} \mathrm{P}_{1 / 2}\right)$ by resonant energy transfer from the excited $\mathrm{O}_{2}\left(\mathrm{a}^{1} \Delta\right)$. Large-diameter optics, situated transverse to the reacting flow and placed out of the reaction zone, form a resonator cavity structure that provides optical feedback, beam-power build up, and output coupling of laser power to the ABL beam control systems and ultimately to the target missile. The COIL lases at a wavelength of $1.315 \mu \mathrm{m}$ (Eq. 18.5). The cross section for stimulated emission on the $\mathrm{I}\left({ }^{2} \mathrm{P}_{1 / 2} \rightarrow{ }^{2} \mathrm{P}_{3 / 2}\right)$ laser transition is about $10^{-17} \mathrm{~cm}^{2}[184, \mathrm{p}$. 330]. Typical gain in a COIL device is about $1 \% / \mathrm{cm}$ [185] and a typical saturation intensity is $10 \mathrm{~kW} / \mathrm{cm}^{2}[184$, p. 334]. A laser system having an aperture of $25 \mathrm{~cm}$ and sufficient gain length and appropriate mirror reflectivity should therefore be capable of generating multi-megawatt output power. Large aperture lasers of this type typically operate with unstable resonator mirror structures, allowing good spatial-mode overlap with the gain region and a relatively highquality (transverse mode quality) output beam. In the ABL, an architecture that uses up to 14 separate laser gain modules is planned. Figure 18.2 shows the layout of the HEL components.

Chemicals are typically consumed in the chemical reactions at a rate of about $100 \mathrm{~kg}$ per $10 \mathrm{MJ}$ of energy produced by a COIL [184, p. 321].

\subsection{Chronology of the Airborne Laser}

The Air Force launched the ABL program in FY 1994. It followed the Airborne Laser Laboratory (ALL) weapons program of the 1970s and early 1980s which used a $\mathrm{CO}_{2}$ laser system. In 1996, the Program Definition and Risk Reduction (PDRR) phase contract was awarded to Team-ABL (Boeing, TRW, and Lockheed Martin).

Milestones of the ABL effort included a Preliminary Design Review (1998), Authority to Proceed-1 (1998); System-Level Critical Design Review (2000); and delivery of the first aircraft, YAL-1A, (2001). Flight tests against a representative TBM in boost phase are planned in 2004. Following successful demonstration of missile destruction, the Engineering and Manufacturing Development (EMD) phase is planned to begin in the middle of this decade. The PDRR phase includes 6 COIL-laser modules for each aircraft, while the EMD phase plans 14 laser modules. Production is planned for seven aircraft. Initial operational capability could be fielded by 2008-10; a date for full operational capability has not yet been determined [178].

Funding and program management for the ABL was transferred to the Ballistic Missile Defense Office (BMDO) in 2001. In 2002, this office was renamed the Missile Defense

\footnotetext{
${ }^{1}$ In practice a mixture of alkaline hydroxides, $\mathrm{LiOH}, \mathrm{KOH}$, and $\mathrm{NaOH}$, are used instead of simply $\mathrm{KOH}$, but for simplicity, this discussion uses only $\mathrm{KOH}$.
} 


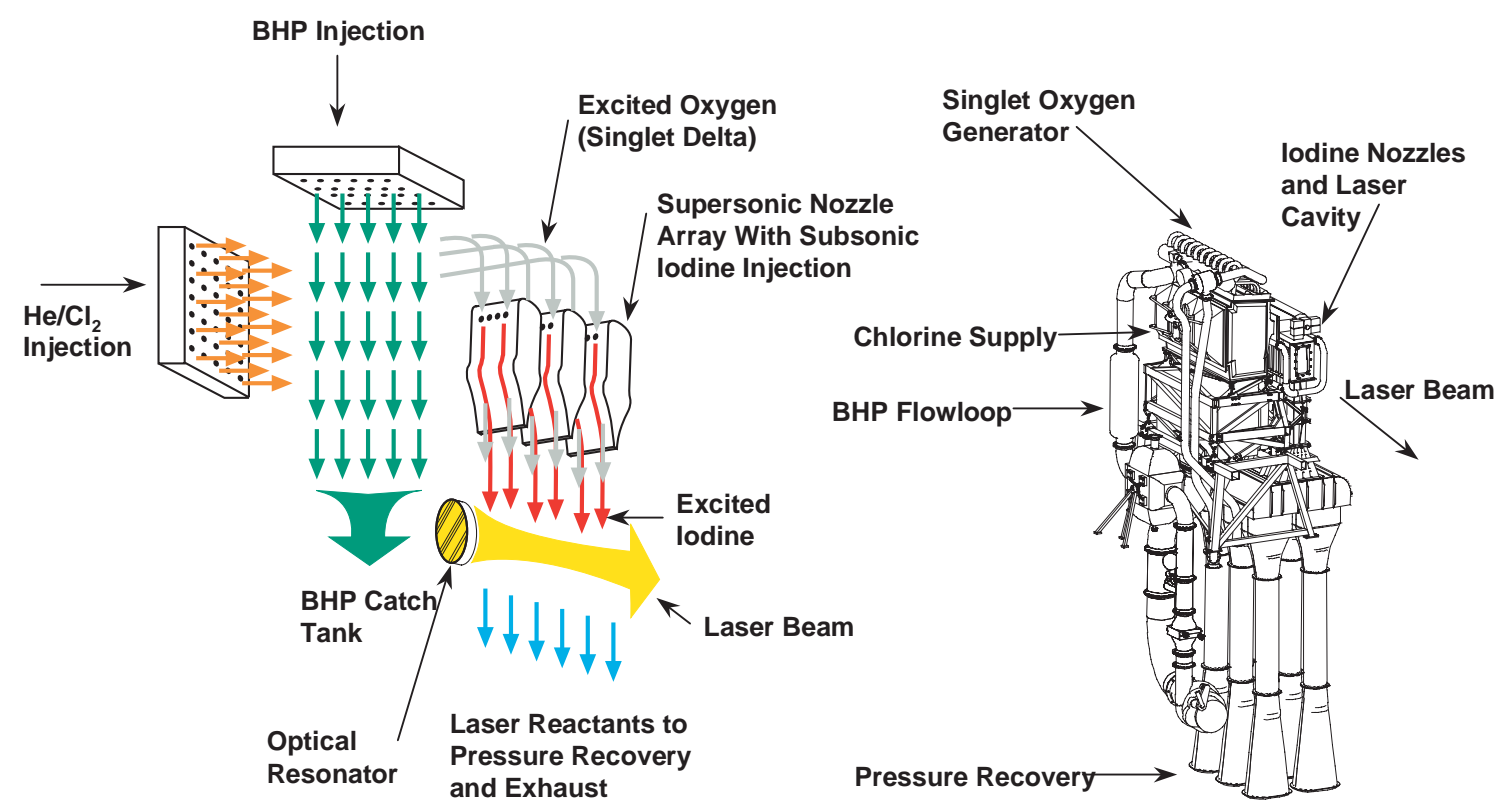

9701148.002.SGD11

Figure 18.2. Elements of the High Energy Laser. Schematic diagram of laser (left); illustration of laser gain module (right). Reproduced with permission from [183].

Agency.

\subsection{Airborne Laser Performance}

Because the measured performance of the laser is classified, little information is available to evaluate how nearly the ABL approaches its design goals. From published information, two areas deserve attention:

- A recent GAO report [186] states that the present laser with 6 modules weighs 180,000 pounds (82 tonnes), compared with the design flight weight of 175,000 pounds (79 tonnes) for the full laser of 14 modules. Each module weighs about 6,000 pounds (2.7 tonnes). Unless or until this weight problem is solved, the power output is likely to be less than half of the design power.

- It has been reported by GAO [187] that the laser modules have achieved power output exceeding the design requirements. However, these tests were performed using a stable resonator rather than the unstable resonator that is called for in the design. A later GAO report [186] states that the laser modules have not yet been tested with an unstable resonator. The unstable resonator is important to achieve the high spatial mode quality and for efficient extraction of energy from the laser medium. 


\section{References for Chapter 18}

[178] Defense Science Board, "Defense Science Board Task Force on High Energy Laser Weapon System Applications." Technical report, Office of the Under Secretary of Defense for Acquisition, Technology, and Logistics, June 2001.

[179] Steven M. Daigneault et al., "ABL Subscale Deformable Mirror Risk Reduction Tests," SPIE 3706, 304 (1998).

[180] Michael E. Davey and Frederick Martin, "The Airborne Laser Anti-Missile Program." Technical Report RL30185, CRS, May 1999. CRS Report to Congress.

[181] Kenneth W. Billman et al., "Development of Low-OPD Windows for Airborne Laser," SPIE 4376, 45 (2001).

[182] G. D. Hager et al., IEEE J. Quantum Electron. 32, 1535 (1996).

[183] S. Lamberson, "The Airborne Laser," in High-Power Laser Ablation IV, SPIE 4760, 25 (2002).

[184] N. G. Basov, "Chemical Lasers," (Springer Verlag), 1990.

[185] R. F. Tate et al., IEEE J. Quantum Electron. 31, 1632 (1995).

[186] General Accounting Office, "Knowledge-Based Decision Making Needed to Reduce Risks in Developing the Airborne Laser." Technical Report GAO-02-631, U.S. Government, July 2002. Available at: http://www.gao.gov/new.items/d02631.pdf.

[187] General Accounting Office, "DOD Efforts to Develop Laser Weapons for Theater Defense." Technical Report GAO/NSIAD-99-50, U.S. Government, March 1999. Available at: http://www.gao.gov/archive/1999/ns99050.pdf. 


\section{Chapter 19}

\section{Airborne Laser Beam Propagation}

\section{Contents}

\begin{tabular}{|c|c|c|}
\hline 19.1 & Vacuum Propagation $\ldots \ldots \ldots \ldots \ldots$ & S303 \\
\hline 19.2 & Atmospheric Effects & S304 \\
\hline 19.3 & Characterization of Turbulence . & S305 \\
\hline 19.4 & Adaptive Optics $\ldots \ldots \ldots \ldots \ldots \ldots \ldots$ & S306 \\
\hline 19.5 & Benchmarking Adaptive Optics Hardware and Algorithms & S307 \\
\hline 19.6 & Other Issues $\ldots \ldots \ldots \ldots \ldots \ldots \ldots \ldots \ldots$ & S309 \\
\hline 19.7 & Engagement Envelope $\ldots \ldots \ldots \ldots \ldots$ & S309 \\
\hline
\end{tabular}

This chapter lays the groundwork for understanding the requirements for an effective laser weapon to function in the atmosphere. Section 19.1 discusses the basic optics of propagation in the absence of atmospheric disturbance. Section 19.2 gives an overview of atmospheric effects and briefly describes measures used to describe the disturbances. Section 19.3 characterizes the atmosphere in the framework of a widely used model. Sections 19.4 and 19.5 discuss the use and parameters of adaptive optics (AO) along with benchmark experiments to test the AO design. Finally, Section 19.7 describes the parameters of the engagement "envelope" compatible with the ABL design presented in Chapter 18. The symbols used in this chapter are summarized on page S310.

We assume that the ABL can deliver the laser beams meeting its specifications. However, the atmosphere is not perfectly homogeneous, which affects the size of the spot on the missile that is illuminated by the laser beam. The beam power and spot size are crucial parameters for delivering the fluence $\left(\mathrm{MJ} / \mathrm{m}^{2}\right)$ required to cause the requisite damage to disable the target. Thus, propagation of those beams through the atmosphere requires particular attention, and we have analyzed this problem in some depth. This chapter provides an overview, and details for the interested reader are given in Appendix D.

\subsection{Vacuum Propagation}

For a total power $P$, an ideal diffraction-limited laser beam of diameter $D$ and wavelength $\lambda$, focused on a target at a distance $L$, will deliver an irradiance $I$, the power per unit area, as a function of transverse coordinates $r$ and $\phi$ of

$$
I(r, \phi)=\frac{\pi}{4}\left(\frac{D}{\lambda L}\right)^{2}\left[\frac{2 J_{1}(\pi r D /(\lambda L))}{\pi r D /(\lambda L)}\right]^{2} P
$$


where $J_{1}$ is the Bessel function of order 1 .

A real ABL laser beam will not have an exactly uniform profile, and there will probably be a "hole" in the center as part of the optical design. The central hole has a minimal effect. $^{1}$ There will be some imperfections in the beam quality, too. ${ }^{2}$ A measure of optical performance of a system is the Strehl ratio $S_{R}$, which is the ratio of the peak irradiance delivered divided by the same quantity for a diffraction-limited system.

\subsection{Atmospheric Effects}

Even very small variations of the index of refraction $n$ of the atmosphere can significantly reduce the Strehl ratio, because the path length is so long. These variations come about because of small fluctuations in the local temperature, which in turn cause variations in the density, and thus the index of refraction.

The simplest manifestation of phase variations is a "tilt" of the beam. ("Tilt" refers to the first moments in transverse angle coordinates around the $x$ and $y$ axes.) The tilt causes the beam spot to move rapidly about its central point - the primary problem ground-based astronomers have with getting a good image. Any research-grade telescope coming close to the diffraction limit must do something to avoid this problem. Adaptive optics have been developed to overcome this problem, as well as some of the higher-order distortions (see Appendix D.3). Correcting for atmospheric effects for the ABL is more difficult than for astronomical telescopes because the laser beam travels through the atmosphere obliquely, giving it a much longer atmospheric path than for astronomical viewing. This is discussed in Section 19.4.

The variation of the index of refraction is a statistical problem. Kolmogorov [188] developed a model of the atmosphere used in many analyses. The parameter $C_{n}^{2}$ is a local measure of the turbulence, and values of $C_{n}^{2}$ vary with time, altitude, and atmospheric conditions. The ABL flies at an altitude $\geq 12 \mathrm{~km}$, above the worst atmospheric turbulence and above most cloud cover. Characteristically, $C_{n}^{2} \approx 5 \times 10^{-18} \mathrm{~m}^{-2 / 3}$ at an altitude of $12 \mathrm{~km}$, but it can fluctuate by an order of magnitude above or below this value with even small changes of altitude.

Maxwell's equations for the beam propagation are solved in the Rytov approximation (see D.1), which is valid for low turbulence. For many calculations, integrals of $C_{n}^{2}$ are computed along the path of propagation $z$ with weighting factors depending on $z$. The following quantities are of primary interest. Appendix D provides additional details.

- $r_{0}$ (see Eq. D.6), the Fried parameter, is a measure of the transverse characteristic coherence length for an engagement. For typical ABL engagements, it ranges from $0.26 \mathrm{~m}$ at low altitudes to $0.8 \mathrm{~m}$ at high altitudes.

- $\sigma_{t x y}$ (see Eq. D.7) is the rms tilt angle of a beam caused by turbulence, and it is the dominant cause of beam spot spreading on target without AO. For comparison, the core of a diffraction-limited intensity profile is similar to a Gaussian having a standard deviation $\sigma=0.45 \lambda / D$. The rms tilt achieves this value for $r_{0} \approx D$, which is not reached, even for the highest altitude engagements.

\footnotetext{
${ }^{1}$ In Eq. $19.1, D^{2}$ is replaced by $D^{2}-d^{2}$, where $d$ is the diameter of the central hole, $\approx 0.3 \mathrm{~m}$, compared to $D=1.5 \mathrm{~m}$.

${ }^{2}$ The beam shape is an octagon, not a circle. It is not clear if the corners are clipped. In addition, the intensity profile across the aperture may not be uniform.
} 
- $\sigma_{\varphi}^{2}$ is the spatial variance of the phase of the beam caused by turbulence. Phasecorrection $\mathrm{AO}$ seek to minimize this quantity (see Eqs. D.8, D.10, D.27, D.29, and D.32).

- $\sigma_{R}^{2}$ (see Eq. D.12), the Rytov variance, is the spatial variance of the log amplitude of the electric field. It is the most widely used single measure of turbulence for an engagement. The region of validity of the Rytov approximation is essentially $\sigma_{R}^{2} \leq 0.5 \mathrm{np}^{2} \cdot{ }^{(3)}$

- $\theta_{0}$ (see Eq. D.28), the isoplanatic angle, is a measure of how rapidly the phase variations change with angle away from the center of view because the beam passes through a different part of the atmosphere. Stated differently, it is a measure of the field angle over which the AO can be effective.

For large phase variances, e.g. before the application of AO, the Strehl ratio is given by an asymptotic series [189, p. 164]

$$
S_{R} \approx\left(\frac{r_{0}}{D}\right)^{2}-0.6159\left(\frac{r_{0}}{D}\right)^{3}+0.05\left(\frac{r_{0}}{D}\right)^{5}+0.00661\left(\frac{r_{0}}{D}\right)^{7} \ldots\left(r_{0} / D<1\right) .
$$

This expression is applicable for engagements of interest and sets a lower bound for the atmospheric part of the Strehl ratio without AO. This limit on $S_{R}$ ranges from 0.02 to 0.2 , depending on the engagement geometry. For small variances, e.g. in low turbulence or after application of AO, the Strehl ratio is given approximately by $[189, \mathrm{p} .46]$

$$
S_{R} \approx \exp \left(-\sigma_{R}^{2}-\sigma_{\varphi}^{2}\right)
$$

This relation is called the extended Maréchal limit, and it underestimates the Strehl ratio; (see Section D.5.3). After AO, $\sigma_{\varphi}^{2}$ is used in Eq. 19.3 as appropriate for the degree of correction achieved.

\subsection{Characterization of Turbulence}

Correctly characterizing the turbulence with which the ABL must contend is critical. The first question is how values of $C_{n}^{2}$ vary with locale, season of year, and day to day. What fraction of the time would the turbulence be low enough for the ABL to perform its mission if called upon?

A standard model of atmospheric turbulence is called "Clear-1" [190]. The ABL specification is that it must function properly despite turbulence as intense as twice this model. The model is based on smoothed thermal measurements made with balloons. The model assumes that the $C_{n}^{2}$ depends only on altitude, and in particular it is independent of time and geographical location. There is a broad plateau of $C_{n}^{2} \approx(0.5-1.0) \times 10^{-17} \mathrm{~m}^{-2 / 3}$ for altitudes between 10 and $17 \mathrm{~km}$. Values fall rapidly at greater altitudes (see Appendix D.2).

There has been some controversy regarding $C_{n}^{2}$. Measurements of $C_{n}^{2}$ have been made using optical methods [191], thermal methods [192, 193], and radar methods [192, 194]. The consistency among various measurements is not very good. At least part of the discrepancy results from the natural variations of $C_{n}^{2}$ itself; its values are not expected to be static in time. Geographical variations are also expected [195]. Two General Accounting Office (GAO) reports [196, 197] and the United States Air Force (USAF) Scientific Advisory

\footnotetext{
${ }^{3}$ The unit $\mathrm{np}=$ neper is used to emphasize the use of natural log instead of common log.
} 
Board [198] commented on this discrepancy and recommended that optical measurements be the primary reference. The GAO report [197] is critical of the state of the knowledge of turbulence in theaters of interest. More measurements have been made using balloons, radar, and stellar scintillometers [199] in southern Asia and the Middle East, but the results have not been published [200]. As we shall see in Section 21.5.2, whether the standard $C_{n}^{2}=2 \times$ "Clear 1 Night" or $4 \times$ as advocated by some critics is used makes no qualitative difference for the engagements of interest to this study, because of the high engagement altitudes.

It should be noted that although the normal ABL operating altitude of $12 \mathrm{~km}$ is above most of the turbulence (which decreases rapidly above the tropopause), the altitude of the tropopause varies with season and location. Greater turbulence and high-altitude cirrus clouds may sometimes present problems (see Section 22.2).

An interesting phenomenon is that of large-scale waves that are created in the atmosphere as air masses flow across mountains [201]. These waves may create unusually intense turbulence even at high altitudes. It is not known to what extent, if any, this effect could influence ABL engagements.

\subsection{Adaptive Optics}

Although astronomers first suggested the use of AO [202], its first use was for military applications in the mid 1970s and early 1980s. ${ }^{4}$ Astronomers now use AO to compensate for the image broadening due to atmospheric turbulence. Deformable mirrors are used in conjunction with a reference signal (guide or reference star, either real or artificial) to largely compensate errors, with impressive results. For example, the Keck telescope's adaptive optics system achieved a Strehl ratio of 0.30 at $\lambda=1.65 \mu \mathrm{m}$ compared to an uncorrected Strehl ratio of 0.006 [204].

Adaptive optics are essential to the ABL program. However, for a number of reasons $\mathrm{AO}$ is more difficult to implement for the ABL than for astronomical applications. Here are some of the AO challenges for ABL and how they differ from the astronomical case:

1. Astronomy deals with optical paths through the atmosphere not far from the zenith (of the order of $15 \mathrm{~km}$ ). The ABL deals with a slant range through the atmosphere of the order of $100 \mathrm{~km}$ to $300 \mathrm{~km}$.

2. The ABL requires a very high bandwidth AO operation. Astronomical AO systems run at a closed-loop bandwidth of $10-100 \mathrm{~Hz}$, whereas the ABL's AO system requires a closed-loop bandwidth of $500 \mathrm{~Hz}$. This bandwidth requirement is more demanding than has been achieved on ground-based telescopes to date.

3. The isoplanatic angle $\theta_{0}$ is a measure of the characteristic angle over which the AO corrections change. A separation of the "guide star" (beacon) from the target by an angle larger than the isoplanatic angle substantially degrades AO performance. The isoplanatic angle is much smaller for the ABL than for astronomical applications. It can be between 1 to 10 times the diffraction limit angle for the ABL, depending on the engagement. For near-infrared 8-10 m telescopes, the isoplanatic angle can be several hundred times the diffraction limit. This difference arises because most of the turbulence is distributed near the ground for the astronomical case, but it is distributed over a much longer length for the ABL.

\footnotetext{
${ }^{4}$ A nice historical account of the development of AO may be found in Ref. [203, p. 16 et seq.].
} 
4. Because of the long flight path from the ABL to target and back, there is a significant delay just from the propagation time of light between receiving an image to correct with $\mathrm{AO}$ and delivering the beam on target. In this time, the ABL and the target move, and the atmosphere may have changed. This is another form of anisoplanatism.

5. For the ABL, atmospheric turbulence causes large fluctuations in the intensity of the beam, which is called scintillation. The ABL program does phase-only corrections for the wavefront. With the large effect of scintillation, the sufficiency of phase-only AO could be an issue.

6. Although astronomical telescopes suffer from vibrations due to wind-shake and other drivers, the ABL has airframe flexure and vibration that are of considerably larger amplitude and that may be of higher frequency as well.

\subsection{Benchmarking Adaptive Optics Hardware and Algorithms}

Several scaled test beds have been set up to simulate the scaled ABL conditions. Those simulations chose the wavelengths of the lasers, the size of the optics, the length of propagation, and the turbulence so that four dimensionless parameters match that of a characteristic ABL TBM engagement [205]:

- Fresnel number $D^{2} /(\lambda L)=6.34,{ }^{5}$

- $D / r_{0}=5.86$

- $\theta_{0} /(\lambda / D)=0.65$, and

- $\sigma_{R}^{2}=0.49 \mathrm{np}^{2}$.

Using such test beds, the performance of the AO can be tested quantitatively.

The MIT Lincoln Lab built the ABL Firepond facility in hilly terrain to test AO over a range of $5.4 \mathrm{~km}$. By choosing the data during a calm period shortly before sunset, turbulence representing appropriate ABL parameters could be obtained. A mock missile, a cylinder having a pointed end, served as the optical target. Beam splitters at the target analyzed the beams incident on the target. Two major results are reported [206, 207, 208, 209] from these measurements:

- Tracking error (tilt) due to scintillation and speckle on the target could be substantially reduced by the use of multiple, incoherent target illuminator (TILL) lasers. This is particularly important to control tilt jitter in the direction along the missile.

- The Strehl ratio could be improved by a factor as great as 4 by the AO, achieving $S_{R}$ as large as 0.15 for $\sigma_{R}^{2}=0.5 \mathrm{np}^{2}$ for a nearly ideal laser source. This is the worst case for the ABL design envelope. The Strehl ratio will be better for engagements having lower values of $\sigma_{R}^{2}$.

The ABL Advanced Concepts Testbed (ABL ACT) at the White Sands Missile Range is also a facility scaled to ABL parameters, like Firepond, but the range is $51 \mathrm{~km}$. In this case the laser was fixed as in Firepond, but the target was carried on the side of an airplane. The target provided a "cooperative beacon," i.e., a laser diode, instead of the uncooperative

\footnotetext{
${ }^{5}$ From the Fresnel number, one can deduce $L=270 \mathrm{~km}$. For simplicity this report uses $300 \mathrm{~km}$.
} 
reflected image of a mock missile. Results from this test stand [210] report the ability of the AO to improve the Strehl ratio and centroid jitter by factors of about 4 .

The Lockheed Martin Sunnyvale "Brassboard" is another very powerful experimental simulator scaled to ABL parameters [205, 211]. It is of laboratory dimensions, having a range of only $16 \mathrm{~m}$. It is also particularly elegant, in that it can "make an atmosphere to order" by means of 7 rotating phase screens in the optical path. The AO closely follows the ABL design, and the simulator employs 80 percent to 90 percent of the ABL beam control system hardware. There are four target-illuminator and four beacon-laser sub-beams. Brassboard's performance has been benchmarked against the ABL ACE and Firepond. In addition to the turbulence correction issues, Brassboard also deals with the motion of the aircraft. Since pointing accuracy is crucial and the flight of the aircraft itself wanders and jitters by much more than allowed, correction is necessary. The Brassboard includes an inertial reference transfer unit (IRTU) providing inputs to the steering mirrors. Two major results are reported from these experiments:

- Firepond results are reproduced, $S_{R} \approx 0.15$ for $\sigma_{R}^{2} \approx 0.5 \mathrm{np}^{2}$.

- Results of propagation code are reproduced.

Another scaled laboratory with a "made-to-order" atmosphere using rotating phase screens similar to the Brassboard is the Advanced Concepts Laboratory (ACL) at MIT's Lincoln Lab. This set-up has a range of 10 to $28 \mathrm{~m}$ with 7 rotating phase screens. Its feedback loop has a bandwidth of $2.5 \mathrm{~Hz}$, which scales to the ABL's design value of $500 \mathrm{~Hz}$. Unfortunately, only results for $\sigma_{R}^{2} \leq 0.25 \mathrm{np}^{2}$, or in a few cases $0.34 \mathrm{np}^{2}$, are presented. Reported results are: [212]

- Results of the propagation code are reproduced.

- The tilt jitter perpendicular to the missile axis is about the same as from a point source, but the jitter along the axis is 2 to 3 times as large, largely due to scintillation.

- The main loss of Strehl ratio comes from tracking (tilt) errors.

- Extended beacon spot sizes have only a modest deleterious effect on the performance of the AO.

It is worth noting that none of these simulations can properly test effects involving propagation delay because of the short baseline (20 $\mathrm{m}$ to a few kilometers) compared with hundreds of kilometers in the actual ABL system. This issue will arise in subsequent discussion. In principle the results should not change, but part of the AO algorithm is not tested.

There are reports that a test facility has demonstrated pointing correction for the aircraft at the level of $100 \mathrm{nrad}$ [213] and [214, p. 4], but the reports have too few details to evaluate. Since no tests in an aircraft are yet possible, this test must be some sort of scaled measurement of the degree to which a feedback system could accommodate some assumed flexing, vibration, and wandering of the aircraft.

In summary, making the ABL beam propagation work to the specifications stated will be a technical challenge, but we see no intrinsic reason these goals cannot be reached if adequate research and engineering resources are given to the project. In particular, if the engagement altitudes are appropriately chosen, slant ranges of 300 to $600 \mathrm{~km}$ against ICBMs 
should be achievable. ${ }^{6}$ We shall assume that a Strehl ratio $\geq 0.13$ can be achieved for an ideal laser in engagements. It can be substantially better for high-altitude engagements where the turbulence is less. A large uncertainty is the engagement time, which depends on the hardness of the threat missile. See Section 20.1.

\subsection{Other Issues}

A potential concern is the occurrence of unusually high-altitude cirrus clouds. See Section 22.2 for more information.

Other issues have been raised, such as

1. The adaptive optics do a phase correction, but no amplitude correction.

2. Branch points in the phase of the wavefront occur at zeros of the amplitude.

3. Chromatic anisoplanatism due to the difference of wavelengths of the TILL, BILL, and HEL.

4. Nonlinear phenomena.

5. Aerosol scattering.

None of these is seen as a serious problem; see Appendix D.6 for discussion.

\subsection{Engagement Envelope}

We define engagement requirements in these terms, making an arbitrary choice that the engagement time $t_{e} \leq 20 \mathrm{~s}:$

- Turbulence $\leq 2 \times$ Clear-1 Night,

- $\sigma_{R}^{2} \leq 0.5 \mathrm{np}^{2}$,

- $r_{0} \geq 0.26 \mathrm{~m}$,

- $\sigma_{t} \leq 1.8(\lambda / D)$

- $\theta_{0} \geq 0.65(\lambda / D)$,

- $t_{e} \leq 20 \mathrm{~s}$.

The limits on the parameters $r_{0}, \sigma_{t}$, and $\theta_{0}$ (see Appendix D.1 and D.4) are limits set by atmospheric turbulence and the ability to do AO corrections. The limit on $t_{e}$ is one of practicality. If the engagement time is too long, the target kinematics can change, such as staging or burnout. In addition, the fuel magazine is limited, and the ABL needs to have the capability of multiple shots.

Further analysis of the problem of beam propagation is presented in Appendix D. We turn now to examining the effect of the laser beam on a missile.

\footnotetext{
${ }^{6}$ This statement does not necessarily apply to the theater defense role in which the burnout altitude of a short range missile may be low enough to interfere with the minimum engagement altitude requirement.
} 


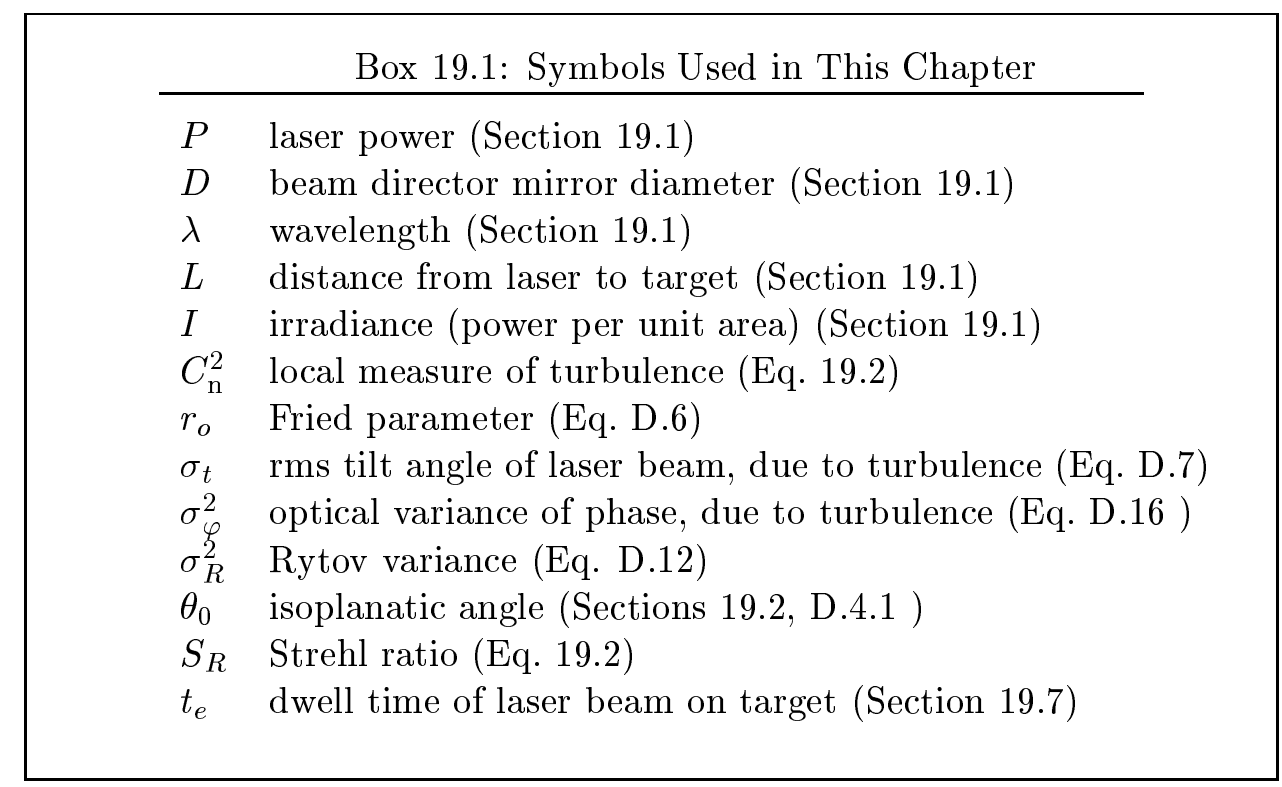

\section{References for Chapter 19}

[188] A. Kolmogorov, Turbulence: Classic Papers on Statistical Theory, page 151. Interscience, 1961.

[189] Richard J. Sasiela, Electromagnetic Wave Propagation in Turbulence. (Springer Verlag) 1994.

[190] Robert R. Beland, The Infrared and Electro-Optical Systems Handbook, Vol. 2, eds. Joseph S. Acetta and David L. Shumaker, International Society for Optical Engineering, 1993.

[191] Russell Butts, "U.S. Air Force Phillips Laboratory Interview." Technical report, United States Air Force, December 1995. Available at: http://www.spie.org/web/oer/december/oer_dec95_1.html.

[192] Frank D. Eaton et al., "Radar and Aircraft Observations of a Layer of Strong Refractivity Turbulence," SPIE 3381, 230 (1998).

[193] Scott Nowlin, Ila Hahn, and Bob Bishop, "Qualitative Comparison of Concurrent Vertical Optical Turbulence Profiles from an Aircraft and Balloons over White Sands Missile Range," SPIE 3706, 95 (1999).

[194] Frank D. Eaton et al., "Variability of Slant-Path Turbulence Parameters and Inner Scale Effects," SPIE 3706, 80 (1999).

[195] Robert I. Reznik and Matthew Bold, "Optical Turbulence Path Integration: Methodology," SPIE 4034, 58 (2000).

[196] General Accounting Office, "Significant Technical Challenges Face the Airborne Laser Program." Technical Report GAO/NSIAD-98-37, U.S. Government, October 1997. Available at: http://www.gao.gov/archive/1998/ns98037.pdf. 
[197] General Accounting Office, "DOD Efforts to Develop Laser Weapons for Theater Defense." Technical Report GAO/NSIAD-99-50, U.S. Government, March 1999. Available at: http://www.gao.gov/archive/1999/ns99050.pdf.

[198] Scientific Advisory Board, "Airborne Laser Scenarios and Concept of Operations." Technical report, U.S. Air Force, February 1998.

[199] Robert R. Beland and John R. Roadcap, "Analysis of Atmospheric Effects on Stellar Scintillation," SPIE 4034, 33 (2000).

[200] Director, Operational Test and Evaluation, "Annual Report FY00 Airborne Laser." Technical report, U.S. Air Force, 2000. Available at: http://www.dote.osd.mil/reports/FY00/airforce/00abl.html.

[201] T. R. Akylas, "Gravity-wave dynamics in the atmosphere." Briefing Presented to the APS Study Group, September 2001.

[202] H. W. Babcock, "The possibility of compensating astronomical seeing," Pub. Astr. Soc. Pac. 65, 229 (1953).

[203] John W. Hardy, Adaptive Optics for Astronomical Telescopes. (Oxford University Press, New York) 1998.

[204] P. Wizinowich et al., "First Light Adaptive Optics Images from the Keck II Telescope: A New Era of High Angular Resolution Imagery," Pub. Astr. Soc. Pac. 112, 315 (2000).

[205] Kenneth W. Billman et al., "ABL Beam Control Laboratory Demonstrator," SPIE 3706, 172 (1999).

[206] Charles Higgs, "Overview of the ABL-Firepond Active-Tracking and Compensation Facility," SPIE 3381, 14 (1998).

[207] C. Higgs et al., "Adaptive-Optics Compensation Using Active Illumination," SPIE 3381, 47 (1998).

[208] C. Higgs et al., "Active Tracking Using Multibeam Illumination," SPIE 3381, 160 (1998).

[209] C. Higgs et al., "Multibeam Laser Illuminator Approach," SPIE 3706, 206 (1999).

[210] C. Higgs et al., "Dynamic Target Board for ABL ACT Performance Characterization," SPIE 3706, 216 (1999).

[211] Kenneth W. Billman et al., "Airborne Laser System Common Path/Common Mode Design Approach," SPIE 3706, 196 (1999).

[212] Mitchell H. Fields et al., "Initial results from the Advanced-Concepts Laboratory for adaptive optics and tracking," SPIE 4034, 116 (2000).

[213] Boeing News Release, "ABL Beam Control System Laboratory Demonstrator Shows Performance Needed for Airborne Laser." Available at: http://www.Boeing.com/news/releases/1998/news_release_980429c.htm, April 1998. 
[214] Michael E. Davey and Frederick Martin, "The Airborne Laser Anti-Missile Program." Technical Report RL30185, CRS, May 1999. CRS Report to Congress. 


\section{Chapter 20}

\section{Disabling Missiles Using the Airborne Laser}

\section{Contents}

20.1 Effects of Laser Beams on Boosters and Warheads . . . . . S314

20.2 Kill Assessment . . . . . . . . . . . . . . . S316

The ABL was designed for theater missile defense. For that purpose, it must engage a variety of missiles, from relatively short-range Scuds to longer-range missiles such as the Taepo Dong 1. See Table 20.1 for a summary of the characteristics of both theater missiles and ICBMs [215, 216, 217, 218, 219, 220, 221].

In some ways, intercepting ICBMs is less difficult than intercepting theater missiles. The time line is longer and the burnout altitude is greater. The short time line for a Scud B is very stressing, but the longer-range missiles are much less troublesome. Because the amount of turbulence decreases at high altitude, a higher burnout altitude reduces the degradation of laser performance by atmospheric turbulence.

Section 20.1 of this chapter describes the requirements for destroying missiles using a laser beam. Estimates for the energy density (fluence) required to disable both liquidand solid-propellant missiles are given. It is important to know when and if an engagement attempt resulted in a successful intercept, and issues related to this verification are discussed in Section 20.2.

Table 20.1. Summary of Missile Properties

\begin{tabular}{|c|c|c|c|}
\hline Name & $\begin{array}{r}\text { Range } \\
(\mathrm{km})\end{array}$ & $\begin{array}{l}t_{b o}^{a} \\
(\mathrm{~s})\end{array}$ & $\begin{array}{r}h_{b o}^{b} \\
(\mathrm{~km})\end{array}$ \\
\hline Scud B & 300 & 60 & 31 \\
\hline No Dong & 1000 & 95 & 69 \\
\hline Taepo Dong 1 & 3000 & 165 & 150 \\
\hline Titan II & 12000 & 320 & 360 \\
\hline Liquid-propellant ICBM $^{c}$ & 12000 & 240 & 175 \\
\hline Solid-propellant ICBM ${ }^{c}$ & 12000 & 170 & 200 \\
\hline
\end{tabular}

${ }^{a}$ Time at burnout.

${ }^{b}$ Altitude at burnout.

${ }^{c}$ Models defined in Section 15.1. 


\subsection{Effects of Laser Beams on Boosters and Warheads}

We begin by using available information for the ABL in theater missile defense as an introduction. Unfortunately, there appears to be no definitive public statement of a theater missile defense engagement for the ABL that could be used to estimate performance of the ABL. Putting together information from various sources, however, provides a consistent scenario of a slant range $L \approx 300 \mathrm{~km}$ and an engagement time $t_{e} \approx 5 \mathrm{~s}$. The exact laser power is classified, but public statements give it as "multi-MW"; we assume $P=3 \mathrm{MW}$. This report deals with ICBM engagements, and as an attack on a liquid-propellant missile requires delivering a fluence to the target of the order of $1 \mathrm{~kJ} / \mathrm{cm}^{2}=10 \mathrm{MJ} / \mathrm{m}^{2}$. For a wavelength $\lambda=1.315 \mu \mathrm{m}$ and a beam-director diameter $D=1.5 \mathrm{~m}$, the characteristic size of the beam spot at the target for a diffraction-limited optical system is of the order of $L \lambda / D$. The irradiance $I_{0}$, the central power density on the target, would be (see Eq. 19.1)

$$
\begin{aligned}
I_{0} & \approx \frac{\pi}{4}\left(\frac{D}{\lambda L}\right)^{2} P \\
& \approx 34\left(\frac{300 \mathrm{~km}}{L}\right)^{2} \mathrm{MW} / \mathrm{m}^{2} .
\end{aligned}
$$

A real system will not be diffraction-limited, and the best peak power density the system can deliver is

$$
I=I_{0} \times S_{R},
$$

where $S_{R}$ is the Strehl ratio. The ABL design goal is for a beam quality of 1.2 "times diffraction limited," [222] which translates into a Strehl ratio of $S_{R}=1 / 1.2^{2}=0.69 .{ }^{1}$ The beam exits the aircraft through a turret window, where it encounters uncorrectable turbulence in the thin boundary layer there. An estimate of a Strehl ratio from this turbulence alone is 0.8. There are other effects associated with propagation in the atmosphere that make $S_{R}$ for the system $\geq 0.15$ after $\mathrm{AO}$ for engagements of interest. The laser beam is unlikely to hit the missile at a right angle, reducing the fluence delivered by a factor of $\cos \theta_{i} \approx 0.5$ (attitude of booster with respect to the ABL plus curvature of booster). Thus an engagement dwell time would be of the order of $t_{e} \approx 10 /(34 \times 0.15 \times 0.5) \approx 4 \mathrm{~s}$.

We turn now to consideration of the use of the ABL against ICBMs, having a possibly longer range.

The laser beam spot focused on the missile may be relatively large. A diffraction-limited spot would have a diameter of about $\lambda L / D$, which is $0.44 \mathrm{~m}$ at a distance of $500 \mathrm{~km}$. The real spot size and shape will be larger, affected by the optical performance of the laser, and particularly by the degree to which the AO can compensate for the deleterious effects of atmospheric turbulence. Because of asymmetric uncompensated jitter, the spot will be elongated along the axis of the missile, with an aspect ratio of roughly 1:2 [223].

Liquid-propellant boosters are large fuel tanks made of thin metal. Structurally, the tanks are under moderate internal pressure for fuel delivery, and they also are under a high axial compression load from the acceleration of the whole rocket. Heating the metal to a critical temperature causes the metal to lose its strength and will likely cause the tank to rupture or the missile to buckle. Buckling may well vent the struck fuel tank, but in any case, the center of mass will be severely affected, and the thrust will no longer be in line,

\footnotetext{
${ }^{1}$ It is not clear what is meant by the specification, in light of the less-than-ideal-parameters, such as the shape and intensity profile (see 19.1). We take 0.69 as an upper limit.
} 
causing the missile to tumble. The critical temperature to disable the missile is well below the melting temperature of the metal. For example, aluminum loses its strength at about $180^{\circ} \mathrm{C}$ and steel loses its strength at about $480^{\circ} \mathrm{C}$ [216]. Relating these temperatures to the absorbed fluence is complicated problem. The air friction during ascent through the atmosphere will have substantially raised the temperature of the missile's metal skin above the ambient launch temperature, but the amount of heating depends on many factors. Convective or radiative cooling will be small for interesting engagements [224, p. 120]. Cooling by the liquid in a tank is not expected to be large, because the metal will rapidly rise above the nucleate boiling point of the liquid, forming an insulating layer [224, p. 125]. We make a conservative assumption that the starting temperature of the metal is only $20^{\circ} \mathrm{C}$. For a wall of $2 \mathrm{~mm}$ of $\mathrm{Al}$, reaching the critical temperature requires absorbing about $0.8 \mathrm{MJ} / \mathrm{m}^{2}$. For a wall of $1 \mathrm{~mm}$ of steel, about $1.6 \mathrm{MJ} / \mathrm{m}^{2}$ must be absorbed.

It is not known what fraction of the fluence would be absorbed, but the reflectivity $R_{e}$ might be as large as 0.9 . Note that $R_{e}$ refers to the total reflectivity, i.e., specular plus diffuse. Perhaps the best measure of this quantity is $R_{e}=1-\epsilon$, where the $\epsilon$ is the emissivity for the metal. Values for $\epsilon$ range from 0.05 to 0.2 [225], depending on the surface condition, temperature, and metal (excepting rusted iron, which is much higher). The absorbed fluence $F\left(\mathrm{MJ} / \mathrm{m}^{2}\right)$ is given by

$$
F=I_{0} S_{R} \cos \theta_{i}\left(1-R_{e}\right) t_{e} .
$$

Table 20.2. Some Material Properties of Threat Missiles

\begin{tabular}{lrrrr}
\hline \hline Property & \multicolumn{4}{c}{ Material $^{a}$} \\
& (a) & (b) & (c) & (d) \\
\hline Thickness $[\mathrm{mm}]$ & 2 & 1 & 5 & 6 \\
$F_{a b s}\left[\mathrm{MJ} / \mathrm{m}^{2}\right]$ & 0.8 & 1.6 & 60 & 50 \\
$F_{c}\left[\mathrm{MJ} / \mathrm{m}^{2}\right]^{b}$ & 15 & 32 & 240 & 100 \\
$R_{e}$ & 0.9 & 0.9 & 0.5 & 0.0 \\
Density $\left[\mathrm{g} / \mathrm{cm}^{3}\right]$ & 2.7 & 7.8 & 2 & 0.25 \\
$C_{p}\left[\mathrm{~J} /\left(\mathrm{cm}^{3} \mathrm{~K}\right)\right]$ & 2.4 & 3.5 & & \\
$\Delta T[\mathrm{~K}]$ & 160 & 460 & & \\
$Q^{*}[\mathrm{~kJ} / \mathrm{g}]$ & & & 6 & 32 \\
\hline
\end{tabular}

${ }^{a}$ Materials: (a) aluminum, (b) steel, (c) composite, (d) cork.

${ }^{b}\left\langle\cos \theta_{i}\right\rangle=0.5$

Table 20.2 summarizes the material properties relevant to attacking missiles. $F_{\text {abs }}$ is the fluence that must be absorbed, and $F_{\mathrm{c}}=F_{\mathrm{abs}} /\left[\left(1-R_{e}\right)\left\langle\cos \theta_{i}\right\rangle\right]$ is the incident fluence required because of reflectivity and the average cosine of the angle of incidence $\left\langle\cos \theta_{i}\right\rangle=0.5$. It should be noted that the material thicknesses given in the table are only rough estimates, but thicknesses for real missiles are not likely to be smaller than those presented in the table. Thus, the values for the fluence required are lower bounds.

Attacking a solid-propellant missile is more difficult and complex because of its structure. The body is a very strong pressure vessel, and the primary load is a hoop stress rather than 
a compressive one. The amount of energy required to damage the wall is much higher than for the liquid-propellant missile, because the composite structure is much thicker and much energy must be expended to melt or vaporize the resin binder. Once sufficient energy is delivered to the wall, it is more likely to blow out than to buckle the whole structure. There are substantial uncertainties in the fluence required to damage the composite structure [224, p. 126]. The critical fluence per unit mass $Q^{*}$ for fiberglass composite ranges from $6 \mathrm{~kJ} / \mathrm{g}$ at lower irradiance to $15 \mathrm{~kJ} / \mathrm{g}$ at higher irradiance, with a rather sharp transition at about $10 \mathrm{MW} / \mathrm{m}^{2}$. For a typical engagement $Q^{*}=6 \mathrm{~kJ} / \mathrm{g}$. The reflectivity is not likely to exceed 0.5 [224, p. 126]. A booster wall is about 1-cm thick, and if half the thickness is damaged over the large area of the beam spot, it probably will blow out. Taking a density of about $2 \mathrm{~g} / \mathrm{cm}^{3}$ and a thickness of $0.5 \mathrm{~cm}$ means an absorbed fluence of $6 \mathrm{~kJ} / \mathrm{cm}^{2}\left(60 \mathrm{MJ} / \mathrm{m}^{2}\right)$ is required.

The fluence required to disable either a liquid- or solid-propellant missile will depend on details of the construction at the aim point of the laser. For example, stiffening members of a liquid-propellant missile would be much more resistant to attack than the values in Table 20.2 indicate. As a consequence, some care may be needed in selecting the aim point for the attack, based on the type of missile. For the purposes of this study, we take the required incident fluence, $F_{\mathrm{c}}$ to be $32 \mathrm{MJ} / \mathrm{m}^{2}$ for a liquid-propellant missile and $240 \mathrm{MJ} / \mathrm{m}^{2}$ for a solid-propellant missile (Table 20.2, Columns (b) and (c)).

With the fluence available from the ABL, attacking a warhead hardened for re-entry through the atmosphere (as opposed to attacking the missile) is simply not feasible, by a large factor.

\subsection{Kill Assessment}

Kill assessment plays an important role, not only to determine if an engagement were successful, but also to know when the engagement can be terminated and the HEL turned off or directed to another target. The latter is particularly important in the case of multiplethreat missile launches. Preventing waste of laser fuel is also important, since the magazine (fuel runtime) is limited.

The impact of a kill vehicle on a target has a very clear signature. In contrast, the effect of the HEL on the target may be subtle. The simplest case is when a liquid-propellant missile suffers catastrophic buckling, which will lead, at minimum, to a drastic change in the trajectory of the missile and probably tumbling, because the thrust is no longer along the center of mass. Buckling any stage of the missile, even one not under power, would probably have similar catastrophic effects. Rupture of the fuel tank will be a less clearcut indication of successful engagement, since some time may be required for the motor to loose thrust.

For the solid-propellant missile, a blowout on the side of the missile will change the optical signature and perhaps also induce tumbling. In the absence of tumbling, the signature of a successful engagement will most likely be marked change in the acceleration of the missile and the optical character of the exhaust, caused by gases exiting from the side of the missile. We have not tried to estimate the difficulty of such observations. 


\section{References for Chapter 20}

[215] Michael E. Davey and Frederick Martin, "The Airborne Laser Anti-Missile Program." Technical Report RL30185, CRS, May 1999. CRS Report to Congress.

[216] Geoffrey Forden, "Ballistic Missile Defense: The Airborne Laser," IEEE Spectrum 34, September 1997.

[217] Jane's Strategic Weapons Systems (JSWS) 36, 12 July 2001. Article: CSS-4 (DF-5).

[218] Thomas C. Cochran, William M. Arkin, and Milton M. Hoenig, Nuclear Weapons Data Book, Volume I: U.S. Nuclear Forces and Capabilities. (Ballinger, Cambridge, MA) 1983.

[219] Dean Wilkening, "Boost-Phase Missile Defense." Briefing Presented to the APS Study Group, July 2001.

[220] Steven J. Isakowitz, Joseph P. Hopkins, and Joshua B. Hopkins, International Reference Guide to Space Launch Systems. American Institute of Aeronautics and Astronautics, 3rd edition, December 1999.

[221] Orbital Sciences Corporation, Minotaur User's Guide. Chandler AZ. http://www.orbital.com/, March 2002. Space launch vehicle using Minuteman II stages 1 and 2 .

[222] Ostap Kosovych and David McGarvey, "The BMDO Boost Phase Intercept Study." POET Briefing, September 1995.

[223] Mitchell H. Fields et al., "Initial results from the Advanced-Concepts Laboratory for adaptive optics and tracking," SPIE 4034, 116 (2000).

[224] N. Bloembergen et al., Rev. Mod. Phys. 59 S1 (1987).

[225] Robert C. Weast, Handbook of Chemistry and Physics. (CRC Press), 1979. 



\section{Chapter 21}

\section{Airborne Laser Engagements}

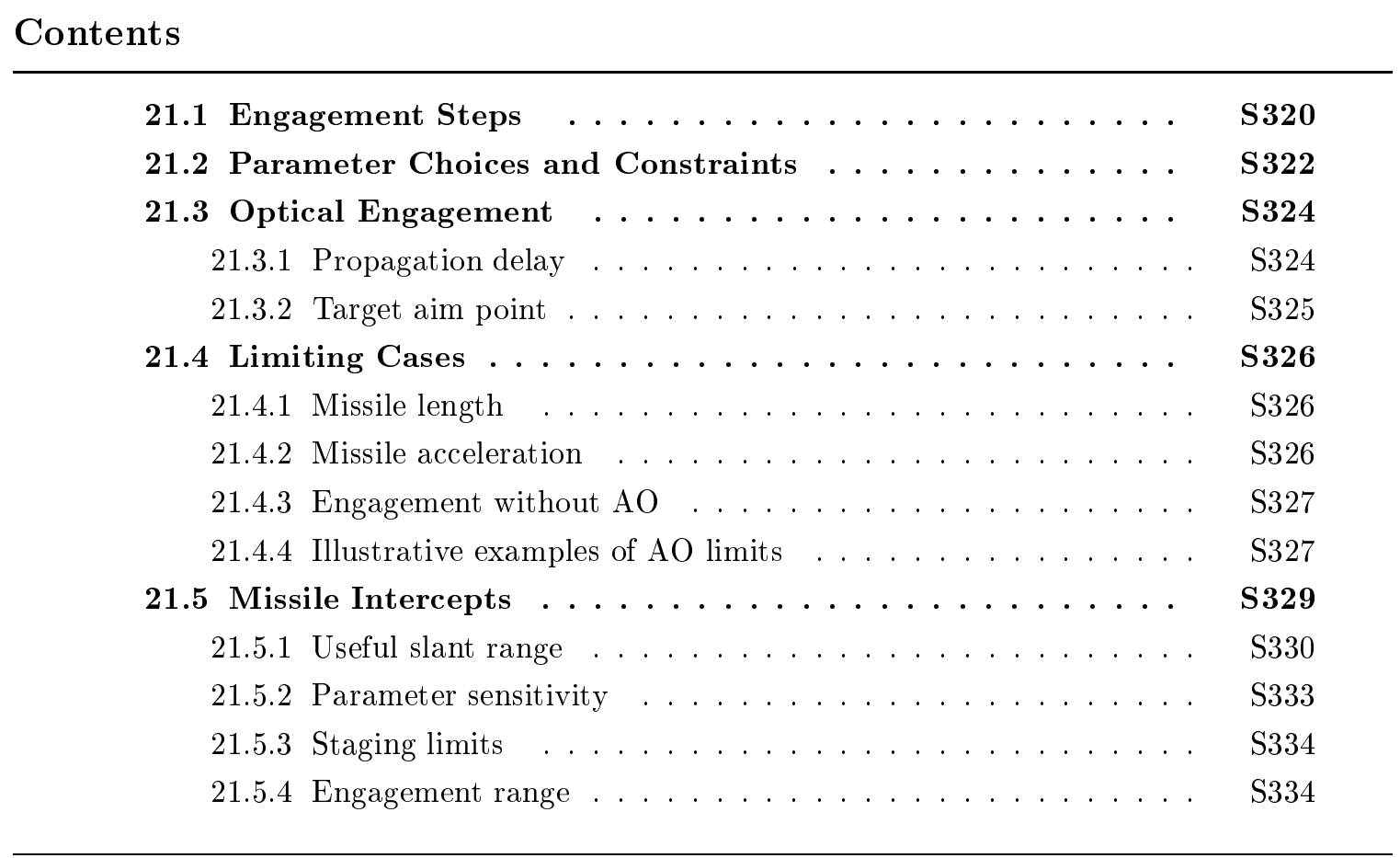

Engaging missiles with a laser is quite different from engaging them with kinetic kill vehicles. The laser beam propagates to the target at the speed of light, i.e., in a few milliseconds, in contrast to the two or three minutes required for a kill vehicle to intercept the target missile. On the other hand, once a kill vehicle reaches the target, the damage is done almost instantaneously, whereas the laser must focus its beam on the target for a minimum of several seconds to deliver enough energy density (fluence) to damage it.

This chapter is devoted to the issue of engaging targets in realistic geographic scenarios. Section 21.1 describes the sequence of events leading up to the delivery of an ABL beam onto the target, and Section 21.2 summarizes the parameters and assumptions made to complete the optical engagement. Some quantities can only be roughly estimated. For example, the laser power of the ABL is classified, and we can make only a reasonable assumption for it. Similarly, there is substantial uncertainty in the fluence required to destroy a missile, even if its type (i.e., liquid- or solid-propellant) is known to the defense.

Even though the laser beam travels at the speed of light, propagation delay time plays an essential role in carrying out an intercept. Section 21.3 describes the optical engagement and its relation to the requirements of adaptive optics (AO). Various limiting cases are 


\begin{tabular}{ll} 
Box 21.1: & Acronyms Used in This Chapter \\
\hline AO & adaptive optics \\
BILL & beacon illuminator laser \\
FOV & field of view \\
HEL & high energy laser \\
IRST & infrared search and track sensor \\
IRTU & inertial reference transfer unit \\
TILL & tracking illuminator laser
\end{tabular}

described in Section 21.4 to illustrate the effect of different constraints on the useful range of the laser.

Section 21.5 describes the various factors that affect the range of the ABL and gives values for the range in various types of engagements. It includes a discussion of the sensitivity of the effective range to the parameters assumed for the engagement. Generally speaking, the results are robust to moderate variations.

Because some key performance parameters of the ABL are not known, it is not possible to make a detailed analysis of the ABL's capabilities in given defense scenarios. Nevertheless, the principles of such an analysis may be illustrated by considering defense possibilities under a set of given assumptions.

\subsection{Engagement Steps}

The term engagement is used here in the same sense as in the kill-vehicle analysis of Chapter 5-an engagement is the sequence of events from first detection of a missile through the firing of the interceptor. However, the term is also used to describe the acquisition of the target by the ABL and the actual ABL firing. For instance, a "20-second engagement" describes an engagement in which the dwell time (the time during which the ABL delivers its fluence to the missile) is $20 \mathrm{~s}$. The sense in which engagement is used will be clear from the context.

The ABL is conceived as an autonomous weapon, yet it is also meant to be integrated into an overall family of theater-missile defense systems [226]. It will loiter above the cloud level (near 12-km altitude) with an air speed of about $600 \mathrm{mph}$ and a 24-hour coverage time of potential missile launch sites, based on multiple-aircraft deployment and a refueling option (Section 22.1).

Numerous acronyms are used in this section, and Box 21.1 summarizes the most common. Symbols used in this chapter are summarized in page S336.

When a threat missile is launched, the ABL may be cued by IR-satellite detection of missile exhaust, by externally based radar, or by multiple IR search and track (IRST) sensors located on the ABL. These surveillance sensors provide wide-angle coverage and detect IR radiation from a missile's booster plume. This cue may appear only after the missile breaks through the cloud layer, or earlier if there are no clouds. An active ranging system (ARS), which uses a $\mathrm{CO}_{2}$ 10.6- $\mu$ m laser illuminator in a pod on top of the airplane, then provides position coordinates of the missile.

Information from the wide field-of-view (FOV) IRST sensors allows the turret-based 
telescope, located in the nose of the aircraft, to slew towards the missile. The telescope will be used to collect both plume light and laser-backscattered light, and to point and focus both sensing and high-energy lasers. The aim-points of the telescope are contained in an angle of about $240^{\circ}$ in the flight plane (nearly wing-tip to wing-tip) [227] and an unspecified angle in altitude, centered on the flight path.

A handoff is then made to the turret's IR co-boresighted acquisition sensor, a mediumFOV IR-sensing array that centers the missile plume in the ABL telescope's FOV. Tracking algorithms capture the target and direct the turret to follow the missile trajectory.

The missile plume then is detected by the IR-sensing, narrower-FOV plume tracker, which looks through the telescope optics. The plume tracker stabilizes the image of the plume, establishes the track, and points a tracking illuminator laser (TILL) at the hardbody of the missile.

The TILL beam is "walked" up the missile to illuminate the tip for the first stage of beam correction. The TILL is a solid-state, $1.030-\mu \mathrm{m}$, multi-kW laser that is diode-pumped at a $5-\mathrm{kHz}$ repetition rate. Multiple beams (probably four) are created with several-nanosecond optical path-length delays to create incoherence of the light, and the multiple beams propagate through different air columns to reduce the deleterious effects of scintillation and speckle in the reflection from the nose of the missile that is observed by the small-FOV fine tracker array.

Return light from the TILL-illuminated missile nose provides edge-feature information to the fine tracker sensor, which stabilizes the image of the illuminated hardbody. The first step in AO corrections (tilt) is done on the TILL beams using the edge of the missile nose. Control is transferred to tracking algorithms in a handover to the fine tracking system, to determine aim points for the beacon illuminating laser (BILL) and the chemical oxygeniodine laser (COIL) high-energy laser (HEL) at appropriate locations on the missile. The locations of these aim points are important in maximizing the vulnerability of the threat missile to the energy deposited by the HEL beam, but their locations are constrained by limitations of the AO system.

The BILL provides an illuminated spot that is used as a beacon to correct for atmospheric distortion. The BILL is a diode-pumped, solid-state, $5-\mathrm{kHz}$ and $\mathrm{kW}$-level laser operating at $1.064 \mu \mathrm{m}$. Again, multiple time-delayed beams reduce coherence effects, as in the case of the TILL.

Light from the TILL and BILL illumination acts as a beacon or guide star for correcting atmospheric distortion; return light samples the atmosphere between the missile and the ABL. A wavefront sensor analyzes distortions of this returned light, which have resulted from propagation through the atmosphere, on a pulse-by-pulse basis at $5 \mathrm{kHz}$. This information is provided at a rate of $500 \mathrm{~Hz}$ to a fast steering mirror and two multiple-element deformable mirrors: one for focus and one for higher-order terms. These mirrors place the conjugate of the distortions on the outgoing HEL beam. To minimize the phase errors, the HEL beam attempts to return in an air column as close as possible to that by which the illumination arrived. The pre-distortion of the outgoing HEL beam causes it to arrive more highly focused on target, as the phase distortion is "undone" by the atmosphere.

The TILL-illuminated nose image provides tilt information, and the BILL image provides the higher-order AO corrections for the HEL. Neither the TILL nor the BILL beams are corrected to compensate for atmospheric distortions. The HEL's separate fast-steering and deformable mirrors thus allow compensation for atmospheric phase distortion as the HEL beam propagates towards the missile, providing an improved focus and smaller spot size for the HEL. Additional deformable and fast steering mirrors pre-compensate the HEL 
beam for any intrinsic phase distortions originating within the high-power laser path in the ABL.

An inertial reference transfer unit (IRTU), employing a $0.83-\mu \mathrm{m}$ laser system and a suite of detectors, allows for alignment through the optical train and along the length of the aircraft. Target coordinates are provided to the IRTU to define the line-of-sight (LOS) toward the target missile. Alignment and pointing at the level of $100 \mathrm{nrad}$ are anticipated to keep the HEL focused on the missile.

The time from missile launch to initial detection is $45 \mathrm{~s}$ for ICBM L and $30 \mathrm{~s}$ for ICBM S2 (see Section 10.1.4). It is claimed that the process of tracking control, from target detection to the handover to the fine tracker, nominally takes less than $10 \mathrm{~s}$ [226]. Depending on the type of missile, boost-phase engagement opportunities will end within several minutes after launch, following missile thrust termination at altitudes and ranges up to several hundred kilometers. It is claimed that the ABL could engage from 20 to 40 missiles [228], sequentially, before requiring refueling of the HEL.

The beam control design is based on a common-path - common-mode approach [229] which provides a shared set of optical components for alignment, targeting, compensation, and high-power laser beams, as well as sensing and control functions for optical components in the beam path. This shared-aperture design offers advantages in tracking, jitter, and alignment control and reduces the complexity and weight of the optics.

The above characterization of the design and function of the ABL is based on available public literature. It is consequently relatively fragmentary and limited in scope. However, it is clear that there are significant challenges for the ABL program, as pointed out in recent reviews, including Refs. [227], [228], [230], [231], [232], and [233].

These challenges include demonstration of simultaneous performance in a variety of areas, including laser output power, beam quality, AO compensation of atmospheric distortion under realistic conditions, lethality on appropriate threat missiles, and integration of all systems.

\subsection{Parameter Choices and Constraints}

Many variables enter into computing possible engagements, and there are substantial uncertainties in some of these quantities.

- We have had to make an arbitrary choice of the HEL power of $3 \mathrm{MW}$, because the design power is classified. The only public statement is "multi-MW" for all 14 gain modules and $>1 \mathrm{MW}$ for 6 modules. We shall show the sensitivity of successful performance of the ABL to this assumption.

- We assume that the laser beam quality of 1.2 times diffraction limited can be achieved. We shall show the sensitivity to this assumption.

- The fluence requirements to disable the missile (see Table 20.2) are only reasonable estimates. The thickness and reflectivity of the walls of the target missiles are unknown, and the precise damage mechanism is uncertain. Measurements of lethality criteria, particularly for solid-propellant missiles, are classified, if they exist. We shall show the sensitivity to this assumption.

- Countermeasures that may increase the fluence requirement have not been taken into account. 


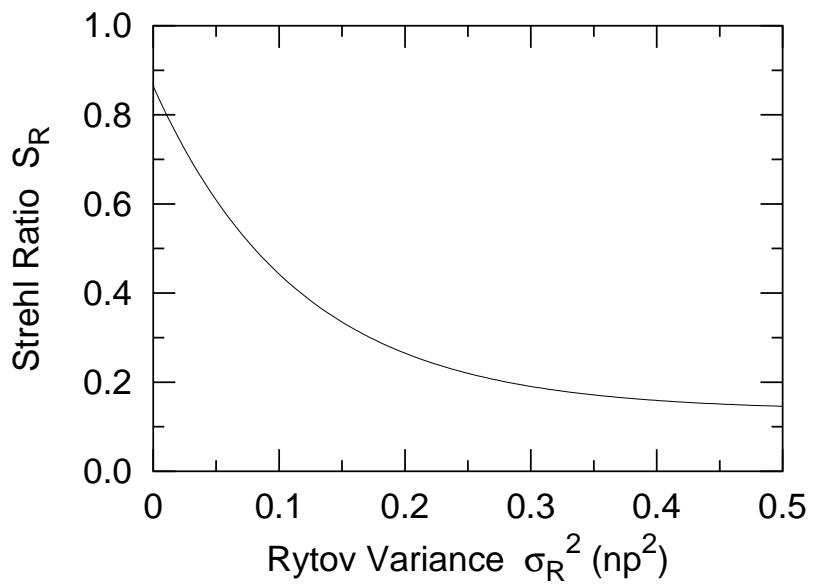

Figure 21.1. Estimated Strehl ratio vs. $\sigma_{R}^{2}$ achievable for adaptive optics.

- The turbulence of the atmosphere is a fundamental variable (see Section 19.2) that the $\mathrm{AO}$ is designed to handle. We assume that the Strehl ratios in the presence of turbulent air that were achieved in the benchmarking experiments ([234], [235]) can be achieved in actual conditions. Most of the engagements of interest to this study take place at higher altitudes than the theater missile defense scenarios for which the ABL is designed. Turbulence effects become less important at our higher engagement altitudes. We shall show the sensitivity to this assumption.

- We assume maximum engagement dwell times $t_{e}$ of $20 \mathrm{~s}$ and $5 \mathrm{~s}$ to explore sensitivity to the choice of time.

- The purpose of the calculations presented herein is to provide a scale, not precise numbers.

In light of these uncertainties, we have tried to explore the possible capability of the ABL for intercepting ICBMs. In case of a question, we have made an optimistic assumption.

Computing engagement times requires combining fluence requirements from Table 20.2 with the geometrical information $h_{t}$ (altitude of the target) and $L$ (slant range from ABL to target), that determine the laser propagation characteristics. The irradiance delivered to the target is taken from Eq. 20.3. The system Strehl ratio is computed using Eq. D.56, with $S_{R-\text { laser }}=0.69, S_{R \text {-window }}=0.8, S_{R-A O}$, the estimated Strehl ratio achievable by the AO, and $S_{R-a n i s o}$, the anisoplanatic contribution. The best estimate of $S_{R-A O}$ without anisoplanatism is from the experimental measurements of Firepond [234] and Brassboard [235]. The experimental points were smoothed, and the result is shown in Figure 21.1. The anisoplanatic contribution to the Strehl ratio $S_{R \text {-aniso }}$ is computed in the extended Maréchal approximation.

There may be unaccounted AO shortcomings, such as uncorrected residual tilts, which could lower the Strehl ratio. For lack of any quantitative information, we make the optimistic assumption of ignoring such losses. The resulting Strehl ratio for the system varies from 0.1 to 0.5 for engagements of interest, depending on the geometry. 


\subsection{Optical Engagement}

\subsubsection{Propagation delay}

The optical engagement is complicated by several factors. First, optical constraints set by the atmospheric turbulence, motion of the ABL and the target, and propagation delay of the laser beam from the ABL to the target, impose limits. Second, considerations of missile vulnerability may limit the location on the missile's surface where the HEL beam should be directed, imposing other restrictions. The optical constraints and missile vulnerability constraints may conflict, requiring compromises. This section discusses the optical constraints.

The simplest optical constraints are set by the limits of the AO's ability to correct for turbulence. The ABL design parameters (see Section 19.5) are $\sigma_{R}^{2} \leq 0.5$, and $\theta_{0} \geq 0.65 \lambda / D$. These are of interest mainly for low-altitude engagements.

Engaging ICBMs is more effective for high altitudes, because of greater maximum, slant range. Here, optimizing the Strehl ratio means minimizing anisoplanatic effects. The isoplanatic length at the missile, $\theta_{0} L$, ranges from $0.2 \mathrm{~m}$ for low-altitude engagements to 3 $\mathrm{m}$ or more at high altitudes. Minimizing the anisoplanatic phase corruption means trying to use the same column of air for the HEL return that the arriving "guide star" image used. Because the target is moving and the propagation delay is significant, the HEL spot will strike a different place on the target than that from which the guide star image left.

This propagation delay offset, $\delta_{p}=2 L v / c$, can be large. For example, an engagement at a range of $L=300 \mathrm{~km}$ has a roundtrip time delay of $2 \mathrm{~ms}$. At an altitude of $30 \mathrm{~km}$, a liquid-propellant missile is moving at about $1.5 \mathrm{~km} / \mathrm{s}$, and a solid-propellant missile is moving at about $2 \mathrm{~km} / \mathrm{s}$. Thus, $\delta_{p}=3 \mathrm{~m}$ to $4 \mathrm{~m}$. For an engagement at $500 \mathrm{~km}$ and an altitude of $50 \mathrm{~km}$, the missiles are moving at about $2 \mathrm{~km} / \mathrm{s}$ and $3 \mathrm{~km} / \mathrm{s}$, respectively, corresponding to $\delta_{p}=6.7 \mathrm{~m}$ to $10 \mathrm{~m}$. The propagation delay offset is correspondingly larger at even higher altitudes, when the missile speed is greater. As subsequent discussion shows, such large offsets can limit the range of an engagement.

Recall that the tilt correction for the HEL uses the nose image for the beacon and the higher-order corrections for the HEL use the BILL image. Although the BILL is not AO-compensated and suffers jitter on the target, the orthogonality of the Zernike functions allows the higher-order terms to be separated from the tilt. Both the TILL and BILL images are sampled at a rate of $5 \mathrm{kHz}$ on a pulse-by-pulse basis. For each pulse, the BILL image is substantially smaller than the time-averaged, tilt-smeared image. (See Section D.4.3 for a discussion of the beacon size).

The optical engagement is somewhat complex, and Figure 21.2 illustrates the situation. The target is a distance $L$ from the ABL and moving at a speed $v$.

- The TILL illuminates the nose at point $\mathrm{N}$, and the image is used for the tilt correction.

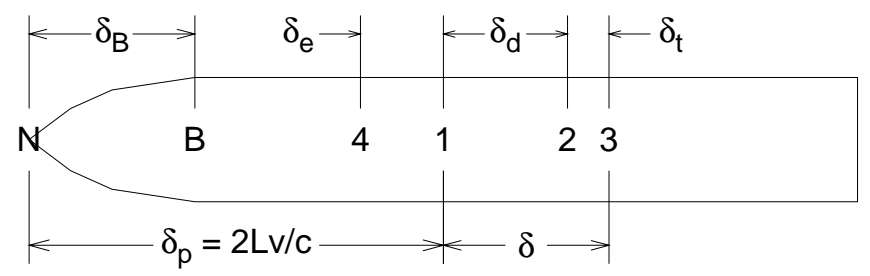

Figure 21.2. Schematic of steps of an optical engagement. 
- Point B is the BILL image. Since AO is not used for the outgoing BILL beam, it can be placed anywhere desired on the target.

- Point 1 , a distance $\delta_{p}=2 L v / c$ from the nose, is tilt-isoplanatic with the nose image because of propagation delay.

Point 1 can be higher-order isoplanatic, as well as tilt-isoplanatic, by placing the BILL at the nose, i.e., $\delta_{B}=0$ (or alternatively by using the nose image itself). Point 1 is optically the ideal attack point, but it may be unfavorable from a missile vulnerability standpoint.

It is important to have some freedom of choice in the aim point for two reasons. First, some parts of the missile are more vulnerable than others. Second, the effect of hitting a part of a booster that is not yet burning, such a later stage, may be very difficult to assess, depending on the kill mechanism.

It is relatively easy to choose an aim point (Point 2) farther down the missile than Point 1, but doing so introduces tilt anisoplanatism. As discussed in Section D.4.4, the characteristic angle for tilt anisoplanatism is $\theta_{t 0} \approx 3 \theta_{0}$. One can, however, extend beyond this by exploiting the separation of the ABL's tilt correcting mirrors from the higherorder AO mirrors to make Point 2 tilt isoplanatic with $\mathrm{N}$. This is done by introducing a programmed delay $\tau=\delta_{d} / v$ between the receipt of the nose image and the command to the tilt mirror. ${ }^{1}$ The cost of doing so is to introduce a delay anisoplanatism caused by the motion of the aircraft during the delay $\tau$. Delay anisoplanatism is discussed in Section D.4.5. Apart from the anisoplanatism introduced by the delay, Point 2 can be made both tilt- and higher-order isoplanatic by choosing $\delta_{B}=\delta_{d}$.

The above choice of $\tau$ is not optimal, because a Point 3 can be chosen with some mixture of delay anisoplanatism and tilt anisoplanatism, having a smaller total phase variance,

$$
\Delta \sigma_{\varphi \text { tot }}^{2}(\delta)=\sigma_{\varphi \text { tilt }}^{2}\left(\delta-\delta_{d}\right)+\sigma_{\varphi \text { del }}^{2}\left(\delta_{d}\right),
$$

for a given total offset $\delta$. As discussed in Section D.4.4, the tilt phase variance can be reduced, particularly for low-altitude engagements. By choosing $\delta_{B}=\delta$, there is no higherorder anisoplanatic contribution.

A more difficult case is placing the beam at Point 4, which is necessarily anisoplanatic in both tilt and higher order, since the BILL image cannot be placed farther upstream than the nose and $\tau$ cannot be negative. The phase variance is given by Eq. D.29 with the normal $\theta_{0}$, with correction for piston removal (see Section D.3).

The result is that there is much freedom to place the HEL spot farther down the missile than Point 1, but much less freedom to move towards the nose from Point 1.

\subsubsection{Target aim point}

Lethality considerations may favor some areas of the target, depending on the type and construction of the target missile. Typing the missile will be important in choosing an appropriate point to attack. This information can be obtained, to some degree, by observation of the acceleration of the target.

Some parts of a missile can be especially resistant to optical attack. For example, structural members or the combustion chamber will be much more resistant than the walls of the tanks of a liquid-propellant booster. The interface between stages is also likely to

\footnotetext{
${ }^{1}$ It is not known if this method is used in the ABL design; such details of the optical engagement do not appear in the public literature.
} 
be rather robust against attack. Similarly, if the kill mechanism assumes column collapse, the attack must be directed to an area under large column loading, not an area near the nose. Alternatively, if the kill mechanism relies on vessel rupture, the effects of attacking a stage that is not yet burning will be difficult to assess, since there will be little effect on the trajectory at that time.

\subsection{Limiting Cases}

There are several limiting cases that are useful to analyze to understand the behavior of the maximum range $L_{\max }$ achievable for engagements. These are propagation delay and the length of the missile, propagation delay and the acceleration of the missile, and an engagement without using AO.

A successful engagement must deliver an incident fluence, $F_{c}$ of about $32 \mathrm{MJ} / \mathrm{m}^{2}$ for liquid-propellant missiles and $240 \mathrm{MJ} / \mathrm{m}^{2}$ for solid-propellant missiles (see Section 20.1) .

$$
F_{\mathrm{c}}=\int_{0}^{t_{e}} d t I_{0} S_{R}(t)
$$

where $S_{R}(t)$ is the total Strehl ratio for all effects, $I_{0}$ is taken from Eq. 20.2, and $S_{R}$ is determined as described in Section 21.2.

\subsubsection{Missile length}

From the preceding discussion, we see that the propagation delay and AO requirements place some limits on the aim point. Obviously, if propagation delay places Point 1 beyond the end of the missile (or, more properly, the vulnerable point farthest from the nose) large anisoplanatic errors ${ }^{2}$ cannot be avoided with respect to Point 1 . Propagation delay effects can rapidly degrade the Strehl ratio. In simple terms, a limiting case is $\delta_{p}=l_{m}=2 L_{\max } v / c$, where $l_{m}$ is the missile length (or the last vulnerable point). Going beyond this limit introduces anisoplanatic phase variance. The real limit occurs when the Strehl ratio is degraded enough that the required fluence can no longer be achieved during a maximum acceptable engagement time $t_{e}$.

Note that although this discussion concentrates on the missile length, in fact the limitation on range is caused by propagation delay, compared with the length of the missile divided by the missile speed. For this discussion, it is more convenient to talk in terms of a length limit, because the aim point must be chosen, and the length changes discontinuously during staging events. The speed of the missile is prescribed by the necessity to deliver its payload to a given target.

\subsubsection{Missile acceleration}

Another constraint arises from the acceleration $a$ of the missile, causing $\delta_{p}$ to change with time $t$ during the engagement,

$$
\delta\left(\delta_{p}(t)\right)=2 L \frac{\delta v}{c}=2 L \frac{a t}{c} .
$$

If the aim point is 3 , i.e., beyond Point 1 , compensating for this movement is possible with little penalty. An attack on Point 4, however, cannot be compensated, and the anisoplanatism will increase during the total engagement time $t_{e}$.

\footnotetext{
${ }^{2}$ All Zernike modes except piston (see App. D.3).
} 
We consider the case in which $\delta_{e}=0$ at $t=0$. As time progresses, an angular offset $\theta_{s}$ develops, and in the extended Maréchal approximation, the contribution to the Strehl ratio $S_{R \theta}$ will be

$$
\begin{aligned}
\theta_{s} & =\frac{\delta\left(\delta_{p}\right)}{L}=\frac{2 a t}{c} \\
S_{R \theta} & =\exp \left[-\rho\left(\frac{\theta_{s}}{\theta_{0}}\right)^{5 / 3}\right] \\
& =\exp \left[-\rho\left(\frac{t}{\tau}\right)^{5 / 3}\right] \\
\tau & =\frac{c \theta_{0}}{2 a}
\end{aligned}
$$

where $\rho$ is the phase-variance reduction factor for piston removal defined in Eq. D.30. It varies from 1 for very small $\theta_{s}$ to about 0.5 during the engagement. The characteristic time $\tau$ ranges from as little as $5 \mathrm{~s}$ to as much as $30 \mathrm{~s}$ for engagements of interest. A consequence is that the fluence delivered is not linear during the engagement time unless $t_{e} \ll \tau$.

\subsubsection{Engagement without AO}

Because the choice of aim point may require very large anisoplanatism, there will be a point where AO produces a smaller Strehl ratio than not using AO, since the AO phase variance saturates at twice the uncompensated value (see App. D.4). Turning off the AO results in a Strehl ratio given by Eq. 19.2.

\subsubsection{Illustrative examples of $\mathrm{AO}$ limits}

Figure 21.3 illustrates the effect of AO limits for the case of a liquid-propellant missile and a dwell time of $20 \mathrm{~s}$; the next section provides specific information on intercept parameters. The figure shows the maximum slant range $L_{\max }$ for an intercept vs. the altitude $h_{t}$ of the target at intercept.

Line (a) shows what would happen if there were no atmospheric turbulence, or alternatively if the AO worked perfectly and the target did not move. Line (a) thus represents the upper bound of the range for a given laser. The decrease of range at low $h_{t}$ is caused by the curvature of Earth. The subsequent lines include turbulence for $2 \times$ Clear- 1 Night. Line (b) assumes that the length and acceleration limits do not apply, or, alternatively, that the target does not move. This line cannot reach line (a) at large $h_{t}$ because the ABL itself is still in the atmosphere. Line (c) applies the acceleration limit, but not the length limit. The kink at $h_{t}=55 \mathrm{~km}$ is a result of the change in acceleration at staging for model ICBM L. Line (d) applies the length limit, but not the acceleration limit. The kink at $h_{t}=90 \mathrm{~km}$ results from $\delta_{p}$ exceeding the length of the missile. Line (e) applies all the limits for a real engagement. Line (f) represents the case where no AO is used at all, and thus the lower bound of the range for a given laser and the assumed $2 \times$ Clear-1 Night turbulence. The difference between line (e) and line (f) shows the improvement from the planned AO. We note in passing that because of the uncorrectable anisoplanatism, the range of the laser above the kink at $h_{t}=90 \mathrm{~km}$ in line (e) would hardly change if the AO performed ideally, rather than the assumed performance.

As we shall see, the useful range against a solid-propellant missile is substantially less than that for a liquid-propellant missile. As a result, the above limits manifest themselves 


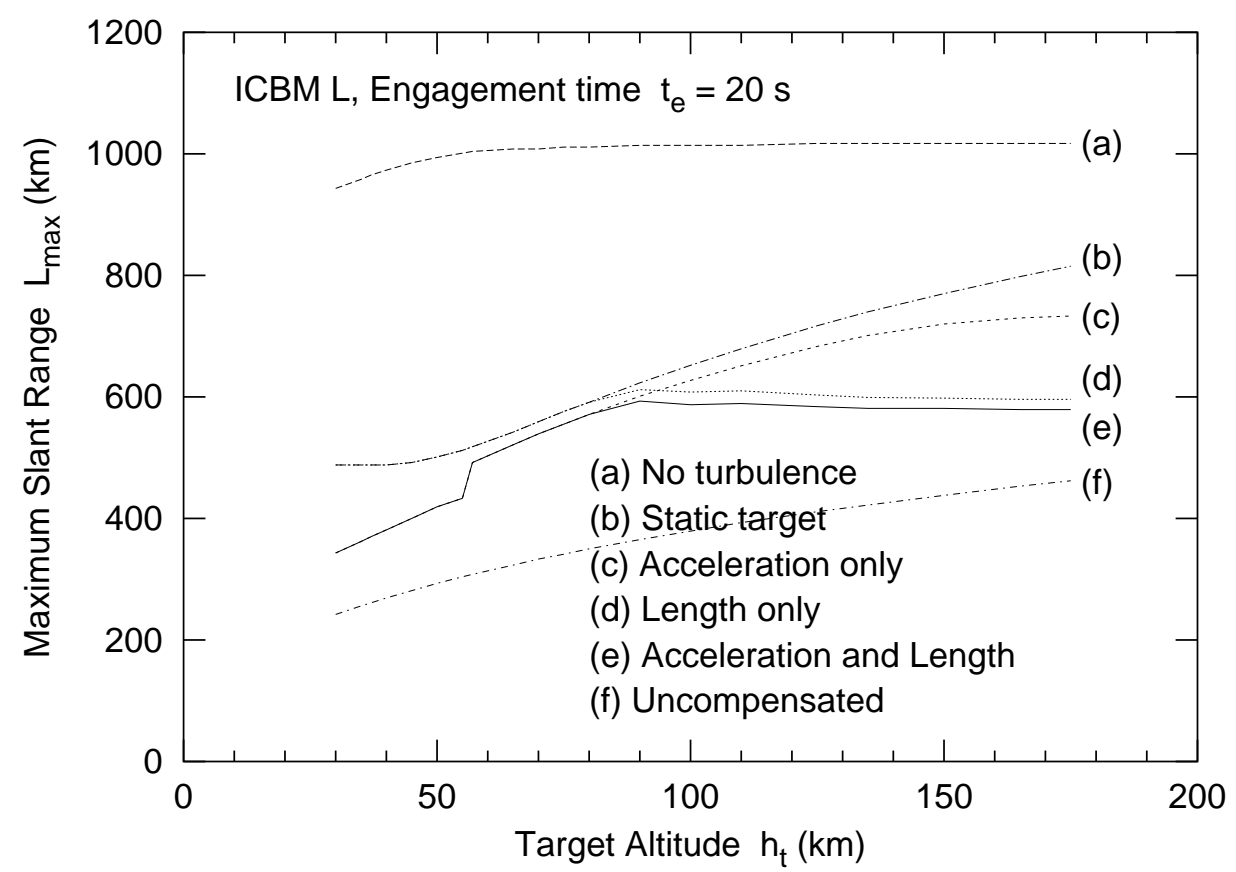

Figure 21.3. Effect on maximum slant range by applying various limits to the optical engagement of a liquid-propellant missile vs. altitude of the target.

in different ways. Figure 21.4 shows the equivalent information for the solid-propellant ICBM-S2. Note in particular that the maximum range for no turbulence is only a little longer than what can be achieved in a real engagement, because the Strehl ratio achievable at high altitude is large.

As an academic point, it is interesting to note that the propagation-delay AO limitations shown in Figures 21.3 and 21.4 could in principle be overcome if a point-ahead beacon were available. That is to say, if an artificial beacon could be provided, such as is used now in astronomy where no natural guide star is available within the isoplanatic patch, or more importantly, for delivering a light beam to a satellite. See Refs. [236, p. 216 et seq.] for an overview of artificial beacons using Rayleigh backscattering (relatively low-altitude atmosphere) and backscattering from the mesospheric sodium layer (altitude about $90 \mathrm{~km}$ ) and [237] for use of the mesospheric sodium layer for correcting a beam to a satellite. Once the trajectory of the missile is available, with the velocity and an estimate of its acceleration, an artificial beacon could be placed ahead of the missile to be in synchronism with the propagation delay of the HEL. Such a technique could substantially enhance the useful range of the ABL against a liquid-propellant missile [curve (b) vs. curve (e) of Figure 21.3], but it would do little to help against a solid-propellant missile. We have not attempted to analyze what would be required technically to realize a useful point-ahead beacon for the ABL nor how effective an implementation might be in overcoming the propagation delay limitation. From public documents, this capability does not appear to be included in the ABL design. 


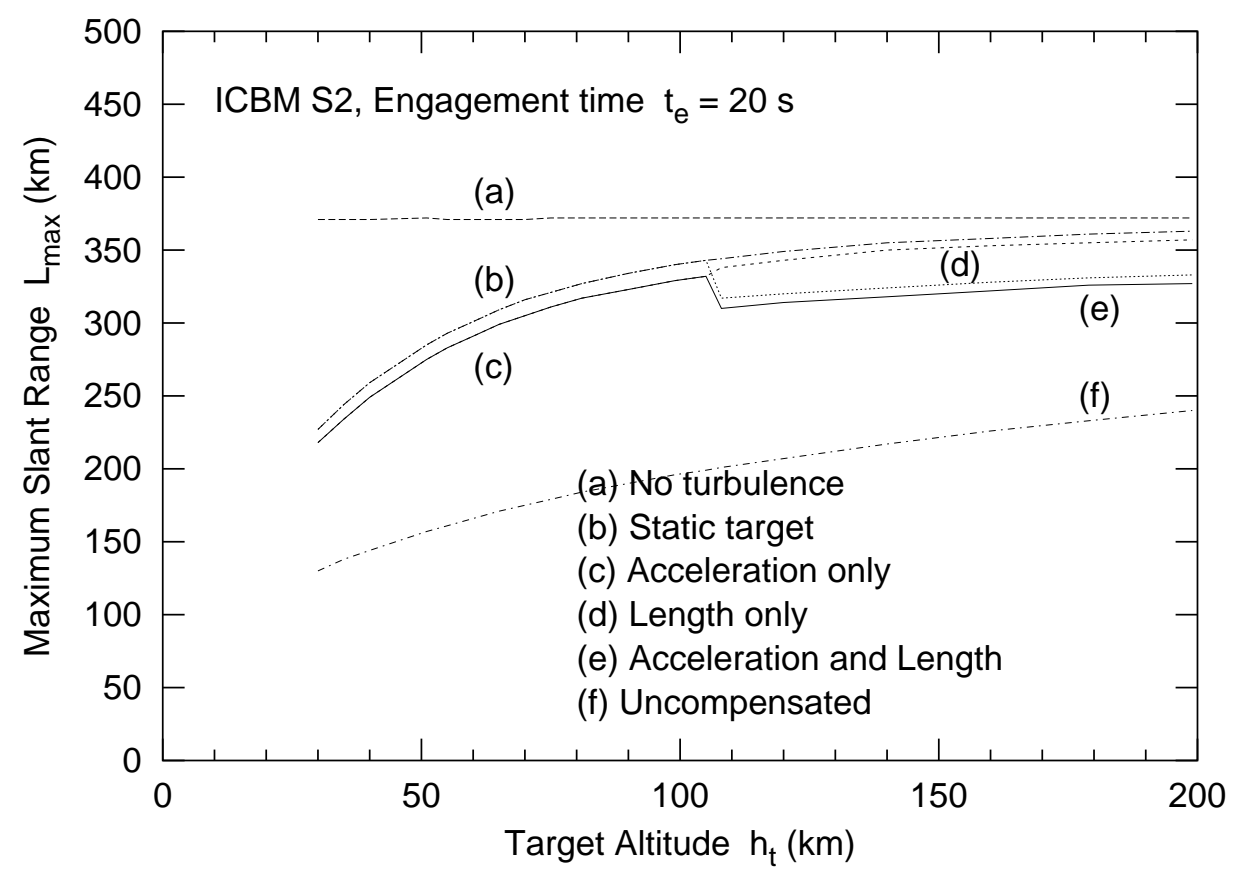

Figure 21.4. Effect on maximum slant range by applying various limits to the optical engagement of a solid-propellant missile vs. altitude of the target.

\subsection{Missile Intercepts}

Engagements of both liquid- and solid-propellant missiles have been modeled where the maximum slant range $L_{\max }$ has been computed for which the requisite critical fluence $F_{c}$ can be delivered in a given engagement time $t_{e}$. Various scenarios to achieve this goal were explored. Of primary interest are the engagements using the standard parameters, such as laser power, for two different engagement times, $20 \mathrm{~s}$ and $5 \mathrm{~s}$. The 20 -second engagement time is viewed as a practical, although somewhat arbitrary, upper limit. The 5-second engagement was chosen to study the sensitivity of $L_{\max }$ to the engagement time.

The liquid-propellant missile (ICBM L) is mechanically modeled after a DF-5 to define the vulnerable dimensions. The vulnerable length is $29 \mathrm{~m}$ for the whole missile and $12 \mathrm{~m}$ for the second stage alone. First-stage separation occurs $120 \mathrm{~s}$ after launch at an altitude of about $55 \mathrm{~km}$. Burnout of the second stage occurs $240 \mathrm{~s}$ after launch at an altitude of about $183 \mathrm{~km}$.

Similarly, the solid-propellant missile (ICBM S2) is mechanically modeled after a Minuteman III. The total length is $17 \mathrm{~m}$; the second and third stages together are $10 \mathrm{~m}$; and the third stage is $6 \mathrm{~m}$. First-stage separation occurs $60 \mathrm{~s}$ after launch at an altitude of about $29 \mathrm{~km}$; second-stage separation occurs at $120 \mathrm{~s}$ at about $105 \mathrm{~km}$, and the third stage burns out at $170 \mathrm{~s}$ and about $199 \mathrm{~km}$.

For both missiles, the flight profiles of acceleration, speed, and altitude were used, as shown in Figures 21.5 and 21.6. Figures 21.7 and 21.8 show the limitations on $L_{\max }$ for laser powers of $1.5 \mathrm{MW}, 3 \mathrm{MW}$, and $6 \mathrm{MW}$ for engagements times of $t_{e}=20 \mathrm{~s}$ and $5 \mathrm{~s}$ respectively. In varying degrees, evidence of the limiting cases previously discussed are apparent.

- For the liquid-propellant missile at $h_{t}=55 \mathrm{~km}$ and the solid-propellant missile at $h_{t}=29 \mathrm{~km}$, a "jump" occurs as a result of the decrease of acceleration during staging 


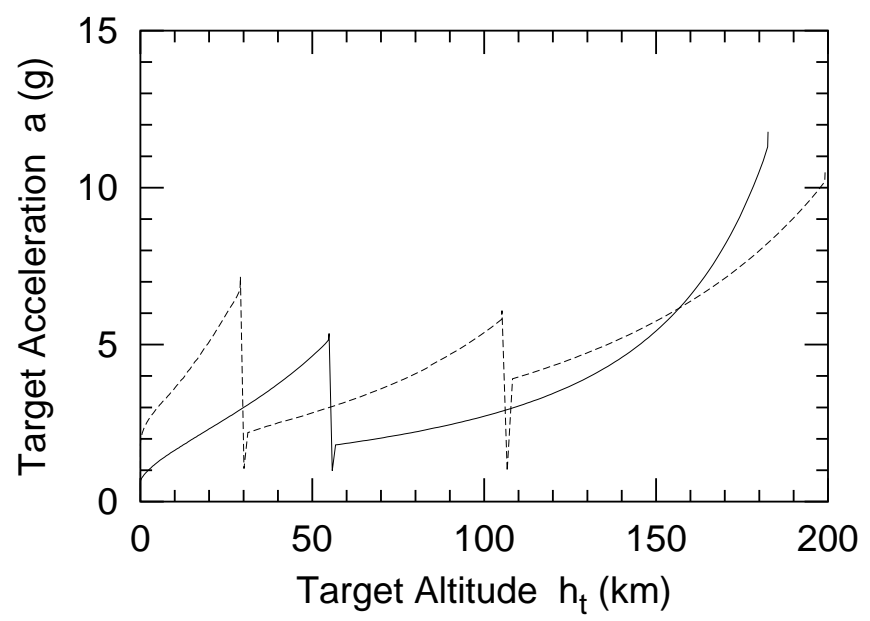

Figure 21.5. Acceleration vs. altitude for liquid-propellant ICBM L (solid line) and solid-propellant ICBM S2 (dashed line).

(Section 21.4.2).

- The length "limit" (Section 21.4.1) can be seen at $h_{t} \approx 90 \mathrm{~km}\left(t_{e}=20 \mathrm{~s}\right.$ ) or $110 \mathrm{~km}$ $\left(t_{e}=5 \mathrm{~s}\right)$ for the liquid- propellant missile and $h_{t} \approx 105 \mathrm{~km}$ for the solid-propellant missile, but for different reasons. For the liquid case, the anisoplanatism due to the length occurs gradually as $\delta_{p}$ moves off the end of the missile. For the solid case, staging suddenly reduces the missile's length.

- The decrease of $L_{\max }$ with speed (altitude) due to the length is visible for the 5-s liquid-propellant missile engagement. For other cases, the ability to engage with little or no AO washes out the length limit at higher altitudes.

- The lines marked $a$ and $b$ in Figures 21.7 and 21.8 show respectively $\theta_{0}=0.65$ $\lambda / D$ and $\sigma_{R}^{2}=0.5 \mathrm{np}^{2}$. The ABL is designed to function in the region below both these curves. These limitations on $L_{\max }$ are important primarily for low-altitude engagements.

The natural parameter for describing ABL performance is the altitude $h_{t}$, but the timesince-launch of the missile is the important parameter for understanding the time line. Figure 21.9 shows $h_{t}$ vs. $t$ for both the liquid-propellant ICBM L and the solid-propellant ICBM S2. The vertical lines (a), (b), and (c) show critical times and their relation to $h_{t}$. For example, a useful engagement of the liquid-propellant missile can begin at $92 \mathrm{~s}$ at which $h_{t}$ is $30 \mathrm{~km}$ and, from Figure 21.8, the useful range of the ABL would be about $320 \mathrm{~km}$. The useful range is more than $500 \mathrm{~km}$ for $t>125 \mathrm{~s}$.

\subsubsection{Useful slant range}

The maximum distance for disabling a missile depends on the engagement time, $t_{e}$, i.e., the dwell time of the engagement during which the laser delivers energy to the missile. In rough terms, liquid-propellant missiles can be intercepted at a slant range of about $600 \mathrm{~km}$ for $t_{e}=20 \mathrm{~s}$ and $450 \mathrm{~km}$ for $t_{e}=5 \mathrm{~s}$. Solid-propellant missiles can be intercepted at about $300 \mathrm{~km}$ for $t_{e}=20 \mathrm{~s}$ and $180 \mathrm{~km}$ for $t_{e}=5 \mathrm{~s}$. 


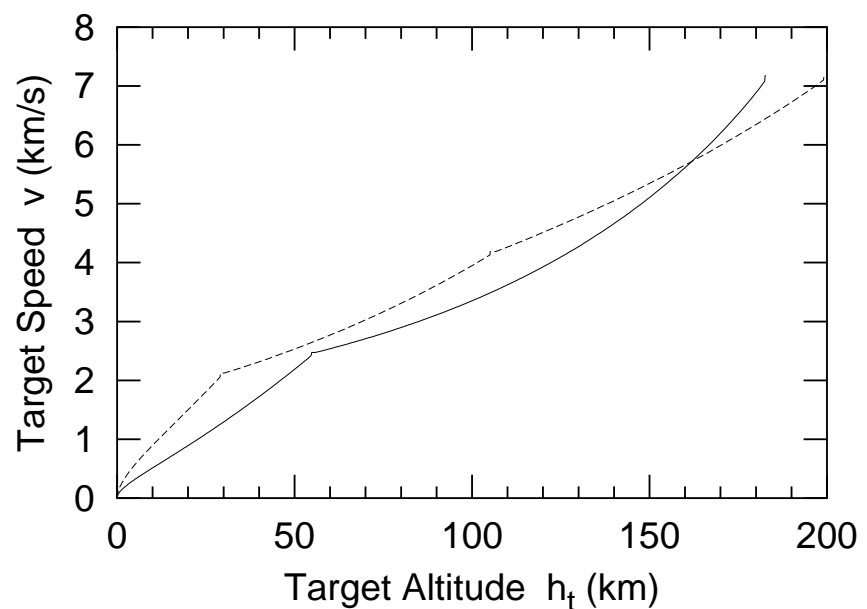

Figure 21.6. Speed versus altitude for liquid-propellant ICBM L (solid line) and solid-propellant ICBM S2 (dashed line).

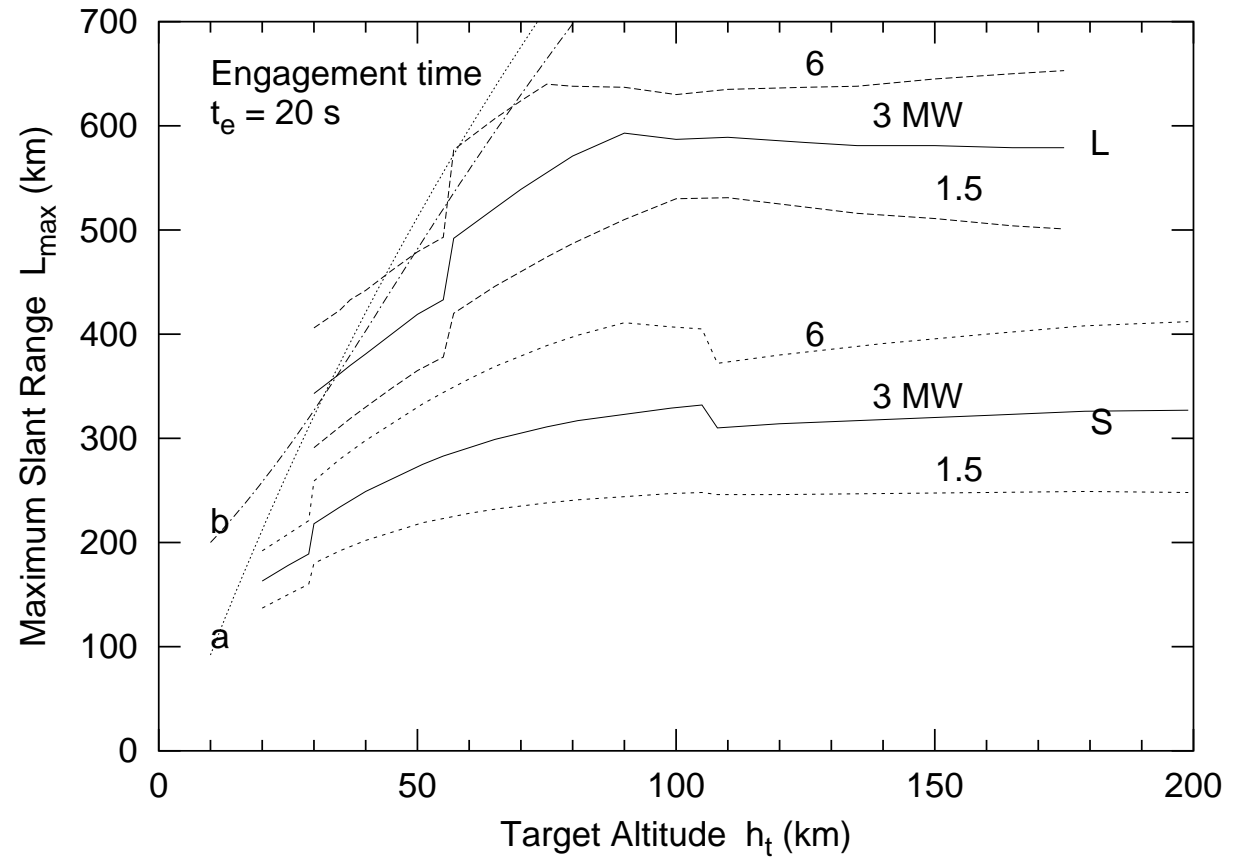

Figure 21.7. Engagement of liquid- and solid-propellant ICBMs for $t_{e}=20 \mathrm{~s}$, in which the laser power is $6 \mathrm{MW}, 3 \mathrm{MW}$, and $1.5 \mathrm{MW}$. The upper three curves correspond to values for $L_{\max }$ for the liquid-propellant ICBM L and the lower three correspond to values for $L_{\max }$ for solid-propellant ICBM S2. The lines marked $a$ and $b$ show respectively $\theta_{0}=0.65 \lambda / D$ and $\sigma_{R}^{2}=0.5 \mathrm{np}^{2}$; see text. 


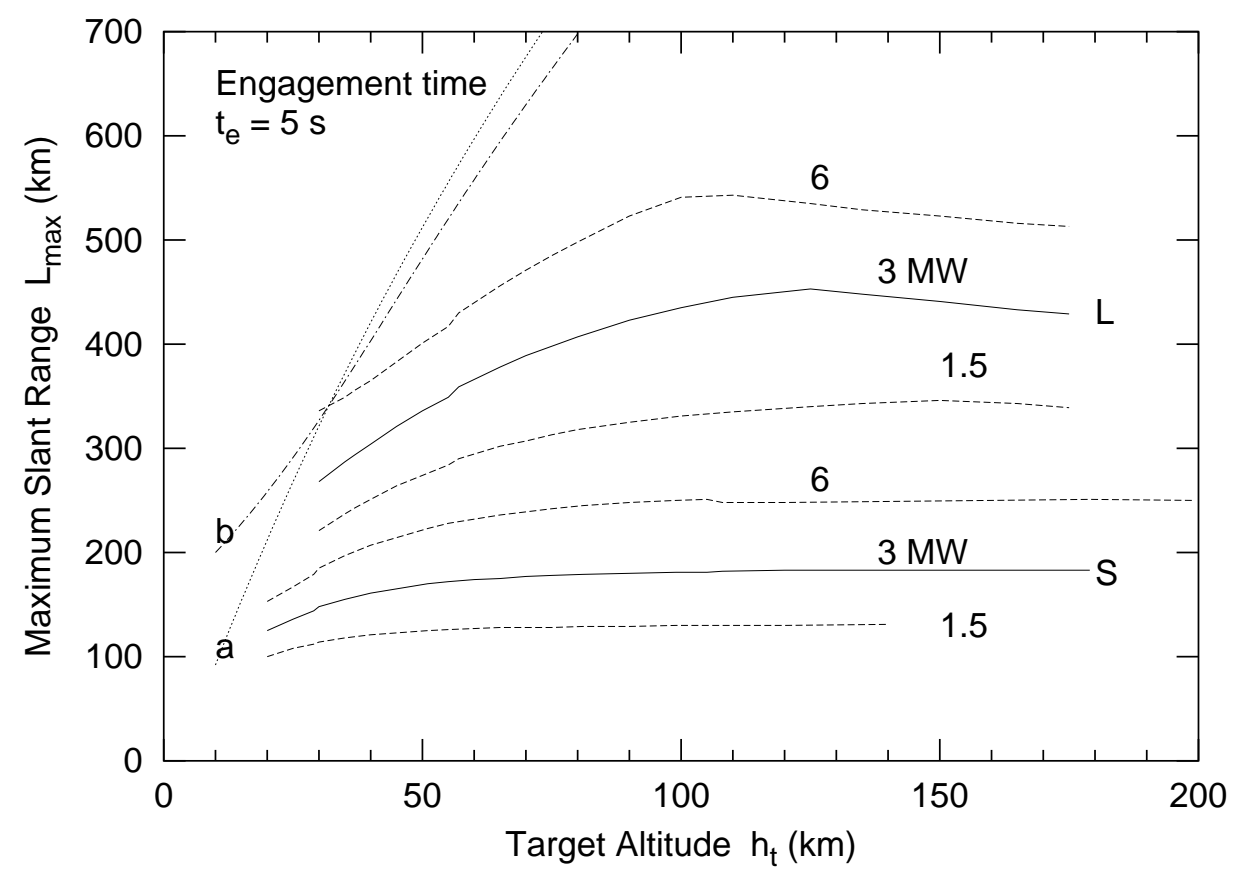

Figure 21.8. Engagement of liquid- and solid-propellant ICBMs for $t_{e}=5 \mathrm{~s}$, in which the laser power is $6 \mathrm{MW}, 3 \mathrm{MW}$, and $1.5 \mathrm{MW}$. The upper three curves are for the liquid-propellant ICBM L and the lower three are for the solid-propellant ICBM S2. The lines marked $a$ and $b$ show respectively $\theta_{0}=0.65 \lambda / D$ and $\sigma_{R}^{2}=0.5 \mathrm{np}^{2}$; see text.

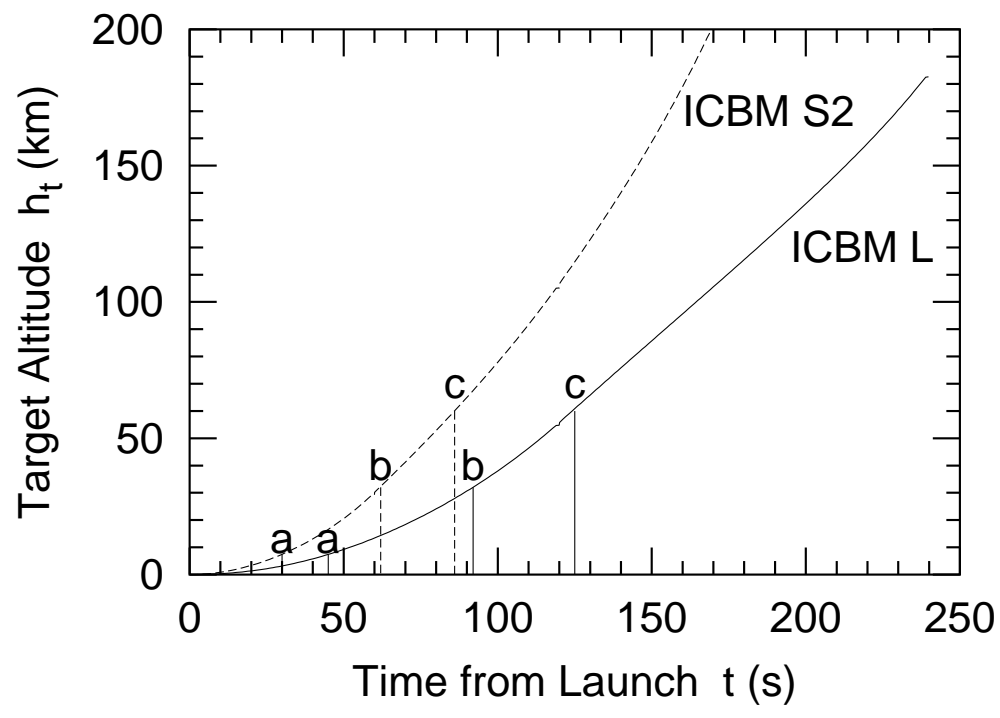

Figure 21.9. Altitude $h_{t}$ vs. time $t$ since launch for the solid-propellant ICBM S2 (dashed line) and the liquid-propellant ICBM L (solid line). (a) The missile is detected (at $45 \mathrm{~s}$ for the liquid and at $30 \mathrm{~s}$ for solid). (b) Earliest useful engagement. (c) Beginning of long-range performance plateau; see Figure 21.7. 
The slant range for 20-s engagements is only moderately larger than for $5 \mathrm{~s}$. The difference is much less than the naive estimate for vacuum propagation $L_{\max } \propto \sqrt{t_{e}}$, because the relation between the fluence and $t_{e}$ is non-linear (Section 21.4.2), and the irradiance does not decrease as $1 / L^{2}$ because of the uncorrected beam spreading caused by turbulence.

Intercepting solid-propellant missiles is substantially more difficult than liquid-propellant missiles for two reasons. The most obvious reason is the greater hardness of the missile body, a factor of 8 in $F_{c}$ required. In addition, the solid-propellant missiles are substantially shorter, causing increased anisoplanatism from the length.

\subsubsection{Parameter sensitivity}

Several assumptions have been made in the choice of engagement parameters. In this section we examine the sensitivity to those assumptions.

The nominal laser power was assumed to be $3 \mathrm{MW}$ and the nominal critical fluence for intercepts was assumed to be $32 \mathrm{MJ} / \mathrm{m}^{2}$ for liquid-propellant missiles and $240 \mathrm{MJ} / \mathrm{m}^{2}$ for solid-propellant missiles. Figures 21.7 and 21.8 show $L_{\max }$ vs. $h_{t}$ for $t_{e}=20 \mathrm{~s}$ and $5 \mathrm{~s}$ respectively. In the figures, the laser power has been varied from $3 \mathrm{MW}$ up to $6 \mathrm{MW}$ and down to 1.5 MW. Alternatively, these variations can be interpreted as varying the critical fluence down and up by a factor of 2 with respect to the nominal values. The results are shown to be relatively insensitive to factor-of- 2 changes. In particular, for factor-of- 2 power variations, $L_{\max }$ changes by \pm 10 percent to \pm 15 percent for liquid-propellant missiles and $t_{e}=20 \mathrm{~s}$, and \pm 20 percent for $t_{e}=5 \mathrm{~s}$. For solid-propellant missiles, the variation is \pm 20 percent for $t_{e}=20 \mathrm{~s}$ and \pm 30 percent for $t_{e}=5 \mathrm{~s}$. In rough terms, $L_{\max }$ scales with the power as $P^{s}$, where $s$ ranges from 0.15 to 0.3 for the liquid-propellant missile and 0.25 to 0.3 for the solid-propellant missile. This behavior can be far from the naive scaling for vacuum propagation for which $s=0.5$. One consequence of this slow variation of $L_{\max }$ with $P$ is that the effectiveness of the defense is decreased less than one might expect if the laser power is less than expected or the targets are somewhat harder than assumed. On the other hand, the same behavior means that it is difficult to change the effective range very much by increasing the laser power. For example, increasing the beam power from $3 \mathrm{MW}$ to $30 \mathrm{MW}$ and using the same algorithm for computing the range increases the range only from about $600 \mathrm{~km}$ to about $750 \mathrm{~km}$ for the liquid-propellant missile and from about $300 \mathrm{~km}$ to about $550 \mathrm{~km}$ for the solid-propellant missile. Even if such a power were available, thermal blooming may prevent its effective utilization (App. D.6.2).

The nominal beam quality of the laser is 1.2 times diffraction limited. Calculations were done assuming 1.4 and 1.6 times diffraction limited to test the sensitivity to this parameter. The loss of range is small. For the case of 1.6 times diffraction limited, the loss of range for the liquid-propellant missile is only 4 percent for $t_{e}=20 \mathrm{~s}$ and 15 percent for $t_{e}=5 \mathrm{~s}$. For solid-propellant missiles the loss is 12 percent for $t_{e}=20 \mathrm{~s}$ and 20 percent for $t_{e}=5 \mathrm{~s}$. For 1.4 times diffraction limited, the fractional loss of range is about half the above. The very low sensitivity for the liquid-propellant missile and $t_{e}=20 \mathrm{~s}$ is because of the relatively low Strehl ratio, which is dominated by factors other than the laser. For the other cases, the loss of Strehl ratio is similar to a loss of roughly a factor of 1.5 in power for the 1.6 times diffraction limited case, and correspondingly less for 1.4 times diffraction limited.

A similar set of calculations was done in which the turbulence was varied from the nominal $2 \times$ "Clear- 1 Night" to $1 \times$ and $4 \times$. The change in $L_{\max }$ was less than that shown in Figures 21.7 and 21.8 for a factor-of- 2 variation of the laser power, sometimes much less. The reason for the modest variation is that for the engagements of interest, which occur at 


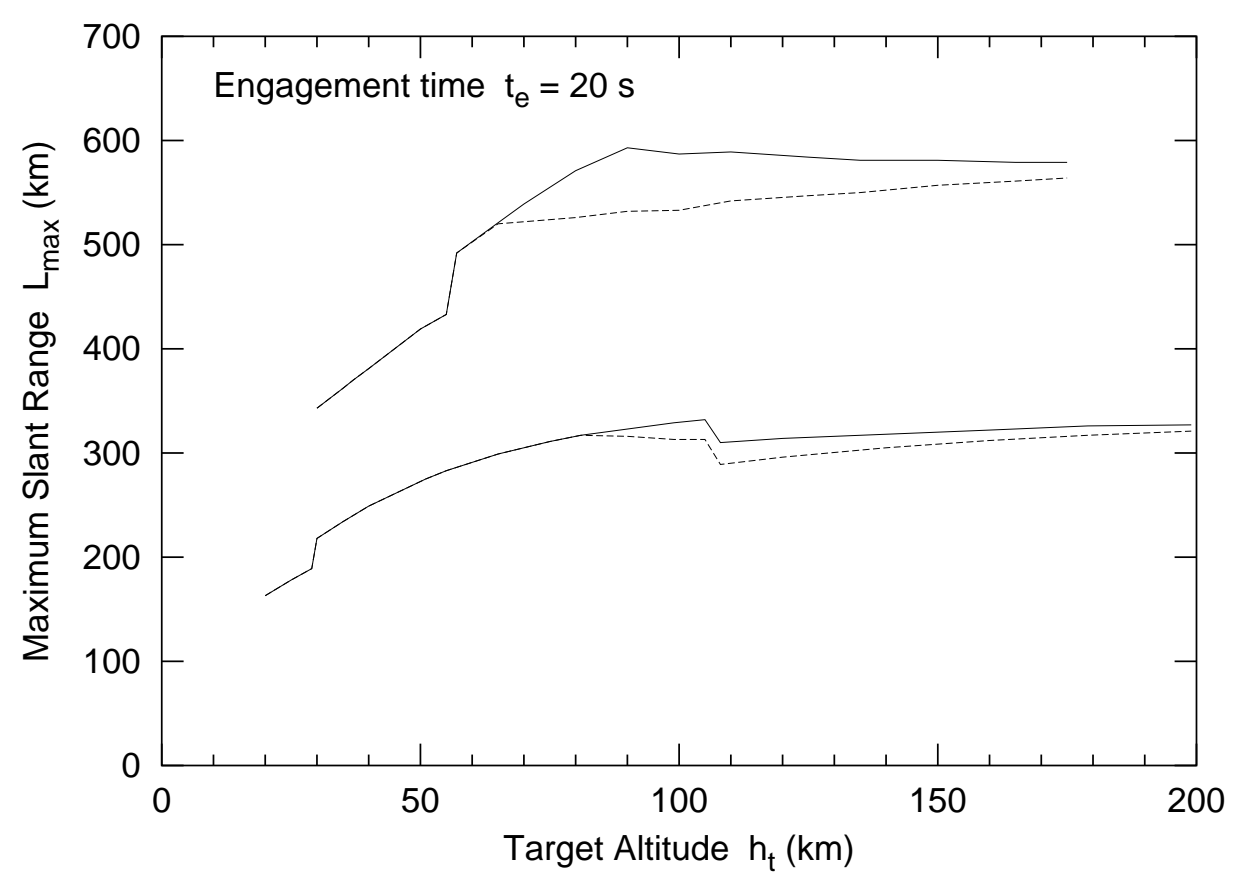

Figure 21.10. Engagement of liquid- (upper lines) and solid-propellant missile (lower lines) boosters for $t_{e}=20 \mathrm{~s}$, in which the nominal length of the missile was used (solid line), and an aim point $3 \mathrm{~m}$ shorter than the length was used (dashed line).

high altitude, the Strehl ratios achieved are relatively high, so the effect of turbulence is modest.

Finally, Figures 21.10 and 21.11 show how sensitive the engagements are to the assumption made of the optimum engagement, i.e., an engagement as close to isoplanatic as permitted by the length of the target. If, for example, the aim point needed to be $3-\mathrm{m}$ shorter than the target, additional anisoplanatism may be introduced, depending on the range. The figures show the effect of a $3-\mathrm{m}$ change. The effect is significant, but not large.

\subsubsection{Staging limits}

Staging events will probably be avoided. Unless one relies on the buckling failure mode of the missile, hitting a stage that is about to burn out is not fruitful. Also, there is an abrupt change of kinematics, and some time will be required to reestablish an acceleration profile to keep the beam on target. Finally, kill assessment may be difficult during the staging event.

\subsubsection{Engagement range}

The possible flying areas for the ABL have a somewhat different character from the equivalent basing areas for boost-phase defense utilizing terrestrial-based interceptors. The useful range depends on the engagement altitude, both for atmospheric propagation reasons, previously discussed, as well as geometric reasons. An important distinction must be made between the slant range $L$ (ABL to target) used in the ABL propagation discussion and the laser ground range, $L G R=\sqrt{L^{2}-h_{t}^{2}}$. A high-altitude attack will use up some of the slant 


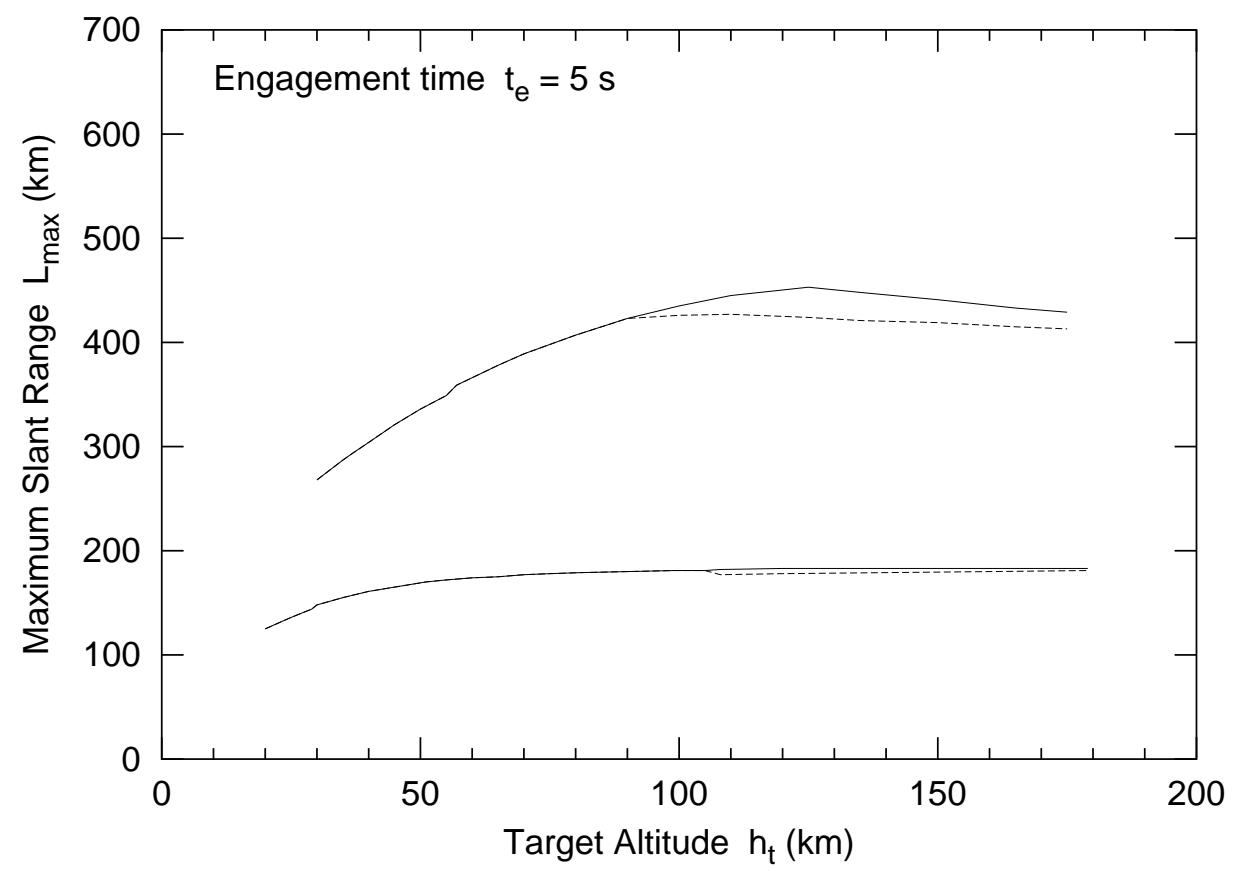

Figure 21.11. Engagement of liquid- (upper lines) and solid-propellant (lower lines) missiles for $t_{e}=5 \mathrm{~s}$, in which the nominal length of the missile was used (solid line), and an aim point $3 \mathrm{~m}$ shorter than the length was used (dashed line).

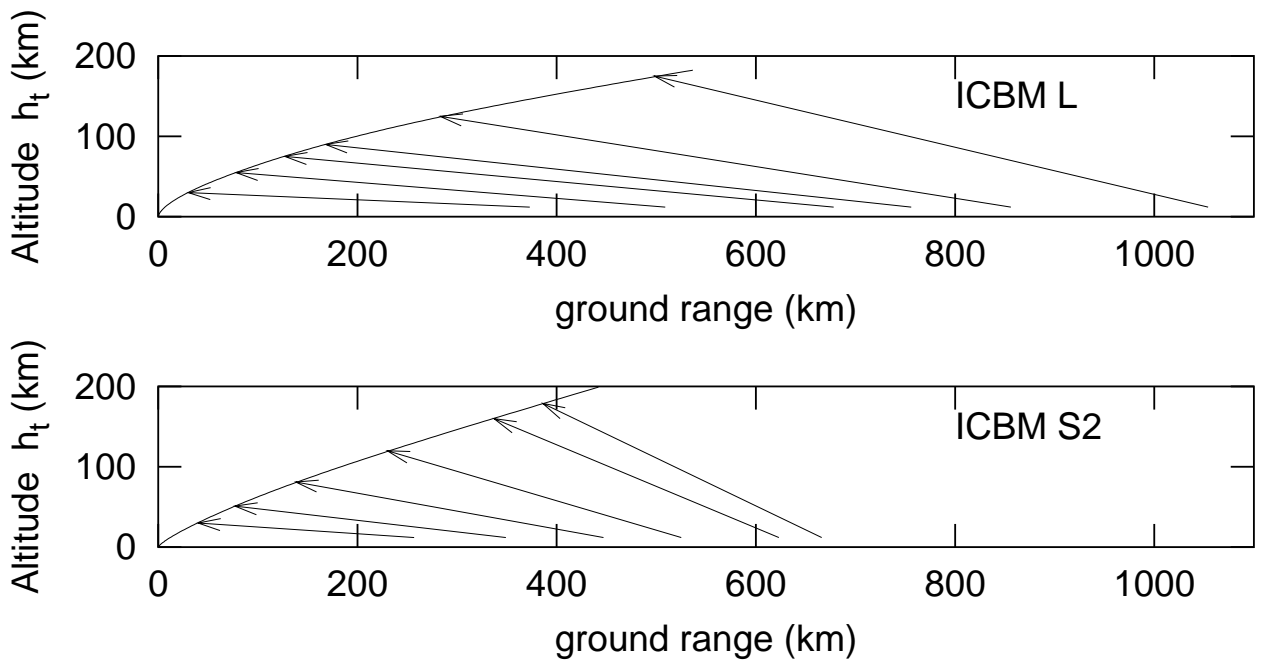

Figure 21.12. Typical engagement geometries for a liquid-propellant missile, ICBM L, (top) and a solid-propellant missile, ICBM S2, bottom, for a planar (maximum standoff distance) engagement. The missile altitude and ground range are shown by the curve rising from the lower left. The arrows show the path of the ABL beam from its 12-km altitude at various distances to the missile. The $\mathrm{ABL}$ ground range (LGR) is the projection of the arrow on the horizontal axis. There are large uncertainties in the ABL range; the values are illustrative only. 


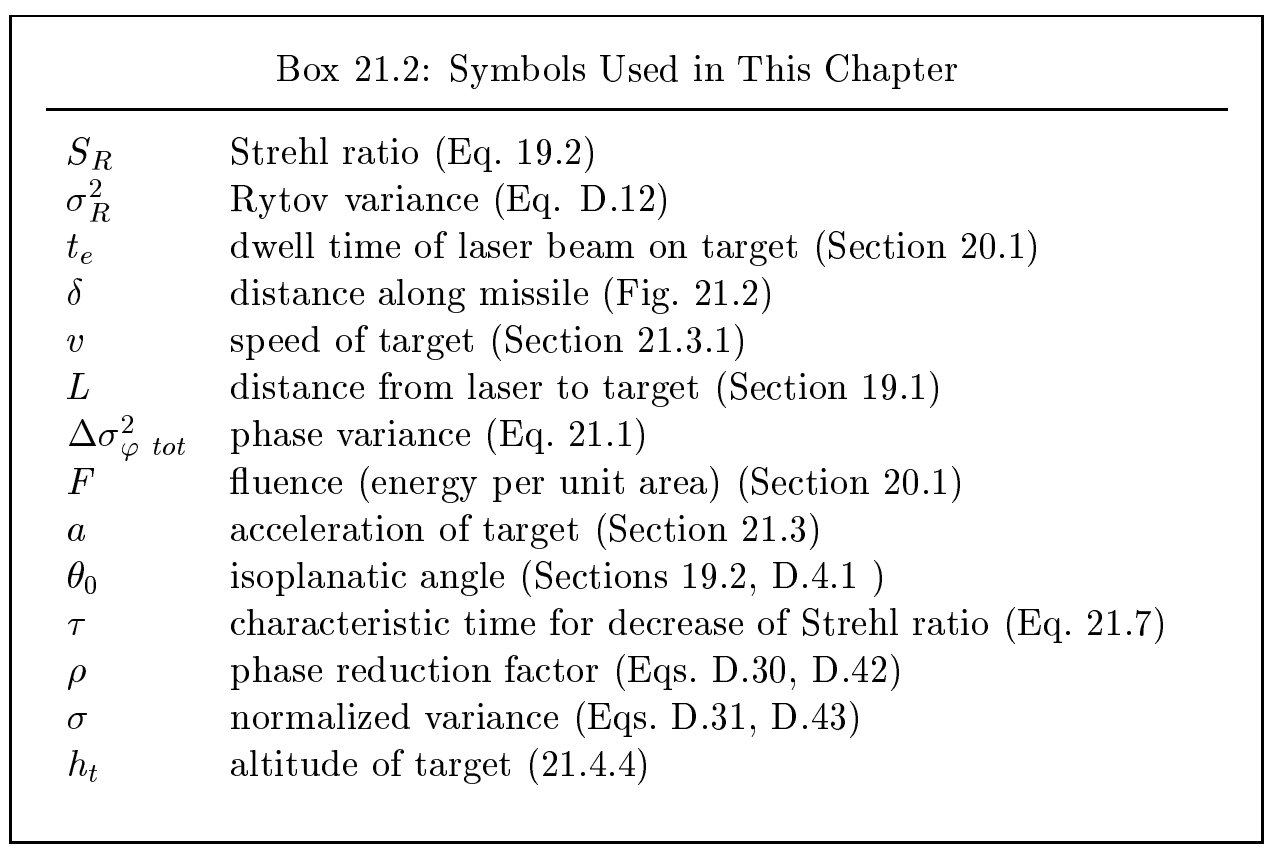

range, reducing the ground range available. There is a limit on the altitude angle to which the ABL may be directed, but a number is not available.

Figure 21.12 (a) and (b) show the maximum ABL range in planar geometry of an ABL engaging a liquid-propellant missile and a solid-propellant missile (ICBM S2), respectively. These are analogous to the engagement figures for kill vehicle intercepts of Section 4.5, showing the altitude vs. range of the missiles with arrows for the ABL laser beam from its altitude of $12 \mathrm{~km}$ to the missile. The values of the ABL range are illustrative only, because of the large uncertainties discussed.

For a liquid-propellant missile, the largest value of $L G R \approx 600 \mathrm{~km}$ is for $h_{t}=90 \mathrm{~km}$ at a standoff range (ground distance from ABL to missile launch point) of about $750 \mathrm{~km}$. The largest standoff range is about $1050 \mathrm{~km}$ for $h_{t}=175 \mathrm{~km}$ and $L G R \approx 550 \mathrm{~km}$.

The range for engaging a solid-propellant missile is considerably more limited, because of the greater hardness of the missile's body. The maximum value of $L G R \approx 300 \mathrm{~km}$ occurs for $h_{t}=105 \mathrm{~km}$ and a standoff range of about $500 \mathrm{~km}$, just before the second stage burns out. The largest standoff range is about $650 \mathrm{~km}$ for $h_{t}=180 \mathrm{~km}$ and $L G R \approx 300 \mathrm{~km}$.

\section{References for Chapter 21}

[226] Bryan L. Kelchner and Ronald Dauk, "ABL Beam Control Segment," SPIE 3381, 8 (1998).

[227] Michael E. Davey and Frederick Martin, "The Airborne Laser Anti-Missile Program." Technical Report RL30185, CRS, May 1999. CRS Report to Congress.

[228] Defense Science Board, "Defense Science Board Task Force on High Energy Laser Weapon System Applications." Technical report, Office of the Under Secretary of Defense for Acquisition, Technology, and Logistics, June 2001. 
[229] Kenneth W. Billman et al., "Airborne Laser System Common Path/Common Mode Design Approach," SPIE 3706, 196 (1999).

[230] General Accounting Office, "Significant Technical Challenges Face the Airborne Laser Program." Technical Report GAO/NSIAD-98-37, U.S. Government, October 1997. Available at: http://www.gao.gov/archive/1998/ns98037.pdf.

[231] General Accounting Office, "DOD Efforts to Develop Laser Weapons for Theater Defense." Technical Report GAO/NSIAD-99-50, U.S. Government, March 1999. Available at: http://www.gao.gov/archive/1999/ns99050.pdf.

[232] Independent Assessment Team, "Report of the Airborne Laser Program." Technical report, Office of the Secretary of Defense, February 1999.

[233] Office of the Secretary of Defense, "Report to Congress: Assessment of the Technical and Operational Aspects of the Airborne Laser Program." Technical report, Office of the Secretary of Defense, March 1999.

[234] C. Higgs et al., "Adaptive-Optics Compensation Using Active Illumination," SPIE 3381, 47 (1998).

[235] Kenneth W. Billman et al., "ABL Beam Control Laboratory Demonstrator," SPIE 3706, 172 (1999).

[236] John W. Hardy, Adaptive Optics for Astronomical Telescopes. (Oxford University Press, New York) 1998.

[237] W. G. Happer et al., "Atmospheric-turbulence compensation by resonant optical backscattering from the sodium layer in the upper atmosphere," J. Opt. Soc. Am. A11, 263 (1994). 



\title{
Chapter 22
}

\section{Deployment Considerations for the Airborne Laser}

\author{
Contents
}

22.1 Parameters of Operations . . . . . . . . . . S S339

22.2 Operating Altitude and Cloud Cover . . . . . . . S S340

22.3 ABL Defense Against Short-Range Missiles . . . . . . . S340

This chapter discusses how ABLs might be deployed in the field. Section 22.1 discusses the flight pattern and coverage. Section 22.2 takes note of possible limitations caused by high-altitude cirrus clouds. Finally, Section 22.3 briefly discusses the use of the ABL against MRBMs launched from ships off the coast of the United States.

\subsection{Parameters of Operations}

The ABL is housed in a modified Boeing 747 that patrols at an altitude of about $12 \mathrm{~km}$ to minimize the effects of the atmospheric turbulence between the ABL and the target. The ABL can direct its beam by varying the polar and roll angles of the turret; pitch angle with respect the LOF must be achieved by means of the polar and roll angles. The beam can be directed anywhere in a fan-like pattern extending about $120^{\circ}$ in polar angle on either side of the LOF (Section 21.1). The available roll angle range is not published in the open literature. A limitation of this angle can seriously restrict some engagements, particularly in the near forward direction. ${ }^{1}$

The optimum path that the aircraft flies depends on the geometrical extent and disposition of the bases of the threat missiles. Most discussions assume two aircraft flying together in a closed loop at some standoff distance from the potential missile launch sites. The choice of standoff distance would be set by the area in which the threat to the aircraft is low. A figure "8" loop, oriented with the long side facing the threat, allows the aircraft to make a turn-around at the ends, such that it never faces away from the threat area. With appropriate phasing of the two aircraft, at least one aircraft will have the threat ahead or to the side of its flight path at all times. The size of the flight pattern will depend on the geometrical extent of the threat area, but the minimum size is set by the aircraft turning radius, about $25 \mathrm{~km}$. The aircraft can stay on station for long periods of time by using in-flight refueling, which requires descent to lower altitudes. The ABL plan is to have seven

\footnotetext{
${ }^{1}$ For example, a target directly ahead in bearing, but higher than the $\mathrm{ABL}$ will require rotating the turret to a $90^{\circ}$ roll angle.
} 
aircraft to be able to provide two aircraft in the air at all times. The strategy for managing the flight operation is not treated in this study.

Just as for kill vehicle interceptors, tail-chase intercepts by the ABL are unfavorable, but for different reasons. It is futile for the ABL to direct its laser beam against the rocket nozzle; the beam must strike the side of the missile. A grazing incidence on the side results in reduced fluence onto the surface. A cone with half angle of $11^{\circ}$ from the missile axis would limit the fluence on target to about 20 percent that of normal incidence. The criteria in Section 20.1 have assumed 50 percent for this factor. A more subtle limitation is the loss of a well-defined feature, such as the missile nose, for the AO compensation. The nose itself may be obscured by an angle as large as $45^{\circ}$ depending on missile geometry, although the visible shoulder hiding the nose may serve the role. This has not been studied. Finally, the exhaust gases of the rocket motor are violently turbulent, and it is possible that this could stress the AO system. A mitigating factor is that the source of the turbulent medium is relatively near the target, so its effect is reduced. This possible problem has not been studied.

The fuels used by the COIL are not exotic (Section 18.2), but they are precious to the mission. The amount of fuel carried (called the magazine) allows a run time of only $100 \mathrm{~s}$ to $200 \mathrm{~s}$ [238]. Depending on the engagement time and the number of engagements a single aircraft must execute, this asset must be used carefully. A magazine of $100 \mathrm{~s}$ with an engagement time of $5 \mathrm{~s}$ would allow up to 20 engagements, but an engagement time of $20 \mathrm{~s}$ would allow only 5 engagements. For two aircraft, the total number of engagements could be doubled.

Protecting the ABL from attack constitutes a major challenge to its deployment. The ABL is vulnerable to attack from the ground by surface-to-air missiles, and also to aircraft attack. This issue is treated in greater detail in Chapter 9.2.

\subsection{Operating Altitude and Cloud Cover}

A high operating altitude for the ABL is necessary to minimize the effects of atmospheric turbulence and to be above as much cloud cover as possible. Cirrus clouds between the ABL and the target would be a serious problem, because the scattering of the beam would be so severe as to make a successful engagement impossible. The ABL cannot "burn through" the clouds.

Sub-visual and thin cirrus clouds occur quite frequently, from 40 percent to 70 percent of the time [239] in mid latitudes. The altitude of these clouds varies with latitude and season, with the highest altitudes occurring in low latitudes and in summer. The mean altitude is at about 80 percent of the tropopause, which varies from $18 \mathrm{~km}$ at the equator to $8 \mathrm{~km}$ at the poles. It is estimated [239] that such clouds exist above $12 \mathrm{~km} 50$ percent of the time, and above $15 \mathrm{~km} 10$ percent of the time. The nominal altitude of the ABL is $12 \mathrm{~km}$. It is not known what the ceiling for the fully loaded ABL would be, but $15 \mathrm{~km}$ may be close to the limit. Commercial 747 operating ceilings are variously reported from 12.4 to $13.6 \mathrm{~km}$ (41,000 to 45,000 feet). Thus, there could be a problem with the fraction of time the ABL is available for engagements.

\subsection{ABL Defense Against Short-Range Missiles}

The discussion has concentrated on the use of the ABL against ICBMs. The threat of TBMs and MRBMs launched from ships off the US coast is an identified problem; see Section 5.7.1. 
These cases have not been analyzed in detail, but low-altitude engagements at close range and very high irradiance, and therefore short engagement times, are possible.

Figure 5.18 shows the trajectories of MRBMs M1 and M2, including time tics. From this and the information in Fig. 21.8, the useful ABL engagement ranges may be estimated. Figure 21.8 strictly applies to the ICBMs whose flight characteristics enter into the detailed ABL performance, but it should be a reasonable approximation to the performance against MRBMs. The M1 presents a more stressing time line than M2, because the total boost time is only $65 \mathrm{~s}$, and the missile burns out at an altitude of only $50 \mathrm{~km}$. For an engagement time of $5 \mathrm{~s}$, a liquid-propellant M1 could be engaged at a range up to $150 \mathrm{~km}$ when it reached an altitude of $20 \mathrm{~km}, 35 \mathrm{~s}$ after launch, or a range exceeding $200 \mathrm{~km}$ once it rose above $30 \mathrm{~km}$, $44 \mathrm{~s}$ after launch. As with the ship-borne case, the shortfall problem has not been analyzed if the missile target is well inland.

Such a mission would likely not subject the ABL to attack from enemy aircraft, but ships could carry surface-to-air missiles which could be a threat. The primary problem is likely to be logistical support. As with ship-borne kill vehicle coastal defense (Section 5.7.1), cueing information would be required to know which ships might be threatening. A disadvantage of the ABL with respect to a ship-borne interceptor is that the ABL could not take out a ship that had already launched a missile and thus prevent any more launches.

\section{References for Chapter 22}

[238] Col. James Forrest, SMDC Media Update. Values reported for the magazine usually lie in the range of $100-200 \mathrm{~s}$. This briefing reported a magazine of $480 \mathrm{~s}$, but this is inconsistent with all other information. July 2001.

[239] D. A. Nahrstedt, "Cloud Modeling for Laser Weapon Propagation Analysis," SPIE 4034, 69 (2000). 



\title{
Appendix A
}

\section{Ballistic Missile Types and Phases of Flight}

\author{
Contents \\ A.1 Classes of Ballistic Missiles _. . . . . . . . . . . S S343 \\ A.1.1 Short- and medium-range ballistic missile (SRBMs and MRBMs) S343 \\ A.1.2 Intermediate-range ballistic missiles (IRBMs) . . . . . . . . S344 \\ A.1.3 Intercontinental ballistic missiles (ICBMs) . . . . . . . . . S344 \\ A.2 Phases of Ballistic Missile Flight . . . . . . . . . . S345

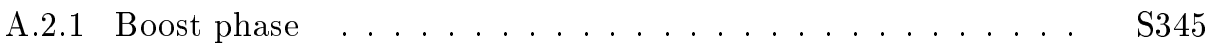 \\ A.2.2 Payload deployment . . . . . . . . . . . . . . S346 \\ A.2.3 Midcourse phase . . . . . . . . . . . . . . . . . S347

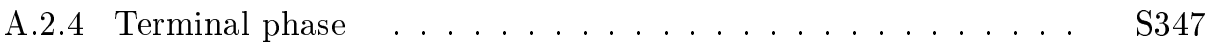

This appendix provides background information that is important for understanding the Report. Section A.1 explains how ballistic missiles are categorized by range and propellant. These categories are used throughout the Report. Section A.2 describes the phases of the flight of a ballistic missile. This information is needed to understand the role of the boost phase in the overall flight of such a missile, and the relation of a boost-phase missile defense to defenses that aim to destroy missiles or warheads in the midcourse or terminal phases of flight. It also provides context for the descriptions of missile trajectories presented in Chapters 15 and 5 and the discussions of the deployment of warheads and penetration aids in Chapters 5, 13, and 9.

\section{A.1 Classes of Ballistic Missiles}

Ballistic missiles can be classified by range, although the boundaries are not rigid, and by the type of propellant used.

\section{A.1.1 Short- and medium-range ballistic missile (SRBMs and MRBMs)}

The United States defines missiles with ranges of less than 1,000 km as short-range ballistic missiles (SRBMs) and those with ranges between 1,000 and 3,000 km as medium-range ballistic missiles (MRBMs) [240]. SRBMs typically have only a single booster stage.

Although SRBMs and MRBMs are usually intended for tactical applications in a military campaign and are therefore sometimes called tactical or theater ballistic missiles (TBMs), they could be used to attack the territory of the United States and therefore can also be 
considered as potential strategic weapons. In the present Study, we categorize missiles by their physical characteristics, such as their range, rather than by the presumed intent of the entity deploying them, which may be unknown and could change on short notice.

The Russian Scud B is an example of a widely available SRBM. Its weight at launch is about $6,000 \mathrm{~kg}$, and it can deliver a 1,000-kg payload to a range of $300 \mathrm{~km}$ [241].

If launched from a ship or other sea platform positioned within range of U.S. coastal regions, SRBMs and MRBMs could attack the territory of the United States.

\section{A.1.2 Intermediate-range ballistic missiles (IRBMs)}

The United States defines missiles with ranges between 3,000 and 5,500 km as intermediaterange ballistic missiles (IRBMs) [240]. These missiles usually have two or more booster stages.

Intermediate-range ballistic missiles can be employed for strategic as well as tactical military purposes. With additional stages added, some IRBMs could be used to launch communications, surveillance, or scientific satellites.

The North Korean No Dong-1 is an example of an IRBM. It can deliver its $1,200-\mathrm{kg}$ payload to a range of $1,500 \mathrm{~km}$. Its launch weight is $16,250 \mathrm{~kg}[241,242]$.

\section{A.1.3 Intercontinental ballistic missiles (ICBMs)}

The United States defines missiles with ranges greater than 5,500 km as intercontinental ballistic missiles (ICBMs) [240]. They can deliver warheads to large areas of Earth from a single launch site. Some authors subdivide the ICBM category into limited-range ICBMs, which have ranges less than $8,000 \mathrm{~km}$, and full-range ICBMs, which have ranges greater than $8,000 \mathrm{~km}$.

There are two fundamentally different types of ballistic missiles: liquid-propellant ICBMs typically have two stages and relatively long boost phases, whereas solid-propellant ICBMs typically have three stages and much shorter boost phases. The Soviet SS-11 is an example of an early, liquid-propellant full-range ICBM. It had a total burn time of $267 \mathrm{~s}$ and could deliver an $800-\mathrm{kg}$ payload to a range of 11,000 to $12,000 \mathrm{~km}$. The Chinese DF-5 deployed in the 1980s is also a liquid-propellant full-range ICBM. It has a total burn time of $231 \mathrm{~s}$ [241] and can deliver a 3,000-kg payload to a range of $12,000 \mathrm{~km}$ [243]. The Russian RT-2PM Topol (SS-25) is an example of a modern, solid-propellant, full-range ICBM. Similar in size to the U.S. Minuteman, it has three stages, probably has a total burn time of less than $200 \mathrm{~s}$, and can deliver its approximately $1,000-\mathrm{kg}$ payload to a range of $13,000 \mathrm{~km}$ [244].

Most liquid-propellant missiles have tankage that is pressurized only to the extent needed to feed fuel and oxidizer to turbo pumps, which in turn feed the combustion chambers of the rocket engines. Accordingly, the structural design of the stages is usually determined by acceleration and bending loads associated with controlling flight in the atmosphere. To minimize the structure weights, the optimum acceleration profiles for these missiles tend to be lower, with correspondingly longer burn times, than a solid-propellant missile having equal performance. These missiles are generally large, like the Titan II or the Russian SS-18 which burn for more than $300 \mathrm{~s}$, or the Chinese DF-5, which reportedly burns between $220 \mathrm{~s}$ and $250 \mathrm{~s}$. The North Korean Taepo Dong 2 ICBM is also reported to burn for about $250 \mathrm{~s}$. The relatively low tankage weight for liquid boosters usually results in an optimum design having two stages to achieve ranges in excess of $12,000 \mathrm{~km}$. 
Long-range ballistic missiles using solid propellant require the propellant tanks to serve as the combustion chamber; as a result, those vessels are designed for chamber pressures on the order of $600 \mathrm{psi}$ or more. The structures that result are inherently much stronger with respect to buckling and bending and are therefore capable of higher acceleration and aerodynamic loads. Because of the density of their propellant and the lack of a fuel and oxidizer expulsion system, these missiles are generally smaller than a liquid system with similar performance, and usually optimize at three stages with much shorter burn times than their liquid counterparts for the same maximum ranges.

Once out of the atmosphere, either type of missile can execute maneuvers to shape the trajectory at the expense of range, but liquid-propellant missiles are somewhat more constrained in this regard than solid boosters due to structural limits and propellant slosh. Without these limitations, solid- propellant missiles can make more radical maneuvers.

Faster-burning solid-propellant ICBMs are a natural countermeasure to any boost-phase defense. Intercontinental-range ballistic missiles based on first-generation (1960s) solidpropellant technology typically have total boost phases of $160 \mathrm{~s}$ to $180 \mathrm{~s}, 50 \mathrm{~s}$ to $100 \mathrm{~s}$ shorter than early liquid propellant ICBMs. However, these ICBMs were not designed to cope with boost-phase defenses. Studies performed in the mid-1980s [245, Section 2.3.3] suggested that with the solid-propellant technology then available, solid-propellant ICBMs having much shorter burn times that would burn out at altitudes of 80 to $100 \mathrm{~km}$ appeared feasible. There appeared to be no physical barriers to achieving such performance; the only issues were engineering tradeoffs. These studies also showed that the payload mass penalty for burning out this quickly would be relatively small. Even ICBMs having total burn times of $130 \mathrm{~s}$ would be much more challenging to a boost-phase defense than any of the model ICBMs considered in this study.

\section{A.2 Phases of Ballistic Missile Flight}

All ballistic missiles that rise above the sensible atmosphere (i.e., above about $100 \mathrm{~km}$ ) have three stages of flight: boost, midcourse, and re-entry ${ }^{1}$. Missiles that deploy their payload from a "post-boost vehicle" (PBV) after the boost phase has ended are considered to have a fourth phase of flight called the post-boost phase. This section provides a brief discussion of the phases of ballistic missile flight and their relevance to missile defense (see also [246]).

\section{A.2.1 Boost phase}

The boost phase of a missile begins when it is launched and lasts until the rocket motor of the missile's final stage is shut down or burns out. Depending on the missile, this stage can last 2 to 5 minutes. During the early part of its boost phase, the missile is moving relatively slowly, but toward the end it is moving at a speed of $7 \mathrm{~km} / \mathrm{s}$ or even faster. An attacking missile will typically execute a series of maneuvers during its boost phase to shape its trajectory so that its munitions reach their intended target or to evade any boost-phase defense (see Chapters 15 and 5).

The boost phases of ICBMs typically end at altitudes of 170-200 km, high above Earth's atmosphere (the sensible atmosphere is generally considered to be negligible for our purposes

\footnotetext{
${ }^{1}$ Some authors use the term "ascent phase" to refer to the first half of the midcourse phase, i.e., from the time the missile's final stage is shut down or burns out until the warheads reach the highest points of their trajectories (their apogees). Others use the term "ascent phase" to refer to the boost phase as well as the first half of the midcourse phase.
} 
at about $100 \mathrm{~km}$ ). Once a missile's boost phase ends, its final stage continues on a ballistic trajectory, acted on primarily by gravity.

A boost-phase intercept (BPI) defense must intercept and disable ICBMs during the few minutes their rocket motors are burning. To accomplish this, rocket interceptors must be based close enough to reach and disable the missiles before they have reached a velocity that will allow their payload to strike a defended area. As they approach the missile, boost-phase interceptors must shift from homing on the bright plume of the rocket motor to homing on the missile body, and thus must be able to hit and disable the missile as it maneuvers. A missile defense system that can disable missiles launched from a particular launch site can, in principle, defend the entire United States from attack by missiles launched from that site.

\section{A.2.2 Payload deployment}

The payload of a missile includes its munitions and any devices to aid in penetrating a missile defense ("penetration aids"). The munitions may consist of one or more chemical explosive warheads, nuclear warheads, or chemical or biological munitions. To disperse chemical or biological agents over a wide area, a missile may deploy dozens or even hundreds of small bomblets called "submunitions."

The components of an ICBM's warhead, or "payload", could be deployed in several ways (see Chapters 15 and 9). One way is to release the payload after the missile's propellant has been exhausted or its thrust has been terminated, but while the attitude of the final boost stage is still under control. In this case, the payload is separated "softly." If the payload is a warhead, then the warhead may be reoriented and spin-stabilized so that its trajectory during re-entry is more predictable. Sometimes a vernier adjustment to the warhead's velocity is made during the process, and the warhead is further separated from the spent final stage.

Of particular significance for boost-phase defenses, warheads and penetration aids could be deployed while the final stage is still in powered flight. Historically, multiple re-entry vehicles (MRVs) were sequentially ejected from the accelerating booster. For example, the final stage of the Soviet SS-9 Mod 2 liquid-propellant ICBM deployed three re-entry vehicles (RVs) as it continued to burn. The final stage of the U.S. Polaris A3 solid-propellant ICBM deployed both RVs and countermeasures while in powered flight. The small eject motors of the RVs had four nozzles that were canted to impart the desired spin to each RV while accelerating the RV to velocities relative to the booster of about a hundred meters per second. In both cases the guidance and control system remained on the final stage and continued to guide it; the RVs lacked their own guidance and propulsion systems. Using the much smaller and cheaper inertial measurement systems and Global Positioning System (GPS) receivers that are widely available today, multiple RVs, each with its own propulsion and guidance, could be deployed sequentially or in parallel at any time during final-stage boost, as long as center-of-gravity symmetry is preserved.

Munitions and penetration aids could also be deployed from a "post-boost vehicle" (PBV) or "bus" [245, Section 2.3.4]. A PBV carries the primary missile guidance system and has its own control and propulsion systems. It separates from the missile's final stage after the final stage has been shut down or burns out and then executes a series of maneuvers to sequentially deploy RVs and penetration aids having different release conditions to attack different targets. Such RVs are called "multiple independently targeted re-entry vehicles" (MIRVs). The period from the time the PBV has separated from the final stage until it has 
deployed all its warheads and penetration aids is considered a fourth phase of flight, called the post-boost phase.

The MIRV systems of both the United States and the former Soviet Union were somewhat inefficient from a propulsion and volumetric standpoint, but they allowed a single large and expensive unit having guidance and control and propulsion systems to target all the RVs. With technology that is widely available today, RVs having their own individual guidance and propulsion systems could be deployed in parallel simply and cheaply. Systems of this type ("multiple third stages") were evaluated in the 1960s during preliminary studies of the feasibility of MIRV systems and were found to be as good in terms of weight and volume efficiency as a bus-type system. The principal drawback at that time was the complexity and cost of multiple guidance systems that had to be accessible for maintenance. This constraint would no longer be a factor.

Deploying munitions during the powered flight of the final stage is a natural countermeasure to boost-phase defense of any kind. When determining the time available to achieve intercept, a boost-phase defense must assume that the missile's warheads could be deployed during the powered flight of the final stage, as well as after the final stage has been shut down or has burned out. No matter how a missile's munitions are deployed, the only way the defense can be certain of intercepting the missile before it has deployed its munitions is to intercept it before it has achieved a velocity that would carry them to the defended area.

\section{A.2.3 Midcourse phase}

The midcourse phase begins once the missile's final stage or PBV has ceased firing and ends when the missile's munitions begin to reenter Earth's atmosphere. This is the longest phase of the flight of a long-range missile and may last 20 minutes or longer for a full-range ICBM.

During the midcourse phase the missile's final stage, its munitions, and - if present - the PBV and any penetration aids fall along ballistic trajectories under the influence of gravity (aerodynamic forces are negligible). These objects, called the "threat cloud," ascend toward their apogees during the first half of the midcourse phase and then descend toward Earth during the latter half.

The components of the threat cloud can be tracked by radars or other sensors during the midcourse phase. With sufficient tracking information, their future positions can be predicted accurately. Because all the components of the threat cloud, including penetration aids such as decoys, remain close to one another during the midcourse phase, a midcourse intercept system must be able to overcome any countermeasures and discriminate between warheads and decoys to intercept and disable the warheads. Correctly identifying warheads and decoys is called the discrimination problem. A single missile defense site supplied with long-range midcourse interceptors could in principle defend a large geographic area against missiles coming from a range of directions.

\section{A.2.4 Terminal phase}

The terminal phase of flight begins when the missile's warheads reenter Earth's atmosphere and ends when they detonate or strike Earth's surface. This phase lasts less than a minute for strategic warheads, which can enter the atmosphere at speeds of about $7 \mathrm{~km} / \mathrm{s}$, and depending on their aerodynamic characteristics (ballistic coefficient), can impact at 1 to $3 \mathrm{~km} / \mathrm{s}$. Warheads can be designed to maneuver during their terminal phase using aero- 
dynamic forces. Their motion is also affected by winds in the upper atmosphere and near Earth's surface.

Since aerodynamic drag causes less-dense objects to fall through the atmosphere more slowly than warheads, lightweight decoys and countermeasures are rendered ineffective during the terminal phase. A missile defense designed to intercept warheads during the terminal phase could defend a limited area against attack by missiles launched from anywhere in the world.

\section{References for Appendix A}

[240] National Intelligence Council, "Foreign Missile Developments and the Ballistic Missile Threat to the United States Through 2015," Unclassified Summary of a National Intelligence Estimate. Available at:

http://www.cia.gov/cia/publications/nie/nie99msl.html\#rtoc7, September 1999.

[241] David Wright, "Ballistic missile systems." Briefing Presented to the APS Study Group, July 2001.

[242] Charles P. Vick, "North Korean No Dong Missile." Available at: http://www.fas.org/nuke/guide/dprk/missile/nd-1.htm, 2002.

[243] Charles P. Vick, "Chinese DF-5 Missile." Available at: http://www.fas.org/nuke/guide/china/icbm/df-5.htm, 2000.

[244] Charles P. Vick, "Russian RT-2PM Topol (SS-25) Missile." Available at: http://www.fas.org/nuke/guide/russia/icbm/rt-2pm.htm, 2000.

[245] N. Bloembergen et al., Rev. Mod. Phys. 59, S1 (1987).

[246] Office of Technology Assessment, "Ballistic Missile Defense Technologies." Technical Report OTA-ISC-254, U.S. Congress, Government Printing Office, Washington, D.C., September 1985. Available at: http://www.fas.org/spp/starwars/ota/. 


\section{Appendix B}

\section{Calculating Missile Trajectories}

\section{Contents}

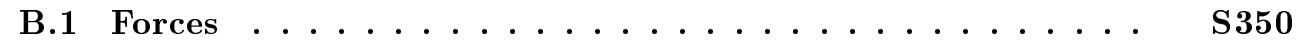

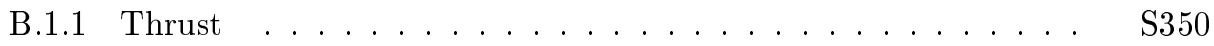

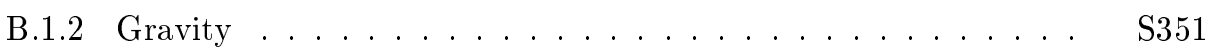

B.1.3 Aerodynamic forces . . . . . . . . . . . . . . S351

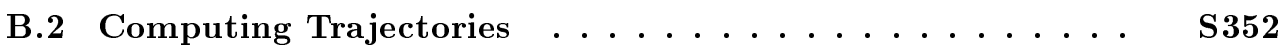

B.2.1 Illustrative equations . . . . . . . . . . . . . . S S352

B.2.2 Solving the trajectory equations . . . . . . . . . S353

B.3 Simple Scaling Relations . . . . . . . . . . . . . S354

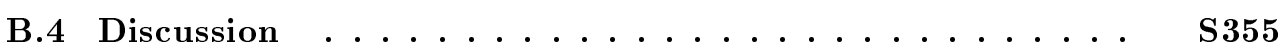

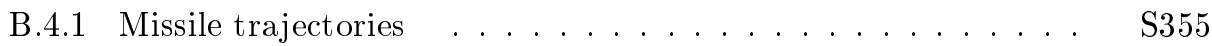

B.4.2 Interceptor trajectories . . . . . . . . . . . S356

Using interceptor missiles to intercept attacking missiles during their boost phases involves hitting rockets in powered flight with other rockets in powered flight. Analyzing such engagements requires computation of the flyout trajectories of both the attacking missiles and the interceptors. The purpose of this appendix is to explain how the rocket trajectories used in the Study were computed.

A variety of different computer codes were used to compute the trajectories needed. These codes were validated by comparing their results with one another and with results of computer calculations carried out at Sandia National Laboratories using trajectory codes that have been validated over many years of use. Illustrative missile and interceptor flyout trajectories are presented in Chapters 15 and 16, respectively. Suites of trajectory calculations are used to analyze engagements in Chapters 4 and 5 and to analyze the problem of managing the shortfall of debris and munitions in Chapter 5.

Section B.1 discusses the forces that act on a rocket in powered flight and describes how they were treated in computing the trajectories used in the Study. Section B.2.1 describes how trajectories were computed by first displaying an illustrative set of differential equations describing the ascent of a rocket through the atmosphere and then discussing the approximations typically adopted in solving such sets of equations. Many of the physical quantities and variables relevant to trajectory calculations are defined in this section. Section B.3 derives some simple scaling relations that are cited or used in various chapters. Section B.4 discusses briefly the trajectories of ICBMs and interceptor missiles for boost-phase defense. Some of the comments and remarks in this section may help readers unfamiliar with rockets 
understand better some of the material in Chapters 15, 16, 4, and 5. Introductions to rocket flight may be found in the recent monographs by Griffin and French [247] and Sutton and Biblarz [248].

\section{B.1 Forces}

The principal forces that act on a rocket during its ascent through Earth's atmosphere are the thrust generated by its motor, the gravitational attraction of Earth, and - when the rocket is within the atmosphere - the aerodynamic lift and drag that it experiences. ${ }^{1}$

\section{B.1.1 Thrust}

A rocket propulsion system accelerates the rocket by burning propellant (fuel and oxidizer) in a combustion chamber and then allowing the burned propellant to escape as hot gas through one or more nozzles. The total force produced by the exhaust is the thrust of the rocket motor. Its magnitude is $[247, \S 5.1]$

$$
T=\dot{m}_{p} \bar{v}_{e}+\left[p_{e}-p_{a}(h)\right] A_{e} .
$$

The first term on the right-hand side of Eq. (B.1) is the momentum thrust and the second is the pressure thrust. The momentum thrust is the product of the propellant mass flow rate $\dot{m}_{p}$ and the average velocity $\bar{v}_{e}$ of the rocket exhaust relative to the vehicle at the exit plane of the rocket nozzle. The pressure thrust is the product of the difference between $p_{e}$, the pressure of the hot exhaust gas at the exit plane, and $p_{a}$, the ambient atmospheric pressure at the altitude $h$ of the rocket, and the cross-sectional area $A_{e}$ of the nozzle exit.

The performance of a rocket propulsion system is usually characterized by its specific impulse $I_{\mathrm{sp}}$, which is the thrust it generates per unit weight (at sea level) of propellant expended, i.e.,

$$
I_{\mathrm{sp}} \equiv T / \dot{m}_{p} g_{s}
$$

Here $g_{s}$ is the acceleration of gravity at mean sea level. Specific impulse $I_{\mathrm{sp}}$ has units of seconds in both the metric and English Engineering systems.

A rocket motor's $I_{\mathrm{sp}}$ depends on the ambient pressure as well as its intrinsic properties (compare Eqs. [B.1] and [B.2]). As a rocket rises through Earth's atmosphere, the ambient pressure decreases, causing the thrust and specific impulse of its motor to increase. The thrust of a motor can be 10 percent- 15 percent greater at high altitudes than at sea level [248, Fig. 2-2]. The sea-level $I_{\mathrm{sp}}$ 's of modern chemical rockets range from about 200 s to about 400 s $[248$, Table 2-1].

Another measure of the performance of a rocket motor is its thrust-to-weight ratio $T / m g_{s}$. This measure is particularly relevant to applications, such as space-based interceptors, where low weight and high acceleration are very desirable.

The magnitude of the thrust generated by a liquid-propellant motor can be adjusted by controlling the flow of propellants into the combustion chamber. Although there is a performance penalty for throttling the engine in this way, it is generally small [248, $\S 3.8]$. The thrust of a liquid-propellant motor can be terminated by shutting off the flow of propellants to the combustion chamber or simply allowing the motor to burn out when the propellant tanks become empty. In contrast, in a solid-propellant rocket motor, the

\footnotetext{
${ }^{1}$ We did not study the rotational dynamics of the rocket body, i.e., the torques that would cause the rocket to rotate about any of its three axes, and the rocket's response to these torques.
} 
propellant container is also the combustion chamber. Consequently, once the motor is started it will burn until the propellant is exhausted ("burnout"). The thrust of a solidpropellant motor can be pre-programmed to some extent by the design of the propellant, and it can also be adjusted by using exhaust deflector devices in the nozzle, but this approach is not typical. The thrust of such a motor can be terminated before burnout by opening thrust termination ports arranged to allow hot gases and other burning products to escape without giving the rocket any net impulse.

The direction of the thrust of a rocket motor can be varied by, for example, gimbaling the motors, using thrust deflector plates, or deforming the exhaust nozzle.

\section{B.1.2 Gravity}

The effect of gravity on a rocket (or any other mass) is given by the acceleration of gravity,

$$
g(h)=g_{s}\left[r_{s} /\left(r_{s}+h\right)\right]^{2} .
$$

Here $g_{s}$ is again the acceleration of gravity at sea level, $r_{s}$ is the radius of mean sea level as measured from Earth's center and $h$ is the altitude of the rocket above sea level. This expression neglects the nonspherical shape of Earth and its seas and the gravitational effects of geographical features.

\section{B.1.3 Aerodynamic forces}

The most important aerodynamic forces acting on a missile rising through the atmosphere are lift and drag. The lift force

$$
L=\frac{1}{2} \rho_{a} V^{\prime 2} A C_{l}(\alpha, M, R e)
$$

acts perpendicular to the rocket's flight path, whereas the drag force

$$
D=\frac{1}{2} \rho_{a} V^{\prime 2} A C_{d}(\alpha, M, R e)
$$

acts parallel to the rocket's flight path, opposing the motion of the rocket through the atmosphere. Here $\rho_{a}$ is the local mass density of the atmosphere, $V^{\prime}$ is the speed of the rocket relative to the atmosphere, $A$ is a reference area usually related to a cross-sectional area of the rocket body, and $C_{d}$ and $C_{l}$ are the coefficients of lift and drag. Both coefficients depend on the rocket's angle of attack $\alpha$, defined as the angle between its centerline and its velocity vector. The angle of attack is positive if the projection of the centerline is above the projection of the velocity vector. The coefficients also depend on the rocket's Mach number $M \equiv V^{\prime} / c_{s}$, where $c_{s}$ is the local speed of sound, the Reynolds number $R e$, and the choice of reference area $A$.

For small angles of attack $\left(\alpha<10^{\circ}\right), C_{d}$ is generally less than 0.5 at subsonic speeds but increases sharply by a factor of about 2 to 3 when $M$ reaches about 1 , and then declines steadily as $M$ increases further. In contrast, $C_{l}$ is roughly independent of $M$ for $M$ less than about 1.5, but then declines steadily with increasing $M$. Both $C_{d}$ and $C_{l}$ increase with increasing $\alpha$ for modest values of $\alpha$. The aerodynamic forces, especially the drag, are also affected by the flow and pressure distribution of the rocket exhaust gases, which tend to increase the drag at subsonic speeds and decrease it at supersonic speeds [248, Chapter 18]. In computing missile trajectories for the Study, tabulated lift and drag coefficients for similar missiles, or fits of simple analytical expressions to such tables, were used. 


\section{B.2 Computing Trajectories}

Equivalent equations describing the ascent of a rocket through the atmosphere can be formulated in terms of different physical variables, with certain formulations being more convenient for some applications than others. The full trajectory equations may also be approximated in different ways, depending on the aspects of the ascent that are of most interest. During the Study, several computer codes using different formulations of the ascent equations, different approximations, and different methods of solution were employed to compute missile trajectories for different purposes. Rather than describing all the different approaches and codes that were used, we present here a single formulation that illustrates most of the features of interest.

All the computer codes that were used were validated by comparing their results with those of other codes developed independently and employing different algorithms. The codes used to compute ICBM and interceptor trajectories were also validated by comparing their results with the results produced by computer codes at Sandia National Laboratories that have been validated over many years of use.

\section{B.2.1 Illustrative equations}

The following equations of motion describe the ascent of a rocket from the surface of a rotating planet in three dimensions relative to Earth (see [249, §IV]):

$$
\begin{aligned}
\frac{d \lambda}{d t}= & \frac{V \cos \gamma \cos \psi}{r \cos \tau} \\
\frac{d \tau}{d t}= & \frac{V \cos \gamma \sin \psi}{r} \\
\frac{d h}{d t}= & V \sin \gamma \\
\frac{d V}{d t}= & \frac{1}{m}[T \cos (\alpha+\delta)-D-m g \sin \gamma] \\
& +r \omega^{2} \cos \tau[\cos \tau \sin \gamma-\sin \tau \cos \gamma \sin \psi] \\
\frac{d \gamma}{d t}= & \frac{1}{m V}\{[T \sin (\alpha+\delta)+L] \cos \mu-m g \cos \gamma\}+\frac{V}{r} \cos \gamma \\
& +2 \omega \cos \gamma \cos \psi+\frac{r \omega^{2}}{V} \cos \tau[\cos \tau \cos \gamma+\sin \tau \sin \gamma \sin \psi] \\
\frac{d \psi}{d t}= & -\frac{1}{m V \cos \gamma}[T \sin (\alpha+\delta)+L] \sin \mu-\frac{V}{r} \tan \tau \cos \gamma \cos \psi \\
& +2 \omega[\cos \tau \tan \gamma \sin \psi-\sin \tau]-\frac{r \omega^{2}}{V \cos \gamma} \cos \tau \sin \tau \cos \psi \\
\frac{d m}{d t}= & -\frac{d m_{p}}{d t} \\
\frac{d m_{p}}{d t}= & \frac{T}{g_{s} I_{\mathrm{sp}}}
\end{aligned}
$$

The variables in these equations are defined in Box B.1. In this formulation, the angle of attack $\alpha$, thrust gimbal angle $\delta$, and velocity roll angle $\mu$ are control variables, i.e., they must be specified functions of the rocket's position during its ascent. 


\begin{tabular}{|ll|}
\hline & Box B.1: Symbols Used in This Appendix \\
\hline$\lambda$ & Longitude \\
$\tau$ & Latitude \\
$h$ & Altitude above mean sea level \\
$r$ & Distance from the center of Earth \\
$\omega$ & Rotational angular velocity of Earth \\
$V$ & Speed relative to Earth \\
$V^{\prime}$ & Speed relative to the atmosphere \\
$\gamma$ & Flight-path angle relative to local horizontal \\
$\psi$ & Heading angle relative to local West \\
$m$ & Total vehicle mass \\
$m_{p}$ & Propellant mass \\
$\dot{m}_{p}$ & Propellant mass flow rate \\
$A_{e}$ & Cross-sectional area of the nozzle exit \\
$p_{e}$ & Rocket exhaust pressure at nozzle exit \\
$v_{e}$ & Average exhaust velocity at nozzle exit \\
$I_{\mathrm{sp}}$ & Specific impulse \\
$T$ & Thrust \\
$L$ & Aerodynamic lift force \\
$D$ & Aerodynamic drag force \\
$M$ & Mach number \\
$C_{l}$ & Aerodynamic lift coefficient \\
$C_{d}$ & Aerodynamic drag coefficient \\
$R e$ & Reynolds number \\
$\rho_{a}$ & Ambient atmospheric density \\
$p_{a}$ & Ambient atmospheric pressure \\
$\alpha$ & Angle of attack \\
$\delta$ & Thrust gimbal angle \\
$\mu$ & Velocity roll angle \\
$g_{s}$ & Acceleration of gravity at sea level \\
$g$ & Acceleration of gravity at the vehicle's altitude \\
& \\
& \\
\hline
\end{tabular}

\section{B.2.2 Solving the trajectory equations}

The missile ascent trajectories used in the Study were computed by solving a variety of trajectory equations equivalent to Eqs. (B.6)-(B.13), in various approximations. The missile and interceptor trajectories shown in Chapters 15 and 16 are planar, i.e., the rocket follows a path that lies entirely in a single two-dimensional plane (the trajectories of the target missile and interceptor missile need not be in the same plane). However, some examples of nonplanar trajectories are shown in Chapter 5 , to illustrate possible large-scale evasive maneuvers by the attacker.

The local trajectories of ICBMs flying out of North Korea shown in Chapter 5 include the effects of Earth's rotation, because for these trajectories, the rotation of Earth is significant for assessing the importance of geographic constraints on interceptor basing. Earth's 
rotation was neglected in plotting the local trajectories of ICBMs launched from Iraq and Iran and in plotting all global trajectories, including those from North Korea to the United States, because its effects would not alter any conclusions derived from these trajectories.

In trajectory computations, the thrust given by Eq. (B.1) can usually be modeled using the simpler expression

$$
T=T_{\mathrm{vac}}-p_{a}(h) A_{e},
$$

where $T_{\mathrm{vac}} \equiv \dot{m}_{p} \bar{v}_{e}+p_{e} A_{e}$, the thrust of the motor in vacuum, is treated as a constant.

With appropriate initial conditions, equations similar to Eqs. (B.6)-(B.13) can be used to compute the trajectories of space-based interceptors when boosted out of orbit. In this case, aerodynamic forces can generally be neglected.

\section{B.3 Simple Scaling Relations}

A simple, one-dimensional analysis is possible when a rocket is operating in a gravity-free vacuum environment and its thrust is always in the same direction. This analysis yields several useful scaling relations and insights that remain approximately true more for more general conditions.

In the absence of gravity and aerodynamic forces, conservation of momentum yields

$$
m(d V / d t)=T,
$$

where $m(t)$ is the total mass of the vehicle at time $t$. The thrust $T$ may be rewritten as

$$
T=\dot{m}_{p} v_{\mathrm{eq}}
$$

where $\dot{m}_{p}$ is the propellant mass flow rate and $v_{\text {eq }} \equiv \bar{v}_{e}+\left[p_{e}-p_{a}(h)\right] A_{e}$ is the so-called equivalent velocity (see Eq. B.1). It is the velocity with which cold propellant would have to be ejected to provide a thrust equal to that provided by the rocket motor. Expulsion of propellant reduces the total mass of the vehicle by the same amount [see Eq. B.12], so $T=-(d m / d t) v_{\text {eq }}$ and hence

$$
m(d V / d t)=-v_{\mathrm{eq}}(d m / d t) .
$$

Equation (B.17) is the standard rocket equation. If the rocket's initial mass and speed are $m_{i}$ and $V_{i}$, the thrust is always in the direction of motion, and if $v_{\text {eq }}$ remains constant as the rocket accelerates, its speed at time $t$ will be

$$
V(t)=V_{i}+v_{\mathrm{eq}} \ln \left[m_{i} / m(t)\right] .
$$

The change $\Delta V(t) \equiv V(t)-V_{i}$ in the rocket's speed is

$$
\Delta V(t)=v_{\mathrm{eq}} \ln \left[m_{i} / m(t)\right] .
$$

The maximum value of $\Delta V$ that a rocket can achieve under the ideal conditions assumed in deriving Eq. (B.19) is

$$
\Delta V(\text { ideal })=v_{\text {eq }} \ln (M R) \text {. }
$$

Here $M R \equiv m_{i} / m_{f}$ is the rocket's mass ratio during its flight, where $m_{f}$ is the its final mass after its motor is shut down or burns out. The final mass includes the mass of all components other than useful propellant, including any unburned propellant as well as the propulsion hardware, fuel tanks, power supplies, guidance system, navigational equipment, 
and payload. Obviously $\Delta V$ (ideal) is greater when a rocket's propellant mass is a larger fraction of its initial mass. A rocket's useful propellant mass is denoted $m_{p}$. If no shroud or other equipment is ejected during flight, $m_{p}=m_{i}-m_{f}$. The ratio $m_{p} / m_{i}=1-m_{f} / m_{i}$ is called the propellant mass fraction. For values of $m_{p} / m_{i}$ near unity, $\Delta V$ (ideal) increases rapidly with increasing $m_{p} / m_{i}$, which puts a high premium on minimizing the mass of a rocket's tanks, motors, unexpended propellant, and other deadweight.

The "ideal" velocity change $\Delta V$ (ideal) is an upper bound on the speed that a missile rising from Earth's surface can achieve and is sometimes used as a measure of the missile's performance. The actual speed that the missile can achieve is less than $\Delta V$ (ideal) because of the retarding effects of gravity and aerodynamic drag. The reductions in the rocket's final speed caused by these two effects are sometimes referred to as "gravity losses" and "drag losses," respectively, and denoted $\Delta V_{g}$ and $\Delta V_{d}$. They depend on the rocket's flight path and atmospheric conditions. For long-range rockets, the drag loss is typically 5 percent to 20 percent of the final velocity [248, §4.2]. Gravity losses are a function of boost time and are greater the longer the boost time. Rather than characterize the performance of a missile by $\Delta V$ (ideal) $\equiv \Delta V_{\max }$, we have preferred to use its speed at burnout on its maximum range trajectory (see below), which we call its "actual" velocity change $\Delta V$ (actual) (see Chapter 15), which is a more accurate indicator of the velocity that the missile will achieve at burnout when flying trajectories of interest in the Study.

The concept of a maximum attainable change in the velocity of a rocket is useful in analyzing the ability of an interceptor to reach a target missile during the latter's boost phase. Equation (B.20) can be rewritten as $m_{i}=m_{f} \exp \left(\Delta V\right.$ (ideal) $\left./ v_{\text {eq }}\right)$, which shows that the required launch mass of an interceptor with a given propulsion system and propellant mass fraction increases exponentially with the velocity change $\Delta V$ that is required to achieve intercept.

For a given propellant mass fraction, the final $\Delta V$ of a rocket can be increased by subdividing it into stages and dropping off each stage as its propellant is exhausted. If a rocket is subdivided into $n$ stages and each stage has the same mass ratio (an unrealistic assumption), $\Delta V$ is increased by a factor of $n$. In reality, a rocket's final speed increases more slowly than this with each additional stage. Moreover, additional stages introduce additional complications and tend to decrease the overall reliability of the system. In practice, longer-range rockets generally have two or three stages, whereas shorter-range rockets have one or two stages. The propellant tanks of solid-propellant rockets, which also serve as combustion chambers, must be more robust and hence are generally more massive than those of liquid-propellant rockets. As a result, long-range solid-propellant rockets often optimize at three stages, whereas liquid-propellant rockets often optimize at two stages.

The weight of a multi-stage rocket at liftoff is approximately proportional to the weight of its payload, even though the mass of the payload is typically a very small fraction of the rocket's liftoff mass $[248, \S 4.7]$. Hence the weight of the booster(s) required to accelerate a given homing kill vehicle to a given velocity is approximately proportional to the kill vehicle's mass.

\section{B.4 Discussion}

\section{B.4.1 Missile trajectories}

The range of a particular ballistic missile depends on the mass of the payload it is carrying; any missile can in principle deliver a lighter warhead to a more distant target. Thus, to 
be useful, a statement of the range of a missile must accompanied by a statement of the payload mass assumed. ${ }^{2}$

For structural reasons, missiles are almost always launched nearly vertically, and once off the ground begin to turn toward the target by making small, very brief adjustments of the thrust gimbal angle $\delta$. Once their flight path angle $\gamma$ is less than $90^{\circ}$, gravity will cause the missile to continue to turn toward the target, even if its thrust vector is aligned with its velocity vector; this phenomenon is called a gravity turn.

Every missile has a so-called maximum-range trajectory, which is the trajectory for which the impact point of the payload is farthest from the launch site, with effects of gravity and aerodynamic forces included. The shape of a missile's maximum range trajectory therefore depends on its shape and structure and its acceleration profile.

The range penalty for deviating by modest amounts from a missile's maximum-range trajectory is small, and hence it may be advantageous for an attacker to program the missile to fly a trajectory that is higher ("lofted") or lower ("depressed") than the maximum-range trajectory, for tactical reasons.

Altitude vs. range and time vs. range plots are shown in Chapter 15 for a variety of lofted and depressed trajectories, as well as for the maximum-range trajectories of the missile models used in the Study.

\section{B.4.2 Interceptor trajectories}

To intercept ICBMs during their boost phase, interceptor missiles must have high accelerations and high burnout velocities so that they can reach the target missile as quickly as possible. A two-stage interceptor may be necessary (as explained above, if there are two stages, the first stage can be dropped after its propellant has been consumed, reducing the inert mass of the missile's final stage and increasing its burnout velocity). There are practical limits to the accelerations and final velocities of interceptor missiles.

High-acceleration, exoatmospheric interceptors reach much higher velocities early in their flight than do ICBMs. Consequently, if they are launched from Earth's surface, they are usually programmed to fly a flight path that is different from the typical flight paths of ICBMs: they initially rise more nearly vertically to get out of the denser part of the atmosphere as quickly as possible and then pitch over and accelerate toward the predicted intercept point. Typical altitude vs. range and time vs. range plots are shown in Chapter 16 for the model interceptors used in the Study.

If the closing velocity between an interceptor or kill vehicle and the target is high and, as in boost-phase intercept applications, the target is maneuvering, the interceptor may have to be capable of large accelerations perpendicular as well parallel to its existing flight path. In principle, these accelerations could be achieved using a single motor, if the interceptor were agile enough and could be rotated in any direction without losing its ability to track the target. However, it is usually more practical to allow the interceptor to remain oriented more or less in the same direction to facilitate tracking the target and to use side thrusters as well as an axial thruster to achieve intercept. Side thrusters are often called divert thrusters, because they divert the vehicle's flight from its current direction. However, from a more

\footnotetext{
${ }^{2}$ In practice, the payload of a missile usually cannot be altered significantly without adjusting its design or flight profile. For example, changing the mass of the payload will affect the center of mass of the missile and may affect its aerodynamic stability. Decreasing the payload mass of a missile will increase its nominal range but, other things being equal, will also increase the acceleration and aerodynamic loads experienced by the missile during its boost phase, which it may or may not be able to withstand.
} 
general point of view, any thrust provides divert capability, in that it will cause the flight path of the vehicle to depart from the ballistic trajectory that it would otherwise follow. If the target maneuvers so that it will arrive at the originally predicted intercept point earlier than expected, axial thrust may be as important as lateral thrust in achieving intercept. All thrusters must be positioned so that the forces they apply are directed through the center of mass of the interceptor or kill vehicle to avoid causing it to spin.

\section{References for Appendix B}

[247] Michael D. Griffin and James R. French, "Space Vehicle Design." AIAA Education Series, 1991.

[248] George P. Sutton and Oscar Biblarz, Rocket Propulsion Elements. (John Wiley \& Sons, Inc.), 7th edition, 2001.

[249] S.-Y. Park and S. R. Vadall, "Touch points in optimal ascent trajectories with first-order state inequality constraints," J. Guidance, Control, and Dynamics 21, 603 (1998). 



\section{Appendix C}

\section{Boost-Phase Tracking Simulations}

\section{Contents}

C.1 Engagement of ICBM S1 and the 10- $\mathrm{km} / \mathrm{s}$ Interceptor I-5 S360

C.1.1 Missile and interceptor trajectories . . . . . . . . . S360

C.1.2 Measurement models . . . . . . . . . . . . . . . S362

C.1.3 Tracking Kalman filter . . . . . . . . . . . . . S S366

C.1.4 Trajectory state estimation . . . . . . . . . . . . . S371

C.1.5 Engagement summary . . . . . . . . . . . . . S S381

C.2 Engagement of ICBM L and the 5-km/s Interceptor I-5 . S381

C.2.1 Missile and interceptor trajectories . . . . . . . . . . . S381

C.2.2 Measurement models . . . . . . . . . . . . . . . S383

C.2.3 Trajectory state estimation . . . . . . . . . . . . S385

C.2.4 Engagement summary . . . . . . . . . . . . . S S386

The boost-phase intercept is a complex problem that includes target missile, interceptor, space sensor system, surface-based sensor system (e.g., radar), kill vehicle, and its suite of on-board sensors. Detailed algorithms for the tracking of the target missile and the interceptor and the guidance and control schemes used by the interceptor and kill vehicle must also be included in the modeling. Using assumptions on the error models for the different sensors and statistical variations for the different systems (rocket motors, aerodynamics, actuator responses, etc.), Monte Carlo simulations can be carried out to obtain the miss-distance distribution of the intercept.

The Study broke the analysis into several parts and worked them somewhat independently of each other. (The general approach of the analyses is described in detail in Chapter 2.) In short, a set of possible target missiles was first identified and trajectories were generated for each of them (Chapter 15). Then a set of surface-based interceptors and their flyout capabilities were constructed to counter these target missiles, based on three geographic scenarios (Chapter 16). These interceptor trajectories were used to analyze the areas of coverage that each could provide (Chapter 5).

Space sensor measurement error models and surface-based radar error models were derived (Chapter 10), so that they could be used to drive the tracking Kalman filter (to be described in this Appendix). Using the results of this error analysis, the kill-vehicle intercept problem was modeled with guidance algorithms, actuator dynamics, time delays, kill-vehicle divert capabilities, and homing sensor errors (Chapter 12). The output from 
those simulations was the divert velocity required along with miss distance as a function of these parameters.

This Appendix describes simulation results for two missile-interceptor engagements. The first engagement discussed in this Appendix is between the ICBM model S1 (referred to as ICBM S1 hereafter) and the $10-\mathrm{km} / \mathrm{s}$ interceptor. The second engagement is between the ICBM model L (referred to as ICBM L hereafter) and the $5-\mathrm{km} / \mathrm{s}$ interceptor. These two engagements were chosen to illustrate the features of a faster-burning solid-propellant missile (the ICBM S1) and a slow-burning liquid-propellant missile (the ICBM L). For these two missiles, an aggressive 10-km/s interceptor is needed for the ICBM S1 missile, while a more feasible $5-\mathrm{km} / \mathrm{s}$ interceptor is sufficient for the ICBM L missile.

Chapter 15 describes three target missiles and their trajectories that were generated by the Study Group. They are the ICBM L, ICBM S1, and ICBM S2 missiles, and their mass properties and characteristics are given in Tables 15.3, 15.4, and 15.5, respectively. Threedegree-of-freedom (3-DOF) simulations were used to generate these trajectories. These trajectories closely matched the ones generated by Sandia National Laboratories, confirming the method of generating missile trajectories used in this Report.

In Chapter 16, three interceptors and their trajectories are discussed in detail. They are the $10 \mathrm{~km} / \mathrm{s}, 6.5 \mathrm{~km} / \mathrm{s}$, and $5 \mathrm{~km} / \mathrm{s}$ interceptors. The mass properties and characteristics of the interceptors are given in Tables 16.1, 16.2, and 16.3.

The ICBM-S1 engagement is presented first, followed by the ICBM-L engagement in a separate section. For each of the two engagements, we first discuss the missile and interceptor trajectories, then the accuracy of tracking the missile using different sensors. Measurement errors of candidate space IR sensors, surface radars, and kill-vehicle IR sensors are discussed. These measurement errors are added to the true states to generate measurements that are used to drive the Kalman filter. Different sensitivity runs are made and the tracking performance is compared to assess the robustness of the Kalman filter.

\section{C.1 Engagement of ICBM S1 and the 10-km/s Interceptor I-5}

In this section, we examine the engagement of the faster-burning solid-propellant missile ICBM S1 and the aggressive 10-km/s interceptor, I-5.

\section{C.1.1 Missile and interceptor trajectories}

Figure C.1 shows this engagement. The intercept at $21 \mathrm{~s}$ before the missile's burnout is chosen for analysis in this section. The trajectory being analyzed is labeled as "c" in the plot, having an angle of attack of $-30^{\circ}$ for the third stage. Note that if an angle of attack of $-45^{\circ}$ were used for the third stage, depicted by trajectory "d", the intercept could take place more than $40 \mathrm{~s}$ before missile burnout.

The space sensor is assumed to be able to detect the missile when it breaks cloud cover at an altitude of $7 \mathrm{~km}$ ( $29 \mathrm{~s}$ after launch of the ICBM S1). Note that this assumption might be slightly optimistic as cloud cover could be as high as $10 \mathrm{~km}$.

When the interceptor is launched $59 \mathrm{~s}$ after the target missile is launched, the space sensor has tracked the target missile for $30 \mathrm{~s}(59 \mathrm{~s}-29 \mathrm{~s})$. The surface-based radar will not be able to acquire the missile until $95 \mathrm{~s}$ after it is launched, after it clears the horizon (but reliable measurements will not be available until $t=99 \mathrm{~s}$ ). Therefore, it is important to analyze the ability of the space-based sensor to track the missile when the radar is not yet available. (Space-based sensor and tracking radars are discussed in Chapter 10. 

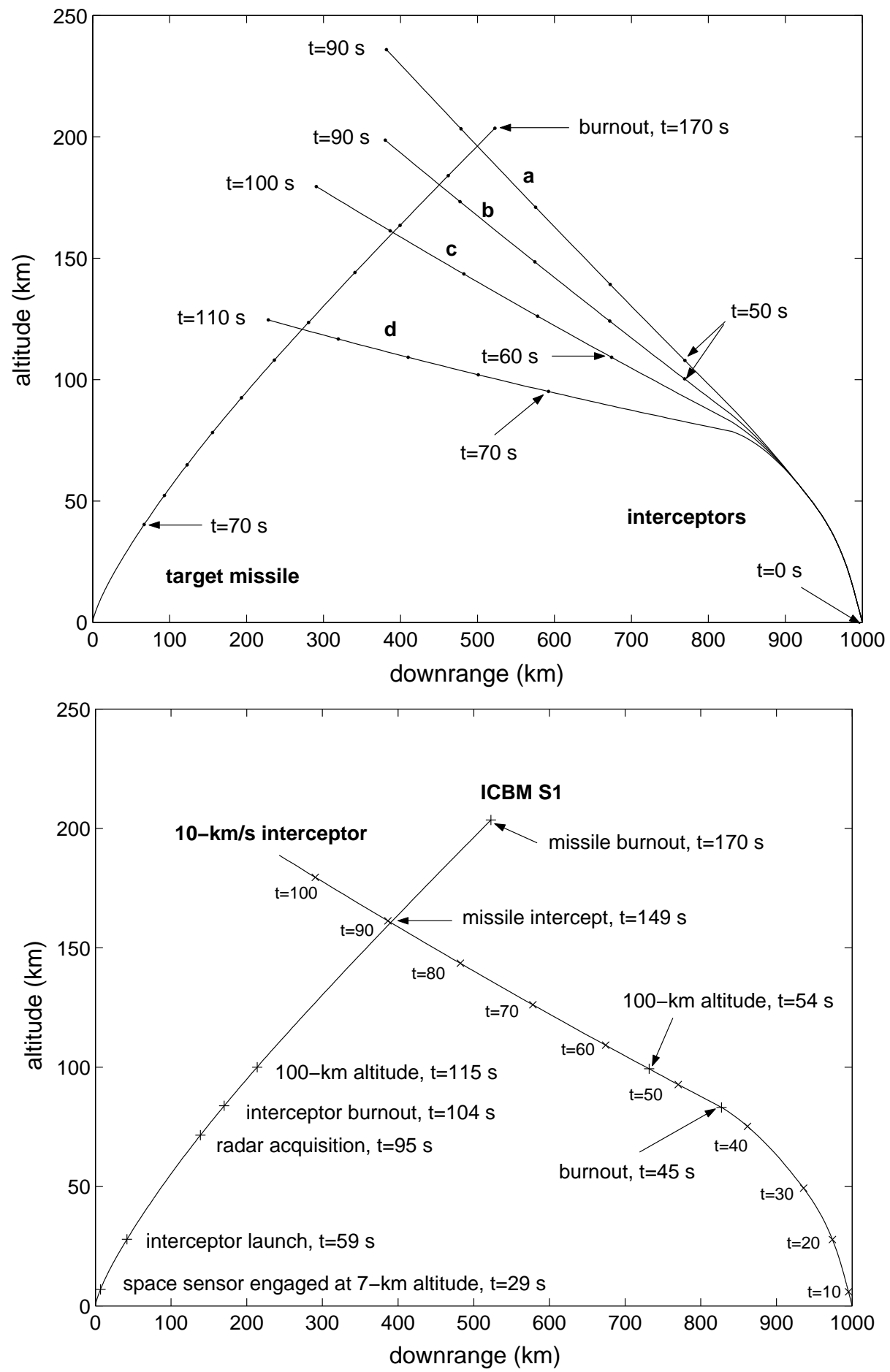

Figure C.1. (top) Engagement of the target missile ICBM S1 (on the left) by multiple $10-\mathrm{km} / \mathrm{s}$ interceptors (on the right). The time ticks on the trajectories are $10 \mathrm{~s}$ apart. Interceptor trajectories labeled a, b, c, and d have angles of attack of $-10^{\circ},-20^{\circ},-30^{\circ}$, and $-45^{\circ}$, respectively, during third-stage burn. The interceptors are launched $59 \mathrm{~s}$ after the target missile is launched, which is $\mathrm{t}=0$ for the interceptors. (bottom) Detail of the engagement. The interceptor trajectory is trajectory "c" in the top plot. 


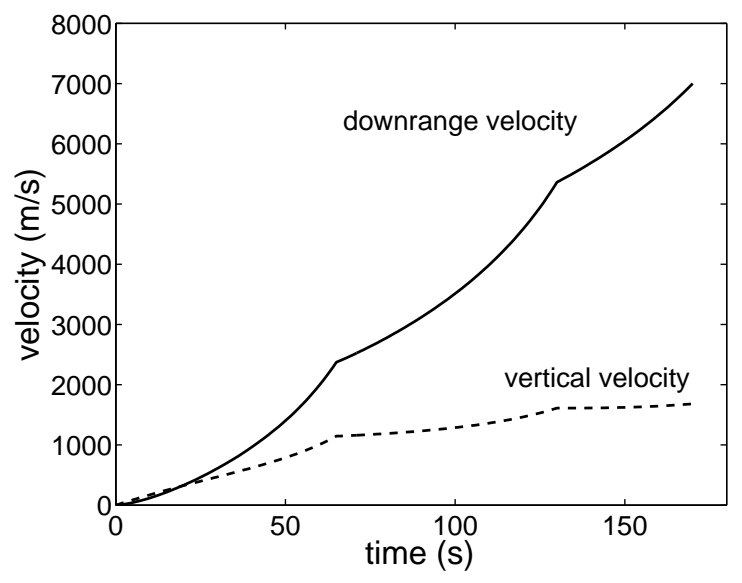

(a)

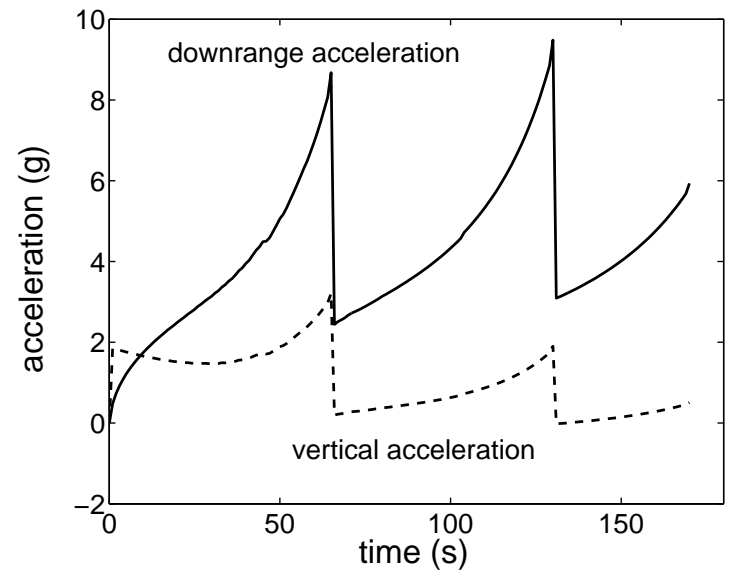

(b)

Figure C.2. (a) Downrange and vertical velocities of the ICBM S1 target missile. (b) Downrange and vertical accelerations of the ICBM S1 target missile.

Actual missile tracking using space sensors and radar is described in the next section.) The interceptor burns out after $45 \mathrm{~s}$ (104 s after target launch) and reaches an altitude of $100 \mathrm{~km} 9 \mathrm{~s}$ later at $t=54 \mathrm{~s}$. About two seconds later, the ICBM S1 also reaches the $100-\mathrm{km}$ altitude. The $100 \mathrm{~km}$ altitude is significant to both the missile and interceptor because the shrouds for both normally come off at about 80 - to $100-\mathrm{km}$ altitude, and countermeasures could be deployed or maneuvers initiated by the target missile and on-board sensors could be used by the kill vehicle along with divert maneuvers after this point.

After reaching 100-km altitude at about the same time, the interceptor gains altitude at about the same rate as the missile, but gains downrange distance at about twice the rate. The two vehicles intercept at $t=149 \mathrm{~s}, 21 \mathrm{~s}$ before missile burnout.

For reference, the velocity and acceleration profiles of the ICBM S1 target missile are shown in Fig. C.2, and those for the 10-km/s interceptor are shown in Fig. C.3.

The question of typing the target missile based on early trajectory information is often raised. Figure C.4 (a) shows that although the ICBM L has a more vertical trajectory profile near cloud-cover altitude $(7 \mathrm{~km})$, it eventually has a flatter trajectory above $100 \mathrm{~km}$, shown in Fig. C.4 (b). This example shows that it is difficult to predict the overall trajectory based on early trajectory-profile information.

\section{C.1.2 Measurement models}

Before going into the formulation of the Kalman filter for this engagement, we will first discuss the measurements and their error characteristics.

\section{C.1.2.1 Space-based IR sensor measurement errors}

Based on the analyses of space-based IR sensors (see Chapter 10), we can count on 500-m 1- $\sigma$ position errors in the direction perpendicular to the sensing direction. We assume that three satellites are at equal intervals in geostationary orbits. With any two sensors, we should have 500-m 1- $\sigma$ in all three axes.

In the simulations, white Gaussian noise with $\sigma=500 \mathrm{~m}$ is added to the true missile positions (in all three axes, although the trajectory is in the downrange-altitude plane) to 


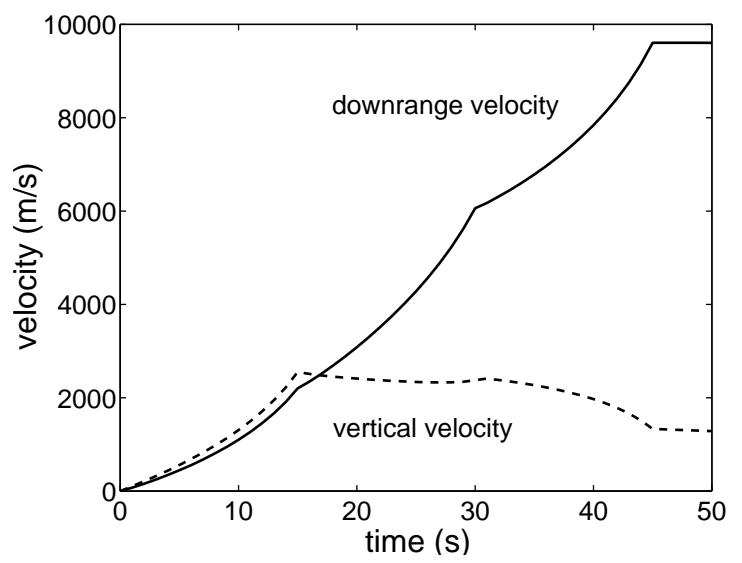

(a)

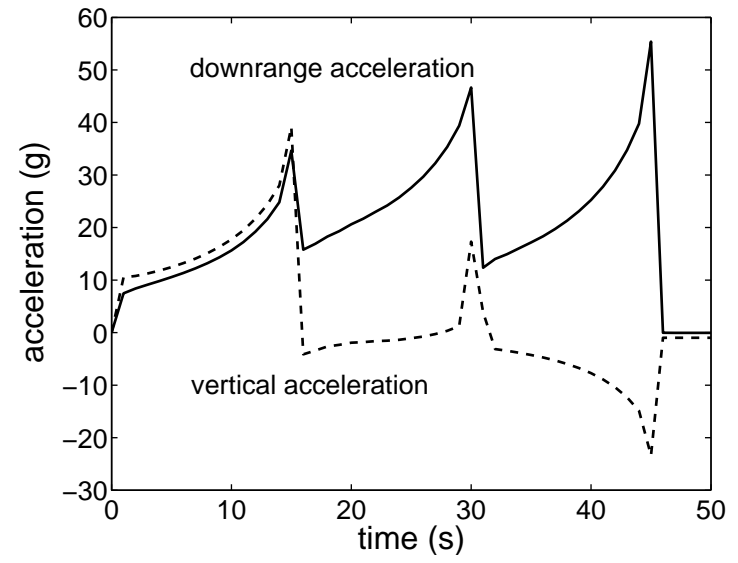

(b)

Figure C.3. (a) Downrange and vertical velocities of the $10-\mathrm{km} / \mathrm{s}$ interceptor. (b) Downrange and vertical accelerations of the $10-\mathrm{km} / \mathrm{s}$ interceptor.

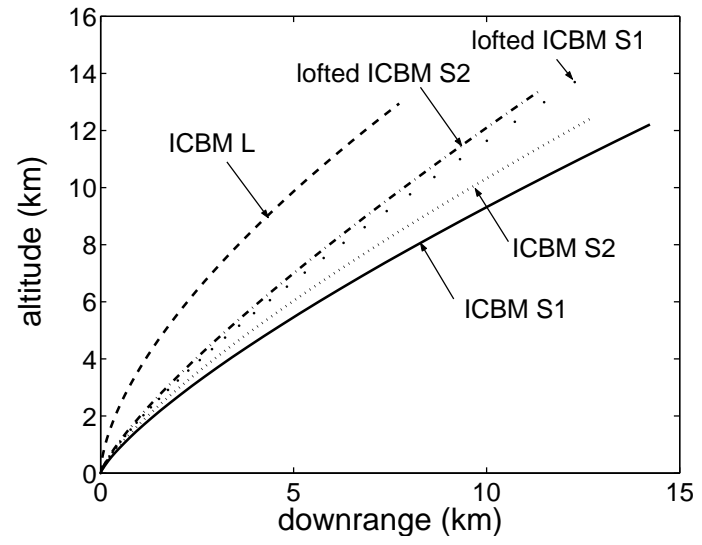

(a)

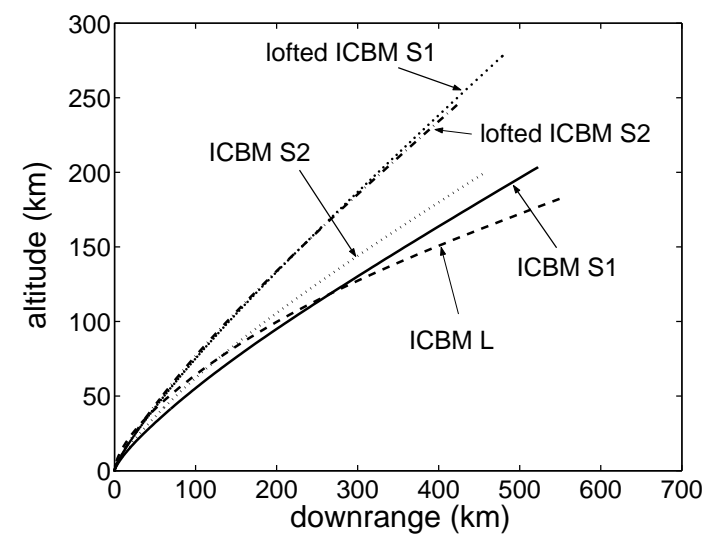

(b)

Figure C.4. (a) Five target missile trajectories near cloud-cover altitude. (b) Five target missile trajectories. 

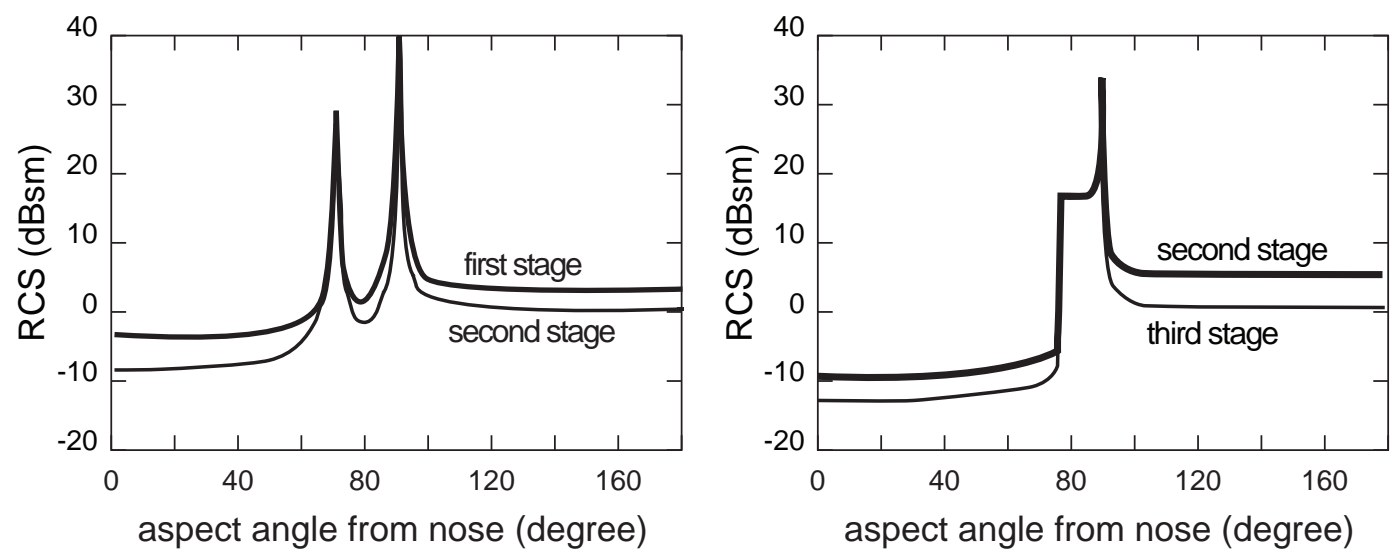

Figure C.5. Estimated average S-band radar cross sections of the liquid-propellant ICBM model L (left) and the solid-propellant ICBM model S1 (right), as functions of aspect angle from the nose.

generate the space IR measurements. This generated measurement sequence is then used as one of the measurement inputs to the Kalman filter.

\section{C.1.2.2 Surface radar measurement errors}

Radar acquisition For operations based $1000 \mathrm{~km}$ or less from the target launching site, the existing Aegis AN/SPY-1B radar is adequate for acquiring and tracking the ICBM target as modeled for this study. The parameters of this radar, as used to provide command guidance data for the interceptor's midcourse guidance phase, were given in Section 10.2. The radar is notified by a space-based IR system and searches a small volume just above the horizon, at the predicted time of appearance of the target, locking onto and then tracking the target until intercept. It also receives data on launch and trajectory of the interceptor, tracking it using a transponder.

Source and accuracy of designation data The designation error of the target relative to the radar position has been conservatively assumed to be $5 \mathrm{~km}$ rms. At the $890-\mathrm{km}$ horizon range of the target, this designation error corresponds to an azimuth accuracy $\sigma_{a z}=5.6 \mathrm{mr}$, and with an added allowance for possible azimuth change during the acquisition process, the radar should scan about four beamwidths at an elevation just above the horizon.

Target cross section The RCS model for this target is based on an outline drawing of the Minuteman III, and the RCS during first and second stages is shown in Fig. C.5, (right). The aspect angle from the nose is near $30^{\circ}$ during acquisition, increasing gradually toward $50^{\circ}$ near the end of powered flight. In this sector, the significant contributions are from the rounded nose cone tip and the irregularities and edges assumed to scatter into the forward hemisphere, totaling $0.045 \mathrm{~m}^{2}$ from the final stage and $0.055 \mathrm{~m}^{2}$ from the second-stage booster. A value $\sigma=0.10 \mathrm{~m}^{2}=-10 \mathrm{dBsm}$ applies during the critical period of radar operation.

Signal-to-noise ratio at horizon The target appears above the horizon at $t=87 \mathrm{~s}$ with an elevation rate of $2.1 \mathrm{mr} / \mathrm{s}$. It spends $11 \mathrm{~s}$ within the $26-\mathrm{mr}$ elevation beamwidth of 

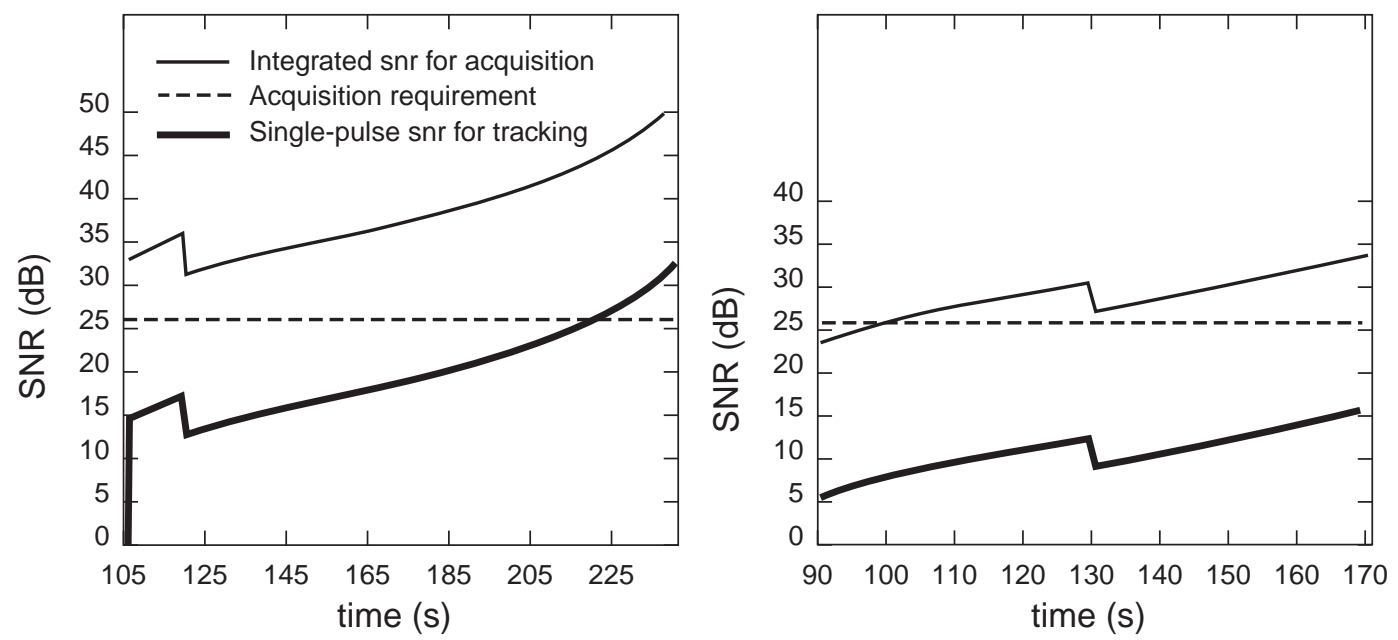

Figure C.6. Signal-to-noise ratio for acquisition and tracking Aegis radar. ICBM L (left) and ICBM S1 (right).

the radar. The single-pulse, beam-center signal-to-noise ratio at the horizon $+5.9 \mathrm{~dB}$ after allowance for tropospheric loss of $3.8 \mathrm{~dB}$ (including the tropospheric lens loss).

Probability of acquisition Acquisition is achieved by devoting essentially all radar resources over an 11-s period to acquisition of this target, using multiple-pulse integration. The resulting integrated signal-to-noise ratio for a 6 -s integration time, allowing for two such attempts during passage of the target through the beam, is shown in Fig. C.6 (right). That process leads to detection of the target with high probability before it leaves the elevation beam at $t=99 \mathrm{~s}$, with a probable time allocation of about $7 \mathrm{~s}$ for detection at $t=94 \mathrm{~s}$. During this 7-s period, the radar would reserve minimal resources for tracking other targets and outgoing interceptors, but would not be able to search for targets other than the single one being designated by the space-based IR system.

Radar tracking error calculations Once the radar has acquired the target, tracking can begin. It has been assumed that the radar will allocate 12 percent of its time and power resources to tracking an ICBM target, providing ten pulses per second with the signal-tonoise ratio shown in Fig. C.6 (right). The sources of error and equations for calculating them were given in Section 10.2. The rms magnitude of errors has been calculated on the basis of averages over a smoothing time of $1 \mathrm{~s}$. Error components that are independent from pulse to pulse, and hence from each 1-s sample to the next, are defined as noise, while those that remain correlated over several seconds are defined as bias. The latter are generally not subject to reduction by smoothing in a tracking filter, but neither are they constant over such long periods that they make no contribution to errors in velocity and acceleration. These noise and bias error components for elevation and azimuth tracking data are shown in Fig. C.7. Errors in range are negligible, amounting to a few meters rms. 

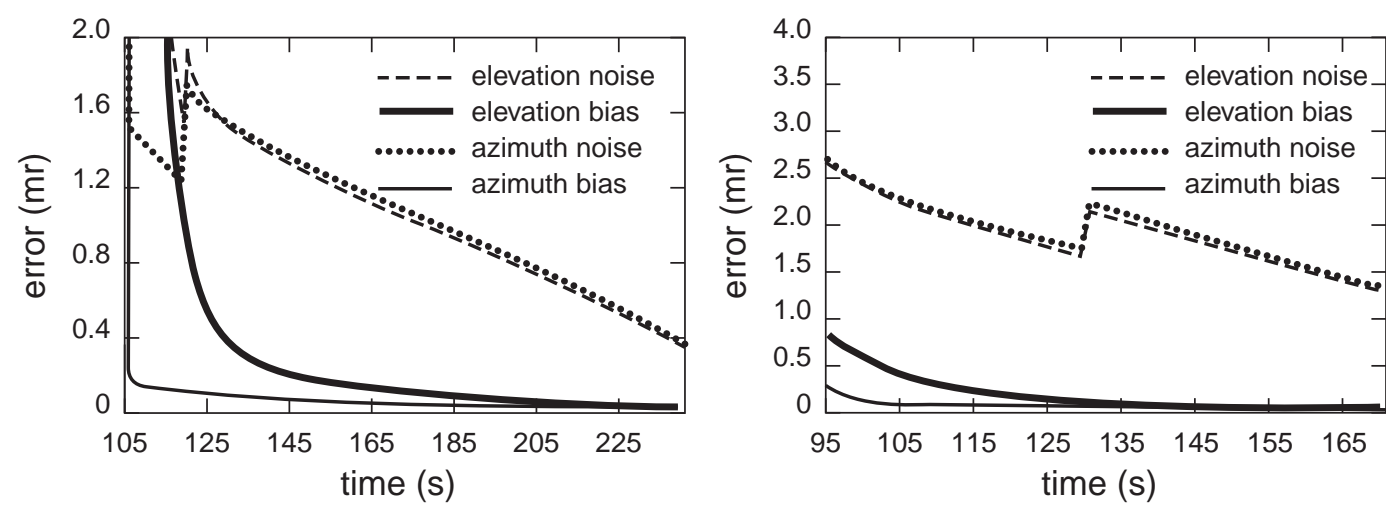

Figure C.7. Aegis tracking errors vs. time for the liquid-propellant ICBM model (left) and the solid-propellant ICBM models (right).

\section{C.1.2.3 KV IR sensor measurement errors}

For the kill-vehicle-based IR sensors, the errors will be completely dominated by our ability to discriminate the hardbody from the plume. We are estimating that we can do this within a 100-m box, or 30-m 1- $\sigma$ accuracy in the azimuth and elevation directions, independent of range, from 100 to $1000 \mathrm{~km}$.

\section{C.1.3 Tracking Kalman filter}

In this section, the missile tracking problem using space IR sensors, surface radar, and the kill-vehicle IR sensor is analyzed. A Kalman filter is used to estimate the "states" of the missile $[250,251]$ i.e., its three positions, velocities, and accelerations. The tracking problem begins when the missile breaks cloud cover at $7-\mathrm{km}$ altitude, $t=29 \mathrm{~s}$, at which time only space-based IR measurements are available. As the radar acquires the missile after $t=99 \mathrm{~s}$, the Kalman filter will use both space IR and radar measurements. As the shroud comes off the interceptor, at $t=113 \mathrm{~s}$, the kill-vehicle IR sensor begins operating, and its measurements will also be used. The tracking will continue until the interceptor enters its endgame, when the kill vehicle uses its on-board sensors for guidance.

The discrete extended Kalman filter equations are

$$
\begin{aligned}
\mathbf{x}(k+1) & =\mathbf{\Phi} \mathbf{x}(k)+\mathbf{w}(k) \\
\mathbf{y}(k) & =\mathbf{H x}(k)+\mathbf{u}(k),
\end{aligned}
$$

where $\mathbf{x}$ is the state vector, $\mathbf{y}$ is the measurement vector, $\mathbf{\Phi}$ is the state transition matrix, $\mathbf{H}$ is the measurement matrix, $\mathbf{w}$ is the process noise, and $\mathbf{u}$ is the measurement noise. The normal conventions for measurement vector and measurement noise are $\mathbf{z}$ and $\mathbf{v}$, respectively, but to avoid confusion with the variables used for altitude $(z)$ and velocity $(v), \mathbf{y}$ and $\mathbf{u}$ are used here[250, 251]. 


\section{C.1.3.1 System model}

Since the missile positions, velocities, and accelerations are needed for the guidance of the interceptor, the nine states of the filter are

$$
\mathbf{x}=\left[\begin{array}{c}
x_{1} \\
x_{2} \\
x_{3} \\
x_{4} \\
x_{5} \\
x_{6} \\
x_{7} \\
x_{8} \\
x_{9}
\end{array}\right]=\left[\begin{array}{c}
d \\
c \\
z \\
v_{d} \\
v_{c} \\
v_{z} \\
a_{d} \\
a_{c} \\
a_{z}
\end{array}\right]
$$

where $d, c$, and $z$ are the downrange, crossrange, and altitude positions, $v_{d}, v_{c}, v_{z}$ are the downrange, crossrange, and altitude velocities, and $a_{d}, a_{c}, a_{z}$ are the downrange, crossrange, and altitude accelerations, respectively. The state transition matrix is

$$
\boldsymbol{\Phi}=\left[\begin{array}{ccccccccc}
1 & 0 & 0 & \Delta t & 0 & 0 & \frac{1}{2}(\Delta t)^{2} & 0 & 0 \\
0 & 1 & 0 & 0 & \Delta t & 0 & 0 & \frac{1}{2}(\Delta t)^{2} & 0 \\
0 & 0 & 1 & 0 & 0 & \Delta t & 0 & 0 & \frac{1}{2}(\Delta t)^{2} \\
0 & 0 & 0 & 1 & 0 & 0 & \Delta t & 0 & 0 \\
0 & 0 & 0 & 0 & 1 & 0 & 0 & \Delta t & 0 \\
0 & 0 & 0 & 0 & 0 & 1 & 0 & 0 & \Delta t \\
0 & 0 & 0 & 0 & 0 & 0 & 1 & 0 & 0 \\
0 & 0 & 0 & 0 & 0 & 0 & 0 & 1 & 0 \\
0 & 0 & 0 & 0 & 0 & 0 & 0 & 0 & 1
\end{array}\right]
$$

where $\Delta t$ is the sample time. The process noise $\mathbf{w}$ is a way to account for the inaccurate modeling of the true system dynamics. Since $\boldsymbol{\Phi}$ is for a constant-acceleration missile, that is,

$$
\begin{aligned}
& a_{d}(k+1)=a_{d}(k) \\
& a_{c}(k+1)=a_{c}(k) \\
& a_{z}(k+1)=a_{z}(k),
\end{aligned}
$$

process noise must be added to these three acceleration states to track an accelerating missile. The amount of noise that is needed depends on how fast the acceleration is changing and the sample time of the filter. For a sample time of $1 \mathrm{~s}$, a standard deviation of $5 \mathrm{~m} / \mathrm{s}^{2}$ is chosen for the process noise for the acceleration states. The process noise covariance matrix is given by

$$
Q=\operatorname{Diag}\left[\begin{array}{lllllllll}
0 & 0 & 0 & 0 & 0 & 0 & \left(5 \mathrm{~m} / \mathrm{s}^{2}\right)^{2} & \left(5 \mathrm{~m} / \mathrm{s}^{2}\right)^{2} & \left(5 \mathrm{~m} / \mathrm{s}^{2}\right)^{2}
\end{array}\right]
$$




\section{C.1.3.2 Measurement models}

The vector of measurement functions in this formulation is given by

$$
\mathbf{h}=\left[\begin{array}{c}
d_{s} \\
c_{s} \\
z_{s} \\
r \\
\theta \\
\phi \\
c_{v} \\
z_{v}
\end{array}\right]
$$

where $d_{s}, c_{s}$, and $z_{s}$ are the downrange $(\mathrm{m})$, crossrange $(\mathrm{m})$, and altitude $(\mathrm{m})$ positions measured by the space IR sensor (where the subscript $s$ stands for space), $r, \theta$, and $\phi$ are the range $(\mathrm{m})$, azimuth angle (mrad), and elevation angle (mrad) measured by the surface radar, and $c_{v}$ and $z_{v}$ are the crossrange $(\mathrm{m})$ and altitude $(\mathrm{m})$ positions measured by the kill-vehicle IR sensor (where the subscript $v$ stands for vehicle), respectively. Note that the measurements are arranged in the order in which they become available, i.e., space IR, radar, then kill-vehicle IR. Thus the measurement vector simply increases in size without having to rearrange the vector components. The angles are kept in milliradians to avoid numerical problems associated with the huge dynamic range of the measurement-error covariance matrix, $\mathbf{R}$, which is given below.

The measurement matrix of the extended Kalman filter is obtained by taking the derivatives of the measurement functions with respect to the states,

$$
\mathbf{H}=\frac{d \mathbf{h}}{d \mathbf{x}} .
$$

The components of $\mathbf{h}$ are

$$
\begin{aligned}
d_{s} & =d \\
c_{s} & =c \\
z_{s} & =z \\
r & =\sqrt{d^{2}+c^{2}+z^{2}} \\
\theta & =1000 \tan ^{-1} \frac{c}{d} \\
\phi & =1000 \sin ^{-1} \frac{z}{\sqrt{d^{2}+c^{2}+z^{2}}} \\
c_{v} & =c \\
z_{v} & =z .
\end{aligned}
$$

Carrying out the differentiations, we get

$$
\begin{aligned}
& H_{11}=\frac{d d_{s}}{d d}=1 \\
& H_{22}=1 \\
& H_{33}=1 \\
& H_{41}=\frac{d r}{d d}=\frac{d}{\sqrt{d^{2}+c^{2}+z^{2}}}
\end{aligned}
$$




$$
\begin{aligned}
& H_{42}=\frac{d r}{d c}=\frac{c}{\sqrt{d^{2}+c^{2}+z^{2}}} \\
& H_{43}=\frac{d r}{d z}=\frac{z}{\sqrt{d^{2}+c^{2}+z^{2}}} \\
& H_{51}=\frac{d \theta}{d d}=1000 \frac{-c}{d^{2}+c^{2}} \\
& H_{52}=\frac{d \theta}{d c}=1000 \frac{d}{d^{2}+c^{2}} \\
& H_{53}=\frac{d \theta}{d z}=0 \\
& H_{61}=\frac{d \phi}{d d}=1000 \frac{-d z}{\left(d^{2}+c^{2}+z^{2}\right) \sqrt{d^{2}+c^{2}}} \\
& H_{62}=\frac{d \phi}{d c}=1000 \frac{-c z}{\left(d^{2}+c^{2}+z^{2}\right) \sqrt{d^{2}+c^{2}}} \\
& H_{63}=\frac{d \phi}{d z}=1000 \frac{\sqrt{d^{2}+c^{2}}}{d^{2}+c^{2}+z^{2}} \\
& H_{72}=1 \\
& H_{83}=1 .
\end{aligned}
$$

All the other elements of $\mathbf{H}$ are zeros.

The space IR sensor errors, as discussed in Section C.1.2.1, have standard deviations of $500 \mathrm{~m}$ in all three axes. The radar range errors, as discussed in Section C.1.2.2, are on the order of a few meters rms, so a somewhat conservative standard deviation of $10 \mathrm{~m}$ is used. The radar azimuth and elevation-angle measurement errors have a noise component (random) and a bias component (correlated). Using the time-varying statistics for the noise and bias components, plotted in Fig. C.7, some sample sequences of angle-measurement errors for the ICBM S1 target are shown in Fig. C.8. The bias components are generated with the half-power point at $0.05 \mathrm{~Hz}$ using the time-varying standard deviations. Note that the noise components are on the order of 10 times larger than the bias components and that the elevation bias errors are larger than the azimuth bias errors. The kill-vehicle IR sensor errors, as discussed in Section C.1.2.3, have standard deviations of $30 \mathrm{~m}$ in the downrange and crossrange directions. Putting all these statistics together, the time-varying measurement-error covariance matrix is given by

$$
\begin{array}{r}
\mathbf{R}(k)=\operatorname{Diag}\left[\begin{array}{rrrr}
(500 \mathrm{~m})^{2} & (500 \mathrm{~m})^{2} & (500 \mathrm{~m})^{2} & (10 \mathrm{~m})^{2} \\
\sigma_{\theta}^{2}(k) & \sigma_{\phi}^{2}(k) & (30 \mathrm{~m})^{2} & (30 \mathrm{~m})^{2}
\end{array}\right]
\end{array}
$$

where $\sigma_{\theta}(k)$ and $\sigma_{\phi}(k)$, in milliradians, are the time-varying standard deviations of the noise components of the azimuth and elevation-angle measurement errors, respectively. The bias components of the angle measurements are not modeled in the filter, resulting in a filter that is slightly more optimistic. Note that if $\sigma_{\theta}(k)$ and $\sigma_{\phi}(k)$ were in radians instead of milliradians, the dynamic range of $\mathbf{R}$ would be increased by six orders of magnitude. Huge dynamic range results in poor numerical stability when the inverse of such a matrix is taken, which is part of the Kalman filter equations (see Eq. C.6 below). 


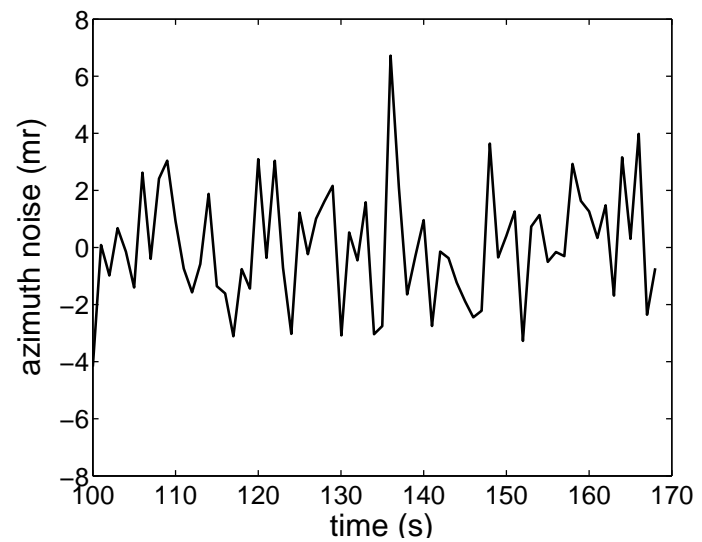

(a)

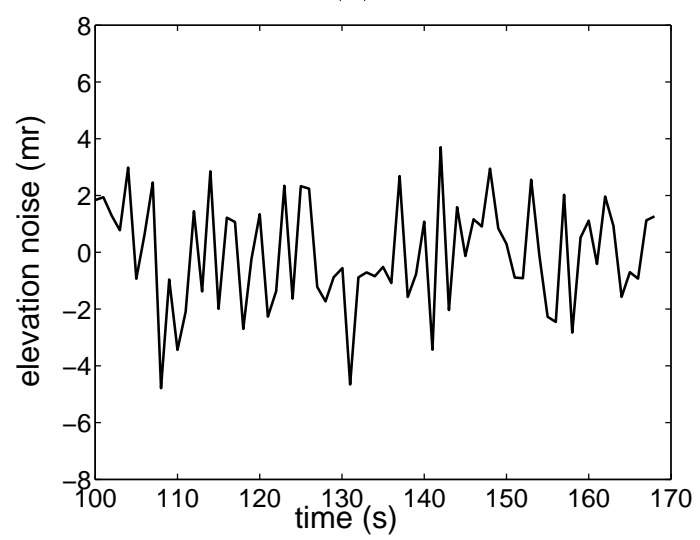

(c)

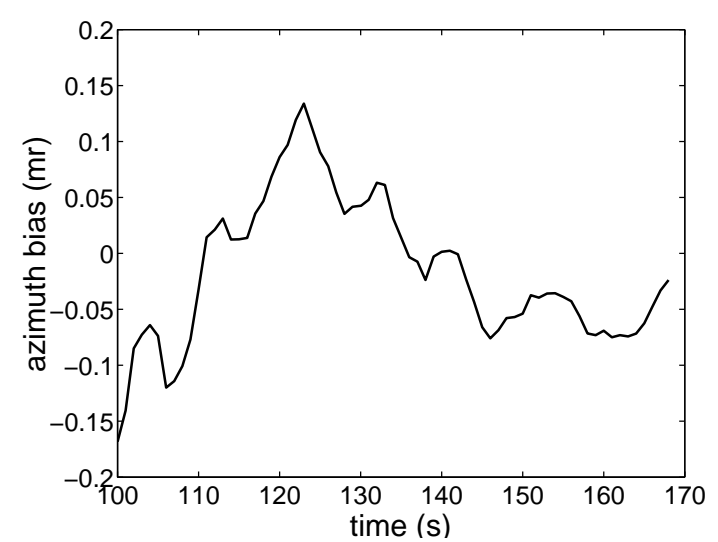

(b)

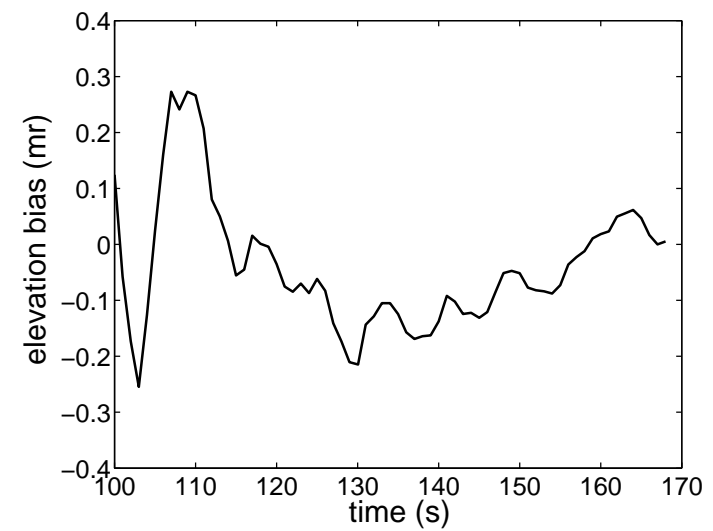

(d)

Figure C.8. (a) Sample sequence of the noise (random) component of the radar azimuth measurement errors. (b) Sample sequence of the bias (correlated) component of the radar azimuth measurement errors. (c) Sample sequence of the noise (random) component of the radar elevation measurement errors. (d) Sample sequence of the bias (correlated) component of the radar elevation measurement errors. 


\section{C.1.3.3 Kalman filter update equations}

With all the necessary components of the Kalman filter defined above, the time-propagation equations (from measurement $k-1$ to measurement $k$ ) are given by

$$
\begin{aligned}
\hat{\mathbf{x}}_{k}(-) & =\boldsymbol{\Phi}_{k-1} \hat{\mathbf{x}}_{k-1}(+) \\
\mathbf{P}_{k}(-) & =\boldsymbol{\Phi}_{k-1} \mathbf{P}_{k-1}(+) \boldsymbol{\Phi}_{k-1}^{T}+\mathbf{Q}_{k-1},
\end{aligned}
$$

where the $\hat{\mathbf{x}}$ denotes the estimate of $\mathbf{x},(-)$ and $(+)$ indicate before and after the measurement, respectively, and $\mathbf{P}$ is the state covariance matrix. Note that the time indices $k-1$ and $k$ are written as subscripts so that $(+)$ and $(-)$ can be used. The measurement-update equations are given by

$$
\begin{aligned}
\mathbf{K}_{k} & =\mathbf{P}_{k}(-) \mathbf{H}_{k}^{T}\left[\mathbf{H}_{k} \mathbf{P}_{k}(-) \mathbf{H}_{k}^{T}+\mathbf{R}_{k}\right]^{-1} \\
\hat{\mathbf{x}}_{k}(+) & =\hat{\mathbf{x}}_{k}(-)+\mathbf{K}_{k}\left[\mathbf{y}_{k}-\mathbf{h}\left(\hat{\mathbf{x}}_{k}(-)\right)\right] \\
\mathbf{P}_{k}(+) & =\left[\mathbf{I}-\mathbf{K}_{k} \mathbf{H}_{k}\right] \mathbf{P}_{k}(-),
\end{aligned}
$$

where $\mathbf{K}$ is the Kalman gain, $\mathbf{y}$ is the actual measurement vector, and $\mathbf{h}\left(\hat{\mathbf{x}}_{k}(-)\right)$ is the measurement vector function computed using $\hat{\mathbf{x}}_{k}(-)$. The initial values for $\hat{\mathbf{x}}$ and $\mathbf{P}$ needed to start the filter are

$$
\begin{aligned}
\hat{\mathbf{x}}_{0} & =\mathrm{E}(\mathbf{x}(0)) \\
\mathbf{P}_{0} & =\mathrm{E}\left[\left(\mathbf{x}(0)-\hat{\mathbf{x}}_{0}\right)\left(\mathbf{x}(0)-\hat{\mathbf{x}}_{0}\right)^{T}\right]
\end{aligned}
$$

\section{C.1.4 Trajectory state estimation}

With the Kalman filter formulation and the measurement errors described above, the simulation is carried out as follows. The space IR measurements are available to the filter after $t=29 \mathrm{~s}$ (when the missile clears cloud cover), and the radar measurements are available after $t=99 \mathrm{~s}$ (when the missile clears the horizon). Infrared measurements are available to the kill vehicle after $t=113 \mathrm{~s}$ (when the shroud comes off at 100-km altitude). As more measurements are incorporated, the matrices $\mathbf{R}, \mathbf{H}$, and $\mathbf{y}$ are adjusted in size to take on the additional measurements.

Three cases are presented below to assess the performance of the filter under different conditions. In the baseline case, the initial state estimates are set to the true states. In the poor-initial-estimates case, the initial state estimates are set such that their errors are two times the standard deviations in the state covariance matrix, $\mathbf{P}$. This case shows how well the filter can handle poor initial state estimates (which occurs when the space IR or surface radar give poorer-than-expected initial states of the missile). In the large-measurementerrors case, the errors used to create the measurements for the simulation are twice those given in the measurement-error covariance matrix, $\mathbf{R}$ (in addition to the large initial state estimate errors). This case shows how well the filter can handle measurement errors that are larger than expected.

\section{C.1.4.1 Baseline case}

In the baseline case, the initial state estimates and the initial covariance matrix are

$$
\hat{\mathbf{x}}(+)=\text { true missile states }
$$


Table C.1. Summary of state-estimate uncertainties (1- $\sigma$ values) for the different sensors used during the target-tracking problem.

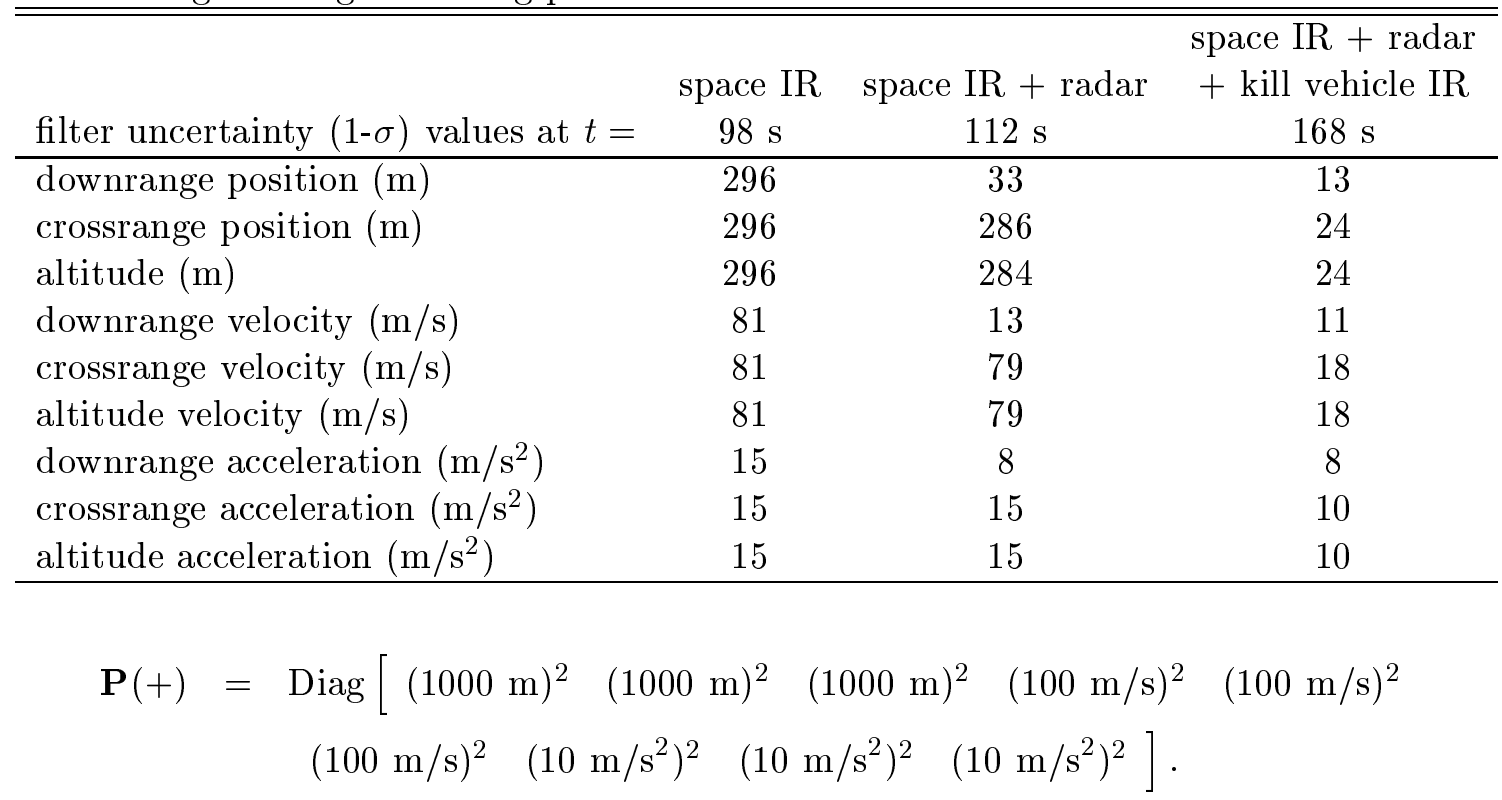

The measurements feeding the simulation are created by adding uncorrelated noises to the true measurements. These uncorrelated noises are normally distributed with standard deviations coming from the $\mathbf{R}$ matrix (Eq. C.3). The only correlated noises come from the bias components of the radar's azimuth and elevation measurements. However, these correlated errors are much smaller than the uncorrelated ones (see Fig. C.8). Therefore, other than the uncorrelated azimuth- and elevation-angle errors, the measurement model matches the measurements feeding the filter. We expect the best performance from the filter in this case.

Shown in Figs. C.9 and C.10 are the errors in the filter's position, velocity, and acceleration estimates, along with their corresponding standard deviations from the filter covariance matrix, plotted as + and - one-standard-deviation $(1-\sigma)$ envelopes. If the filter were tuned properly, that is, not overly optimistic or pessimistic, the 1- $\sigma$ values should be good estimates of the uncertainties (or true errors) in the filter state estimates. The true errors should be inside the 1- $\sigma$ envelopes most the time, but could be outside occasionally. Figures C.9 and C.10 show that the filter is tuned properly. Table C.1 summarizes the state-estimate uncertainties (1- $\sigma$ values) for the different sensors used during the target-tracking problem.

When the filter starts to process its first measurements at $t=29 \mathrm{~s}$, the $1-\sigma$ error estimates of the position states drop quickly from the initial values of $1000 \mathrm{~m}$ to around $300 \mathrm{~m}$. This drop is reasonable, as the filter is told that the space IR sensor can measure the positions in all three axes to 500-m accuracy. However, the uncertainties of the velocity state estimates improve only from $100 \mathrm{~m} / \mathrm{s}$ to around $80 \mathrm{~m} / \mathrm{s}$ using the space IR sensor alone. In fact, with the space IR sensor only, the uncertainties of the acceleration state estimates actually grow from $10 \mathrm{~m} / \mathrm{s}^{2}$ to more than $15 \mathrm{~m} / \mathrm{s}^{2}$ (see Fig. C.10). One reason for this growth is the process noise of $5 \mathrm{~m} / \mathrm{s}^{2}$ (standard deviation) adds to the acceleration states, which keeps their variances from diminishing too quickly. The other reason is that the space IR sensor measurements are not accurate enough to improve the acceleration state estimates.

When the radar measurements are available from $t=99 \mathrm{~s}$, the downrange position un- 


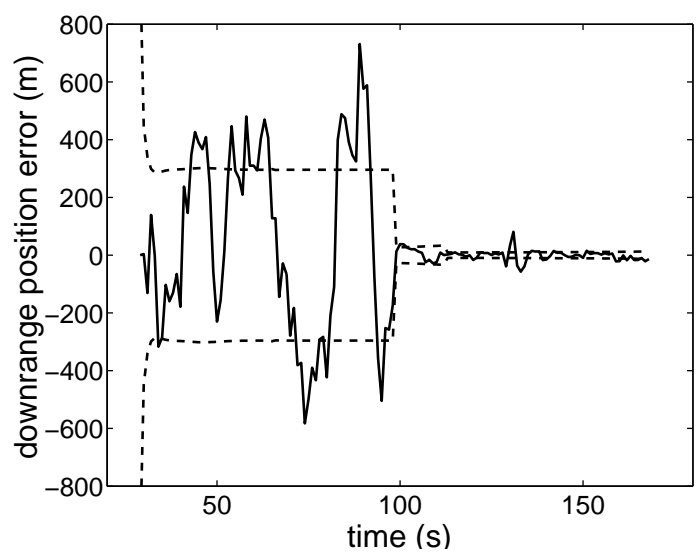

(a)

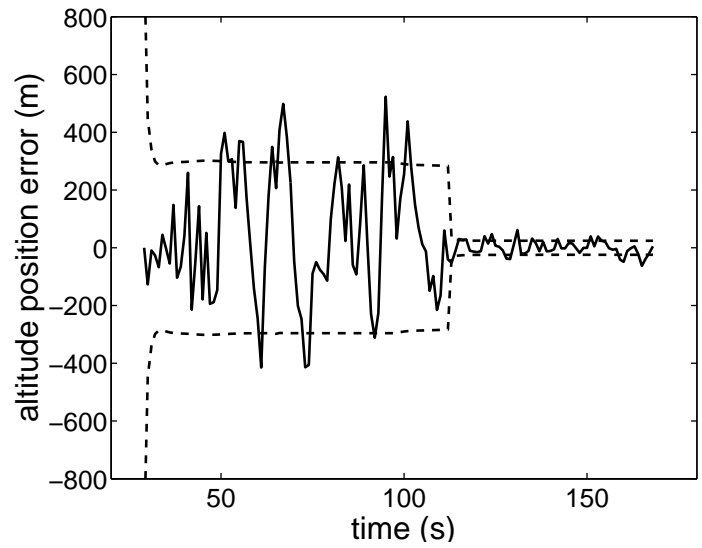

(c)

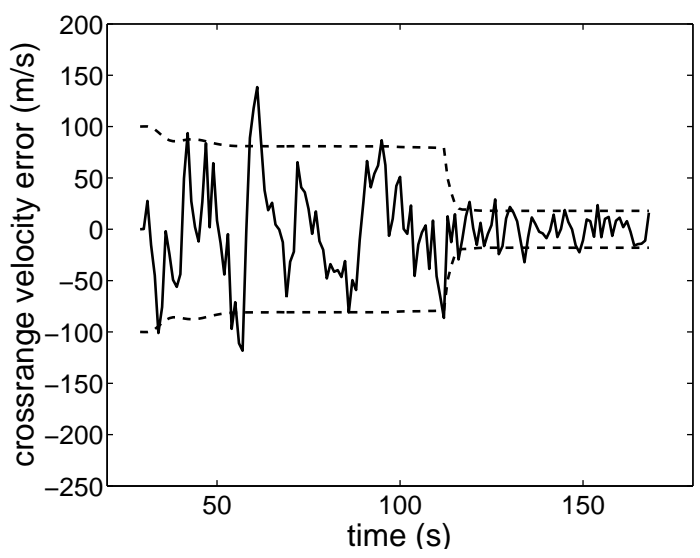

(e)

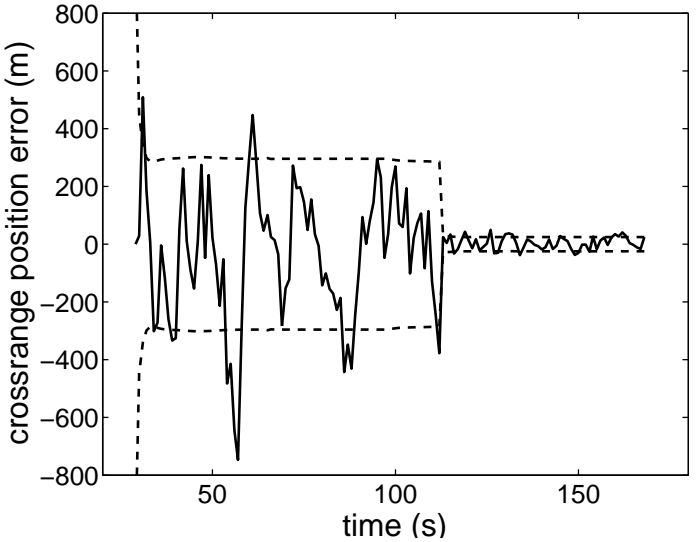

(b)

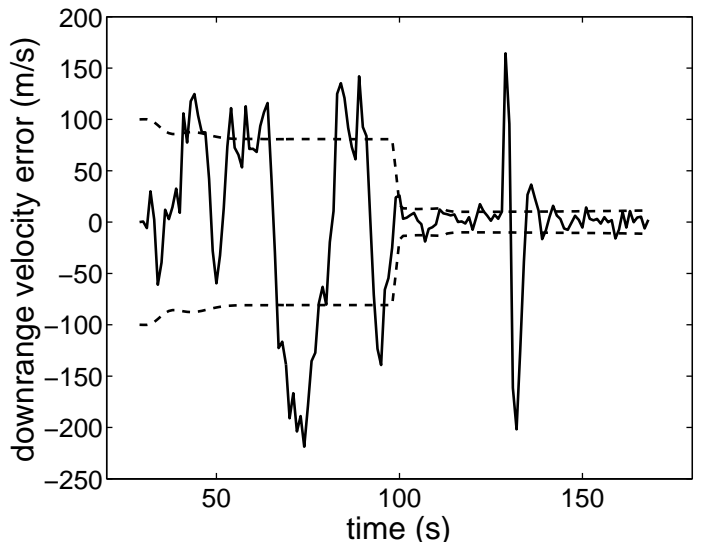

(d)

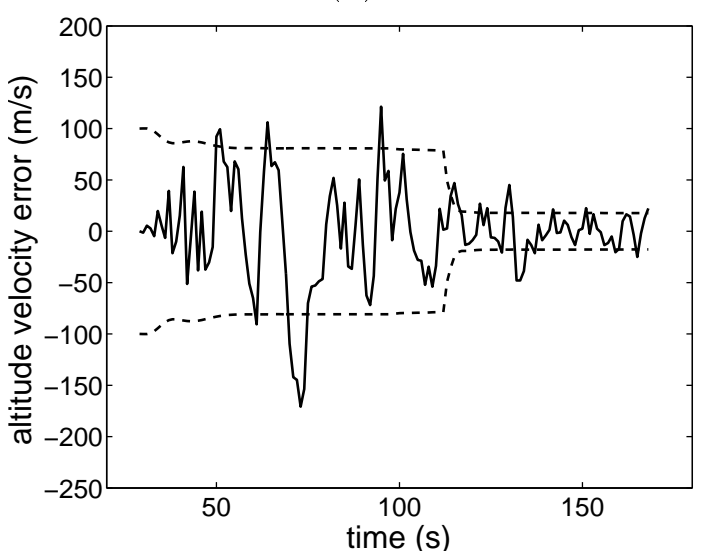

(f)

Figure C.9. (a), (b), (c) Baseline case: Downrange, crossrange, and altitude position errors and filter's estimates of 1- $\sigma$ values. (d), (e), (f) Downrange, crossrange, and altitude velocity errors and filter's estimates of 1- $\sigma$ values. 


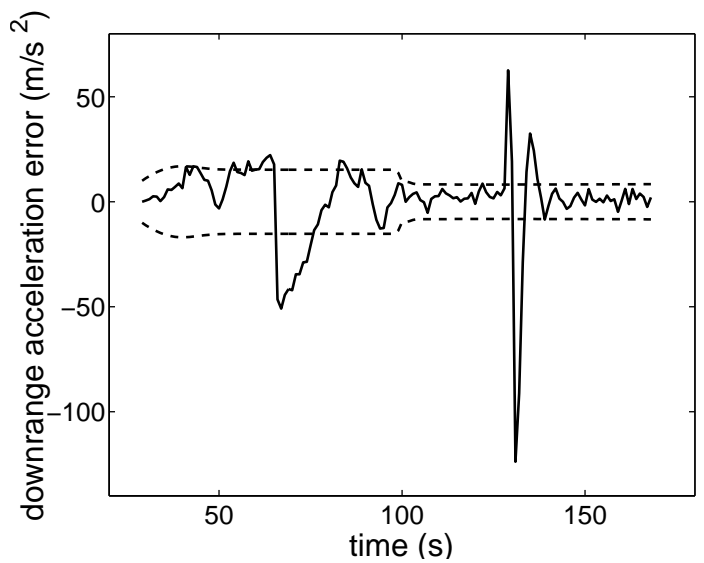

(a)

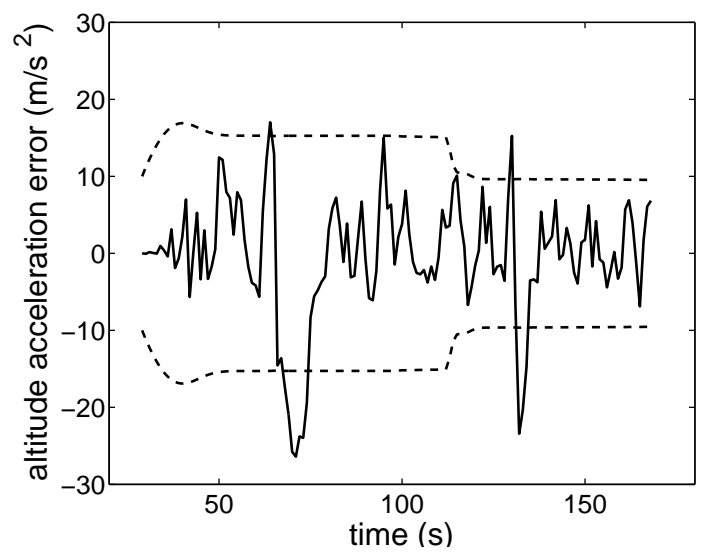

(c)

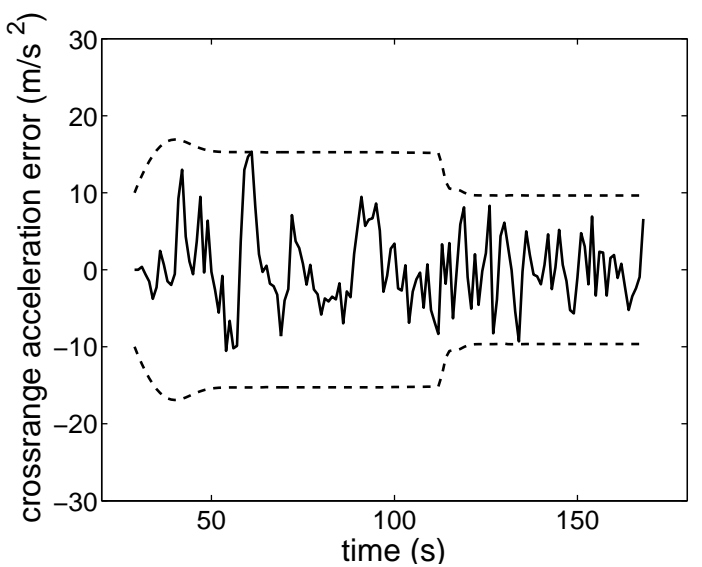

(b)

Figure C.10. (a), (b), (c) Baseline case: Downrange, crossrange, and altitude acceleration errors and filter's estimates of $1-\sigma$ values. 
certainty improves dramatically to around $30 \mathrm{~m}$ (Fig. C.9 (a)). This improvement is due to the accurate range measurements, which are mainly in the downrange direction. However, the crossrange position and the altitude estimates do not improve, because these directions are dominated by the azimuth noise and elevation noise of the radar, which still have standard deviations of more than $2 \mathrm{mr}$ during this part of the trajectory. At a downrange distance of $800 \mathrm{~km}$, the azimuth and elevation uncertainties translate roughly to $1600 \mathrm{~m}$ of measurement error in crossrange and altitude positions. Therefore, the space IR sensor is still providing better measurements. Similarly for the velocity estimates, the downrange velocity estimates improve from around $80 \mathrm{~m} / \mathrm{s}$ to $13 \mathrm{~m} / \mathrm{s}$, but the uncertainties of the crossrange and vertical velocity estimates stay around $80 \mathrm{~m} / \mathrm{s}$. The downrange acceleration estimates improve to around $8 \mathrm{~m} / \mathrm{s}^{2}$, while the crossrange and vertical acceleration uncertainties remain near $15 \mathrm{~m} / \mathrm{s}^{2}$.

When the kill-vehicle IR measurements come on at $t=113 \mathrm{~s}$, the downrange positionestimate uncertainty improves to $13 \mathrm{~m}$ (the scale of the plot does not show this level of detail), and the crossrange position and altitude uncertainties both improve to $24 \mathrm{~m}$. Again this behavior is reasonable, as the standard deviation of the kill-vehicle IR measurement errors is $30 \mathrm{~m}$. The downrange velocity estimates further improve to $11 \mathrm{~m} / \mathrm{s}$. The crossrange and vertical velocity uncertainties decrease from around $80 \mathrm{~m} / \mathrm{s}$ to $18 \mathrm{~m} / \mathrm{s}$. The downrange acceleration uncertainty does not see any further improvement since the kill-vehicle IR does not provide range information. Both the crossrange and altitude acceleration estimates improve to $10 \mathrm{~m} / \mathrm{s}^{2}$.

It should be pointed out that the target missile stages at $65 \mathrm{~s}$ and $130 \mathrm{~s}$ (see Fig. C.2). The effects are that the downrange acceleration changes significantly, from about $8 \mathrm{~g}$ to $2 \mathrm{~g}$ and from about $9 \mathrm{~g}$ to $3 \mathrm{~g}$ for the two stagings, respectively. The errors in the downrange acceleration estimates (see Fig. C.10 (a)) during these two times (greater than $40 \mathrm{~m} / \mathrm{s}^{2}$ and $120 \mathrm{~m} / \mathrm{s}^{2}$ at the two staging times, respectively) are much larger than the filter's prediction. This discrepancy is to be expected, since the filter is set up with a constant-acceleration model and the missile is accelerating. However, because of the $5-\mathrm{m} / \mathrm{s}^{2}$ (standard deviation) process noise added to the acceleration states, the filter is able to track the huge changes in acceleration during staging. Note that the downrange velocity state also sees errors on the order of $200 \mathrm{~m} / \mathrm{s}$. The vertical acceleration changes are less dramatic (from about $3 \mathrm{~g}$ to $0 \mathrm{~g}$ and from about $2 \mathrm{~g}$ to $0 \mathrm{~g}$, respectively), but the effects on the vertical acceleration estimates at 65 and $130 \mathrm{~s}$ are also obvious (Fig. C.10 (c)). Since we are working with a planar trajectory, the staging events do not affect the crossrange acceleration (Fig. C.10 (b)). (With these observations, we could reduce the process noise on the altitude acceleration state, and perhaps even set it to zero for the crossrange acceleration state, to further improve the filter's performance if we wished. However, since our goal here is to demonstrate the simulation procedures, we did not try to optimize the filter's performance any further.) The ability to track an accelerating target through staging is a concern within the missile-tracking community. However, in this example, given our assumptions, the filter is able to track the missile through the two staging events. 


\section{C.1.4.2 Poor-initial-estimates case}

In this case, the initial state estimates are set so that their errors are two times the standard deviations in the state covariance matrix,

$$
\hat{\mathbf{x}}(+)=\text { true missile states }-\left[\begin{array}{c}
2000 \mathrm{~m} \\
2000 \mathrm{~m} \\
2000 \mathrm{~m} \\
200 \mathrm{~m} / \mathrm{s} \\
200 \mathrm{~m} / \mathrm{s} \\
200 \mathrm{~m} / \mathrm{s} \\
20 \mathrm{~m} / \mathrm{s}^{2} \\
20 \mathrm{~m} / \mathrm{s}^{2} \\
20 \mathrm{~m} / \mathrm{s}^{2}
\end{array}\right],
$$

and the initial covariance matrix is the same as the one in the baseline case,

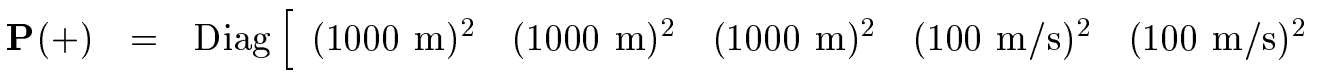

$$
\begin{aligned}
& \left.(100 \mathrm{~m} / \mathrm{s})^{2} \quad\left(10 \mathrm{~m} / \mathrm{s}^{2}\right)^{2} \quad\left(10 \mathrm{~m} / \mathrm{s}^{2}\right)^{2} \quad\left(10 \mathrm{~m} / \mathrm{s}^{2}\right)^{2}\right] .
\end{aligned}
$$

Figures C.11 and C.12 show that even with the large errors in the initial-state estimates when the filter is started, the filter is still able to converge to the correct state estimates. Once the initial-error transients are gone, the filter has similar performance as in the baseline case.

\section{C.1.4.3 Large-measurement-errors case}

In this case, the poor initial-state estimates from the previous case are kept. In addition, the standard deviations (from the $\mathbf{R}$ matrix) used to generate the measurements are doubled, but the filter still uses the same $\mathbf{R}$. (The bias components of the radar azimuth and elevation measurements are also doubled.) This case simulates the scenario in which the measurement errors are actually larger than expected. Besides setting up a dynamics model that represents the state dynamics correctly, the level at which $\mathbf{R}$ truly represents the measurement errors is the most important factor that determines the filter performance. If the $\mathbf{R}$ values are set too low, the filter would trust the measurements too much which could lead to filter instability (state estimates diverge). If the $\mathbf{R}$ values are set too high, the filter would not trust the measurement enough and it could take a long time to converge to the correct state estimates. It would also have difficulties in tracking transients, such as the staging events.

Figures C.13 and C.14 show that although the state estimates have larger errors than the other two cases and the state estimate errors are outside the 1- $\sigma$ envelopes much more often, the filter still provides correct estimates. Again, the staging effects are apparent in the downrange acceleration and downrange velocity state estimates. Even with mismatched measurement error model (by 100 percent), the filter is able to track through the two staging events, indicating that the filter is fairly robust.

\section{C.1.4.4 Tracking with two radars}

When radar jamming is deployed, the range measurements may be assumed to be invalid, and two or more radars must be used to provide angle-only measurements. A Kalman filter 


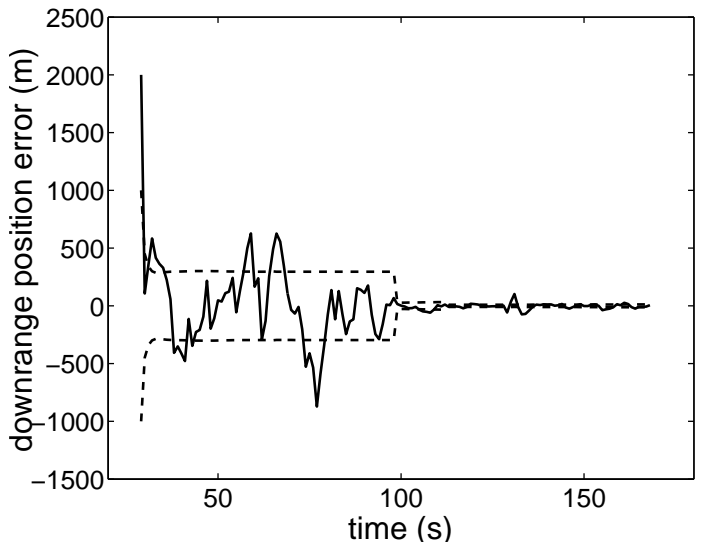

(a)

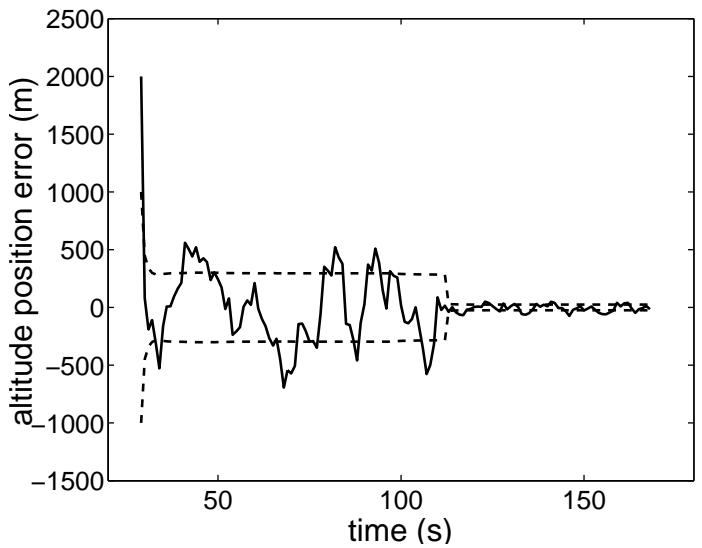

(c)

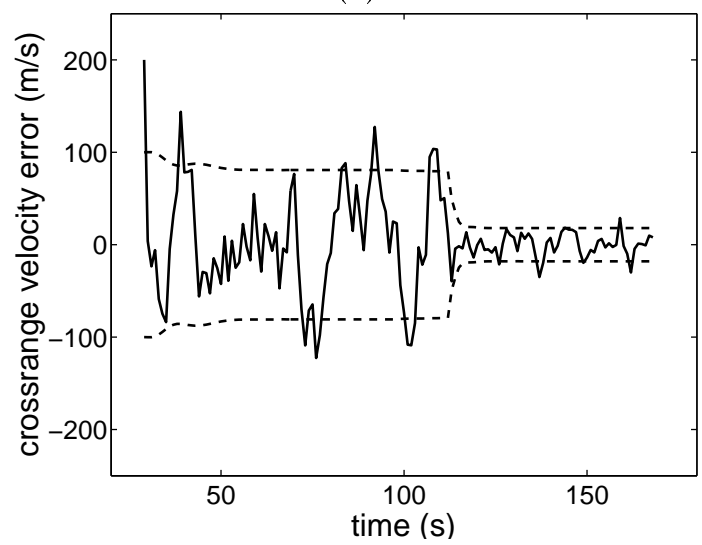

(e)

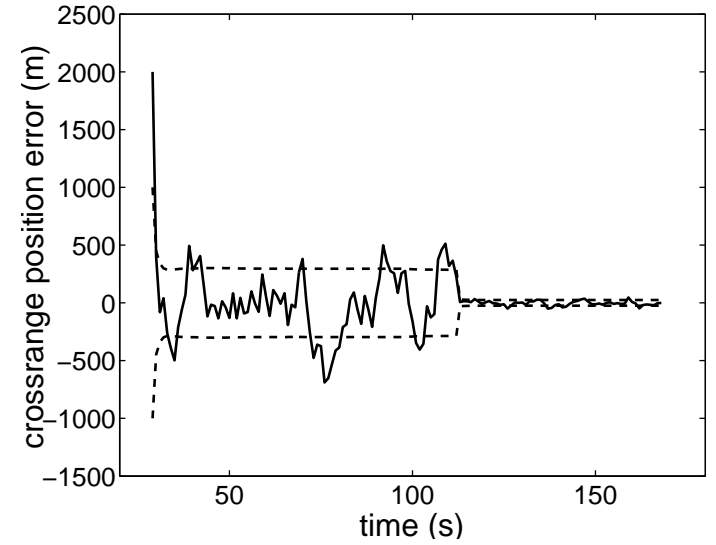

(b)

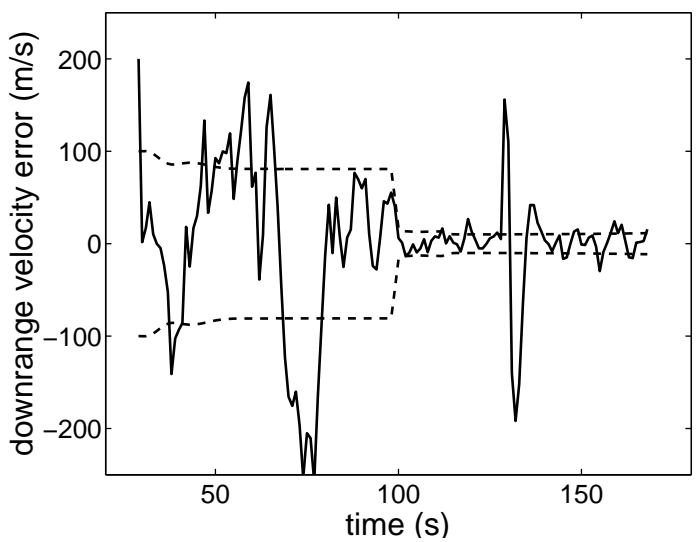

(d)

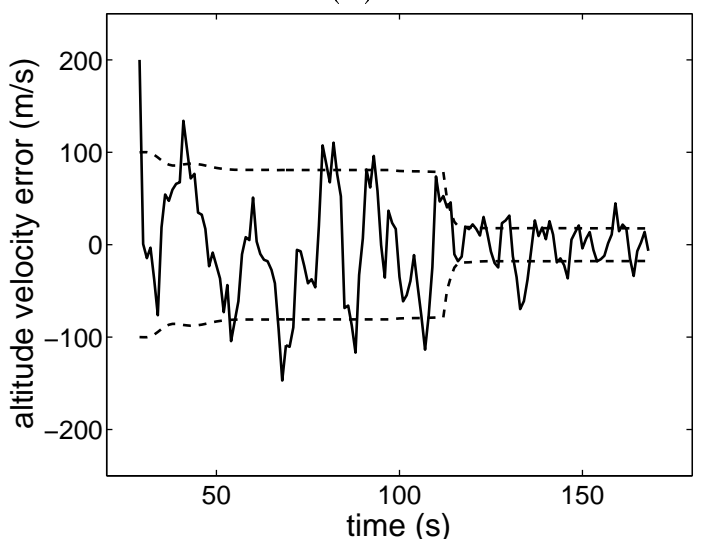

(f)

Figure C.11. (a), (b), (c) Poor-initial-estimates case: Downrange, crossrange, and altitude position errors and filter's estimates of 1- $\sigma$ values. (d), (e), (f) Downrange, crossrange, and altitude velocity errors and filter's estimates of 1- $\sigma$ values. Note the poor downrange velocity estimates in (d) during staging events at $t=65 \mathrm{~s}$ and $t=130 \mathrm{~s}$. 


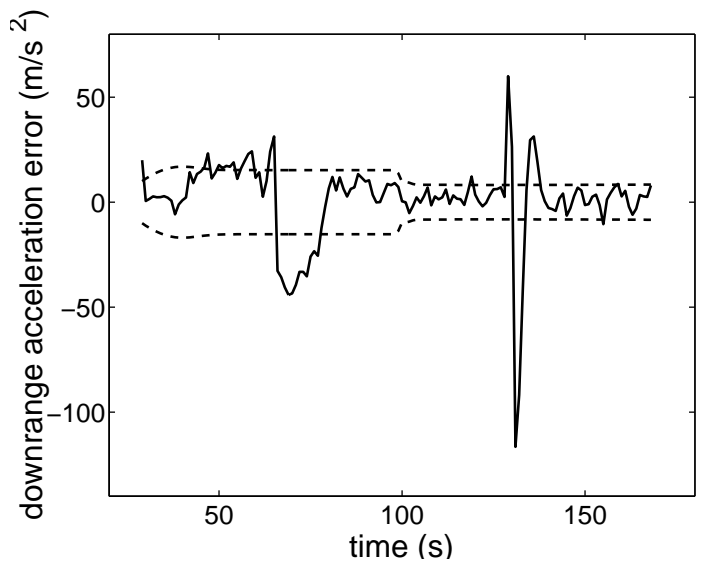

(a)

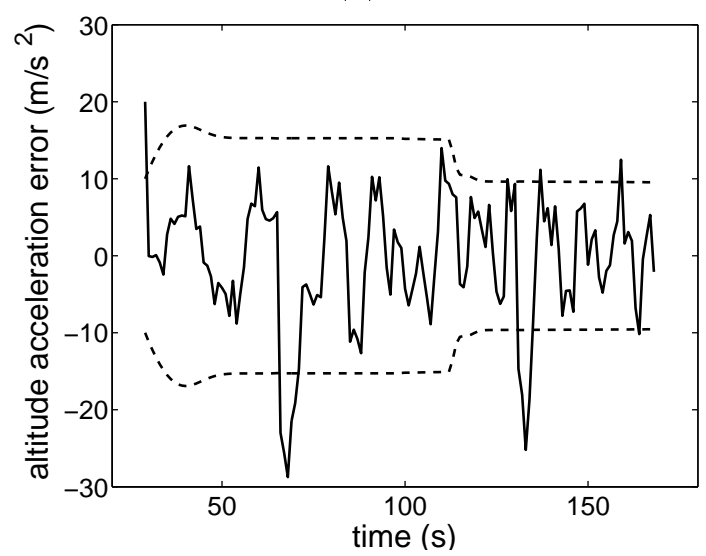

(c)

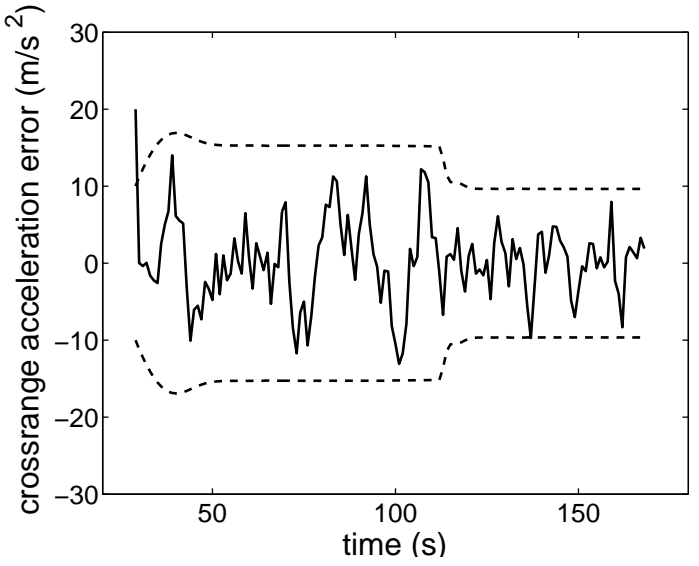

(b)

Figure C.12. (a), (b), (c) Poor-initial-estimates case: Downrange, crossrange, and altitude acceleration errors and the filter's estimates of $1-\sigma$ values. Note the poor downrange acceleration estimates in (a) during staging events at $t=65 \mathrm{~s}$ and $t=130 \mathrm{~s}$. 


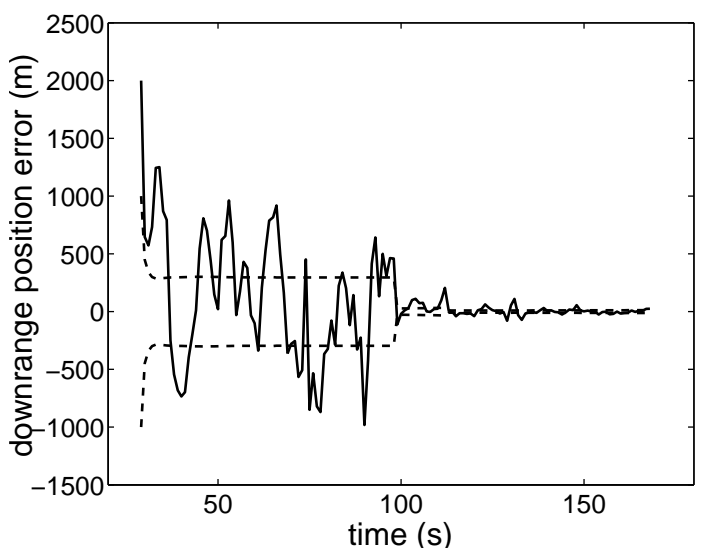

(a)

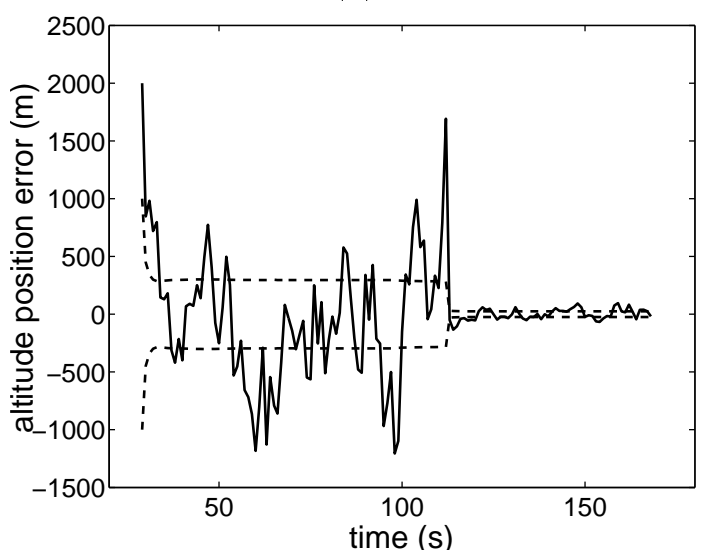

(c)

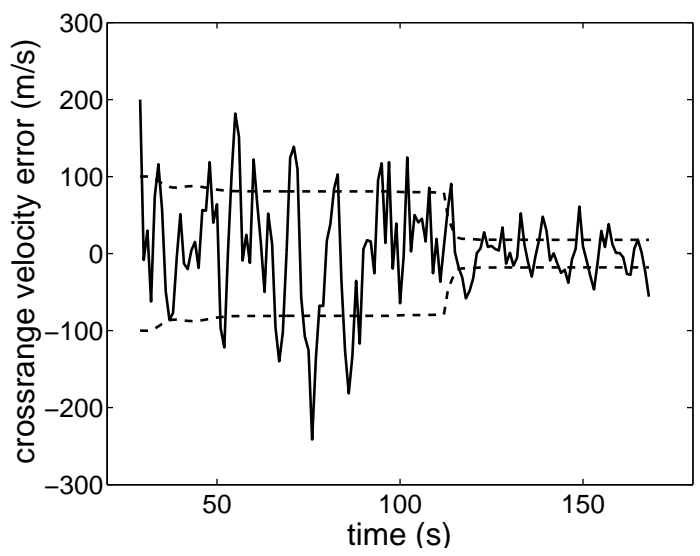

(e)

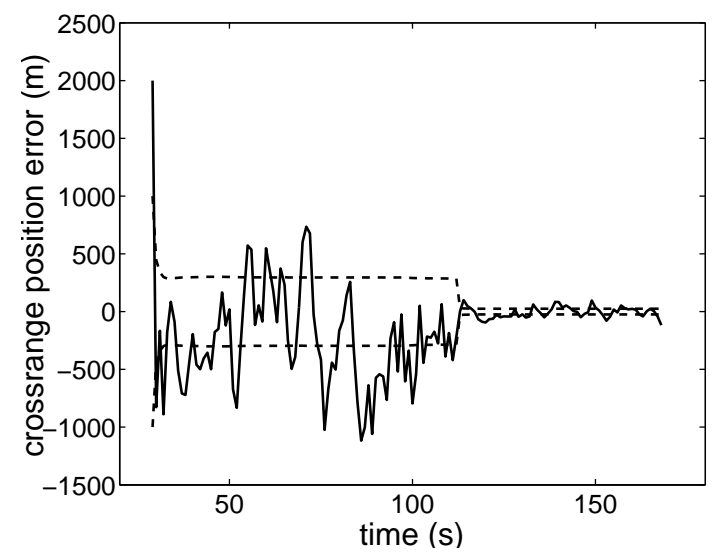

(b)

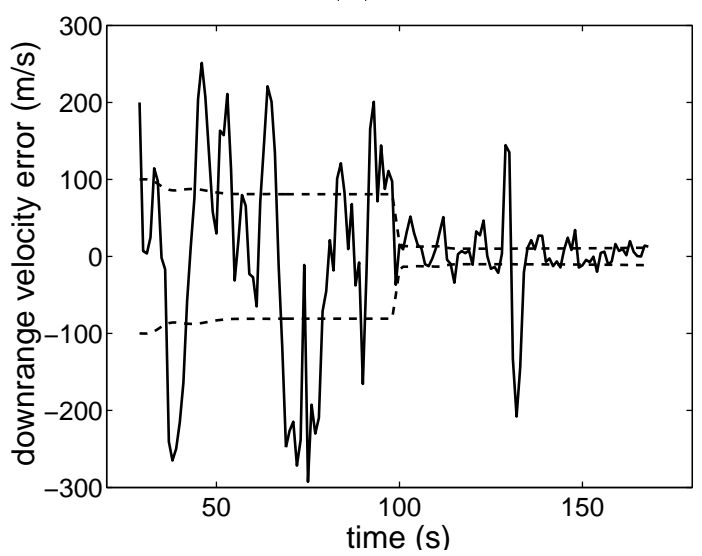

(d)

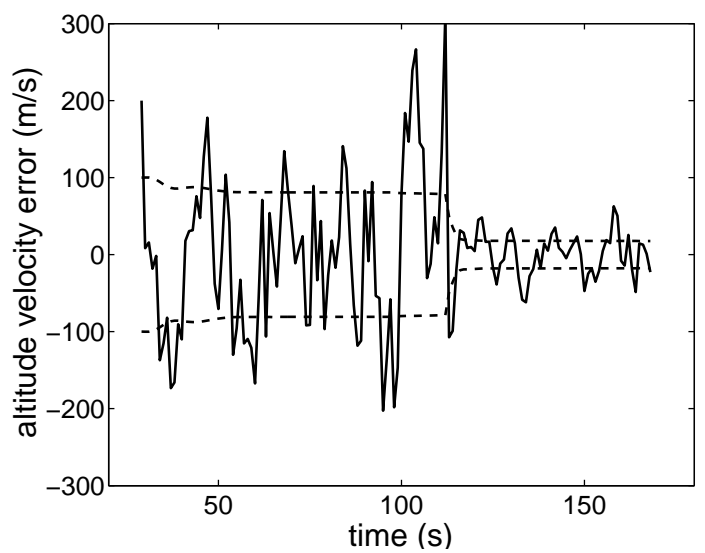

(f)

Figure C.13. (a), (b), (c) Large-measurement-errors case: Downrange, crossrange, and altitude position errors and filter's estimates of 1- $\sigma$ values. (d), (e), (f) Downrange, crossrange, and altitude velocity errors and filter's estimates of $1-\sigma$ values. 


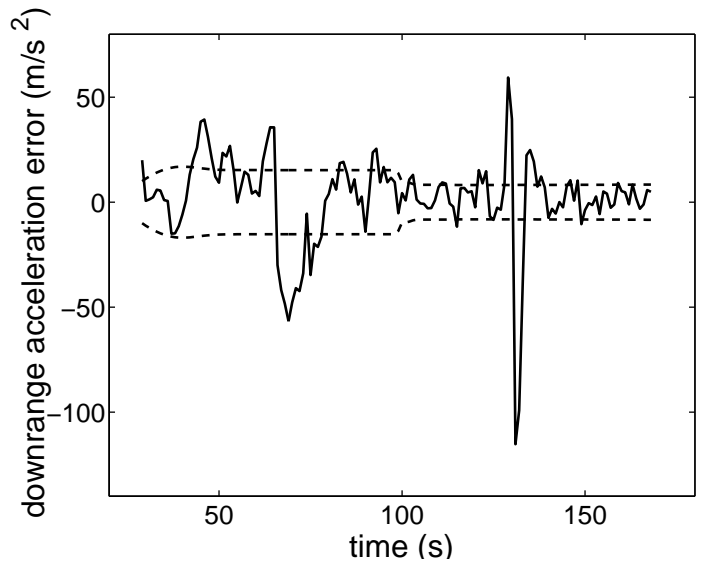

(a)

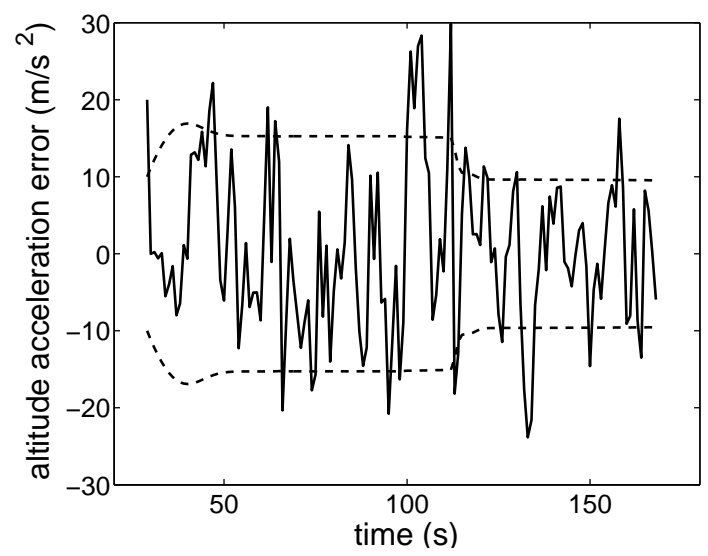

(c)

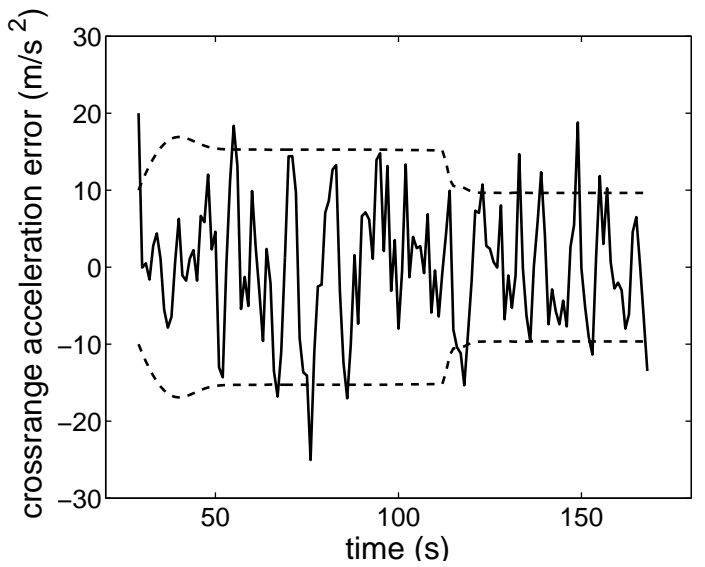

(b)

Figure C.14. (a), (b), (c) Large-measurement-errors case: Downrange, crossrange, and altitude acceleration errors and filter's estimates of 1- $\sigma$ values. 
is formulated in which two radars are separated by $1000 \mathrm{~km}$, which is also the distance between the missile and interceptor launch sites. Although the look angles from these two radars are different from the single radar at the interceptor launch site, the same error models for the azimuth and elevation measurements are used. The results show that insignificant degradation in state estimates occurs when angle-only measurements are used from two radars, when compared with range and two-angle measurements from one radar.

\section{C.1.5 Engagement summary}

In this section, we examined the engagement between the faster-burning solid-propellant missile (ICBM S1) and an aggressive 10-km/s interceptor. After looking at the trajectories for the missile and interceptor, the measurement models for the space IR, surface radar, and kill-vehicle IR were discussed. A Kalman filter was formulated to estimate the missile's position, velocity, and acceleration. Three sensitivity cases were studied to evaluate the robustness of filter performance. With zero error for the initial state estimates and measurement error models matching the actual measurements, the filter performed very well, providing state estimates that are consistent with the measurement errors. With large errors for the initial state estimates (twice the values given in the state covariance matrix), the filter essentially gave the same kind of estimates as the previous case after a few measurements were processed. In the third case, the measurement errors were twice as large as those given to the filter. Although the estimate errors are larger, and the covariance matrix is somewhat optimistic about its estimates (estimate errors are larger than the 1- $\sigma$ values), the state estimates are still acceptable. In all three cases, the filter was able to track through two staging events, a concern shared by many experts in this area. These cases indicate that based on the assumptions outlined in this section, the trajectory estimation problem is solvable.

\section{C.2 Engagement of ICBM L and the 5-km/s Interceptor I-5}

In this section, the engagement of the slower liquid-propellant ICBM L and the $5-\mathrm{km} / \mathrm{s}$ interceptor I-5 is examined.

\section{C.2.1 Missile and interceptor trajectories}

Figure C.15 shows the engagement between the ICBM L target missile and three $5-\mathrm{km} / \mathrm{s}$ interceptors. (The time marks on the trajectories indicate times since launch.) The three interceptor trajectories are labeled $(0,30),(30,30)$, and $(45,60)$. They denote the angles of attack used during the third stage of the interceptors (between 36 and $56 \mathrm{~s}$ after interceptor launch). For example, $(0,30)$ means that the angle of attack is $0^{\circ}$ from $t=36$ to $t=46 \mathrm{~s}$ and is $-30^{\circ}$ from $t=46 \mathrm{~s}$ to $t=56 \mathrm{~s}$. The $(45,60)$ designation means $-45^{\circ}$ for $10 \mathrm{~s}$ and $-60^{\circ}$ for another $10 \mathrm{~s}$. All three interceptors are launched having a $55^{\circ}$ launch angle. The ICBM L has a burnout time of $240 \mathrm{~s}$. The two launch points are $800 \mathrm{~km}$ apart (compared with $1000 \mathrm{~km}$ for the ICBM S1 case).

Instead of maximizing the time between missile burnout and time of intercept as we did for the ICBM S1-engagement (because the solid-propellant missile has a shorter burn time), we try to maximize the allowable interceptor launch delay in this case, achieving a 115-s launch delay. (The launch delay for the ICBM S1 engagement is $59 \mathrm{~s}$.) The interceptor that intercepts the missile $16 \mathrm{~s}$ before the missile's burnout is chosen for the analysis in 

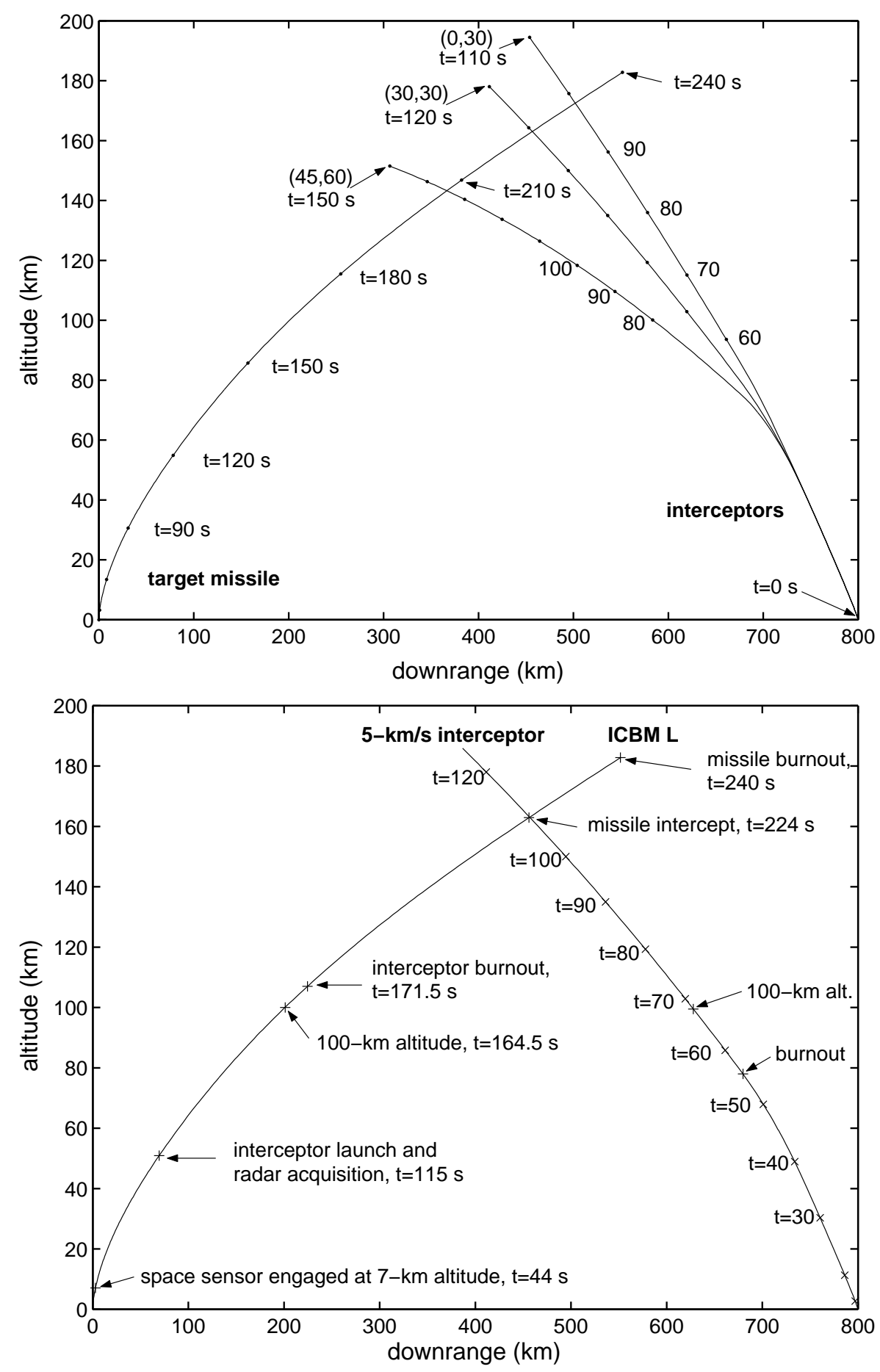

Figure C.15. (top) Engagement between the target missile ICBM L and three $5-\mathrm{km} / \mathrm{s}$ interceptors. (bottom) Details of the engagement. The interceptor trajectory is the same as interceptor trajectory $(30,30)$ in the top plot.

this section. This is the interceptor launched with $-30^{\circ}$ angle of attack for the entire third stage, denoted by $(30,30)$ in Fig. C.15. If the $(45,60)$ interceptor were used, the intercept could take place $33 \mathrm{~s}$ before missile burnout, but it would give an interceptor-launch delay 


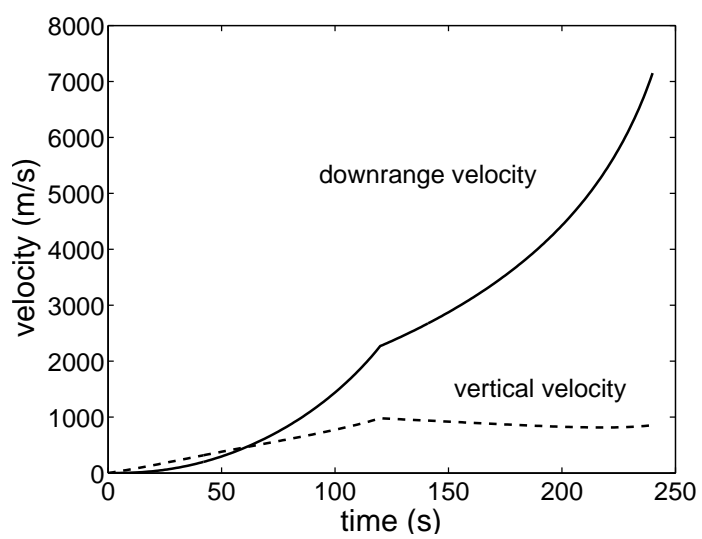

(a)

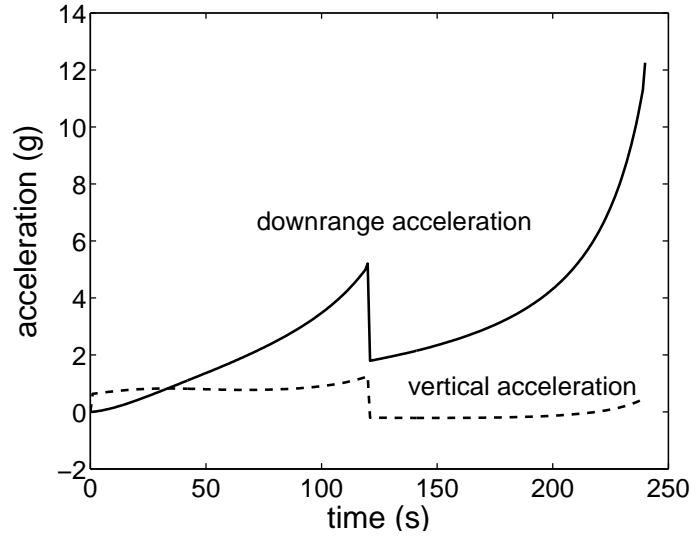

(b)

Figure C.16. (a) Downrange and vertical velocities of the ICBM L target missile. (b) Downrange and vertical accelerations of the ICBM L target missile.

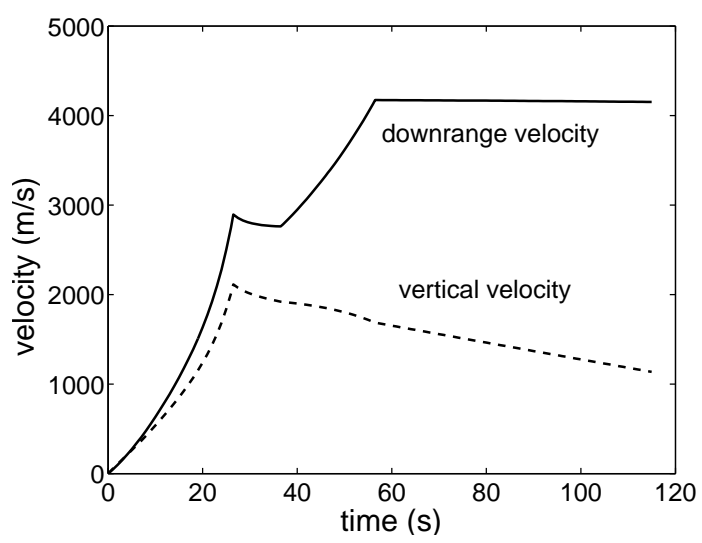

(a)

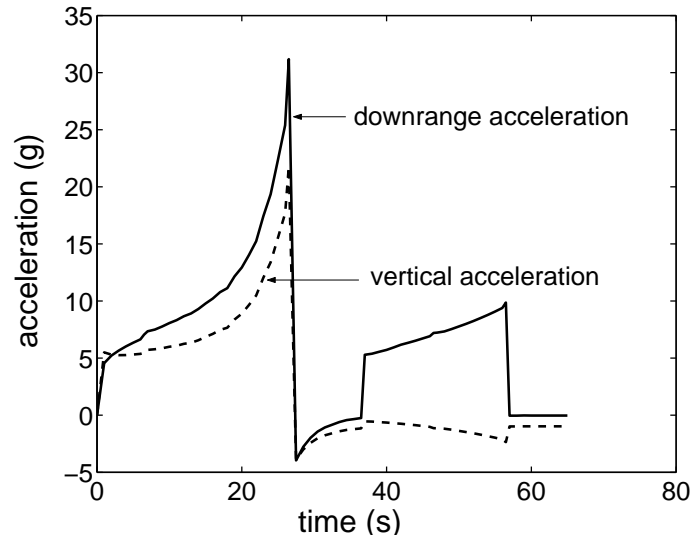

(b)

Figure C.17. (a) Downrange and vertical velocities of the $5-\mathrm{km} / \mathrm{s}$ interceptor. (b) Downrange and vertical accelerations of the $5-\mathrm{km} / \mathrm{s}$ interceptor.

of only $72 \mathrm{~s}$.

Details of the ICBM L missile and the chosen $5-\mathrm{km} / \mathrm{s}$ interceptor trajectories are shown in Fig. C.15. The missile reaches an altitude of $7 \mathrm{~km}$ after $44 \mathrm{~s}$ into flight, at which time the space IR could see the missile. The interceptor is launched at $t=115 \mathrm{~s}$ and around the same time the surface radar starts to acquire the missile, but reliable radar measurements are not available until after $t=125 \mathrm{~s}$. The interceptor reaches $100-\mathrm{km}$ altitude $68 \mathrm{~s}$ after launch, which means the kill-vehicle IR sensor could see the missile about $183 \mathrm{~s}(115+68 \mathrm{~s})$ into the ICBM L's flight.

The velocities and accelerations for both the ICBM L and the interceptor are shown in Figs. C.16 and C.17, respectively.

\section{C.2.2 Measurement models}

The same error models from the ICBM S1 engagement are used here. However, since the radar-tracking range is different in this engagement $(800 \mathrm{~km}$ vs. $1000 \mathrm{~km})$ and the radar 
cross section of the ICBM L is different from the ICBM S1, different radar measurement errors are used. These are discussed in the sections below.

\section{C.2.2.1 Space-based and kill-vehicle IR sensor measurement errors}

We assume that the space IR and kill-vehicle IR measurement errors are the same, although the IR signatures of the solid- and liquid-propellant missile are different. The space IR measurements are assumed to have 500-m 1- $\sigma$ errors in all three axes. The kill-vehicle IR is assumed to have 30-m 1- $\sigma$ accuracy in the azimuth and elevation directions.

\section{C.2.2.2 Surface radar measurement errors}

Radar acquisition For operations based $800 \mathrm{~km}$ from the target launching site, the existing Aegis AN/SPY-1B radar is adequate for acquisition and tracking of the ICBM targets modeled for this study. The parameters of this radar, as used to provide command guidance data for the interceptor's midcourse guidance phase, were given in Section 10.2 (shipboard radar for boost-phase detection and tracking). The radar is notified by a space-based IR system and searches a small volume just above the horizon, at the predicted time of appearance of the target, locking onto and then tracking the target until intercept. It also receives data on launch and trajectory of the interceptor, tracking it using a transponder.

Source and accuracy of designation data The designation error of the target relative to the radar position has been conservatively assumed to be $5 \mathrm{~km} \mathrm{rms}$. At the $747-\mathrm{km}$ horizon range of the target, this designation error corresponds to an azimuth accuracy $\sigma_{a z}=6.8 \mathrm{mr}$, which for a high probability of acquisition requires coverage of an azimuth sector $A_{m}=5 \sigma_{a z}=34 \mathrm{mr}$. This is slightly larger than the azimuth beamwidth, so only a small scan is required and the radar acquires the target in one of two beams just above the horizon.

Target cross section The RCS model for this target is based on an outline drawing of the ICBM L, and the RCS during the two stages of propulsion is shown in Fig. C.5, (left). The aspect angle from the nose is near $30^{\circ}$ during acquisition, increasing gradually toward $60^{\circ}$ near the end of powered flight. In this sector, the significant contributions are from the rounded nose cone tip and the irregularities and edges assumed to scatter into the forward hemisphere, totaling $0.17 \mathrm{~m}^{2}$ from the final stage and $0.31 \mathrm{~m}^{2}$ from the secondstage booster. A value $\sigma=0.48 \mathrm{~m}^{2}=-3.1 \mathrm{dBsm}$ applies during the initial acquisition period, but the transition to the second boost stage results in a reduction in RCS soon after acquisition, with a constant $\sigma=0.17 \mathrm{~m}^{2}=-8 \mathrm{dBsm}$ for the remainder of radar operation.

Signal-to-noise ratio at horizon The target appears above the horizon at $t=106 \mathrm{~s}$ with an elevation rate of $1.3 \mathrm{mr} / \mathrm{s}$. It spends $20 \mathrm{~s}$ within the $26-\mathrm{mr}$ elevation beamwidth of the radar. The single-pulse, beam-center signal-to-noise ratio at the horizon is calculated to be $+14.4 \mathrm{~dB}$ after allowance for tropospheric loss of $5 \mathrm{~dB}$ (including the tropospheric lens loss).

Probability of acquisition Because the radar scan cannot be planned on the basis of a target RCS larger than $0.1 \mathrm{~m}^{2}$ during the initial attempt at acquisition, the scan is designed to devote essentially all radar resources over a $16 \mathrm{~s}$ period to acquisition of the target, using 
multiple-pulse integration. The resulting integrated signal-to-noise ratio for a 8-s integration time, allowing for two such attempts during passage of the target through the beam, is shown in Fig. C.6. That process leads to detection of the target with high probability during the first scan, with a probable time allocation of half a scan period, or $4 \mathrm{~s}$, for detection at $t=110 \mathrm{~s}$. During this 4 -s period, the radar would reserve minimal resources for tracking other targets and outgoing interceptors, but would not be able to search for targets other than the single one being designated by the space-based IR system.

Radar tracking error calculations Once the radar has acquired the target, tracking can begin. It has been assumed that the radar will allocate 12 percent of its time and power resources to tracking an ICBM target, providing ten pulses per second with the signal-tonoise ratio shown in Fig. C.6. The sources of error and equations for calculating them were given in Section 10.2. The rms magnitude of errors has been calculated on the basis of averages over a smoothing time of $1 \mathrm{~s}$. Error components that are independent from pulse to pulse, and hence from each 1-s sample to the next, are defined as noise, while those that remain correlated over several seconds are defined as bias. The latter are generally not subject to reduction by smoothing in a tracking filter, but neither are they constant over such long periods that they make no contribution to errors in velocity and acceleration. These noise and bias error components for elevation and azimuth tracking data are shown in Fig. C.7 (left). The large initial error in elevation is the result of multipath signals, but this component drops rapidly as the target rises above one beamwidth in elevation. Errors in range are negligible, amounting to a few meters rms.

\section{C.2.3 Trajectory state estimation}

The same Kalman filter formulation developed in Section C.1.3 is used for this engagement. The time-varying measurement-error covariance matrix, initial covariance matrix, and initial state estimates are

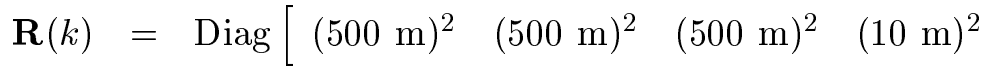

$$
\begin{aligned}
& \left.\sigma_{\theta}^{2}(k) \quad \sigma_{\phi}^{2}(k) \quad(30 \mathrm{~m})^{2} \quad(30 \mathrm{~m})^{2}\right]
\end{aligned}
$$

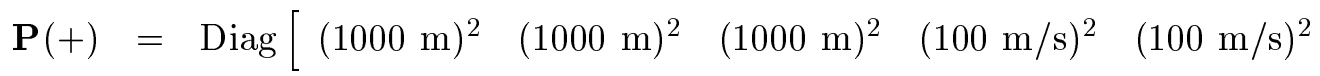

$$
\begin{aligned}
& \left.(100 \mathrm{~m} / \mathrm{s})^{2} \quad\left(10 \mathrm{~m} / \mathrm{s}^{2}\right)^{2} \quad\left(10 \mathrm{~m} / \mathrm{s}^{2}\right)^{2} \quad\left(10 \mathrm{~m} / \mathrm{s}^{2}\right)^{2}\right] \\
& \hat{\mathbf{x}}(+)=\text { true missile states }-\left[\begin{array}{c}
2000 \mathrm{~m} \\
2000 \mathrm{~m} \\
2000 \mathrm{~m} \\
200 \mathrm{~m} / \mathrm{s} \\
200 \mathrm{~m} / \mathrm{s} \\
200 \mathrm{~m} / \mathrm{s} \\
20 \mathrm{~m} / \mathrm{s}^{2} \\
20 \mathrm{~m} / \mathrm{s}^{2} \\
20 \mathrm{~m} / \mathrm{s}^{2}
\end{array}\right] .
\end{aligned}
$$

The space IR measurements are available to the filter after $t=44 \mathrm{~s}$ (after the missile reaches $7-\mathrm{km}$ altitude). Solid radar measurements are available after $t=125 \mathrm{~s}$. The kill-vehicle IR 
measurements are available after $t=183 \mathrm{~s}$ (after the interceptor shroud comes off at $100-\mathrm{km}$ altitude).

Figures C.18 and C.19 show that the errors in the filter's position, velocity, and acceleration estimates are very similar to the results for the ICBM S1 case. The estimate errors are well predicted by the covariance matrix, as the errors are within the 1- $\sigma$ envelopes most of the time, indicating that the filter is tuned well. The large errors in the initial state estimates, although twice that of the initial covariance matrix values, are quickly reduced after a few measurements are processed. The staging at $t=120 \mathrm{~s}$ causes large errors in the downrange and altitude acceleration state estimates, but the filter is able to track through the event and maintain small estimation errors thereafter.

\section{C.2.4 Engagement summary}

The slower-burning ICBM L missile allowed a longer interceptor launch delay than the faster-burning solid-propellant missile (115 s compared with $59 \mathrm{~s}$ ). With the chosen interceptors $(10-\mathrm{km} / \mathrm{s}$ and $5-\mathrm{km} / \mathrm{s})$, the times between missile burnout and time of intercept are about the same for both cases, $21 \mathrm{~s}$ for the ICBM S1 and $16 \mathrm{~s}$ for the ICBM L. Using the same Kalman filter formulation and same measurement models (except for the radar errors, since tracking ranges and radar cross sections are different for the two missiles), similar missile state estimates were obtained for the ICBM L missile. The ICBM L had one staging at $t=120 \mathrm{~s}$, and the filter was able to track through the event without difficulty. Therefore, as expected, the state estimation problem is also solvable for the slower missile, based on our assumptions.

Using fixed-length polynomial filters instead of Kalman filters, estimate uncertainties very similar to the ones obtained in this Appendix were obtained in Chapter 12. Two simple maneuvers, a sudden increase or decrease in the angle of attack of the target missile by $15^{\circ}$, were assumed for the target missile to stress the kill vehicle's $\Delta V$ capabilities. Using Monte Carlo simulations, the required total $\Delta V$ was determined to be at least $2.0 \mathrm{~km} / \mathrm{s}$ to $2.5 \mathrm{~km} / \mathrm{s}$. This total $\Delta V$ required is the most significant result from the engagement simulations. 


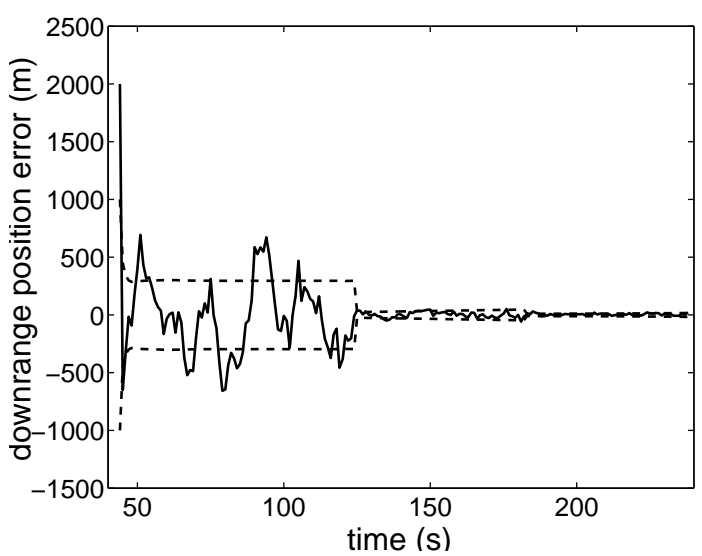

(a)

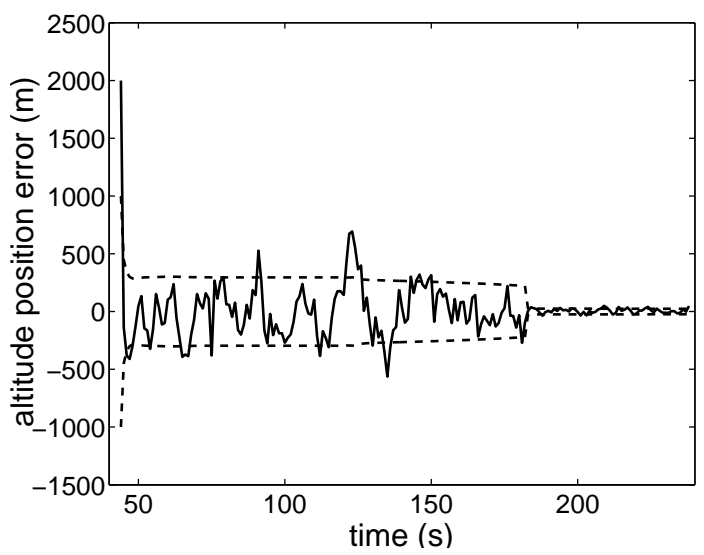

(c)

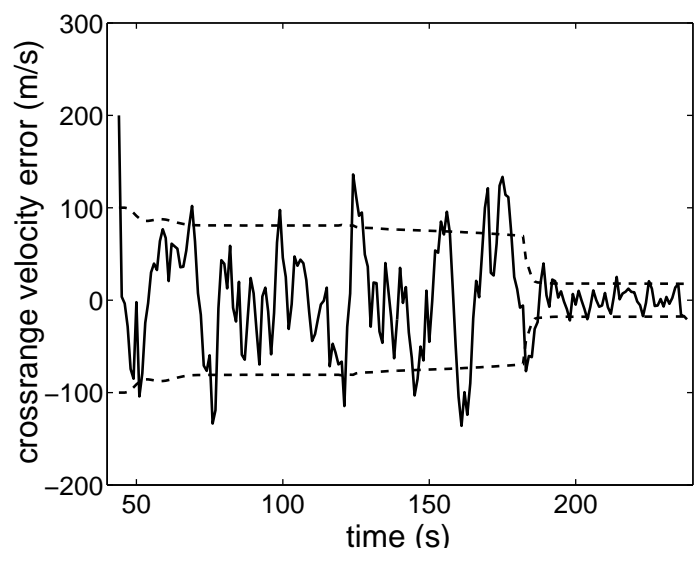

(e)

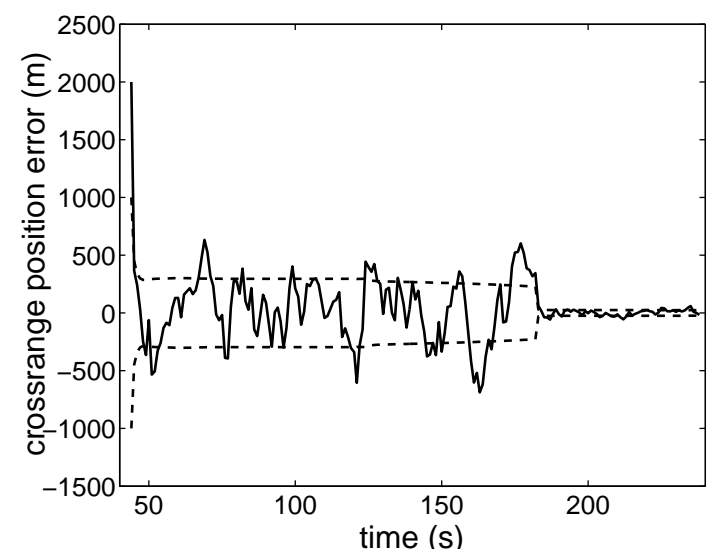

(b)

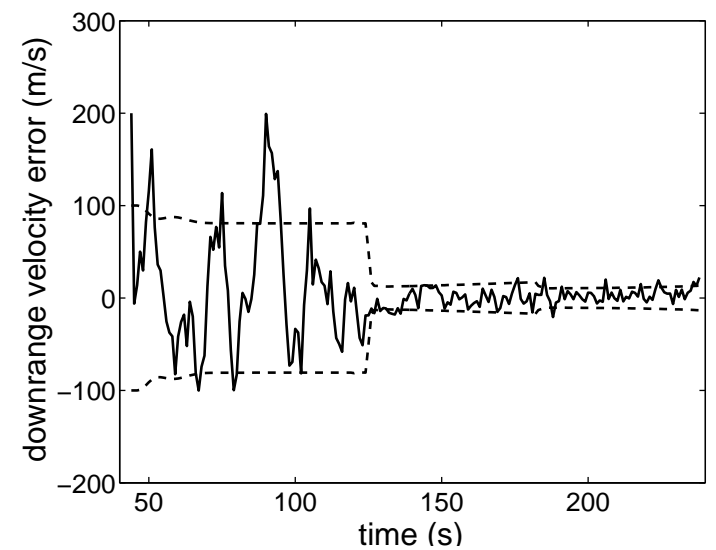

(d)

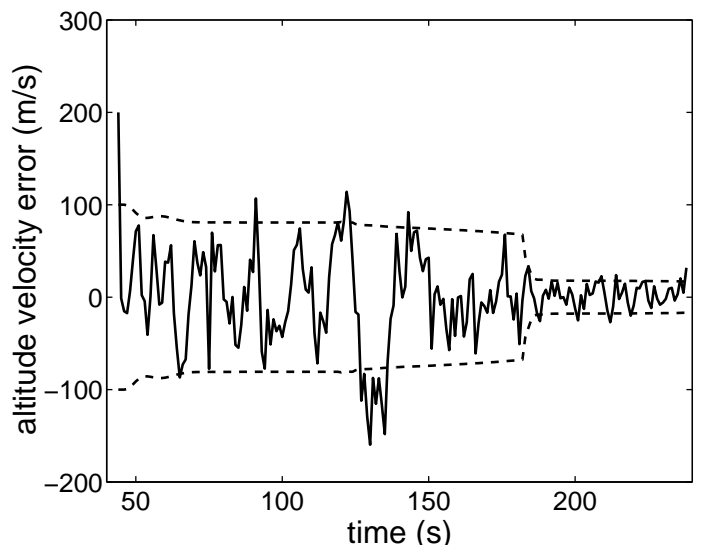

(f)

Figure C.18. (a), (b), (c) Downrange, crossrange, and altitude position errors and filter's estimates of 1- $\sigma$ values. (d), (e), (f) Downrange, crossrange, and altitude velocity errors and filter's estimates of $1-\sigma$ values. 


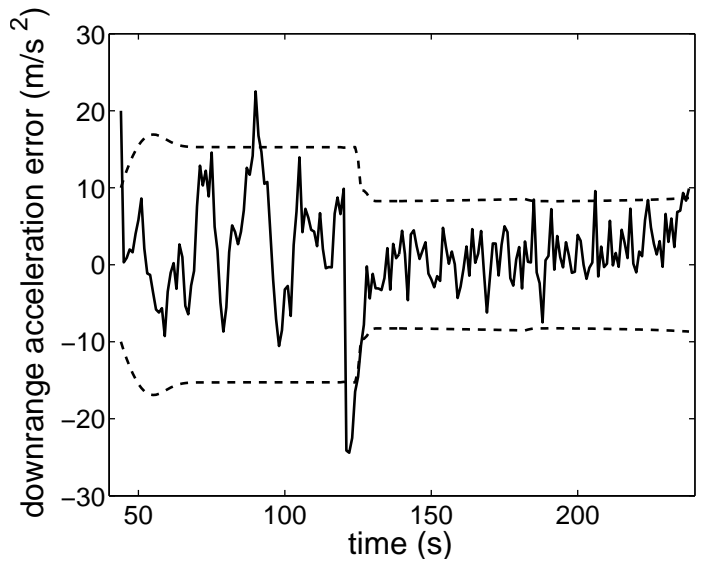

(a)

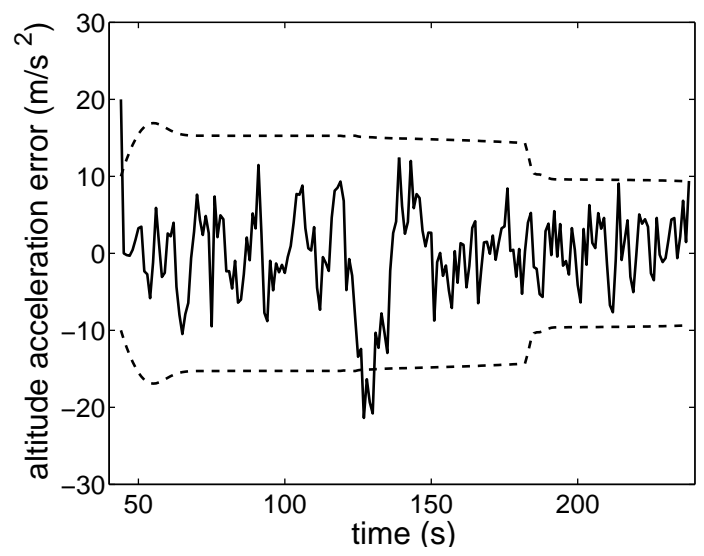

(c)

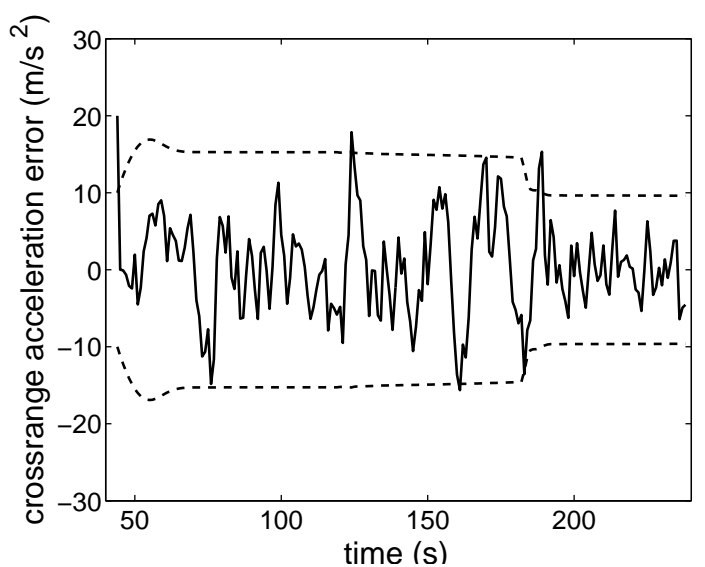

(b)

Figure C.19. (a), (b), (c) Downrange, crossrange, and altitude acceleration errors and filter's estimates of $1-\sigma$ values. 


\section{References for Appendix C}

[250] Arthur Gelb, Applied Optimal Estimation. (The M.I.T. Press), 1974.

[251] Mohinder S. Grewal and Angus P. Andrews, "Kalman Filtering - Theory and Practice." Prentice Hall Information and System Sciences Series, 1993. 



\section{Appendix D}

\section{Beam Propagation and Corrections}

\section{Contents}

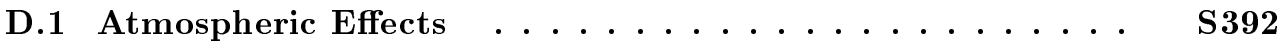

D.2 Characterization of $C_{n}^{2} \ldots \ldots \ldots \ldots$ S394

D.3 Characterizing the Wavefront Aberrations . . . . . . S S396

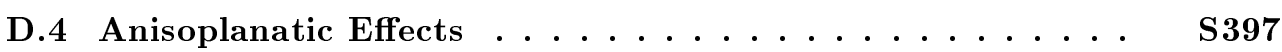

D.4.1 Angle offset . . . . . . . . . . . . . . . . S400

D.4.2 Feedback bandwidth and ABL and target motion . . . . . . S401

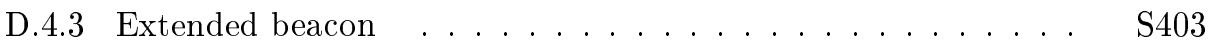

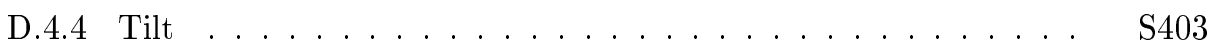

D.4.5 Time delay . . . . . . . . . . . . . . . . . S404

D.5 Strehl Ratio Details . . . . . . . . . . . . S405

D.5.1 Beam shape and partial AO compensation . . . . . . . S405

D.5.2 Combining Strehl ratios . . . . . . . . . . . . . . S407

D.5.3 Strehl ratio beyond the Maréchal limit . . . . . . . . S408

D.6 Minor Issues . . . . . . . . . . . . . . . . . S408

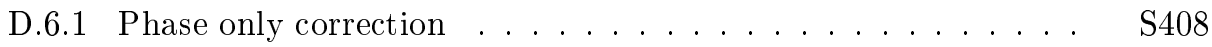

D.6.2 Thermal blooming . . . . . . . . . . . . . . S408

D.6.3 Branch points . . . . . . . . . . . . . . . . S410

D.6.4 Chromatic anisoplanatism . . . . . . . . . . . S411

D.6.5 Ionization . . . . . . . . . . . . . . . . S411

D.6.6 Aerosols . . . . . . . . . . . . . . . . . . S411

This appendix provides details for many topics touched on in the body of Part D of this report. It assumes knowledge of Chapter 19 on beam propagation as an introduction.

Sections D.1 and D.2 describe the optical properties of a turbulent atmosphere. Section D.3 defines a standard decomposition of optical aberrations in terms of Zernike modes. An extensive development of anisoplanatic effects appears in Section D.4. These results are used heavily in the analysis of Chapter 21 on engagements.

Throughout the analysis, it is necessary to combine various sources of degradation of beam intensity on the target caused by optical distortions. Section D.5 discusses this issue.

Finally, a number of minor issues are discussed in Section D.6. A list of symbols used in this appendix can be found on page S412. 


\section{D.1 Atmospheric Effects}

The variation of the index of refraction is essentially a statistical problem. Kolmogorov [252] developed a model of the atmosphere starting with a structure function $\mathcal{D}_{n}(\mathbf{z}, \mathbf{r})$,

$$
\mathcal{D}_{n}(\mathbf{z}, \mathbf{r})=<[n(\mathbf{z}+\mathbf{r})-n(\mathbf{z})]^{2}>.
$$

The structure function measures how rapidly the index of refraction $n$ varies with $\mathbf{r}$ about a point $\mathbf{z}$. The direction of propagation is along $\mathbf{z}$. The brackets indicate an ensemble average. If the atmosphere is locally homogeneous and isotropic, then $\mathcal{D}_{n}$ will depend only on the magnitudes $z=|\mathbf{z}|$ and $r=|\mathbf{r}|$,

$$
\mathcal{D}_{n}(z, r)=C_{n}^{2}(z) r^{2 / 3} .
$$

The $r^{2 / 3}$ dependence arises essentially for dimensional reasons [253, p. 21], and $C_{n}^{2}(z)$ is a local measure of the turbulence itself. Values of $C_{n}^{2}$ vary with time and vary greatly for different altitudes and atmospheric conditions.

The ABL flies at an altitude of $\geq 12 \mathrm{~km}$, above the worst of the turbulence and above most cloud cover. Characteristically, $C_{n}^{2} \approx 5 \times 10^{-18} \mathrm{~m}^{-2 / 3}$ at an altitude of $12 \mathrm{~km}$, but it can vary by an order of magnitude above or below this value within small changes of altitude [254].

Almost all the work in adaptive optics (AO) is done in the Rytov approximation [253, p. 30], in which the propagating electric field $\mathbf{E}(\mathbf{r}, t)$ is expressed as

$$
\mathbf{E}(\mathbf{r}, t)=\mathbf{E}_{0}(\mathbf{r}, t) \exp (\Phi(\mathbf{r})),
$$

where $\mathbf{E}_{\mathbf{0}}$ is the unperturbed electric field in a uniform medium. The propagation equations are satisfied by a scalar $\Phi$, and the equations are solved by a perturbation calculation in terms of the small variation $\delta n$. The Rytov approximation uses only the first order,

$$
\Phi_{1}=\chi+i \varphi .
$$

The variable $\chi$ is the $\log$-amplitude variation, and $\varphi$ is the phase variation from the unperturbed field. Many of the results are stated in terms of variances of these quantities, either with or without AO corrections. In the discussion, integrals will be made along the path of propagation, taken along the $z$ direction over a slant range $L$. The Rytov approximation should be good provided

$$
\begin{gathered}
k^{2} \int_{0}^{L} C_{n}^{2}(z) z^{5 / 6} d z<1 \\
k=\frac{2 \pi}{\lambda},
\end{gathered}
$$

although in some cases the range of validity is greater.

A result of this statistical model is that the characteristic size of the beam is $r_{0}$, the Fried parameter [255], [253, p. 61], ${ }^{1}$ which is a measure of the global turbulence, being integrated along the entire path of propagation.

$$
r_{0}=\left[0.423 k^{2} \int_{0}^{L} C_{n}^{2}(z)\left(1-\frac{z}{L}\right)^{5 / 3} d z\right]^{-3 / 5} .
$$

\footnotetext{
${ }^{1}$ The form used by Sasiela assumes a target at infinity.
} 
For a low-altitude engagement, $r_{0}$ is about $0.26 \mathrm{~m}$, which is much smaller than $D$. For highaltitude engagements $r_{0}$ may be as large as $0.8 \mathrm{~m}$, still smaller than $D$. This expression, along with all future discussion, unless otherwise noted, assumes that $\mathcal{D}_{n}$ is in the "inertial region," $l_{0} \leq r \leq L_{0}$, where $l_{0}$ is the "inner scale," and $L_{0}$ is the "outer scale." The value of $l_{0}$ is of the order of $1 \mathrm{~mm}$ near the ground and $10 \mathrm{~mm}$ at the tropopause [256, p. 48], [257, p. 81]. The value of $L_{0}$ is somewhat controversial; [256, p. 49] states that it is of the order of $0.2 \times$ the height above the ground or $100 \mathrm{~m}$, whichever is smaller. See reference [257, p. 81] for further discussion.

The rms tilt angle ${ }^{2}$ is given by [253, p. 63]

$$
\sigma_{t x, y} \approx 0.427\left(\frac{D}{r_{0}}\right)^{5 / 6} \frac{\lambda}{D} \text { (each axis) }
$$

For most engagements, the rms tilt angle is substantially larger than the diffraction limit, for which the rms of the core is $\approx 0.45 \lambda / D$. For large $D$, the phase variance with piston removed (see subsequent discussion in Section D.3) is [253, p. 65]

$$
\sigma_{\varphi c p r}^{2}=1.033\left(\frac{D}{r_{0}}\right)^{5 / 3}
$$

Outer scale effects reduce this value somewhat; see [253, p. 114]. The phase variance for tilt only (both axes) is [253, p. 64]

$$
\sigma_{\varphi c t}^{2}=0.899\left(\frac{D}{r_{0}}\right)^{5 / 3}
$$

Note that $\sigma_{\varphi}^{2}$ with both piston and tilt removed,

$$
\sigma_{\varphi c p t r}^{2}=0.134\left(\frac{D}{r_{0}}\right)^{5 / 3}
$$

is only about 13 percent of the piston-removed variance, so tilt is the largest contribution.

One of the most commonly used parameters to characterize the global turbulence is the Rytov variance, which is the variance of the amplitude of the electric field $(\chi)$ in the limit of an infinite aperture,

$$
\begin{aligned}
\sigma_{R}^{2} & =\sigma_{\chi \infty}^{2} \\
& =0.5631 k^{7 / 6} \times \int_{0}^{L} C_{n}^{2}(z)\left[z\left(1-\frac{z}{L}\right)\right]^{5 / 6} d z .
\end{aligned}
$$

This expression is quite similar to the left side of Eq. D.5. The validity of the Rytov approximation is essentially the same as requiring $\sigma_{R}^{2}<0.5 \mathrm{np}^{2} ;^{3}$ the $\mathrm{ABL}$ is designed to work in this region.

For large phase variances, e.g. before the application of AO, the Strehl ratio is given by an asymptotic series [253, p. 164]

$$
S_{R} \approx\left(\frac{r_{0}}{D}\right)^{2}-0.6159\left(\frac{r_{0}}{D}\right)^{3}+0.05\left(\frac{r_{0}}{D}\right)^{5}+0.00661\left(\frac{r_{0}}{D}\right)^{7} \ldots\left(r_{0} / D<1\right) .
$$

\footnotetext{
${ }^{2}$ The singularity for $D \rightarrow 0$ comes about from neglecting the inner scale. This not a concern here. See $[253$, p. 118] for a discussion.

${ }^{3}$ The Rytov variance $\sigma_{R}^{2}$ is dimensionless, but sometimes expressed as having units $\mathrm{np}^{2}$ to emphasize the use of a natural log rather than a common log.
} 


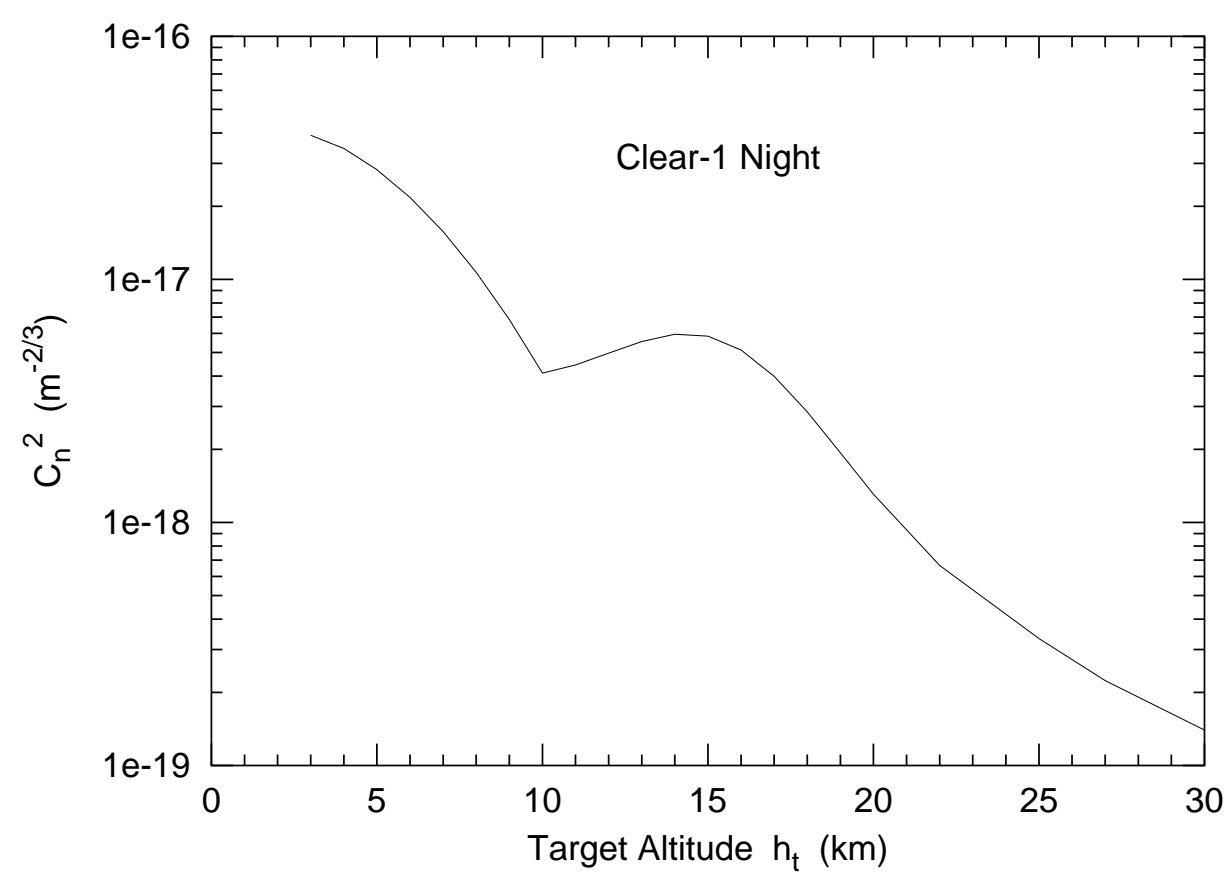

Figure D.1. $C_{n}^{2}$ vs. target altitude $h_{t}$ in Clear-1 Night model.

This expression is applicable to engagements of interest and sets a lower bound for the atmospheric part of the Strehl ratio without AO. This limit ranges from 0.02 to 0.2 , depending on the engagement geometry. For small variances, e.g. in low turbulence or after application of AO, the Strehl ratio is given approximately in the extended Maréchal limit by $[253$, p. 46]

$$
S_{R} \approx \exp \left(-\sigma_{R}^{2}-\sigma_{\varphi}^{2}\right)
$$

After AO, Eq. D.14 applies, where $\sigma_{\varphi}^{2}$ is appropriate for the degree of correction achieved.

\section{D.2 Characterization of $C_{n}^{2}$}

A standard model of turbulence is called "Clear-1" [258]. The ABL specification is that it must function for turbulence as intense as twice this model. The model assumes that $C_{n}^{2}$ depends only on altitude, and in particular that it is independent of time and geographical location. Figure D.1 shows $C_{n}^{2}$ vs. altitude.

Because turbulence decreases at higher altitudes, the effect of turbulence can be reduced by making the engagement at higher altitudes. Figure D.2 shows $\sigma_{R}^{2}$ vs. engagement altitude for five different target ranges, $100 \mathrm{~km}$ to $500 \mathrm{~km}$. The line at $\sigma_{R}^{2}=0.5 \mathrm{np}^{2}$ is the maximum value contemplated for $\mathrm{ABL}$ operations. Note, for example, that for $L=300 \mathrm{~km}$, the engagement altitude needs to be above about $30 \mathrm{~km}$, and for $L=500 \mathrm{~km}$, at an altitude above $50 \mathrm{~km}$.

Figure D.3 shows $r_{0}$ vs. engagement altitude for five different target ranges, 100 to $500 \mathrm{~km}$. The line at $r_{0}=0.26 \mathrm{~m}$ is the minimum value contemplated.

Figure D.4 shows $\theta_{t x y}$ vs. engagement altitude for five different target ranges, 100 to $500 \mathrm{~km}$. The line at $1.8 \lambda / D$ is the upper limit for engagements, and the line at $0.45 \lambda / D$ represents the jitter, which is about the same as diffraction-limited spreading. 


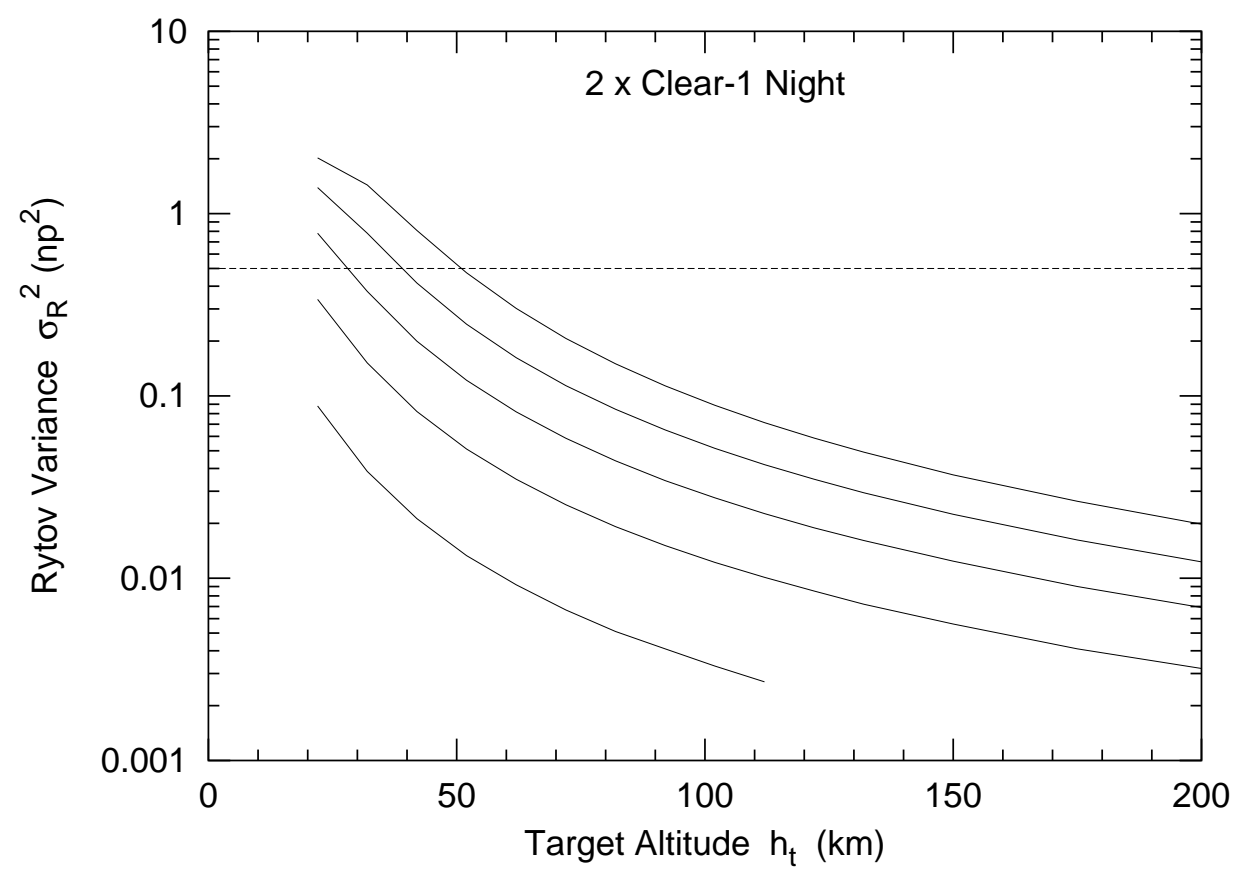

Figure D.2. Rytov variance $\sigma_{R}^{2}$ vs. target altitude $h_{t}$ for ranges, from bottom to top, $L=100,200$, 300,400 , and $500 \mathrm{~km}$. The dotted horizontal line represents the maximum value contemplated for the ABL operations.

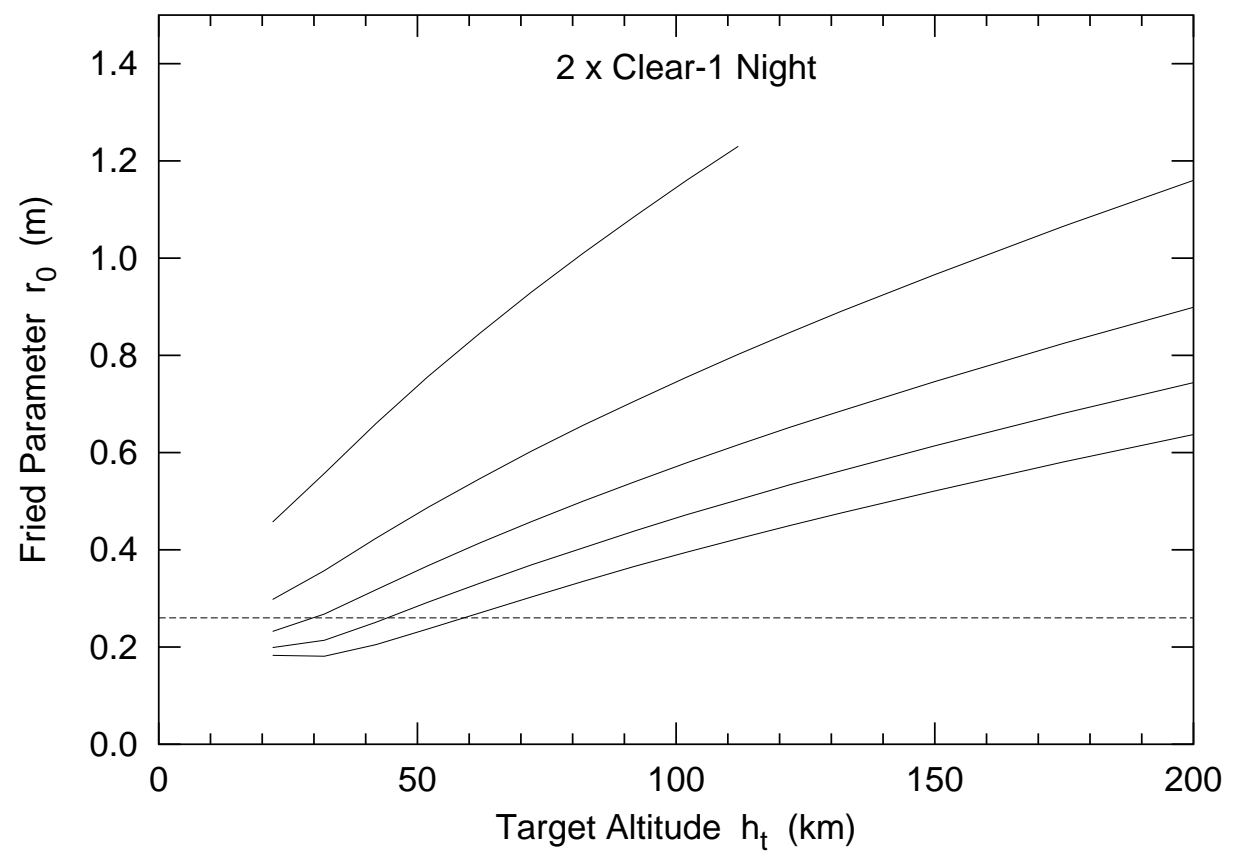

Figure D.3. Fried parameter $r_{0}$ vs. target altitude $h_{t}$ for ranges, from top to bottom, $L=100$, $200,300,400$, and $500 \mathrm{~km}$. The dotted horizontal line is the minimum value considered for ABL operations. 


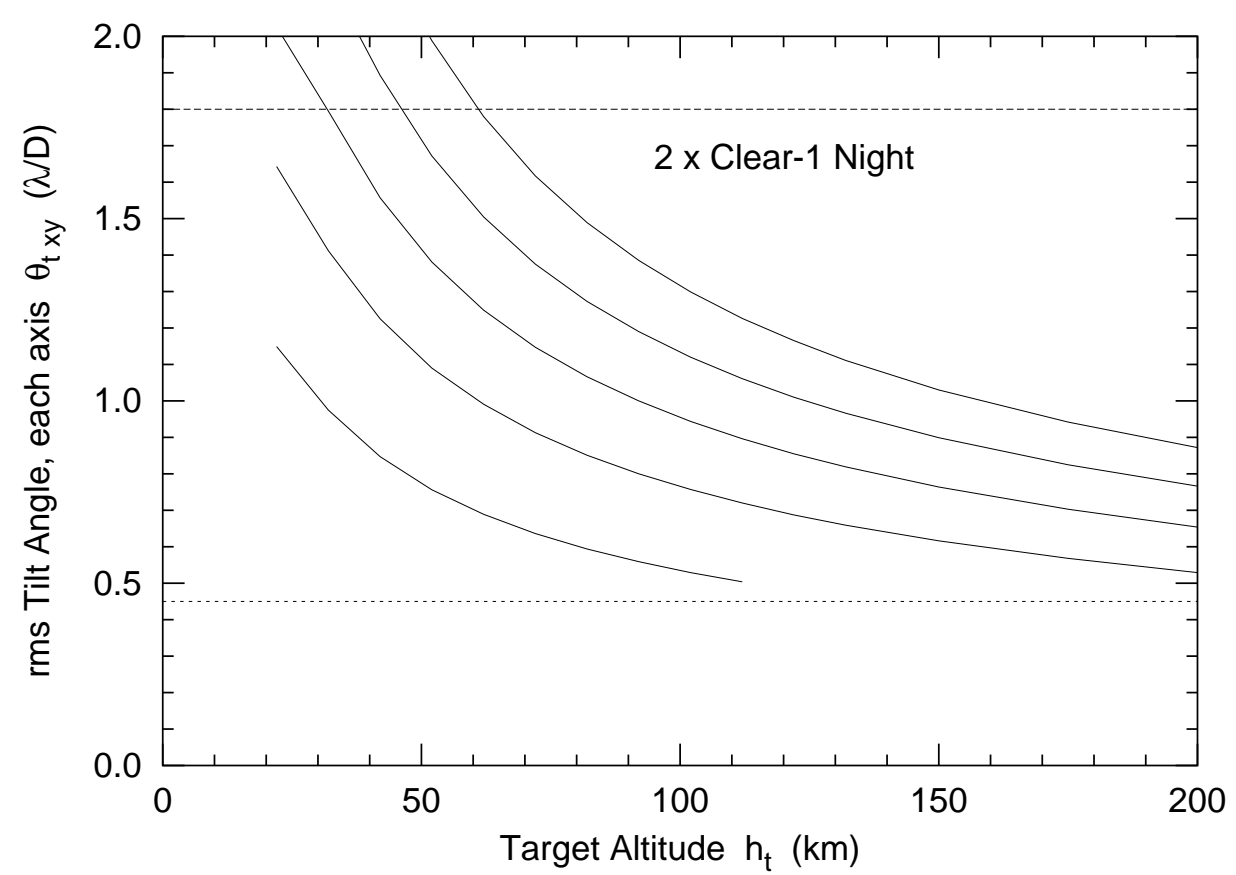

Figure D.4. Root mean square tilt angle $\theta_{t} x y$ vs. target altitude $h_{t}$ for ranges, from bottom to top, $L=100,200,300,400$, and $500 \mathrm{~km}$. The upper dotted horizontal line represents the upper limit for engagements. The lower dotted horizontal line represents the rms size of a diffraction limited beam.

Another useful measure of the turbulence is the isoplanatic angle $\theta_{0}$; (see Section D.4 for discussion). Figure D.5 shows $\theta_{0}$ vs. engagement altitude for five different target ranges, 100 to $500 \mathrm{~km}$. The line at $\theta_{0}=0.65$ is the minimum value contemplated for ABL operations. Note, for example, that for $L=300 \mathrm{~km}$, the engagement altitude must to be above about $30 \mathrm{~km}$, and for $L=500 \mathrm{~km}$, at an altitude above $50 \mathrm{~km}$.

\section{D.3 Characterizing the Wavefront Aberrations}

Conventional AO usually involves correcting the phase of the wavefront at the entrance to an astronomical telescope or the exit of the ABL mirror (or equivalent). To do this, it is convenient to decompose the phase as a function of position into modes. One commonly used decomposition is in terms of Zernike functions, $Z_{j}(r, \theta)[259,260,253,257]$. These are orthonormal functions in normalized radius $r(0 \leq r \leq 1)$ and angle $\theta$ at the pupil of the laser/telescope. They are functions of indices radial order $n$, and azimuthal order $m$, and are commonly referred to in terms of a single sequential index $j$. They satisfy the following orthogonality condition.

$$
\int_{0}^{2 \pi} \int_{0}^{1} Z_{j}(r, \theta) Z_{k}(r, \theta) r d r d \theta=\delta_{j k}
$$

The first few functions with descriptions are shown in Table D.1.

In astronomy, the biggest atmospheric effect is wavefront tilt, which causes the image to move; see Eq. D.7. For astronomy, good seeing will have $r_{0} \approx 0.1 \mathrm{~m}$ at $\lambda=0.5 \mu \mathrm{m}$, or $r_{0} \approx$ $0.42 \mathrm{~m}$ at $\lambda=1.65 \mu \mathrm{m}$. Thus, for the Keck telescope $(D=10 \mathrm{~m} \lambda=1.65 \mu \mathrm{m}) \sigma_{t} \approx 6 \lambda / D$. For the ABL at a low-altitude engagement, $r_{0} \approx 0.26 \mathrm{~m}$ and $\sigma_{t}=1.8 \lambda / D$. Correcting for 


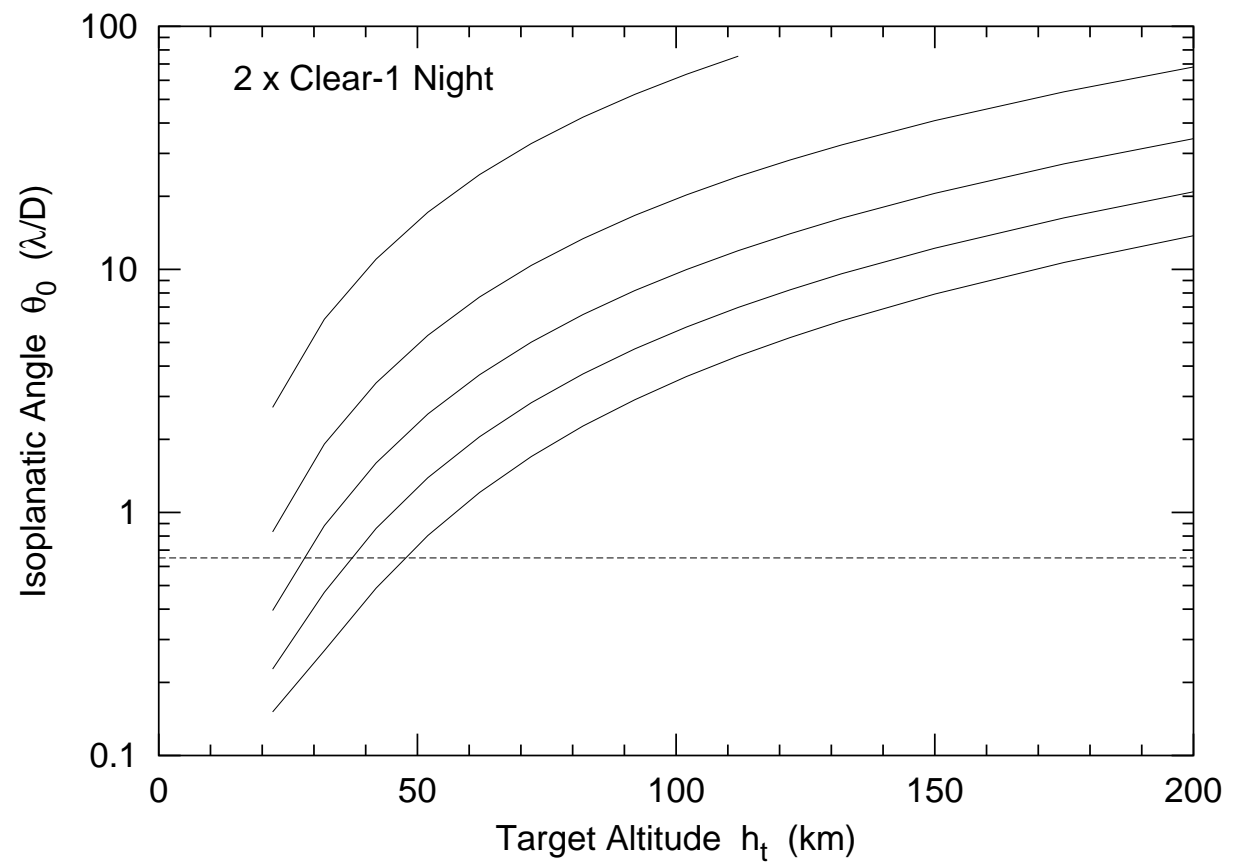

Figure D.5. Isoplanatic angle $\theta_{0}$ vs. target altitude $h_{t}$ for ranges, from top to bottom, $L=100,200$, 300,400 , and $500 \mathrm{~km}$. The dotted horizontal line represents the minimum value contemplated for the ABL operations.

a tilt is the easiest correction, and it may be done with a tilting flat mirror before using a deformable mirror for the higher-order modes.

\section{D.4 Anisoplanatic Effects}

There are limits on how well the AO can correct for phase distortions. One such limit is when the path traversed by the beacon beam does not exactly coincide with the return path of the high-energy laser (HEL) beam. The starting point for computing the phase variance is this relation $([261]$ and $[253$, p. 72,130$])$ :

$$
\begin{aligned}
\sigma_{\varphi c}^{2} & =0.2073 k^{2} \int_{0}^{L} d z C_{n}^{2}(z) \int \frac{d^{2} \kappa}{\kappa^{11 / 3}} \times F_{Z}(x) \times 2\left[1-\cos \left(\kappa d_{z} \cos \phi\right)\right] \\
x & =\kappa D_{z} / 2
\end{aligned}
$$

where $\kappa$ is the two-dimensional transverse wave number describing the spatial frequency of the turbulence for which a Kolmogorov spectrum $\kappa^{-11 / 3}$ is used, $\phi$ is the azimuthal coordinate for the wave number, $d_{z}(z)$ is the distance between the paths, $D_{z}(z)$ is the beam diameter, with $D_{z}=D(1-z / L)$ for a beam focused ${ }^{4}$ at $z=L$, and $F_{Z}(x)$ is a Zernike moment extraction filter. The integral on $\phi$ may be done using

$$
J_{n}(z)=\frac{1}{2 \pi} \int_{0}^{2 \pi} d \phi \cos (n \phi) e^{i z \cos \phi}
$$

\footnotetext{
${ }^{4}$ Note that this expression is only an approximation, since in practice the beam cannot be focused to a point, for several reasons. Because of this, the actual $z$-dependence of $D_{z}$ is complex, particularly near the target. Fortunately, for the present work the "focus" is in a region of low turbulence, and the small value of $C_{n}^{2}$ will reduce the influence of the non-ideal beam spreading.
} 
Table D.1. Zernike Functions $Z_{j}(r, \theta)$

\begin{tabular}{cccll}
\hline \hline$j$ & $n$ & $m$ & $\sqrt{\pi} \times$ Function & Description \\
\hline 1 & 0 & 0 & 1 & Piston \\
2 & 1 & 1 & $2 r \cos \theta$ & Tip \\
3 & 1 & 1 & $2 r \sin \theta$ & Tilt \\
4 & 2 & 0 & $\sqrt{3}\left(2 r^{2}-1\right)$ & Defocus \\
5 & 2 & 2 & $\sqrt{6} r^{2} \sin 2 \theta$ & Astigmatism \\
6 & 2 & 2 & $\sqrt{6} r^{2} \cos 2 \theta$ & Astigmatism \\
\hline & & & \\
& & & 1 \\
& & $=$ & \\
&
\end{tabular}

where $J_{n}(z)$ are the ordinary Bessel functions. Then the generic form becomes

$$
\begin{aligned}
\sigma_{\varphi c}^{2} & =2.605 k^{2} \int_{0}^{L} d z C_{n}^{2}(z) \times \int_{0}^{\infty} \frac{d \kappa}{\kappa^{8 / 3}} F_{Z}(x)\left[1-J_{0}\left(\kappa d_{z}\right)\right] \\
& =2.914 k^{2} \int_{0}^{L} d z C_{n}^{2}(z) d_{z}^{5 / 3} A_{F}(a) \\
A_{F}(a) & =\frac{2.605}{2.914} \int_{0}^{\infty} \frac{d y}{y^{8 / 3}} F_{Z}(a y)\left[1-J_{0}(y)\right] \\
y & =\kappa d_{z} \\
a & =D_{z} /\left(2 d_{z}\right) .
\end{aligned}
$$

The choice of the factor $2.605 / 2.914$ is such that $A_{F}(a)$ becomes 1 in the case $F_{Z}(x)=1$ (i.e., independent of $a$ ), corresponding to accepting all Zernike modes.

The general form of a filter to select all Zernike modes of order $n$, i.e., the sum on $m$, is

$$
F_{n}(x)=(n+1)^{2}\left[\frac{2 J_{n+1}(x)}{x}\right]^{2} .
$$

Special cases are [253, p. 49]

$$
\begin{aligned}
F_{\text {piston }} & =\left[\frac{2 J_{1}(x)}{x}\right]^{2} \\
F_{\text {pr }} & =1-F_{\text {piston }} \\
F_{\text {tilt }} & =\left[\frac{4 J_{2}(x)}{x}\right]^{2},
\end{aligned}
$$

where $F_{p r}$ is Piston Removed, which is a case often of interest, and $F_{\text {tilt }}$ refers to both axes, not just one. The Piston Removed filter $F_{p r}$ has the property that for the purpose of integration $F_{p r} \rightarrow 1$ when $D_{z} / d_{z} \rightarrow \infty$, meaning that the infinite aperture limit corresponds to taking all modes; alternatively, the piston component vanishes in this limit. ${ }^{5}$ Figure D.6 shows these filter functions. Note the different wave number sensitivity for the various

\footnotetext{
${ }^{5}$ Note: The vanishing of the piston component applies in this discussion of anisoplanatism because the term $\left[1-J_{0}\left(\kappa d_{z}\right)\right]$ suppresses the low wave number contributions. It does not apply to the case of uncorrected phase variance, where the low wave number dominance makes the piston component diverge because of the $\kappa^{-8 / 3}$ from the spectrum.
} 


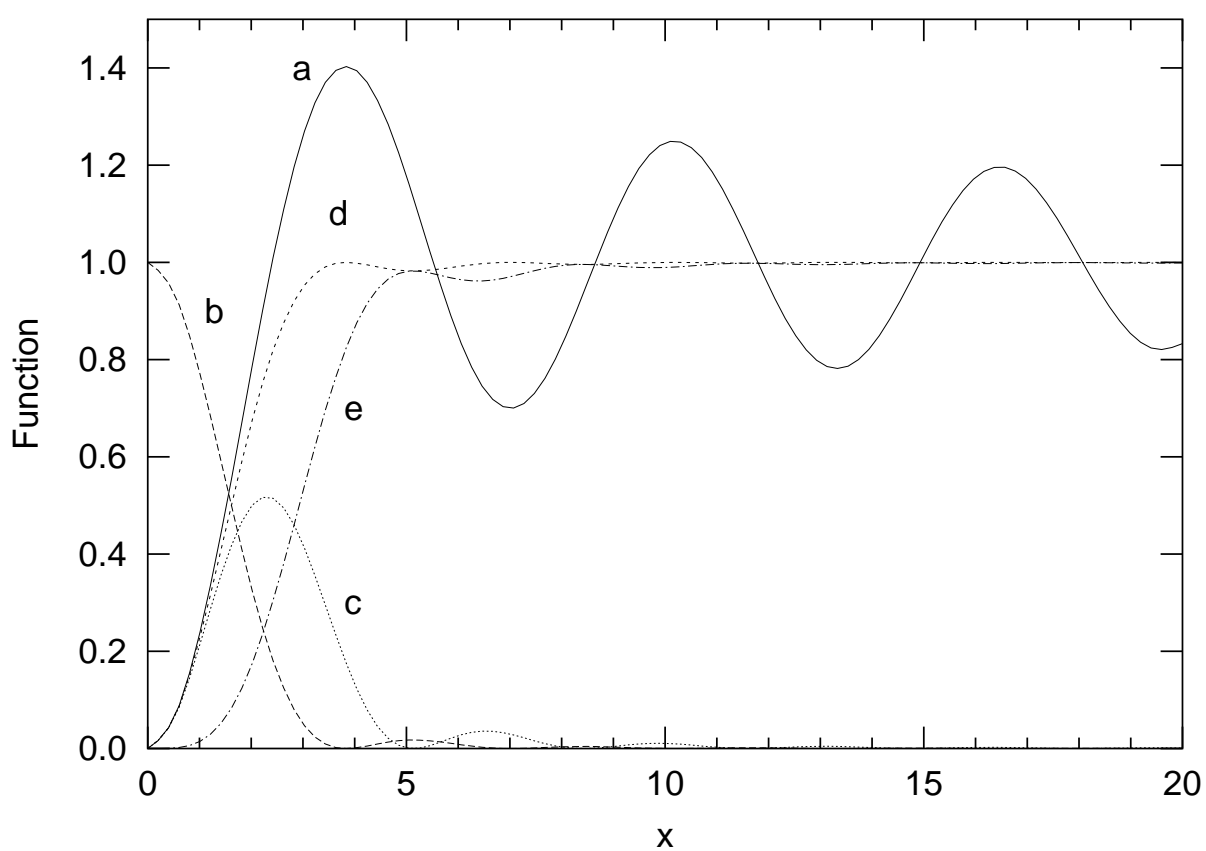

Figure D.6. Filter functions, (a) Anisoplanatism $\left[1-J_{0}(x)\right]$ (b) Piston, (c) Tilt, (d) Piston Removed, and (e) Higher Order (beyond Tilt).

filters. "Piston" is a low pass filter, passing only the lowest spatial frequencies, starting at 0 . "Tilt" is a bandpass filter having upper and lower cutoffs. Higher-order modes move the band to higher and higher frequencies. "Piston Removed" is a high pass filter; "Higher Order" is a high-pass filter that cuts off at a higher frequency than "Piston Removed." See lines (d) and (e) of Fig. D.6.

Figure D.7 shows in dimensionless form the equivalent of $d_{z}^{5 / 3} A_{F}(a)$ vs. $d_{z}$ in Eq. D.20 for the phase variance contributions of Zernike modes (a) Piston Removed, (b) Tilt, and (c) Higher Order $(n>1)$. Note in particular the different dependence on $d_{z}$. For small $d_{z}$, the tilt contribution is proportional to $d_{z}^{2}$, while the others are proportional to $d_{z}^{5 / 3}$. See Section D.4.4 for further discussion of this dependence.

The infinite aperture limit leads to simple results, because $A_{F}=1$, particularly when $d_{z}(z)$ depends on a multiplicative number characterizing the effect. For example, an angular anisoplanatism has $d_{z}=\theta z$, and $\theta$ is the multiplicative number. Much of the literature concentrates on expressions in the infinite aperture limit because of the simplicity. The reader is cautioned that although the result is simple, it is not necessarily a very accurate representation of the physically interesting piston removed mode, unless the anisoplanatism is very small, often not the case for ABL applications. The simple form overestimates the true piston removed phase variance by roughly a factor of 2 in many cases of interest. Taken to extremes, the simple forms diverge for large anisoplanatism, but the correct expression will saturate at twice the uncompensated piston removed phase variance, Eq. D.8, since the AO result becomes the difference of two uncorrelated variances. One can see this mathematically from Eq. D.16. It is identical to the equation used (not shown) to derive Eq. D.8 for the uncorrected, piston removed phase variance, if the factor $2\left[1-\cos \left(\kappa d_{z} \cos \phi\right)\right]$ is replaced by 1 . When $d_{z}$ becomes large, the cosine term oscillates to zero, leaving just 2 . 


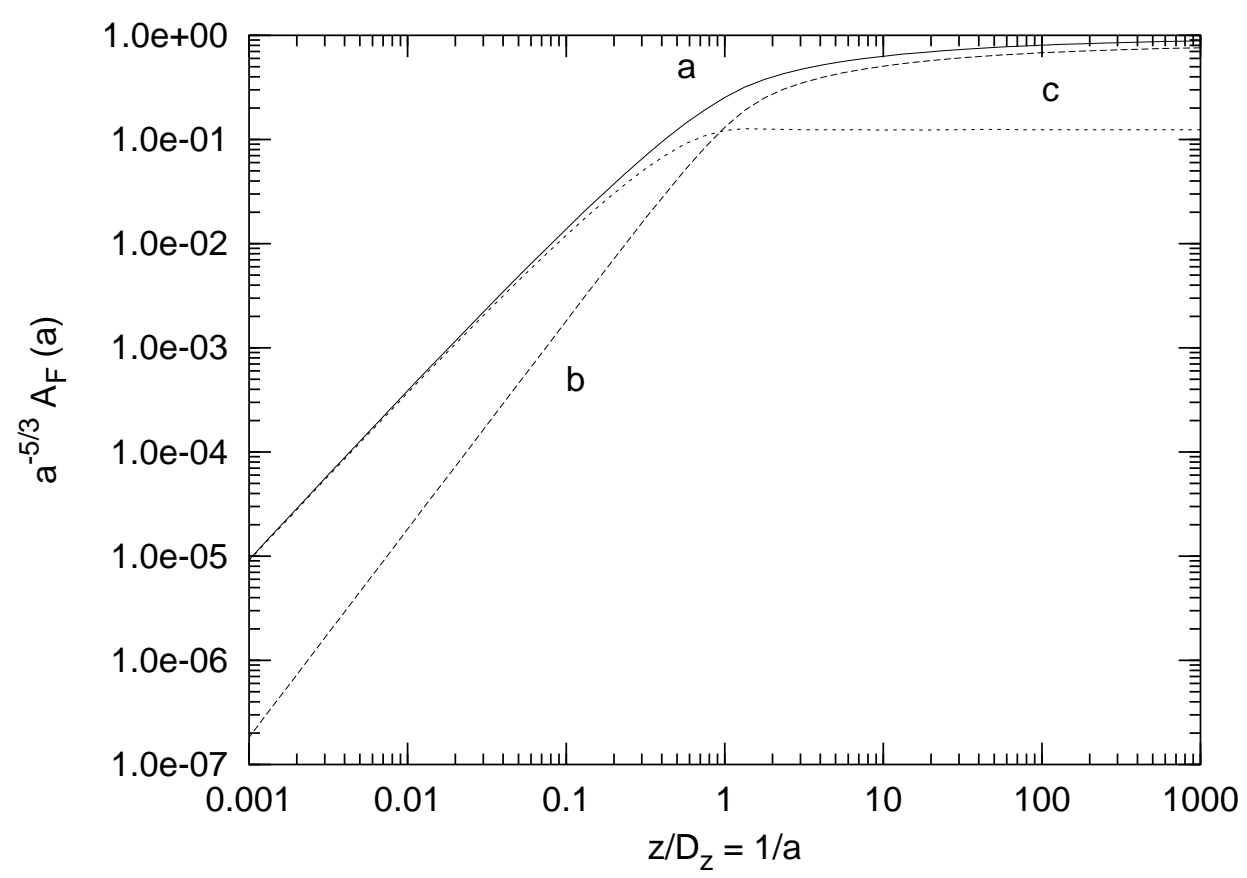

Figure D.7. Contributions to the anisoplanatic integrand of Eq. D.21 for (a) Piston Removed, (b) Tilt, and (c) Higher Order (beyond Tilt).

For any given Zernike mode, the AO corrected phase variance will saturate at twice the uncorrected variance for the same mode.

\section{D.4.1 Angle offset}

The phase variance for an angular offset after AO correction is given by Eq. D.20, where $d_{z}=\theta z[253$, p. $61,73,174]$,

$$
\begin{aligned}
\sigma_{\varphi c \theta}^{2} & =2.914 k^{2} \theta^{5 / 3} \int_{0}^{L} C_{n}^{2}(z) z^{5 / 3} d z \\
& =\left(\frac{\theta}{\theta_{0}}\right)^{5 / 3} \\
\theta_{0}^{-5 / 3} & =2.914 k^{2} \int_{0}^{L} C_{n}^{2}(z) z^{5 / 3} d z,
\end{aligned}
$$

where $\theta_{0}$ is the isoplanatic angle.

From Eq. D.27, if the aim point is displaced by a distance $d_{s}$ along the missile away from the ideal, a phase variance [261]

$$
\sigma_{\varphi c d}^{2}=\left(\frac{d_{s}}{L \theta_{0}}\right)^{5 / 3}
$$

results.

Equations D.26 and D.27 are simple, but in practical applications for the ABL they lead to a substantial overestimate of the phase variance. They work reasonably well only for small phase variance, where the piston removed variance is about the same as the total variance; 


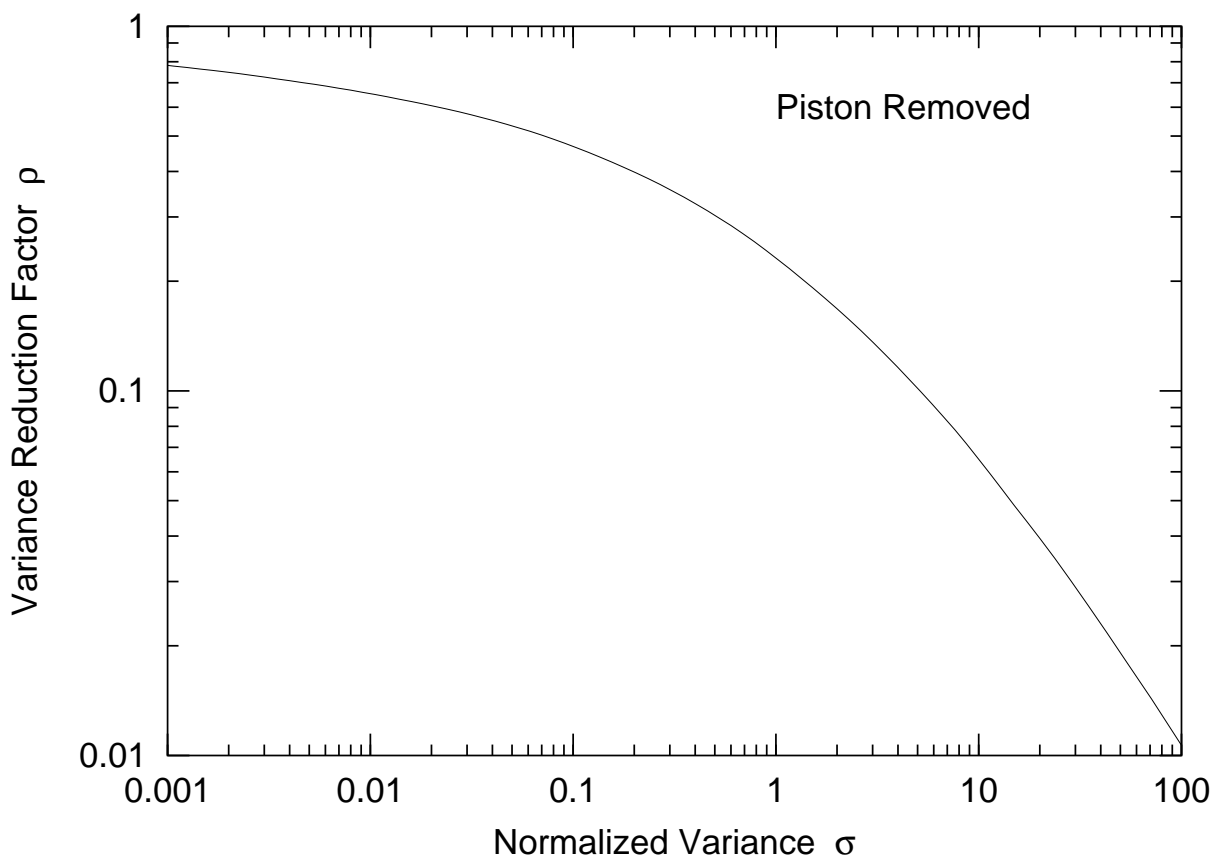

Figure D.8. Variance reduction factor $\rho$ vs. normalized variance $\sigma$ for piston removed mode; see Eqs. D.30 and D.31.

only piston removed is physically interesting. For large displacement, the variance must saturate at twice that of Eq. D.8. The expression in Eq. D.20 in principle is complicated, depending on $\theta_{0}$ and details of the turbulence profile and not having a simple scaling with angle. In practice, a reasonable approximation exists for a scaling relation of $\rho$, the ratio of the phase variance for piston removed to the phase variance for all modes (Eq. D.27), as a function of $\sigma$, the ratio of the total variance to the total uncorrected variance (Eq. D.8),

$$
\begin{aligned}
\rho & =\frac{\sigma_{\varphi c ~ p r}^{2}}{\left(\theta / \theta_{0}\right)^{5 / 3}} \\
\sigma & =\frac{\left(\theta / \theta_{0}\right)^{5 / 3}}{1.033\left(D / r_{0}\right)^{5 / 3}} .
\end{aligned}
$$

It turns out that the relationship between $\rho$ and $\sigma$ is nearly independent of the engagement parameters, as was pointed out in Refs. [261, 262], although expressed in slightly different form. Figure D.8 shows this relation. Since the $\rho-\sigma$ relationship scaling is only approximate and depends on details of the engagement $\left(h_{t}\right.$ and $\left.L\right)$ the line is an average over engagements of interest, but the differences are small $( \pm<10$ percent). Thus, the piston removed variance may be estimated using the simple equation D.27 and multiplying it by $\rho$ determined from $\sigma$. Characteristically, the piston removed variance is half or less that of the total variance.

\section{D.4.2 Feedback bandwidth and $A B L$ and target motion}

The motion of the ABL and missile through the atmosphere causes the air path through which the beams travel to change with time. This variation puts requirements on the feedback bandwidth for the AO corrections. We consider a model of an effective wind speed 


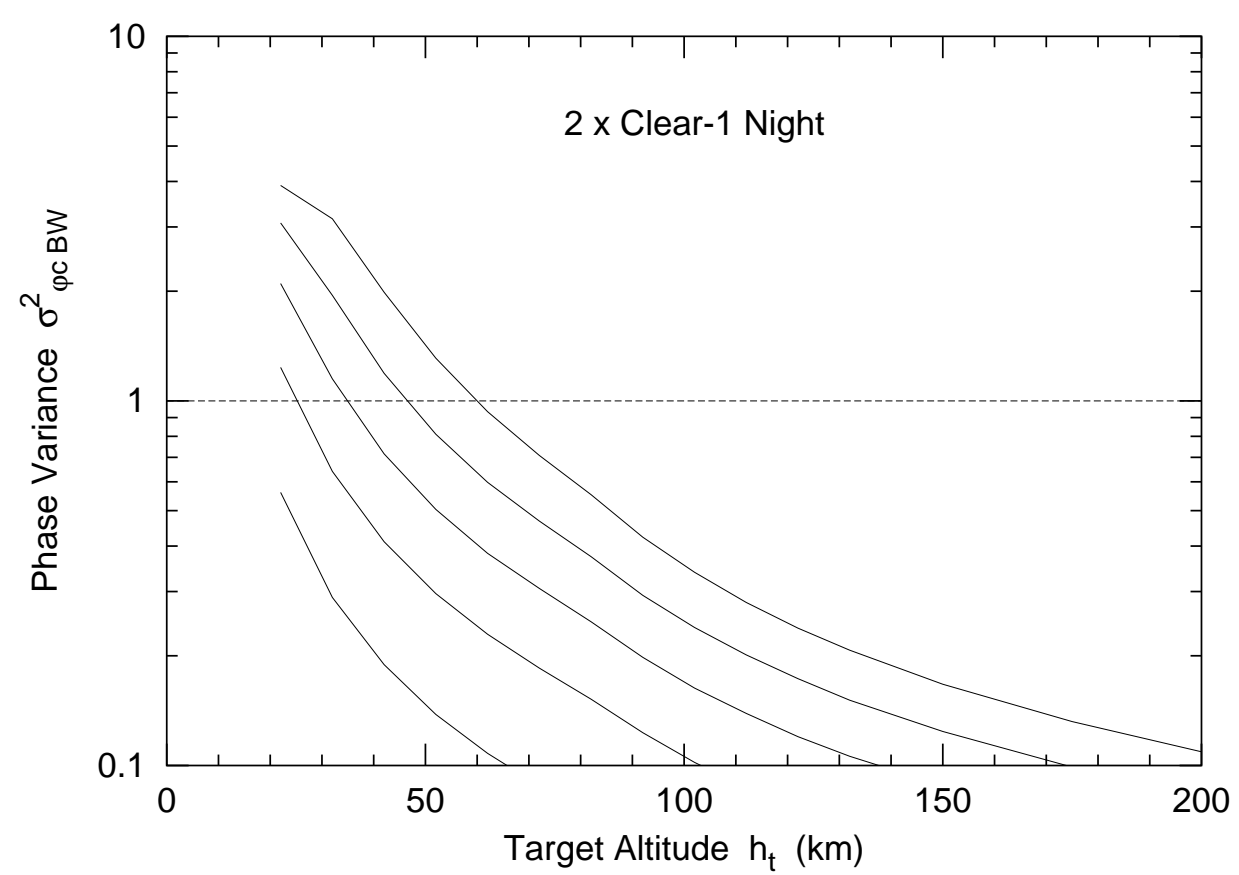

Figure D.9. $\sigma_{\varphi c B W}^{2}$ for $n_{f}=1$ vs. target altitude $h_{t}$ for ranges, from bottom to top, $L=100,200$, 300,400 , and $500 \mathrm{~km}$.

$w$, which transports the turbulent atmosphere through the image path or beam path. With respect to the ground, $w$ is linear in the propagation distance $z$ and varies as a vector from $200 \mathrm{~m} / \mathrm{s}$ horizontally at the ABL to $1.5 \mathrm{~km} / \mathrm{s}$ to $7 \mathrm{~km} / \mathrm{s}$ in the direction of missile flight at the location of the missile. This effective wind determines how rapidly the feedback must respond.

The feedback 3 - $\mathrm{dB}$ bandwidth of $f_{B}=500 \mathrm{~Hz}$ leads to a phase variance (all modes) for a bandpass filter of order $n_{f}$ [263], [253, p. 71], [262],

$$
\begin{aligned}
\sigma_{\varphi c B W}^{2} & =\left(\frac{f_{G}}{f_{B}}\right)^{5 / 3} \\
f_{G}^{3 / 5} & =K_{f} k^{2} \int_{0}^{L} C_{n}^{2} w^{5 / 3} d z \\
K_{f} & =\frac{0.051}{n_{f} \sin \left(5 \pi / 6 n_{f}\right)} \\
& =0.102 \quad\left(n_{f}=1\right) \\
& =0.0196 \quad\left(n_{f} \rightarrow \infty\right) .
\end{aligned}
$$

Figure D.9 shows the piston removed $\sigma_{\varphi c ~ B W}^{2}$ for $n_{f}=1$ as a function of altitude for various ranges. ${ }^{6}$ The piston removed variance is smaller than the result of Eq. D.32 by a factor of about 2 .

\footnotetext{
${ }^{6}$ The feedback filter used for the ABL does not appear in the literature. A value $n_{f}=2$ filter would reduce $K_{f}$ by nearly a factor of 4 , lowering $\sigma_{\varphi c B W}^{2}$ by a factor of 10 . Higher-order filters make little additional improvement.
} 


\section{D.4.3 Extended beacon}

An extended beacon leads to another form of anisoplanatism. The outgoing beacon illuminator laser (BILL) is not AO-compensated, and its spot on the target will be larger than optimum; however, the extended nature is relatively benign. The result for a uniform circular beacon [261] is the same as Eq. D.29, with $d_{s}$ replaced by $\approx 0.1 \times d_{\text {spot }}$, where $d_{\text {spot }}$ is the diameter of the beacon spot. Because the BILL is pulsed, the beam spot for each pulse will be much smaller than the time average of an uncorrected beam moving because of tilt jitter. Section D.5.1 discusses the spot for a "short exposure" and concludes that the FWHM will be, at worst, about $2 \times \lambda / D$ for a low-altitude engagement and will be about $1 \times \lambda / D$ for high-altitude engagements.

For a low-altitude engagement, $h_{t}=30 \mathrm{~km}, L=300 \mathrm{~km}$, and $\theta_{0} \approx 0.9 \lambda / D$. For the beacon $\mathrm{FWHM}=2 \times \lambda / D$, the resulting piston removed phase variance will be less than 0.07 , resulting in less than a 7 percent loss of the Strehl ratio. ${ }^{7}$ For a high altitude engagement, $h_{t}=50 \mathrm{~km}, L=500 \mathrm{~km}, \theta_{0} \approx 8 \lambda / D$, and the beacon FWHM $=1 \times \lambda / D$; the piston removed phase variance is less than $10^{-3}$, for a negligible loss of Strehl ratio.

More serious is the scintillation that may accompany such a beacon. This intensity modulation can degrade AO performance. The tilt correction along the missile is degraded by a factor of the order of 2 to 3 [264].

\section{D.4.4 Tilt}

The anisoplanatic phase variance for any Zernike mode may be computed from Eq. D.19 using the appropriate mode filter $F_{n}$ from Eq. D.22. The filter $F_{n}(x)$ selects certain ranges of frequencies $x \approx n$. Consider first the case which corresponds to $\left|\kappa d_{z}\right| \ll 1$ and $\left|D_{z} /\left(2 n d_{z}\right)\right| \gg 1,{ }^{8}$ and expand $1-J_{0}(y) \approx y^{2} / 4$. Then

$$
\begin{aligned}
I_{d} & =\frac{2.605}{2.914} \int_{0}^{\infty} \frac{d \kappa}{\kappa^{8 / 3}} \times F_{z}\left(\kappa D_{z} / 2\right)\left[1-J_{0}\left(\kappa d_{z}\right)\right] \\
& \approx \frac{2.605}{2.914} \frac{d_{z}^{2}}{4} \int_{0}^{\infty} \frac{d \kappa}{\kappa^{2 / 3}} F_{z}\left(\kappa D_{z} / 2\right) .
\end{aligned}
$$

Specializing to the case of tilt, $F_{n}=F_{\text {tilt }}=\left(4 J_{2}(x) / x\right)^{2}$, and for an angular offset $\theta$ making $d_{z}=\theta z$

$$
I_{d} \approx 0.2291 \frac{\theta^{2} z^{2}}{D_{z}^{1 / 3}}
$$

Equation D.19 becomes

$$
\begin{aligned}
\sigma_{\varphi c t i l t}^{2} & \approx 0.688 k^{2} \int_{0}^{L} d z C_{n}^{2} \frac{d_{z}^{2}}{D_{z}^{1 / 3}} \\
& \approx 0.688 k^{2} \theta^{2} \int_{0}^{L} d z C_{n}^{2} \frac{z^{2}}{D_{z}^{1 / 3}} \\
& \approx\left[\frac{\theta}{\theta_{t 0}}\right]^{2}
\end{aligned}
$$

\footnotetext{
${ }^{7}$ The effective value of $D$ may be less than $1.5 \mathrm{~m}$ because of aperture sharing to make separate beams to minimize the scintillation on the target; see Section 21.1. No information is available to quantify how much the phase variance would be increased because of this.

${ }^{8}$ In practice, $D_{z} /\left(2 n d_{z}\right)>1$ is not a bad approximation.
} 


$$
\theta_{t 0}^{-2}=0.688 \frac{k^{2}}{D^{1 / 3}} \int_{0}^{L} d z C_{n}^{2} \frac{z^{2}}{(1-z / L)^{1 / 3}} .
$$

The expression for the characteristic angle $\theta_{t 0}$ is similar to the expression for $\theta_{0}$, having a different multiplicative factor and slightly different $z$ weighting of $C_{n}^{2}$. Actual computations show that for the engagements considered, $\theta_{t 0} / \theta_{0}=2.7$ to 3.0 , depending only slightly on the slant range or engagement altitude.

Instead of the small anisoplanatism case, the opposite extreme $\kappa d_{z} \gg 1$ makes $J_{0} \rightarrow 0$ and the phase variance saturates, becoming independent of $\theta$ at twice the uncorrected tilt variance of Eq. D.10.

It is worth noting that the $d_{z}^{2}$ dependence of the small anisoplanatic behavior of the tilt mode is not specific to this mode. Higher modes simply become increasingly restrictive of the region of applicability, and the sum of all modes changes to $d^{5 / 3}$ dependence.

Equation D.40 is an approximation to Eq. D.20 for small angles. An argument similar to that used to extract the phase variance for piston removed anisoplanatism can be made to extend the result of Eq. D.40 to compute the large-angle-tilt anisoplanatism. In particular, the ratio $\rho$ of the correct tilt phase variance to the approximate result of Eq. D.40 is related to the ratio $\sigma$ of the approximate variance to the total uncompensated variance

$$
\begin{aligned}
\rho & =\frac{\sigma_{\varphi c t i l t}^{2}}{\left(\theta / \theta_{t 0}\right)^{2}} \\
\sigma & =\frac{\left(\theta / \theta_{t 0}\right)^{2}}{1.033\left(D / r_{0}\right)^{5 / 3}} .
\end{aligned}
$$

Again, this relationship depends only slightly on the details of the engagement parameters. Figure D.10 shows the correction factor averaged over characteristic engagements. The correct tilt phase variance is obtained by using the result of Eq. D.40 multiplied by $\rho$, which is determined from the corresponding value of $\sigma$.

\section{D.4.5 Time delay}

In Section 21.3.1, a method is discussed to reduce the tilt anisoplanatism by introducing a delay between the receipt of the nose image and the command to the outgoing tilt mirror. This section quantifies the tilt anisoplanatism caused by this delay $\tau=\delta_{d} / v$, where $\delta_{d}$ is the desired offset down the missile, and $v$ is the speed of the missile.

Using Eq. D.19, the phase variance can be computed for $d_{z}=w(z) \tau$, where $w$ is the effective wind speed as a function of $z$. Since the optical engagement attempts to use the same air column for the return beam as that taken by the nose image, $w(z)=w_{0}(1-z / L)$, where $w_{0}$ is the speed of the ABL through the air. The desired phase variance is obtained by using an appropriate filter function.

The case usually treated in the literature is $F_{Z}=1$, i.e., all modes, which results in

$$
\begin{aligned}
\sigma_{\varphi c \tau}^{2} & =2.914 k^{2} \int_{0}^{L} d z C_{n}^{2} d_{z}^{5 / 3} \\
& =2.914 k^{2}\left(w_{0} \tau\right)^{5 / 3} \int_{0}^{L} d z C_{n}^{2}(1-z / L)^{5 / 3} \\
& =6.89\left[\frac{w_{0} \tau}{r_{0}}\right]^{5 / 3},
\end{aligned}
$$

where use has been made of Eq. D.6. 


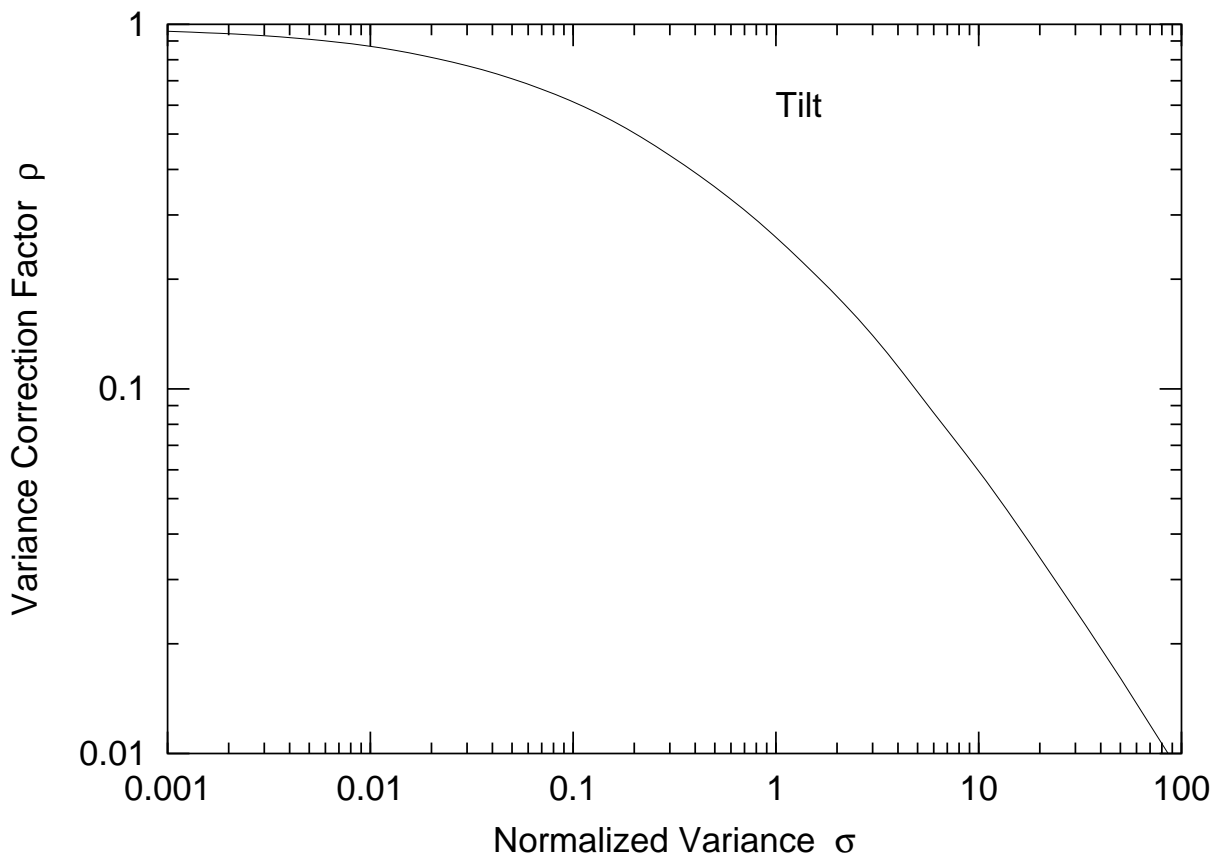

Figure D.10. Variance correction factor $\rho$ vs. normalized variance $\sigma$ for tilt mode; see Eqs. D.42 and D.43.

Of interest here is the variance for tilt only. Using Eq. D.38, we obtain

$$
\begin{aligned}
\sigma_{\varphi c \tau \text { tilt }}^{2} & =0.688 k^{2} \frac{\left(\tau w_{0}\right)^{2}}{D^{1 / 3}} \times \int_{0}^{L} d z C_{n}^{2} \frac{(1-z / L)^{2}}{(1-z / L)^{1 / 3}} \\
& =1.58\left(\frac{w_{0} \tau}{r_{0}}\right)^{2}\left(\frac{r_{0}}{D}\right)^{1 / 3}
\end{aligned}
$$

again using Eq. D.6. For most engagements, especially low-altitude ones, the ratio of the result of Eq. D.40 to Eq. D.48 for the same offset $\delta_{d}$ at the missile, i.e., $\theta=\delta_{d} / L$ and $\tau=\delta_{d} / v$, is large, meaning that the use of the delay can significantly reduce the tilt variance.

\section{D.5 Strehl Ratio Details}

\section{D.5.1 Beam shape and partial AO compensation}

The Strehl ratio $S_{R}$ is given by Eq. D.14 for small phase variances and by Eq. D.13 for large phase variances. Sometimes $S_{R}$ is simply related to the beam shape, and sometimes it is not. This section examines the relationship.

The core of the diffraction-limited intensity profile (Eq. 19.1) can be approximated by a Gaussian with a standard deviation $\sigma_{0}$,

$$
\begin{aligned}
I_{G}(\alpha, \beta) & =\frac{1}{2 \pi \sigma_{0}^{2}} e^{-\frac{\alpha^{2}+\beta^{2}}{2 \sigma_{0}^{2}}} \\
\sigma_{0} & =0.45 \lambda / D
\end{aligned}
$$


For present purposes, a Cartesian coordinate system is more convenient, in which $\alpha=x / L$ and $\beta=y / L$. We investigate how this profile changes when the system is not diffraction limited because of incomplete AO compensation. References [253, p. $155 \mathrm{ff}$, and $269 \mathrm{ff}]$ and [257, p. $115 \mathrm{ff}]$ discuss this issue in more depth.

Uncompensated (or partially compensated) tilt rapidly moves the centroid of the beam spot. If the only $\mathrm{AO}$ error were tilt, then the response would be (in this approximation) the convolution of Eq. D.49 with a Green's function derived from Eq. D.7 for single-axis tilt, which is Gaussian

$$
G(\alpha, \beta ; u, v)=\frac{1}{2 \pi \sigma_{t x} \sigma_{t y}} e^{-\frac{(\alpha-u)^{2}}{2 \sigma_{t x}^{2}}-\frac{(\beta-v)^{2}}{2 \sigma_{t y}^{2}}},
$$

where we allow different single-axis tilt for the $x$ and $y$ axes. Since the convolution of two Gaussians is another Gaussian (in each dimension), with a new variance being the sum of the component variances, computing the new beam profile is easy.

$$
\begin{aligned}
I_{t}(\alpha, \beta) & =\frac{1}{2 \pi \sigma_{x} \sigma_{y}} e^{-\frac{\alpha^{2}}{2 \sigma_{x}^{2}}-\frac{\beta^{2}}{2 \sigma_{y}^{2}}} \\
\sigma_{x}^{2} & =\sigma_{0}^{2}+\sigma_{t x}^{2} \\
\sigma_{y}^{2} & =\sigma_{0}^{2}+\sigma_{t y}^{2} .
\end{aligned}
$$

The Strehl ratio in this approximation then becomes

$$
S_{R} \approx \frac{\sigma_{0}^{2}}{\sigma_{x} \sigma_{y}} .
$$

Reference [253, p. 168] analyzes the case $\sigma_{t x}=\sigma_{t y}=\sigma_{t}$ using

$$
S_{R} \approx \frac{1}{1+\left(\frac{\pi}{\sqrt{2}} \frac{\sigma_{t}}{\lambda / D}\right)^{2}}
$$

(to which Eq. D.52 reduces) and says that this approximation is asymptotically correct for very small $\sigma_{t}$ or very large $\sigma_{t}$. For intermediate values, it overestimates the correct Strehl ratio by $\leq 10$ percent.

The behavior of the beam shape for uncorrected higher-order errors can be different. This is of interest if the tilt is well corrected or irrelevant, such as the image of a single pulse from the BILL (or "short exposure" in astronomy). Reference [253, p. 271] discusses the former case, in particular for anisoplanatism. The result is that for small angular anisoplanatism, the beam power on axis decreases without substantial change in the core beam width. The lost power is distributed into a large halo. As the degree of anisoplanatism increases from zero, the beam intensity at an angle $\theta \approx 0.85 \lambda / D$ remains approximately constant at a value of 10 percent of the central diffraction-limited value. For larger anisoplanatism, the core disappears, and only a roughly Gaussian halo remains.

Reference [257, p. 93] discusses the short exposure resolution, i.e., spot size, as a function of $r_{0} / D$. The beam size is shown to follow the diffraction-limited value for $r_{0} / D \geq 1$, then broadens to about twice the diffraction limit for $r_{0} / D=0.2$, eventually approaching the uncompensated size as $r_{0} / D$ decreases. Our cases of interest are $0.26 \mathrm{~m} \leq r_{0} \leq 0.8 \mathrm{~m}$, or $0.17 \leq r_{0} / D \leq 0.53$, so the core width will be between 1 and 2 times the diffraction-limited width. 


\section{D.5.2 Combining Strehl ratios}

There are many different sources of degradation of the Strehl ratio, and it is not clear how to best estimate the aggregate effect, apart from direct measurements. A commonly used practice, with no particular justification, is to simply take the product of the Strehl ratios,

$$
S_{R}=\prod_{i=1, N} S_{i}
$$

In reality, what should be done is to make a convolution of all of the point spread functions including phase correlations for each of the effects. In practice, the point spread functions are not well known, but their phase variances can be calculated, from which a Strehl ratio may be estimated.

Section D.5.1 discusses the beam profile and associated Strehl ratio for different cases of partial AO correction. Residual tilt errors are expected to be a substantial contribution to the loss of ABL AO performance. Such errors are expected to produce Gaussian smearing of the beam spot, although perhaps asymmetric in orthogonal directions. The Strehl ratio for one symmetric contribution, $S_{1}$, is given by Eq. D.52 for $\sigma_{1}=\sigma_{x}=\sigma_{y}$, resulting in

$$
S_{1}=\frac{\sigma_{0}^{2}}{\sigma_{0}^{2}+\sigma_{1}^{2}}
$$

Suppose another such contribution $S_{2}$ has the same form with $\sigma_{2}$. The combined Strehl ratio of the two is

$$
S_{R}^{-1}=1+\left(S_{1}^{-1}-1\right)+\left(S_{2}^{-1}-1\right),
$$

which generalizes for $N$ contributions to

$$
S_{R}^{-1}=1+\sum_{i=1, N}\left(S_{i}^{-1}-1\right) .
$$

Note that Eqs. D.56 and D.54 give similar results if all the Strehl ratios are near 1, but the results can be quite different otherwise.

Although Eq. D.55 was derived assuming symmetric distributions, numerically it gives a result close to Eq. D.52, even for rather asymmetric cases. It is expected that the ABL tilt errors are about twice as large along the missile axis as across it, for which the error made by the use of Eq. D.55 is very small.

On the other hand, uncorrected anisoplanatic errors have a different behavior. For small anisoplanatic errors, a narrow core is preserved whose peak simply decreases with increasing anisoplanatism, but whose width stays constant. Combining such cases might be more reasonably done with Eq. D.54. At large anisoplanatism, however, the core disappears, and the profile simply broadens, suggesting the use of Eq. D.56.

An example of the complexity of combining contributions to the Strehl ratio is discussed in [253, p. 180], which combines three different anisoplanatic errors. The result is that there is no unambiguous rule-of-thumb method for combining Strehl ratios because of correlations between the various contributions. Equation D.54 may substantially underestimate the true Strehl ratio, or even overestimate it, depending on the correlations between the sources of the phase variance. This reference does not examine Eq. D.56, but from the information provided, the same conclusion may be reached for this form, although the extreme deviations are smaller. 
Reference [264] used Eq. D.55 and compared calculations to measurements made with the Advanced Concepts Laboratory. This form represented the measurements well, but Eq. D.54 did not do as well. We have chosen to use Eq. D.56 throughout in the interest of simplicity and the apparent agreement with measurements.

\section{D.5.3 Strehl ratio beyond the Maréchal limit}

The simple form for the Strehl ratio given in the extended Maréchal limit by Eq. 19.3 or D.14 can substantially underestimate the Strehl ratio for many cases of interest for the ABL. Reference [253, p. 168 et seq.] discusses this, in which the phase contribution to the Strehl ratio with anisoplanatism is given by

$$
\begin{aligned}
S_{R} \approx & \exp \left(-\sigma_{\varphi c}^{2}\right) \times \\
& {\left[1+0.9736 E+0.5133 E^{2}+0.2009 E^{3}+0.06970 E^{4}+0.02744 E^{5}\right], }
\end{aligned}
$$

where $E$ depends on the anisoplanatism in the form of moments similar to, but different from, those used for $\sigma_{\varphi c}^{2}$. In its dependence on the anisoplanatic parameter, such as the angle offset, $E \propto\left(\sigma_{\varphi c}^{2}\right)^{6 / 5}$, and the proportionality constant may not be small compared to 1 . The reader is directed to this reference for further discussion of this topic for angular and time-delay anisoplanatism.

\section{D.6 Minor Issues}

\section{D.6.1 Phase only correction}

The ABL AO system does only phase correction, and at this time there are no implemented methods available to do amplitude corrections. Proposals exist to do amplitude correction $[265,266]$. Not doing an amplitude correction degrades the Strehl ratio by a factor of about $\exp \left(-\sigma_{R}^{2}\right)$, which is significant, but not intolerable, since $\sigma_{R}^{2} \leq 0.5 \mathrm{np}^{2}$ for ABL engagements. Scintillation in and of itself does not change the engagement. The hot and cold spots move rapidly within the engagement time, averaging out.

\section{D.6.2 Thermal blooming}

Thermal blooming was a major concern for the ground-based cw lasers considered for the Strategic Defense Initiative, but it is of very little concern for the ABL. The phenomenon [256] is a non-linear effect that arises from the absorption of a small part of the beam energy by the atmosphere. This causes the air to heat up, and in turn, the index of refraction is reduced in a non-uniform fashion. Because of the variation of the index of refraction induced across the beam profile, the propagation is disturbed from that occurring in a homogeneous medium. Absorption at $\lambda=1.315 \mu \mathrm{m}$ is very small, and the amount of absorption decreases rapidly with altitude with respect to the value at ground level. In addition, for the ABL the effect is greatly mitigated by the motion of the aircraft carrying the laser, because a fresh column of air is constantly being swept into the beam line. The exception occurs for very forward engagements, i.e., along the line of flight of the aircraft. In this case, the motion does little to bring fresh air into the beam path.

The method used to estimate the effect is adapted from Ref. [256]. Equation D.58 describes the heat flow

$$
\rho C_{p} \mathbf{w} \cdot \nabla T+k_{c} \nabla^{2} T-\alpha I=0
$$


where $\rho$ is the density of air, $C_{p}$ is the heat capacity at constant pressure, $\mathbf{w}$ is the effective wind velocity coming from the motion of the ABL and the slewing of the beam as it follows the target, $T$ is the temperature, $k_{c}$ is the thermal conductivity, $\alpha$ is the absorption coefficient per unit length, and $I(\mathbf{r})$ is the beam intensity. For the ABL, the heat transport by the effective wind greatly exceeds the transport by conduction. Take the wind to be in the $x$ direction. Then

$$
\rho C_{p} w \frac{\partial T}{\partial x}=\alpha I
$$

Changes in temperature cause changes in air density, which in turn cause changes in the index of refraction.

$$
\begin{aligned}
\frac{\Delta(n-1)}{n-1} & =\frac{\Delta \rho}{\rho}=-\frac{\Delta T}{T} \\
\frac{d n}{d T} & =-\frac{n-1}{T} .
\end{aligned}
$$

Gradients in $n$ cause deflections of the light according to the eikonal equation

$$
\frac{d^{2} \mathbf{r}}{d z^{2}}=\frac{1}{n} \nabla_{\perp} n
$$

Approximating $n$ by 1 and combining Eqs. D.59 and D.62 we have

$$
\begin{aligned}
\frac{d^{2} x}{d z^{2}} & =\frac{\partial n}{\partial x}=\frac{d n}{d T} \frac{\partial T}{\partial x} \\
& =\frac{d n}{d T} \frac{\alpha I}{\rho C_{p} w} .
\end{aligned}
$$

Integrating Eq. D.63 depends on the intensity profile, which in turn can change with $z$ because of focusing as well as the thermal blooming itself.

Reference [256] quotes a result for a Gaussian beam having a $1 / e$ radius $a$. It cites the ratio of the intensity at the target with blooming to that without blooming as

$$
\begin{aligned}
\frac{I(\text { Bloom })}{I(\text { no Bloom })} & =\frac{1}{1+B^{2}} \\
B & =B_{0} I_{B} .
\end{aligned}
$$

Taking this form and allowing $\alpha, T$, a, and $w$ to vary along the path $((1 / \rho)(d n / d T)$ is constant)

$$
\begin{aligned}
B_{0} & =-\frac{d n_{0}}{d T} \frac{\alpha_{0} P L^{2}}{4 \pi \rho_{0} C_{p} w_{0} a_{0}^{3}} \\
I_{B} & =\frac{2}{L^{2}} \int_{0}^{L} \frac{a_{0}}{a(z)} d z \times \int_{0}^{z} \frac{a_{0}^{2} w_{0} \alpha\left(z^{\prime}\right) T_{0}}{a^{2}\left(z^{\prime}\right) w\left(z^{\prime}\right) \alpha_{0} T\left(z^{\prime}\right)} d z^{\prime},
\end{aligned}
$$

where the subscript 0 refers to the value of the parameter at the ABL, i.e., $z=0, L$ is the distance from the ABL to the target, and $P$ is the total power. By construction, if none of the variables in Eq. D.67 depended on $z$, then $I_{B}=1$. In fact $I_{B} \ll 1$ for a high engagement because $\alpha$ falls rapidly for altitudes above $22 \mathrm{~km}$; in addition $w$ increases with $z$. The changes of $a$ and $T$ along the path are not large where $\alpha$ is large. 
Values for the extinction coefficient (which is larger than the absorption coefficient because of scattering) vs. altitude for $\lambda \approx 1.02 \mu \mathrm{m}$ are available from Ref. [267]. They fall from $6 \times 10^{-4} \mathrm{~km}^{-1}$ at $6 \mathrm{~km}$ to about $3 \times 10^{-4} \mathrm{~km}^{-1}$ at $h=10 \mathrm{~km}$, followed by a plateau of about 2 to $1 \times 10^{-4} \mathrm{~km}^{-1}$ from 12 to $22 \mathrm{~km}$. Above $22 \mathrm{~km}$, they fall rapidly.

Consider $B_{0}$ for a characteristic engagement. We ignore the scattering component contained in the extinction coefficient and use it for $\alpha$. Use these values of the parameters:

- $T_{0}=-50 \mathrm{C}=223 \mathrm{~K}$

- $C_{p}=1.0 \mathrm{~J} /(\mathrm{g} \mathrm{K})$

- $P=3 \mathrm{MW}$

- $w_{0}=0.2 \mathrm{~km} / \mathrm{s}$

- $L=500 \mathrm{~km}$

- $(1 / \rho)(d n / d T)=-1.0 \times 10^{-3} \mathrm{~cm}^{3} /(g K)$

- $\alpha_{0}=2 \times 10^{-4} \mathrm{~km}^{-1}$

- $a_{0}=0.75 \mathrm{~m}$

This gives $B_{0}=0.14$, which is a pessimistic limit, because the true beam profile is more nearly uniform than Gaussian, resulting in lower gradients.

A simple estimate of $I_{B}$ can be made for a high-altitude engagement. Suppose $\alpha=\alpha_{0}$ for $h_{a} \leq h \leq h_{c}$, and 0 elsewhere, where $h_{a}=12 \mathrm{~km}$ is the altitude of the ABL, and $h_{c}=22 \mathrm{~km}$ is a cutoff altitude for $\alpha$. Let $\tan \theta_{e}$ be the tangent of the engagement-altitude angle. Then the inner integral of Eq. D.67 will saturate at $L_{c} \approx\left(h_{c}-h_{a}\right) / \tan \theta_{e}$. Then

$$
\begin{aligned}
I_{B} & \approx \frac{2}{L^{2}} L L_{c} \\
& \approx \frac{2\left(h_{c}-h_{a}\right)}{L \tan \theta_{e}} .
\end{aligned}
$$

For the case considered, and a target altitude of $h_{t}=100 \mathrm{~km}, \tan \theta_{e} \approx 0.2$ and $I_{B} \approx 0.2$. The product $B=B_{0} I_{B} \approx 0.029$, which in Eq. D.64 results in a negligible effect for thermal blooming. A low-altitude engagement, $h_{t}=30 \mathrm{~km}$ and $L=300 \mathrm{~km}$ gives $B_{0}=0.05$, $I_{B} \approx 1$, and $B \approx 0.05$. This also results in negligible blooming. Stated differently, the beam power is at least an order of magnitude below that which could begin to raise concern for engagements of interest.

From this discussion, it is clear that the dead region in the very forward region for which thermal blooming could be a concern is very small, since the effective wind would scale as the component transverse to the beam.

\section{D.6.3 Branch points}

The phase information is typically reconstructed from wavefront sensor data using a leastsquares approach, which may not represent the true phase. A characteristic of the strong turbulence with which the ABL must contend is that the phase may not be smooth; it may have discontinuities or branch points [268, 255, 269, 270]. When the intensity vanishes, e.g. because of scintillation, the phase is undefined. Experimental phase conjugation of 
discontinuous wavefronts cannot be done by means of a continuous faceplate mirror. This effect has been taken into account in the Firepond, Brassboard and ACL measurements. It seems that the problem looks worse at first sight than it really is. The number of branch points increases not quite linearly with $\sigma_{R}^{2}$ [268], but they appear to come in positivenegative pairs which are close together, so that they tend to cancel [271]. More elaborate wavefront reconstruction techniques could result in a significant improvement in Strehl ratio, as large as a factor of 2 [272].

\section{D.6.4 Chromatic anisoplanatism}

Because the wavelengths of the BILL, TILL, and HEL are different, an error may be introduced in the AO applied to the HEL beam. This is a chromatic anisoplanatism that has not been analyzed here, but it is expected to be a small effect. See [253, p. 177].

\section{D.6.5 Ionization}

Ionization and stimulated Raman scattering [273, p. 108] were issues for pulsed lasers considered in the Strategic Defense Initiative in the mid-1980s. These non-linear effects arise from the peak power. The $\mathrm{cw}$ nature of the ABL laser simplifies things greatly. In particular, these non-linear effects are not an issue because of the relatively low peak power densities compared to pulsed lasers of similar or even lower average power.

\section{D.6.6 Aerosols}

A controversy arose at a SPIE meeting in April 2001 [274] and subsequent news coverage [275] over the possibility that aerosols in the atmosphere, even at the ABL altitude, could have a deleterious effect on ABL performance. This claim appears to be at variance with other observations of aerosol concentrations [276, 267], and there appear to be some internal inconsistencies as well. We do not regard aerosols to be a significant problem. 


\begin{tabular}{|ll|}
\hline & \multicolumn{1}{c|}{ Box D.1: Symbols Used in This Appendix } \\
\hline $\mathcal{D}$ & Kolmogarov structure function (Eq. D.1) \\
$C_{n}^{2}$ & local measure of turbulence (Section 19.2) \\
$\lambda$ & wavelength (Section 19.1) \\
$r_{o}$ & Fried parameter (Eq. D.6) \\
$\sigma_{t}$ & rms tilt angle of laser beam, due to turbulence (Eq. D.7) \\
$\sigma_{\varphi}^{2}$ & optical variance of phase, due to turbulence (Eq. D.16) \\
$S_{R}$ & Strehl ratio (Eq. 19.2) \\
$\sigma_{R}^{2}$ & Rytov variance (Eq. D.12) \\
$D_{z}$ & diameter of laser beam at distance $z$ from laser (Section D.4) \\
$t_{e}$ & dwell time of laser beam on target (Section 20.1) \\
$L$ & distance from laser to target (Section 19.1) \\
$v$ & speed of target (21.3.1) \\
$\delta$ & distance along missile (Fig. 21.2) \\
$\Delta \sigma_{\varphi}^{2}$ tot & phase variance (Eq. 21.1) \\
$F$ & fluence (energy per unit area) (Section 20.1) \\
$F_{z}$ & Zernike moment extraction filter (Eq. D.22) \\
$a$ & acceleration of target ( Section 21.3) \\
$\theta_{0}$ & isoplanatic angle (Sections 19.2, D.4.1 ) \\
$\rho$ & phase reduction factor (Eqs. D.30, D.42) \\
$\rho$ & density of air (Section D.6.2, only) \\
$\sigma$ & normalized variance (Eqs. D.31 and D.43) \\
$\tau$ & characteristic time for decrease of Strehl ratio (Eq. 21.7) \\
$h_{t}$ & altitude of target (21.4.4) \\
& \\
\hline & \\
\hline
\end{tabular}

\section{References for Appendix D}

[252] A. Kolmogorov, Turbulence: Classic Papers on Statistical Theory, Interscience, p. 151 (1961).

[253] Richard J. Sasiela, Electromagnetic Wave Propagation in Turbulence, (Springer Verlag), 1994.

[254] Scott Nowlin, Ila Hahn, and Bob Bishop, "Qualitative Comparison of Concurrent Vertical Optical Turbulence Profiles from an Aircraft and Balloons over White Sands Missile Range," SPIE 3706, 95 (1999).

[255] D. L. Fried, "Limiting Resolution Looking Down Through the Atmosphere," J. Opt. Soc. Am. 56 (10), 1380 (1966).

[256] Hugo Weichel, Laser Beam Propagation in the Atmosphere, Vol. TT 3. (SPIE Optical Engineering Press, Bellingham, WA), 1990.

[257] John W. Hardy, Adaptive Optics for Astronomical Telescopes. (Oxford University Press, New York), 1998. 
[258] Robert R. Beland, The Infrared and Electro-Optical Systems Handbook, Vol. 2, eds. Joseph S. Acetta and David L. Shumaker, International Society for Optical Engineering, 1993.

[259] F. Zernike, Physica 1, 689 (1934).

[260] Max Born and Emil Wolf, Principles of Optics. (Cambridge Oxford Press), 1959.

[261] Phillip D. Stroud, "Anisoplanatism in Adaptive Optics Compensation of a Focused Beam Using Distributed Beacons," J. Opt. Soc. Am. A 13, 868 (1996).

[262] Phillip D. Stroud, "LANL report LAUR-92-693." Technical report, Los Alamos National Laboratory, 1992.

[263] D. P. Greenwood, "Bandwidth Specifications for Adaptive Optics Systems," J. Opt. Soc. Am. 67(3), 390 (1977).

[264] Mitchell H. Fields et al., "Initial results from the Advanced-Concepts Laboratory for adaptive optics and tracking," SPIE 4034, 116 (2000).

[265] Michael C. Roggerman and David J. Lee, "A Two Deformable Mirror Concept for Correcting Scintillation Effects in Laser Projection," SPIE 3381, 66 (1998).

[266] Glenn A. Tyler, Private communication, 2001.

[267] G. S. Kent et al., J. Geophys. Res. 103, 28863 (1998). Also see http://www-sage2.larc.nasa.gov/data/aerosol/tropospheric/.

[268] E-O Le Bigot, W. J. Wild, and E. J. Kibblewhite, "Branch Point Reconstructors for Discontinuous Light Phase Functions," SPIE 3381, 76 (1998).

[269] Thomas R. Brown and Timothy L. Berkopek, "Adaptive Optics Performance Comparisons for Various Phase Reconstruction Algorithms," SPIE 3381, 136 (1998).

[270] Byron M. Welsh, Michael C. Roggermann, and Andrew Schepler, "Tracking and Higher Order Correction Tradeoffs for Beam Correction Through Strong Turbulence," SPIE 4125, 87 (2000).

[271] David L. Fried, "The Nature of the Branch Point Problem in Adaptive Optics," SPIE 3381, 38 (1998).

[272] Glenn A. Tyler, "Reconstruction and Assessment of the Least Squares and Slope Discrepancy Components of the Phase," J. Opt. Soc. Am. 17, 1828 (2000).

[273] N. Bloembergen et al., Rev. Mod. Phys. 59, S1 (1987).

[274] A. Zilberman, N. S. Kopeika, and Y. Sorani, "Laser Beam Widening as a Function of Elevation in the Atmosphere for Horizontal Propagation," SPIE 4376, 177 (2001).

[275] Dan Drollette, "Is the Airborne Laser Allergic to Dust?" Photonics Today, July 2001.

[276] D. A. Nahrstedt, "Cloud Modeling for Laser Weapon Propagation Analysis," SPIE 4034, 69 (2000). 



\section{Glossaries}

\section{Acronyms}

AAM air-to-air missile

ABL The Airborne Laser System

ABM anti-ballistic missile

ACS attitude control system

AIT Advanced Interceptor Technology

ALCM air-launched cruise missile

ALEXIS Los Alamos R\&D satellite

ALL Airborne Laser Laboratory

AN/APY-2 air surveillance radar used in the current E-3 AWACS aircraft

AN/SPY-1B multifunction radar of the U.S. Aegis system

AO adaptive optics

APD avalanche photodiode

APN augmented proportional navigation

APS American Physical Society

ARS active ranging system

ASAT a weapon designed to destroy satellites in space

ATKV Advanced Technology Kill Vehicle, Livermore Laboratory conceptual design

AWACS airborne warning and command system

BHP basic hydrogen peroxide

BILL beacon illuminating laser

BMD ballistic missile defense

BMDO Ballistic Missile Defense Office (now Missile Defense Agency)

BPI boost-phase intercept 
CCD charge-coupled device

COIL chemical oxygen-iodine laser

cw continuous wave

DACS divert and attitude control system

DF-5 a Chinese liquid-propellant ICBM

DSP Defense Support Program, missile launch warning satellite

ECM electronic countermeasure

EELV evolved expendable launch vehicle (USAF term)

EKV exoatmospheric kill vehicle

ERINT/PAC3 Extended Range Interceptor of Patriot Advanced Capability-3 system

ERIS Exoatmospheric Reentry Intercept System

FLAGE Flexible Light-weight Agile Guided Experiment

FOV field of view

GAO General Accounting Office

GEMS generalized energy-management steering

GEO geosynchronous Earth orbit

GIP Ground intercept point, i.e., the point on Earth's surface vertically below the intercept point

GPS Global Positioning System

HEDI High Endoatmospheric Defense Interceptor

HEL high energy laser

HIT spinning kill vehicle prototyped and tested in the 1970s

HKV homing kill vehicle (alternative: KV)

HOE Homing Overlay Experiment

I-1 the first of two constrained interceptor models used in this study; similar to the Standard Missile SM-2

I-2 the second of two constrained interceptor models used in this study, characteristics of which are listed in Table 16.4

I-3 the first of three interceptor models used in this study, characteristics of which are listed in Table 16.1 
I-4 the second of three interceptor models used in this study, characteristics of which are listed in Table 16.2

I-5 the third of three interceptor models used in this study, characteristics of which are listed in Table 16.3

ICBM intercontinental ballistic missile

ICBM model $\mathbf{L}$ the liquid-propellant missile model used in this study, characteristics of which are listed in Table 15.3; similar to the Chinese DF-5

ICBM model S1 the first of two solid-propellant missile models used in this study, characteristics of which are listed in Table 15.4

ICBM model S2 the second of two solid-propellant missile models used in this study, characteristics of which are listed in Table 15.5

IFUs in-flight updates

IGR interceptor ground range, ground distance from firing point to intercept point

IMU inertial measurement unit

IOC initial operational capability

IP intercept point of KV with threat missile

IR infrared

IRBM intermediate-range ballistic missile

IRST infrared search and track

IRTU inertial-reference transfer unit

ISP specific impulse of propellant

KEW kinetic-energy weapon

KV kill vehicle, the last stage of an interceptor with guidance and maneuvering capability for homing in on and hitting the missile

KKV kinetic kill vehicle, synonymous with KV

LEAP Lightweight Exoatmospheric Projectile

LEO low-Earth orbit

LIDAR light detection and ranging

LLNL Lawrence Livermore National Laboratory

LOF line of flight

LOS line of sight

LWIR long-wavelength infrared 
M-1 same as missile model TBM-1

M-2 same as missile model TBM-2

MCT mercury cadmium telluride

MHV Miniature Homing Vehicle

MIRV multiple independently targeted re-entry vehicle

MRBM medium-range ballistic missile

MWIR medium-wavelength infrared

NIE National Intelligence Estimate

NK North Korea

NMD National Missile Defense

NSBS Notional space-based sensor system

PDRR program definition and risk reduction

PG predictive guidance

PN proportional navigation

POCA point of closest approach

RCS radar cross section

$\mathbf{R F}$ radio frequency

rms root mean square

RULLI remote ultra low light imaging

RV re-entry vehicle

S-200 Russian long-range SAM system; also referred to as SA-5

SA-5 Russian long-range SAM system

SA-12 Russian long-range SAM system

SAM surface-to-air missile

S-band the radar band 2.7 to $3.3 \mathrm{GHz}$

SBIRS space-based infrared system

SBIs space-based kinetic energy interceptors

SLBM submarine launched ballistic missile

SLV space-launch vehicle 
SM standard missile

SPIE International Society of Optical Engineers

SPY-1B Aegis radar (complete designation: AN/SPY-1B)

SS-18 largest ICBM in former Soviet inventory credited with carrying 10 RVs

SWIR short wavelength infrared

TBM theater or tactical ballistic missile (short range)

TBM-1 the first of two medium-range ballistic missiles models used in this study, characteristics of which are listed in Table 15.6

TBM-2 the second of two medium-range ballistic missiles models used in this study, characteristics of which are listed in Table 15.7

TGR threat missile ground range, ground distance from launching point to intercept point

THAAD Theater High-Altitude Area Defense system

TILL target illuminator laser

TLP threat missile launch point

USAF United States Air Force

UV ultraviolet

VLS vertical launch system used on Aegis ships

X-band the radar band from 8.5 to $10.7 \mathrm{GHz}$

YAG yttrium-aluminum-garnet (laser material)

ZEM zero effort miss, distance of kill vehicle miss if guidance ceases 


\section{Technical Terms}

angle of attack the angle between a vehicle's centerline and its velocity vector

barrage noise jammer a jammer in which random noise is emitted over the expected tuning range of the victim radar

basket the volume (relative to the target position) within which the kill vehicle must lie (1) for its seeker to acquire the target or (2) for the endgame to be initiated successfully

boost phase the initial phase of a missile's flight during which its rocket motors are burning

closing velocity the rate of change of range between the kill vehicle and the target rocket

cloud optical depth cloud thickness in units of the mean free path for photon scattering

command guidance guidance of the interceptor by a command link from an external station using external information about the trajectories of the target and interceptor

cutoff time the time at which a rocket's thrust is terminated

decision time The time interval between the moment an acceptable firing solution is first obtained and the moment the first interceptor is fired; see also maximum decision time

divert capability the ability of an interceptor or kill vehicle to change its velocity to reach a different intercept point, usually in response to a change in the target's trajectory

divert velocity the absolute magnitude of the total change in speed and direction of the upper stage of an interceptor from its original aim point

dry mass the mass of the kill vehicle exclusive of its fuel

endgame the final few seconds of homing

engagement a sequence of events in which a target rocket is detected and tracked and an attempt is made to intercept it; see intercept

endoatmospheric inside the atmosphere, typically at an altitude below $100 \mathrm{~km}$

exoatmospheric outside the atmosphere, typically used to refer to altitudes greater than 80 to $100 \mathrm{~km}$.

firing time as used here, the time between launch of the target rocket and firing of the interceptor

flight path angle angle of a rocket's velocity vector from the local horizontal

flyout fan a map of interceptor flyout possibilities and limits showing possible trajectories from the interceptor's base or station and contours of constant time from the time of launch; also referred to as flyout characteristics

ground intercept point (GIP) point on the ground directly below the intercept point 
hardbody the mechanical structure of a rocket, as distinct from its exhaust plume

homing the phase of kill vehicle guidance in which on-board sensors continually observe the target and guide the kill vehicle to an intercept

interceptor ground range (IGR) distance from interceptor firing point to ground intercept point

impact range the distance around Earth's surface from a missile's launch site to the location where its payload strikes the ground; see also range

in-band luminosity as used here, power radiated in all directions within a prescribed bandpass (referring to IR)

in-band radiance as used here, power per unit area and solid angle within a prescribed bandpass (referring to IR)

intensity as used here, power per unit area and solid angle

intercept the portion of an engagement in which the interceptor attempts to damage or destroy the target

interceptor basing area the area within interceptor ground range of the intercept points for successfully defending a particular area against a particular missile

intercept point (a) the location in space where an interceptor will attempt to intercept the target; (b) the location in space where an interceptor succeeds in intercepting the target

intercontinental ballistic missiles (ICBMs) missiles with a range of more than 5,500 km

intermediate-range ballistic missiles (IRBMs) missiles with a range of 3,000 to $5,500 \mathrm{~km}$

intrinsic core the altitude-invariant emission from a rocket exhaust plume produced by the exhaust products as they expand into a low-pressure environment; also called the vacuum core

irradiance power per unit area

isoplanatic angle a measure of how rapidly the $\mathrm{AO}$ phase corrections change with angle from the center of view because the beam passes through a different portion of the atmosphere

jinking maneuver a periodic (square-wave or sinusoidal) acceleration of a target missile

Kalman filter a recursive algorithm that computes the conditional mean and covariance of the probability distribution of the state of a linear stochastic system with process noise and measurement noise that are both uncorrelated and Gaussian

kill vehicle the last stage of an interceptor with guidance and maneuvering capability for homing on and hitting the target rocket

latency the time delay of the target state estimate formed in the tracking system and the actual target state 
line of flight as used here, the instantaneous direction of flight of an Airborne Laser aircraft

line of sight (LOS) line of sight between (1) a kill vehicle and its target or (2) a sensor and its target

line-of-sight angle the angle between a kill vehicle's line of sight and a fixed reference direction

luminosity power radiated in all directions in all wavebands

lunge maneuver an abrupt step in acceleration of the target missile, defined here as being normal to the kill vehicle's line of sight

mass fraction ratio of propellant mass to total propulsion system mass

maximum decision time The time interval between the moment an acceptable firing solution is first obtained and the last moment an interceptor could be fired and still achieve intercept early enough to prevent munitions from falling on the defended area; see also decision time

medium-range ballistic missiles (MRBMs) missiles with a range of 1,000 to $3,000 \mathrm{~km}$

midcourse phase the phase of a long-range missile's flight when it has risen above the atmosphere and begins to deploy its munitions or penetration aids; the midcourse phase ends when the missile's munitions re-enter the atmosphere

Minuteman U.S. solid-propellant ICBM with three models, range 13,000 km

miss distance the closest approach of the kill vehicle to the aimpoint on the target rocket

MODTRAN a moderate-resolution atmospheric transmission code developed by the Air Force Research Laboratory

No Dong North Korean medium-range ballistic missile

penetration aid any countermeasure designed to help a warhead penetrate defenses

Pegasus an air-launched space vehicle developed by Orbital Sciences Corporation

plume see rocket exhaust plume

Poseidon a class of U.S. nuclear-submarine-launched ballistic missiles

predicted intercept point the location in space where an interceptor is expected to intercept the target

radiance as used here, power per unit area, solid angle, and wavelength

range (a) the distance around Earth's surface from a missile's launch site to the location where it's payload strikes the ground; (b) the distance between a sensor (or kill vehicle) and its target

rocket exhaust plume the cloud of hot, chemically active gas ejected from the nozzle of a rocket motor 
shortfall impact of a missile's payload at a range short of its target caused by premature termination of its thrust, possibly causing live nuclear, chemical, or biological munitions or debris to strike the United States or countries friendly to the United States

short-range ballistic missiles (SRBMs) missiles with a range less than 1,000 km

shroud an aerodynamic structure that protects the payload of a missile while it is ascending through the atmosphere

sine alpha steering a homing system in which a single axial thruster is used on the kill vehicle and the kill vehicle's acceleration normal to the line of sight is controlled by the angle of the thrust axis relative to the closing velocity vector

slant range distance from $\mathrm{ABL}$ to target (as distinct from ground range)

specific impulse the ratio of thrust to propellant mass flow

spectral intensity power per unit area, solid angle, and wavelength or frequency

staging ratio ratio of propulsion stage mass to the mass of successive propulsion stages of a multi-stage rocket

standoff distance distance between the launch site of a target missile and location where a defensive interceptor or tracking sensor is based or stationed

Strehl ratio ratio of the peak irradiance delivered by a particular laser to the peak irradiance delivered by a diffraction-limited system with the same power

successful engagement an engagement in which the target missile is intercepted and disabled or destroyed before it can inflict damage on the defended area

successful intercept an intercept that disables or destroys the target missile before it can inflict damage on the defended area

switchback maneuver an abrupt reversal of a missile's angle of attack

terminal phase the final phase of a missile's flight; it begins when its munitions re-enter the atmosphere and ends when they detonate or strike the ground

terrestrial interceptor land-, sea-, or air-based interceptor

thermal blooming transverse growth of a laser beam's size resulting from heating by the beam of the air through which the beam passes

threat missile ground range (TGR) distance around Earth's surface from a missile's launch site to the ground intercept point

time-to-go the time remaining before the closest approach of the interceptor to the target rocket

tn abbreviation for tonne (metric ton)

tonne metric ton $=1,000 \mathrm{~kg}$ 
total velocity change $\Delta \mathbf{V}$ the integral of the absolute magnitude of the kill vehicle's acceleration from the time it is deployed to the end of an engagement

Trident (a) a class of U.S. ballistic missile submarine; (b) the class of U.S. nuclearsubmarine-launched ballistic missiles carried by Trident submarines

trough region the altitude interval during the rise of a rocket launched from near the surface of Earth during which the IR emission from its exhaust plume is relatively low

vacuum core the altitude-invariant emission from a rocket exhaust plume produced by the exhaust products as they expand into a low-pressure environment; also called the intrinsic core

wallplug efficiency the ratio of useful power delivered by a laser to the total input power wet mass the mass of the kill vehicle when fully loaded with fuel. 